\title{
Antipsychotic combinations for schizophrenia (Review)
}

Ortiz-Orendain J, Castiello-de Obeso S, Colunga-Lozano LE, Hu Y, Maayan N, Adams CE

Ortiz-Orendain J, Castiello-de Obeso S, Colunga-Lozano LE, Hu Y, Maayan N, Adams CE.

Antipsychotic combinations for schizophrenia.

Cochrane Database of Systematic Reviews 2017, Issue 6. Art. No.: CD009005.

DOI: 10.1002/14651858.CD009005.pub2.

www.cochranelibrary.com 
TABLE OF CONTENTS

HEADER

ABSTRACT

PLAIN LANGUAGE SUMMARY

SUMMARY OF FINDINGS

BACKGROUND

OBJECTIVES

METHODS

Figure 1.

Figure 2.

RESULTS

Figure 3.

DISCUSSION

AUTHORS' CONCLUSIONS

ACKNOWLEDGEMENTS

REFERENCES

CHARACTERISTICS OF STUDIES

DATA AND ANALYSES

Analysis 1.1. Comparison 1 ANTIPSYCHOTIC COMBINATIONS vs ANTIPSYCHOTIC MONOTHERAPY, Outcome 1 Clinical response:

1. No clinically important response - not improved.

Analysis 1.2. Comparison 1 ANTIPSYCHOTIC COMBINATIONS vs ANTIPSYCHOTIC MONOTHERAPY, Outcome 2 Clinical response: 2. Relapse.

Analysis 1.3. Comparison 1 ANTIPSYCHOTIC COMBINATIONS vS ANTIPSYCHOTIC MONOTHERAPY, Outcome 3 Leaving the study early.

Analysis 1.4. Comparison 1 ANTIPSYCHOTIC COMBINATIONS vs ANTIPSYCHOTIC MONOTHERAPY, Outcome 4 Service utilisation: Hospital admission.

Analysis 1.5. Comparison 1 ANTIPSYCHOTIC COMBINATIONS vs ANTIPSYCHOTIC MONOTHERAPY, Outcome 5 Clinical response: 3. Global state - i. average severity score (CGI-S scale, high = bad).

Analysis 1.6. Comparison 1 ANTIPSYCHOTIC COMBINATIONS vS ANTIPSYCHOTIC MONOTHERAPY, Outcome 6 Clinical response: 3. Global state - ii. change in severity score (CGI-S scale, high = bad).

Analysis 1.7. Comparison 1 ANTIPSYCHOTIC COMBINATIONS vs ANTIPSYCHOTIC MONOTHERAPY, Outcome 7 Clinical response: 4. Global state - average improvement score (CGI-I scale, high = bad).

Analysis 1.8. Comparison 1 ANTIPSYCHOTIC COMBINATIONS vs ANTIPSYCHOTIC MONOTHERAPY, Outcome 8 Clinical response: 5. Global state $-i$. average functioning score (GAF scale, high = good).

Analysis 1.9. Comparison 1 ANTIPSYCHOTIC COMBINATIONS vS ANTIPSYCHOTIC MONOTHERAPY, Outcome 9 Clinical response: 5. Global state - ii. change in functioning score (GAF scale, high = good).

Analysis 1.10. Comparison 1 ANTIPSYCHOTIC COMBINATIONS vS ANTIPSYCHOTIC MONOTHERAPY, Outcome 10 Mental state: 1. Overall - a.i average total score (PANSS scale, high $=$ bad).

Analysis 1.11. Comparison 1 ANTIPSYCHOTIC COMBINATIONS vs ANTIPSYCHOTIC MONOTHERAPY, Outcome 11 Mental state: 1. Overall - a.ii change in total score (PANSS scale, high $=$ bad).

Analysis 1.12. Comparison 1 ANTIPSYCHOTIC COMBINATIONS vS ANTIPSYCHOTIC MONOTHERAPY, Outcome 12 Mental state: 1. Overall - b.i. average total score (BPRS scale, high $=$ bad).

Analysis 1.13. Comparison 1 ANTIPSYCHOTIC COMBINATIONS vs ANTIPSYCHOTIC MONOTHERAPY, Outcome 13 Mental state: 1. Overall - b.ii change total score (BPRS scale, high = bad).

Analysis 1.14. Comparison 1 ANTIPSYCHOTIC COMBINATIONS vs ANTIPSYCHOTIC MONOTHERAPY, Outcome 14 Mental state: 2. Specific - a. positive symptoms - no clinical improvement.

Analysis 1.15. Comparison 1 ANTIPSYCHOTIC COMBINATIONS vS ANTIPSYCHOTIC MONOTHERAPY, Outcome 15 Mental state: 2. Specific - b. positive symptoms $-i$. average score (PANSS scale, high = bad).

Analysis 1.16. Comparison 1 ANTIPSYCHOTIC COMBINATIONS vS ANTIPSYCHOTIC MONOTHERAPY, Outcome 16 Mental state: 2. Specific - b. positive symptoms - ii. change score (PANSS scale, high = bad).

Analysis 1.17. Comparison 1 ANTIPSYCHOTIC COMBINATIONS vs ANTIPSYCHOTIC MONOTHERAPY, Outcome 17 Mental state: 2. Specific - b. positive symptoms - iii. average score (BPRS scale, high = bad).

Analysis 1.18. Comparison 1 ANTIPSYCHOTIC COMBINATIONS vs ANTIPSYCHOTIC MONOTHERAPY, Outcome 18 Mental state: 2. Specific - b. positive symptoms - iv. change data (BPRS scale, high = bad). 
Analysis 1.19. Comparison 1 ANTIPSYCHOTIC COMBINATIONS vs ANTIPSYCHOTIC MONOTHERAPY, Outcome 19 Mental state: 2. Specific - b. positive symptoms - v. average score (SAPS scale, high = bad).

Analysis 1.20. Comparison 1 ANTIPSYCHOTIC COMBINATIONS vs ANTIPSYCHOTIC MONOTHERAPY, Outcome 20 Mental state: 2. Specific - b. positive symptoms - vi. change score (SAPS scale, high = bad).

Analysis 1.21. Comparison 1 ANTIPSYCHOTIC COMBINATIONS vs ANTIPSYCHOTIC MONOTHERAPY, Outcome 21 Mental state: 3. Specific - a. negative symptoms - no clinical improvement.

Analysis 1.22. Comparison 1 ANTIPSYCHOTIC COMBINATIONS vs ANTIPSYCHOTIC MONOTHERAPY, Outcome 22 Mental state: 3. Specific - b. negative symptoms - i. average score (PANSS scale, high = bad).

Analysis 1.23. Comparison 1 ANTIPSYCHOTIC COMBINATIONS vs ANTIPSYCHOTIC MONOTHERAPY, Outcome 23 Mental state: 3. Specific - b. negative symptoms - ii. change score (PANSS scale, high = bad).

Analysis 1.24. Comparison 1 ANTIPSYCHOTIC COMBINATIONS vs ANTIPSYCHOTIC MONOTHERAPY, Outcome 24 Mental state: 3. Specific - b. negative symptoms - iii. average score (BPRS scale, high = bad).

Analysis 1.25. Comparison 1 ANTIPSYCHOTIC COMBINATIONS vs ANTIPSYCHOTIC MONOTHERAPY, Outcome 25 Mental state: 3. Specific - b. negative symptoms - iv. change score (BPRS scale, high = bad).

Analysis 1.26. Comparison 1 ANTIPSYCHOTIC COMBINATIONS vS ANTIPSYCHOTIC MONOTHERAPY, Outcome 26 Mental state: 3. Specific - b. negative symptoms - v. average score (SANS scale, high = bad).

Analysis 1.27. Comparison 1 ANTIPSYCHOTIC COMBINATIONS vs ANTIPSYCHOTIC MONOTHERAPY, Outcome 27 Mental state: 3. Specific - b. negative symptoms - vi. average score (SANS scale, high = bad).

Analysis 1.28. Comparison 1 ANTIPSYCHOTIC COMBINATIONS vS ANTIPSYCHOTIC MONOTHERAPY, Outcome 28 Mental state: 4. Specific - aggression/agitation - average score (BPRS scale, high = bad).

Analysis 1.29. Comparison 1 ANTIPSYCHOTIC COMBINATIONS vs ANTIPSYCHOTIC MONOTHERAPY, Outcome 29 Adverse events: 1. General - a. serious event or requiring discontinuation.

Analysis 1.30. Comparison 1 ANTIPSYCHOTIC COMBINATIONS vS ANTIPSYCHOTIC MONOTHERAPY, Outcome 30 Adverse events: 1. General - b. death (suicide or non-suicide deaths).

Analysis 1.31. Comparison 1 ANTIPSYCHOTIC COMBINATIONS vs ANTIPSYCHOTIC MONOTHERAPY, Outcome 31 Adverse events: 2. Movement disorders - a. any.

Analysis 1.32. Comparison 1 ANTIPSYCHOTIC COMBINATIONS vs ANTIPSYCHOTIC MONOTHERAPY, Outcome 32 Adverse events: 2. Movement disorders - b.i. average scores (SAS, high = bad).

Analysis 1.33. Comparison 1 ANTIPSYCHOTIC COMBINATIONS vS ANTIPSYCHOTIC MONOTHERAPY, Outcome 33 Adverse events: 2. Movement disorders - b.ii. change scores (SAS, high $=$ bad).

Analysis 1.34. Comparison 1 ANTIPSYCHOTIC COMBINATIONS vs ANTIPSYCHOTIC MONOTHERAPY, Outcome 34 Adverse events: 2. Movement disorders - b.iii. average scores (TESS, high = bad).

Analysis 1.35. Comparison 1 ANTIPSYCHOTIC COMBINATIONS vS ANTIPSYCHOTIC MONOTHERAPY, Outcome 35 Adverse events: 2. Movement disorders - b.iv. average scores (DIEPSS, high = bad).

Analysis 1.36. Comparison 1 ANTIPSYCHOTIC COMBINATIONS vs ANTIPSYCHOTIC MONOTHERAPY, Outcome 36 Adverse events: 2. Movement disorders - b.v. change scores (BAS, high = bad).

Analysis 1.37. Comparison 1 ANTIPSYCHOTIC COMBINATIONS vs ANTIPSYCHOTIC MONOTHERAPY, Outcome 37 Adverse events: 2. Movement disorders - b.vi. change scores (AIMS, high = bad).

Analysis 1.38. Comparison 1 ANTIPSYCHOTIC COMBINATIONS vS ANTIPSYCHOTIC MONOTHERAPY, Outcome 38 Adverse events: 3. Endocrine - prolactin level (high = bad).

Analysis 1.39. Comparison 1 ANTIPSYCHOTIC COMBINATIONS vs ANTIPSYCHOTIC MONOTHERAPY, Outcome 39 Adverse events: 4. Metabolic - a. weight gain (binary).

Analysis 1.40. Comparison 1 ANTIPSYCHOTIC COMBINATIONS vs ANTIPSYCHOTIC MONOTHERAPY, Outcome 40 Adverse events: 4. Metabolic - b. average weight gain $(\mathrm{kg})$.

Analysis 1.41. Comparison 1 ANTIPSYCHOTIC COMBINATIONS vs ANTIPSYCHOTIC MONOTHERAPY, Outcome 41 Adverse events: 5. Blood - a. decreased white cell counts (binary).

Analysis 1.42. Comparison 1 ANTIPSYCHOTIC COMBINATIONS vS ANTIPSYCHOTIC MONOTHERAPY, Outcome 42 Adverse events: 5. Blood - b. average white cell counts (10-3/mm3).

Analysis 1.43. Comparison 1 ANTIPSYCHOTIC COMBINATIONS vS ANTIPSYCHOTIC MONOTHERAPY, Outcome 43 Adverse events: 6. Central nervous system (CNS) - a. drowsiness.

Analysis 1.44. Comparison 1 ANTIPSYCHOTIC COMBINATIONS vs ANTIPSYCHOTIC MONOTHERAPY, Outcome 44 Adverse events: 6. Central nervous system (CNS) - b. tremor.

Analysis 1.45. Comparison 1 ANTIPSYCHOTIC COMBINATIONS vS ANTIPSYCHOTIC MONOTHERAPY, Outcome 45 Quality of life: 1a. Average score (QLS high=good).

Analysis 1.46. Comparison 1 ANTIPSYCHOTIC COMBINATIONS vs ANTIPSYCHOTIC MONOTHERAPY, Outcome 46 Quality of life: 1b. Average score (SWN, high=good). 
Analysis 1.47. Comparison 1 ANTIPSYCHOTIC COMBINATIONS vs ANTIPSYCHOTIC MONOTHERAPY, Outcome 47 Quality of Life: 1c. Average score - Mental component summary (SF-36, high = good).

Analysis 1.48. Comparison 1 ANTIPSYCHOTIC COMBINATIONS vs ANTIPSYCHOTIC MONOTHERAPY, Outcome 48 Quality of Life: 1d. Average score - Physical component summary (SF-36, high = good).

Analysis 1.49. Comparison 1 ANTIPSYCHOTIC COMBINATIONS vs ANTIPSYCHOTIC MONOTHERAPY, Outcome 49 SUBGROUP ANALYSIS Clinical Response: Not clinically improved - Patients enrolled in the studies.

Analysis 1.50. Comparison 1 ANTIPSYCHOTIC COMBINATIONS vs ANTIPSYCHOTIC MONOTHERAPY, Outcome 50 SUBGROUP ANALYSIS Clinical Response: Not clinically improved - Treatment duration.

Analysis 1.51. Comparison 1 ANTIPSYCHOTIC COMBINATIONS vS ANTIPSYCHOTIC MONOTHERAPY, Outcome 51 SUBGROUP ANALYSIS Clinical Response: Not clinically improved - Use of clozapine in both groups.

Analysis 1.52. Comparison 1 ANTIPSYCHOTIC COMBINATIONS vs ANTIPSYCHOTIC MONOTHERAPY, Outcome 52 SUBGROUP ANALYSIS Clinical Response: Not clinically improved - Drug added to clozapine.

Analysis 1.53. Comparison 1 ANTIPSYCHOTIC COMBINATIONS vs ANTIPSYCHOTIC MONOTHERAPY, Outcome 53 SUBGROUP ANALYSIS Leaving the study early - Patients enrolled in the studies.

Analysis 1.54. Comparison 1 ANTIPSYCHOTIC COMBINATIONS vS ANTIPSYCHOTIC MONOTHERAPY, Outcome 54 SUBGROUP ANALYSIS Leaving the study early - Treatment duration.

Analysis 1.55. Comparison 1 ANTIPSYCHOTIC COMBINATIONS vs ANTIPSYCHOTIC MONOTHERAPY, Outcome 55 SUBGROUP ANALYSIS Leaving the study early - Use of clozapine in both groups.

Analysis 1.56. Comparison 1 ANTIPSYCHOTIC COMBINATIONS vS ANTIPSYCHOTIC MONOTHERAPY, Outcome 56 SUBGROUP ANALYSIS Leaving the study early - Drug added to clozapine.

Analysis 1.57. Comparison 1 ANTIPSYCHOTIC COMBINATIONS vs ANTIPSYCHOTIC MONOTHERAPY, Outcome 57 SENSITIVITY ANALYSIS Clinical Response: Not clinically improved - Randomisation.

Analysis 1.58. Comparison 1 ANTIPSYCHOTIC COMBINATIONS vS ANTIPSYCHOTIC MONOTHERAPY, Outcome 58 SENSITIVITY ANALYSIS Clinical Response: Not clinically improved - Double blind.

Analysis 1.59. Comparison 1 ANTIPSYCHOTIC COMBINATIONS vs ANTIPSYCHOTIC MONOTHERAPY, Outcome 59 SENSITIVITY ANALYSIS Clinical response: 1 . No clinically important response - not improved - Fixed effect.

Analysis 1.60. Comparison 1 ANTIPSYCHOTIC COMBINATIONS vs ANTIPSYCHOTIC MONOTHERAPY, Outcome 60 SENSITIVITY ANALYSIS Leaving the study early - Randomisation.

Analysis 1.61. Comparison 1 ANTIPSYCHOTIC COMBINATIONS vs ANTIPSYCHOTIC MONOTHERAPY, Outcome 61 SENSITIVITY ANALYSIS Leaving the study early - Double blind.

Analysis 1.62. Comparison 1 ANTIPSYCHOTIC COMBINATIONS vs ANTIPSYCHOTIC MONOTHERAPY, Outcome 62 SENSITIVITY ANALYSIS Leving the study early - Fixed effect.

ADDITIONAL TABLES

APPENDICES

WHAT'S NEW

HISTORY

CONTRIBUTIONS OF AUTHORS 
[Intervention Review]

\section{Antipsychotic combinations for schizophrenia}

Javier Ortiz-Orendain ${ }^{1}$, Santiago Castiello-de Obeso², Luis Enrique Colunga-Lozano ${ }^{3}$, Yue Hu ${ }^{4}$, Nicola Maayan ${ }^{5}$, Clive E Adams ${ }^{6}$

${ }^{1}$ Centro Universitario de Ciencias de la Salud, University of Guadalajara, Guadalajara, Mexico. ${ }^{2}$ Center for Behavior Studies and Investigations, University of Guadalajara, Guadalajara, Mexico. ${ }^{3}$ Departments of Health Research Methods, Evidence, and Impact and of Medicine, McMaster University, Hamilton, Canada. ${ }^{4}$ Graduate School, Tianjin University of Traditional Chinese Medicine, Tianjin, China. ${ }^{5}$ Cochrane Response, Cochrane, London, UK. ${ }^{6}$ Cochrane Schizophrenia Group, The University of Nottingham, Nottingham, UK

Contact address: Javier Ortiz-Orendain, Centro Universitario de Ciencias de la Salud, University of Guadalajara, Sierra Mojada 950, Col. Independencia, Guadalajara, Jalisco,44340, Mexico.jaortzor@gmail.com, javierortizzz@hotmail.com.

Editorial group: Cochrane Schizophrenia Group.

Publication status and date: Edited (no change to conclusions), published in Issue 10, 2017.

Citation: Ortiz-Orendain J, Castiello-de Obeso S, Colunga-Lozano LE, Hu Y, Maayan N, Adams CE. Antipsychotic combinations for schizophrenia. Cochrane Database of Systematic Reviews 2017, Issue 6. Art. No.: CD009005. DOI: 10.1002/14651858.CD009005.pub2.

Copyright @ 2017 The Cochrane Collaboration. Published by John Wiley \& Sons, Ltd.

\section{A B S T R A C T}

\section{Background}

Many people with schizophrenia do not achieve a satisfactory treatment response with their initial antipsychotic drug treatment. Sometimes a second antipsychotic, in combination with the first, is used in these situations.

\section{Objectives}

To examine whether:

1. treatment with antipsychotic combinations is effective for schizophrenia; and

2. treatment with antipsychotic combinations is safe for the same illness.

\section{Search methods}

We searched the Cochrane Schizophrenia Group's register which is based on regular searches of CINAHL, BIOSIS, AMED, Embase, PubMed, MEDLINE, PsycINFO, and registries of clinical trials. There are no language, time, document type, or publication status limitations for inclusion of records in the register. We ran searches in September 2010, August 2012 and January 2016. We checked for additional trials in the reference lists of included trials.

\section{Selection criteria}

We included all randomised and quasi-randomised controlled trials comparing antipsychotic combinations with antipsychotic monotherapy for the treatment of schizophrenia and/or schizophrenia-like psychoses.

\section{Data collection and analysis}

We independently extracted data from the included studies. We analysed dichotomous data using risk ratios (RR) and the $95 \%$ confidence intervals (CI). We analysed continuous data using mean difference (MD) with a 95\% Cls. For the meta-analysis we used a random-effects model. We used GRADE to complete a 'Summary of findings' table and assessed risk of bias for included studies.

\section{Main results}

Sixty-two studies are included in the review, 31 of these compared clozapine monotherapy with clozapine combination. We considered the risk of bias in the included studies to be moderate to high. The majority of trials had unclear allocation concealment, method of randomisation and blinding, and were not free of selective reporting. 
There is some limited evidence that combination therapy may be superior to monotherapy in reducing the risk of no clinical response ( $R R 0.73 \mathrm{Cl} 0.64$ to 0.83 ; participants $=2398$; studies $=29$; very low-quality evidence), subgroup analyses show that the positive result was due to the studies with clozapine in both the monotherapy and combination groups $(\mathrm{RR} 0.66 \mathrm{Cl} 0.53$ to 0.83 ; participants $=1127$; studies $=$ 17 ) and typical in both groups (RR $0.64 \mathrm{Cl} 0.49$ to 0.84 ; participants $=597$; studies $=5$ ). The subgroup with atypical antipsychotics in both groups did not showed a difference between the two interventions (RR $0.95 \mathrm{Cl} 0.83$ to 1.09; participants $=674 ;$ studies $=7$ ). Three studies provided data regarding relapse, the pooled data showed high heterogeneity $\left(I^{2}=82 \%\right)$ and therefore the results were not pooled. Two studies showed no difference between the interventions and one study showed that antipsychotics combination might decrease the risk of relapse. A combination of antipsychotics was not superior or inferior to antipsychotic monotherapy in reducing the number of participants discontinuing treatment early (RR $0.90 \mathrm{Cl} 0.76$ to 1.07 ; participants $=3137$; studies $=43$, low-quality evidence). No difference was found between treatment groups in the number of participants hospitalised ( $R R 0.96 \mathrm{Cl} 0.36$ to 2.55; participants $=202$; studies $=3$, very low-quality evidence). We did not find evidence of a difference between treatment groups in serious adverse events or those requiring discontinuation (RR $1.05 \mathrm{Cl} 0.65$ to 1.69; participants = 2398; studies $=30$, very low-quality evidence). There is a lack of evidence on clinically important change in quality of life, with only four studies reporting average endpoint or change data for this outcome on three different scales, none of which showed a difference between treatment groups.

\section{Authors' conclusions}

Currently, most evidence regarding the use of antipsychotic combinations comes from short-term trials, limiting the assessment of longterm efficacy and safety. We found very low-quality evidence that a combination of antipsychotics may improve the clinical response. We also found very low-quality evidence that a combination of antipsychotics may make no difference at preventing participants from leaving the study early, preventing relapse and/or causing more serious adverse events than monotherapy.

\section{PLAIN LANGUAGE SUMMARY}

\section{Combining antipsychotic medication for the treatment of schizophrenia}

\section{Background}

Antipsychotic medication was introduced in the 1950s to reduce or alleviate the symptoms of schizophrenia, such as the psychotic states of hearing voices, visual hallucinations and strange thoughts such as paranoia (feeling singled-out or put upon by others). Medication for mental illness also helped to establish care in the community, because people could take medication in their homes or by regularly visiting the hospital. But this also led to new issues such as the effectiveness of different medication (taken alone or in combination) and compliance (the willingness of service users to take their medication without being supervised).

The range of antipsychotic medication available is wide and their effectiveness can also vary from individual to individual. In addition, not all patients fully respond to a single antipsychotic, and in these situations, a combination of antipsychotics are often prescribed. The evidence for the benefits of taking one or more antipsychotics in combination is often unclear. There are also differing profiles of typical (first generation) and atypical (second generation) antipsychotics adding to a confusing array of terminology and dilemma of what is the best medication for service users.

\section{Searches}

This review investigates the effects of different antipsychotic combinations compared with single antipsychotics for people with schizophrenia. Searches for randomised controlled trials have now been run by the Information Specialist of the Cochrane Schizophenia Group in 2010, 2012 and 2016. Sixty-two trials, reporting useable data, are included in the review.

\section{Main results}

The review of available evidence found that combinations of antipsychotics may be more effective in treating symptoms of schizophrenia compared with taking one antipsychotic. In particular, combination treatments that included clozapine and typical antipsychotic in both groups were found to be effective. Few studies reported on this central issue of relapse rates (service users becoming unwell again), but this was because most of the studies were of short length (whereas schizophrenia is a long-term health problem that requires studies of an equally long duration). No real differences were found between combinations of antipsychotics and single antipsychotics for preventing relapse and roughly equal numbers of people discontinued their treatment. There was also no difference between combination therapy and monotherapy regarding hospital admission and/or occurrence of serious adverse events. Numbers leaving the studies early were similar. Clinically meaningful data for quality of life were not reported.

\section{Conclusions}

These results show that there may be some clinical benefit for combination therapy in that more people receiving a combination of antipsychotic showed an improvement in symptoms. For other important outcomes such as relapse, hospitalisation, adverse events, discontinuing treatment or leaving the study early, no clear differences between the two treatment options were observed. However, these results are based on very low or low-quality evidence and more research providing high-quality evidence is needed before firm conclusions can be made. 
This plain language summary has been adapted from an original summary by Benjamin Gray, Service User and Service User Expert, Rethink Mental Illness. Email: ben.gray@rethink.org 
SUMMARY OF FINDINGS

Summary of findings for the main comparison. Combinations of antipsychotic drugs compared to single antipsychotic drugs for schizophrenia

Combinations of antipsychotic drugs compared to single antipsychotic drugs for schizophrenia

Patient or population: schizophrenia or related disorders

Setting: outpatients and inpatients

Intervention: combinations of antipsychotic drugs

Comparison: single antipsychotic drugs

\begin{tabular}{|c|c|c|c|c|c|c|}
\hline \multirow[t]{2}{*}{ Outcomes } & \multicolumn{2}{|c|}{$\begin{array}{l}\text { Anticipated absolute effects }{ }^{\star}(95 \% \\
\mathrm{CI})\end{array}$} & \multirow[t]{2}{*}{$\begin{array}{l}\text { Relative effect } \\
(95 \% \mathrm{Cl})\end{array}$} & \multirow{2}{*}{$\begin{array}{l}\text { № of partici- } \\
\text { pants } \\
\text { (studies) }\end{array}$} & \multirow{2}{*}{$\begin{array}{l}\text { Quality of the } \\
\text { evidence } \\
\text { (GRADE) }\end{array}$} & \multirow[t]{2}{*}{ Comments } \\
\hline & $\begin{array}{l}\text { Risk with sin- } \\
\text { gle antipsy- } \\
\text { chotic drugs }\end{array}$ & $\begin{array}{l}\text { Risk with com- } \\
\text { binations of an- } \\
\text { tipsychotic drugs }\end{array}$ & & & & \\
\hline $\begin{array}{l}\text { Clinical response: No clinically important } \\
\text { response }\end{array}$ & \multicolumn{2}{|c|}{ Study population } & $\begin{array}{l}\text { RR } 0.73 \\
(0.64 \text { to } 0.83)\end{array}$ & $\begin{array}{l}2398 \\
(29 \mathrm{RCTs})\end{array}$ & $\begin{array}{l}\oplus \ominus \ominus \ominus \\
\text { VERY LOW } 123 \\
4\end{array}$ & \\
\hline \multirow{2}{*}{$\begin{array}{l}\text { Relapse - as defined by each of the studies } \\
\text { follow up: range } 2 \text { months to } 36\end{array}$} & \multicolumn{2}{|c|}{ Study population } & \multirow[t]{2}{*}{-} & \multirow{2}{*}{$\begin{array}{l}512 \\
(3 \mathrm{RCTs})\end{array}$} & \multirow[t]{2}{*}{-} & \multirow{2}{*}{$\begin{array}{l}\text { Data were not pooled due } \\
\text { to high heterogeneity }(12= \\
82 \%) \text {. Two studies showed } \\
\text { no difference between } \\
\text { the interventions and one } \\
\text { study favoured antipsy- } \\
\text { chotic combinations. }\end{array}$} \\
\hline & see comment & see comment & & & & \\
\hline \multirow{2}{*}{$\begin{array}{l}\text { Leaving the study early } \\
\text { follow up: range } 6 \text { weeks to } 52 \text { weeks }\end{array}$} & \multicolumn{2}{|c|}{ Study population } & \multirow{2}{*}{$\begin{array}{l}\text { RR } 0.90 \\
\text { (0.76 to } 1.07 \text { ) }\end{array}$} & \multirow{2}{*}{$\begin{array}{l}3137 \\
(43 \mathrm{RCTs})\end{array}$} & \multirow{2}{*}{$\begin{array}{l}\oplus \oplus \odot \odot \\
\text { LOW } 257\end{array}$} & \\
\hline & 183 per 1,000 & $\begin{array}{l}164 \text { per } 1,000 \\
(139 \text { to } 195)\end{array}$ & & & & \\
\hline \multirow{2}{*}{$\begin{array}{l}\text { Service utilisation: Hospital admission } \\
\text { follow up: range } 12 \text { weeks to } 26 \text { weeks }\end{array}$} & \multicolumn{2}{|c|}{ Study population } & \multirow{2}{*}{$\begin{array}{l}\text { RR } 0.96 \\
\text { (0.36 to } 2.55)\end{array}$} & \multirow{2}{*}{$\begin{array}{l}202 \\
\text { (3 RCTs) }\end{array}$} & \multirow{2}{*}{$\begin{array}{l}\oplus \ominus \ominus \ominus \\
\text { VERY LOW } 256\end{array}$} & \\
\hline & 69 per 1,000 & $\begin{array}{l}67 \text { per } 1,000 \\
(25 \text { to } 177)\end{array}$ & & & & \\
\hline $\begin{array}{l}\text { Service utilisation: Change in hospital sta- } \\
\text { tus - not reported }\end{array}$ & \multicolumn{2}{|c|}{ Study population } & not estimable & (studies) & - & $\begin{array}{l}\text { No studies provided data } \\
\text { for this outcome. }\end{array}$ \\
\hline
\end{tabular}




\begin{tabular}{|c|c|c|c|c|c|c|}
\hline \multirow{3}{*}{$\begin{array}{l}\text { Adverse events: Serious event or requiring } \\
\text { discontinuation } \\
\text { follow up: range } 6 \text { weeks to } 8 \text { months }\end{array}$} & 0 per 1,000 & $\begin{array}{l}0 \text { per } 1,000 \\
(0 \text { to } 0)\end{array}$ & & & & \\
\hline & \multicolumn{2}{|c|}{ Study population } & \multirow{2}{*}{$\begin{array}{l}\text { RR } 1.05 \\
\text { (0.65 to } 1.69)\end{array}$} & \multirow{2}{*}{$\begin{array}{l}2398 \\
\text { (30 RCTs) }\end{array}$} & \multirow{2}{*}{$\begin{array}{l}\oplus \ominus \Theta \ominus \\
\text { VERY LOW } 125 \\
7\end{array}$} & \\
\hline & 47 per 1,000 & $\begin{array}{l}49 \text { per } 1,000 \\
(31 \text { to } 80)\end{array}$ & & & & \\
\hline $\begin{array}{l}\text { Quality of life } \\
\text { assessed with: QLS, SWN and SF-36 } \\
\text { follow up: range } 6 \text { weeks to } 16\end{array}$ & see comment & see comment & - & $\begin{array}{l}398 \\
\text { (4 RCTs) }\end{array}$ & - & $\begin{array}{l}\text { Data were not pooled, as } \\
\text { they were presented in } \\
\text { both change and endpoint } \\
\text { data for } 3 \text { different scales. } \\
\text { None of the scales showed } \\
\text { a difference between the } \\
\text { two groups. }\end{array}$ \\
\hline
\end{tabular}

*The risk in the intervention group (and its $95 \%$ confidence interval) is based on the assumed risk in the comparison group and the relative effect of the intervention (and its $95 \% \mathrm{Cl})$.

Cl: Confidence interval; RR: Risk ratio; OR: Odds ratio;

\section{GRADE Working Group grades of evidence}

High quality: We are very confident that the true effect lies close to that of the estimate of the effect

Moderate quality: We are moderately confident in the effect estimate: The true effect is likely to be close to the estimate of the effect, but there is a possibility that it is substantially different

Low quality: Our confidence in the effect estimate is limited: The true effect may be substantially different from the estimate of the effect

Very low quality: We have very little confidence in the effect estimate: The true effect is likely to be substantially different from the estimate of effect

1 Downgraded one level due to risk of bias.

2 Downgraded one level due to inconsistency.

3 Although there is a concern about the timeframe to measure the outcome we decided not to downgrade due to overall quality assessment.

4 Downgraded one level due to publication bias.

5 Downgraded one level due to imprecision.

6 Downgraded one level due to indirectness.

7 Although there is a concern about the influence on industry we decided not to downgrade due to overall quality assessment.

8 Downgraded two levels due to inconsistency.

Please see Checklist to aid consistency and reproducibility of GRADE assessments Appendix 1. 


\section{B A C K G R O U N D}

\section{Description of the condition}

Schizophrenia is a chronic disorder with a lifetime prevalence of four per 1000 persons (McGrath 2008). It is characterised by emotional, cognitive, and behavioural dysfunctions. In order to meet the diagnostic criteria of schizophrenia, patients require two or more positive, disorganised, or negative symptoms that persist for at least six months, with at least one of them being a positive symptom or disorganised speech (APA 2013). Positive symptoms include delusions (e.g. a false belief that is resistant to change, immune to contradictory evidence, and without correlation to the sociocultural background) and hallucinations (e.g. a sensory experience in the absence of external stimulus to the corresponding sensory organ). Negative symptoms are characterised by deficits in normal behaviour, which consist of five domains: blunted affect, alogia, asociality, anhedonia, and avolition (Kirkpatrick 2006).

Schizophrenia is difficult to treat and significantly burdens an individual's daily life. Despite the introduction of antipsychotics in the 1950 s and the reintroduction of clozapine to the Western world, the mean recovery rate of schizophrenia is $13.5 \%$ (Jääskeläinen 2012). In clinical practice, multiple augmentation strategies such as adding another antipsychotic, mood-stabiliser, benzodiazepines, lithium, electroconvulsive therapy, or repetitive transcranial magnetic stimulation have been used for these patients in order to improve their clinical state, but the evidence for the use of these interventions is lacking (Hasan 2012).

\section{Description of the intervention}

Antipsychotic medications are the cornerstone for the treatment of schizophrenia. They were originally classified on the basis of their risk for the development of extrapyramidal side effects (EPS) as typical (e.g. chlorpromazine, haloperidol, fluphenazine) or atypical (e.g. clozapine, olanzapine, risperidone) if the risk for the development of EPS is low (Grunder 2009).

Antipsychotic polypharmacy/combination treatment, e.g. concurrent treatment with more than one antipsychotic medication, is a common strategy for the management of disturbed behaviour, poor response to antipsychotic monotherapy, or acute positive symptom exacerbation (Paton 2008). Concerning this practice, recommendations are varied. While some countries justify this practice (e.g. Finland, France, the UK), others recommend against it (e.g. Canada, Denmark, Spain), and many abstain from making any recommendation (Gaebel 2005). Regardless of the recommendations and lack of evidence, this practice has shown a trend towards increased use over time (Gangluy 2004). It is estimated that $19.6 \%$ of patients with schizophrenia across the world receive antipsychotic polypharmacy/combination treatment (Gallego 2009).

\section{How the intervention might work}

Currently, there is not a current understanding of how the combination of antipsychotics might work. Plausible hypotheses include (Freudenreich 2002):

1. achieving optimal receptor occupancy;

2. targeting different receptors with the added drug (Kapur 2001); and
3. reducing the dose-related side-effects by using lower doses of the two drugs.

\section{Why it is important to do this review}

A number of potential concerns regarding antipsychotic combinations have been identified. These include the possibility of unnecessarily high doses, an increased acute and/or chronic sideeffect burden, adverse pharmacodynamic and pharmacokinetic interactions, increased rates of non-compliance, difficulties in determining cause and effect of multiple treatments, potential increased mortality, higher costs and poorly documented risks and benefits of this practice (Centorrino 2005; Meltzer 2000; Misawa 2011; Rupnow 2007; Waddington 1998; Weiden 1999). In this review, we examine the evidence for the efficacy and safety of antipsychotic combinations in the treatment of schizophrenia and schizophrenia-like psychoses. We are aware of the sister Cochrane review investigating the effects of different clozapine-antipsychotic combinations (Barber 2017). However, this review is different in its scope as it investigates any combinations of antipsychotic therapy versus any antipsychotic monotherapy.

\section{O B JECTIVES}

To examine whether:

1. treatment with antipsychotic combinations is effective for schizophrenia; and

2. treatment with antipsychotic combinations is safe for the same illness.

\section{METHODS}

\section{Criteria for considering studies for this review}

Types of studies

We included all relevant randomised controlled trials (RCTs) and quasi-RCTs. Where a trial was described as 'double-blind' and it implied that the study was randomised and the demographic details of each group were similar, those trials were also included. After debate we decided to maintain the same inclusion criteria determined by previous authors to include quasi-RCTs (see Differences between protocol and review).

\section{Types of participants}

Adults, however defined, with schizophrenia or related disorders, including schizophreniform disorder, schizoaffective disorder and delusional disorder, again, by any means of diagnosis.

\section{Types of interventions}

\section{Treatment with more than one antipsychotic medication}

Any dose and route of administration.

\section{Treatment with only one antipsychotic medication}

Any dose and route of administration.

\section{Types of outcome measures}

We grouped outcomes into long term (over 26 weeks, A), medium term (13 to 26 weeks, B) and short term (up to 12 weeks, C). 


\section{Primary outcomes}

\section{Clinical response}

1.1 No clinically important response - as defined by each of the studies

1.2 Relapse - as defined by each of the studies

\section{Leaving the study early}

\section{Secondary outcomes}

\section{Service utilisation}

1.1 Hospital admission

1.2 Days in hospital

1.3 Change in hospital status

\section{Clinical response}

2.1 No clinically important improvement of global state

2.2 Average score/change in global state

2.3 No clinically important improvement in mental state - as defined by each of the studies

2.4 Average score/change in mental state

2.5 No clinically important response on positive symptoms - as defined by each of the studies

2.6 Average score/change in positive symptoms

2.7 No clinically important response on negative symptoms - as defined by each of the studies

2.8 Average score/change in negative symptoms

2.9 No clinically important response on aggression/agitation symptoms - as defined by each of the studies

2.10 Average score/change in aggression/agitation symptoms

\section{Behaviour}

3.1 General behaviour

3.2 Specific behaviours

3.2.1 Social functioning

3.2.2 Employment status during trial (employed / unemployed)

3.2.3 Occurrence of violent incidents (to self, others, or property)

3.2.4 Level of substance abuse

\section{Adverse events}

4.1 Serious adverse events

4.2 Adverse events requiring hospitalisation

4.3 Specific adverse events

4.3.1 Allergic reactions

4.3.2 Blood dyscrasia such as agranulocytosis

4.3.3 Central nervous system (ataxia, nystagmus, drowsiness, fits, diplopia, tremor)

4.3.4 Death (suicide and non-suicide deaths)

4.3.5 Endocrinological dysfunction (hyperprolactinaemia)

4.3.6 Weight gain

4.3.7 Movement disorders (extrapyramidal side effects (EPS))

\section{Quality of life}

\section{Economic burden (cost of care)}

\section{'Summary of findings' table}

We used the GRADE approach to interpret findings (Schünemann 2011), and used GRADE profiler (GRADEPRO) to import data from RevMan 5 (Review Manager) in order to create a 'Summary of findings' table. This table provides outcome-specific information concerning the overall quality of evidence from each included study in the comparison, the magnitude of effect of the interventions examined, and the sum of available data on all outcomes we rated as important to patient care and decision-making. Also, we prepared an appendix (Appendix 1) to help with the standardisation of the 'Summary of findings' table (please see Differences between protocol and review). We aimed to select the following main outcomes for inclusion in the 'Summary of findings' table.

\section{Clinical response}

1.1 No clinically important response - as defined by each of the studies

1.2 Relapse - as defined by each of the studies

\section{Leaving the study early}

3. Service utilisation

3.1 Hospital admission

3.2 Change in hospital status

4. Adverse events: clinically important - as defined by individual studies*

5. Quality of life: clinically important response - as defined by individual studies ${ }^{\star}$

\section{Search methods for identification of studies}

\section{Electronic searches}

\section{Cochrane Schizophrenia Group's Trials Register}

The Information Specialist searched the Cochrane Schizophrenia Group's Study-Based Register of Trials (June 2010, August 2012 and 25 January, 2016) using the following search strategy, which has been developed based on literature review and consulting with the authors of the review:

(((antipsychot* or neuroleptic* or drug*) and combin*) or *add-on* or *addition*or *supplement*or * supplementation*or ${ }^{*}$ cotreatment ${ }^{*}$ or * ${ }^{*}$-treatment ${ }^{*}$ or *adjunctive ${ }^{*}$ or ${ }^{*}$ concurrent ${ }^{*}$ or ${ }^{*}$ concomitant ${ }^{*}$ or * simultaneous* or $^{*}$ parallel ${ }^{*}$ or * polypharmacy) in title, abstract or index terms of REFERENCE or ("polytherapy* or *augmentation ${ }^{*}$ or ${ }^{*}$ parallel $^{*}$ or * ${ }^{*}$ combined ${ }^{\star}$ ) in interventions of STUDY

In such a study-based register, searching the major concept retrieves all the synonym keywords and relevant studies because all the studies have already been organised based on their interventions and linked to the relevant topics.

The Cochrane Schizophrenia Group's Register of Trials is compiled by systematic searches of major resources (including AMED, BIOSIS, CINAHL, Embase, MEDLINE, PsycINFO, PubMed, and registries of clinical trials) and their monthly updates, handsearches, grey literature, and conference proceedings (see Group's Module). There is no language, date, document type, or publication status limitations for inclusion of records into the register.

\section{Searching other resources}

\section{Reference searching}

We inspected references of all included studies for further relevant studies. 


\section{Personal contact}

Where necessary, we contacted the first author of each included study for information regarding unpublished trials. We noted the outcome of this contact in the Characteristics of included studies, Characteristics of studies awaiting classification tables.

\section{Data collection and analysis}

The text below describes data collection and analysis for the 2016 search; the previous data collection and analysis can be seen in Appendix 2.

\section{Selection of studies}

Two review authors JO and SC inspected all abstracts of studies identified as above and identified potentially relevant reports. $\mathrm{YH}$ screened the Chinese language studies, and one study in Korean language was inspected by $\mathrm{HH}$. We resolved disagreements by discussion, or where there was still doubt, we acquired the full-text article for further inspection. We acquired the full-text articles of relevant reports/abstracts meeting initial criteria for reassessment and carefully inspected for a final decision on inclusion (see Criteria for considering studies for this review). JO and SC were not blinded to the names of the authors, institutions or journal of publication. Where difficulties or disputes arose, we asked author LC for help, and where it was impossible to decide or if adequate information was not available to make a decision, we added these studies to those awaiting assessment and contacted the authors of the papers for clarification.

\section{Data extraction and management}

\section{Extraction}

Review authors JO and SC independently extracted data from all included studies and $\mathrm{YH}$ extracted data for Chinese studies. In addition, to ensure reliability, LC extracted data from a random sample of these studies, comprising $10 \%$ of the total. Again, we discussed any disagreement and documented decisions. With any remaining problems, LC helped clarify issues and we documented these final decisions. We extracted data presented only in graphs and figures whenever possible, but included only if two review authors independently had the same result. We attempted to contact authors through an open-ended request in order to obtain missing information or for clarification whenever necessary. If studies were multi-centre, where possible, we extracted data relevant to each component centre separately.

\section{Management}

\subsection{Forms}

We adapted the 'Data collection form for intervention reviews' provided by Cochrane to collect data.

\subsection{Scale-derived data}

We included continuous data from rating scales only if:

a) the psychometric properties of the measuring instrument have been described in a peer-reviewed journal (Marshall 2000); and b) the measuring instrument has not been written or modified by one of the trialists for that particular trial.

Ideally, the measuring instrument should either be: i. a selfreport or ii. completed by an independent rater or relative (not the therapist). We realise that this is not often reported clearly, therefore we noted in Description of studies if this was the case or not.

\subsection{Endpoint versus change data}

There are advantages of both endpoint and change data. Change data can remove a component of between-person variability from the analysis. On the other hand, calculation of change needs two assessments (baseline and endpoint), which can be difficult in unstable and difficult to measure conditions such as schizophrenia. We decided primarily to use endpoint data, and only use change data if the former were not available. We did not combined endpoint data and change data, we decided to present the data in the analysis separately (see Differences between protocol and review).

\subsection{Skewed data}

Continuous data on clinical and social outcomes are often not normally distributed. To avoid the pitfall of applying parametric tests to non-parametric data, we applied the following standards to relevant data before inclusion.

Please note, we entered data from studies of at least 200 participants in the analysis irrespective of the following rules, because skewed data pose less of a problem in large studies. We also entered all relevant change data, as when continuous data are presented on a scale that includes a possibility of negative values (such as change data), it is difficult to tell whether data are skewed or not.

For endpoint data:

(a) when a scale started from the finite number zero, we subtracted the lowest possible value from the mean, and divided this by the standard deviation (SD). If this value was lower than 1 , it strongly suggests a skew and we excluded these data. If this ratio was higher than one but below 2, there is suggestion of skew. We entered these data and tested whether their inclusion or exclusion changed the results substantially. Finally, if the ratio was larger than 2 we included these data, because skew is less likely (Altman 1996; Higgins 2011).

(b) if a scale starts from a positive value (such as the Positive and Negative Syndrome Scale (PANSS), (Kay 1986)) which can have values from 30 to 210), we modified the calculation described above to take the scale starting point into account. In these cases skew is present if $2 \mathrm{SD}>$ (S-S min), where $\mathrm{S}$ is the mean score and 'S min' is the minimum score.

\subsection{Common measure}

Where relevant, to facilitate comparison between trials, we converted variables that can be reported in different metrics, such as days in hospital (mean days per year, per week or per month) to a common metric (e.g. mean days per month).

\subsection{Conversion of continuous to binary}

Where possible, we converted continuous outcome measures to dichotomous data. This can be done by identifying cut-off points on rating scales and dividing participants accordingly into 'clinically improved' or 'not clinically improved'. It is generally assumed that if there is a $50 \%$ reduction in a scale-derived score such as the Brief Psychiatric Rating Scale (BPRS, Overall 1962), or the Positive and 
Negative Syndrome Scale (PANSS, Kay 1986), this can be considered as a clinically significant response (Leucht 2005; Leucht 2005a). If data based on these thresholds were not available, we used the primary cut-off presented by the original authors.

\subsection{Direction of graphs}

Where possible, we entered data in such a way that the area to the left of the line of no effect indicated a favourable outcome for treatment with antipsychotic combinations. Where keeping to this made it impossible to avoid outcome titles with clumsy doublenegatives (e.g. 'Not un-improved'), we presented data where the left of the line indicates an unfavourable outcome and noted this in the relevant graphs.

\section{Assessment of risk of bias in included studies}

Review authors, JO and SC independently assessed the risk of bias of each trial published in English and $\mathrm{YH}$ assessed trials published in Chinese by using criteria described in the Cochrane Handbook for Systematic Reviews of Interventions to assess trial quality (Higgins 2011a). This set of criteria is based on evidence of associations between overestimate of effect and high risk of bias of the article such as sequence generation, allocation concealment, blinding, incomplete outcome data and selective reporting.

If the raters disagreed, we made the final rating by consensus, with the involvement of LC. Where inadequate details of randomisation and other characteristics of trials were provided, we contacted authors of the studies in order to obtain further information. If nonconcurrence occurred, we reported this.

We noted the level of risk of bias in the text of the review and in Figure 1, Figure 2 and Summary of findings for the main comparison.

Figure 1. 'Risk of bias' graph: review authors' judgements about each risk of bias item presented as percentages across all included studies.

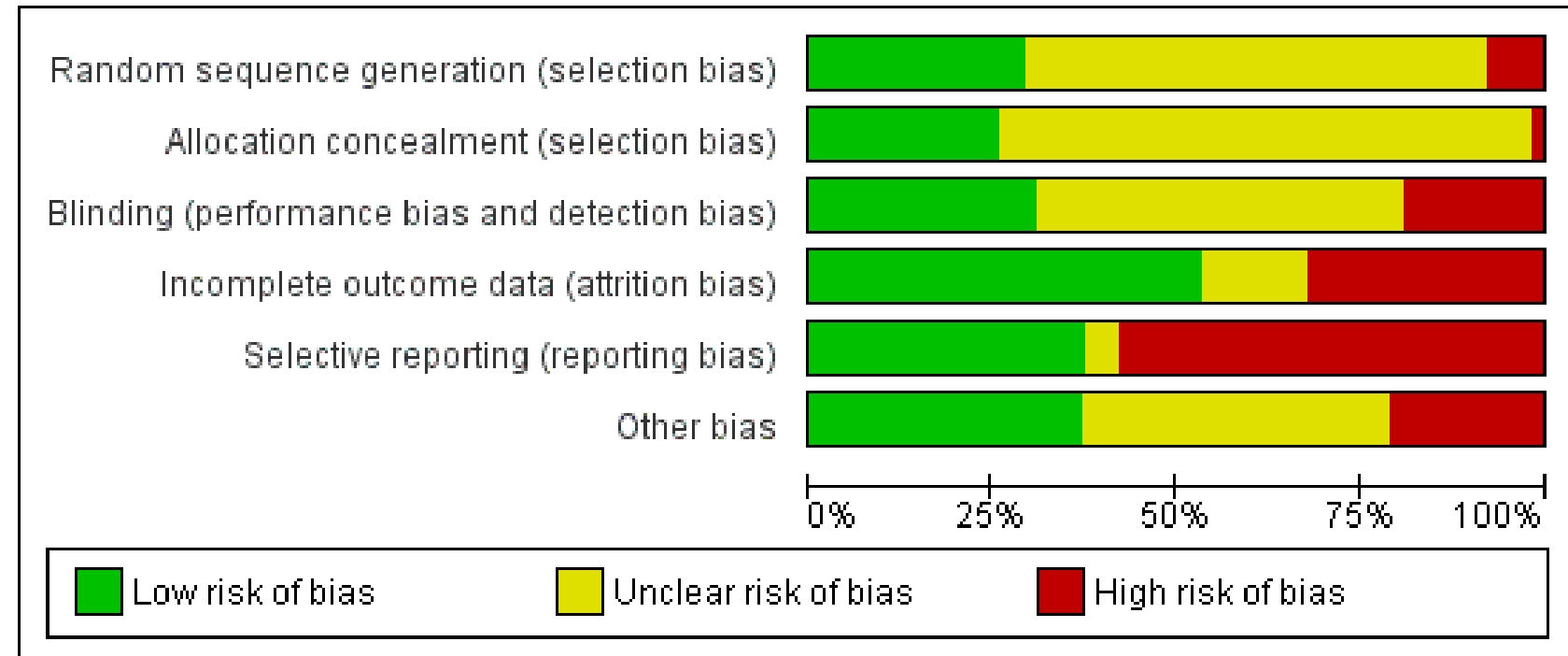


Figure 2. 'Risk of bias' summary: review authors' judgements about each risk of bias item for each included study.

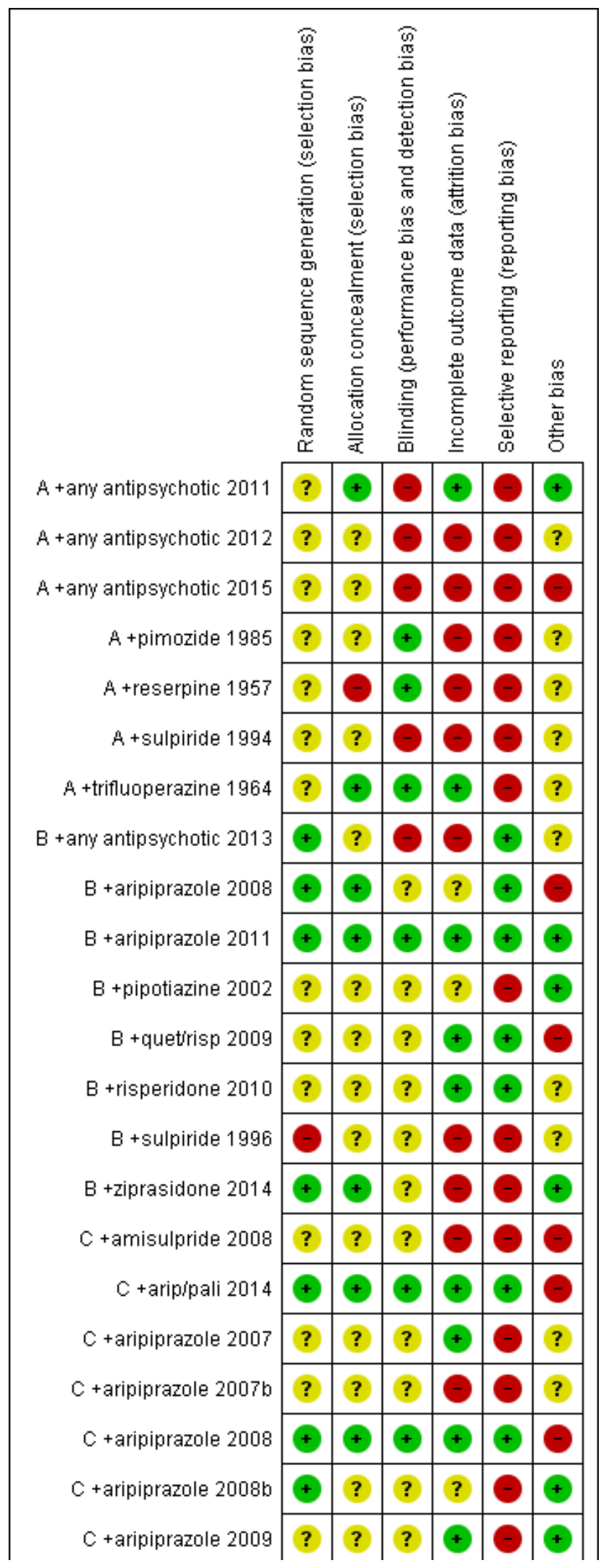


Figure 2. (Continued)

\begin{tabular}{|c|c|c|c|c|c|c|}
\hline C + aripiprazole 2009 & $?$ & $?$ & $?$ & + & $\odot$ & $\odot$ \\
\hline C +aripiprazole 2012 & $?$ & $?$ & $?$ & $?$ & $\odot$ & $\odot$ \\
\hline C +aripiprazole 2013 & $?$ & $?$ & $?$ & $\odot$ & $\odot$ & $\odot$ \\
\hline C +aripiprazole 2013b & $?$ & $?$ & $?$ & $\oplus$ & $\odot$ & $?$ \\
\hline C +aripiprazole 2014 & $\odot$ & 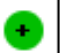 & 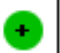 & $\oplus$ & $\odot$ & $\odot$ \\
\hline C + aripiprazole 2015 & $\odot$ & 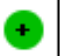 & 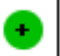 & $\oplus$ & $\odot$ & $\odot$ \\
\hline C + aripiprazole $2015 b$ & $\odot$ & + & + & $?$ & $\odot$ & $\odot$ \\
\hline c +aripiprazole $2015 \mathrm{c}$ & $\odot$ & $?$ & $\odot$ & $\odot$ & $\odot$ & $\odot$ \\
\hline C +aripiprazole 2016 & $\odot$ & $?$ & $\odot$ & 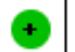 & $\odot$ & $\odot$ \\
\hline C +clozapine 2001 & $?$ & $?$ & ? & + & - & $?$ \\
\hline C+clozapine 2013 & $?$ & $?$ & $\odot$ & + & $\oplus$ & $\odot$ \\
\hline$C+C P Z 1973$ & $?$ & $?$ & $?$ & $\Theta$ & - & $?$ \\
\hline$C+C P Z 1989$ & $?$ & $?$ & $?$ & $?$ & - & $?$ \\
\hline$C+C P Z 1999$ & $\odot$ & $?$ & ? & + & $\odot$ & $\odot$ \\
\hline $\mathrm{C}+$ fluphen dec 2009 & $\odot$ & $?$ & $\odot$ & $\oplus$ & $\odot$ & $?$ \\
\hline C +haloperidol 2006 & $?$ & $?$ & ? & $?$ & - & $?$ \\
\hline C + haloperidol 2010 & $?$ & $?$ & ? & + & $\odot$ & $\odot$ \\
\hline c +levomepromazine 2004 & $?$ & $?$ & - & - & $\odot$ & $?$ \\
\hline C +olanirisp 2014 & + & + & - & $\Theta$ & + & $?$ \\
\hline C+olanzapine 2012 & $?$ & $\odot$ & - & + & $\odot$ & $\odot$ \\
\hline $\mathrm{c}+$ olanzapine $2012 \mathrm{~b}$ & $?$ & $?$ & + & $\Theta$ & $\Theta$ & $\odot$ \\
\hline$C+$ perphenazine 1976 & $?$ & $?$ & + & ? & $\odot$ & $?$ \\
\hline C +pimozide 2011 & $?$ & $?$ & $?$ & + & + & $\odot$ \\
\hline C +pimozide 2013 & $?$ & $?$ & $\odot$ & $\odot$ & $\Theta$ & $\Theta$ \\
\hline C +pipotiazine 2000 & $?$ & ? & $?$ & $\odot$ & $?$ & $\odot$ \\
\hline C+risperidone 2001 & $?$ & ? & $?$ & + & $?$ & + \\
\hline $\mathrm{C}+$ risperidone $2001 \mathrm{~b}$ & $?$ & $?$ & $?$ & + & $\odot$ & $?$ \\
\hline C +risperidone 2001c & ? & ? & $?$ & + & ? & + \\
\hline C+risperidone 2005 & + & + & + & - & $\Theta$ & $\odot$ \\
\hline $\mathrm{C}+$ risperidone $2005 \mathrm{~b}$ & $?$ & + & + & + & - & $\odot$ \\
\hline C +risperidone 2006 & $\odot$ & + & + & + & 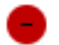 & ? \\
\hline
\end{tabular}


Figure 2. (Continued)

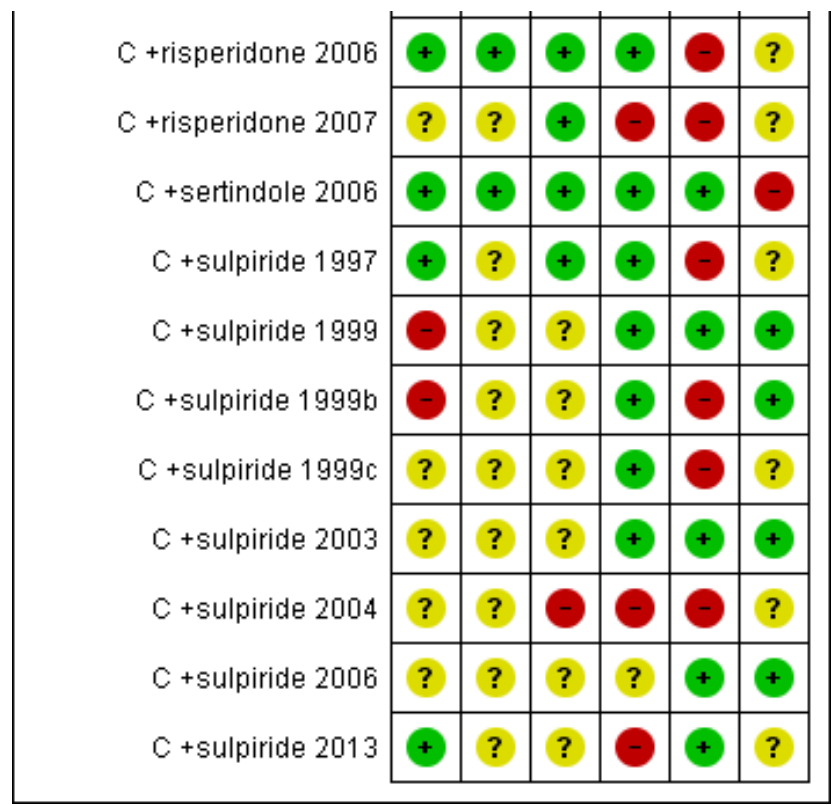

\section{Measures of treatment effect}

\section{Binary data}

For binary outcomes, we calculated a standard estimation of the risk ratio (RR) and its 95\% confidence interval ( $\mathrm{Cl}$ ). It has been shown that RR is more intuitive (Boissel 1999) than odds ratios and that odds ratios tend to be interpreted as RR by clinicians (Deeks 2000).

\section{Continuous data}

For continuous outcomes, we estimated mean difference (MD) between groups. We preferred not to calculate effect size measures (standardised mean difference (SMD)). However, if scales of very considerable similarity were used, we presumed there was a small difference in measurement, and calculated the effect size and transformed the effect back to the units of one or more of the specific instruments.

\section{Unit of analysis issues}

\section{Cluster trials}

Studies increasingly employ 'cluster randomisation' (such as randomisation by clinician or practice), but analysis and pooling of clustered data poses problems. Firstly, authors often fail to account for intra-class correlation in clustered studies, leading to a 'unit of analysis' error (Divine 1992), whereby $P$ values are spuriously low, confidence intervals unduly narrow and statistical significance overestimated. This causes type I errors (Bland 1997; Gulliford 1999).

Where clustering was not accounted for in primary studies, we presented such data in a table, with a $\left(^{\star}\right)$ symbol to indicate the presence of a probable unit of analysis error. In subsequent versions of this review we will seek to contact first authors of studies to obtain intra-class correlation coefficients (ICCS) for their clustered data and to adjust for this by using accepted methods (Gulliford 1999). Where clustering has been incorporated into the analysis of primary studies, we will present these data as if from a non-cluster randomised study, but adjust for the clustering effect.

We have sought statistical advice and have been advised that the binary data as presented in a report should be divided by a 'design effect'. This is calculated using the mean number of participants per cluster $(\mathrm{Bm})$ and the ICC [Design effect $=1+(\mathrm{m}-1)^{\star} \mathrm{ICC}$ ] (Donner 2002). If the ICC is not reported it will be assumed to be 0.1 (Ukoumunne 1999).

If cluster studies have been appropriately analysed taking into account ICCs and relevant data documented in the report, synthesis with other studies would be possible using the generic inverse variance technique.

\section{Cross-over trials}

A major concern of cross-over trials is the carry-over effect. It occurs if an effect (e.g. pharmacological, physiological or psychological) of the treatment in the first phase is carried over to the second phase. As a consequence, on entry to the second phase the participants can differ systematically from their initial state despite a wash-out phase. For the same reason cross-over trials are not appropriate if the condition of interest is unstable (Elbourne 2002). As both effects are very likely in severe mental illness, we only used data from the first phase of cross-over studies.

\section{Studies with multiple treatment groups}

Where a study involved more than two treatment arms, if relevant, we presented the additional treatment arms in comparisons. If data were binary, we simply added and combined within the two-bytwo table. If data were continuous, we combined data following the formula in section 7.7.3.8 (Combining groups) of the Cochrane Handbook for Systematic Reviews of Interventions ( Higgins 2011). We did not use data where the additional treatment arms were not relevant. 


\section{Dealing with missing data}

\section{Overall loss of credibility}

At some degree of loss of follow-up, data must lose credibility (Xia 2009). We chose that, for any particular outcome, should more than $50 \%$ of data be unaccounted for, we would not reproduce these data or use them within analyses. If, however, more than $50 \%$ of those in one arm of a study were lost, but the total loss was less than $50 \%$, we addressed this within the 'Summary of findings' table by down-rating quality. We also downgraded quality within the 'Summary of findings' table should loss be $25 \%$ to $50 \%$ in total.

\section{Binary}

In the case where attrition for a binary outcome was between $0 \%$ and $50 \%$ and where these data were not clearly described, we presented data on a 'once-randomised-always-analyse' basis (an intention-to-treat (ITT) analysis). We assumed all those leaving the study early to have the same rates of negative outcome as those who completed -except for the outcomes of death and adverse effects- for these outcomes we used the rate of those who stayed in the study (in that particular arm of the trial) for those who did not. We undertook a sensitivity analysis to test how prone the primary outcomes were to change by comparing data only from people who completed the study to that point to the ITT analysis using the above assumptions.

\section{Continuous}

\subsection{Attrition}

We reported and used data where attrition for a continuous outcome was between $0 \%$ and $50 \%$, and data only from people who completed the study to that point were reported.

\subsection{Standard deviations}

If standard deviations (SDs) were not reported, we first tried to obtain the missing values from the authors. If not available, where there were missing measures of variance for continuous data, but an exact standard error (SE) and confidence intervals available for group means, and either $\mathrm{P}$ value or $\mathrm{T}$ value available for differences in mean, we calculated them according to the rules described in the Cochrane Handbook for Systematic Reviews of Interventions (Deeks 2011). When only the SE is reported, SDs) are calculated by the formula SD $=\mathrm{SE}$ * square root (N). Chapters 7.7.3 and 16.1.3 of the Cochrane Handbook for Systematic Reviews of Interventions (Deeks 2011) present detailed formulae for estimating SDs from P values, $t$ or $F$ values, confidence intervals, ranges or other statistics. If these formulae did not apply, we calculated the SDs according to a validated imputation method, which is based on the SDs of the other included studies (Furukawa 2006). Although some of these imputation strategies can introduce error, the alternative would be to exclude a given study's outcome and thus to lose information. We nevertheless examined the validity of the imputations in a sensitivity analysis excluding imputed values.

\subsection{Assumptions about participants who left the trials early or were lost to follow-up}

Various methods are available to account for participants who left the trials early or were lost to follow-up. Some trials just present the results of study completers, others use the method of last observation carried forward (LOCF), while more recently methods such as multiple imputation or mixed-effects models for repeated measurements (MMRM) have become more of a standard. While the latter methods seem to be somewhat better than LOCF (Leon 2006), we feel that the high percentage of participants leaving the studies early and differences in the reasons for leaving the studies early between groups is often the core problem in randomised schizophrenia trials. We therefore did not exclude studies based on the statistical approach used. However, we preferred to use the more sophisticated approaches. (e.g. MMRM or multipleimputation) and only presented completer analyses if some kind of ITT data were not available at all. Moreover, we addressed this issue in the item "incomplete outcome data" of the 'Risk of bias' tool.

\section{Assessment of heterogeneity}

\section{Clinical heterogeneity}

We considered all included studies initially, without seeing comparison data, to judge clinical heterogeneity. We simply inspected all studies for clearly outlying people or situations which we had not predicted would arise and discussed in the text if they arose.

\section{Methodological heterogeneity}

We considered all included studies initially, without seeing comparison data, to judge methodological heterogeneity. We simply inspected all studies for clearly outlying methods which we had not predicted would arise and discussed in the text if they arose.

\section{Statistical heterogeneity}

\subsection{Visual inspection}

We visually inspected graphs to investigate the possibility of statistical heterogeneity.

\subsection{Employing the $\mathrm{I}^{2}$ statistic}

We investigated heterogeneity between studies by considering the $\mathrm{I}^{2}$ method alongside the $\mathrm{Chi}^{2} \mathrm{P}$ value. The $\mathrm{I}^{2}$ provides an estimate of the percentage of inconsistency thought to be due to chance (Higgins 2003). The importance of the observed value of $\mathrm{I}^{2}$ depends on i. magnitude and direction of effects and ii. strength of evidence for heterogeneity (e.g. P value from $\mathrm{Chi}^{2}$ test, or a confidence interval for $\mathrm{I}^{2}$ ). An $\mathrm{I}^{2}$ estimate greater than or equal to around $50 \%$ accompanied by a statistically significant $\mathrm{Chi}^{2}$ statistic, can be interpreted as evidence of substantial levels of heterogeneity (Section 9.5.2 - Deeks 2011). We explored and discussed in the text potential reasons for substantial levels of heterogeneity (Subgroup analysis and investigation of heterogeneity).

\section{Assessment of reporting biases}

Reporting biases arise when the dissemination of research findings is influenced by the nature and direction of results (Egger 1997). These are described in Section 10 of the Cochrane Handbook for Systematic Reviews of Interventions (Sterne 2011). We are aware that funnel plots may be useful in investigating reporting biases but are of limited power to detect small-study effects. We did not use funnel plots for outcomes where there are 10 or fewer studies, or where all studies were of similar sizes. In future versions of this review, if funnel plots are possible, we will seek statistical advice in their interpretation. 


\section{Data synthesis}

We understand that there is no closed argument for preference for use of fixed-effect or random-effects models. The random-effects method incorporates an assumption that the different studies are estimating different, yet related, intervention effects. This often seems to be true to us and the random-effects model takes into account differences between studies even if there is no statistically significant heterogeneity. There is, however, a disadvantage to the random-effects model. It puts added weight onto small studies which often are the most biased ones. Depending on the direction of effect, these studies can either inflate or deflate the effect size. We chose random-effects model for all analyses.

\section{Subgroup analysis and investigation of heterogeneity}

\section{Subgroup analyses}

We presented data in the analyses grouped by the type of antipsychotic used: trials with clozapine in both the monotherapy and combination arm, trials with other atypical drugs in both the monotherapy and combination arms, trials with typical antipsychotic drugs in both arms, or any antipsychotics in both groups, in order to facilitate subgroup analyses (see Differences between protocol and review).

\subsection{Primary outcomes}

In addition, we also undertook subgroup analyses comparing the results for the following:

1. enrolment of acutely exacerbated or chronically ill patients;

2. treatment duration $<12$ weeks vs $\geq 12$ weeks;

3. clozapine vs non-clozapine combinations; and

4. drug added to clozapine treatment.

\section{Investigation of heterogeneity}

If inconsistency was high first, we investigated whether data were entered correctly. Second, if data were correct, we visually inspected the graph and successively removed outlying studies to see if homogeneity was restored. For this review, we decided that should this occur with data contributing to the summary finding of no more than around $10 \%$ of the total weighting, we would present data. If not, we would not pool such data but discuss issues. We know of no supporting research for this $10 \%$ cut-off but are investigating use of prediction intervals as an alternative to this unsatisfactory state.

We performed a meta-regression for the primary outcome 'No clinically important response' (Please see Differences between protocol and review).

When unanticipated clinical or methodological heterogeneity were obvious, we simply discussed these. We did not undertake sensitivity analyses relating to these.

\section{Sensitivity analysis}

\section{Implication of randomisation}

If trials were described in some way as to imply randomisation, we undertook a sensitivity analyses for the primary outcomes. We included these studies in the analyses and if there was no substantive difference when the implied randomised studies were added to those with better description of randomisation, then we used relevant data from these studies.

\section{Assumptions for lost binary data}

Where assumptions had to be made regarding people lost to followup (see Dealing with missing data), we compared the findings of the primary outcomes when we used our assumption compared with completer data only. If there was a substantial difference, we reported and discussed these results, but continued to employ our assumption.

Where assumptions had to be made regarding missing SDs data (see Dealing with missing data), we compared the findings of the primary outcomes when we used our assumption compared with completer data only. We undertook a sensitivity analysis to test how prone results were to change when 'completer' data only were compared to the imputed data using the above assumption. If there was a substantial difference, we reported and discussed these results, but continued to employ our assumption.

\section{Risk of bias}

We analysed the effects of excluding trials that we judged to be at high risk of bias across one or more of the domains of randomisation (implied as randomised with no further details available) allocation concealment, blinding and outcome reporting for the meta-analysis of the primary outcome. If the exclusion of trials at high risk of bias did not substantially alter the direction of effect or the precision of the effect estimates, we included data from these trials in the analysis

\section{Imputed values}

We undertook a sensitivity analysis to assess the effects of including data from trials where we used imputed values for ICCS in calculating the design effect in cluster-randomised trials.

If we found substantial differences in the direction or precision of effect estimates in any of the sensitivity analyses listed above, we did not pool data from the excluded trials with the other trials contributing to the outcome, but presented them separately

\section{Fixed and random effects}

We synthesised data using a random-effects model, however, we also synthesised data for the primary outcome using a fixed-effect model to evaluate whether this altered the significance of the results

\section{RES U L T S}

\section{Description of studies}

Please also see Characteristics of included studies, Characteristics of excluded studies, Characteristics of studies awaiting classification, and Characteristics of ongoing studies. To try and aid clarity, we have named the studies in an unusual manner. The study tag starts with the duration category $(A=$ long term (over 26 weeks); $\mathrm{B}=$ medium term ( 13 to 26 weeks) and $\mathrm{C}=$ short term (up to 12 weeks); the remainder of the tag is the additional drug in the combination antipsychotic group (chlorpromazine has to be shortened to 'CPZ'). Finally, if two studies had similar names an alphabetical tag ( - b, - c) was added. 


\section{Results of the search}

Searches were originally carried out in 2010 and 2012. We supplemented these with a January 2016 search of the Cochrane Schizophrenia Group's Register of trials. Another trial (Xu 2006) was added as it appeared as a reference in one of the included trials (C +aripiprazole 2014). We included one trial (C +aripiprazole 2015b) that was found by methods not described in the protocol (please see Differences between protocol and review). From these searches 62 trials met the inclusion criteria. Sixty-two trials were excluded. There are three trials awaiting assessment (Characteristics of studies awaiting classification) and there are three ongoing studies (Figure 3).

Figure 3. Naming of the subgroups: The studies were arranged into four subgroups according to the type of antipsychotics used in both arms: clozapine, atypical antipsychotics other than clozapine, typical antipsychotics and any antipsychotics. Naming of the studies: The study tag starts with the duration category ( $\mathrm{A}=$ long term (over 
26 weeks); B = medium term (13 to 26 weeks) and C = short term (up to 12 weeks); the remainder of the tag is the additional drug in the combination antipsychotic group.

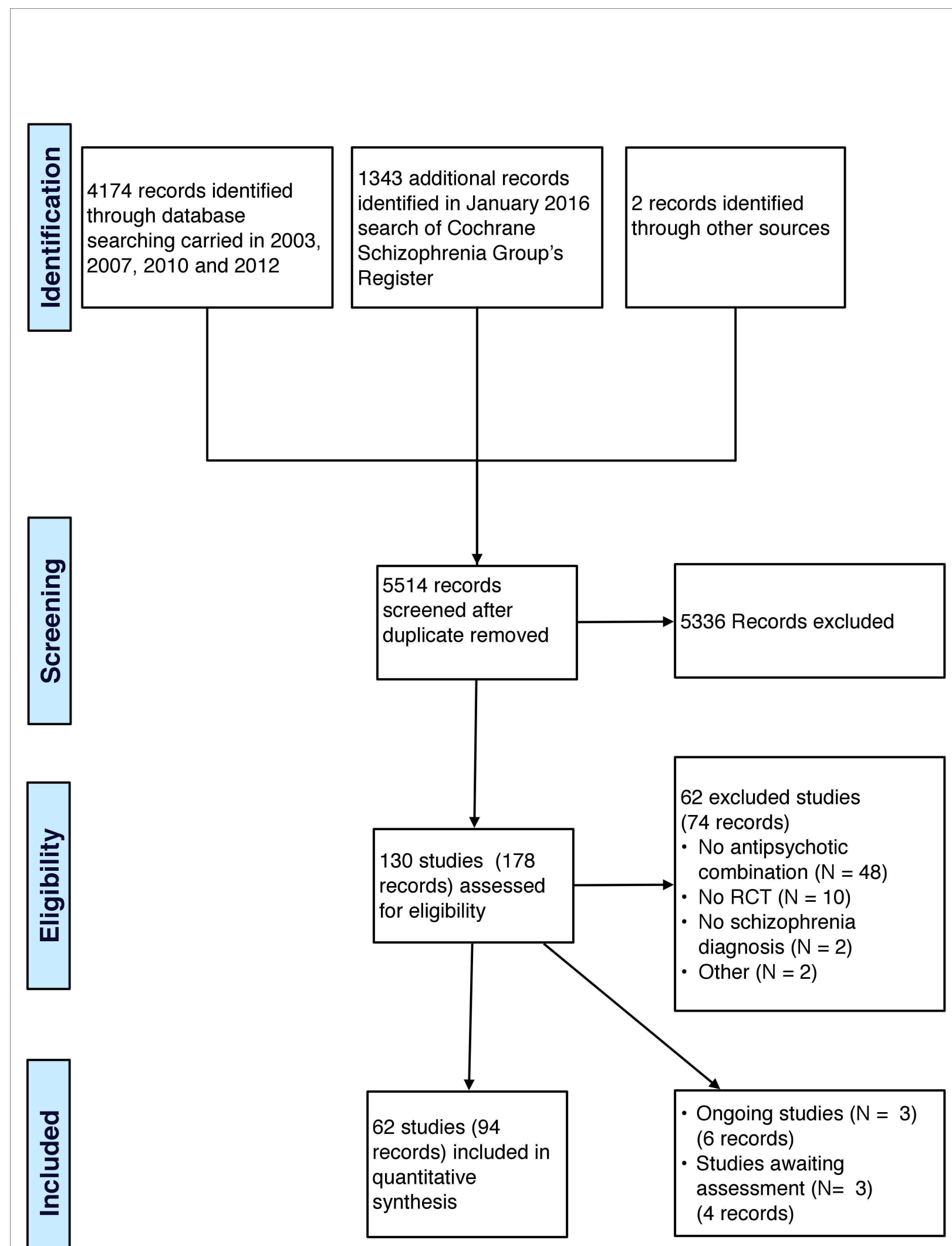


Figure 3. (Continued)

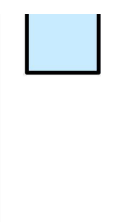

\section{Included studies}

The current review includes 94 reports describing 62 trials (4833 participants); 41 studies were two-arm trials comparing an antipsychotic monotherapy with a combination therapy; 12 trials were three-arm studies comparing two monotherapies with the combination therapy; four were three-arm trials comparing one monotherapy with two combinations, and three studies were fourarm trials comparing two monotherapies with two combinations. $C$ +aripiprazole 2015 was a four-arm trial comparing one combination therapy at three different doses against monotherapy, and, finally, A +pimozide 1985 was an eight-arm trial comparing two monotherapies at two different doses with one combination therapy at four different doses.

\section{Study duration}

Forty-seven of the included studies were short term in duration (less than 12 weeks, C). Eight were of medium term (13 to 26 weeks, B) and seven were long term (over 26 weeks, A).

\section{Design}

Most of the included studies presented a parallel longitudinal design. However A +reserpine 1957 and C +olanzapine 2012b, were cross-over trials and we only used data from the first phase of these trials until the point of the first cross-over. Nine were multi-centre trials; B +aripiprazole 2008 had centres across Europe and in South Africa, C +risperidone 2006 centres in Canada, Germany, China and the UK, and the other seven within their respective countries include (A +any antipsychotic 2011: USA; B +quet/risp 2009: USA; C +perphenazine 1976: Japan; C +aripiprazole 2013b: Korea; A +any antipsychotic 2015: USA; C + pimozide 2013: USA; C +olan/risp 2014; Japan).

\section{Participants}

A total of 4833 participants are included (average $\sim 78$ people per study). C +haloperidol 2006 and A +any antipsychotic 2012 did not report the country of origin. See also Appendix 3.

All studies included people with schizophrenia, schizophreniform psychoses, delusional disorder and schizoaffective psychoses. Several means of diagnoses were used. See Appendix 4.

Most studies included people that had chronic schizophrenia and/ or had experienced treatment failure while taking monotherapy antipsychotics. The average age was about 36 years old.

\section{Settings}

Thirty studies included inpatients, 16 studies included outpatients and seven studies both inpatients and outpatients. Two studies (C +aripiprazole 2009; C +sulpiride 1999) included participants in a community setting. Seven studies did not report the setting (C +aripiprazole 2007b, C +aripiprazole 2014, C +haloperidol 2006, C +pipotiazine 2000, C +sertindole 2006, C +sulpiride 1997, and C +sulpiride 2006).

\section{Interventions}

Full details of the doses used are reported in Characteristics of included studies and Appendix 5. We arranged the studies into four subgroups according to the type of antipsychotics used in both the monotherapy and combination group: clozapine, atypical antipsychotics other than clozapine, typical antipsychotics and any antipsychotics.

In order to determine if the doses used for the antipsychotics in the monotherapy groups were standard, we compared the dosages used in the clinical trials versus dosages suggested by Hasan 2012 and Gardner 2010. We decided not to appraise the interventions in the combination group since there is no evidence for the optimal regimen.

\section{Clozapine in both groups}

Thirty-one studies tested clozapine in both the monotherapy and combination arms of the trial. In 26 of these studies an atypical antipsychotic was added to clozapine in the combination therapy, and in five studies a typical antipsychotic was added to clozapine.

Three studies (B +risperidone 2010, C +olanzapine 2012b and C +risperidone 2005) and of the 31 clozapine studies did not report the doses used. One study ( $C+$ sulpiride 2006) used belowstandard doses of clozapine in the monotherapy group. In 25 studies, standard doses of clozapine were used. C +pimozide 2013 reported blood levels and showed higher blood levels of clozapine in the combination group. All except two studies used only oral antipsychotics; B +pipotiazine 2002 and C +pipotiazine 2000 included oral clozapine and pipotiazine administered through muscle injection.

\section{Other atypical antipsychotics in both groups}

Eighteen studies tested atypical antipsychotics (other than clozapine) in both the monotherapy and combination therapy arms of the trial. In two of these trials, a typical antipsychotic was added to an atypical one in the combination therapy, and in the other 16 studies, the combination therapy consisted of two atypical antipsychotics. One study (C +clozapine 2013) did not report the doses used. The rest of the studies used a standard dose of the antipsychotic in the monotherapy group. All except one study used only oral antipsychotics; C +fluphen dec 2009 included oral olanzapine and fluphenazine decanoate administered through muscle injection. 


\section{Typical antipsychotic in both groups}

Nine studies tested typical antipsychotics in both arms. In four of these trials, an atypical antipsychotic was added to the typical antipsychotic in the combination therapy, and in five studies, the combination therapy consisted of two typical antipsychotics. Three studies (C +aripiprazole 2007b; C +aripiprazole 2009; C +levomepromazine 2004) did not report the doses used. Regarding the monotherapy group, three studies used standard doses, two studies (A +pimozide 1985; C +perphenazine 1976) used belowstandard doses, and one study (C +CPZ 1973) used above-standard doses. All except one study used only oral antipsychotics; C +CPZ 1973 included oral chlorpromazine and fluphenazine decanoate administered through muscle injection.

\section{Any antipsychotic in both groups}

Four trials are included in this subgroup. All except one study included participants already on any combination of antipsychotics who were randomised to monotherapy by discontinuation of one of their current antipsychotics and therefore included any combination of two antipsychotics in the combination arm and any one antipsychotic in the monotherapy arm. Doses were reported as haloperidol, chlorpromazine or olanzapine equivalent. Two trials used standard doses, one study ( $A$ +any antipsychotic 2015), used above-standard doses in both groups. A tany antipsychotic 2012 included participants treated with monotherapy who were randomised to switch to combination therapy by adding another antipsychotic or to continue receiving monotherapy. The medication to be added was decided by the prescriber and the patient; no doses were reported for this study.

\section{Outcomes}

The included studies provided data for the following outcomes: leaving the study early, clinical improvement, relapse, adverse events (serious or requiring discontinuation, death, movement disorders, prolactin level and weight gain), and used various scales to assess treatment effects in global state, mental state general and specific symptoms, movement disorders and quality of life.

\subsection{Outcome scales}

Only details of scales that provided usable data are shown below. Fifteen different instruments were used to collect scale data. Overall, scale data were poorly presented.

\section{Global state}

i. Clinical Global Impression Scale - CGI Scale (Guy 1976) This is used to assess both severity of illness and clinical improvement, by comparing the conditions of the person standardised against other people with the same diagnosis. A seven-point scoring system is usually used with low scores showing decreased severity and/or overall improvement. CGI-Severity (CGI$\mathrm{S}$ ) is one component of the $\mathrm{CGI}$, which rates illness severity and $\mathrm{CGI}$-Improvement (CGI-I) rates improvement. High scores indicate a worse outcome.

ii. Global Assessment Scale of Functioning Scale (GAF) (APA 2000). This is a modified version of the Global Assessment Scale (GAS) (Endicott 1976), an observer-rated scale for evaluating the overall functioning of a patient during a specified time period on a continuum from psychological or psychiatric sickness to health. Score ranges from zero to 100 , where a higher score indicates a better outcome.

\section{Mental state}

i. Positive and Negative Syndrome Scale - PANSS (Kay 1987) This schizophrenia scale has 30 items, each of which can be defined on a seven-point scoring system varying from one - absent to seven - extreme. This scale can be divided into three sub-scales for measuring the severity of general psychopathology, positive symptoms (PANSS-P), and negative symptoms (PANSS-N). A low score indicates lesser severity.

\section{ii. Brief Psychiatric Rating Scale - BPRS (Overall 1962)}

This is used to assess the severity of abnormal mental state. The original scale has 16 items, but a revised 18 -item scale is commonly used. Each item is defined on a seven-point scale varying from 'not present' to 'extremely severe', scoring from zero to six or one to seven. Scores can range from zero to 126 , with high scores indicating more severe symptoms.

iii. Scale for the Assessment of Positive Symptoms - SAPS (Andreasen 1984)

This six-point scale gives a global rating of positive symptoms such as delusions, hallucinations and disordered thinking. Higher scores indicate more symptoms.

iv.Scale for the Assessment of Negative Symptoms - SANS (Andreasen 1983)

This scale allows a global rating of the following negative symptoms: alogia (impoverished thinking), affective blunting, avolition-apathy, anhedonia-asociality, and attention impairment. Assessments are made on a six-point scale from zero (not at all) to five (severe). Higher scores indicate more symptoms.

\section{Movement disorders}

i. Barnes Akathisia Scale - BAS (Barnes 1989)

A scale consisting of four sub-scales to assess the severity of akathisia: objective rating (zero to three), subjective awareness of restlessness (zero to three), subjective distress related to restlessness (zero to three), and global clinical assessment of akathisia (zero to five). Higher scores indicate more severe akathisia.

ii. Abnormal Involuntary Movement Scale - AIMS (Guy 1976)

The AIMS is a 12-item scale consisting of a standardised examination followed by questions rating the orofacial, extremity and trunk movements, as well as three global measurements. Each of these 10 items can be scored from zero (none) to four (severe). Two additional items assess the dental status. The AIMS ranges from zero to 40 , with higher scores indicating greater severity.

\section{iii. Simpson Agnus Scale - SAS (Simpson 1970)}

This scale contains 10 items: gait, arm dropping, shoulder shaking. elbow rigidity, wrist rigidity, leg pendulousness, head dropping, glabella tap, tremor and salivation. Each item is rated between zero and four. A total score is obtained by adding the items and dividing by 10 . Scores of up to 0.3 are considered within the normal range. Higher scores indicate greater severity.

iv. Udvalg for Kliniske Undersøgelser Side Effect Rating Scale - UKU (Lingjaerde 1987)

A comprehensive, clinician-rated scale, designed to assess the side effects in patients treated with psychotropic medications. The UKU consists of 48 questions. Zero indicates normal; one indicates mild 
symptoms; two indicates moderate symptoms; and three indicates severe symptoms. Higher scores indicate greater severity.

v. Drug-Induced Extrapyramidal Symptoms Scale - DIEPSS (Kim 2002)

The DIEPSS developed in Japan consists of four sub-scales for Parkinsonism (five items), akathisia, dystonia, and dyskinesia in combination with a global evaluation. Each item of assessment is rated on a five-point scale. The severity of each item is graded from zero (normal) to four (severe), higher scores indicate more severe symptoms.

vi. Extrapyramidal Symptom Rating Scale - ESRS (Chouinard 1980) The ESRS measures movement disorders and scores range from zero to 246. There are sub-scales for parkinsonism (zero to 108), dystonia (zero to 96), and dyskinesia (zero to 42). Higher scores indicate more severe symptoms.

\section{Quality of life}

i. Quality of Life Scale - QLS (Heinrich 1984)

This six-point quality of life scale has been designed as an outcome instrument for schizophrenic deficit syndrome as well as to measure impaired functioning in studies of chronic schizophrenia, to assess the deficit syndrome's impact on the patient's life. There are seven severity steps (zero to six, six being adequately functioning and zero being deficient). The time frame is one month. Four item categories have been identified by factor analysis 1 ) interpersonal relationships (seven items), 2) instrumental role (four items), 3) intrapsychic function (seven items) and 4) commonplace objects and activities.

ii. Subjective well-being under neuroleptic treatment scale - SWN (Naber 1995)

This 38-item scale with five factors self-rating scale measures subjective well-being on neuroleptics. The 20 positively- and 18 negatively-phrased items are rated on a zero to five scale, from not at all, to very much. The five factors are 1) emotional regulation, 2) self-control, 3) mental functioning, 4) social integration and 5) physical functioning. Low scores predict non-compliance or discontinuation of treatment in maintenance periods.

\section{iii. Short form-36 - SF-36 (Ware 1992)}

This is a 36-item scale with two components, one measures the physical component and the other the mental component, the scores range from zero to 100 . Each scale is subdivided in four factors. For the physical component: 1) physical functioning, 2) role-physical, 3) bodily pain, and 4) general health; and for the mental component: 1) vitality, 2) social functioning, 3) roleemotional, and 4) mental health. Lower scores indicate more disability.

\section{Excluded studies}

We excluded 62 studies from the review (Characteristics of excluded studies). Three trials (Barbui 2011, Zink 2009, JPRNUMIN000017047) compared two combinations of antipsychotics but did not include a monotherapy. Wu 2015 compared two combinations of antipsychotics with the addition of a systematic nursing intervention. Eighteen studies were randomised control trials testing an antipsychotic combination, but the combinations did not include two antipsychotics. Twelve studies were randomised control trials comparing different antipsychotic monotherapies with another intervention. Seven studies were randomised control trials evaluating switching strategies to a different antipsychotic. Four trials (Sukegawa 2008, Sukegawa 2014, Yamanouchi 2015 and DRKS00008018) did not evaluate the combination of antipsychotics. Semenikhin 2013, NCT01939548 and NCT02477670 did not test antipsychotic drugs. Mantovani 2013 and Mythri 2013 did not evaluate participants with a diagnosis of schizophrenia. Ten studies were not randomised controlled trials. Henderson 2009 was a crossover trial that did not report the results separately for each phase. JPRN-UMIN000011710 ended without enrolling any patient.

\section{Awaiting classification}

There are three trials awaiting classification (Studies awaiting classification):

NCT01450514 is a clinical trial, which according to the principal investigator, enrolled patients but was concluded prematurely due to funding problems. We tried to obtain the data from the patients that started the trial, but the sponsor decided to keep the data confidential.

Xu 2006 is a clinical trial that evaluated the effects of aripiprazole compared with placebo on females with hyperprolactinaemia induced by antipsychotics. The placebo used for this trial was vitamin C (100 mg/day), which might have a significant effect on the symptoms of schizophrenia (Magalhães 2016).

Yuan 2014 is a clinical trial with multiple treatment stages. In the third stage, participants were able to receive a combinations of antipsychotics. We tried to obtain data regarding the participants who were enrolled on this stage but no response was received.

\section{Ongoing studies}

There are three ongoing studies (Characteristics of ongoing studies). One tests amisulpiride augmentation in clozapineunresponsive schizophrenia (ISRCTN68824876), one olanzapine and amisulpiride (Schmidt-Kraepelin 2013), and one aripiprazole augmentation for participants with weight problems treated with clozapine (CTRI-02-003397).

\section{Risk of bias in included studies}

We prepared a 'Risk of bias' assessment for each trial. For multicentre trials providing data for a single centre, we did not assess the risk of bias for each centre. Our judgments regarding the overall risk of bias in individual studies is illustrated in Figure 1 and Figure 2.

\section{Allocation}

Of the 62 trials analysed in this review, 18 reported an adequate generation of allocation sequence. In two studies ( $B$ +sulpiride 1996; C +fluphen dec 2009) the risk of bias was high for sequence generation as a quasi-randomised method was used, and three studies (C +CPZ 1999, C +sulpiride 1999, C +sulpiride 1999b) had a high risk of bias as they randomised according to hospital admission order or time. In all remaining studies, the method of assignment was unclear. Similarly, methods used to conceal allocation had a low risk of bias in 16 trials, high risk of bias in one and unclear in the remainder (please see Differences between protocol and review). 


\section{Blinding}

In 19 studies, participants, care providers, and outcome assessors were blinded, 12 studies were high risk of bias for blinding as they were either open-label studies or the participants and personnel were not blinded; the risk of bias was unclear for the remaining 31 trials.

\section{Incomplete outcome data}

There was a low risk of bias for incomplete data in 33 studies, an unclear risk of bias in nine studies, and a high risk of bias in 20 trials.

\section{Selective reporting}

Twenty-three studies were free from selective reporting, 36 studies had a high risk of bias for selective reporting, and three had an unclear risk of bias.

\section{Other potential sources of bias}

Twenty-three studies were free from other biases, eight were subject to other biases and in the remaining studies the risk of bias was unclear.

\section{Effects of interventions}

See: Summary of findings for the main comparison Combinations of antipsychotic drugs compared to single antipsychotic drugs for schizophrenia

Where data were available, they were arranged into four subgroups according to the type of antipsychotics used in both arms: clozapine, atypical antipsychotics other than clozapine, typical antipsychotics and any antipsychotics. Studies were also named according to the add-on antipsychotic (see Description of studies), so it is possible to see in each analysis more information about the combination of antipsychotics used in each study, as well as the length of follow-up.

Where data were missing, such as standard deviations for continuous outcomes, we imputed these data using trials with similar means for that scale. We used the mean difference and reported the data separately for different scales within an outcome (Appendix 6).

For studies with more than two comparison groups we combined data, i.e. if the study tested different antipsychotics in two monotherapy groups or two combination groups. Where studies had two monotherapy groups, for studies with typical drugs in both groups data from the monotherapy groups were combined; for studies with clozapine in both groups, only data from the clozapine monotherapy group was added to the data analysis.

\section{COMPARISON 1: ANTIPSYCHOTIC COMBINATIONS vS ANTIPSYCHOTIC MONOTHERAPY}

This particular comparison has 63 outcomes.

\subsection{Clinical response: 1 . No clinically important response - not improved}

We found twenty-nine trials ( $N=2398)$, with six weeks to three years follow-up. We found that the use combination of antipsychotics may reducing the risk of no clinical response (RR $0.73 \mathrm{Cl} 0.64$ to 0.83; Analysis 1.1; very low quality evidence). Results showed an important heterogeneity $\left(I^{2}=54 \%\right)$. When we split the trials by length of follow-up, the results remain but there is no heterogeneity for the longer-term trials - the heterogeneity may be due to the short-term trials.

\subsection{1 clozapine in both groups}

Trials with clozapine ( $N=1127)$; in both groups also favoured the combination therapy (RR $0.66 \mathrm{Cl} 53$ to 0.83), but had high heterogeneity $\left(I^{2}=64 \%\right)$ and no obviously outlying trials.

\subsection{2 other atypical in both groups}

Seven trials tested atypical in both groups $(N=674)$. There was not a clear difference between antipsychotic combinations and antipsychotic monotherapy within this subgroup (RR $0.95 \mathrm{Cl} 0.83$ to 1.09).

\subsection{3 typical drugs in both groups}

We found five trials to be relevant to this subgroup, which included a total of 597 participants. For this outcome, we did find evidence that antipsychotic combinations reduced the risk of no response when compared with antipsychotic monotherapy (RR $0.64 \mathrm{Cl} 0.49$ to 0.84$)$. For this subgroup heterogeneity is moderately high $\left(1^{2}=\right.$ 47\%) but when the outlying trial C + perphenazine 1976 is removed the results show no heterogeneity.

\subsection{Clinical response: 2. Relapse}

Three trials ( $N=512)$, with follow-up durations of eight weeks, one year and three years, respectively provided data regarding relapse. Results showed high heterogeneity $\left(I^{2}=81 \%\right)$, when the outlying study (A + pimozide 1985) is removed heterogeneity is restored. But as this trial carries more than $10 \%$ of the weighting for this outcome, the results were not pooled. A +sulpiride 1994 and C + perphenazine 1976 found no difference between the two interventions. A +pimozide 1985 found that antipsychotics combinations is more effective for preventing relapse when compared to monotherapy.

\subsection{Leaving the study early}

Forty-three trials ( $\mathrm{N}=3137$ ), with six weeks to one year followup found no difference in the number of people leaving the study early (RR $0.90 \mathrm{Cl} 0.76$ to 1.07 ; Analysis 1.3, low-quality evidence). Subgroup analysis showed no important difference between groups.

\subsection{Service utilisation: Hospital admission}

Three trials ( $N=202)$, with follow-up duration of eight, ten weeks and six months, respectively, provided data on hospital admission. Two trials tested clozapine in both groups, and the other tested any antipsychotics in both groups. A combination of antipsychotics was not superior or inferior to antipsychotic monotherapy in preventing hospital admission (RR $0.96 \mathrm{Cl} 0.36$ to 2.55; Analysis 1.4, very lowquality evidence). None of the subgroups showed different results.

\subsection{Clinical response: 3 . Global state - i. average severity score (CGI-S scale, high = bad)}

For this outcome we found seven relevant studies which provided endpoint data regarding global state on the severity component of the CGI scale, with six weeks to three years follow-up involving 496 participants. For this outcome, we did not find evidence that antipsychotic combinations was different in its effects compared with antipsychotic monotherapy (MD $-0.13 \mathrm{Cl}-0.31$ to 0.06 , Analysis 
1.5). This outcome had moderate levels of heterogeneity $(12=44 \%)$. None of the subgroups showed different results.

\subsection{Clinical response: 3 . Global state - ii. change in severity score (CGI-S scale, high = bad)}

Three relevant $(\mathrm{N}=233$ ) studies involving 233 participants only provided change data regarding this scale. For this outcome, we did not find evidence that antipsychotic combinations was clearly different in its effects compared with antipsychotic monotherapy (MD 0.11 Cl-0.09 to 0.32; Analysis 1.6).

\subsection{Clinical response: 4. Global state - average improvement score $(\mathrm{CGI}-\mathrm{I}$ scale, high = bad)}

Four trials, with ten to 16 weeks follow-up, measured global state on the improvement component of the CGI scale. We found that the combination therapy may improve clinical response when compared to monotherapy (MD $-0.36 \mathrm{Cl}-0.58$ to -0.13 ; Analysis 1.7).

\subsection{Clinical response: 5 . Global state - $i$. average functioning score (GAF scale, high = good)}

We identified three studies relevant to this outcome, with 6 to 12 weeks follow-up, involving 107 participants. For this outcome heterogeneity is high $(12=80 \%)$. When $C$ +risperidone 2005 is removed, heterogeneity is restored but as this trial carries more than $10 \%$ of the weighting for this outcome, the results were not pooled and we only presented the data for the subgroups:

\subsubsection{Clozapine in both groups}

There is a single trial in this subgroup, which included a total of 30 participants. We found evidence that antipsychotics combination is worse than monotherapy for improvement of the global state (MD $-4.5 \mathrm{Cl}-8.38$ to -0.62 ; Analysis 1.8$)$.

\subsubsection{Other atypical drugs in both groups}

There are two relevant trials in this subgroup, which included a total of 77 participants. We found evidence that the use antipsychotic combinations when compared to antipsychotic monotherapy improves the global state when assessed with the GAF scale (MD $8.73 \mathrm{Cl} 1.56$ to 15.9 ; Analysis 1.8).

\subsection{Clinical response: 5 . Global state - ii. change in functioning} score (GAF scale, high = good)

We found three studies ( $\mathrm{N}=349$ ) which provided only change data for the GAF scale, we did not find evidence of a clear difference between the two treatments in this comparison (MD $0.27 \mathrm{Cl}-1.42$ to 1.97; Analysis 1.9).

\subsection{Mental state: 1. Overall - a.i average total score (PANSS scale, high $=$ bad)}

We identified 11 studies relevant to this outcome involving 721 participants. We did not find evidence of a clear difference between the two treatments in this comparison. This outcome had important levels of heterogeneity $(12=58 \%)$. When $B+$ ziprasidone 2014 and C +risperidone 2001 are removed, heterogeneity is decreased but as these trials carry more than $10 \%$ of the weighting for this outcome, the results were not pooled.
1.11 Mental state: 1. Overall - a.ii change in total score (PANSS scale, high = bad)

Eight studies ( $N=406)$ only provided change data for the PANSS scale, we did not find evidence of a clear difference between the two treatments in this comparison (MD $-1.05 \mathrm{Cl}-3.42$ to 1.32 ; Analysis 1.11). Subgroup analysis showed no difference.

\subsection{Mental state: 1. Overall - b.i. average total score (BPRS scale, high $=$ bad)}

We found 21 trials $(N=1082)$, with six weeks to six months follow-up, who reported data for mental state on the BPRS scale, but results showed high heterogeneity $\left(I^{2}=92 \%\right.$; Analysis 1.12$)$. Removal of the outlying studies C + sulpiride 1999b and C + sulpiride 2003 reduces heterogeneity for the clozapine subgroup $\left(I^{2}=47 \%\right)$, but not for the pooled results $\left(I^{2}=81 \%\right)$. Data were, therefore, not pooled for this outcome.

\subsection{Mental state: 1. Overall - b.ii change total score (BPRS scale, high $=$ bad)}

We identified one study which only provided change data for this outcome involving 100 participants. We did find evidence that antipsychotic combinations improved the overall mental state when evaluated with the BPRS scale (MD $-2.72 \mathrm{Cl}-5.37$ to -0.07 ; Analysis 1.13).

\subsection{Mental state: 2 . Specific - a. positive symptoms - no clinical improvement}

Two trials, with six and 10 weeks follow-up, reported binary data for no clinical improvement on positive symptoms, but the results showed high heterogeneity $\left(I^{2}=80\right.$; Analysis 1.14). Data were, therefore, not pooled for this outcome. None of the studies showed a difference between the two groups.

1.15 Mental state: 2. Specific - b. positive symptoms - i. average score (PANSS scale, high = bad)

For this outcome we found four relevant studies involving 158 participants. We found evidence that participants assigned to antipsychotics combinations had a poorer response to the positive symptoms than patients assigned to antipsychotics monotherapy (MD $2.02 \mathrm{Cl} 0.90$ to 3.14; Analysis 1.15). The results are due to the trials in the subgroup where clozapine was used in both groups.

1.16 Mental state: 2. Specific - b. positive symptoms - ii. change score (PANSS scale, high $=$ bad)

We identified nine studies who reported only change data for the positive symptoms assessed with the PANSS scale. We did not find evidence of a clear difference between antipsychotic combinations and antipsychotic monotherapy (MD $0.01 \mathrm{Cl}-0.45$ to 0.47 ; Analysis 1.16).

\subsection{Mental state: 2 . Specific - b. positive symptoms - iii. average score (BPRS scale, high $=$ bad)}

We identified three studies, with a follow-up time between eight and 16 weeks, we did not find evidence of a clear difference between antipsychotic combinations and antipsychotic monotherapy (MD $-1.02 \mathrm{Cl}-2.42$ to 0.38 ; Analysis 1.17). This outcome had moderate levels of heterogeneity $\left(I^{2}=41 \%\right)$. We did not find a difference in the results between subgroups. 
1.18 Mental state: 2 . Specific - b. positive symptoms - iv. change data (BPRS scale, high $=$ bad)

We identified one study $(\mathrm{N}=17)$ which only reported change data for the positive symptoms when assessed with the BPRS scale. We did not find evidence of a clear difference between antipsychotic combinations and antipsychotic monotherapy (MD $-0.3 \mathrm{Cl}-1.16$ to 0.56; Analysis 1.18)

\subsection{Mental state: 2. Specific - b. positive symptoms - v. average score (SAPS scale, high $=$ bad)}

We identified one study relevant to this outcome involving 28 participants. For this outcome, we did find evidence that antipsychotic combinations is better than monotherapy for the positive symptoms when assessed with the SAPS scale (MD $-6.76 \mathrm{Cl}$ -11.91 to -1.61 , Analysis 1.19)

1.20 Mental state: 2 . Specific - b. positive symptoms - vi. change score (SAPS scale, high = bad)

One study provided only change data for this scale. We found evidence that antipsychotic combinations is better at reducing the positive symptoms when compared to monotherapy (MD $-5.8 \mathrm{Cl}$ -11.33 to -0.27 , Analysis 1.20 )

\subsection{Mental state: 3 . Specific - a. negative symptoms - no clinical improvement}

Three trials, with six to ten weeks follow-up, reported binary data for no clinical improvement on negative symptoms, but the results showed high heterogeneity $\left(\mathrm{I}^{2}=65\right.$; Analysis 1.21). Removal of $C$ +risperidone 2005 restores homogeneity and the results become significant (RR $0.80 \mathrm{Cl} 0.65$ to 0.98 ), but as this trial carries more than $10 \%$ of the weighting for this outcome, the results were not pooled.

1.22 Mental state: 3. Specific - b. negative symptoms - i. average score (PANSS scale, high $=$ bad)

For this outcome we found five relevant studies involving 194 participants. For this outcome heterogeneity is high $(12=57 \%)$. The heterogeneity is due to $B$ +any antipsychotic 2013 which belongs to the subgroup 'Any antipsychotic in both groups', when this trial is removed heterogeneity is resolved. Because this trial carries more than $10 \%$ of the weighting for this outcome, the results were only presented in subgroups.

\subsection{1 clozapine in both groups}

We found three trials to be relevant to this subgroup $(\mathrm{N}=119)$. For this subgroup, we did not find evidence of a clear difference between the two treatments (MD $0.31 \mathrm{Cl}-1.18$ to 1.8 ; Analysis 1.22).

\subsection{2 other atypical drugs in both groups}

We found one trial to be relevant to this subgroup, with a total of 36 people. For this subgroup, we did not find evidence of a clear difference between the two treatments (MD $1.2 \mathrm{Cl}-1.51$ to 3.91 ; Analysis 1.22).

\subsubsection{Any antipsychotic in both groups}

We found one trial to be relevant to this subgroup, with a total of 39 people. We found evidence that antipsychotics combinations is worse for improving the negative symptoms when compared to antipsychotic monotherapy (MD $3.3 \mathrm{Cl} 1.6$ to 5.0; Analysis 1.22).
1.23 Mental state: 3 . Specific - b. negative symptoms - ii. change score (PANSS scale, high = bad)

We identified nine studies involving 891 participants. The studies only reported change score. We did not find evidence of a clear difference between antipsychotic combinations and antipsychotic monotherapy (MD $0.02 \mathrm{Cl}-0.54$ to 0.58 , Analysis 1.23 ). No important difference was found between subgroups.

1.24 Mental state: 3. Specific - b. negative symptoms - iii. average score (BPRS scale, high = bad)

We identified two studies relevant to this outcome. We did not pooled the data because heterogeneity was high $\left(1^{2}=56 \%\right)$ and we only present the results by subgroups.

\subsection{1 clozapine in both groups}

There is a single trial in this subgroup, which included a total of 61 participants. There was not a clear difference between antipsychotic combinations and antipsychotic monotherapy within this subgroup (MD $-4.3 \mathrm{Cl}-12.25$ to 3.65 ; Analysis 1.24).

\subsection{2 other atypical drugs in both groups}

There is a single trial in this subgroup, which included a total of 40 participants. We found evidence that antipsychotic combinations is worse at improving the negative symptoms when compared to antipsychotic monotherapy (MD $1.9 \mathrm{Cl} 0.69$ to 3.11; Analysis 1.24)

1.25 Mental state: 3. Specific - b. negative symptoms - iv. change score (BPRS scale, high = bad)

For this outcome we found a single trial $(N=12)$. We did not find evidence of a clear difference between antipsychotic combinations and antipsychotic monotherapy (MD $0.2 \mathrm{Cl}-0.29$ to 0.69 ; Analysis 1.25)

1.26 Mental state: 3 . Specific - b. negative symptoms - v. average score (SANS scale, high $=$ bad)

Eleven trials, with six to 16 weeks follow-up, measured negative symptoms on the SANS scale (Analysis 1.26). These results were not pooled in the analysis as they showed high heterogeneity $\left(I^{2}\right.$ $=96 \%$ ). Most studies tested clozapine in both groups, but again with high heterogeneity $\left(I^{2}=93 \%\right)$. Removing the outlying trials C +risperidone 2005b, B +sulpiride 1996, C +sulpiride 1999b and C +sulpiride 2003 does restore heterogeneity for the clozapine subgroup but does not affect the overall results. They account for more than $10 \%$ of the weighting and so again, results are not pooled. No important difference was found for other atypical drugs or typical in both groups.

\subsection{Mental state: 3 . Specific - b. negative symptoms - vi. average score (SANS scale, high $=$ bad)}

We found one trial who only reported change data for this scale, which included a total of 28 participants. We found evidence that the use of antipsychotic combinations is better at improving the negative symptoms when compared to antipsychotic monotherapy (MD $-6.80 \mathrm{Cl}-12.65$ to -0.95 ; Analysis 1.27 ).

1.28 Mental state: 4. Specific - aggression/agitation - average score (BPRS scale, high $=$ bad)

One trial ( $N=12)$, with a follow-up duration of eight weeks, only provided data for change in aggression/agitation symptoms when 
assessed with the BPRS scale. We found evidence in favour of combination therapy in improving aggression/agitation symptoms (MD - $1.30 \mathrm{Cl}-2.32$ to -0.28; Analysis 1.28).

\subsection{Adverse events: 1. General - a. serious event or requiring discontinuation}

Thirty trials ( $N=2398)$, with six weeks to eight months followup, did not find an important difference in the number of adverse events that were serious or required discontinuation (RR $1.05 \mathrm{Cl}$ 0.65 to 1.69; Analysis 1.29, very low quality of evidence). None of the subgroups of antipsychotics showed a significant difference.

\subsection{Adverse events: 1. General - b. death (suicide or non- suicide deaths)}

Only four trials reported on deaths, with follow-up durations of eight to 12 weeks. There was only one death reported in the combination group and no deaths in the monotherapy group Analysis 1.30 .

\subsection{Adverse events: 2. Movement disorders - a. any}

Twenty trials, with 30 days to three years follow-up, provided binary data regarding movement disorders. No difference was found between combination therapy and monotherapy in the number of participants experiencing movement disorders (RR $1.07 \mathrm{Cl} 0.92$ to $1.25 ; \mathrm{N}=1868$; studies = 20; Analysis 1.31 ), and none of the subgroups of antipsychotics showed a significant difference.

Movement disorders were also measured on eight scales (Barnes Akathisia Scale (BAS), Abnormal Involuntary Movement Scale (AIMS), Simpson Angus Scale (SAS), Udvalg for Kliniske Undersøgelser (UKU), Treatment Emergent Symptom Scale (TESS), Extrapyramidal SymptomRating Scale (ESRS) and Drug-Induced Extrapyramidal Symptoms Scale (DIEPSS). However, much of the data were considerably skewed and are reported in Appendix 7.

\subsection{Adverse events: 2 . Movement disorders - b.i. average scores (SAS, high = bad)}

Nine trials reported on movement disorders using the SAS, but in six of them, the data were very skewed and not added to the analysis. The pooled data for the three remaining trials showed very high heterogeneity $\left(I^{2}=99 \%\right.$; Analysis 1.32) and so were not pooled. The heterogeneity might be due to the difference in the properties of the drugs, as C +fluphen dec 2009 is using a typical antipsychotic.

\subsection{Adverse events: 2. Movement disorders - b.ii. change scores (SAS, high $=$ bad)}

We found one trial $(N=63)$ who only reported change data for the SAS scale, which also did not showed any difference between the two intervention groups (Analysis 1.33).

\subsection{Adverse events: 2 . Movement disorders - b.iii. average scores (TESS, high $=$ bad)}

Again, three out of the five trials that reported movement disorders on the TESS reported skewed data. Heterogeneity was very high for the other two trials $\left(I^{2}=99 \%\right)$ and so they were not pooled. Similarly to the findings on the SAS, the trial ( $C+C P Z$ 1999) using a typical antipsychotic resulted in worse movement disorders for the combination group (MD $5.80 \mathrm{Cl} 5.03$ to 6.57; Analysis 1.34); the trial with other atypical drugs showed no difference between monotherapy and combination.
1.35 Adverse events: 2 . Movement disorders - b.iv. average scores (DIEPSS, high = bad)

One trial (C +aripiprazole 2008), with a follow-up duration of eight weeks, which tested clozapine in both the combination and monotherapy groups, found no important difference in movement disorders when measured on the DIEPSS scale (MD $0.30 \mathrm{Cl}-0.49$ to 1.09; participants $=61$; studies $=1$; Analysis 1.35).

\subsection{Adverse events: 2. Movement disorders - b.v. change scores (BAS, high = bad)}

Ten trials reported on movement disorders using the BAS, but in eight, data were very skewed and were not added to the analysis. Two trials, with follow-up duration of six weeks, which tested atypical antipsychotics in both the combination and monotherapy groups, found no significant difference in movement disorders when measured on the BAS scale (MD $-0.70 \mathrm{Cl}-1.54$ to $0.14 ; \mathrm{N}=91$; studies $=2$; Analysis 1.36). C +aripiprazole 2015b reported that all participants scored zero at follow-up.

\subsection{Adverse events: 2 . Movement disorders - b.vi. change scores (AIMS, high = bad)}

Five trials reported on movement disorders using the AIMS, but in four studies data were very skewed and not added to the analysis. One trial ( $N=63)$, with follow-up duration of six weeks, which tested atypical antipsychotics in both the combination and monotherapy groups, found no significant difference in movement disorders when measured on the AIMS scale (MD $0.10 \mathrm{Cl}-0.84$ to 1.04; Analysis 1.37).

\subsection{Adverse events: 3 . Endocrine - prolactin level (high = bad)}

Fifteen trials reported prolactin levels, but in eight, data were very skewed and not added to the analysis. In the seven remaining trials with six to 16 weeks follow-up, the pooled data regarding change in prolactin level had very high heterogeneity $\left(I^{2}=98 \%\right.$, Analysis 1.38). For studies with clozapine in both groups, data were also highly heterogeneous $\left(I^{2}=98 \%\right)$. When we split the data by trials using aripiprazole as the add-on antipsychotic, the heterogeneity is reduced $\left(1^{2}=66 \%\right)$ for the trials not using aripiprazole as an intervention. Data were not pooled for this outcome.

\subsection{Adverse events: 4. Metabolic - a. weight gain (binary)}

Six trials $(\mathrm{N}=804)$, with six to 16 weeks follow-up, provided data regarding the number of participants experiencing weight gain. No significant difference was found in the number of participants experiencing weight gain ( $\mathrm{RR} 1.00 \mathrm{Cl} 0.66$ to 1.53 ; Analysis 1.39).

\subsection{Adverse events: 4. Metabolic - b. average weight gain (kg)}

Nine trials, with six to 16 weeks follow-up, provided data regarding average weight gain, but results showed high heterogeneity $\left(1^{2}=\right.$ $53 \%$; Analysis 1.40). When B +aripiprazole 2008 is removed from the analysis, the heterogeneity is reduced $\left(I^{2}=4 \%\right)$, but as this trial carries more than $10 \%$ of the weighting for this outcome, the results were not pooled.

\subsection{Adverse events: 5. Blood - a. decreased white cell counts (binary)}

Two trials $(\mathrm{N}=315)$, tested clozapine in both groups with eight to 12 weeks follow-up provided data regarding number of participants experiencing decreased white cell counts. A significant difference 
was found in favour of the combination therapy (RR $0.18 \mathrm{Cl}$ 0.04 to 0.82 ; Analysis 1.41). The result could be explained by a dose-dependant side effect. The studies used higher doses in the clozapine monotherapy group when compared with the doses used in the combination group.

\subsection{Adverse events: 5 . Blood - b. average white cell counts (10-3/mm3)}

One trial ( $N=61$ ) with eight weeks follow-up reported data for white blood cell counts. No significant difference was found between the monotherapy and combination groups (MD $0.66 \mathrm{Cl}-0.20$ to 1.52 ; Analysis 1.42). A further four trials measured white blood counts but did not report any data: C +aripiprazole 2008 also found no difference between groups, $C+$ risperidone 2005b and C + sulpiride 1997 reported that there were no changes in white blood cell counts.

\subsection{Adverse events: 6. Central nervous system (CNS) - a. drowsiness}

Eleven trials, with six to 16 weeks follow-up, provided data regarding drowsiness, but results showed high heterogeneity $\left(I^{2}\right.$ $=67 \%$; Analysis 1.43). When the outlying trials $C$ +risperidone 2001 and C +sertindole 2006 are removed from the analysis heterogeneity restores both for the clozapine group and overall. As these trials carry more than $10 \%$ of the weighting for this outcome, the results were not pooled.

\subsection{Adverse events: 6. Central nervous system (CNS) - b. tremor}

Four trials $(\mathrm{N}=22)$, with six to 12 weeks follow-up, found no significant difference in the number of patients in the monotherapy or combination therapy groups experiencing tremors (RR $0.87 \mathrm{Cl}$ 0.47 to 1.62 ; Analysis 1.44 )

\subsection{Quality of life: 1a. Average score (QLS high=good)}

C +risperidone 2005 with 30 participants and a follow-up of six weeks, tested clozapine in both groups. This trial measured quality of life on the QLS scale and found no important difference between treatment groups (MD $0.80 \mathrm{Cl}-5.44$ to 7.04 ; Analysis 1.45).

\subsection{Quality of life: 1b. Average score (SWN, high=good)}

Two trials with eight to 16 weeks follow-up, which tested clozapine in both groups measured quality of life on the SWN scale and found no significant difference between treatment groups (MD $2.05 \mathrm{Cl}$ -1.08 to 5.18; Analysis 1.46). B +quet/risp 2009 also used the SWN scale, but did not report SDs and no suitable means were available to impute the data.

\subsection{Quality of Life: 1c. Average score - Mental component summary (SF-36, high = good)}

C +sulpiride $2013(N=60)$ with a follow-up of six weeks, measured the mental component of quality of life on the SF-36 scale and found no significant difference between treatment groups (MD 0.60 $\mathrm{Cl}-4.28$ to 5.48 ; Analysis 1.47 ).

\subsection{Quality of Life: 1d. Average score - Physical component summary (SF-36, high = good)}

Again, $C$ +sulpiride $2013(N=60)$ with a follow-up of six weeks, measured the physical component of quality of life on the SF-36 scale and found no significant difference between treatment groups (MD $-1.70 \mathrm{Cl}-4.71$ to 1.31 ).

\section{Other outcomes}

Although A +reserpine 1957, B +any antipsychotic 2013, C+CPZ 1973 and $C+$ pimozide 2011 measured behaviour and social functioning (on four different scales), none reported data that could be used in the analysis. Data were also not available on number of days in hospital, change in hospital status, employment status during trial, occurrence of violent incidents, levels of substance abuse, adverse events requiring hospitalisation, and allergic reactions. Studies did not report economic burden (cost of care), although two trials reported the cost of the therapies; $\mathrm{C}$ +haloperidol 2010 reported the cost of the combination therapy ( $2 \mathrm{mg}$ per day risperidone plus $2 \mathrm{mg}$ per day haloperidol, $\$ 1.26$ per day) was approximately half the cost of the monotherapy (4 mg per day risperidone, \$2.40) and $\mathrm{C}$ +sulpiride 2013 reported the cost of the combination therapy (amisulpride $400 \mathrm{mg}$ per/day plus sulpiride $800 \mathrm{mg} /$ day, US\$2.82/ day) was also approximately half of the cost of monotherapy (amisulpride $800 \mathrm{mg} /$ day, $\$ 4.88 /$ day).

\section{Subgroup analyses for clinical response: not clinically improved}

We used random-effects model for subgroup analyses. No subgroup differences were found for clinical response for subgroup analyses of chronic versus acutely ill people, length of treatment less than 12 weeks versus more than 12 weeks and between studies that tested clozapine in both groups versus all other studies (Analysis 1.49; Analysis 1.50; Analysis 1.51). A subgroup difference was found between the drugs added to clozapine ( $P$ $=0.009$; Analysis 1.52) with pipotiazine and sulpiride favouring the combination of antipsychotics and risperidone showing no differences in the number of participants not clinically improved. The pooled results showed high heterogeneity $\left(I^{2}=64 \%\right)$. However, when the risperidone subgroup is removed heterogeneity is restored.

In the meta-regression, we did not found an effect or an interaction by the potential modifiers: year of publication and Chinesse origin. The meta-regression model adjust was poor $(0.00 \%)$ (Table 1 ).

\section{Subgroup analyses for leaving the study early}

No subgroup differences were found for leaving the study early for the subgroup analyses of chronic versus acutely ill people, length of treatment less than 12 weeks versus more than 12 weeks, between studies that tested clozapine in both groups versus all other studies and between the drugs added to clozapine (Analysis 1.53; Analysis 1.54; Analysis 1.55; Analysis 1.56).

\section{Sensitivity analyses for clinical response: not clinically improved}

Three studies (B +sulpiride 1996; C +sulpiride 1999; C +sulpiride $1999 b)$ which reported data for clinical response had a high risk of bias for sequence generation. A significant subgroup difference $(P=0.03$; Analysis 1.57) was found between studies with a low or unclear risk of bias versus those with a high risk of bias for randomisation, with both groups favouring the combination of antipsychotics. No subgroup difference was found between studies with a low or unclear risk of bias versus those with a high risk of bias for blinding (Analysis 1.57). When data are synthesised using 
a fixed-effect model, results remain unchanged (RR $0.74 \mathrm{Cl} 0.68$ to 0.81; Analysis 1.59).

\section{Sensitivity analyses for leaving the study early}

Only one study that reported data for leaving the study early had a high risk of bias for sequence generation ( $C+$ fluphen dec 2009). This small study reported no losses to follow-up and so no sensitivity analysis was possible (Analysis 1.60). No subgroup difference was found between studies with a low or unclear risk of bias versus those with a high risk of bias for blinding (Analysis 1.61). When data for is synthesised using a fixed-effect model the results remain unchanged (RR $0.87,95 \% \mathrm{Cl} 0.76$ to 1.00 , Analysis 1.62) except for studies that tested typical drugs in both groups and any antipsychotic in both groups in which the combination therapy was favoured.

\section{DISCUSSION}

\section{Summary of main results}

The summary below reflects the outcomes chosen for the 'Summary of findings' table, and considers the main findings of this review that can support evidence-based decision making. For all outcomes included in the 'Summary of findings' table the quality of evidence was found to be either low or very low. Overall, findings from this review are that combination therapy does not have clear differences in its effects compared with monotherapy.

\section{Clinical response: not clinically improved}

Overall, we found evidence that combination therapy may be superior to monotherapy in improving clinical response. Subgroup analyses of different combinations of antipsychotics showed that this effect was due to the trials who included either clozapine in both groups or atypical drugs in both groups. It is important to note that these findings are mostly from short-term trials (22 trials of 12 weeks or less). Only two long-term trials (one year and three years) were identified. We, therefore, do not know what the efficacy might be if more trials had measured this outcome in the long term. Also, the definition of clinical response varied considerably across the studies hindering the interpretation of the findings. The finding that typical drugs in both groups may improve clinical response should be taken with caution, as all of the studies were undertaken more than 30 years ago, and translating these data to the actual clinical setting may be problematic. Nonetheless, the finding that adjunctive antipsychotic drug to either clozapine or a typical antipsychotics remains an interesting finding and worth considering to be the focus of further work. The very low quality of the evidence, as assessed with GRADE, diminish the confidence that can be placed in the magnitude of the effect.

\section{Clinical response: relapse}

There was a lack of information within the studies regarding relapse, most likely due to the duration of the trials (see above), and failure to report this outcome in the trials that were longer. Only three studies reported relapse - two of which were older studies comparing typical antipsychotics in both groups (A + pimozide 1985; C +perphenazine 1976) and one, a more recent trial, testing clozapine in both groups (A +sulpiride 1994). There was a lot of heterogeneity for this outcome, perhaps unsurprisingly given such different treatment drugs and lengths of trials: A +pimozide 1985 showed an important efficacy in favour of the combination treatment group for typical drugs over one year, and $C$ +perphenazine 1976 showed no difference over the course of eight weeks. A+sulpiride 1994, however, showed no difference over three years for clozapine in both groups. Currently, there is only sparse evidence on relapse for typical antipsychotic combination therapy and clozapine combination therapy, and oddly, a complete lack of evidence regarding other atypical drugs in combination.

\section{Leaving the study early}

In 43 RCTs between 0\% and 74\% (average 16\%) of people receiving antipsychotics (combination or monotherapy) left the study early. Overall, there was not convincing evidence that, at least within trials, combined antipsychotic treatment was any different to monotherapy for helping people stay longer. People do leave early for a variety of reasons, but the combination therapies did not clearly prevent or encourage this. A common precursor to relapse is stopping medication. Combining antipsychotics does not seem to prevent or encourage cessation, although generalising from these studies to the real world is problematic. Nine studies - four with clozapine in both groups (C +risperidone 2005b; C +risperidone 2001b; C +sulpiride 1997; C +sulpiride 1999c), four with other atypical drugs in both groups (C +arip/pali 2014; C +clozapine 2001; C +clozapine 2013; C +fluphen dec 2009), and one with typical drugs in both groups (A +trifluoperazine 1964) - had no participants leave early at all and were therefore not accounted for in the general analysis.

\section{Hospital admission}

There was a lack of studies contributing to this outcome, most likely attributed to the setting of the trials as most were performed in an inpatient setting. Of the three included trials only one was long term (A +any antipsychotic 2011) and contributed to $80.7 \%$ of the weight for this outcome. No difference was found between treatment groups in the number of participants hospitalised.

\section{Adverse events}

We did not find evidence of a difference between treatment groups in serious adverse events or those requiring discontinuation; neither subgroup analysis showed a significant difference. There were no reports of agranulocytosis. Although there were no differences in serious adverse events in the short term, we are not able to say whether there is a difference in serious adverse events when using these therapies in the long term as most of the data is from short-term trials (22 trials of 12 weeks or less). Nine studies had no serious adverse events or an event requiring discontinuation at all and were therefore not accounted for in this general analysis. There was no evidence of a difference in the number of patients experiencing movement disorders or weight gain.

\section{Quality of life}

There is a lack of evidence on quality of life, with only four studies reporting data for this outcome on three different scales, none of which showed a difference between treatment groups.

\section{Overall completeness and applicability of evidence}

\section{Completeness}

We did not find any trials with data for the following outcomes: days in hospital, change in hospital status, general behaviour, specific behaviours, social functioning, employment status during the trial, 
occurrence of violent incidents, and level of substance abuse. Quality of life, independence and the ability to work are important outcomes for those living with schizophrenia. Very few studies reported data for these outcomes and, those that did, reported data that are not really clinically meaningful.

We are aware of one study (NCT01450514) that looked at the effects of a combination therapy versus monotherapy. We tried to obtain data from this trial but the sponsor decided not to provide data as they wanted to keep it confidential.

For studies that had both clozapine and another antipsychotic in two monotherapy groups, we only included the data from the clozapine arm, as we considered this to be the more important comparison and in order to facilitate subgroup analyses.

This review compares only monotherapy with combination therapy, and we do not make direct comparisons between different types of combinations of antipsychotics, e.g. combinations including typical antipsychotics versus those containing clozapine.

\section{Applicability}

The majority of studies were less than 12 weeks in duration, with 10 trials longer or equal to six months and, as schizophrenia is a chronic disease with a long-term course, there is only limited information about the long-term safety and efficacy of antipsychotic combination therapy. Most trials were undertaken in very formalised settings - not at all reflective of the everyday circumstances in which people with schizophrenia live. This must also reduce applicability. Long trials set in very real-world circumstances are needed.

\section{Quality of the evidence}

As can be seen graphically in Figure 1 we felt the risk of bias in the included studies to be moderate to high. The majority of trials had unclear allocation concealment, method of randomisation and blinding, and were not free of selective reporting. Only around half of the studies addressed incomplete data adequately and in most it was unclear if they were free from other biases. There is a real danger in unfairly judging studies of the past by today's standards. However, this may not be as unfair as it seems. For mental health there is some evidence that reporting of trials was as good if not better in the 1960s and 1970s than it was two decades later (Ahmed 1998). However, the CONSORT initiative was formalised in 1996 (Begg 1996), and only eight of the 62 included trials predated this. That only 18 trials reported adequate generation and 16 the methods used to conceal allocation reflects poor quality reporting, probably poor [biased] conduct, and is certainly associated with exaggerated estimates of effect (Schulz 1995).

The sensitivity analysis for the quality of reporting of randomisation did not suggest that the less convincingly randomised studies produced discernibly different results, nor did the sensitivity analysis for the blinding of studies.

\section{Potential biases in the review process}

In our search strategy we tried to identify all relevant trials. However, there is the possibility that we may have failed to identify some studies. We have worked only with published reports. By doing this we may be perpetuating a reporting and publishing bias. It would be better to have original individual patient data.
The extraction of data and the risk of bias for the Chinese language studies were completed by only one review author. There is the possibility that this may have introduced some bias into the results as it was not possible to cross-check these data.

\section{Agreements and disagreements with other studies or reviews}

We know of seven other reviews that compare various combinations of antipsychotics. Correll 2009 addressed the same topic as the current review and reported that antipsychotic combination therapy was superior to monotherapy for inefficacy and leaving the study. In our analysis we also found an effect in favour of the combination therapy for inefficacy, but did not find a clear difference between treatments for leaving the study early. This difference in findings was due to our inclusion of more data from recently published studies. For example, for the outcome of 'leaving the study early', in Correll 2009 the weighting for data from the trial A + pimozide 1985 was $90 \%$. For our review the weighting for these data from A +pimozide 1985 was only $12.6 \%$. This illustrates how fast evidence can change with emergence of new data and the need for regular updating of review.

The relevant Canadian Agency for Drugs and Technologies in Health overview (CADTH 2012) presented results in subgroups for clozapine in both groups and other atypical drugs in both groups. Our findings were the same for efficacy, serious adverse events and leaving the study early.

A further five reviews looked specifically at the augmentation of clozapine with another antipsychotic. Barber 2017 included participants with treatment-resistant schizophrenia and found efficacy in favour of the clozapine combination in open-label studies but not double-blind studies. This contrasts with our results, as in this review trials with a low or an unclear risk of bias for blinding favoured the combination of antipsychotics. Sommer 2012 reviewed pharmacological augmentation of clozapine. Our results did not differ from this review in regards to aripiprazole, haloperidol, risperidone and sulpiride augmentation. C +aripiprazole 2009 reviewed sulpiride augmentation of clozapine and includes the same studies as our review, and the findings are the same. Taylor 2009 also found efficacy in favour of combination therapy; however, they dealt with data differently from our review by combining data from the Brief Psychiatric Rating Scale (BPRS) and Positive and Negative Syndrome Scale (PANSS), so it is not possible to compare the findings. They found no difference in leaving the study early, which is the same as our findings. Meng 2015 reviewed pharmacological augmentation with aripiprazole for participants with antipsychotic-induced hyperprolactinaemia. They reported data differently from our review as they presented a dichotomous outcome for the proportion of participants whose prolactin levels returned to normal; their results favoured the use of aripiprazole. They also found no difference in leaving the study early.

\section{AUTHORS' CONCLUSIONS}

\section{Implications for practice}

\section{For people with schizophrenia}

Currently, there is very low-quality evidence that the use of antipsychotic combinations results in a clinical improvement when compared with monotherapy. There is no evidence that 
combination antipsychotic therapy does that much, either in terms of leaving the study early and/or adverse events. Quality of evidence is low and unique positive reactions to combinations of drugs do occur. All we can say is that combinations do not have a clear advantage over monotherapy from within the context of trials.

\section{For clinicians}

As a clinician it is common to find a person on a combination of antipsychotics or to be tempted to add other drugs to an already established, but clearly inadequate antipsychotic regimen. This review does not preclude adding an additional antipsychotic, nor does this review suggest that discontinuation of one of the two combination antipsychotics is necessarily indicated. All should be done with caution due to lack of sufficient evidence. It would be beneficial if clinicians that face the clinical question addressed in this review, began to randomise their patients to contribute to the body of evidence.

\section{For policymakers}

It would seem sensible that the fewer antipsychotics, the better - but some people do seem to do well on combinations of antipsychotics and there is a risk of upsetting this group of people by stipulating that it is going against policy to have people on more than one antipsychotic. Furthermore, there is not good quality evidence for this stipulation.

\section{Implications for research}

\section{General}

Registration of trials before anyone is randomised would ensure that participants could be confident that people would know that the study had at least taken place. Unique study numbers would help researchers identify single studies from multiple publications and reduce the risk of duplicating the reporting of data.

Compliance with CONSORT would help clarify methodology and many outcomes. Failure to do this results in both loss of data and confusion in the results.

\section{Specific}

\subsection{Trials}

It would be beneficial to people with schizophrenia if there was a long-term (e.g. > one year) trial comparing antipsychotics combination with monotherapy. The outcomes measured should include leaving the study early, clinical improvement, relapse, adverse events and quality of life, which is an important outcome that has been overlooked in most trials. We do realise that such a study is a considerable undertaking and that we have only reviewed others' work in this area. However, that does give a perspective and we have suggested a design of study in Table 2.The fact that the last clinical trials which used a combination of typical antipsychotics was more than 30 years ago, is concerning as this review suggests the use of these drugs might have a place in the care of the patients with schizophrenia. Overlooking the typical antipsychotics in the design of new trials could potentially mislead the consumers to use more atypical antipsychotics.

\section{ACKNOWLEDGEMENTS}

Many thanks to Farhad Shokraneh for the trials search. We are most grateful for the ongoing support of Claire Irving and Tracey Roberts at the Cochrane Schizophrenia Group's editorial base. We acknowledge Ha Hyung Jung for screening one trial from the Korean literature and Liliya Eugenevna from Cochrane Russia for helping us screen one Russian trial plus Jun Xia for help with translating Chinese trials. We would also like to acknowledge Karla Soares-Weiser for her significant contributions to previous versions of this review.

We would also like to thank the editorial base of the Cochrane Schizophrenia Group for their help and acknowledge the use of their template for the methods section of this review. We used their template and adapted it for our review.

We would like to thank Timothy Kariotis and Vivek Agarwal for peer reviewing this version of the review.

We are grateful to the authors who responded to our requests for further information on the trials in which they were involved.

This project was supported by the National Institute for Health Research, via Cochrane Infrastructure funding to the Schizophrenia Group. The views and opinions expressed therein are those of the authors and do not necessarily reflect those of the Systematic Reviews Programme, NIHR, NHS or the Department of Health.

Parts of this review were generated using RevMan HAL v 4.0. You can find more information about RevMan here. 


\section{R E F E R E N C E S}

\section{References to studies included in this review}

\section{A +any antipsychotic 2011 \{published data only\}}

Essock SM, Schooler NR, Stroup TS, McEvoy JP, Rojas I, Jackson C, et al. Effectiveness of switching from antipsychotic polypharmacy to monotherapy. American Journal of Psychiatry 2011;168(7):702-8. [DOI: 10.1176/appi.ajp.2011.10060908]

\section{A +any antipsychotic 2012 \{published data only\}}

Fricchione Parise V, Balletta G, Addeo L, Manna G. Effectiveness of antipsychotic polypharmacy or monotherapy: real-world study outcomes. European Neuropsychopharmacology 2012;22(Suppl 2):323. [DOI: 10.1016/S0924-977X(12)70495-9]

\section{A +any antipsychotic 2015 \{published data only\}}

Constantine RJ, Andel R, McPherson M, Tandon R. The risks and benefits of switching patients with schizophrenia or schizoaffective disorder from two to one antipsychotic medication: a randomized controlled trial. Schizophrenia Research 2015;166(1-3):194-200. [DOI: 10.1016/ j.schres.2015.05.038]

\section{A +pimozide 1985 \{published data only\}}

Nishikawa T, Tsuda A, Tanaka M, Koga I, Uchida Y. Prophylactic effects of neuroleptics in symptom-free schizophrenics: roles of dopaminergic and noradrenergic blockers. Biological Psychiatry 1985;20(11):1161-6. [0006-3223: (Print); Nishikawa 1985]

\section{A +reserpine 1957 \{published data only\}}

Barrett WW, Ellsworth RB, Clark LD, Enniss J. Study of the differential behavioral effects of reserpine, chlorpromazine, and a combination of these drugs in chronic schizophrenic patients. Diseases of the Nervous System 1957; Vol. 18, issue 6:209-15. [0012-3714: (Print); Barrett 1957]

\section{A +sulpiride 1994 \{published data only\}}

Wang CH, Qin TF, Lin YL, Zhao XF. A clinical effect and followingup study about sulpiride and clozapine for 105 cases of the schizophrenia type. Journal of Xinxiang Medical College 1994;11(2):148-51. [MK_007527]

\section{A +trifluoperazine 1964 \{published data only\}}

Talbot DR. Are tranquilizer combinations more effective than a single tranquilizer?. American Journal of Psychiatry 1964;121:597-600. [0002-953X: (Print); MEDLINE: Talbot 1964]

\section{B + any antipsychotic 2013 \{published data only\}}

Hori H, Yoshimura R, Katsuki A, Sugita Al, Atake K, Nakamura J. Switching to antipsychotic monotherapy can improve attention and processing speed, and social activity in chronic schizophrenia patients. Journal of Psychiatric Research 2013;47(12):1843-8. [DOI: 10.1016/j.jpsychires.2013.08.024]

\section{B +aripiprazole 2008 \{published data only\}}

* Fleischhacker WW, Heikkinen ME, Olie JP, Landsberg W, Dewaele P, McQuade RD, et al. Effects of adjunctive treatment with aripiprazole on body weight and clinical efficacy in schizophrenia patients treated with clozapine:a randomized, double-blind, placebo-controlled trial. International Journal of
Neuropsychopharmacology 2010;13(8):1115-25. [DOI: 10.1017/ S1461145710000490]

Fleischhacker WW, Heikkinen ME, Olié JP, Landsberg W, Dewaele P, McQuade R, et al. Weight change on aripiprazoleclozapine combination in schizophrenic patients with weight gain and suboptimal response on clozapine: 16-week doubleblind study. European Psychiatry 2008;23(Suppl 2):114-5. [DOI: 10.1016/j.eurpsy.2008.01.784; 0924-9338]

Kriel W. A multicenter, comparative, randomized, double-blind placebo controlled study on the effect on weight of adjunctive treatment with aripiprazole in patients with schizophrenia. www.sanctr.gov.za/SAClinicalTrials/tabid/169/Default.aspx (first received 15 November 2006).

Millar H, Felter C, Landsberg W. The effects of aripiprazole in combination with clozapine: patient functioning results from a double-blind, 16-week study in patients with schizophrenia (CN138-170). Journal of Psychopharmacology 2008;22(5):A17. [MEDLINE: Millar 2008 a]

\section{B +aripiprazole 2011 \{published data only\}}

Muscatello MRA, Bruno A, Pandolfo G, Mico U, Scimeca G, Di Nardo F, et al. Effect of aripiprazole augmentation of clozapine in schizophrenia: a double-blind-placebo-controlled study. Schizophrenia Research 2011;127(1-3):93-9. [DOI: 10.1016/j.schres.2010.12.011]

\section{B + pipotiazine 2002 \{published data only\}}

Zhu H, Deng D. A study of clozapine combined with or without pipotiazine palmitate in refractory schizophrenia. Journal of Clinical Psychological Medicine 2002;12:15-7.

B +quet/risp 2009 \{published data only\}

* Kane JM, Correll CU, Goff DC, Kirkpatrick B, Marder SR, VesterBlokland E, et al. A multicenter, randomized, double-blind, placebo-controlled, 16-week study of adjunctive aripiprazole for schizophrenia or schizoaffective disorder inadequately treated with quetiapine or risperidone monotherapy. Journal of Clinical Psychiatry 2009;70(10):1348-57. [1555-2101: (Electronic); DOI: 10.4088/JCP.09m05154yel; Kane 2009]

NCT00325689. A multicenter, randomized, doubleblind, placebo-controlled, 16 week study of aripiprazole used as dual therapy in the treatment of patients with chronic stable schizophrenia or schizoaffective disorder.. www.clinicaltrials.gov/ct2/show/NCT00325689? term=NCT00325689\&rank=1 (first received May 11 2006). [MEDLINE: Bristol-Myers 2006]

\section{B +risperidone 2010 \{published data only\}}

Richardson CM, Feldman S, Kelly DL, Ball MP, Boggs DL, Weiner $\mathrm{E}$, et al. Metabolic side effects of combined antipsychotic treatment: results from a double blind trial of adjunctive risperidone in clozapine treated people with treatmentresistant schizophrenia. Schizophrenia Bulletin 2009;35(Suppl 1):38-9. [MEDLINE: Richardson 2009] 
* Weiner E, Conley RR, Ball MP, Feldman S, Gold JM, Kelly DL, et al. Adjunctive risperidone for partially responsive people with schizophrenia treated with clozapine. Neuropsychopharmacology 2010;35(11):2274-83. [DOI: 10.1038/ npp.2010.101]

\section{B +sulpiride 1996 \{published data only\}}

Liu QH, Li XL, Zhang YQ, Jin SL, Li ZC, Wang NS, et al. A control study of clozapine in combination with sulpiride in alleviating the negative symptoms of schizophrenia. Chinese Journal of Psychiatry 1996;29(2):87-90. [MEDLINE: Liu 1996]

\section{B +ziprasidone 2014 \{published data only\}}

Muscatello MR, Pandolfo G, Mico U, Lamberti Castronuovo E, Abenavoli E, Scimeca G, et al. Augmentation of clozapine with ziprasidone in refractory schizophrenia: a doubleblind, placebo-controlled study. Journal of Clinical Psychopharmacology 2014;34(1):129-33. [DOI: 10.1097/ JCP.0000000000000042]

\section{C +amisulpride 2008 \{published data only\}}

Assion HJ, Reinbold H, Lemanski S, Basilowski M, Juckel G. Amisulpiride augmentation in patients with schizophrenia partially responsive or unresponsive to clozapine. A randomized, double-blind, placebo-controlled trial. Pharmacopsychiatry 2008;41(1):24-8. [0176-3679: (Print); DOI: 10.1055/s-2007-993209; Assion 2008]

\section{C +arip/pali 2014 \{published data only\}}

Mayabhate M, Badar V, Somani A. Cognitive and psychomotor effects of adjunctive aripiprazole or paliperidone in patients of schizophrenia receiving olanzapine: a double-blind placebo controlled clinical study. International Journal of Basic and Clinical Pharmacology 2014;3(1):130-8. [DOI: 10.5455/2319-2003.ijbcp20140216]

\section{C +aripiprazole 2007 \{published data only\}}

Chen HZ, Yu BR, Yang SG, Shen ZX, Mi Q, Jiang YH. Effect of aripiprazole on the hyperprolactinemia caused by sulpiride in the treatment of patients with schizophrenia: a clinical observation. Herald of Medicine 2007;26(10):1145-6. [Chen 2007]

\section{C +aripiprazole 2007b \{published data only\}}

Shim J, Shin J, Kelly DL, Jung D, Seo Y, Conley RR. Adjunctive treatment with aripiprazole for haloperidol induced hyperprolactinemia: double blind, placebo controlled study. Schizophrenia Bulletin 2007;33:460. [Shim 2006 a]

Shim JC, Jae YM, Shin JG, Jung DW, Seo YS, Liu KH, et al. Drug interactions between aripiprazole and haloperidol: double blind placebo controlled study. European Neuropsychopharmacology 2007;17:S438. [Shim 2007 d]

* Shim JC, Shin JG, Kelly DL, Jung DU, Seo YS, Liu KH, et al. Adjunctive treatment with a dopamine partial agonist, aripiprazole, for antipsychotic-induced hyperprolactinemia: a placebo-controlled trial. American Journal of Psychiatry 2007;164(9):1404-10. [0002-953X: (Print); DOI: 10.1176/ appi.ajp.2007.06071075; Shim 2006 a1]

\section{C +aripiprazole 2008 \{published data only\}}

Chang JS, Ahn YM, Park HJ, Lee KY, Kim SH, Kang UG, et al. Aripiprazole augmentation in clozapine-treated patients with refractory schizophrenia: an 8-week, randomized, double-blind, placebo-controlled trial. Asian Psychiatric Educational Workshop 2007;5:720-31. [MEDLINE: Chang 2008]

* Chang JS, Ahn YM, Park HJ, Lee KY, Kim SH, Kang UG, et al. Aripiprazole augmentation in clozapine-treated patients with refractory schizophrenia: an 8-week, randomized, doubleblind, placebo-controlled trial. Journal of Clinical Psychiatry 2008;69(5):720-31. [1555-2101: (Electronic); MEDLINE: Kim 2006 g2]

Chang JS, Lee NY, Ahn YM, Kim YS. The sustained effects of aripiprazole-augmented clozapine treatment on the psychotic systems and metabolic profiles of patients with refractory schizophrenia. Journal of Clinical Psychopharmacology 2012;32(2):282-4. [DOI: 10.1097/JCP.0b013e3182485871]

Kim YS. A double-blind randomized placebo controlled study of aripiprazole augmentation for clozapine-treated patients with refractory schizophrenia. www.clinicaltrials.gov/ct2/show/ NCT00328367?term=NCT00328367\&rank=1 (first received 17 May 2006). [MEDLINE: Kim 2006 g]

\section{C +aripiprazole 2008b \{published data only\}} Jin JF, Chao YQ, Xu L, Song ZY, et al. Combined lowdose aripiprazole improved due to hyperprolactinemia chlorpromazine clinical study [合并小剂量阿立哌唑改善氯 丙嗪所致高催乳素血症的临床研究]. Journal of Psychiatry 2008;21(6):455-6.

\section{C +aripiprazole 2009 \{published data only\}}

Wang L, Zhang B, Xu L, et al. A clinical study on aripiprazole in the treatment of female hyperprolactinemia by haloperidol. China Journal of Health Psychology 2009;17(2):194-5.

\section{C +aripiprazole 2012 \{published data only\}}

Yasui-Furukori N, Kaneda A, Sugawara N, Tomita T, Kaneko S. Effect of adjunctive treatment with aripiprazole to atypical antipsychotics on cognitive function in schizophrenia patients. Journal of Psychopharmacology 2012;26(6):806-12. [DOI: 10.1177/0269881111405555]

\section{C + aripiprazole 2013 \{published data only\}}

Fan X, Borba CP, Copeland P, Hayden D, Freudenreich O, Goff DC, et al. Metabolic effects of adjunctive aripiprazole in clozapine-treated patients with schizophrenia. Acta Psychiatrica Scandinavica 2013;127(3):217-26. [DOI: 10.1111/acps.12009]

\section{C +aripiprazole 2013b \{published data only\}}

Lee BJ, Lee SJ, Kim MK, Lee JG, Park SW, Kim GM, et al. Effect of aripiprazole on cognitive function and hyperprolactinemia in patients with schizophrenia treated with risperidone. Clinical Psychopharmacology and Neuroscience 2013; Vol. 11, issue 2:60-6. [DOI: 10.9758/cpn.2013.11.2.60]

C +aripiprazole 2014 \{published data only\}

Chen JX, Zhang RZ, Li W, Liu YH, Jiang LY, Bian QT, et al. Adjunctive treatment of risperidone-induced hyperprolactinemia with aripiprazole: a randomized, double- 
blind, placebo-controlled study. Chinese Journal of New Drugs 2014; Vol. 23:811-4.

\section{C +aripiprazole 2015 \{published and unpublished data\}}

Chen JX, Su YA, Bian QT, Wei LH, Zhang RZ, Liu YH, et al. Adjunctive aripiprazole in the treatment of risperidone-induced hyperprolactinemia: a randomized, double-blind, placebocontrolled, dose-response study. Psychoneuroendocrinology 2015;58:130-40. [DOI: 10.1016/j.psyneuen.2015.04.011]

\section{C +aripiprazole 2015b \{published and unpublished data\}}

Raghuthaman G, Venkateswaran R, Krishnadas R. Adjunctive aripiprazole in risperidone-induced hyperprolactinaemia: double-blind, randomised, placebo-controlled trial. British Journal of Psychiatry Open 2015;1(2):172-7. [DOI: 10.1192/ bjpo.bp.115.001248]

\section{C +aripiprazole 2015c \{published data only\}}

ChiCTR-IOR-15006278. Adjunctive aripiprazole treatment for risperidone-induced hyperprolactinemia: an 8-week randomized, open-label, comparative clinical trial. www.chictr.org.cn/showprojen.aspx?proj=10830 (first received 22 April 2015).

* Zhao J, Song X, Ai X, Gu X, Huang G, Li X, et al. Adjunctive aripiprazole treatment for risperidone-induced hyperprolactinemia: an 8-week randomized, open-label, comparative clinical trial. PLoS One 2015;10(10):e0139717. [DOI: 10.1371/journal.pone.0139717]

\section{C +aripiprazole 2016 \{published data only\}}

ChiCTR-TRC-14004186. Research of the variance on patients with schizophrenia treated with aripiprazole on hyperprolactinemia induced by risperidone or paliperidone. www.chictr.org.cn/showprojen.aspx?proj=5382. China, (first received 8 January 2014).

* Qiao Y, Yang F, Li C, Guo Q, Wen H, Zhu S, et al. Add-on effects of a low-dose aripiprazole in resolving hyperprolactinemia induced by risperidone or paliperidone. Psychiatry Research 2016;237:83-9. [DOI: 10.1016/j.psychres.2015.12.033]

\section{C +clozapine 2001 \{published data only\}}

Xie C, Ni XL. The compared study of treating schizophrenia with risperidone combining clozapine. Journal of Preventative Medicine 2001;17(4):245. [MEDLINE: Xie 2001]

\section{C +clozapine 2013 \{published data only\}}

Jiang L, Lei J, Peng L, et al. Analysis of efficacy of antipsychotics combined with clozapine orally disintegrating tablets in the treatment of schizophrenia with negative symptoms [氯氮平联 合抗精神病药治疗精神分裂症阴性症状的效果分析]. Journal of Bethune Military Medical College 2013;11(4):310-11.

\section{C +CPZ 1973 \{published data only\}}

Chien CP, Cole JO. Depot phenothiazine treatment in acute psychosis: a sequential comparative clinical study. American Journal of Psychiatry 1973;130(1):13-8. [0002-953X: (Print); DOI: 10.1176/ajp.130.1.13; MEDLINE: Chien 1973]

\section{C +CPZ 1989 \{published data only\}}

* Potter WZ, Ko GN, Zhang LD, Yan WW. Clozapine in China: a review and preview of US/PRC collaboration. Psychopharmacology 1989;99(Suppl):87-91. [0033-3158: (Print); MEDLINE: Potter 1989]

Zhang L, Xu Y. A comparison study on treatment effect of clozapine, chlorpromazine and the combination of clozapine and chlorpromazine in schizophrenia. Journal of Nervous and Mental Disease 1989; Vol. 5, issue 5:306-8. [MEDLINE: Zhang 1989]

\section{C +CPZ 1999 \{published data only\}}

Cha C, Hui G, Quing G. Evaluation of therapeutic effect with chlorpromazine and clozapine for treatment of schizophrenia. Xinxiang Med Stud 1999;16:311-6.

\section{C +fluphen dec 2009 \{published data only\}}

Shafti SS. Augmentation of olanzapine by fluphenazine decanoate in poorly responsive schizophrenia. Clinical Schizophrenia and Related Psychoses 2009;3(2):97-102. [DOI: 10.3371/CSRP.3.2.4]

\section{C +haloperidol 2006 \{published data only\}}

Mossaheb N, Sacher J, Wiesegger G, Klein N, Spindelegger C, Asenbaum S, et al. Haloperidol in combination with clozapine in treatment-refractory patients with schizophrenia. European Neuropsychopharmacology 2006;16(suppl 4):416. [DOI: 10.1016/ s0924-977X(06)70524-7]

\section{C +haloperidol 2010 \{published data only\}}

Lin CH, Kuo CC, Chou LS, Chen YH, Chen CC, Huang KH, et al. A randomized, double-blind comparison of risperidone versus low-dose risperidone plus low-dose haloperidol in treating schizophrenia. Journal of Clinical Psychopharmacology 2010;30(5):518-25. [DOI: 10.1097/JCP.0b013e3181f28dff]

\section{C +levomepromazine 2004 \{published data only\}}

Higashima M, Takeda T, Nagasawa T, Hirao N, Oka T, Nakamura M, et al. Combined therapy with low-potency neuroleptic levomepromazine as an adjunct to haloperidol for agitated patients with acute exacerbation of schizophrenia. European Psychiatry 2004;19(6):380-1. [0924-9338: (Print); DOI: 10.1016/j.eurpsy.2004.07.001; Higashima 2004]

\section{C +olan/risp 2014 \{published data only\}}

Hatta K, Otachi T, Fujita K, Morikawa F, Ito S, Tomiyama H, et al. Antipsychotic switching versus augmentation among early non-responders to risperidone or olanzapine in acute-phase schizophrenia. Schizophrenia Research 2014;158(1-3):213-22. [DOI: 10.1016/j.schres.2014.07.015]

\section{C +olanzapine 2012 \{published data only\}}

Hatta K, Otachi T, Sudo Y, Kuga H, Takebayashi H, Hayashi H, et al. A comparison between augmentation with olanzapine and increased risperidone dose in acute schizophrenia patients showing early non-response to risperidone. Psychiatry Research 2012;198(2):194-201. [DOI: 10.1016/j.psychres.2012.01.006] 


\section{C +olanzapine 2012b $\{$ published data only\}}

Repo-Tiihonen E, Hallikainen T, Kivisto P, Tiihonen J. Antipsychotic polypharmacy in clozapine resistant schizophrenia: a randomized controlled trial of tapering antipsychotic co-treatment. Mental Illness 2012; Vol. 4:1-4. [DOI: 10.4081/mi.2012.e1]

\section{C +perphenazine 1976 \{published data only\}}

Yagi G. A double-blind controlled study on the usefulness of carpipramine-chlorpromazine combination in the pharmacotherapy of chronic schizophrenic patients. Clincal Evaluation 1976; Vol. 3:351-403. [MEDLINE: Yagi 1976]

\section{C +pimozide 2011 \{published data only\}}

Bergmann M, Golembo S, Friedman JI, Trammel-Fisher A, Siever LJ. Pimozide augmentation of clozapine in schizophrenia. www.clinicaltrials.gov/ct2/show/NCT00158223? term=NCT00158223\&rank=1 (first received 7 September 2005). [MEDLINE: Bergmann 2005]

* Friedman JI, Lindenmayer JP, Alcantara F, Bowler S, Parak M, White $L$, et al. Pimozide augmentation of clozapine inpatients with schizophrenia and schizoaffective disorder unresponsive to clozapine monotherapy. Neuropsychopharmacology 2011;36(6):1289-95. [DOI: 10.1038/npp.2011.14]

\section{C +pimozide 2013 \{published data only\}}

Gunduz-Bruce H. Efficacy of pimozide augmentation for clozapine partial response. www.clinicaltrials.gov/ct2/show/ NCT00374244?term=Efficacy+of+pimozide+augmentation +for+clozapine+partial+response\&rank=1 (first received 7 September 2006). [MEDLINE: Gunduz-Bruce 2006]

Gunduz-Bruce H. Pimozide for Schizophrenia. Stanley Foundation Research Programs 2009. [MEDLINE: Gunduz-Bruce 2006]

* Gunduz-Bruce H, Oliver S, Gueorguieva R, Forselius-Bielen K, D'Souza DC, Zimolo Z, et al. Efficacy of pimozide augmentation for clozapine partial responders with schizophrenia. Schizophrenia Research 2013;143(2):344-7. [DOI: 10.1016/ j.schres.2012.11.008]

\section{C +pipotiazine 2000 \{published data only\}}

Jia Z, Zhang Z, Jin S. A controlled trial for comparing clozapine combined with pipothiazine palmitate to clozapine alone in the treatment of negative symptoms in schizophrenic patients. Herald of Medicine 2000;19:142-3.

\section{C +risperidone 2001 \{published data only\}}

Ni J, Jang L, Hong X. Therapeutic effects of clozapine, risperidone and their combination in the treatment of schizophrenia. Health Psychology 2001;3:181-2.

\section{C +risperidone 2001b \{published data only\}}

Peng $\mathrm{H}$, Kuang Y, Huang X. A control study of risperidone in combination with clozapine in treating refractory schizophrenia. Journal of Modern Clinical Medical Bioengineering 2001;7(2):100-2. [MEDLINE: Peng 2001]

\section{C +risperidone 2001c \{published data only\}}

Xin X, Du B, Zeng Z. A controlled clinical study of risperidone and low dose of clozapine in treating schizophrenia. Herald of Medicine 2001;20:501-2.

\section{C +risperidone 2005 \{published data only\}}

Akdede BB, Anil Yagcioglu AE, Alptekin K, Turgut TI, Tumuklu M, Yazici MK, et al. A double-blind study of combination of clozapine with risperidone in patients with schizophrenia: effects on cognition. Journal of Clinical Psychiatry 2006;67(12):1912-9. [1555-2101: (Electronic); DOI: 10.4088/JCP.v67n1211; MEDLINE: Anil Yagcioglu 2005]

Anil E. Risperidone augmented with clozapine for schizophrenia. Stanley Foundation Research Programs 2009. [MEDLINE: Anil Yagcioglu 2005]

* Anil Yagcioglu AE, Kivircik Akdede BB, Turgut TI, Tumuklu M, Yazici MK, Alptekin K, et al. A double-blind controlled study of adjunctive treatment with risperidone in schizophrenic patients partially responsive to clozapine: efficacy and safety. Journal of Clinical Psychiatry 2005; Vol. 66, issue 1:63-72. [0160-6689: (Print); DOI: 10.4088/JCP.v66n0109; MEDLINE: Anil Yagcioglu 2005]

Yagcioglu AEA, Akdede BBK, Turgut TI, Tumuklu M, Yazici MK, Alptekin $\mathrm{K}$, et al. A double-blind controlled study of adjunctive treatment with risperidone in schizophrenic patients partially responsive to clozapine: efficacy and safety. 24th Congress of the Collegium Internationale Neuro-Psychopharmacologicum (CINP); 2004 Jun 20-24; Paris, France. 2004:63-72. [MEDLINE: Yagcioglu 2004]

\section{C +risperidone 2005b \{published data only\}}

Josiassen RC, Joseph A, Kohegyi E, Stokes S, Dadvand M, Paing WW, et al. Clozapine augmented with risperidone in the treatment of schizophrenia: a randomized, doubleblind, placebo-controlled trial. 9th International Congress on Schizophrenia Research; 2003 Mar 29-Apr 2; Colorado Springs, CO. 2003:130-6. [MEDLINE: Josiassen 2003]

* Josiassen RC, Joseph A, Kohegyi E, Stokes S, Dadvand M, Paing WW, et al. Clozapine augmented with risperidone in the treatment of schizophrenia: a randomized, double-blind, placebo-controlled trial. American Journal of Psychiatry 2005;162(1):130-6. [0002-953X: (Print); DOI: 10.1176/ appi.ajp.162.1.130; MEDLINE: Josiassen 2005]

\section{C +risperidone 2006 \{published data only\}}

Gerson SL. Clozapine alone versus clozapine and risperidone for refractory schizophrenia. New England Journal of Medicine 2006;354(17):1846-8. [1533-4406: (Electronic); MEDLINE: Honer 2006]

Grass G, Hellmich M, Leweke FM. Clozapine alone versus clozapine and risperidone for refractory schizophrenia. New England Journal of Medicine 2006;354(17):1846-8. [1533-4406: (Electronic); MEDLINE: Honer 2006 b2]

Honer W. A randomized controlled trial of antipsychotic polypharmacy: clozapine plus risperidone. European Neuropsychopharmacology 2007;17(Suppl 4):S200. [DOI: 10.1016/S0924-977X(07)70237-7; MEDLINE: Honer 2006 b6] 
Honer W. International study of improving treatment for the most severely ill with schizophrenia. www.clinicaltrials.gov/ct2/ show/NCT00272584?term=NCT00272584\&rank=1 (first received 3 January 2006). [MEDLINE: Honer 2006 a]

* Honer WG, Thornton AE, Chen EY, Chan RC, Wong JO, Bergmann A, et al. Clozapine alone versus clozapine and risperidone with refractory schizophrenia. New England Journal of Medicine 2006;354(5):472-82. [1533-4406: (Electronic); DOI: 10.1056/NEJMoa053222; MEDLINE: Honer 2006]

Meltzer HY, Anil Yagcioglu AE, Akdede BB. Clozapine alone versus clozapine and risperidone for refractory schizophrenia. New England Journal of Medicine 2006;354(17):1846-8. [1533-4406: (Electronic); MEDLINE: Honer 2006 b3]

Procyshyn RM, Wasan KM, Thornton AE, Barr AM, Chen EY, Pomarol-Clotet $\mathrm{E}$, et al. Changes in serum lipids, independent of weight, are associated with changes in symptoms during longterm clozapine treatment. Journal of Psychiatry Neuroscience 2007;32(5):331-8. [1180-4882: (Print); MEDLINE: Procyshyn 2007]

\section{C +risperidone 2007 \{published data only\}}

Freudenreich $\mathrm{O}$. Risperidone added to clozapine for schizophrenia. Stanley Foundation Research Programs 2009. [Walsh 2006 b1]

* Freudenreich O, Henderson DC, Walsh JP, Culhane MA, Goff DC. Risperidone augmentation for schizophrenia partially responsive to clozapine: a double-blind, placebo-controlled trial. Schizophrenia Research 2007;92(1-3):90-4. [0920-9964: (Print); DOI: 10.1016/j.schres.2006.12.030; Walsh 2006 b2]

Walsh JP, Freudenreich O, Goff DC. Risperidone augmentation in patients with schizophrenia partially responsive to clozapine. www.clinicaltrials.gov/ct2/show/NCT00289861? term=NCT00289861\&rank=1 (first received 8 February 2006). [MEDLINE: Walsh 2006 b]

\section{C +sertindole 2006 \{published data only\}}

* Nielsen J, Emborg C, Gydesen S, Dybbro J, Aagaard J, Haderup K, et al. Augmenting clozapine with sertindole: a double-blind, randomized, placebo-controlled study. Journal Clincal Psychopharmacology 2012;32(2):173-8. [DOI: 10.1097/ JCP.0b013e318248dfb8]

Nielsen J, Munk-Jorgensen P. Augmenting clozapine with sertindole - a double blinded randomized placebo study. www.clinicaltrials.gov/ct2/show/NCT00345982? term=NCT00345982\&rank=1 (first received 28 June 2006). [Nielsen 2006]

Nielsen RE, Levander S, Thode D, Nielsen J. Effects of sertindole on cognition in clozapine-treated schizophrenia patients. Acta Psychiatrica Scandinavica 2012;126(1):31-9. [DOI: 10.1111/ j.1600-0447.2012.01840.x]

\section{C +sulpiride 1997 \{published data only\}}

* Shiloh R, Zemishlany Z, Aizenberg D, Radwan M, Schwartz B, Dorfman-Etrog $P$, et al. Sulpiride augmentation in people with schizophrenia partially responsive to clozapine. A doubleblind, placebo-controlled study. British Journal of Psychiatry 1997;171:569-73. [0007-1250: (Print); Shiloh 1997]
Shiloh R, Zemishlany Z, Aizenberg D, Weizman A. Sulpiride adjunction to clozapine in treatment-resistant schizophrenic patients: a preliminary case series study. European Psychiatry 1997;12(3):152-5. [0924-9338: (Print); MEDLINE: Shiloh 1997]

C +sulpiride 1999 \{published data only\}

Si S, Yuan C. A comparative trial of the effects of sulpiride combined with clozapine in the treatment of schizophrenia. Shandong Archives of Psychiatry 1999;12:17-20.

C +sulpiride 1999b \{published data only\}

Xao H. A double-blind comparative study of the effects of sulpiride combined with clozapine in the treatment of schizophrenia. Sichuan Mental Health 1999;12:250-1.

\section{C +sulpiride 1999c \{published data only\}}

Zhu Y, Zhang S, Zhang D. A controlled trial comparing chlorimipramine and sulpiride as adjunct to clozapine in the treatment of negative symptoms of schizophrenia. Journal of Clinical Psychology in Medical Settings 1999;9(4):204-5. [MEDLINE: Zhu 1999]

\section{C +sulpiride 2003 \{published data only\}}

Zou G, Huang Y, Zou S, Yang Y. A comparative trial of the beneficial effects of sulpiride combined with clozapine in the treatment of refractory schizophrenia. Journal of Yichun University 2003;25:94-6.

\section{C +sulpiride 2004 \{published data only\}}

Kotler M, Strous RD, Reznik I, Shwartz S, Weizman A, Spivak B. Sulpiride augmentation of olanzapine in the management of treatment-resistant chronic schizophrenia: evidence for improvement of mood symptomatology. International Clinical Psychopharmacology 2004;19(1):23-6. [0268-1315: (Print); MEDLINE: Kotler 2004]

\section{C +sulpiride 2006 \{published data only\}}

Xu B. Observation on the effect of clozapine and sulpiride on negative symptom schizophrenia patients. China Tropical Medicine 2006;6(5):806.

\section{C +sulpiride 2013 \{published and unpublished data\}}

Lin CH, Wang FC, Lin SC, Huang YH, Chen CC, Lane HY. Antipsychotic combination using low-dose antipsychotics is as efficacious and safe as, but cheaper, than optimal-dose monotherapy in the treatment of schizophrenia: a randomized, double-blind study. International Clinical Psychopharmacology 2013;28(5):267-74. [DOI: 10.1097/YIC.0b013e3283633a83.]

\section{References to studies excluded from this review}

\section{Ahn 2002 \{published data only\}}

Ahn YM, Kweon YS, Kwon JS, Min SH, Park DB, Yang MJ, et al. The study for switching methods to olanzapine in Korean schizophrenic patients treated with other antipsychotics (II): comparison of safety [항정신병약물 사용 중인 정신분 열병 환자에서 올란자핀으로의 교체 방법에 관한 연구: 안전 성 비교]. Journal of Korean Neuropsychiatric Association 2002;41(5):890-4. 
Alptekin 2012 \{published data only\}

Alptekin K, Akdede B, Soygur H. TRIES: An open, randomized, prospective, multicenter study, searching the best switch policy of sertindole in patients with schizophrenia. Schizophrenia Research 2012;136:S164-5. [DOI: 10.1016/ s0920-9964(12)70519-4]

\section{Awad 2014 \{published data only\}}

Awad G, Hassan M, Loebel A, Hsu J, Pikalov A, Rajagopalan K. Health-related quality of life among patients treated with lurasidone: results from a switch trial in patients with schizophrenia. BMC Psychiatry 2014;14(1):53. [DOI: 10.4088/ jcp.12m07992]

\section{Barbui 2011 \{published data only\}}

* Barbui C, Accordini S, Nose M, Stroup S, Purgato M, Girlanda F, et al. Aripiprazole versus haloperidol in combination with clozapine for treatment-resistant schizophrenia in routine clinical care a randomized, controlled trial. Journal of Clinical Psychopharmacology 2011;31(3):266-73. [DOI: 10.1097/ JCP.0b013e318219cba3.]

Cipriani A, Accordini S, Nose M, Purgato M, Girlanda F, Tansella M, et al. Aripiprazole versus haloperidol in combination with clozapine for treatment-resistant schizophrenia: a 12month, randomized, naturalistic trial. Journal of Clinical Psychopharmacology 2013;33(4):533-7. [DOI: 10.1097/ JCP.0b013e318296884f.]

Nose M, Accordini S, Artioli P, Barale F, Barbui C, Beneduce R, et al. Rationale and design of an independent randomised controlled trial evaluating the effectiveness of aripiprazole or haloperidol in combination with clozapine for treatmentresistant schizophrenia. Trials 2009;10(31):31. [1745-6215: (Electronic); MEDLINE: Barbui 2006]

\section{Cazorla 2012 \{published data only\}}

Cazorla P, Mackle M, Zhao J, Ha X, Szegedi A. Safety and tolerability of switching to asenapine from other antipsychotic agents: pooled results from two randomized multicenter trials in stable patients with persistent negative symptoms in schizophrenia. Neuropsychiatric Disease and Treatment 2012;8:247-57. [DOI: 10.2147/NDT.S29891]

\section{ChiCTR-TRC-14004854 \{published data only\}}

ChiCTR-TRC-14004854. A randomized, controlled, multicenter clinical trial to study the treatment resistant schizophrenia (TRS) in Zhejiang province. www.chictr.org.cn/ showprojen.aspx?proj=4719 (first received 26 June 2014).

\section{Citrome 2012 \{published data only\}}

Citrome L, Kianifard F, Meng X, Winseck A, Hochfeld M, Stahl S. Discontinuations following a switch from risperidone, olanzapine, or aripiprazole to iloperidone in patients with schizophrenia: The i-fans study. Neuropsychopharmacology 2012;38(S1):S413-S4.

\section{Dai 2012 \{published data only\}}

Dai J, Gao H, Sheng J. The effect of synthetical intervention to schizophrenic and hyperlipidemia patients [精神分裂症并发高 脂血症患者的综合干预对生活质量的影响]. Medical Journal of Chinese People's Health 2012;24(5):541-2. [DOI: 10.3969/

j.issn.1672-0369.2012.05.011]

Dai 2012a \{published data only\}

Dai T, Xu L, Houzhan L, Chen R, Fan R, Zhao H, et al. Group therapy in combination with antipsychotic medication for the treatment of schizophrenia patients in recovery period [团体治 疗联合抗精神病药物对精神分裂症康复期治疗的 效果观察]. Chinese Journal of Clinical Rational Drug Use 2012;5(12A):66.

DRKS00008018 \{published data only\}

DRKS00008018. Are antipsychotics neurotoxic or neuroprotective? A long-term comparison of two treatment strategies. drks-neu.uniklinik-freiburg.de/ drks_web/navigate.do? navigationld=trial.HTML\&TRIAL_ ID=DRKS00008018 (first received 24 April 2015).

Fang 2012 \{published data only\}

Fang M, Chen H, Li LH, Wu R, Li Y, Liu L, et al. Comparison of risperidone oral solution and intramuscular haloperidol with the latter shifting to oral therapy for the treatment of acute agitation in patients with schizophrenia. International Clinical Psychopharmacology 2012;27(2):107-13. [DOI: 0.1097/ YIC.0b013e32834fc431.]

\section{Fleischhacker 2012 \{published data only\}}

Fleischhacker WW, Sanchez R, Perry PP, Jin N, PetersStrickland T, Johnson BR, et al. Aripiprazole once-monthly for the treatment of schizophrenia: A double-blind, randomized, non-inferiority study versus oral aripiprazole. Neuropsychopharmacology 2012;38(S1):S339.

Fleischhacker 2013 \{published data only\}

Fleischhacker WW, Sanchez R, Johnson B, Jin N, Forbes RA, McQuade R, et al. Long-term safety and tolerability of aripiprazole once-monthly in maintenance treatment of patients with schizophrenia. International Clinical Psychopharmacology 2013;28:171-6. [DOI: 10.1097/ YIC.0b013e3283615dba]

\section{Goff 2008 \{published data only\}}

Goff DC, Lamberti JS, Leon AC, Green MF, Miller AL, Patel J, et al. A placebo-controlled add-on trial of the Ampakine, CX516, for cognitive deficits in schizophrenia. Neuropsychopharmacology 2008;33(3):465-72. [0893-133X: (Print); DOI: 10.1038/ sj.npp.1301444; MEDLINE: Goff 2008]

Henderson 2009 \{published data only\} Henderson DC, Fan X, Copeland PM, Sharma B, Borba CP, Boxill R, et al. Aripiprazole added to overweight and obese olanzapine-treated schizophrenia patients. Joural of Clinical Psychopharmacology 2009;29(2):165-9. [1533-712X: (Electronic); DOI: 10.1097/JCP.0b013e31819a8dbe; MEDLINE: Henderson $2009 \mathrm{c}]$

Hwang 2015 \{published data only\}

Hwang TJ, Lo WM, Chan HY, Lin CF, Hsieh MH, Liu CC, et al. Fast versus slow strategy of switching patients with schizophrenia to aripiprazole from other antipsychotics. Journal of Clinical Psychopharmacology 2015;35(6):635-44. [DOI: 10.1097/ JCP.0000000000000426.] 
JPRN-UMIN000011710 \{published and unpublished data\} JPRN-UMIN000011710. New Chiba Refractory Schizophrenia Treatment (CREST)-LAI study: Effectiveness of risperidone long-acting injectable for treatment-resistant schizophrenia. apps.who.int/trialsearch/Trial.aspx?TrialID=JPRNUMIN000011710 (first received 2013).

\section{JPRN-UMIN000012729 \{published data only\}}

JPRN-UMIN000012729. A comparison of medication adherence of blonanserin and aripiprazole in schizophrenic patients: a multicenter, randomized, open-label study. apps.who.int/ trialsearch/Trial.aspx?TrialID=JPRN-UMIN000012729 (first received 2013).

\section{JPRN-UMIN000017047 \{published data only\}}

JPRN-UMIN000017047. A randomized, open-label clinical trial on the efficacy of blonanserin and olanzapine in patients with schizophrenia and dopamine supersensitivity psychosis. apps.who.int/trialsearch/Trial.aspx?TrialID=JPRNUMIN000017047 (first received 2015).

\section{Kelly 2005 \{published data only\}}

Kelly D, Fischer G, Suppes T. Divalproex extended release and placebo, lithium, or quetiapine for mania. www.clinicaltrials.gov/ct2/show/NCT00183443? term=Divalproex+extended+release+and+placebo $\% 2 \mathrm{C}+$ lithium $\% 2 \mathrm{C}+$ or+quetiapine+for+mania\&rank=1 (first received 13 September 2005). [MEDLINE: Kelly 2005]

\section{Kreinin 2006 \{published data only\}}

Kreinin A, Novitski D, Weizman A. Amisulpiride treatment of clozapine-induced hypersalivation in schizophrenia patients: a randomized, double-blind, placebo-controlled cross-over study. International Clinical Psychopharmacology 2006;21:99-103.

\section{Kwon 2012 \{published data only\}}

Kwon JS, Mittoux A, Hwang JY, Ong A, Cai ZJ, Su TP. The efficacy and safety of 12 weeks of treatment with sertindole or olanzapine in patients with chronic schizophrenia who did not respond successfully to their previous treatments: A randomized, double-blind, parallel-group, flexible-dose study. International Clinical Psychopharmacology 2012;27(6):326-35. [DOI: 10.1097/YIC.0b013e32835767a0]

\section{Lerner 2004 \{published data only\}}

Lerner V, Libov I, Kotler M, Strous RD. Combination of "atypical" antipsychotic medication in the management of treatment-resistant schizophrenia and schizoaffective disorder. Progress in Neuro-Psychopharmacology and Biological Psychiatry 2004;28(1):89-98. [0278-5846: (Print); DOI: 10.1016/ j.pnpbp.2003.09.024; MEDLINE: Lerner 2004]

\section{Li 2013 \{published data only\}}

Li J. Liqi xingshen method for the treatment of TanQi Yujie type schizophrenia: a random parallel control study [理气醒神方 治疗痰气郁结型精神分裂症随机平行对照研究]. Journal of Practical Traditional Chinese Internal Medicine 2013;27(5):19-20. [DOI: 10.3969/j.issn.1671-7813.2013.05(s).84]

\section{Lieberman 2009 \{published data only\}}

Lieberman J, Farr G, Seoane F. TC-5619 as augmentation therapy to improve cognition in outpatients with cognitive dysfunction in schizophrenia. www.clinicaltrials.gov (first received 27 October 2009). [MEDLINE: Lieberman 2009]

NCT01003379. TC-5619 as augmentation therapy to improve cognition in outpatients with cognitive dysfunction in schizophrenia. clinicaltrials.gov/ct2/show/NCT01003379 (first received 27 October 2009). [MEDLINE: Anon 2009]

\section{Lieberman 2013 \{published data only\}}

Lieberman JA, Cutler AJ, Lu K, Laszlovszky I, Migliore R, Durgam S. Cariprazine in acute exacerbation of schizophrenia: a fixed-dose phase III, randomized, double-blind, placebo- and active-controlled trial. CNS Spectrums 2013;18(6):368.

Lieberman JA, Cutler AJ, Wan S, Migliore R, Ruth A, Laszlovszky I, et al. Cariprazine in acute exacerbation of schizophrenia: a fixed-dose, randomised, doubleblind, placebo- and active-controlled trial. European Neuropsychopharmacology 2013;23:S477-8.

\section{Lundbeck 2004 \{published data only\}}

Lundbeck H. A prospective randomised double-blind parallelgroup active-controlled cognition study of bifeprunox in schizophrenia (add-on study to studies 10199 and 10200). www.lundbecktrials.com (first accessed 2004). [MEDLINE: Lundbeck 2004]

\section{Lundbeck 2008 \{published data only\}}

Lundbeck H. Efficacy study exploring the effect of Lu AE58054 as augmentation therapy in patients with schizophrenia. www.clinicaltrials.gov/ct2/show/NCT00810667? term=Efficacy+study+exploring+the+effect+of+Lu +AE58054+as+augmentation+therapy+in+patients+with +schizophrenia\&rank=1 (first received 17 December 2008). [MEDLINE: Lundbeck 2008]

\section{Mantovani 2013 \{published data only\}}

Mantovani C, Labate CM, Sponholz AJ, de Azevedo Marques JM, Guapo V G, de Simone Brito dos Santos ME, et al. Are low doses of antipsychotics effective in the management of psychomotor agitation? A randomized, rated-blind trial of four intramuscular interventions. Journal of Clinical Psychopharmacology 2013;33:306-12.

\section{Meltzer 2008 \{published data only\}}

Johnson, Johnson. Randomized, Double-blind, Placeboand Active-controlled Parallel Group, Dose-response Study to Evaluate the Efficacy and Safety of 3 Fixed Dosages of Paliperidone Extended Release (6, 9, and $12 \mathrm{mg} /$ Day) and Olanzapine (10 mg/Day) With Open-label Extension in Treatment of Schizophrenia. www.clinicaltrials.gov/ct2/show/ NCT00078039?term=Randomized\%2C+Double-blind\%2C +Placebo-+and+Active-controlled+Parallel+Group $\% 2 C+$ Doseresponse+Study+to+Evaluate+the+Efficacy+and+Safety+of +3+Fixed+Dosages+of+Paliperidone+Extended+Release\&rank=1 (first received 17 February 2004).

* Meltzer H, Bobo W, Nuamah I, Lane R, Hough D, Kramer M, et al. Efficacy and tolerability of oral paliperidone extended- 
release tablets in the treatment of acute schizophrenia: pooled data from three 6-week, placebo-controlled studies. Journal of Clinical Psychiatry 2008;69(5):817-29.

\section{Meltzer 2012 \{published data only\}}

Meltzer HY, Elkis H, Vanover K, Weiner DM, Van Kammen DP, Peters P, et al. Pimavanserin, a selective serotonin (5-ht)2ainverse agonist, enhances the efficacy and safety of risperidone, $2 \mathrm{mg}$ /day, but does not enhance efficacy of haloperidol, $2 \mathrm{mg}$ / day: comparison with reference dose risperidone, $6 \mathrm{mg} /$ day. Schizophrenia Research 2012;141:144-52. [DOI: 10.1016/ j.schres.2012.07.029.; MEDLINE: 22954754]

\section{Mi 2013 \{published data only\}}

MI Z, Shi S. Antipsychotic medication combined psychological intervention for the treatment of patients with schizophrenia [药物联合心理干预治疗精神分裂症患者的临床研究]. Chinese Medicine Guide 2013;11(4):505-6.

\section{Mir 2008 \{published data only\}}

Mir A, Shivakumar K, Williamson RJ, McAllister V, O'Keane V, Aitchison KJ. Change in sexual dysfunction with aripiprazole: A switching or add-on study. Journal of Psychopharmacology 2008;22(3):244-53. [DOI: 10.1177/0269881107082901]

\section{Mythri 2013 \{published data only\}}

Mythri SV, Tharyan P, Sunder S, Kattula D, Kirubakaran R, Adams CE. Rapid tranquillization of violent or agitated patients in a psychiatric emergency setting: Pragmatic, randomized, allocation concealed, participant and assessor blinded trial of intramuscular zuclopenthixol acetate versus intramuscular haloperidol plus promethazine [A comparative study of the effects of an intramuscular injection of zuclopenthixol acetate versus an intramuscular injection of a combination of haloperidol plus promethazine in people with violence or agitation presenting to a psychiatric hospital as an emergency]. Study Protocol (as supplied 2013).

\section{NCT01003379 \{published data only\}}

NCT01003379. TC-5619 as augmentation therapy to improve cognition in outpatients with cognitive dysfunction in schizophrenia. clinicaltrials.gov/ct2/show/record/NCT01003379 (first received 27 October 2009).

\section{NCT01234779 \{published data only\}}

NCT01234779. A study of ro4917838 in patients with acute exacerbation of schizophrenia. clinicaltrials.gov/show/ NCT01234779 (first received 3 November 2010).

\section{NCT01939548 \{published data only\}}

NCT01939548. A 12-week, randomized, phase 2, doubleblind, parallel-group study of two dose levels of PF-02545920 compared to placebo in the adjunctive treatment of outpatients with sub-optimally controlled symptoms of schizophrenia. clinicaltrials.gov/show/NCT01939548 (first received 9 August 2013).

\section{NCT02477670 \{published data only\}}

NCT02477670. Efficacy, safety, and tolerability of AVP-786 for the treatment of residual schizophrenia. clinicaltrials.gov/show/ NCT02477670 (first received 18 June 2015).
Pfizer 2009 \{published data only\}

Pfizer. A study of PF-03463275 as add-on therapy in outpatients with persistent negative symptoms of schizophrenia. clinicaltrials.gov/ct2/show/NCT00977522?term=A+study+of +PF-03463275+as+add-on+therapy+in+outpatients+with +persistent+negative+symptoms+of+schizophrenia\&rank $=1$ (first received 14 September 2009). [MEDLINE: Pfizer 2009]

Ruiz-Doblado 2010 \{published data only\}

Ruiz-Doblado S, Baena-Baldomero A, Esparrago-Llorca G. Pharmacological augmentation strategies in clozapine-resistant schizophrenia: Overcoming the resistance. Psiquiatria Biologica 2010;17(3):96-101. [DOI: 10.1016/j.psiq.2010.07.002]

\section{Rupnow 2005 \{published data only\}}

Rupnow M, Greenspan A, Kosik-Gonzalez C, Bossie C, Zhu Y, Gharabawi G, et al. Polypharmacy in schizophrenia: data from a randomized, double-blind study. 158th Annual Meeting of the American Psychiatric Association; 2005 May 21-26; Atlanta, Georgia, USA. 2005. [MEDLINE: Rupnow 2005]

\section{Sacchetti 2006 \{published data only\}}

Sacchetti E, Galluzzo A, Valsecchi P, Romeo F, Gorini B, Warrington L. Comparative efficacy and safety of ziprasidone and clozapine in treatment refractory schizophrenic patients: results of a randomized, double-blind, 18-week trial. 13th Biennial Winter Workshop on Schizophrenia Research; 2006 Feb 4-10; Davos, Switzerland 2006. [MEDLINE: Sacchetti 2006]

\section{Semenikhin 2013 \{published data only\}}

Semenikhin DG, Kuchaeva AV, Karpov AM, Mikhailova EB, Murav'eva AV. Additional possibilities for correction of movement disorders induced by neuroleptics in patients with schizophrenia. Zhurnal Nevrologii I Psikhiatrii Imeni S.S. Korsakova 2013;113(11):78-80.

\section{Sofronov 2013 \{published data only\}}

Sofronov A, Spikina A, Savelyev A. The role of modern antipsychotics in correction of neurocognitive deficit in schizophrenia patients. European Psychiatry 2013;28:1247. [DOI: 10.1016/S0924-9338(13)76320-X]

\section{Spyker 2015 \{published data only\}}

Spyker DA, Riesenberg RA, Cassella JV. Multiple dose pharmacokinetics of inhaled loxapine in subjects on chronic, stable antipsychotic regimens. Journal of Clinical Pharmacology 2015;55(9):985-94. [DOI: 10.1002/jcph.502]

\section{Stahl 2010b \{published data only\}}

Stahl S, Cucchiaro J, Simonelli D, Loebel A. Long-term safety and tolerability of lurasidone in subjects with schizophrenia: results of a 6-month, open-label study. 49th Annual meeting of the Americam College of Neuropsychopharmacology; 5-9 December 2010; Miami, Florida. 2010.

\section{Sukegawa 2008 \{published data only\}}

Sukegawa T, Ito T, Hasegawa M, Mizuno Y, Inagaki A, Sakamoto $\mathrm{H}$, et al. A randomized controlled trial on the dose reduction and simplification for polypharmacy of antipsychotics. Tottori Journal of Clinical Research 2008;1(1):169-81. 


\section{Sukegawa 2014 \{published data only\}}

Sukegawa T, Inagaki A, Yamanouchi Y, Inada T, Yoshio T, Yoshimura R, et al. Study protocol: safety correction of high dose antipsychotic polypharmacy in Japan. BMC Psychiatry 2014;14(1):103. [DOI: 10.1186/1471-244X-14-103]

\section{UMIN000004931 \{published data only\}}

* Hirano J, Watanabe K, Suzuki T, Uchida H, et al. An open-label study of algorithm-based treatment versus treatment-as-usual for patients with schizophrenia. Neuropsychiatric Disease and Treatment 2013;9:1553-64. [DOI: 10.2147/ndt.s46108]

UMIN000004931. An intervention study of optimizing algorism-based pharmacological treatment for schizophrenia. www.umin.ac.jp/ctr/index.htm (first received 22 January 2011).

Wang 2013 \{published data only\}

Wang W, Chen H, Zhao L. Antipsychotic medication combined with acupuncture therapy for the treatment of schizophrenia [抗精神病药物联合针刺治疗精神分裂症 60 例疗效分析]. Medical Journal of Chinese People's Health 2013;25(10):63-4. [DOI: 10.3969/j.issn.1672-0369.2013.10.043]

Weiden 2013 \{published data only\}

Weiden PJ, Citrome L, Glick ID, Alva G, Winseck A, Kianifard F, et al. Initial 2-week outcomes following 2 methods of switching to iloperidone from risperidone in patients with schizophrenia. CNS Spectrums 2013;18(6):348.

\section{Wilson 1994 \{published data only\}}

Wilson WH. Open clozapine treatment following a controlled clinical trial of lithium augmentation of haloperidol for refractory schizophrenia. Lithium 1994;5(2):113-4.

\section{Winseck 2013 \{published data only\}}

Winseck A, Glick ID, Weiden PJ, Citrome L, Alva G, Kianifard F, et al. Initial 2-week outcomes following 2 methods of switching to iloperidone from aripiprazole in patients with schizophrenia. CNS Spectrums 2013;18(6):362-3.

\section{Wu 2002 \{published data only\}}

Wu L. A control study of risperidone and clozapine combination for the treatment of refractory schizophrenia. Health Psychology 2002;10:135-7.

\section{Wu 2015 \{published data only\}}

吴士玲, 张金香. Clinical study of aripiprazole combined with ziprasidone for schizophrenia [阿立哌唑结合齐拉西酮治疗精

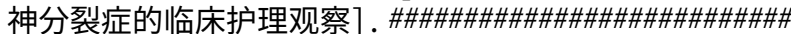
2015;22(1):145.

\section{Xia 2014 \{published data only\}}

Xia SY, Zhang YR, Yu H, Meng X, Zhang P, Liu J. Treatment of antipsychotic drug-induced phlegm dampness type amenorrhea by Wuji Powder and a small dose aripiprazole: a clinical study. Chinese Journal of Integrated Traditional and Western Medicine 2014;34(12):1440-3.

\section{Xu 2008 \{published data only\}}

Xu. Clinical observation of sertraline combined with sulpiride for first onset schizophrenia [舍曲林合并舒必利治疗首发
精神分裂症临床观察]. Ningxia Medical Journal [\#\#\#\#\#] 2008;30(6):542-3.

Yamanouchi 2015 \{published data only\}

UMIN000004511. The clinical study to correct multiple and large amount of administering to antipsychotic safely and effectively. www.umin.ac.jp/ctr/index.htm (first received 10 November 2010).

* Yamanouchi Y, Sukegawa T, Inagaki A, Inada T, Yoshio T, Yoshimura R, et al. Evaluation of the individual safe correction of antipsychotic agent polypharmacy in Japanese patients with chronic schizophrenia: validation of safe corrections for antipsychotic polypharmacy and the high-dose method. International Journal of Neuropsychopharmacology 2015;18:5. [DOI: http://dx.doi.org/10.1093/ijnp/pyu016]

\section{Yue 2004 \{published data only\}}

Yue $\mathrm{H}$, Song L, Xu Y. A comparative trial of risperidone in the treatment of schizophrenia over two years. Shangai Archive of Psychiatry 2004;16:165-7.

\section{Zhang 2012 \{published data only\}}

Zhang L, Wang J, Zhang Z. Shugan-Jieyu capsule combined with antipsychotics for the treatment of negative symptoms of schizophrenia [舒肝解郁胶糞合并抗精神病药物治疗精神分 裂症阴性症状的疗效]. Zhejiang Journal of Traditional Chinese Medicine 2012;10(4):228-9.

\section{Zhang 2013 \{published data only\}}

Zhang B, Chang L. Venlafaxine combined antipsychotics in the treatment of negative symptoms of schizophrenia [文拉法辛 联合抗精神病药治疗精神分裂症阴性症状疗效分析]. Shanxi Medical Journal 2013;42(6):685-6.

\section{Zink 2009 \{published data only\}}

Kuwilsky A, Krumm B, Englisch S, Dressing H, Zink M. Long-term efficacy and tolerability of clozapine combined with ziprasidone or risperidone. Pharmacopsychiatry 2010;43(6):216-20.

Zink M. Clozapine-augmentation with ziprasidone or risperidone, a randomized, prospective trial. www.clinicaltrials.gov (first received 15 September 2005). [MEDLINE: Zink 2005_a]

* Zink M, Kuwilsky A, Krumm B, Dressing H. Efficacy and tolerability of ziprasidone versus risperidone as augmentation in patients partially responsive to clozapine: a randomised controlled clinical trial. Journal of Psychopharmacology 2009;23(3):305-14. [0269-8811: (Print); MEDLINE: Zinc 2009]

\section{References to studies awaiting assessment}

\section{NCT01450514 \{published data only\}}

NCT01450514. Proof-of-concept study of pipamperone 15mg added to stable risperidone or paliperidone treatment in chronic schizophrenic and schizoaffective patients with residual symptoms: a Phase I/Ila, randomized, double-blind, placebocontrolled trial of 7 weeks. clinicaltrials.gov/show/NCT01450514 (first received 7 October 2011). 


\section{Xu 2006 \{published data only\}}

Xu L, Ji Jue H, Shi H. A control study of aripiprazole in the treatment of hyperprolactinemia by antipsychotics origin [阿立 呱哩治疗抗精神病药物所致 高催乳素血症对照研究]. Chinese Journal of Behavioral Medical Science 2006;15(8):718-20.

Yuan 2014 \{published data only\}

Han X, Yuan YB, Yu X. The Chinese first-episode schizophrenia trial: Background and study design [中國首發精神分裂症 臨床試驗(CNFEST):背景和研究設計] . East Asian Archives of Psychiatry 2014;24:169-73.

Yuan Y, Yang F, Lu Z, Wang CY, Deng H, Zhao J, et al. Effectiveness of three atypical antipsychotic-initiated treatments in chinese first-episode schizophrenia: An open randomized clinical trial. Schizophrenia Research 2014;153(Suppl. 1):S218. [DOI: 10.1016/S0920-9964(14)70628-0]

\section{References to ongoing studies}

\section{CTRI-02-003397 \{published data only\}}

CTRI-02-003397. Efficacy of Aripiprazole adjunctive treatment on body weight, metabolic parameters, clinical efficacy, and adverse events in people with psychotic disorders on treatment with Clozapine. A randomized, double-blind, placebocontrolled trial. www.ctri.nic.in/Clinicaltrials/pmaindet2.php? trialid=5862 (first received 14 February 2013).

ISRCTN68824876 \{published data only\}

ISRCTN68824876. Amisulpiride augmentation in clozapineunresponsive schizophrenia. www.controlled-trials.com/ ISRCTN68824876 (first received 2 March 2010).

NCT01246232. Amisulpiride augmentation in clozapineunresponsive schizophrenia. clinicaltrials.gov/ct2/show/ NCT01246232 (first received 22 November 2010).

\section{Schmidt-Kraepelin 2013 \{published data only\}}

DRKS00003603. A randomized double-blind controlled trial to assess the benefits of olanzapine and amisulpiride combination treatment in acutely ill schizophrenia patients - COMBINE. ichgcp.net/clinical-trials-registry/NCT01609153 (first received 1 June 2012).

NCT01609153. A randomized double-blind controlled trial to assess the benefits of olanzapine and amisulpride combination treatment in acutely ill schizophrenia patients - COMBINE. http://ClinicalTrials.gov/show/NCT01609153 (first received 23 May 2012).

Schmidt-Kraepelin C. A RCT to assess the benefits of olanzapine and amisulpride combination treatment (COMBINE): design and methods. European Archives of Psychiatry and Clinical Neuroscience 2013;263(Suppl. 1):S15-6.

\section{Additional references}

\section{Ahmed 1998}

Ahmed I, Soares KV, Seifas R, Adams CE. Randomized controlled trials in Archives of General Psychiatry (1959-1995): a prevalence study. Archives of General Psychiatry 1998;55(8):754-5. [PUBMED: 9707389]

\section{Altman 1996}

Altman DG, Bland JM. Detecting skewness from summary information. BMJ 1996;313(7066):1200.

\section{Andreasen 1983}

Andreasen NC. The Scale for the Assessment of Negative Symptoms (SANS). lowa City, lowa: University of lowa, 1983.

\section{Andreasen 1984}

Andreasen NC. The Scale for the Assessment of Positive Symptoms (SAPS). Iowa City, lowa: University of lowa, 1984.

\section{APA 2000}

American Psychiatric Association. Diagnostic and Statistical Manual of Mental Disorders. 4th Edition. Washington DC: American Psychiatric Publishing Inc., 2000.

\section{APA 2013}

American Psychiatric Association. Diagnostic and Statistical Manual of Mental Disorders: DSM-5. Washington, DC: American Psychiatric Publishing, 2013. [DOI: 10.1176/ appi.books.9780890425596]

\section{Barber 2017}

Barber S, Olotu U, Corsi M, Cipriani A. Clozapine combined with different antipsychotic drugs for treatment-resistant schizophrenia. Cochrane Database of Systematic Reviews 2017, Issue 3. [DOI: 10.1002/14651858.CD006324.pub3; CD006324]

\section{Barnes 1989}

Barnes TRE. A rating scale for drug-induced akathisia. British Journal of Psychiatry 1989;154:672-6.

\section{Begg 1996}

Begg C, Cho M, Eastwood S, Horton R, Moher D, Olkin I, et al. Improving the quality of reporting of randomized controlled trials. The CONSORT statement. JAMA 1996;276(8):637-9. [PUBMED: 8773637]

\section{Bland 1997}

Bland JM. Statistics notes. Trials randomised in clusters. BMJ 1997;315:600.

\section{Boissel 1999}

Boissel JP, Cucherat M, Li W, Chatellier G, Gueyffier F, Buyse $M$, et al. The problem of therapeutic efficacy indices. 3. Comparison of the indices and their use [Apercu sur la problematique des indices d'efficacite therapeutique, 3 : comparaison des indices et utilisation. Groupe d'Etude des Indices D'efficacite]. Therapie 1999;54(4):405-11. [PUBMED: 10667106]

\section{CADTH 2012}

[No authors listed]. Combination and high-dose atypical antipsychotic therapy in patients with schizophrenia: systematic review. Canadian Agency for Drugs and Technologies in Health overviews 2012;2(3):e2301. [PUBMED: 23002379] 


\section{Centorrino 2005}

Centorrino F, Fogarty KV, Sani G, Salvatore P, Cincotta SL, Hennen J, et al. Use of combinations of antipsychotics: McLean Hospital inpatients, 2002. Human Psychopharmacology 2005;20(7):485-92. [DOI: 10.1002/hup.719]

\section{Chouinard 1980}

Chouinard G, Ross-Chouinard A, Annable L, Jones BD. The extrapyramidal symptom rating scale. Canadian Journal of Neurological Sciences 1980;7:233.

\section{Correll 2009}

Correll CU, Rummel-Kluge C, Corves C, Kane JM, Leucht S. Antipsychotic combinations vs monotherapy in schizophrenia: A meta-analysis of randomized controlled trials. Schizophrenia Bulletin 2009;35(2):443-57. [DOI: 10.1093/schbul/sbn018]

\section{Deeks 2000}

Deeks J. Issues in the selection for meta-analyses of binary data. 8th International Cochrane Colloquium; 2000 Oct 25-28; Cape Town. Cape Town: The Cochrane Collaboration, 2000.

\section{Deeks 2011}

Deeks JJ, Higgins JPT, Altman DG, editor(s). Chapter 9: Analysing data and undertaking meta-analyses. In: Higgins JPT, Green S, editor(s). Cochrane Handbook for Systematic Reviews of Interventions Version 5.1.0 (updated March 2011). The Cochrane Collaboration, 2011. Available from www.handbook.cochrane.org.

\section{DerSimonian 1986}

DerSimonian R, Laird N. Meta-analysis in clinical trials. Controlled clinical trials 1986;7(3):177-88.

\section{Divine 1992}

Divine GW, Brown JT, Frazier LM. The unit of analysis error in studies about physicians' patient care behavior. Journal of General Internal Medicine 1992;7(6):623-9.

\section{Donner 2002}

Donner A, Klar N. Issues in the meta-analysis of cluster randomized trials. Statistics in Medicine 2002;21:2971-80.

\section{Egger 1997}

Egger M, Davey Smith G, Schneider M, Minder C. Bias in meta-analysis detected by a simple, graphical test. $B M J$ 1997;315:629-34.

\section{Elbourne 2002}

Elbourne D, Altman DG, Higgins JPT, Curtina F, Worthingtond HV, Vaile A. Meta-analyses involving crossover trials: methodological issues. International Journal of Epidemiology 2002;31(1):140-9.

\section{Endicott 1976}

Endicott J, Spitzer RL, Fleiss JL, Cohen J. The Global Assessment Scale: A procedure for measuring overall severity of psychiatric disturbance. Archives of General Psychiatry 1976;33(6):766-71.

\section{Freudenreich 2002}

Freudenreich O, Goff D. Antipsychotic combination therapy in schizophrenia. A review of efficacy and risks of current combinations. Acta Psychiatrica Scandinavica 2002;106:320-30. [DOI: 10.1034/j.1600-0447.2002.01331.x]

\section{Furukawa 2006}

Furukawa TA, Barbui C, Cipriani A, Brambilla P, Watanabe N. Imputing missing standard deviations in meta-analyses can provide accurate results. Journal of Clinical Epidemiology 2006;59(7):7-10.

\section{Gaebel 2005}

Gaebel W, Weinmann S, Sartorius N, Rutz W, McIntyre JS. Schizophrenia practice guidelines: international survey and comparison. British Journal of Psychiatry 2005;187(3):248-55. [DOI: 10.1192/bjp.187.3.248]

\section{Gallego 2009}

Gallego JA, Bonetti J, Zhang J, Kane JM, Correll CU. Prevalence and correlates of antipsychotic polypharmacy: A systematic review and meta-regression of global and regional trends from the 1970s to 2009. Schizophrenia Research 2012;138(1):18-28. [DOI: 10.1016/j.schres.2012.03.018]

\section{Gangluy 2004}

Ganguly R, Kotzan JA, Miller LS, Kennedy K, Martin BC. Prevalence, trends, and factors associated with antipsychotic polypharmacy among Medicaid-eligible schizophrenia patients, 1998-2000. Journal of Clinical Psychiatry 2004;65(10):1377-88. [DOI: 10.4088/JCP.v65n1013]

\section{Gardner 2010}

Gardner DM, Murphy AL, O'Donnell H, Centorrino F, Baldessarini RJ. International consensus study of antipsychotic dosing. American Journal of Psychiatry 2010;167(6):686-93. [DOI: 10.1176/appi.ajp.2009.09060802]

\section{Grunder 2009}

Gründer G, Hippius H, Carlsson A. The 'atypicality' of antipsychotics: a concept re-examined and re-defined. Nature Reviews Drug Discovery 2009;8(3):197-202. [DOI: 10.1038/ nrd2806]

\section{Gulliford 1999}

Gulliford MC. Components of variance and intraclass correlations for the design of community-based surveys and intervention studies: data from the Health Survey for England 1994. American Journal of Epidemiology 1999;149:876-83.

\section{Guy 1976}

Guy U. Early Clinical Drug Evaluation (ECDEU) Assessment Manual for Psychopharmacology. National Institute of Mental Health, 1976.

\section{Hasan 2012}

Hasan A, Falkai P, Wobrock T, Lieberman J, Glenthoj B, Gattaz WF, et al. World Federation of Societies of Biological Psychiatry (WFSBP) Guidelines for Biological Treatment of Schizophrenia, part 1: update 2012 on the acute treatment of schizophrenia and the management of treatment resistance. 
World Journal of Biological Psychiatry 2012;13(5):318-78. [DOI: 10.3109/15622975.2012.696143]

\section{Heinrich 1984}

Heinrich DW, Hanlon TE, Carpenter WT. The quality of life scale: an instrument for rating the schizophrenic deficit syndrome. Schizophrenia Bulletin 1984;10:388-98.

\section{Higgins 2003}

Higgins JP, Thompson SG, Deeks JJ, Altman DG. Measuring inconsistency in meta-analyses. BMJ 2003;327:557-60.

\section{Higgins 2011}

Higgins JPT, Green S, editor(s). Chapter 7: Selecting studies and collecting data. In: Higgins JPT, Green S, editor(s), Cochrane Handbook for Systematic Reviews of Interventions Version 5.1.0 (updated March 2011). The Cochrane Collaboration, 2011. Available from www.handbook.cochrane.org.

\section{Higgins 2011a}

Higgins JPT, Altman DG, Sterne JAC, editor(s). Chapter 8: Assessing risk of bias in included studies. In: Higgins JPT, Green S, editor(s). Cochrane Handbook for Systematic Reviews of Interventions Version 5.1.0 (updated March 2011). The Cochrane Collaboration, 2011. Available from www.handbook.cochrane.org.

\section{Jääskeläinen 2012}

Jääskeläinen, Juola P, Hirvonen N, McGrath J, Saha S, Isohanni M, et al. A systematic review and meta-analysis of recovery in schizophrenia. Schizophrenia Bulletin 2012;39(6):1296-306. [DOI: 10.1093/schbul/sbs130]

\section{Kapur 2001}

Kapur S, Remington G. Dopamine D2 receptors and their role in atypical antipsychotic action: still necessary and may even be sufficient. Biological Psychiatry 2001;50(11):873-83. [DOI: 10.1016/S0006-3223(01)01251-3]

\section{Kay 1986}

Kay SR, Opler LA, Fiszbein A. Positive and Negative Syndrome Scale (PANSS) Manual. North Tonawanda (NY): Multi-Health Systems, 1986.

\section{Kay 1987}

Kay SR, Fiszbein A, Opler LA. The positive and negative syndrome scale (PANSS) for schizophrenia. Schizophrenia Bulletin 1987;13(2):261-76.

\section{Kim 2002}

Kim JH, Jung HD, Kang UG, Jeong SH, Ahn YM, Byun HJ, et al. Metric characteristics of the drug-induced extrapyramidal symptoms scale (DIEPSS): A practical combined rating scale for drug-induced movement disorders. Movement Disorders 2002;17:1354-9.

\section{Kirkpatrick 2006}

Kirkpatrick B, Fenton WS, Carpenter WT, Marder SR. The NIMH-MATRICS consensus statement on negative symptom. Schizophrenia bulletin 2006;32(2):214-9. [DOI: 10.1093/schbul/ sbj053]

\section{Leon 2006}

Leon AC, Mallinckrodt CH, Chuang-Stein C, Archibald DG, Archer GE, Chartier K. Attrition in randomized controlled clinical trials: methodological issues in psychopharmacology. Biological Psychiatry 2006;59(11):1001-5. [PUBMED: 16905632]

\section{Leucht 2005}

Leucht S, Kane JM, Kissling W, Hamann J, Etschel E, Engel RR. What does the PANSS mean?. Schizophrenia Research 2005;79(2-3):231-8. [PUBMED: 15982856]

\section{Leucht 2005a}

Leucht S, Kane JM, Kissling W, Hamann J, Etschel E, Engel R. Clinical implications of brief psychiatric rating scale scores. British Journal of Psychiatry 2005;187:366-71. [PUBMED: 16199797]

\section{Lingjaerde 1987}

Lingjaerde O, Ahlfors UG, Bech P, Dencker SJ, Elgen K. The UKU side effect rating scale. A new comprehensive rating scale for psychotropic drugs and across-sectional study of side effects in neuroleptic-treated patients. Acta Psychiatrica Scandinavica 1987;334(Suppl.):1-100.

\section{Magalhães 2016}

Magalhães PVS, Dean O, Andreazza AC, Berk M, Kapczinski F. Antioxidant treatments for schizophrenia. Cochrane Database of Systematic Reviews 2016, Issue 2. [DOI: 10.1002/14651858.CD008919.pub2]

\section{Marshall 2000}

Marshall M, Lockwood A, Bradley C, Adams C, Joy C, Fenton M. Unpublished rating scales: a major source of bias in randomised controlled trials of treatments for schizophrenia. British Journal of Psychiatry 2000;176:249-52.

\section{McGrath 2008}

McGrath J, Saha S, Welham J. Schizophrenia: A concise overview of incidence, prevalence, and mortality. Epidemiologic Reviews 2008;30(1):67-76. [DOI: 10.1093/epirev/mxn001]

\section{Meader 2014}

Meader N, King K, Llewellyn A, Norman G, Brown J, Rodgers M, et al. A checklist designed to aid consistency and reproducibility of GRADE assessments: development and pilot validation. Systematic Reviews 2014;3:82. [DOI: 10.1186/2046-4053-3-82]

\section{Meltzer 2000}

Meltzer HY, Kostakoglu AE. Combining antipsychotics: is there evidence for efficacy?. Psychiatric Times 2000;17:25-34.

\section{Meng 2015}

Meng M, Li W, Zhang S, Wang HY, Sheng JH, Wang J, et al. Using aripiprazole to reduce antipsychotic-induced hyperprolactinemia: meta-analysis of currently available randomized controlled trials. Shanghai Archives of Psychiatry 2015;27(1):4-17. [DOI: 10.11919/j.issn.1002-0829.215014]

\section{Misawa 2011}

Misawa F, Shimizu K, Fujii Y, Miyata R, Koshiishi F, Kobayashi M, et al. Is antipsychotic polypharmacy associated with metabolic 
syndrome even after adjustment for lifestyle effects?: a cross-sectional study. BMC Psychiatry 2011;11(1):118. [DOI: 10.1186/1471-244X-11-118]

\section{Naber 1995}

Naber D. A self-rating to measure subjective effects of neuroleptic drugs. Relationships to objective psychopathology, quality of life, compliance and other clinical variables. International Clinical Psychopharmacology 1995;10:133-8.

\section{Overall 1962}

Overall JE, Gorham DR. The brief psychiatric rating scale. Psychological Reports 1962;10:790-812.

\section{Paton 2008}

Paton C, Barnes TR, Cavanagh MR, Taylor D, Lelliott P. Highdose and combination antipsychotic prescribing in acute adult wards in the UK: the challenges posed by prn prescribing. British Journal of Psychiatry 2008;192(6):435-9. [DOI: 10.1192/ bjp.bp.107.042895]

\section{Rupnow 2007}

Rupnow MF, Greenspan A, Gharabawi GM, Kosik-Gonzalez C, Zhu Y, Stahl SM. Incidence and costs of polypharmacy: data from a randomized, double-blind, placebo-controlled study of risperidone and quetiapine in patients with schizophrenia or schizoaffective disorder. Current Medical Research and Opinion 2007;23(11):2815-22. [DOI: 10.1185/030079907X233359]

\section{Schulz 1995}

Schulz KF, Chalmers I, Hayes RJ, Altman DG. Empirical evidence of bias: dimensions of methodological quality associated with estimates of treatment effects in controlled trials. JAMA 1995;273:408-12.

\section{Schünemann 2011}

Schünemann HJ, Oxman AD, Vist GE, Higgins JPT, Deeks JJ, Glasziou P, et al. Chapter 12: Interpreting results and drawing conclusions. In Higgins JPT, Green S, editor(s), Cochrane Handbook for Systematic Reviews of Interventions Version 5.1.0 (updated March 2011). The Cochrane Collaboration, 2011. Available from www.cochrane-handbook.org.

\section{Simpson 1970}

Simpson GM, Angus JWS. A rating scale for extrapyramidal side effects. Acta Psychiatrica Scandinavica 1970;(Suppl 212):11-9.

\section{Sommer 2012}

Sommer IE, Begemann MJ, Temmerman A, Leucht S. Pharmacological augmentation strategies for schizophrenia patients with insufficient response to clozapine: a quantitative literature review. Schizophrenia Bulletin 2012;38(5):1003-11. [PUBMED: 21422107]

\section{CHARACTERISTICS OF STUDIES}

\section{Characteristics of included studies [ordered by study ID]}

\section{Sterne 2011}

Sterne JAC, Egger M, Moher D, editor(s). Chapter 10: Addressing reporting biases. In: Higgins JPT, Green S, editor(s). Cochrane Handbook for Systematic Reviews of Intervention. Version 5.1.0 (updated March 2011). The Cochrane Collaboration, 2011. Available from www.handbook.cochrane.org.

\section{Taylor 2009}

Taylor DM, Smith L. Augmentation of clozapine with a second antipsychotic--a meta-analysis of randomized, placebo-controlled studies. Acta Psychiatrica Scandinavica 2009;119(6):419-25. [PUBMED: 19245679]

\section{Ukoumunne 1999}

Ukoumunne OC, Gulliford MC, Chinn S, Sterne JAC, Burney PGJ. Methods for evaluating area-wide and organisation-based intervention in health and health care: a systematic review. Health Technology Assessment 1999;3(5):1-75.

\section{Waddington 1998}

Waddington JL, Youssef HA, Kinsella A. Mortality in schizophrenia. Antipsychotic polypharmacy and absence of adjunctive anticholinergics over the course of a 10-year prospective study. British Journal of Psychiatry 1998;173:325-9.

\section{Ware 1992}

Ware JE, Sherbourne CD. The MOS 36-Item short-form health survey $\left(\mathrm{SF}-36^{\oplus}\right)$ : I. conceptual framework and item selection. Medical Care 1992;30(6):473-83.

\section{Weiden 1999}

Weiden PJ, Casey DE. "Polypharmacy": Combining antipsychotic medications in the treatment of schizophrenia. Journal of Practical Psychiatry and Behavioral Health 1999;5:229-33.

\section{Xia 2009}

Xia J, Adams CE, Bhagat N, Bhagat V, Bhoopathi P, El-Sayeh H, et al. Loss to outcomes stakeholder survey: the LOSS study. Psychiatric Bulletin 2009;33(7):254-7.

\section{References to other published versions of this review \\ Maayan 2011}

Maayan N, Soares-Weiser K, Xia J, Adams CE. Antipsychotic combinations for schizophrenia. Cochrane Database of Systematic Reviews 2011, Issue 2. [DOI: 10.1002/14651858.CD009005]

* Indicates the major publication for the study

\section{A +any antipsychotic 2011}

Methods Allocation: randomised. 
A +any antipsychotic 2011 (Continued)

Blinding: open-label.

Duration: 6 months.

Setting: outpatients.

Design: parallel.

Country: USA, multi-centre.

Darticipants
Diagnosis: schizophrenia or schizoaffective disorder (DSM-IV).
Sex: 127.
Age: $>18$ years. 43.
History: patients taking any two prescribed antipsychotic medications with persistent psychopatholo-
gy or significant side effects and no exacerbation within the past 3 months.

Interventions $\quad$ 1. Combination therapy: stay on antipsychotic combination (any combination of anti-psychotic medication $)^{\star}(\mathrm{N}=62)$.

2. Monotherapy: switch to antipsychotic monotherapy within 30 days $^{\star \star}(\mathrm{N}=65)$.

Schedule: no details of doses.

- Usable data -
$\begin{aligned} & \text { 1. Leaving the study early. } \\ & \text { 2. Service utilisation: hospital admission. } \\ & \text { 3. Adverse events: serious event or requiring discontinuation, movement disorders. }\end{aligned}$

- Unable to use -

1. Change in prolactin level (not reported).

2. Adverse events: AIMS, SAS (not reported).

3. Adverse events: weight gain (not reported).

4. Adverse events: blood levels (not reported).

\section{- Not used in review -}

1. Time to all-cause treatment discontinuation, ASEX physiological measurements, BMI.

Notes $\quad$ The most common antipsychotic combinations were quetiapine and risperidone, quetiapine and a
first-generation antipsychotic, risperidone and a first-generation antipsychotic, olanzapine and a first-
generation antipsychotic, ziprasidone and a first-generation antipsychotic, aripiprazole and quetiapine
and olanzapine and risperidone.

${ }^{* *} 12(21 \%)$ discontinued quetiapine, $10(17 \%)$ discontinued risperidone, nine (15\%) discontinued olan-
zapine, eight (14\%) discontinued haloperidol, and the remaining $19(33 \%)$ discontinued other antipsy-
chotics (each at less than $10 \%$ of discontinuations).

\section{Risk of bias}

\begin{tabular}{lll}
\hline Bias & Authors' judgement & Support for judgement \\
\hline $\begin{array}{l}\text { Random sequence genera- } \\
\text { tion (selection bias) }\end{array}$ & Unclear risk & $\begin{array}{l}\text { "The study's project director used a single predetermined randomisation } \\
\text { stream (i.e., without stratification)", no further details provided. }\end{array}$ \\
\hline $\begin{array}{l}\text { Allocation concealment } \\
\text { (selection bias) }\end{array}$ & Low risk & $\begin{array}{l}\text { Central allocation, "to maintain blinding, randomisation was managed cen- } \\
\text { trally". }\end{array}$ \\
\hline $\begin{array}{l}\text { Blinding (performance } \\
\text { bias and detection bias) } \\
\text { All outcomes }\end{array}$ & High risk & $\begin{array}{l}\text { Open-label, participants and personnel were not blinded, Assessment by } \\
\text { blinded clinical raters, precautions were taken not to reveal treatment alloca- } \\
\text { tion to raters. }\end{array}$ \\
\hline
\end{tabular}


A +any antipsychotic 2011 (Continued)

Incomplete outcome data Low risk ITT method was used for primary analyses. 14\% discontinued from the combi(attrition bias) nation group and $31 \%$ from the monotherapy group, reasons for discontinuaAll outcomes tion were provided.

Selective reporting (re- High risk Not all pre-stated outcomes were fully reported. porting bias)

Other bias Low risk Supported by NIMH.

A +any antipsychotic 2012

\begin{tabular}{ll}
\hline Methods & Allocation: randomised. \\
& Blinding: open-label. \\
Duration: 8 months. & Setting: outpatient. \\
Design: parallel. & Country: not reported.
\end{tabular}

Participants

Diagnosis: schizophrenia or delusional disorder diagnosis (DSM-IV).

$\mathbf{N}=60$.

Sex: M 36, F 24.

Age: Not specified.

History: Taking 1 antipsychotic medication when they entered study.

Interventions

1. Combination therapy: switch to dual antipsychotics by adding up another medication* $(\mathrm{N}=30)$.

2. Monotherapy: assigned to continue receiving monotherapy $(\mathrm{N}=30)$.

Outcomes

\section{- Usable data -}

1. Leaving the study early.

- Unable to use -

1. Clinical response: GAF (Not reported).

2. Mental state: BPRS (Not reported).

\section{- Not used in this review -}

1. BMI and Lunsers scale.

Notes $\quad$ *Fom report: "Choice of medication to add was left to prescribe and patient and also at their discre-
tion the dose of all drug could be raised or lowered."

\section{Risk of bias}

\begin{tabular}{lll}
\hline Bias & Authors' judgement & Support for judgement \\
\hline $\begin{array}{l}\text { Random sequence genera- } \\
\text { tion (selection bias) }\end{array}$ & Unclear risk & "randomly allocated." No other information provided. \\
\hline $\begin{array}{l}\text { Allocation concealment } \\
\text { (selection bias) }\end{array}$ & Unclear risk & No information provided. \\
\hline $\begin{array}{l}\text { Blinding (performance } \\
\text { bias and detection bias) }\end{array}$ & High risk & "Choice of medication to join was left to prescriber and patient \\
\hline $\begin{array}{l}\text { Antipsychotic combinations for schizophrenia (Review) } \\
\text { Copyright } \odot 2017 \text { The Cochrane Collaboration. Published by John Wiley \& Sons, Ltd. }\end{array}$
\end{tabular}


A +any antipsychotic 2012 (Continued)

All outcomes
"Outcomes were performed by trained assessor masked to allocated treatment."
Incomplete outcome data High risk

(attrition bias)

All outcomes
Dropout: 18 (30\%) not equally subdivided between the two groups.

\begin{tabular}{lll}
\hline $\begin{array}{l}\text { Selective reporting (re- } \\
\text { porting bias) }\end{array}$ & High risk & No mean and SD deviation reported for BPRS and GAF. \\
\hline Other bias & Unclear risk & None obvious. \\
\hline
\end{tabular}

A +any antipsychotic 2015

\begin{tabular}{ll}
\hline Methods & Allocation: randomised. \\
Blinding: open-label. \\
Duration: 360 days. \\
Setting: outpatient. \\
Design: parallel. \\
Country: USA, multi-centre. \\
Diagnosis: schizophrenia or schizoaffective (DSM IV-TR). \\
N $=104$. \\
Age: $~$ \\
Participants years. \\
Sex: male and female. \\
History: chronic, stable patients, who had been receiving 2 antipsychotic medications concurrently for \\
at least 90 days (average duration of antipsychotic polypharmacy was 2.5 years).
\end{tabular}

Interventions

1. Combination therapy (Stay): stay participants were required to remain on the two antipsychotic medications they were currently receiving $(N=52)$.

2. Monotherapy (Switch): required to switch from the two antipsychotics they were currently receiving to one of these two within 60 days of baseline assessments ( $N=52$ ).

Outcomes

- Usable data -

1. Leaving the study early.

\section{- Not able to use -}

1. Clinical response: $\mathrm{CGI}-\mathrm{S}, \mathrm{CGI}-\mathrm{I}$ (Unable to impute).

2. Mental state: PANSS (Unable to impute).

3. Adverse events: BAS, SAS, AIMS (Unable to impute).

\section{- Not used in this review -}

1. BMI, lipids, HbA1C.

\section{Notes}

\section{Risk of bias}


A +any antipsychotic 2015 (Continued)

\title{
Bias Authors' judgement Support for judgement
}

Random sequence genera- Unclear risk tion (selection bias)

"The research coordinator at each site applied a site-specific random assignment protocol."

Insufficient information about the sequence generation process.

Allocation concealment $\quad$ Unclear risk Insufficient information to permit judgement.
(selection bias)

(selection bias)

High risk

Blinding (performance

bias and detection bias)

All outcomes
Incomplete outcome data High risk

(attrition bias)

All outcomes
"While treatment was open-label, baseline and subsequent assessments were conducted by "independent assessors" blinded to the research status of participants."

Selective reporting (re- High risk No usable data for CGI-S, CGI-I, PANSS, BAS, SAS and AIMS.
porting bias)

porting bias)

" $21 \%$ discontinued from the combination group and 52\% from the monotherapy group, reasons for discontinuation were not provided for all participants."

Other bias High risk

\begin{abstract}
"the switch and stay groups differed significantly on baseline antipsychotic dose with stay participants receiving an average of $8 \mathrm{mg}$ more olanzapine equivalents per day" Baseline dose imbalances.

This study was funded by the Florida Agency for Health Care Administration.
\end{abstract}

A + pimozide 1985

\begin{tabular}{|c|c|}
\hline Methods & $\begin{array}{l}\text { Allocation: randomised. } \\
\text { Blinding: double-blind. } \\
\text { Duration: } 1 \text { year. } \\
\text { Setting: outpatients. } \\
\text { Design: parallel. } \\
\text { Country: Japan. }\end{array}$ \\
\hline Participants & $\begin{array}{l}\text { Diagnosis: remitted schizophrenics (DSM-III). } \\
\text { N = 106. } \\
\text { Sex: M 78, F } 28 . \\
\text { Age: } \sim 39 \text { years. } \\
\text { History: recovery stage of remission or residual phase, had reported reg }\end{array}$ \\
\hline Interventions & $\begin{array}{l}\text { 1. Combination therapy: thioridazine } 25 \mathrm{mg}+\text { pimozide } 2 \mathrm{mg}(\mathrm{N}=11) \text {. } \\
\text { 2. Combination therapy: thioridazine } 25 \mathrm{mg}+\text { pimozide } 6 \mathrm{mg}(\mathrm{N}=12) \text {. } \\
\text { 3. Combination therapy: thioridazine } 75 \mathrm{mg}+\text { pimozide } 2 \mathrm{mg}(\mathrm{N}=11) \text {. } \\
\text { 4. Combination therapy: thioridazine } 75 \mathrm{mg}+\text { pimozide } 6 \mathrm{mg}(\mathrm{N}=13) \text {. } \\
\text { 5. Monotherapy: thioridazine } 25 \mathrm{mg}(\mathrm{N}=12) \text {. } \\
\text { 6. Monotherapy: thioridazine } 75 \mathrm{mg}(\mathrm{N}=10) \text {. } \\
\text { 7. Monotherapy: pimozide } 2 \mathrm{mg}(\mathrm{N}=13) \text {. } \\
\text { 8. Monotherapy: pimozide } 6 \mathrm{mg}(\mathrm{N}=11) \text {. }\end{array}$ \\
\hline
\end{tabular}

Schedule: Daily doses.

Outcomes

- Usable data -

1. Leaving the study early. 
A +pimozide 1985 (Continued)

2. Clinical response: not clinically improved.

3. Adverse events: relapse.

\section{- Unable to use -}

1. Adverse events: prolactin levels (data not reported).

\begin{tabular}{|c|c|c|}
\hline Notes & ${ }^{\star}$ Data from a previous & tudy were used as a retrospective placebo group. \\
\hline \multicolumn{3}{|l|}{ Risk of bias } \\
\hline Bias & Authors' judgement & Support for judgement \\
\hline $\begin{array}{l}\text { Random sequence genera- } \\
\text { tion (selection bias) }\end{array}$ & Unclear risk & $\begin{array}{l}\text { Each patient was randomly assigned to one drug treatment in a double-blind } \\
\text { design. }\end{array}$ \\
\hline $\begin{array}{l}\text { Allocation concealment } \\
\text { (selection bias) }\end{array}$ & Unclear risk & No details provided. \\
\hline $\begin{array}{l}\text { Blinding (performance } \\
\text { bias and detection bias) } \\
\text { All outcomes }\end{array}$ & Low risk & $\begin{array}{l}\text { Drug appearance, with respect to powder colour, taste, and volume, was made } \\
\text { identical by adding a common gastric aid, SMP. }\end{array}$ \\
\hline $\begin{array}{l}\text { Incomplete outcome data } \\
\text { (attrition bias) } \\
\text { All outcomes }\end{array}$ & High risk & $\begin{array}{l}6 \text { patients who took drugs irregularly were excluded from the final analy- } \\
\text { sis. Other patients discontinued designated use of the assigned drugs either } \\
\text { through overdosage }(N=21) \text { or because of relapse }(N=55) \text {. }\end{array}$ \\
\hline $\begin{array}{l}\text { Selective reporting (re- } \\
\text { porting bias) }\end{array}$ & High risk & Not all expected outcomes reported. \\
\hline Other bias & Unclear risk & Source of funding not reported. \\
\hline
\end{tabular}

A +reserpine 1957

$\begin{array}{ll}\text { Methods } & \text { Allocation: randomised. } \\ \text { Blinding: double-blind. } \\ \text { Duration: } 6 \text { months ( } 2 \text { months baseline, } 3 \text { months before cross-over to a second follow up of } 1 \text { month). } \\ \text { Setting: inpatients. } \\ \text { Design: cross-over. } \\ \text { Country: USA. }\end{array}$
Participants Diagnosis: regressed schizophrenic patients (regression, withdrawal, and intellectual disorganisation). $\mathbf{N}=32$.
Sex: not reported.
Age: average 35 years.
History: chronic, regressed schizophrenia, had received prolonged courses of ECT, insulin, and "total push" programs, without lasting benefit.
Interventions
1. Combination therapy: reserpine + chlorpromazine $(N=10)$.
2. Monotherapy: reserpine $(\mathrm{N}=10)$.
3. Monotherapy: chlorpromazine $(\mathrm{N}=10)$.

Schedule: reserpine $1 \mathrm{mg}$ to $4 \mathrm{mg}$ /day + chlorpromazine $100 \mathrm{mg}$ to $400 \mathrm{mg} /$ day, reserpine $4 \mathrm{mg}$ to 8 $\mathrm{mg}$ /day, chlorpromazine $200 \mathrm{mg}$ to $1200 \mathrm{mg}$ /day.

The medications were given at noon and 8:00 each day. 
A +reserpine 1957 (Continued)

Outcomes

\section{- Usable data -}

1. Leaving the study early.

2. Clinical response: not clinically improved.

3. Adverse events: serious or requiring discontinuation.

\section{- Unable to use -}

1. Behaviour: MACC Behavioural Adjustment Scale, observation of behaviour (results illegible).

Notes

\section{Risk of bias}

\begin{tabular}{lll}
\hline Bias & Authors' judgement & Support for judgement \\
\hline $\begin{array}{l}\text { Random sequence genera- } \\
\text { tion (selection bias) }\end{array}$ & Unclear risk & No details provided. \\
\hline $\begin{array}{l}\text { Allocation concealment } \\
\text { (selection bias) }\end{array}$ & High risk & Medications were designated with an alphabetical code. \\
\hline $\begin{array}{l}\text { Blinding (performance } \\
\text { bias and detection bias) }\end{array}$ & Low risk & $\begin{array}{l}\text { Neither raters nor participants were aware of the nature or quantity of drug } \\
\text { given. Participants were given an identical number of capsules regardless of } \\
\text { the individual dosage. }\end{array}$ \\
\hline $\begin{array}{l}\text { Incomplete outcome data } \\
\text { (attrition bias) } \\
\text { All outcomes }\end{array}$ & High risk & $\begin{array}{l}\text { Two patients dropped out because of adverse events and were replaced by } \\
\text { two reserve patients who had previously been receiving placebos. }\end{array}$ \\
\hline $\begin{array}{l}\text { Selective reporting (re- } \\
\text { porting bias) }\end{array}$ & High risk & Not all expected outcomes reported. \\
\hline \begin{tabular}{l} 
Other bias \\
\hline
\end{tabular} & Unclear risk & Funding not reported. \\
\hline
\end{tabular}

\section{A +sulpiride 1994}

$\begin{array}{ll}\text { Methods } & \text { Allocation: randomised. } \\ \text { Blinding: open-label. } \\ \text { Duration: 3 years. } \\ \text { Setting: inpatients. } \\ \text { Country: China. }\end{array}$

\begin{tabular}{ll}
\hline Participants & Diagnosis: schizophrenia (CCMD-III). \\
N $=105^{\star}$. \\
Sex: $\mathrm{F} 105 . .0$ \\
Age: $18-64$ years, average 30 years. \\
History: illness duration, range of $0.25-12$ years, average 3 SD 3 years. \\
\hline Interventions
\end{tabular}

Outcomes

\section{- Usable data -}

1. Clinical response: no clinical improvement, relapse. 


\section{-Unable to use -}

1. Leaving the study early (no information about the number who dropped out in each treatment group).

\begin{tabular}{ll}
\hline Notes & *Number of reported cases. \\
& Clozapine dosage was higher for the clozapine alone group. \\
& Abstract in English, report in Chinese.
\end{tabular}

\section{Risk of bias}

\begin{tabular}{lll}
\hline Bias & Authors' judgement & Support for judgement \\
\hline $\begin{array}{l}\text { Random sequence genera- } \\
\text { tion (selection bias) }\end{array}$ & Unclear risk & 'Randomised into groups' without further detail. \\
\hline $\begin{array}{l}\text { Allocation concealment } \\
\text { (selection bias) }\end{array}$ & Unclear risk & No information provided. \\
\hline $\begin{array}{l}\text { Blinding (performance } \\
\text { bias and detection bias) }\end{array}$ & High risk & Open-label study. \\
$\begin{array}{l}\text { All outcomes } \\
\begin{array}{l}\text { Incomplete outcome data } \\
\text { (attrition bias) } \\
\text { All outcomes }\end{array}\end{array}$ High risk & $\begin{array}{l}\text { 5 people left the study early but no information about from which treatment } \\
\text { group. Reasons for leaving early not described. }\end{array}$ \\
\hline $\begin{array}{l}\text { Selective reporting (re- } \\
\text { porting bias) }\end{array}$ & High risk & Not all expected outcomes reported. \\
\hline \begin{tabular}{l} 
Other bias \\
\hline
\end{tabular} & Unclear risk & Source of funding not reported. \\
\hline
\end{tabular}

\section{A +trifluoperazine 1964}

\begin{tabular}{ll}
\hline Methods & Allocation: randomised. \\
& Blinding: double-blind. \\
& Duration: 8 months. \\
& Setting: inpatients. \\
& Design: parallel. \\
Country: USA.
\end{tabular}

Participants Diagnosis: schizophrenia.

$\mathbf{N}=77$.

Sex: $M 77$.

Age: not reported.

History: chronic, most severely ill patients on the ward.
Interventions
1. Combination therapy: chlorpromazine + trifluoperazine $(N=27)$.
2. Monotherapy: chlorpromazine + placebo $(N=25)$.
3. Monotherapy: trifluoperazine + placebo $(N=25)$.

Schedule: chlorpromazine $150 \mathrm{mg}$ to $300 \mathrm{mg}$, trifluoperazine $5 \mathrm{mg}$ to $10 \mathrm{mg}$. Lower dosage used for the first two months and then the higher dosage for the next 6 months.

Outcomes - Usable data -


A +trifluoperazine 1964 (Continued)

1. Leaving the study early.

2. Clinical response: not clinically improved.

Notes

Risk of bias

\begin{tabular}{lll}
\hline Bias & Authors' judgement & Support for judgement \\
\hline $\begin{array}{l}\text { Random sequence genera- } \\
\text { tion (selection bias) }\end{array}$ & Unclear risk & $\begin{array}{l}\text { Assigned randomly to one of three drug groups and placed on a regimen of } \\
\text { capsules and tablets. }\end{array}$ \\
\hline
\end{tabular}

Allocation concealment $\quad$ Low risk
(selection bias)

Both capsules containing these supplies were coded with colour labels to conceal the identity of the regimens but to permit administrator to increase dosage from one range to a higher one (after two months and six months) without disrupting the study.

\begin{tabular}{|c|c|c|}
\hline $\begin{array}{l}\text { Blinding (performance } \\
\text { bias and detection bias) } \\
\text { All outcomes }\end{array}$ & Low risk & $\begin{array}{l}\text { Placebo capsules and placebo tablets matched the appearance of the cap- } \\
\text { sules and tablets of the two drugs to insure no knowledge of which group was } \\
\text { receiving which drug or drugs. }\end{array}$ \\
\hline
\end{tabular}

All outcomes

All included patients completed the trial.

Incomplete outcome data $\quad$ Low risk $\quad$ All included patients completed the trial.
(attrition bias)

All outcomes

\begin{tabular}{lll}
\hline $\begin{array}{l}\text { Selective reporting (re- } \\
\text { porting bias) }\end{array}$ & High risk & Not all expected outcomes reported. \\
\hline Other bias & Unclear risk & Source of support not reported. \\
\hline
\end{tabular}

\section{B +any antipsychotic 2013}

\begin{tabular}{|c|c|}
\hline Methods & $\begin{array}{l}\text { Allocation: randomised. } \\
\text { Blinding: single-blind. } \\
\text { Duration: } 24 \text { weeks }{ }^{\star} \text {. } \\
\text { Setting: outpatient. } \\
\text { Design: parallel. } \\
\text { Country: Japan. }\end{array}$ \\
\hline Participants & $\begin{array}{l}\text { Diagnosis: schizophrenia (DSM-IV-TR). } \\
\text { N=39. } \\
\text { Sex: M } 19 \text { F } 16 \text { (data from participants who ended the study). } \\
\text { Age: } 36 \text { years. } \\
\text { History: chronic patients without acute exacerbation on a stable dose of } 2 \text { antipsychotics without pre- } \\
\text { scription changes in the past } 3 \text { months. }\end{array}$ \\
\hline Interventions & $\begin{array}{l}\text { 1. Combination therapy: continuing antipsychotics combination }{ }^{\star \star} \\
\text { 2. Monotherapy: switch to monotherapy, each participant and physician decided together which of the } \\
\text { two antipsychotics to discontinue. Discontinuation had to occur within } 12 \text { weeks }\end{array}$ \\
\hline Outcomes & $\begin{array}{l}\text { - Usable data - } \\
\text { 1. Mental state: PANSS (Total, Positive, Negative). } \\
\text { 2. Adverse events: requiring discontinuation. }\end{array}$ \\
\hline
\end{tabular}


B +any antipsychotic 2013 (Continued)

\section{- Not usable data -}

1. Leaving the study early (dates of discontinuation where not presented).

\section{- Not used in this review -}

1. Brief Assessment of Cognition in Schizophrenia and the Life Assessment Scale for the Mentally Ill.

Notes

* From 0-12 weeks to discontinue polypharmacy, from 13-24 weeks to evaluate monotherapy versus combinations.

** The most common baseline polypharmacy combinations were risperidone and a first-generation antipsychotic $(\mathrm{N}=10)$, olanzapine and a first-generation antipsychotic $(\mathrm{N}=9)$, olanzapine and risperidone $(\mathrm{N}=5)$, risperidone and quetiapine $(\mathrm{N}=3)$, olanzapine and aripiprazole $(\mathrm{N}=3)$, aripiprazole and a firstgeneration antipsychotic $(N=3)$, quetiapine and aripiprazole $(N=2)$, blonanserin and a first-generation antipsychotic $(N=2)$, blonanserin and olanzapine $(N=1)$, blonanserin and quetiapine $(N=1)$.

No protocol- a priori was published.

\section{Risk of bias}

\begin{tabular}{|c|c|c|}
\hline Bias & Authors' judgement & Support for judgement \\
\hline $\begin{array}{l}\text { Random sequence genera- } \\
\text { tion (selection bias) }\end{array}$ & Low risk & $\begin{array}{l}\text { The patients were randomly divided into either the switching group or the } \\
\text { continuing group using StatView. }\end{array}$ \\
\hline $\begin{array}{l}\text { Allocation concealment } \\
\text { (selection bias) }\end{array}$ & Unclear risk & No information provided. \\
\hline $\begin{array}{l}\text { Blinding (performance } \\
\text { bias and detection bias) } \\
\text { All outcomes }\end{array}$ & High risk & $\begin{array}{l}\text { "For participants who were assigned to switch to monotherapy, each partici- } \\
\text { pant and physician decided together which of the two anti- psychotics to dis- } \\
\text { continue." } \\
\text { "The raters were blinded about which group the patients belonged to." }\end{array}$ \\
\hline $\begin{array}{l}\text { Incomplete outcome data } \\
\text { (attrition bias) } \\
\text { All outcomes }\end{array}$ & High risk & $\begin{array}{l}\text { Reasons for missing data differ in both group and this was not addressed in the } \\
\text { data analysis. }\end{array}$ \\
\hline $\begin{array}{l}\text { Selective reporting (re- } \\
\text { porting bias) }\end{array}$ & Low risk & All measured outcomes were reported. \\
\hline Other bias & Unclear risk & $\begin{array}{l}\text { Supported by Grant in Ministry of Education, Culture, Sports, Science and } \\
\text { Technology Japan. }\end{array}$ \\
\hline
\end{tabular}

\section{B +aripiprazole 2008}

\begin{tabular}{ll} 
Methods & Allocation: randomised. \\
& Blinding: double-blind. \\
& Duration: 16 week. \\
Setting: outpatients. \\
Design: parallel. \\
Country: Europe, multi-centre. \\
\hline Participants & Diagnosis: schizophrenia (DSM-IV-TR criteria). \\
& Sex: M 134, F 73. \\
& Age: average 39 years.
\end{tabular}


B +aripiprazole 2008 (Continued)

History: experienced at least $2.5 \mathrm{~kg}$ weight gain and sub-optimal efficacy and/or safety on clozapine.

Interventions 1 . Combination therapy: aripiprazole + clozapine $(N=108)$.

2. Monotherapy: clozapine + placebo $(\mathrm{N}=99)$.

Schedule: aripiprazole $5 \mathrm{mg}$ to $15 \mathrm{mg} /$ day, stable dose of clozapine (163 mg to $900 \mathrm{mg} /$ day).

- Usable data -
1. Leaving the study early.
2. Clinical response: GAF, CGI-I, CGI-S.
3. Mental state: PANSS.
4. Adverse events: serious or requiring discontinuation, weight gain, average weight gain, deaths, move-
ment disorders.
5. Quality of Life: Subjective Well Being under Neuroleptics (SWN - short form).

\section{- Not used in review -}

1. Investigator Assessment Questionnaire (IAQ), GEOPTE social cognition scale, ESS alertness scale, FSI fatigue scale, blood tests.

\begin{tabular}{lll}
\hline Notes & www.clinicaltrials.gov: NCT00300846 \\
\hline Risk of bias & & \\
\hline Bias & Authors' judgement & Support for judgement \\
\hline $\begin{array}{l}\text { Random sequence genera- } \\
\text { tion (selection bias) }\end{array}$ & Low risk & Computer-generated randomisation schedule. \\
\hline $\begin{array}{l}\text { Allocation concealment } \\
\text { (selection bias) }\end{array}$ & Low risk & "Randomization was achieved by a call-in interactive voice response system, \\
\hline $\begin{array}{l}\text { Blinding (performance } \\
\text { bias and detection bias) } \\
\text { All outcomes }\end{array}$ & Unclear risk & "Double-blind", no further details reported. \\
\hline $\begin{array}{l}\text { Incomplete outcome data } \\
\text { (attrition bias) } \\
\text { All outcomes }\end{array}$ & Unclear risk & \begin{tabular}{l} 
The LOCF method was used. \\
\hline $\begin{array}{l}\text { Selective reporting (re- } \\
\text { porting bias) }\end{array}$
\end{tabular} \\
\hline $\begin{array}{l}\text { Other bias } \\
\text { Low risk }\end{array}$ & \begin{tabular}{l} 
All pre-specified outcomes were reported. \\
\hline
\end{tabular} & $\begin{array}{l}\text { Supported by Bristol-Myers Squibb (Princeton, NJ, USA) and Otsuka Pharma- } \\
\text { ceutical Co., Ltd (Tokyo, Japan). }\end{array}$ \\
\hline
\end{tabular}

\section{B +aripiprazole 2011}

\begin{tabular}{ll}
\hline Methods & Allocation: randomised. \\
& Blinding: double-blind. \\
& Duration: 24 weeks. \\
Setting: outpatient. & Design: parallel. \\
Country: Italy.
\end{tabular}


B +aripiprazole 2011 (Continued)

$$
\mathbf{N}=40 \text {. }
$$

Sex: M 23, F 17 .

Age: 25 to 38 years.

History: patients demonstrated persistent positive and negative symptoms despite an adequate trial of clozapine at the highest tolerable range ( $200 \mathrm{mg}$ to $450 \mathrm{mg} /$ day), for at least 1 year.

Interventions

1. Combination therapy: clozapine ( $200 \mathrm{mg}$ to $450 \mathrm{mg} /$ day $)+$ aripiprazole $(10 \mathrm{mg}$ to $15 \mathrm{mg} / \mathrm{day})(\mathrm{N}=20)$.

2. Monotherapy: clozapine ( $200 \mathrm{mg}$ to $450 \mathrm{mg} /$ day $)+$ placebo $(\mathrm{N}=20)$.

Schedule: not reported

Outcomes
- Usable data -
1. Leaving the study early.
2. Mental state: BPRS.
3. Adverse events: movement disorders.

\section{- Unable to use-}

1. Mental state: SANS, SAPS (Skewed data).

\section{- Not used in review -}

1. Neurocogitive functioning: WCST; Neurocogitive functioning: the Verbal Fluency Task; Neurocogitive functioning: Stroop Colour-word Test, Calgary Depression Scale for Schizophrenia (CDSS).

\section{Notes}

\section{Risk of bias}

\begin{tabular}{lll}
\hline Bias & Authors' judgement & Support for judgement \\
\hline $\begin{array}{l}\text { Random sequence genera- } \\
\text { tion (selection bias) }\end{array}$ & Low risk & Randomisation - automated system. \\
\hline $\begin{array}{l}\text { Allocation concealment } \\
\text { (selection bias) }\end{array}$ & Low risk & $\begin{array}{l}\text { During the study, the randomisation list was held securely, and released only } \\
\text { after study completion. }\end{array}$ \\
\hline $\begin{array}{l}\text { Blinding (performance } \\
\text { bias and detection bias) } \\
\text { All outcomes }\end{array}$ & Low risk & $\begin{array}{l}\text { Aripiprazole and placebo were dispensed in identical-appearing capsules; pa- } \\
\text { tients randomise to placebo took the same number of capsules as those as- } \\
\text { signed to aripiprazole. }\end{array}$ \\
\hline $\begin{array}{l}\text { Incomplete outcome data } \\
\text { (attrition bias) }\end{array}$ & Low risk & $\begin{array}{l}\text { "There were nine premature dropouts, six in the aripiprazole group and three } \\
\text { in the placebo group. Of the aripiprazole group, three dropouts were due to } \\
\text { concurrent illness, and three due to non-compliance with the visits. Of the } \\
\text { placebo group, two dropouts were due to non-compliance and one changed } \\
\text { his mind about participating in the study". }\end{array}$ \\
\hline $\begin{array}{l}\text { Selective reporting (re- } \\
\text { porting bias) }\end{array}$ & Low risk & \begin{tabular}{l} 
All pre-specified outcomes were reported. \\
\hline \begin{tabular}{l} 
Other bias \\
\hline
\end{tabular}
\end{tabular} \\
\hline
\end{tabular}

B +pipotiazine 2002

Methods Allocation: randomised (no further information).

Blinding: not stated. 
B +pipotiazine 2002 (Continued)

Duration: 6 months.

Setting: inpatients.

Design: parallel.

Country: China.

$\begin{array}{ll}\text { Participants } & \text { Diagnosis: chronic treatment resistant schizophrenia (CCMD-2-R). } \\ \mathbf{N}=84 . & \\ \text { Age: } 27^{\sim} 58 \text { years. } \\ \text { Sex: male and female. } \\ \text { Average length of illness: } 14.8 \pm 11.4 \text { years. }\end{array}$

Interventions

1. Combination therapy: clozapine + pipotiazine: $50 \mathrm{mg}$ of pipotiazine was given by intramuscular injection, after that $50 \mathrm{mg}^{\sim} 100 \mathrm{mg}$ once every 4 weeks $\left(\mathrm{N}=\right.$ unclear $\left.^{\star}\right)$.

2. Monotherapy: clozapine $200 \mathrm{mg}^{\sim} 450 \mathrm{mg} /$ day $\left(\mathrm{N}=\right.$ unclear $\left.^{\star}\right)$.

Schedule: not reported

\begin{tabular}{ll}
\hline Outcomes & -Usable data - \\
& 1. Clinical response: not clinically improved \\
2. Mental state: BPRS*
\end{tabular}

- Unable to use -

1. Adverse events: movement disorders (TESS) (no data reported).

\begin{tabular}{|c|c|c|}
\hline Notes & ${ }^{\star} \mathrm{N}$ assumed to be $42 \mathrm{i}$ & each treatment group. \\
\hline \multicolumn{3}{|l|}{ Risk of bias } \\
\hline Bias & Authors' judgement & Support for judgement \\
\hline $\begin{array}{l}\text { Random sequence genera- } \\
\text { tion (selection bias) }\end{array}$ & Unclear risk & Randomised, no further information. \\
\hline $\begin{array}{l}\text { Allocation concealment } \\
\text { (selection bias) }\end{array}$ & Unclear risk & Not stated. \\
\hline $\begin{array}{l}\text { Blinding (performance } \\
\text { bias and detection bias) } \\
\text { All outcomes }\end{array}$ & Unclear risk & Not stated. \\
\hline $\begin{array}{l}\text { Incomplete outcome data } \\
\text { (attrition bias) } \\
\text { All outcomes }\end{array}$ & Unclear risk & $\begin{array}{l}\text { Author did not report on the number of people that completed the trial, thus } \\
\text { we are unable to judge if there is incomplete outcome data. }\end{array}$ \\
\hline $\begin{array}{l}\text { Selective reporting (re- } \\
\text { porting bias) }\end{array}$ & High risk & $\begin{array}{l}\text { TESS scores were measured, but not reported. Author only reported that there } \\
\text { is no significant difference between groups. }\end{array}$ \\
\hline Other bias & Low risk & None obvious. \\
\hline
\end{tabular}

$\begin{array}{ll}\text { Methods } & \text { Allocation: randomised. } \\ & \text { Blinding: double-blind. } \\ & \text { Duration: } 16 \text { weeks. }\end{array}$


B +quet/risp 2009 (Continued)

Setting: outpatients.

Design: parallel.

Country: USA, multi-centre.

\begin{tabular}{ll}
\hline Participants & Diagnosis: schizophrenia or schizoaffective disorder (DSM-IV-TR). \\
$\mathbf{N}=323$ & \\
Sex: $\mathrm{M} 198, \mathrm{~F} 125$. & \\
Age: average $\sim 44$ years. & History: chronic, stable. Currently receiving a stable dose of quetiapine $(400-800 \mathrm{mg} / \mathrm{d})$ or risperidone \\
& (4 mg to $8 \mathrm{mg} /$ day) for $\geq 4$ weeks but with an inadequate response; must not have shown significant im- \\
provement or worsening of symptoms within 1 month of screening.
\end{tabular}

Interventions Combination therapy: aripiprazole + quetiapine $(N=78)$.
2. Combination therapy: aripiprazole + risperidone $(N=90)$.
3. Monotherapy: quetiapine + placebo $(N=68)$.
4. Monotherapy: risperidone + placebo $(N=87)$.

Schedule: aripiprazole $2-15 \mathrm{mg} / \mathrm{d}$, quetiapine $400 \mathrm{mg}$ to $800 \mathrm{mg} /$ day, risperidone $4 \mathrm{mg}$ to $8 \mathrm{mg} /$ day.

- Usable data -
1. Leaving the study early.
2. Clinical response: not clinically improved.
3. Mental state: PANSS positive and PANSS negative.
4. Adverse events: serious or requiring discontinuation, weight gain, movement disorders, deaths.
- Unable to use -
1. Global state: CGI (no SDs reported, no suitable mean to impute data).
2. Mental state: PANSS total score, MADRS (no SDs reported, no suitable mean to impute data).
3. Adverse events: AIMS, SAS, BAS, average weight gain, prolactin (no SDs reported, no suitable mean
to impute data).
4. Quality of life: SWN (no SDs reported, no suitable mean to impute data).

\section{- Not used in review -}

1. Calgary Depression Scale for Schizophrenia (CDSS), Arizona Sexual Experience Scale (ASEX), Fatigue Symptom Inventory (FSI), Brief Assessment of cognition in schizophrenia (BACS), Investigator's Assessment Questionnaire (IAQ), HDL, LDL, fasting glucose, triglycerides.

\section{Risk of bias}

\begin{tabular}{lll}
\hline Bias & Authors' judgement & Support for judgement \\
\hline $\begin{array}{l}\text { Random sequence genera- } \\
\text { tion (selection bias) }\end{array}$ & Unclear risk & No details provided. \\
\hline $\begin{array}{l}\text { Allocation concealment } \\
\text { (selection bias) }\end{array}$ & Unclear risk & No details provided. \\
\hline $\begin{array}{l}\text { Blinding (performance } \\
\text { bias and detection bias) } \\
\text { All outcomes }\end{array}$ & Unclear risk & No details provided. \\
\hline
\end{tabular}

\begin{tabular}{|c|c|c|}
\hline $\begin{array}{l}\text { Incomplete outcome data } \\
\text { (attrition bias) }\end{array}$ & Low risk & $\begin{array}{l}\text { All patients were analysed for safety. For efficacy analysis LOCF method was } \\
\text { used. }\end{array}$ \\
\hline
\end{tabular}


B +quet/risp 2009 (Continued)

All outcomes

Selective reporting (re- Low risk All expected outcomes reported.

porting bias)

Other bias High risk $\quad \begin{aligned} & \text { Supported by Bristol-Mywers Squibb (USA) and Otsuka Pharmaceutical Co } \\ & \text { (Japan) }\end{aligned}$

\section{B +risperidone 2010}

\begin{tabular}{ll}
\hline Methods & Allocation: randomised. \\
Blinding: double-blind. \\
Duration: 16 weeks. \\
Setting: inpatients and outpatients. \\
Design: parallel. \\
Country: USA. \\
\hline
\end{tabular}

\begin{tabular}{|c|c|}
\hline Participants & $\begin{array}{l}\text { Diagnosis: schizophrenia or schizoaffective disorder (DSM-IV). } \\
\mathbf{N}=69^{\star} \\
\text { Sex: M 44, F21. } \\
\text { Age: average } 45 \text { years. } \\
\text { History: moderate illness severity and persistent psychosis despite adequate prior clozapine treat- }\end{array}$ \\
\hline
\end{tabular}
ment.

$\begin{array}{ll}\text { Interventions } & \text { Combination therapy: risperidone + clozapine }(\mathrm{N}=30) . \\ \text { 2. Monotherapy: clozapine + placebo }(\mathrm{N}=35) .\end{array}$

Schedule: risperidone $4 \mathrm{mg} /$ day, clozapine dosage not reported.

- Usable data -
1. Leaving the study early.
2. Clinical response: CGI-S, CGI-I.
3. Mental state: BPRS, SANS.
4. Adverse events: serious or requiring discontinuation, prolactin level, average weight gain.

\section{- Unable to use -}

1. Adverse events; AIMS, BAS, SAS.

2. Social functioning: LOF (No data reported).

\section{- Not used in review -}

1. Cognitive scales, fasting glucose, DAI subjective response to treatment scale, vital signs.

\begin{tabular}{|c|c|}
\hline \multirow[t]{2}{*}{ Notes } & $\begin{array}{l}\text { *69 participants were randomised, } 4 \text { participants dropped out before the intervention started, } 65 \text { par- } \\
\text { ticipants entered the active phase of the study. }\end{array}$ \\
\hline & ClinicalTrials.gov Identifier: NCT00056498.. \\
\hline
\end{tabular}

\begin{tabular}{lll}
\hline Risk of bias & \\
\hline Bias & Authors' judgement & Support for judgement \\
\hline $\begin{array}{l}\text { Random sequence genera- } \\
\text { tion (selection bias) }\end{array}$ & Unclear risk & $\begin{array}{l}\text { "Randomization was stratified by in-patient status", no further details provid- } \\
\text { ed. }\end{array}$ \\
\hline
\end{tabular}


B +risperidone 2010 (Continued)

Allocation concealment $\quad$ Unclear risk
(selection bias)

Blinding (performance Unclear risk

"Double-blind", no further details provided for blinding of participants and

bias and detection bias) personnel, raters were blinded to treatment assignment.

All outcomes

$\begin{array}{ll}\begin{array}{l}\text { Incomplete outcome data } \\ \text { (attrition bias) }\end{array} & \text { Low risk } \\ \text { All outcomes } & \begin{array}{l}\text { ITT analysis was carried out and included all participants who received at least } \\ \text { one dose of study medication. }\end{array}\end{array}$

All outcomes

\begin{tabular}{lll}
$\begin{array}{l}\text { Selective reporting (re- } \\
\text { porting bias) }\end{array}$ & Low risk & All expected outcomes reported. \\
\hline Other bias & Unclear risk & $\begin{array}{l}\text { Supported by a NIMH grant and a University of Maryland General Clinical Re- } \\
\text { search Center grant, Janssen Pharmaceuticals provided study medication. ID: } \\
551129 .\end{array}$ \\
\hline
\end{tabular}

B +sulpiride 1996

\begin{tabular}{|c|c|}
\hline Methods & $\begin{array}{l}\text { Allocation: randomised. Stratified randomisation using hospital admission time. } \\
\text { Blinding: not stated. } \\
\text { Duration: } 3 \text { months. } \\
\text { Setting: inpatient. } \\
\text { Design: parallel. } \\
\text { Country: China. }\end{array}$ \\
\hline Participants & $\begin{array}{l}\text { Diagnosis: schizophrenia (DSM-II-R). } \\
\mathbf{N}=102 . \\
\text { Sex: male and female. } \\
\text { Age: } 16-54 \text { years. } \\
\text { Average length of illness: mean } \sim 7 \text { years, SD } 4 \text { years. }\end{array}$ \\
\hline Interventions & $\begin{array}{l}\text { 1. Combination therapy: clozapine + sulpiride, } 436.57 \mathrm{mg}+\backslash-89.85 \mathrm{mg}, 1127.23 \mathrm{mg}+\backslash-156.55 \mathrm{mg} \text { per } \\
\text { day }(\mathrm{N}=31) \text {. } \\
\text { 2. Monotherapy: clozapine, } 486.77 \mathrm{mg}+\backslash-29.81 \mathrm{mg} \text { per day }(\mathrm{N}=32) \text {. } \\
\text { 3. Monotherapy: sulpiride, } 1296.86 \mathrm{mg}+\backslash-105.11 \mathrm{mg} \text { per day }(\mathrm{N}=29) \text {. } \\
\text { Schedule: } \text { not reported. }\end{array}$ \\
\hline Outcomes & $\begin{array}{l}\text { - Usable data - } \\
\text { 1. Clinical response: not clinically improved. } \\
\text { 2. Mental state: BPRS, SANS. } \\
\text { 3. Adverse events: serious or requiring discontinuation. } \\
\text { - Unable to use - } \\
\text { 1. Leaving the study early (the treatment groups that participants dropped out from not reported). } \\
\text { 2. Clinical response: CGI (Not reported). } \\
\text { 3. Adverse events: TESS (Not reported). }\end{array}$ \\
\hline
\end{tabular}


B +sulpiride 1996 (Continued)

Notes

\section{Risk of bias}

\begin{tabular}{|c|c|c|}
\hline Bias & Authors' judgement & Support for judgement \\
\hline $\begin{array}{l}\text { Random sequence genera- } \\
\text { tion (selection bias) }\end{array}$ & High risk & $\begin{array}{l}\text { Stratified randomisation using hospital admission time. No further informa- } \\
\text { tion given. }\end{array}$ \\
\hline $\begin{array}{l}\text { Allocation concealment } \\
\text { (selection bias) }\end{array}$ & Unclear risk & Not described. \\
\hline $\begin{array}{l}\text { Blinding (performance } \\
\text { bias and detection bias) } \\
\text { All outcomes }\end{array}$ & Unclear risk & Not described. Unclear if outcome assessment was blinded. \\
\hline $\begin{array}{l}\text { Incomplete outcome data } \\
\text { (attrition bias) } \\
\text { All outcomes }\end{array}$ & High risk & $\begin{array}{l}10 \text { patients left the study early and were not included in the final analysis. The } \\
\text { number lost to follow-up in each groups was not reported. }\end{array}$ \\
\hline $\begin{array}{l}\text { Selective reporting (re- } \\
\text { porting bias) }\end{array}$ & High risk & TESS and CGI scale scores were measured but not reported. \\
\hline Other bias & Unclear risk & None obvious. \\
\hline
\end{tabular}

\section{B +ziprasidone 2014}

$\begin{array}{ll}\text { Methods } & \text { Allocation: randomised. } \\ & \text { Blinding: double-blind. } \\ \text { Duration: } 16 \text { weeks. } & \text { Setting: outpatients. } \\ \text { Design: parallel. } \\ \text { Country: Italy. }\end{array}$
Participants Diagnosis: schizophrenia (DSM-IV).
$\mathbf{N}=40$.
Sex: M 13, F 27.
Age: 35 years.
History: chronic, treatment resistant patients that demonstrated persistent positive and negative symptoms despite an adequate trial of clozapine.
Interventions
1. Combination: clozapine ( $350 \mathrm{mg}$ to $600 \mathrm{mg} /$ day) + ziprasidone $(80 \mathrm{mg} /$ day) $(\mathrm{N}=20)$.
2. Monotherapy: clozapine (350 $\mathrm{mg}$ to $600 \mathrm{mg} /$ day) + placebo $(\mathrm{N}=20)$.

$\begin{array}{ll}\text { Outcomes } & \text { - Usable data - } \\ \text { 1. Leaving the study early. } \\ \text { 2. Mental State: PANSS (Total), BPRS. } \\ \text { 3. Adverse event: requiring discontinuation. }\end{array}$

\footnotetext{
- Not able to use -

1. Mental state: PANSS (Positive, Negative) (Skewed data).

2. Adverse event: white blood cell count (No data reported).
} 
B +ziprasidone 2014 (Continued)

\title{
- Not used in this review -
}

1. CDSS (Calgary Depression Scale for Schizophrenia).

Notes

\section{Risk of bias}

\begin{tabular}{lll}
\hline Bias & Authors' judgement & Support for judgement \\
\hline $\begin{array}{l}\text { Random sequence genera- } \\
\text { tion (selection bias) }\end{array}$ & Low risk & "Prerandomized codes generated by computer." \\
\hline $\begin{array}{l}\text { Allocation concealment } \\
\text { (selection bias) }\end{array}$ & Low risk & $\begin{array}{l}\text { "Coded treatments were allocated sequentially to subjects in order of their } \\
\text { registration for the trial. The randomization list was held securely." }\end{array}$ \\
\hline $\begin{array}{l}\text { Blinding (performance } \\
\text { bias and detection bias) } \\
\text { All outcomes }\end{array}$ & Unclear risk & $\begin{array}{l}\text { "None of the research personnel, who enrolled, assessed, and treated the pa- } \\
\text { tients, were aware of the patient assignments until the study was concluded. } \\
\text { Ziprasidone and placebo were dispensed in identical-appearing capsules; pa- } \\
\text { tients randomized to placebo took the same number of capsules as those as- } \\
\text { signed to ziprasidone." }\end{array}$
\end{tabular}

Incomplete outcome data High risk (attrition bias)

All outcomes

\begin{abstract}
"Two discontinuations in the ziprasidone group were all attributed to treatment-emergent adverse events (akathisia and sedation) and 2 withdrew for perceived lack of efficacy. Among a total of 3 dropouts in the placebo group, 2 were due to non-compliance with the visits and 1 withdrew due to a subjectively assessed lack of efficacy." The reasons for leaving the study early differ across groups.

"An intention-to-treat analysis with last-observation-carried forward was performed."
\end{abstract}

\begin{tabular}{lll}
\hline $\begin{array}{l}\text { Selective reporting (re- } \\
\text { porting bias) }\end{array}$ & High risk & $\begin{array}{l}\text { No protocol available. } \\
\text { "No clinically significant changes" reported for haematological parameters. }\end{array}$ \\
\hline Other bias & Low risk & None obvious. \\
\hline
\end{tabular}

\section{C +amisulpride 2008}

$\begin{array}{ll}\text { Methods } & \text { Allocation: randomised. } \\ & \text { Blinding: double-blind. } \\ & \text { Duration: } 6 \text { weeks. } \\ \text { Setting: inpatients. } \\ \text { Design: parallel. } \\ \text { Country: Germany. }\end{array}$

\footnotetext{
Participants Diagnosis: schizophrenia (DSM-IV - 295.32; 295.34).

$\mathbf{N}=16$.

Sex: M 12, F 4.

Age: average 43 years.

History: chronically ill, had already received clozapine for at least three months on a stable dose and were only partially or even non-respondent.
}

Interventions Combination therapy: clozapine $300 \mathrm{mg} / \mathrm{day}+$ amisulpride $400 \mathrm{mg} / \mathrm{day}(\mathrm{N}=7)$.
2. Combination therapy: clozapine $300 \mathrm{mg} / \mathrm{day}+$ amisulpride $600 \mathrm{mg} / \mathrm{day}(\mathrm{N}=6)$.
3. Monotherapy: clozapine $300 \mathrm{mg} /$ day + placebo $(\mathrm{N}=3)$.


C +amisulpride 2008 (Continued)

Schedule: not reported.

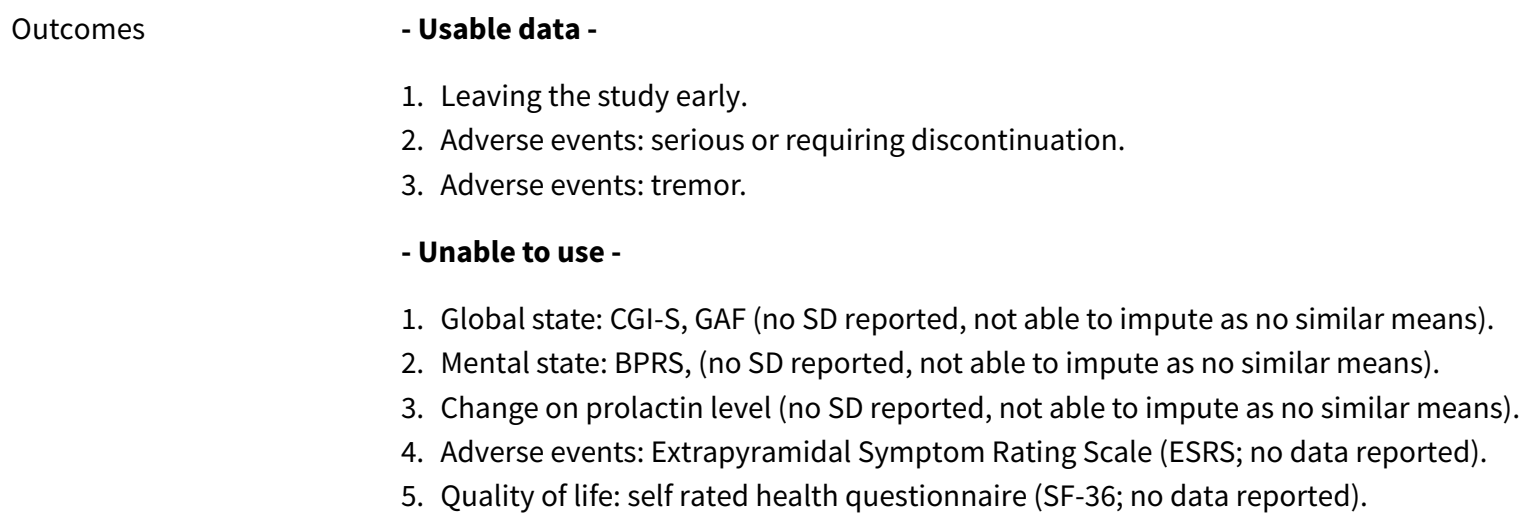

1. Leaving the study early.

2. Adverse events: serious or requiring discontinuation.

3. Adverse events: tremor.

\section{- Unable to use -}

1. Global state: CGI-S, GAF (no SD reported, not able to impute as no similar means).

2. Mental state: BPRS, (no SD reported, not able to impute as no similar means).

3. Change on prolactin level (no SD reported, not able to impute as no similar means).

4. Adverse events: Extrapyramidal Symptom Rating Scale (ESRS; no data reported).

5. Quality of life: self rated health questionnaire (SF-36; no data reported).

\section{- Not used in review -}

1. Montgomery Asberg Depression Rating Scale (MADRS), pulse and blood pressure, laboratory results, ECG, physical and neurological examinations.

Notes

\section{Risk of bias}

\begin{tabular}{lll}
\hline Bias & Authors' judgement & Support for judgement \\
\hline $\begin{array}{l}\text { Random sequence genera- } \\
\text { tion (selection bias) }\end{array}$ & Unclear risk & No details provided. \\
\hline $\begin{array}{l}\text { Allocation concealment } \\
\text { (selection bias) }\end{array}$ & Unclear risk & No details provided. \\
\hline $\begin{array}{l}\text { Blinding (performance } \\
\text { bias and detection bias) } \\
\text { All outcomes }\end{array}$ & Unclear risk & No details provided. \\
\hline $\begin{array}{l}\text { Incomplete outcome data } \\
\text { (attrition bias) }\end{array}$ & High risk & \\
$\begin{array}{l}\text { All outcomes } \\
\text { Selective reporting (re- } \\
\text { porting bias) }\end{array}$ & High risk & $\begin{array}{l}\text { Two patients from combination group and one from monotherapy did not } \\
\text { complete study because of lack of efficacy and unknown reasons. }\end{array}$ \\
\hline $\begin{array}{l}\text { Other bias } \\
\text { High risk }\end{array}$ & Not all expected outcomes reported. \\
\hline
\end{tabular}

\section{C +arip/pali 2014}

$\begin{array}{ll}\text { Methods } & \text { Allocation: randomised. } \\ & \text { Blinding: double-blind. } \\ \text { Duration: } 6 \text { weeks. } \\ \text { Setting: outpatient. }\end{array}$

Design: parallel. 
C +arip/pali 2014 (Continued)

Country: India.

\begin{tabular}{ll}
\hline Participants & $\begin{array}{l}\text { Diagnosis: Schizophrenia (DSM-IV). } \\
\mathbf{N}=90 . \\
\text { Sex: }{ }^{*} \\
\text { Age: } \\
\text { History: } 36 \text { years. }\end{array}$ \\
\hline Interventions & $\begin{array}{l}\text { 1. Combination therapy: olanzapine }(10 \mathrm{mg})+\text { aripiprazole }(10 \mathrm{mg})(\mathrm{N}=30) . \\
\text { 2. Combination therapy: olanzapine }(10 \mathrm{mg})+\text { paliperidone }(3 \mathrm{mg})(\mathrm{N}=30) . \\
\text { 3. Monotherapy: olanzapine }(3 \mathrm{mg} \text { to } 6 \mathrm{mg})+\text { placebo }(\mathrm{N}=30) .\end{array}$ \\
\hline
\end{tabular}

\section{Outcomes}

\section{- Usable data -}

1. Leaving the study early.

2. Mental State: PANSS total.

\section{- Not used in this review -}

1. Digit symbol substitution test, six digit cancellation test, critical flicker fusion test, arithmetic ability, verbal fluency test, digit span test, hand steadiness test, finger tapping test.

Notes clinical registration..
Funding: no funding sources.
Conflict of interest: none declared.
* The gender of the participants in the monotherapy group does not correspond to the number of par-
ticipants included in the trial. No response when we tried contacting the author to clarify this issue.

\section{Risk of bias}

\begin{tabular}{|c|c|c|}
\hline Bias & Authors' judgement & Support for judgement \\
\hline $\begin{array}{l}\text { Random sequence genera- } \\
\text { tion (selection bias) }\end{array}$ & Low risk & $\begin{array}{l}\text { "The randomized treatment allocation sequence was generated by statistician } \\
\text { using random number table." }\end{array}$ \\
\hline $\begin{array}{l}\text { Allocation concealment } \\
\text { (selection bias) }\end{array}$ & Low risk & $\begin{array}{l}\text { "The code of this random allocation sequence was retained in the sealed enve- } \\
\text { lope by this person and was opened only after the completion of study during } \\
\text { analysis of data." }\end{array}$ \\
\hline $\begin{array}{l}\text { Blinding (performance } \\
\text { bias and detection bias) } \\
\text { All outcomes }\end{array}$ & Low risk & $\begin{array}{l}\text { "Double blinding was done by inserting aripiprazole or paliperidone or place- } \\
\text { bo tablet in a non-transparent capsule." } \\
\text { "It was handed over along with identical plastic containers filled with the } \\
\text { study drugs ( } 45 \text { capsules each of aripiprazole or paliperidone or placebo) to a } \\
\text { third person not directly involved in this study." } \\
\text { "The patient as well as the investigator was unaware of the treatment being } \\
\text { administered." }\end{array}$ \\
\hline $\begin{array}{l}\text { Incomplete outcome data } \\
\text { (attrition bias) } \\
\text { All outcomes }\end{array}$ & Low risk & $\begin{array}{l}\text { "All the randomized patients in each group have satisfactorily completed the } \\
\text { study." }\end{array}$ \\
\hline $\begin{array}{l}\text { Selective reporting (re- } \\
\text { porting bias) }\end{array}$ & Low risk & All outcomes reported in methods are presented in the results. \\
\hline Other bias & High risk & Baseline imbalance in PANSS (total) values. \\
\hline
\end{tabular}




$\begin{array}{ll}\text { Methods } & \text { Allocation: randomised. } \\ \text { Blinding: double-blind. } \\ \text { Duration: } 9 \text { weeks. } \\ \text { Setting: inpatients. } \\ \text { Design: parallel. } \\ \text { Country: China. }\end{array}$

\begin{tabular}{ll}
\hline Participants & Diagnosis: schizophrenia. \\
& $\mathbf{N}=61$. \\
& Sex: $\mathrm{M} 61$. \\
& Age: $18-50$ years, average $\sim 35$ years. \\
& History: treated with sulpiride for 6 weeks prior to the study.
\end{tabular}

Interventions Combination therapy: sulpiride $600 \mathrm{mg}$ to $900 \mathrm{mg}$ + aripiprazole $10 \mathrm{mg}(\mathrm{N}=31)$.
2. Monotherapy: sulpiride $500 \mathrm{mg}$ to $900 \mathrm{mg}(\mathrm{N}=30)$.

Schedule: daily dose.

Outcomes - Usable data -

1. Adverse events: movement disorders, TESS and prolactin.

\begin{tabular}{|c|c|c|}
\hline Notes & ${ }^{\star}$ Abstract in English, a & icle in Chinese. \\
\hline \multicolumn{3}{|l|}{ Risk of bias } \\
\hline Bias & Authors' judgement & Support for judgement \\
\hline $\begin{array}{l}\text { Random sequence genera- } \\
\text { tion (selection bias) }\end{array}$ & Unclear risk & Randomised (no further information provided). \\
\hline $\begin{array}{l}\text { Allocation concealment } \\
\text { (selection bias) }\end{array}$ & Unclear risk & Not reported. \\
\hline $\begin{array}{l}\text { Blinding (performance } \\
\text { bias and detection bias) } \\
\text { All outcomes }\end{array}$ & Unclear risk & Not reported. \\
\hline $\begin{array}{l}\text { Incomplete outcome data } \\
\text { (attrition bias) } \\
\text { All outcomes }\end{array}$ & Low risk & No incomplete data. \\
\hline $\begin{array}{l}\text { Selective reporting (re- } \\
\text { porting bias) }\end{array}$ & High risk & Not all expected outcomes reported. \\
\hline Other bias & Unclear risk & No information about source of funding. \\
\hline
\end{tabular}

\section{C +aripiprazole 2007b}

$\begin{array}{ll}\text { Methods } & \text { Allocation: randomised. } \\ \text { Blinding: double-blind. } & \text { Duration: } 8 \text { weeks. } \\ \text { Setting: not reported. } & \text { Design: parallel. }\end{array}$


C +aripiprazole 2007b (Continued)

Country: USA.

\begin{tabular}{ll}
\hline Participants & Diagnosis: schizophrenia (DSM-IV). \\
& N $=56$. \\
& Sex: $\mathrm{M} 22, \mathrm{~F} 32$. \\
& Age: average $\sim 39$ years. \\
& History: treated with haloperidol monotherapy and were taking the same dosage of haloperidol for at \\
& least 3 months.
\end{tabular}

Interventions

1. Combination therapy: aripiprazole + haloperidol $(\mathrm{N}=26)$.

2. Monotherapy: haloperidol + placebo $(\mathrm{N}=28)$.

Schedule: aripiprazole dose fixed at $15 \mathrm{mg} /$ day for first four weeks, then $30 \mathrm{mg} /$ day for following four weeks, haloperidol dose remained fixed throughout study.

- Usable data -
1. Leaving the study early.
2. Clinical response: CGI-S.
3. Mental state: BPRS, SANS.
4. Adverse events: serious or requiring discontinuation, drowsiness.

\section{- Unable to use -}

1. Adverse events: prolactin, BAS, SAS (Skewed data).

\section{Notes}

\section{Risk of bias}

\begin{tabular}{lll}
\hline Bias & Authors' judgement & Support for judgement \\
\hline $\begin{array}{l}\text { Random sequence genera- } \\
\text { tion (selection bias) }\end{array}$ & Unclear risk & No details provided. \\
\hline $\begin{array}{l}\text { Allocation concealment } \\
\text { (selection bias) }\end{array}$ & Unclear risk & No details provided. \\
\hline $\begin{array}{l}\text { Blinding (performance } \\
\text { bias and detection bias) } \\
\text { All outcomes }\end{array}$ & Unclear risk & No details provided. \\
\hline $\begin{array}{l}\text { Incomplete outcome data } \\
\text { (attrition bias) } \\
\text { All outcomes }\end{array}$ & High risk & No ITT analysis performed. \\
\hline $\begin{array}{l}\text { Selective reporting (re- } \\
\text { porting bias) }\end{array}$ & High risk & Not all expected outcomes reported. \\
\hline $\begin{array}{l}\text { Other bias } \\
\text { Und }\end{array}$ & Unclear risk & $\begin{array}{l}\text { Supported by a grant from the Korea Health R\&D Project, Ministry of Health } \\
\text { and Welfare, and Republic of Korea (0412-CT02-0704-0006). }\end{array}$ \\
\hline
\end{tabular}

\section{C +aripiprazole 2008}

$\begin{array}{ll}\text { Methods } & \text { Allocation: randomised. } \\ \text { Blindning: double-blind. } \\ \text { Duration: } 8 \text { weeks. }\end{array}$


C +aripiprazole 2008 (Continued)

Setting: inpatients and outpatients.

Design: parallel.

Country: Korea.

\begin{tabular}{|c|c|}
\hline Participants & $\begin{array}{l}\text { Diagnosis: schizophrenia (DSM-IV). } \\
\text { N =61. } \\
\text { Sex: M 48, F } 13 . \\
\text { Age: average } 32 \text { years. } \\
\text { History: treatment failure prior to clozapine; clozapine treatment for more than } 1 \text { year with at least } 8 \\
\text { weeks at a stable daily dose of } 400 \mathrm{mg} \text { or more; no change in clozapine daily dose or other concomitant } \\
\text { medication for more than } 3 \text { months. }\end{array}$ \\
\hline
\end{tabular}

Interventions

1. Combination therapy: clozapine + aripiprazole $(\mathrm{N}=30)$.

2. Monotherapy: clozapine + placebo $(\mathrm{N}=32)$.

Schedule: clozapine $400+\mathrm{mg} /$ day, aripiprazole $5 \mathrm{mg}$ to $30 \mathrm{mg} /$ day.

- Usable data -
1. Leaving the study early.
2. Clinical response: CGI-S.
3. Mental state: BPRS, SANS.
4. Adverse events: serious or requiring disc
- Unable to use -
1. UKU (no means and SDs reported).
2. Prolactin level (Skewed data).
3. White blood cell count (Not reported).

\section{- Not used in review -}

1. Montgomery-Asberg Depression Rating Scale (MADRS), Yale-Brown Obsessive Compulsive Scale (YBOCS), vital signs (pulse rate and systolic/diastolic blood pressure),ECG, liver function tests, measurement of electrolyte levels, urinalysis, fasting blood sugar, 2-hour postprandial blood sugar, total cholesterol, triglycerides, low-density lipoprotein (LDL) cholesterol, and high-density lipoprotein (HDL) cholesterol.

Notes

*LOCF method was used for any patients who did not complete the 8-week double-blind phase.

Trial Registration: clinicaltrials.gov Identifier: NCT00328367

\section{Risk of bias}

\begin{tabular}{lll}
\hline Bias & Authors' judgement & Support for judgement \\
\hline $\begin{array}{l}\text { Random sequence genera- } \\
\text { tion (selection bias) }\end{array}$ & Low risk & $\begin{array}{l}\text { Random-numbers chart in blocks of 4. The allocation sequence was generated } \\
\text { and monitored by faculty members who were not involved in any part of this } \\
\text { study. }\end{array}$ \\
\hline $\begin{array}{l}\text { Allocation concealment } \\
\text { (selection bias) }\end{array}$ & Low risk & $\begin{array}{l}\text { The investigators were not involved in any part of this study. The investigators } \\
\text { were unaware of the block size. }\end{array}$ \\
\hline $\begin{array}{l}\text { Blinding (performance } \\
\text { bias and detection bias) } \\
\text { All outcomes }\end{array}$ & Low risk & $\begin{array}{l}\text { All the participants and investigators remained blind throughout the study, } \\
\text { and the data analyses were also performed by investigators blind to the identi- } \\
\text { ty of the participants. }\end{array}$ \\
\hline
\end{tabular}

Incomplete outcome data Low risk (attrition bias)

All outcomes
One patient with a history of arteriovenous malformation was mistakenly included in the random assignment to aripiprazole and was consequently ex- 
cluded from the itt analysis. LOCF method was used for any patients who did not complete the 8-week double-blind phase.

\begin{tabular}{lll}
\hline $\begin{array}{l}\text { Selective reporting (re- } \\
\text { porting bias) }\end{array}$ & Low risk & All planned outcomes reported except for the one person who dropped out. \\
\hline Other bias & High risk & $\begin{array}{l}\text { Funded by the Ministry of Science and Technology, Republic of Korea, and } \\
\text { partly by a research grant from Korea Otsuka Pharmaceutical to Dr. Y. S. Kim. }\end{array}$ \\
\hline
\end{tabular}

\section{C +aripiprazole 2008b}

\begin{tabular}{|c|c|}
\hline Methods & $\begin{array}{l}\text { Allocation: randomised (using random number table). } \\
\text { Blinding: not stated. } \\
\text { Duration: } 6 \text { weeks. } \\
\text { Setting: community and inpatients. } \\
\text { Design: parallel. } \\
\text { Country: China. }\end{array}$ \\
\hline Participants & $\begin{array}{l}\text { Diagnosis: schizophrenia (in remission, BPRS score }<25 \text { ), diagnosed with CCMD- } 3 \text {. } \\
\mathbf{N}=80 . \\
\text { Age: } 18-52 \text { years. } \\
\text { Sex: male and female. } \\
\text { History: average length of illness: aripiprazole group, median }=7.8 \text { years, range }=0.2 \text { to } 22 \text { years; chlor- } \\
\text { promazine group = not stated; placebo group, median }=9.4 \text { years, range }=0.1 \text { to } 18 \text { years. }\end{array}$ \\
\hline
\end{tabular}

\begin{tabular}{ll}
\hline Interventions & Combination therapy: aripiprazole $(5 \mathrm{mg} / \mathrm{day})+$ chlorpromazine $(200 \mathrm{mg} \sim 450 \mathrm{mg} / \mathrm{day})(\mathrm{N}=40)$. \\
2. Monotherapy: placebo + chlorpromazine $(200 \mathrm{mg} \sim 450 \mathrm{mg} / \mathrm{day})+$ placebo $(100 \mathrm{mg} / \mathrm{day})(\mathrm{N}=40)$.
\end{tabular}

\begin{tabular}{ll}
\hline Outcomes & - Usable data - \\
& 1. Mental state: BPRS. \\
2. Adverse events: prolactin level, drowsiness.
\end{tabular}

\begin{tabular}{|c|c|c|}
\hline Notes & Article in Chinese. & \\
\hline \multicolumn{3}{|l|}{ Risk of bias } \\
\hline Bias & Authors' judgement & Support for judgement \\
\hline $\begin{array}{l}\text { Random sequence genera- } \\
\text { tion (selection bias) }\end{array}$ & Low risk & Randomised using random number table. \\
\hline $\begin{array}{l}\text { Allocation concealment } \\
\text { (selection bias) }\end{array}$ & Unclear risk & Not stated. \\
\hline $\begin{array}{l}\text { Blinding (performance } \\
\text { bias and detection bias) } \\
\text { All outcomes }\end{array}$ & Unclear risk & Not stated. \\
\hline $\begin{array}{l}\text { Incomplete outcome data } \\
\text { (attrition bias) } \\
\text { All outcomes }\end{array}$ & Unclear risk & It is unclear if there are incomplete data. \\
\hline
\end{tabular}


C +aripiprazole 2008b (Continued)

Selective reporting (re- High risk TESS score was measured, but not reported. porting bias)

Other bias Low risk None obvious.

C +aripiprazole 2009

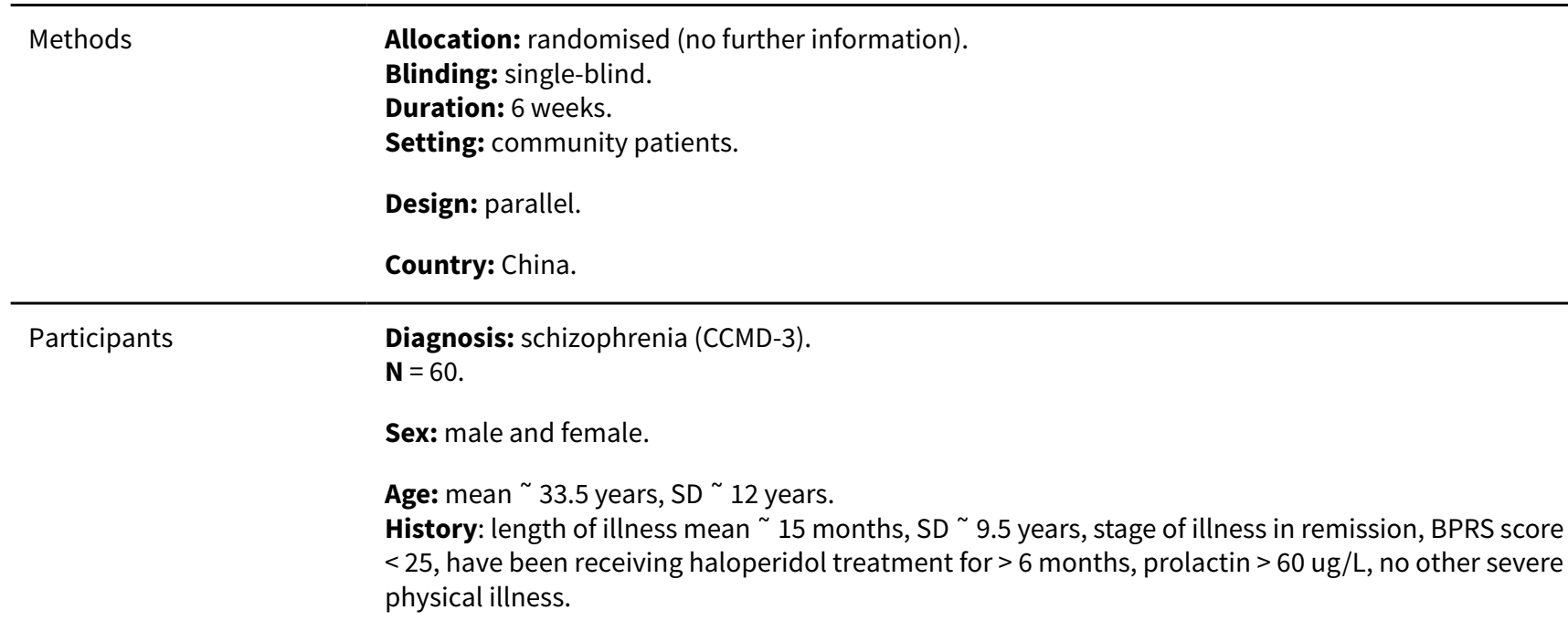

Interventions

1. Combination therapy: haloperidol plus aripiprazole $(5 \mathrm{mg}$ per capsule, one capsule per day for 6 months) $(\mathrm{N}=30)$.

2. Monotherapy: haloperidol plus placebo (vitamin $\mathrm{C} 100 \mathrm{mg}$ per capsule, one capsule per day for 6 month) $(\mathrm{N}=30)$.

Schedule: not reported.

\begin{tabular}{ll}
\hline Outcomes & - Usable data - \\
& 1. Mental state: BPRS. \\
2. Adverse events: prolactin level, drowsiness.
\end{tabular}

Notes

\section{Risk of bias}

\begin{tabular}{lll}
\hline Bias & Authors' judgement & Support for judgement \\
\hline $\begin{array}{l}\text { Random sequence genera- } \\
\text { tion (selection bias) }\end{array}$ & Unclear risk & Randomised, but no further information. \\
\hline $\begin{array}{l}\text { Allocation concealment } \\
\text { (selection bias) }\end{array}$ & Unclear risk & Not stated. \\
\hline $\begin{array}{l}\text { Blinding (performance } \\
\text { bias and detection bias) } \\
\text { All outcomes }\end{array}$ & Unclear risk & Single-blind, untested. \\
\hline $\begin{array}{l}\text { Incomplete outcome data } \\
\text { (attrition bias) } \\
\text { All outcomes }\end{array}$ & Low risk & No incomplete outcome data. \\
\hline \hline
\end{tabular}


C +aripiprazole 2009 (Continued)

Selective reporting (re- High risk TESS score was measured, but not reported. porting bias)

\begin{tabular}{lll}
\hline Other bias Low risk obvious. & None
\end{tabular}

\section{C +aripiprazole 2012}

\begin{tabular}{|c|c|}
\hline Methods & $\begin{array}{l}\text { Allocation: randomised. } \\
\text { Blinding: double-blind. } \\
\text { Duration: } 12 \text { weeks. } \\
\text { Setting: outpatient. } \\
\text { Design: parallel. } \\
\text { Country: Japan. }\end{array}$ \\
\hline Participants & $\begin{array}{l}\text { Diagnosis: schizophrenia (DSM-IV). } \\
\mathbf{N}=36 . \\
\text { Sex: M } 12, \mathrm{~F} 24 . \\
\text { Age: average } 35.3 \text {. } \\
\text { History: no details. }\end{array}$ \\
\hline \multirow[t]{2}{*}{ Interventions } & $\begin{array}{l}\text { 1. Combination therapy: risperidone ( } 2 \mathrm{mg} \text { to } 12 \mathrm{mg} / \text { day })+ \text { aripiprazole }(6 \mathrm{mg} \text { to } 30 \mathrm{mg} / \text { day) OR olan- } \\
\text { zapine }(2.5 \mathrm{mg} \text { to } 20 \mathrm{mg} / \text { day })+\text { aripiprazole }(6 \mathrm{mg} \text { to } 30 \mathrm{mg} / \text { day) }(\mathrm{N}=18) \text {. }\end{array}$ \\
\hline & $\begin{array}{l}\text { 2. Monotherapy: risperidone ( } 2 \mathrm{mg} \text { to } 12 \mathrm{mg} / \text { day })+ \text { placebo OR olanzapine }(2.5 \mathrm{mg} \text { to } 20 \mathrm{mg} / \text { day })+ \\
\text { placebo }(\mathrm{N}=18) \text {. }\end{array}$ \\
\hline
\end{tabular}

Outcomes

\section{- Usable data -}

1. Mental state: PANSS.

\section{- Unable to use -}

1. Adverse events: PANSS positive, UKU (Skewed data).

\section{- Not used in review -}

1. Neurocognitive outcome: Brief Assessment of Cognition in Schizophrenia (BACS).

\section{Notes}

\section{Risk of bias}

\begin{tabular}{lll}
\hline Bias & Authors' judgement & Support for judgement \\
\hline $\begin{array}{l}\text { Random sequence genera- } \\
\text { tion (selection bias) }\end{array}$ & Unclear risk & Randomised, no further details. \\
\hline $\begin{array}{l}\text { Allocation concealment } \\
\text { (selection bias) }\end{array}$ & Unclear risk & No information provided. \\
\hline $\begin{array}{l}\text { Blinding (performance } \\
\text { bias and detection bias) } \\
\text { All outcomes }\end{array}$ & Unclear risk & $\begin{array}{l}\text { "Patients were evaluated by the same investigator. This investigator was from } \\
\text { a different hospital and was not involved in patient care. In addition, the in- } \\
\text { vestigator was blind to drug regimens and the drug concentrations. However, } \\
\text { he had access to the nursing charts." No details as to blindness of participants } \\
\text { and personnel other than "double-blind". }\end{array}$ \\
\hline $\begin{array}{l}\text { Incomplete outcome data } \\
\text { (attrition bias) }\end{array}$ & Unclear risk & \begin{tabular}{l} 
No information provided. \\
\hline
\end{tabular}
\end{tabular}


C +aripiprazole 2012 (Continued)

All outcomes

Selective reporting (re- Low risk $\quad$ All pre-specified outcomes were reported.

porting bias)

Other bias Low risk Funded by the Hirosaki Research Institute for Neurosciences.

$\begin{array}{ll}\text { Methods } & \text { Allocation: randomised. } \\ \text { Blinding: double-blind. } \\ \text { Duration: } 8 \text { weeks. } \\ \text { Setting: outpatient. } \\ \text { Design: parallel. } \\ \text { Country: USA. } \\ \text { Diagnosis: schizophrenia or schizoaffective disorder (DSM-IV). } \\ \text { N = 38. } \\ \text { Sex: M 22, F 8. (Only reported the group that completed the } 8 \text { weeks). } \\ \text { Age: } \sim 44 \text { years. } \\ \text { History: treatment with clozapine for at least } 1 \text { year and on a stable dose of for at least } 1 \text { month. Strati- } \\ \text { fied by the presence or absence of diabetes. }\end{array}$

$\begin{array}{ll}\text { Interventions } & \text { Combination therapy: clozapine (Mean }=397 \mathrm{mg} / \text { day })+ \text { aripiprazole }(15 \mathrm{mg} / \mathrm{day})(\mathrm{N}=20) \text {. } \\ \text { 2. Monotherapy: clozapine (Mean }=400 \mathrm{mg} / \text { day })+ \text { placebo }(\mathrm{N}=18) \text {. }\end{array}$

Outcomes

- Usable data -

1. Leaving the study early.

2. Mental state: PANSS.

3. Adverse event: drowsiness, weight gain.

\section{- Not able to use -}

1. Adverse event: AIMS, SAS (Not reported).

\section{- Not used in this review -}

1. Fasting plasma glucose, Fasting serum insulin, homeostasis model of assessment of insulin resistance; haemoglobin A1c; insulin sensitivity index (SI); glucose effectiveness (SG); acute insulin response to glucose, disposition index, HDL, LDL, VLDL and anthropometric assessment.

Notes ClinicalTrials.gov identifier NCT00345033.

\section{Risk of bias}

\begin{tabular}{lll}
\hline Bias & Authors' judgement & Support for judgement \\
\hline $\begin{array}{l}\text { Random sequence genera- } \\
\text { tion (selection bias) }\end{array}$ & Unclear risk & "Randomized” No other information provided. \\
\hline $\begin{array}{l}\text { Allocation concealment } \\
\text { (selection bias) }\end{array}$ & Unclear risk & No information provided. \\
\hline
\end{tabular}


C +aripiprazole 2013 (Continued)

Blinding (performance Unclear risk "Double blind" No other information provided. bias and detection bias)

All outcomes

\begin{tabular}{lll}
$\begin{array}{l}\text { Incomplete outcome data } \\
\text { (attrition bias) } \\
\text { All outcomes }\end{array}$ & High risk & The analysis does not account for the losses. \\
\hline $\begin{array}{l}\text { Selective reporting (re- } \\
\text { porting bias) }\end{array}$ & High risk & $\begin{array}{l}\text { Results for SAS and AIMS were reported on methods but do not have published } \\
\text { results. }\end{array}$ \\
\hline Other bias & Low risk & $\begin{array}{l}\text { Funded by National Institutes of Health and by National Center for Research } \\
\text { Resources General Clinical Research Centers Program. }\end{array}$ \\
\hline
\end{tabular}

\section{C +aripiprazole 2013b}

Methods
Blinding: double-blind.
Duration: 24 weeks (first 12 weeks using antipsychotic combinations).
Setting: inpatient.
Design: parallel.
Country: Korea, multi-centre.

Diagnosis: schizophrenia (DSM-IV-TR).
P $=35$.
Sex: M $26, \mathrm{~F} 9$.
Age: $\sim 50$ years.
History: Chronic participants, stabilized on their current dose of risperidone (3 mg to $6 \mathrm{mg} / \mathrm{day})$ for a
minimum of 3 months prior to enrolment in the study.

Interventions

1. Combination therapy: risperidone (3 mg to $6 \mathrm{mg})+$ aripiprazole $(10 \mathrm{mg} /$ day) for 12 weeks $(\mathrm{N}=17)$. From weeks 13 to 24 participants in the combination group received aripiprazole and tapered risperidone.

2. Monotherapy: risperidone $(3 \mathrm{mg}$ to $6 \mathrm{mg})+$ placebo for 12 weeks $(\mathrm{N}=18)$. From weeks 13 to 24 participants in the the monotherapy group received their current risperidone dose without placebo.

- Not usable data -
1. Mental state: PANSS (Skewed data).
2. Adverse events: ESRS (Skewed data).
3. Leaving the study early (Presented only at 24 weeks).
4. Adverse events: requiring discontinuation, prolactin levels (Presented only at 24 weeks).

Notes No clinical registration, no information regarding funds and no declaration of interest.

\section{Risk of bias}

\begin{tabular}{lll}
\hline Bias & Authors' judgement & Support for judgement \\
\hline $\begin{array}{l}\text { Random sequence genera- } \\
\text { tion (selection bias) }\end{array}$ & Unclear risk & "Randomly assigned". No other information provided \\
\hline
\end{tabular}


C +aripiprazole 2013b (Continued)

\begin{tabular}{lll}
$\begin{array}{l}\text { Allocation concealment } \\
\text { (selection bias) }\end{array}$ & Unclear risk & No other information provided \\
\hline $\begin{array}{l}\text { Blinding (performance } \\
\text { bias and detection bias) } \\
\text { All outcomes }\end{array}$ & Unclear risk & "Double-blind". No further details
\end{tabular}

Incomplete outcome data $\quad$ Low risk $\quad$ For missing data, the LOCF analysis was used.
(attrition bias)

All outcomes

Selective reporting (re- Low risk All outcome reported in methods, were presented. porting bias)

Other bias Unclear risk Non detected.

$\begin{array}{ll}\text { Methods } & \text { Allocation: randomised. } \\ \text { Blinding: double-blind. } \\ \text { Duration: } 8 \text { weeks. } \\ \text { Setting: unclear. } \\ \text { Design: parallel. } \\ \text { Country: China. }\end{array}$

\begin{tabular}{|c|c|}
\hline Participants & $\begin{array}{l}\text { Diagnosis: schizophrenia (ICD-10) } \\
\text { N = } 116 \\
\text { Age: } 34 \\
\text { Sex: M 54, F62 } \\
\text { History: Use of stable dose of risperidone }(3 \mathrm{mg} \text { to } 8 \mathrm{mg} / \text { day) monotherapy for at least } 6 \text { months. }\end{array}$ \\
\hline Interventions & $\begin{array}{l}\text { 1. Combination therapy: risperidone }(3 \mathrm{mg} \text { to } 8 \mathrm{mg} / \text { day })+\text { aripiprazole }(10 \mathrm{mg} \text { to } 20 \mathrm{mg} / \mathrm{day})(\mathrm{N}=59) \\
\text { 2. Monotherapy: risperidone ( } 3 \mathrm{mg} \text { to } 8 \mathrm{mg} / \mathrm{day})+ \text { placebo }(\mathrm{N}=57)\end{array}$ \\
\hline
\end{tabular}

Outcomes - Usable data -

1. Leaving the study early

2. Clinical response: $\mathrm{CGI}-\mathrm{S}$

3. Mental state: PANSS

4. Adverse events: requiring discontinuation

\section{- Not able to use -}

1. Adverse events: Prolactin levels (Skewed data)

\begin{tabular}{ll}
\hline Notes & Abstract in English, report in Chinese. \\
\hline Risk of bias & \\
\hline Bias & Authors' judgement Support for judgement \\
\hline
\end{tabular}




\section{C +aripiprazole 2014 (Continued)}

Random sequence genera- Low risk $\quad$ From correspondence: "Random numbers table is used for randomization." tion (selection bias)

Allocation concealment Low risk
(selection bias)

Blinding (performance Low risk

bias and detection bias)

Double blind. Placebo, which have the same colour, shape and taste with arip-

All outcomes iprazole.

Incomplete outcome data Low risk ITT.
(attrition bias)
All outcomes

\begin{tabular}{lll}
\hline $\begin{array}{l}\text { Selective reporting (re- } \\
\text { porting bias) }\end{array}$ & Low risk & All measured outcomes were reported. \\
\hline Other bias & High risk & Supported by Chendu Kanghong Pharmaceutical company \\
\hline
\end{tabular}

C +aripiprazole 2015

Methods
Allocation: randomised.
Blinding: double-blind.
Duration: 8 weeks.
Setting: Inpatient and outpatient.
Design: parallel.
Country: China.
Diagnosis: The diagnosis of schizophrenia was determined by the Structured Clinical Interview for
DSM-IV Axis I Disorders, Clinician Version.
N $=119$.
Sex: M $58, F 61$.
Age: average 33.5.
History: Chronic participants, stable during the screening phase, as indicated by a total score $<70$
on the Positive and Negative Syndrome Scale (PANSS) and had to experience hyperprolactinaemia in-
duced by risperidone.

Interventions
2. Combination therapy: risperidone (mean $=4.63 \mathrm{mg} / \mathrm{day})+\operatorname{aripiprazole}(5 \mathrm{mg})(\mathrm{N}=30)$.
3. Combination therapy: $r$ risperidone (mean $=4.79 \mathrm{mg} / \mathrm{day})+\operatorname{aripiprazole}(10 \mathrm{mg})(\mathrm{N}=29)$.
4. Monotherapy: Risperidone (mean $=4.93 \mathrm{mg} / \mathrm{day})+$ Placebo $(\mathrm{N}=30)$.

\section{Outcomes}

\section{- Usable data -}

1. Leaving the study early.

2. Clinical response: CGI-S.

3. Adverse events: requiring discontinuation.

\section{- Unable to use -}

1. Mental state: PANSS (Skewed data).

2. Adverse events: prolactin levels, BAS and SAS (Skewed data).

\section{- Not used in review -}


1. Body weight, waist circumference, hip circumference, fasting glucose, triglycerides, total cholesterol, LDL cholesterol, HDL cholesterol and QTc interval.

Notes

Data from correspondence used for 'Risk of bias' assessment.

Trial Registration: clinicaltrials.gov Identifier: NCT02013232

.

\section{Risk of bias}

\begin{tabular}{|c|c|c|}
\hline Bias & Authors' judgement & Support for judgement \\
\hline $\begin{array}{l}\text { Random sequence genera- } \\
\text { tion (selection bias) }\end{array}$ & Low risk & From correspondence: "Random numbers table is used for randomization." \\
\hline $\begin{array}{l}\text { Allocation concealment } \\
\text { (selection bias) }\end{array}$ & Low risk & From correspondence: "Central telephone randomization system." \\
\hline $\begin{array}{l}\text { Blinding (performance } \\
\text { bias and detection bias) } \\
\text { All outcomes }\end{array}$ & Low risk & $\begin{array}{l}\text { "Double-blind" } \\
\text { "Placebo and aripiprazole tablets were physically indistinguishable." }\end{array}$ \\
\hline $\begin{array}{l}\text { Incomplete outcome data } \\
\text { (attrition bias) } \\
\text { All outcomes }\end{array}$ & Low risk & $\begin{array}{l}\text { "Among the } 12 \text { non-completers (10.1\%), } 4 \text { had been assigned to placebo, } 2 \text { to } \\
5 \mathrm{mg} / \text { day of aripiprazole, } 2 \text { to } 10 \mathrm{mg} / \text { day of aripiprazole, and } 4 \text { to } 20 \mathrm{mg} / \text { day of } \\
\text { aripiprazole. Of these, } 7(5.9 \%) \text { were lost to follow-up, } 2(1.7 \%) \text { withdrew con- } \\
\text { sent, } 3 \text { ( } 2.5 \% \text { ) withdrew because of adverse event" } \\
\text { "Using data from all randomized patients with at least } 1 \text { follow-up test (modi- } \\
\text { fied intent-to treat analysis)." }\end{array}$ \\
\hline $\begin{array}{l}\text { Selective reporting (re- } \\
\text { porting bias) }\end{array}$ & Low risk & All pre-specified outcomes were reported. \\
\hline Other bias & High risk & $\begin{array}{l}\text { This study was supported from Funding for Beijing Outstanding Talent Train- } \\
\text { ing Projects, China. } \\
\text { Kanghong Pharmaceutical provided the medication and placebo. }\end{array}$ \\
\hline
\end{tabular}

C +aripiprazole 2015b

$\begin{array}{ll}\text { Methods } & \text { Allocation: randomised. } \\ \text { Blinding: double-blind. } \\ \text { Duration: 8-weeks. } \\ \text { Setting: outpatient. } \\ \text { Design: parallel. } \\ \text { Country: India. }\end{array}$

Participants

Diagnosis: schizophrenia (DSM-IV).

$\mathbf{N}=30$.

Sex: $M 15, F 15$.

Age: 32 years. 
C +aripiprazole 2015b (Continued)

History: participants on a stable dose of risperidone.

I. Combination therapy: risperidone $($ median $=6 \mathrm{mg})+$ aripiprazole $(10 \mathrm{mg} / \mathrm{day})(\mathrm{N}=15)$.
2. Monotherapy: risperidone $($ median $=6 \mathrm{mg})+$ placebo $(\mathrm{N}=15)$.

\section{Outcomes}

\section{- Usable data -}

1. Leaving the study early.

2. Adverse event: BAS.

\section{- Unable to use -}

1. Metal state: BPRS* (Skewed data).

2. Adverse event: prolactin levels*, SAS* (Skewed data).

\section{- Not used in review -}

1. Galactorrhoea

2. Arizona sexual experience scale

3. Prolactin-related symptoms

Notes

Funding by: Indian Medical Council of Research (ICMR), India.

* Unpublished data.

\section{Risk of bias}

\begin{tabular}{|c|c|c|}
\hline Bias & Authors' judgement & Support for judgement \\
\hline $\begin{array}{l}\text { Random sequence genera- } \\
\text { tion (selection bias) }\end{array}$ & Low risk & $\begin{array}{l}\text { "computer-generated randomisation schedule using permuted blocks of ran- } \\
\text { dom sizes, generated at the pharmacy." }\end{array}$ \\
\hline $\begin{array}{l}\text { Allocation concealment } \\
\text { (selection bias) }\end{array}$ & Low risk & $\begin{array}{l}\text { "Allocation concealment was ensured as the randomisation code was not re- } \\
\text { leased until all the recruitment and assessments were completed" }\end{array}$ \\
\hline $\begin{array}{l}\text { Blinding (performance } \\
\text { bias and detection bias) } \\
\text { All outcomes }\end{array}$ & Low risk & $\begin{array}{l}\text { "placebo tablets that were identical to the aripiprazole tablets..." AND "The } \\
\text { investigators, participants and their carers were masked to the medications } \\
\text { throughout the study..." }\end{array}$ \\
\hline $\begin{array}{l}\text { Incomplete outcome data } \\
\text { (attrition bias) } \\
\text { All outcomes }\end{array}$ & Unclear risk & Reasons for dropouts not reported. \\
\hline $\begin{array}{l}\text { Selective reporting (re- } \\
\text { porting bias) }\end{array}$ & Low risk & $\begin{array}{l}\text { No mean and SD reported for BPRS, prolactin levels, and SAS; data obtained } \\
\text { through correspondence. }\end{array}$ \\
\hline Other bias & Low risk & $\begin{array}{l}\text { Concern about baseline imbalances in prolactin levels, but this outcome was } \\
\text { not used in the analysis. }\end{array}$ \\
\hline
\end{tabular}

\section{C +aripiprazole $2015 c$}

Methods

Allocation: randomised.

Blinding: open-label.

Duration: 8-weeks.

Setting: inpatient and outpatient.

Design: parallel. 
C +aripiprazole 2015c (Continued)

Country: China.

\begin{tabular}{|c|c|}
\hline Participants & $\begin{array}{l}\text { Diagnosis: Schizophrenia or schizoaffective disorder (DSM-IV). } \\
\mathbf{N}=113 . \\
\text { Sex: M 46, F } 67 . \\
\text { Age: } 30 \text { years. } \\
\text { History: Stable patients with elevated serum prolactin level }(>324 \mathrm{mIU} / \mathrm{L} \text { in males and }>496 \mathrm{mIU} / \mathrm{L} \text { in } \\
\text { females). }\end{array}$ \\
\hline Interventions & $\begin{array}{l}\text { 1. Combination therapy: risperidone }(4 \mathrm{mg} \text { to } 6 \mathrm{mg} / \text { day })+\text { aripiprazole }(10 \mathrm{mg} / \mathrm{day})(\mathrm{N}=56) \text {. } \\
\text { 2. Monotherapy: risperidone ( } 4 \mathrm{mg} \text { to } 6 \mathrm{mg} / \mathrm{day})(\mathrm{N}=57) \text {. }\end{array}$ \\
\hline Outcomes & $\begin{array}{l}\text { - Usable data - } \\
\text { 1. Leaving the study early. } \\
\text { 2. Adverse events: requiring discontinuation, any movement disorders. } \\
\text { - Not able to use - } \\
\text { 1. Mental state: PANSS Total }{ }^{\star}, \text { PANSS Positive* }{ }^{\star} \text { PANSS Negative* (Skewed data). } \\
\text { 2. Adverse events: prolactin levels }{ }^{\star \star} \text { (Skewed data), BAS (Not reported), UKU (Not reported). } \\
\text { 3. Rating Scale for Extrapyramidal Side Effects }{ }^{\star \star} \text { (Not reported). }\end{array}$ \\
\hline Notes & $\begin{array}{l}{ }^{\star} \text { Extracted from a graph. } \\
{ }^{\star \star} \text { This scale corresponds to SAS. } \\
\text { Chinese Clinical Trial Registry: ChiCTR-IOR-15006278. }\end{array}$ \\
\hline
\end{tabular}

\section{Risk of bias}

\begin{tabular}{|c|c|c|}
\hline Bias & Authors' judgement & Support for judgement \\
\hline $\begin{array}{l}\text { Random sequence genera- } \\
\text { tion (selection bias) }\end{array}$ & Low risk & $\begin{array}{l}\text { From the protocol: "The generation of random sequence by method of ran- } \\
\text { dom number table". }\end{array}$ \\
\hline $\begin{array}{l}\text { Allocation concealment } \\
\text { (selection bias) }\end{array}$ & Unclear risk & No information provided. \\
\hline $\begin{array}{l}\text { Blinding (performance } \\
\text { bias and detection bias) } \\
\text { All outcomes }\end{array}$ & High risk & "Open-label trial". \\
\hline $\begin{array}{l}\text { Incomplete outcome data } \\
\text { (attrition bias) } \\
\text { All outcomes }\end{array}$ & High risk & $\begin{array}{l}\text { Reason for leaving the study early differ between groups. Only participants } \\
\text { that completed the trial were included for the analysis. }\end{array}$ \\
\hline $\begin{array}{l}\text { Selective reporting (re- } \\
\text { porting bias) }\end{array}$ & High risk & No mean and SD reported for BAS, UKU and SAS. \\
\hline Other bias & Low risk & $\begin{array}{l}\text { Funding by the National Natural Science Foundation of China and the Natural } \\
\text { Science Foundation of Henan. }\end{array}$ \\
\hline
\end{tabular}


C +aripiprazole 2016

Allocation: randomised.
Blinding: open-label.
Duration: 8-weeks.
Setting: inpatient.
Design: parallel.
Country: China.

Diagnosis: schizophrenia (DSM-IV).
Participants 60.
Sex: M 0, F 60.
Age: average 33.3 years.
History: Female participants were randomised if they presented hyperprolactinaemia (> 496 mIU/L)
and a decrease in the PANSS score by $>50 \%$. Previous to randomisation participants received risperi-
done or paliperidone treatment combined with modified electroconvulsive therapy (if antipsychotic
drug treatment was not effective).

Interventions

1. Combination therapy: paliperidone $(6 \mathrm{mg}$ to $12 \mathrm{mg} /$ day $)+$ aripiprazole $(5 \mathrm{mg})(\mathrm{N}=11)$.

2. Combination therapy: risperidone $(3 \mathrm{mg}$ to $6 \mathrm{mg} /$ day) + aripiprazole $(5 \mathrm{mg})(\mathrm{N}=19)$.

3. Monotherapy: paliperidone ( $6 \mathrm{mg}$ to $12 \mathrm{mg} /$ day) $(\mathrm{N}=16)$.

4. Monotherapy: risperidone ( $3 \mathrm{mg}$ to $6 \mathrm{mg} /$ day) $(\mathrm{N}=14)$.

- Usable data -
1. Leaving the study early.
2. Mental state: PANSS Total.
3. Adverse events: requiring discontinuation, any movement disorders.

\section{- Not able to use -}

1. Mental state: PANSS Positive and negative (Skewed data).

2. Adverse events: prolactin levels (Not able to impute), TESS (Not reported), weight gain (Not reported).

\section{- Not used in this review -}

1. Estradiol levels.

$\begin{array}{ll}\text { Notes } & \text { Chinese clinical trial register: ChiCTR-TRC-14004186. } \\ & \text { Funding by: Shanghai Natural Science Research Foundation, National Key Clinical Disciplines at Shang- } \\ \text { hai Mental Health Center, and Shanghai Clinical Center for Mental Disorders. }\end{array}$

\section{Risk of bias}

\begin{tabular}{lll}
\hline Bias & Authors' judgement & Support for judgement \\
\hline $\begin{array}{l}\text { Random sequence genera- } \\
\text { tion (selection bias) }\end{array}$ & Low risk & $\begin{array}{l}\text { From the protocol: “Using SAS9.3 version software and making block random- } \\
\text { ization with PROC PLAN process”. }\end{array}$ \\
\hline $\begin{array}{l}\text { Allocation concealment } \\
\text { (selection bias) }\end{array}$ & Unclear risk & No information provided. \\
\hline
\end{tabular}


C +aripiprazole 2016 (Continued)
Blinding (performance
High risk
Open-label trial.
bias and detection bias)
All outcomes

$\begin{array}{ll}\begin{array}{l}\text { Incomplete outcome data } \\ \text { (attrition bias) }\end{array} & \text { "fow riske participants were lost during follow-up in the treatment group. In the } \\ \text { All outcomes } & \text { control group, two participants were lost during the follow-up and withdrew } \\ \text { because of adverse events." }\end{array}$

\begin{tabular}{|c|c|c|}
\hline $\begin{array}{l}\text { Selective reporting (re- } \\
\text { porting bias) }\end{array}$ & High risk & $\begin{array}{l}\text { The outcomes mentioned in the protocol differ from those presented in the } \\
\text { published report. }\end{array}$ \\
\hline
\end{tabular}

\begin{tabular}{ll}
\hline Other bias $\quad$ High risk $\quad$ Baseline imbalances in PANSS values. \\
\hline
\end{tabular}

\section{C +clozapine 2001}

\begin{tabular}{ll}
\hline Methods & Allocation: randomised. \\
Blinding: double-blind. \\
Duration: 8 weeks. \\
Setting: inpatients. \\
Design: parallel. \\
Country: China. \\
\hline Participants & Diagnosis: schizophrenia. \\
& N = 40. \\
& Sex: M and F (not reported). \\
& Age: average $~ 32$ years. \\
History: not clear.
\end{tabular}

Interventions Combination therapy: risperidone $4 \mathrm{mg}$ to $6 \mathrm{mg}$ + clozapine $50 \mathrm{mg}$ to $300 \mathrm{mg}(\mathrm{N}=20)$.
2. Monotherapy: risperidone $4 \mathrm{mf}$ to $6 \mathrm{mg}(\mathrm{N}=20)$.

Schedule: daily dose.

\begin{tabular}{|c|c|c|}
\hline Outcomes & \multicolumn{2}{|c|}{$\begin{array}{l}\text { - Usable data - } \\
\text { 1. Leaving the study early. } \\
\text { 2. Clinical response: not clinically improved. } \\
\text { 3. Mental state: BPRS. } \\
\text { 4. Adverse events: movement disorders, drowsiness. }\end{array}$} \\
\hline Notes & \multicolumn{2}{|c|}{ *Abstract in English, report in Chinese. } \\
\hline \multicolumn{3}{|l|}{ Risk of bias } \\
\hline Bias & Authors' judgement & Support for judgement \\
\hline $\begin{array}{l}\text { Random sequence genera- } \\
\text { tion (selection bias) }\end{array}$ & Unclear risk & "Sequential trial randomisation". \\
\hline $\begin{array}{l}\text { Allocation concealment } \\
\text { (selection bias) }\end{array}$ & Unclear risk & No information provided. \\
\hline $\begin{array}{l}\text { Blinding (performance } \\
\text { bias and detection bias) } \\
\text { All outcomes }\end{array}$ & Unclear risk & No information provided. \\
\hline
\end{tabular}


C +clozapine 2001 (Continued)

Incomplete outcome data Low risk All included patients completed the trial.

(attrition bias)

All outcomes

Selective reporting (re- High risk $\quad$ Not all expected outcomes reported.
porting bias)

\begin{tabular}{ll}
\hline Other bias $\quad$ Unclear risk & No source of funding reported. \\
\hline
\end{tabular}

C +clozapine 2013

\begin{tabular}{|c|c|}
\hline Methods & $\begin{array}{l}\text { Allocation: randomised. } \\
\text { Blinding: unclear. } \\
\text { Duration: } 8 \text { weeks. } \\
\text { Setting: inpatient. } \\
\text { Design: parallel. } \\
\text { Country: China. }\end{array}$ \\
\hline Participants & $\begin{array}{l}\text { Diagnosis: schizophrenia (CCMD-3). } \\
\text { N=50. } \\
\text { Sex: M } 23 \text {, F } 27 . \\
\text { Age: } ~ 34 \text { years. } \\
\text { History: Chronic participants with predominantly negative symptoms. }\end{array}$ \\
\hline Interventions & $\begin{array}{l}\text { 1. Combination therapy: any antipsychotic }{ }^{\star}+\text { clozapine (orally disintegrated tablet) }(300 \mathrm{mg} \text { to } 400 \mathrm{mg} / \\
\text { day) }(\mathrm{N}=25) \text {. } \\
\text { 2. Monotherapy: any antipsychotic }{ }^{\star \star}(\mathrm{N}=25) \text {. }\end{array}$ \\
\hline
\end{tabular}

\begin{tabular}{ll}
\hline Outcomes & - Usable data - \\
1. Clinical response: not clinically improved. & 2. Leaving the study early. \\
3. Adverse events: drowsiness.
\end{tabular}

\section{- Not usable data -}

1. Mental state: PANSS (Total, Negative) (Skewed data).

Notes $\quad{ }^{*}$ quetiapine fumarate $(N=6)$, ziprasidone $(N=6)$, risperidone $(N=5)$, aripiprazole $(N=4)$, sulpiride $(N=$
4).
${ }^{\star \star}$ quetiapine fumarate $(N=6)$, ziprasidone $(N=5)$, risperidone $(N=6)$, aripiprazole $(N=4)$, sulpiride $(N=$
4).
Abstract in English, report in Chinese.

\section{Risk of bias}

\begin{tabular}{lll}
\hline Bias & Authors' judgement & Support for judgement \\
\hline $\begin{array}{l}\text { Random sequence genera- } \\
\text { tion (selection bias) }\end{array}$ & Unclear risk & Not specified. \\
\hline
\end{tabular}


C +clozapine 2013 (Continued)

\begin{tabular}{lll}
$\begin{array}{l}\text { Allocation concealment } \\
\text { (selection bias) }\end{array}$ & Unclear risk & Not specified. \\
\hline $\begin{array}{l}\text { Blinding (performance } \\
\text { bias and detection bias) } \\
\text { All outcomes }\end{array}$ & High risk & Not specified. \\
\hline
\end{tabular}

Incomplete outcome data Low risk $\quad$ All included participants completed the trial.
(attrition bias)

All outcomes

Selective reporting (re- Low risk $\quad$ All outcomes were reported. porting bias)

\begin{tabular}{ll}
\hline Other bias $\quad$ Low risk $\quad$ None obvious \\
\hline
\end{tabular}

C + CPZ 1973

$\begin{array}{ll}\text { Methods } & \text { Allocation: randomised. } \\ \text { Blinding: double-blind. } \\ \text { Duration: } 30 \text { days. } \\ \text { Setting: inpatients. } \\ \text { Design: parallel. } \\ \text { Country: China. }\end{array}$

Participants Diagnosis: $75 \%$ of the participants likely to be chronic schizophrenia patients with an acute episode. $\mathbf{N}=46$.

Sex: M 24, F 22.

Age: average 37 years.

History: acute psychotics recently admitted to the two health centres, required antipsychotic medication.

Interventions Combination therapy: fluphenazine enanthate + chlorpromazine $(N=15)$.
2. Monotherapy: fluphenazine enanthate $(N=16)$.
3. Monotherapy: chlorpromazine $(N=15)$.

Schedule (on average): fluphenazine enanthate $26 \mathrm{mg}$ every 11.5 days + chlorpromazine $349.6 \mathrm{mg} /$ day, fluphenazine enanthate $28.5 \mathrm{mg}$ every 11.5 days, chlorpromazine $388 \mathrm{mg} / \mathrm{day}$.

Doses administered at clinically determined intervals.

Outcomes - Usable data -

1. Clinical response: not clinically improved.

2. Adverse events: movement disorders.

\section{- Unable to use -}

1. Behaviour: Nurses Observation Scale for Inpatient Evaluation (NOSIE; no data reported).

\section{Notes}

\section{Risk of bias}

Bias Authors' judgement Support for judgement




\section{C +CPZ 1973 (Continued)}

Random sequence genera- Unclear risk $\quad$ No details provided.
tion (selection bias)

Allocation concealment $\quad$ Unclear risk No details provided.
(selection bias)

\begin{tabular}{|c|c|c|}
\hline $\begin{array}{l}\text { Blinding (performance } \\
\text { bias and detection bias) } \\
\text { All outcomes }\end{array}$ & Unclear risk & $\begin{array}{l}\text { At both centres, all patients involved in the study had their dosage regulated } \\
\text { by the usual clinical staff. The research psychiatrist at each centre scrupulous- } \\
\text { ly avoided influencing the amount or frequency of administration of antipsy- } \\
\text { chotic medication used during the course of the study, except to specify that } \\
\text { fluphenazine enanthate dosage should not exceed } 75 \mathrm{mg} \text { in a single dose. }\end{array}$ \\
\hline
\end{tabular}

\begin{tabular}{|c|c|c|}
\hline $\begin{array}{l}\text { Incomplete outcome data } \\
\text { (attrition bias) }\end{array}$ & High risk & $\begin{array}{l}\text { More than } 50 \% \text { of the patients from each treatment group were discharged } \\
\text { (lost to follow-up) within } 30 \text { days after admission. }\end{array}$ \\
\hline
\end{tabular}

All outcomes

(lost to follow-up) within 30 days after admission.

\begin{tabular}{lll}
\hline $\begin{array}{l}\text { Selective reporting (re- } \\
\text { porting bias) }\end{array}$ & High risk & Not all expected outcomes reported. \\
\hline Other bias & Unclear risk & Funding not reported. \\
\hline
\end{tabular}

\section{C +CPZ 1989}

\begin{tabular}{ll}
\hline Methods & Allocation: randomised. \\
Blinding: double-blind. & Duration: 8 weeks. \\
Setting: inpatients. \\
Design: parallel. \\
Country: China.
\end{tabular}

Outcomes - Usable data -

1. Mental state: BPRS.

\section{- Not used in review -}

1. Plasma homovanillic acid (HVA) levels.

Notes Data extrapolated from figure 2.

\section{Risk of bias}

Authors' judgement Support for judgement 


\section{C +CPZ 1989 (Continued)}

Random sequence genera- Unclear risk $\quad$ No details provided.
tion (selection bias)

\begin{tabular}{lll}
\hline $\begin{array}{l}\text { Allocation concealment } \\
\text { (selection bias) }\end{array}$ Unclear risk & No details provided.
\end{tabular}

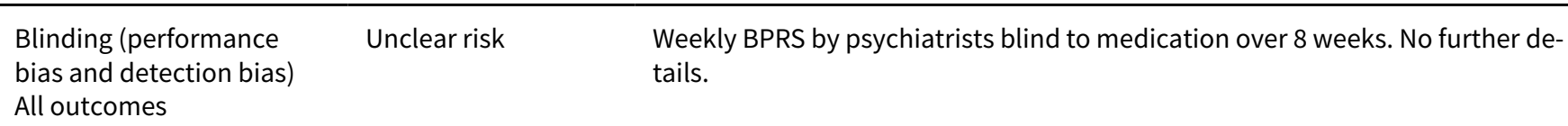

\begin{tabular}{lll}
\hline $\begin{array}{l}\text { Incomplete outcome data } \\
\text { (attrition bias) } \\
\text { All outcomes }\end{array}$ & No details provided. \\
\hline $\begin{array}{l}\text { Selective reporting (re- } \\
\text { porting bias) }\end{array}$ & High risk & Not all expected outcomes reported. \\
\hline Other bias & Unclear risk & Funding not reported. \\
\hline
\end{tabular}

Methods Allocation: 'randomised' according to hospital admission order.

Blinding: not stated.

Duration: 6 weeks.

Setting: inpatients.

Design: parallel.

Country: China.

\begin{tabular}{|c|c|c|}
\hline Participants & \multicolumn{2}{|c|}{$\begin{array}{l}\text { Diagnosis: schizophrenia (CCMD-2-R). } \\
\mathbf{N}=115 . \\
\text { Age: mean } 28 \text { years, SD } 10 \text { years. } \\
\text { Sex: male and female. } \\
\text { History: length of illness mean } \sim 11 \text { months, SD } 9 \text { months. }\end{array}$} \\
\hline Interventions & \multicolumn{2}{|c|}{$\begin{array}{l}\text { 1. Monotherapy: chlorpromazine }(400 \mathrm{mg} / \mathrm{day})(\mathrm{N}=35) \text {. } \\
\text { 2. Monotherapy: clozapine }(300 \mathrm{mg} / \mathrm{day})(\mathrm{N}=40) \text {. } \\
\text { 3. Combination therapy: chlorpromazine }(100 \mathrm{mg} / \text { day })+\text { clozapine }(300 \mathrm{mg} / \mathrm{day})(\mathrm{N}=40) \text {. }\end{array}$} \\
\hline Outcomes & \multicolumn{2}{|c|}{$\begin{array}{l}\text { - Usable data - } \\
\text { 1. Mental state: BPRS. } \\
\text { 2. Adverse events: TESS. }\end{array}$} \\
\hline \multicolumn{3}{|l|}{ Notes } \\
\hline \multicolumn{3}{|l|}{ Risk of bias } \\
\hline Bias & Authors' judgement & Support for judgement \\
\hline $\begin{array}{l}\text { Random sequence genera- } \\
\text { tion (selection bias) }\end{array}$ & High risk & $\begin{array}{l}\text { 'Randomised' according to hospital admission order, but no further informa- } \\
\text { tion. }\end{array}$ \\
\hline
\end{tabular}




\section{C +CPZ 1999 (Continued)}

\begin{tabular}{lll}
$\begin{array}{l}\text { Allocation concealment } \\
\text { (selection bias) }\end{array}$ & Unclear risk & Not stated. \\
\hline $\begin{array}{l}\text { Blinding (performance } \\
\text { bias and detection bias) } \\
\text { All outcomes }\end{array}$ & Unclear risk & Not stated.
\end{tabular}

Incomplete outcome data Low risk No incomplete outcome data.
(attrition bias)

All outcomes

Selective reporting (re- Low risk All measured outcomes were reported.
porting bias)

Other bias Low risk None obvious.

$\begin{array}{ll}\text { Methods } & \text { Allocation: randomised 1:1 ratio. } \\ \text { Blinding: double-blind. } \\ \text { Duration: } 12 \text { weeks. } \\ \text { Setting: inpatients. } \\ \text { Design: parallel. } \\ \text { Country: Iran. }\end{array}$

$\begin{array}{ll}\text { Participants } & \text { Diagnosis: schizophrenia (DSM). } \\ & \mathbf{N}=28 . \\ & \text { Sex: females only. } \\ & \text { History: poor response to olanzapine, chronic schizophrenia. }\end{array}$
Interventions
1. Combination therapy: Olanzapine plus fluphenazine decanoate $(N=14)$.
2. Monotherapy: Olanzapine plus placebo $(\mathrm{N}=14)$.

Schedule: olanzapine $15 \mathrm{mg}$ to $25 \mathrm{mg}$ daily, fluphenazine decanoate week zero $6.25 \mathrm{mg} / 2$ weeks IM, and increased by $6.25 \mathrm{mg}$ increments, as needed or tolerated, in biweekly intervals, to a maximum of $25 \mathrm{mg} / 2$ weeks by week eight.

- Usable data -
1. Leaving the study early.
2. Clinical response: CGI-S.
3. Mental state: SAPS, SANS.
4. Adverse events: serious or requiring discontinuation, SAS.

\section{- Not used in review -}

1. Schedule for Assessment of Insight (SAI).

\section{Notes}

\section{Risk of bias}

Authors' judgement Support for judgement 
C +fluphen dec 2009 (Continued)

Random sequence genera- High risk Quasi-randomised "Randomly entered in one of the two matching contemtion (selection bias)

poraneous groups, alternately one patient after the other (one into the experiment group and the next into the control group, in sequence and back-toback)."

Allocation concealment $\quad$ Unclear risk No details provided.
(selection bias)

\begin{tabular}{|c|c|c|}
\hline $\begin{array}{l}\text { Blinding (performance } \\
\text { bias and detection bias) } \\
\text { All outcomes }\end{array}$ & Low risk & $\begin{array}{l}\text { "Double-blind". "The placebo had been arranged in the shape of comparable } \\
\text { vials, like the target drug". "The evaluators, as well, were unaware concerning } \\
\text { the partition and the type of medications arranged for each group." }\end{array}$ \\
\hline $\begin{array}{l}\text { Incomplete outcome data } \\
\text { (attrition bias) } \\
\text { All outcomes }\end{array}$ & Low risk & No losses to follow-up reported. \\
\hline $\begin{array}{l}\text { Selective reporting (re- } \\
\text { porting bias) }\end{array}$ & High risk & Not all expected outcomes reported. \\
\hline Other bias & Unclear risk & Funding not reported. \\
\hline
\end{tabular}

\section{C +haloperidol 2006}

\begin{tabular}{ll}
\hline Methods & Allocation: randomised. \\
Blinding: double-blind. \\
Duration: 10 weeks.
\end{tabular}

Diagnosis: schizophrenia (criteria not reported).
$\mathbf{N}=10$.
Sex: not reported.
Age: not reported.
History: Treatment-resistant schizophrenia, resistant to 2 adequate trials with 2 different antipsy-
chotics and to a trial with clozapine during a minimum of $6-8$ weeks in adequate dosage.

Interventions

1. Combination therapy: clozapine (mean $450 \mathrm{mg} /$ day SD 70.7) + haloperidol (4 mg/day) ( $\mathrm{N}=$ unclear ${ }^{\star}$ ).

2. Monotherapy: clozapine (mean $500 \mathrm{mg} /$ day SD 81.6) + placebo $\left(\mathrm{N}=\right.$ unclear $\left.^{\star}\right)$.

Schedule: not reported.

\begin{tabular}{ll}
\hline Outcomes & - Usable data - \\
& 1. Mental state: PANSS. \\
\hline Notes & ${ }^{\star} \mathrm{N}$ randomised not reported. \\
& Conference proceeding.
\end{tabular}

\section{Risk of bias}

\section{Authors' judgement Support for judgement}


C +haloperidol 2006 (Continued)

Random sequence genera- Unclear risk "Randomised" no further details reported. tion (selection bias)

\begin{tabular}{ll}
\hline $\begin{array}{l}\text { Allocation concealment } \\
\text { (selection bias) }\end{array}$ & Unclear risk information reported.
\end{tabular}

Blinding (performance
bias and detection bias) Unclear risk "Double-blind" no further details reported.

All outcomes

$\begin{array}{ll}\begin{array}{l}\text { Incomplete outcome data } \\ \text { (attrition bias) }\end{array} & \text { Unclear risk } \\ \begin{array}{l}\text { All outcomes } \\ \text { ed the study". N randomised to each group not reported. }\end{array}\end{array}$

\begin{tabular}{lll}
\hline $\begin{array}{l}\text { Selective reporting (re- } \\
\text { porting bias) }\end{array}$ & High risk & Not all expected outcomes reported. \\
\hline Other bias & Unclear risk & Source of funding not reported. \\
\hline
\end{tabular}

C +haloperidol 2010

$\begin{array}{ll}\text { Methods } & \text { Allocation: randomised. } \\ \text { Blinding: double-blind. } & \text { Duration: } 6 \text { weeks. } \\ \text { Setting: inpatient. } & \text { Design: parallel. } \\ \text { Country: Taiwan. }\end{array}$

\begin{tabular}{ll}
\hline Participants & Diagnosis: schizophrenia (DSM-IV). \\
& N $=88$. \\
& Sex: M 51, F 37. \\
& Age: average 38 years. \\
& History: newly hospitalised schizophrenic patients with acute exacerbation.
\end{tabular}

I. Combination therapy: $2 \mathrm{mg} / \mathrm{d}$ risperidone $+2 \mathrm{mg} /$ day haloperidol $(\mathrm{N}=46)$.
2. Monotherapy: $4 \mathrm{mg} /$ day risperidone $(\mathrm{N}=42)$.

Schedule: not reported.

Outcomes - Usable data -

1. Leaving the study early.

2. Clinical response: not clinically improved ( $<30 \%$ reduction in total PANSS scores), CGI-S, GAF.

3. Mental state: PANSS.

4. Adverse events: Serious adverse events, weight gain, movement disorders.

5. Economic burden: cost of care.

\section{- Unable to use -}

1. Quality of life: SF-36 (No total scores reported).

2. Adverse events: AIMS, BAS, SAS, prolactin (Skewed data).

\section{- Not used in review -}

1. CDSS (Calgary Depression Scale for Schizophrenia) time to clinical response; time to discontinuation; $\mathrm{BMI}$, pulse rate, blood pressure, fasting glucose, liver function, renal function, lipid profiles. 
C +haloperidol 2010 (Continued)

Notes Clinical Trials.gov identifier: NCT00998608.

\section{Risk of bias}

\begin{tabular}{|c|c|c|}
\hline Bias & Authors' judgement & Support for judgement \\
\hline $\begin{array}{l}\text { Random sequence genera- } \\
\text { tion (selection bias) }\end{array}$ & Unclear risk & Randomised, no further details. \\
\hline $\begin{array}{l}\text { Allocation concealment } \\
\text { (selection bias) }\end{array}$ & Unclear risk & No information provided. \\
\hline $\begin{array}{l}\text { Blinding (performance } \\
\text { bias and detection bias) } \\
\text { All outcomes }\end{array}$ & Unclear risk & Double-blind, no further details. \\
\hline $\begin{array}{l}\text { Incomplete outcome data } \\
\text { (attrition bias) } \\
\text { All outcomes }\end{array}$ & Low risk & $\begin{array}{l}\text { Efficacy analysis was carried out with the LOCF method for participants that } \\
\text { did not stay in the study at week } 6 \text {. Analyses of safety assessments were con- } \\
\text { ducted on all randomised patients }(N=88) \text {. }\end{array}$ \\
\hline $\begin{array}{l}\text { Selective reporting (re- } \\
\text { porting bias) }\end{array}$ & Low risk & All pre-specified outcomes were reported. \\
\hline Other bias & Low risk & $\begin{array}{l}\text { Funded by the Kai-Suan Psychiatry Hospital, the National Science Council (Tai- } \\
\text { wan), National Health Research Institutes (Taiwan), Department of Health (Tai- } \\
\text { wan), Department of Health Clinical Trial and Research Center of Excellence, } \\
\text { and China Medial University Hospital (Taiwan). }\end{array}$ \\
\hline
\end{tabular}

\section{C +levomepromazine 2004}

$\begin{array}{ll}\text { Methods } & \text { Allocation: randomised. } \\ \text { Blinding: open-label. } \\ \text { Duration: 8 weeks. } \\ \text { Setting: inpatients. } \\ \text { Design: parallel. } \\ \text { Country: Japan. }\end{array}$

$\begin{array}{ll}\text { Participants } & \text { Diagnosis: schizophrenia (DSM-IV). } \\ & \mathbf{N}=19 . \\ & \text { Sex: M 12, F 7. } \\ & \text { Age: average } 29 \text { years. }\end{array}$

History: not medicated before this trial..

$\begin{array}{ll}\text { Interventions } & \text { Combination therapy: haloperidol + levomepromazine }(N=9) \text {. } \\ \text { 2. Monotherapy: haloperidol }(N=10) \text {. }\end{array}$

Schedule: Dose adjustment was made on the basis of clinical response and tolerance, except that the dose ratio was fixed at 1:10 for the combined therapy.

$\begin{array}{ll}\text { Outcomes } & \text { - Usable data - } \\ \text { 1. Leaving the study early. } & \text { 2. Mental state: BPRS. }\end{array}$

\section{- Not used in review -}


C +levomepromazine 2004 (Continued)

1. ECGs, blood pressure or heart rate.

Notes *Data were extrapolated from figures.

\section{Risk of bias}

\begin{tabular}{lll}
\hline Bias & Authors' judgement & Support for judgement \\
\hline $\begin{array}{l}\text { Random sequence genera- } \\
\text { tion (selection bias) }\end{array}$ & Unclear risk & No details provided. \\
\hline $\begin{array}{l}\text { Allocation concealment } \\
\text { (selection bias) }\end{array}$ & Unclear risk & No details provided. \\
\hline $\begin{array}{l}\text { Blinding (performance } \\
\text { bias and detection bias) } \\
\text { All outcomes }\end{array}$ & High risk & Open-label trial. \\
\hline $\begin{array}{l}\text { Incomplete outcome data } \\
\text { (attrition bias) } \\
\text { All outcomes }\end{array}$ & High risk & Four patients were taken off the monotherapy, because of little improvement \\
\hline $\begin{array}{l}\text { Selective reporting (re- } \\
\text { porting bias) }\end{array}$ & High risk & Not all expected outcomes reported. \\
\hline \begin{tabular}{l} 
Other bias \\
\hline
\end{tabular} & Unclear risk & Funding not reported. \\
\hline
\end{tabular}

C +olan/risp 2014

\begin{tabular}{|c|c|}
\hline Methods & $\begin{array}{l}\text { Allocation: randomised. } \\
\text { Blinding: single-blind. } \\
\text { Duration: } 12 \text { weeks. (10 weeks randomised). } \\
\text { Setting: inpatient. } \\
\text { Design: parallel. } \\
\text { Country: Japan, multi-centre. }\end{array}$ \\
\hline Participants & $\begin{array}{l}\text { Diagnosis: schizophrenia, schizophreniform disorder, or schizoaffective disorder (DSM-IV-TR). } \\
\text { N=51*. } \\
\text { Sex: M } 22, \mathrm{~F} 29 . \\
\text { Age: } 45 . \\
\text { History: acute/non responders. Most patients were behavioral emergencies and about } 60 \% \text { were } \\
\text { brought in by the police. Only early non responders (CGI-I score } \geq 4 \text { ) were randomised after a } 2 \text { week tri- } \\
\text { al with either risperidone or olanzapine. }\end{array}$ \\
\hline Interventions & $\begin{array}{l}\text { 1. Combination therapy: olanzapine (Mean }=19.0 \mathrm{mg} \text { ) augmented with risperidone (Max }=8.1 \mathrm{mg} / \mathrm{day}) \\
(\mathrm{N}=11) \text {. } \\
\text { 2. Combination therapy: risperidone (Mean }=8.7 \mathrm{mg} / \text { day) augmented with olanzapine (Max. dose } 16.1 \\
\mathrm{mg} / \text { day) }(\mathrm{N}=14) \text {. } \\
\text { 3. Monotherapy: olanzapine }(\mathrm{Max}=18.8)(\mathrm{N}=13) \text {. } \\
\text { 4. Monotherapy: risperidone ( } \mathrm{Max}=8.2)(\mathrm{N}=11) \text {. }\end{array}$ \\
\hline
\end{tabular}

\section{Outcomes}

\section{- Usable data -}

1. Clinical Response: not clinically improved, CGI-I and GAF 
2. Leaving the study early.

3. Mental state: PANSS (Total, Positive, Negative).

4. Adverse event: requiring discontinuation, any movement disorder, weight gain.

\section{- Not able to use -}

1. Adverse event: prolactin levels (Skewed data).

\section{- Not used in this review -}

1. Five factor model of the PANSS, triglycerides, LDL, fasting glucose and BMI. the two intervention groups.

Clinical register: UMIN000007145.

\section{Risk of bias}

\begin{tabular}{lll}
\hline Bias & Authors' judgement & Support for judgement \\
\hline $\begin{array}{l}\text { Random sequence genera- } \\
\text { tion (selection bias) }\end{array}$ & Low risk & "For randomization, we referred to a random number table". \\
\hline $\begin{array}{l}\text { Allocation concealment } \\
\text { (selection bias) }\end{array}$ & Low risk & $\begin{array}{l}\text { "Sequentially numbered, opaque, sealed envelopes used to conceal the allo- } \\
\text { cation sequence." }\end{array}$ \\
\hline $\begin{array}{l}\text { Blinding (performance } \\
\text { bias and detection bias) } \\
\text { All outcomes }\end{array}$ & High risk & Rater-blinded. Participants where not blinded to the intervention. \\
\hline $\begin{array}{l}\text { Incomplete outcome data } \\
\text { (attrition bias) }\end{array}$ & High risk & Almost 50\% (25/51) of participants did not completed the study. \\
\hline $\begin{array}{l}\text { Selective reporting (re- } \\
\text { porting bias) }\end{array}$ & Low risk & $\begin{array}{l}\text { The study protocol was published but is not available. The published report in- } \\
\text { clude all expected outcomes, including those that were pre-specified. }\end{array}$ \\
\hline $\begin{array}{l}\text { Other bias } \\
\text { flis }\end{array}$ & Unclear risk & $\begin{array}{l}\text { Funded by the Ministry of Health, Welfare, and Labor of the Japanese Govern- } \\
\text { ment and Intramural Research Grant for Neurological and Psychiatric Disor- } \\
\text { ders. }\end{array}$ \\
\hline
\end{tabular}

\section{C +olanzapine 2012}

$\begin{array}{ll}\text { Methods } & \text { Allocation: randomised. } \\ \text { Blinding: rater blinded. } \\ \text { Duration: } 10 \text { weeks. } \\ \text { Setting: inpatient. } \\ \text { Design: parallel. } \\ \text { Country: Japan. }\end{array}$

Participants

Diagnosis: schizophrenia, schizophreniform disorder, or schizoaffective disorder (DSM IV-TR).

$\mathbf{N}=26^{*}$.

Sex: M 13, F 13.

Age: average 39.5 years.

History: newly admitted patients with acute schizophrenia who were early non-responders to risperidone. 
C +olanzapine 2012 (Continued)

Interventions

1. Combination therapy: risperidone $(<6 \mathrm{mg} /$ day $)+$ Olanzapine $(<20 \mathrm{mg} /$ day $)(\mathrm{N}=13)$.

2. Monotherapy: risperidone, starting at $3 \mathrm{mg} /$ day, at 2 weeks $<6 \mathrm{mg} /$ day was allowed and at 8 weeks $<12 \mathrm{mg} /$ day $(\mathrm{N}=13)$.

Outcomes

\section{- Usable data -}

1. Leaving the study early.

2. Clinical response: not clinically improved (<50\% improvement in PANSS total), CGI-I, GAF.

3. Mental state: PANSS.

4. Adverse events: serious event or requiring discontinuation, movement disorders, average weight gain.

\section{- Not used in review -}

Time to treatment discontinuation, blood levels change from baseline $(\mathrm{mg} / \mathrm{dL})$; fasting glucose, cholesterol, triglycerides.

Notes $\quad{ }^{\star} 26 / 78$ participants were early non-responders to risperidone and were randomised to the two intervention groups.

UMIN Clinical Trials Registry: UMIN000003531.

\section{Risk of bias}

\begin{tabular}{|c|c|c|}
\hline Bias & Authors' judgement & Support for judgement \\
\hline $\begin{array}{l}\text { Random sequence genera- } \\
\text { tion (selection bias) }\end{array}$ & Unclear risk & Randomised, no further details. \\
\hline $\begin{array}{l}\text { Allocation concealment } \\
\text { (selection bias) }\end{array}$ & Low risk & $\begin{array}{l}\text { Opaque, sealed envelopes "We referred to a random number table, with se- } \\
\text { quentially numbered, opaque, sealed envelopes used to conceal the allocation } \\
\text { sequence". }\end{array}$ \\
\hline $\begin{array}{l}\text { Blinding (performance } \\
\text { bias and detection bias) } \\
\text { All outcomes }\end{array}$ & High risk & $\begin{array}{l}\text { Participants and personnel were not blinded. Blind outcome assessment } \\
\text { "Rater blinded". }\end{array}$ \\
\hline $\begin{array}{l}\text { Incomplete outcome data } \\
\text { (attrition bias) } \\
\text { All outcomes }\end{array}$ & Low risk & $\begin{array}{l}\text { Although } 13 \text { participants discontinued treatment, ITT analysis was carried out } \\
\text { of all } 26 \text { participants. }\end{array}$ \\
\hline $\begin{array}{l}\text { Selective reporting (re- } \\
\text { porting bias) }\end{array}$ & Low risk & All pre-specified outcomes were reported. \\
\hline Other bias & Low risk & $\begin{array}{l}\text { Supported by grants from the Ministry of Health, Welfare, and Labor of the } \\
\text { Japanese Government. }\end{array}$ \\
\hline
\end{tabular}

C +olanzapine 2012b

$\begin{array}{ll}\text { Methods } & \text { Allocation: randomised. } \\ \text { Blinding: double-blind. } \\ \text { Duration: } 24 \text { weeks }\left(12 \text { weeks for each phase }{ }^{\star}\right) . \\ \text { Setting: inpatients. } \\ \text { Design: cross-over. } \\ \text { Country: Finland. }\end{array}$


C +olanzapine 2012b (Continued)
Participants
Diagnosis: schizophrenia.
$\mathbf{N}=14$.
Sex: M 11, F1 (data from participants who ended the study).
Age: 48 years.
History: Treatment resistant schizophrenic participants with a GAF score $<25$ who were currently on therapy with clozapine-olanzapine combination.

$\begin{array}{ll}\text { Interventions } & \text { 1. Combination therapy: clozapine }+ \text { olanzapine }(N=7) . \\ \text { 2. Monotherapy: clozapine + placebo }(N=7) .\end{array}$

Outcomes $\quad$ - Usable data -
1. Leaving the study early.

\section{- Not able to use -}

1. Clinical response: CGI-S and GAF (Unable to extract the data from table 2, as it is unclear which results belong which group).

\begin{tabular}{ll}
\hline Notes & * The first 4 weeks of the study were used for tapering the group from clozapine + olanzapine to \\
monotherapy with clozapine.
\end{tabular}

\begin{tabular}{|c|c|c|}
\hline Risk of bias & & \\
\hline Bias & Authors' judgement & Support for judgement \\
\hline $\begin{array}{l}\text { Random sequence genera- } \\
\text { tion (selection bias) }\end{array}$ & Unclear risk & "Randomized" No other information provided. \\
\hline $\begin{array}{l}\text { Allocation concealment } \\
\text { (selection bias) }\end{array}$ & Unclear risk & No information provided. \\
\hline $\begin{array}{l}\text { Blinding (performance } \\
\text { bias and detection bias) } \\
\text { All outcomes }\end{array}$ & Low risk & $\begin{array}{l}\text { Double blind: "Olanzapine and placebo were dispensed in similar gelatin cap- } \\
\text { sules that were formulated for this trial." } \\
\text { "The rater was blind to the study medication (i.e., either olanzapine or place- } \\
\text { bo) until the completion of the trial in each case." }\end{array}$ \\
\hline $\begin{array}{l}\text { Incomplete outcome data } \\
\text { (attrition bias) } \\
\text { All outcomes }\end{array}$ & High risk & $\begin{array}{l}\text { Imbalance in the number of missing data, as one group lost } 4(57 \%) \text { patients } \\
\text { while the other had no losses. }\end{array}$ \\
\hline $\begin{array}{l}\text { Selective reporting (re- } \\
\text { porting bias) }\end{array}$ & High risk & $\begin{array}{l}\text { Unable to use data for GAF and CGI as the results are presented without speci- } \\
\text { fying the group to which they correspond. }\end{array}$ \\
\hline Other bias & Low risk & $\begin{array}{l}\text { The study was supported by funding from the Annual EVO Financing (special } \\
\text { government subsidies from the Ministry of Health and Welfare, Finland). }\end{array}$ \\
\hline
\end{tabular}

\section{C +perphenazine 1976}

\begin{tabular}{ll}
\hline Methods & Allocation: randomised. \\
& Blinding: double-blind. \\
& Duration: 8 weeks. \\
Setting: inpatients. \\
Design: parallel. \\
Country: Japan, multi-centre.
\end{tabular}

Participants Diagnosis: schizophrenia.


C +perphenazine 1976 (Continued)

$\mathbf{N}=317$.

Sex: M 196, F 155.

Age: range 26-31 years.

History: chronic.

Interventions
2. Combination therapy: carpipramine + chlorpromazine $(N=118)$
3. Monotherapy: chlorpromazine + placebo $(N=107)$

Schedule: chlorpromazine $75 \mathrm{mg}$ to $200 \mathrm{mg} /$ day (fixed), carpipramine and perphenazine not reported.

- Usable data -
1. Leaving the study early.
2. Clinical response: not clinically improved, relapse.
3. Adverse events: serious or requiring discontinuation, movement disorders, death.

\section{- Unable to use -}

1. Keio Univ Psychiatric Symptom Scale, Keio Univ Behaviour Rating Scale (Not reported).

\begin{tabular}{lll}
\hline Notes & *Abstract in English, report in Japanese. \\
\hline Risk of bias & Authors' judgement & Support for judgement \\
\hline Bias & Randomly assigned, no further information. \\
\hline $\begin{array}{l}\text { Random sequence genera- } \\
\text { tion (selection bias) }\end{array}$ & Unclear risk & No information provided. \\
\hline $\begin{array}{l}\text { Allocation concealment } \\
\text { (selection bias) }\end{array}$ & Unclear risk & Identical tablets were used for active drugs and placebo. \\
\hline $\begin{array}{l}\text { Blinding (performance } \\
\text { bias and detection bias) } \\
\text { All outcomes }\end{array}$ & Low risk & \\
\hline $\begin{array}{l}\text { Incomplete outcome data } \\
\text { (attrition bias) } \\
\text { All outcomes }\end{array}$ & Unclear risk & No information provided. \\
\hline $\begin{array}{l}\text { Selective reporting (re- } \\
\text { porting bias) }\end{array}$ & High risk & Not all expected outcomes reported. \\
\hline \begin{tabular}{l} 
Other bias \\
\hline
\end{tabular} & Unclear risk & No source of funding reported. \\
\hline
\end{tabular}

\section{C +pimozide 2011}

$\begin{array}{ll}\text { Methods } & \text { Allocation: randomise } \\ \text { Blinding: double-blind } & \\ & \text { Duration: } 12 \text { weeks. } \\ \text { Setting: inpatient. } \\ \text { Design: parallel. } \\ \text { Country: USA. }\end{array}$

Participants

Diagnosis: schizophrenia, schizoaffective disorder (DSM-IV). 
C +pimozide 2011 (Continued)

$\mathbf{N}=53$.

Age: average 32 years.

History: stable dose of clozapine demonstrated to have been associated with a clozapine plasma level greater than $378 \mathrm{Cg} / \mathrm{mL}$ for at least eight weeks and partially or completely unresponsive to clozapine monotherapy.

Interventions

1. Combination therapy: clozapine (mean 518.8 SD 117.3) + pimozide (mean $6.48 \mathrm{mg} /$ day, SD 2.18, $\max$ $8 \mathrm{mg})(\mathrm{N}=25)$.

2. Monotherapy: clozapine (mean $478.1 \mathrm{mg} /$ day, $\mathrm{SD} 150.2)+$ placebo $(\mathrm{N}=28)$.

- Usable data -
1. Leaving the study early.
2. Clinical response: CGI-S.
3. Mental state: PANSS.
4. Adverse events: serious or requiring discontinuation.

\section{- Unable to use -}

1. Specific Level of Function scale (SLOF) (no overall scores reported).

2. Extrapyramidal Symptom Rating Scale (ESRS) (no overall scores reported).

\section{- Not used in review -}

1. Blood pressure, pulse, ECG.

\section{Risk of bias}

\begin{tabular}{|c|c|c|}
\hline Bias & Authors' judgement & Support for judgement \\
\hline $\begin{array}{l}\text { Random sequence genera- } \\
\text { tion (selection bias) }\end{array}$ & Unclear risk & Randomised, no further details. \\
\hline $\begin{array}{l}\text { Allocation concealment } \\
\text { (selection bias) }\end{array}$ & Unclear risk & No information provided. \\
\hline $\begin{array}{l}\text { Blinding (performance } \\
\text { bias and detection bias) } \\
\text { All outcomes }\end{array}$ & Unclear risk & $\begin{array}{l}\text { Double-blind, "Given that the increased risk of extrapyramidal symptoms as- } \\
\text { sociated with pimozide threatened the integrity of the blind, assignment of as- } \\
\text { sessments ensured that the rating of the ESRS was carried out by personnel } \\
\text { different from those performing the PANSS and functional competence rat- } \\
\text { ings." }\end{array}$ \\
\hline $\begin{array}{l}\text { Incomplete outcome data } \\
\text { (attrition bias) } \\
\text { All outcomes }\end{array}$ & Low risk & $\begin{array}{l}\text { Although } 12 \% \text { of combined group participants and } 18 \% \text { of placebo group par- } \\
\text { ticipants discontinued treatment, all participants randomised were included } \\
\text { in the analyses. }\end{array}$ \\
\hline $\begin{array}{l}\text { Selective reporting (re- } \\
\text { porting bias) }\end{array}$ & Low risk & All pre-specified outcomes were reported. \\
\hline Other bias & Low risk & Source of funding NIMH. \\
\hline
\end{tabular}

C +pimozide 2013

$\begin{array}{ll}\text { Methods } & \begin{array}{l}\text { Allocation: randomised. } \\ \text { Blinding: double-blind. }\end{array}\end{array}$


C +pimozide 2013 (Continued)

Duration: 12 weeks.

Setting: outpatients.

Design: parallel.

Country: USA, multi-centre.

\begin{tabular}{ll}
\hline Participants & Diagnosis: schizophrenia and schizoaffective disorder (DSM-IV). \\
& $\mathbf{N}=32^{\star}$. \\
Age: $\sim 42.9$. & $\begin{array}{l}\text { History: currently taking clozapine with blood level of at least } 350 \mathrm{ng} / \mathrm{ml} \text { and on stable dose of clozap- } \\
\text { ine for past } 2 \text { weeks. }\end{array}$ \\
\hline Interventions & $\begin{array}{l}\text { 1. Combination therapy: clozapine }(\text { Mean blood levels: } 650 \mathrm{ng} / \mathrm{mL})+\text { pimozide }(\text { Max dose } 4 \mathrm{mg})(\mathrm{N}=14) . \\
\text { 2. Monotherapy: clozapine }(\text { Mean blood levels } 519 \mathrm{ng} / \mathrm{dL})+\text { placebo }(\mathrm{N}=14) .\end{array}$
\end{tabular}

Outcomes

\section{- Usable data -}

1. Leaving the study early.

2. Adverse event: requiring discontinuation.

3. Mental state: BPRS.

\section{- Unable to use -}

1. Clinical Response: CGI-S and CGI-I (Skewed data).

2. Mental state: SANS (Skewed data).

3. Adverse events: SAS, AIMS (Not reported).

4. Service utilisation: hospital admission (Unable to impute).

\section{- Not used in this review -}

1. Neurocognitive measures: Controlled Word Association Test, Digit Symbol Coding, Rey Auditory Verbal Learning Test, Digit Span and Letter-Number Sequencing and Trail Making Test.

Notes $\quad{ }^{\star}$ Only reported outcomes for 28 patients as 4 patients withdrew prior to receiving medication. ClinicalTrials.gov Identifier: NCT00374244.

\section{Risk of bias}

\begin{tabular}{|c|c|c|}
\hline Bias & Authors' judgement & Support for judgement \\
\hline $\begin{array}{l}\text { Random sequence genera- } \\
\text { tion (selection bias) }\end{array}$ & Unclear risk & "Randomized". No other information provided. \\
\hline $\begin{array}{l}\text { Allocation concealment } \\
\text { (selection bias) }\end{array}$ & Unclear risk & No information provided. \\
\hline $\begin{array}{l}\text { Blinding (performance } \\
\text { bias and detection bias) } \\
\text { All outcomes }\end{array}$ & Low risk & $\begin{array}{l}\text { Double-blind: "Patients were randomized to identical looking pimozide or } \\
\text { placebo capsules by the research pharmacist who was not involved with the } \\
\text { subjects." }\end{array}$ \\
\hline $\begin{array}{l}\text { Incomplete outcome data } \\
\text { (attrition bias) } \\
\text { All outcomes }\end{array}$ & Low risk & Reasons for missing outcome data unlikely to be related to true outcome. \\
\hline $\begin{array}{l}\text { Selective reporting (re- } \\
\text { porting bias) }\end{array}$ & High risk & $\begin{array}{l}\text { Adverse events reported as: "no significant medication effects for AIMS, SAS, } \\
\text { systolic or diastolic blood pressure, heart rate, weight or QTc values over time." }\end{array}$ \\
\hline Other bias & High risk & $\begin{array}{l}\text { Imbalance of clozapine blood levels: "subjects in the augmentation arm show- } \\
\text { ing significantly higher clozapine levels". }\end{array}$ \\
\hline
\end{tabular}


C + pipotiazine 2000

\begin{tabular}{|c|c|}
\hline Methods & $\begin{array}{l}\text { Allocation: randomised (no further information). } \\
\text { Blinding: not stated. } \\
\text { Duration: } 3 \text { months. } \\
\text { Setting: unclear if community or inpatient. } \\
\text { Design: parallel. } \\
\text { Country: China. }\end{array}$ \\
\hline Participants & $\begin{array}{l}\text { Diagnosis: schizophrenia (CCMD-2-R). } \\
\mathbf{N}=50 \text {. } \\
\text { Age: average age } 25 \pm 9 \text { years. } \\
\text { Sex: male and female. } \\
\text { Average length of illness: } 6 \pm 4 \text { years. }\end{array}$ \\
\hline Interventions & $\begin{array}{l}\text { 1. Combination therapy: pipotiazine }(25 \mathrm{mg} \text { administered though muscle injection at the start of the } \\
\text { trial, a further } 50 \mathrm{mg} \text { was administered two weeks later. After that, } 50 \mathrm{mg} \text { to } 100 \mathrm{mg} / \mathrm{month} \text { until the } \\
\text { end of trial) }+ \text { clozapine }(491.62 \mathrm{mg} \pm 30.68 \mathrm{mg})(\mathrm{N}=26) \text {. } \\
\text { 2. Monotherapy: clozapine ( } 489.81 \mathrm{mg} \pm 29.73 \mathrm{mg} / \text { day })(\mathrm{N}=24) \text {. }\end{array}$ \\
\hline
\end{tabular}

\section{- Usable data -}

1. Clinical response: not clinically improved.

2. Mental state: BPRS, SANS*.

\section{- Unable to use -}

1. Adverse events: movement disorders $(\mathrm{TESS})^{\star}$ (Skewed data).

\section{- Not used in review -}

1. ECG.

Notes $\quad$ *N not reported, assumed to be the same as for the outcome 'not clinically improved'.

\section{Risk of bias}

\begin{tabular}{lll}
\hline Bias & Authors' judgement & Support for judgement \\
\hline $\begin{array}{l}\text { Random sequence genera- } \\
\text { tion (selection bias) }\end{array}$ & Unclear risk & Randomised, but no further information. \\
\hline $\begin{array}{l}\text { Allocation concealment } \\
\text { (selection bias) }\end{array}$ & Unclear risk & Not stated. \\
\hline $\begin{array}{l}\text { Blinding (performance } \\
\text { bias and detection bias) }\end{array}$ & Unclear risk & Not stated. \\
All outcomes & \\
\hline $\begin{array}{l}\text { Incomplete outcome data } \\
\text { (attrition bias) } \\
\text { All outcomes }\end{array}$ & Low risk & No incomplete outcome data. \\
\hline \hline
\end{tabular}


C +pipotiazine 2000 (Continued)

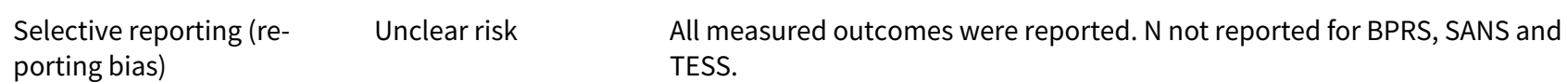
porting bias) TESS.

Other bias Low risk None obvious.

\section{C +risperidone 2001}

\begin{tabular}{|c|c|}
\hline Methods & $\begin{array}{l}\text { Allocation: randomised (no further information). } \\
\text { Blinding: not stated. } \\
\text { Duration: } 8 \text { weeks. } \\
\text { Setting: inpatients. } \\
\text { Design: parallel. } \\
\text { Country: China. }\end{array}$ \\
\hline Participants & $\begin{array}{l}\text { Diagnosis: schizophrenia (CCMD-2). } \\
\mathbf{N}=326 \text {. } \\
\text { Age: clozapine group } 40.2 \pm 8.7 \text { years, risperidone group } 38.9 \pm 9.4 \text { years, combination group } 39.4 \pm 9.2 \\
\text { years. } \\
\text { Sex: male and female. } \\
\text { Average length of illness: clozapine group } 9.1 \pm 4.2 \text { years, risperidone group } 8.6 \pm 3.4 \text { years, combina- } \\
\text { tion group } 8.9 \pm 3.7 \text { years. }\end{array}$ \\
\hline Interventions & $\begin{array}{l}\text { 1. Combination therapy: clozapine }(150 \mathrm{mg} \pm 72 \mathrm{mg} / \text { day })+\text { risperidone }(1.5 \mathrm{mg} \pm 1.3 \mathrm{mg} / \text { day })(\mathrm{N}=109) \text {. } \\
\text { 2. Monotherapy: clozapine }(375 \mathrm{mg} \pm 112 \mathrm{mg} / \text { day })(\mathrm{N}=106) \text {. } \\
\text { 3. Monotherapy: risperidone }(4.3 \mathrm{mg} \pm 1.2 \mathrm{mg} / \text { day })(\mathrm{N}=111) \text {. }\end{array}$ \\
\hline
\end{tabular}

\section{Outcomes}

\section{- Usable data -}

1. Clinical response: not clinically improved ${ }^{\star}$.

2. Mental state: PANSS*

3. Adverse events: drowsiness, white cell count*.

Notes $\quad{ }^{\star} \mathrm{N}$ not reported, assumed to be same as the number randomised.

\section{Risk of bias}

\begin{tabular}{lll}
\hline Bias & Authors' judgement & Support for judgement \\
\hline $\begin{array}{l}\text { Random sequence genera- } \\
\text { tion (selection bias) }\end{array}$ & Unclear risk & Randomised, no further information. \\
\hline $\begin{array}{l}\text { Allocation concealment } \\
\text { (selection bias) }\end{array}$ & Unclear risk & Not stated. \\
\hline $\begin{array}{l}\text { Blinding (performance } \\
\text { bias and detection bias) } \\
\text { All outcomes }\end{array}$ & Unclear risk & Not stated. \\
\hline $\begin{array}{l}\text { Incomplete outcome data } \\
\text { (attrition bias) } \\
\begin{array}{l}\text { All outcomes } \\
\hline\end{array}\end{array}$ & Low risk & No incomplete outcome data. \\
\hline
\end{tabular}


C +risperidone 2001 (Continued)

Selective reporting (re- Unclear risk $\quad$ All measured outcomes were reported. N not reported for outcomes. porting bias)

Other bias Low risk None obvious.

C +risperidone $2001 b$

\begin{tabular}{ll}
\hline Methods & Allocation: randomised. \\
& Blinding: double-blind. \\
& Duration: 8 weeks. \\
Setting: inpatients. \\
Design: parallel. \\
Country: China.
\end{tabular}

\begin{tabular}{ll}
\hline Participants & Diagnosis: schizophrenia. \\
& N = 101. \\
& Sex: $M$ and F (numbers not reported). \\
Age: $16-58$ years, average $~ 25$ years. \\
History: refractory, chronic.
\end{tabular}

1. Combination therapy: clozapine $(200 \mathrm{mg})+$ risperidone $(6 \mathrm{mg})(\mathrm{N}=32)$.
2. Monotherapy: clozapine $(\leq 600 \mathrm{mg})(\mathrm{N}=34)$.
3. Monotherapy: risperidone $(\leq 8 \mathrm{mg})(\mathrm{N}=35)$.

Schedule: daily dose.

Outcomes
- Usable data -
1. Clinical response: not clinically improved.
2. Adverse events: movement disorders.
3. Leaving the study early.

\section{- Unable to use -}

1. PANSS, CGI and TESS (not reported).

Notes *Abstract in English, report in Chinese.

\section{Risk of bias}

\begin{tabular}{lll}
\hline Bias & Authors' judgement & Support for judgement \\
\hline $\begin{array}{l}\text { Random sequence genera- } \\
\text { tion (selection bias) }\end{array}$ & Unclear risk & $\begin{array}{l}\text { Randomly divided into 3 groups (while claiming to be randomised - partici- } \\
\text { pants were matched on the type and stage of illness). }\end{array}$ \\
\hline $\begin{array}{l}\text { Allocation concealment } \\
\text { (selection bias) }\end{array}$ & Unclear risk & No information provided. \\
\hline $\begin{array}{l}\text { Blinding (performance } \\
\text { bias and detection bias) } \\
\text { All outcomes }\end{array}$ & Unclear risk & No information provided. \\
\hline $\begin{array}{l}\text { Incomplete outcome data } \\
\text { (attrition bias) } \\
\text { All outcomes }\end{array}$ & Low risk & All included patients completed the trial. \\
\hline
\end{tabular}


C +risperidone 2001b (Continued)

Selective reporting (re- High risk PANSS, CGI and TESS scores were measured, but not reported. porting bias)

Other bias Unclear risk Source of funding not reported.

\section{C +risperidone $2001 C$}

\begin{tabular}{|c|c|}
\hline Methods & $\begin{array}{l}\text { Allocation: randomised (no further information on randomisation method used). } \\
\text { Blinding: not stated. } \\
\text { Duration: } 12 \text { weeks. } \\
\text { Setting: inpatients. } \\
\text { Design: parallel. } \\
\text { Country: China. }\end{array}$ \\
\hline Participants & $\begin{array}{l}\text { Diagnosis: schizophrenia (CCMD-2-R). } \\
\mathbf{N}=96 . \\
\text { Age: } 18-60 \text { years. } \\
\text { Sex: male and female. } \\
\text { History: average length of illness, mean } \sim 4.5 \text { years, SD } ~ 3.5 \text { years. }\end{array}$ \\
\hline Interventions & $\begin{array}{l}\text { 1. Combination therapy: clozapine (average } \sim 100 \mathrm{mg} / \text { day) }+ \text { risperidone }(1 \mathrm{mg} \text { to } 4 \mathrm{mg} / \text { day) }(\mathrm{N}=32) \text {. } \\
\text { 2. Monotherapy: clozapine ( } 50 \mathrm{mg} \text { to } 400 \mathrm{mg} / \text { day) }(\mathrm{N}=32) \text {. } \\
\text { 3. Monotherapy: risperidone ( } 1 \mathrm{mg} \text { to } 6 \mathrm{mg} / \text { day) }(\mathrm{N}=32) \text {. }\end{array}$ \\
\hline
\end{tabular}

\section{Outcomes}

\section{- Usable data -}

1. Clinical response: not clinically improved.

2. Mental state: BPRS*.

\section{Notes \\ Risk of bias}

*N not reported, assumed to be the same as for the outcome 'not clinically improved'.

\begin{tabular}{lll}
\hline Bias & Authors' judgement & Support for judgement \\
\hline $\begin{array}{l}\text { Random sequence genera- } \\
\text { tion (selection bias) }\end{array}$ & Unclear risk & Randomised, no further information. \\
\hline $\begin{array}{l}\text { Allocation concealment } \\
\text { (selection bias) }\end{array}$ & Unclear risk & Not stated. \\
\hline $\begin{array}{l}\text { Blinding (performance } \\
\text { bias and detection bias) } \\
\text { All outcomes }\end{array}$ & Unclear risk & Not stated. \\
\hline $\begin{array}{l}\text { Incomplete outcome data } \\
\text { (attrition bias) }\end{array}$ & Low risk & No incomplete outcome data. \\
$\begin{array}{l}\text { All outcomes } \\
\text { Selective reporting (re- } \\
\text { porting bias) }\end{array}$ & Unclear risk & All measured outcomes were reported. N not reported for BPRS. \\
\hline \begin{tabular}{l} 
Other bias \\
\hline \hline
\end{tabular}
\end{tabular}




\begin{tabular}{|c|c|}
\hline Methods & $\begin{array}{l}\text { Allocation: randomised. } \\
\text { Blinding: double-blind. } \\
\text { Duration: } 6 \text { weeks. } \\
\text { Setting: } 6 \text { inpatients and } 24 \text { outpatients. } \\
\text { Design: parallel. } \\
\text { Country: Turkey. }\end{array}$ \\
\hline Participants & $\begin{array}{l}\text { Diagnosis: schizophrenia (DSM-IV). } \\
\text { N = 30. } \\
\text { Sex: M 20, F } 10 . \\
\text { Age: average } 33 \text { years. } \\
\text { History: received clozapine treatment ( } 300 \mathrm{mg} \text { to } 900 \mathrm{mg} / \mathrm{day} \text { ) for at least } 6 \text { months, had previously } \\
\text { failed to respond adequately, i.e. had persistent positive symptoms, to at least } 2 \text { trials of adequate du- } \\
\text { ration and dose of antipsychotic drugs other than clozapine. }\end{array}$ \\
\hline Interventions & $\begin{array}{l}\text { 1. Combination therapy: clozapine }(600 \mathrm{mg} \text { to } 900 \mathrm{mg} / \text { day })+\text { risperidone }(2 \mathrm{mg} \text { to } 6 \mathrm{mg} / \text { day })(\mathrm{N}=16) \text {. } \\
\text { 2. Monotherapy: clozapine }(600 \mathrm{mg} \text { to } 900 \mathrm{mg} / \text { day })+\text { placebo }(\mathrm{N}=14)^{\star} \text {. }\end{array}$ \\
\hline Outcomes & $\begin{array}{l}\text { - Usable data - } \\
\text { 1. Leaving the study early. } \\
\text { 2. Clinical response: not clinically improved. } \\
\text { 3. Mental state: no clinical improvement, PANSS. } \\
\text { 4. Quality of life: QLS. } \\
\text { 5. Adverse events: serious or requiring discontinuation, weight gain, extrapyramidal, change in prolactin } \\
\text { level, SAS. }\end{array}$ \\
\hline
\end{tabular}

\section{-Unable to use -}

1. Clinical response: CGI-S, GAF (Skewed data).

2. Adverse events: AIMS, BAS, UKU (Skewed data); white blood count (Not reported).

\section{- Not used in review -}

1. Calgary Depression Scale (CDS).

Notes

* Initially received 1 identical pill administered after the evening meal, increased to 2 after first week and then to 3 ( 1 after breakfast, 2 after evening meal) after second week.

\section{Risk of bias}

\begin{tabular}{lll}
\hline Bias & Authors' judgement & Support for judgement \\
\hline $\begin{array}{l}\text { Random sequence genera- } \\
\text { tion (selection bias) }\end{array}$ & Low risk & $\begin{array}{l}\text { Randomisation planned by unblinded investigators, a pre-assigned random } \\
\text { sequence was determined for each site. }\end{array}$ \\
\hline $\begin{array}{l}\text { Allocation concealment } \\
\text { (selection bias) }\end{array}$ & Low risk & Pre-assigned random sequence in order with their enrolment. \\
\hline $\begin{array}{l}\text { Blinding (performance } \\
\text { bias and detection bias) } \\
\text { All outcomes }\end{array}$ & Low risk & $\begin{array}{l}\text { Identical pills containing either risperidone or placebo were added to clozap- } \\
\text { ine. }\end{array}$ \\
\hline $\begin{array}{l}\text { Incomplete outcome data } \\
\text { (attrition bias) }\end{array}$ & High risk & $\begin{array}{l}\text { One patient from combination group did not complete the study. No further } \\
\text { information given. }\end{array}$ \\
\hline
\end{tabular}


C +risperidone 2005 (Continued)

Selective reporting (re- High risk Not all expected outcomes reported. porting bias)

Other bias

High risk

Not all patients suggested from sample size calculation were recruited. Protocol not available and Janssen Pharmaceuticals.

C +risperidone 2005b

$\begin{array}{ll}\text { Methods } & \text { Allocation: randomised. } \\ \text { Blinding: double-blind. } \\ \text { Duration: } 12 \text { weeks. } \\ \text { Setting: inpatients and outpatients. } \\ \text { Design: parallel } \\ \text { Country: USA. } \\ \text { Diagnosis: schizophrenia or schizoaffective disorder (DSM-IV). } \\ \text { N }=40 . \\ \text { Sex: M 35, F 5. } \\ \text { Age: average } 40 \text { years. } \\ \text { History: before treatment with clozapine, documented treatment failure after two antipsychotics ap- } \\ \text { proved by the U.S. Food and Drug Administration were administered for an adequate duration in a suf- } \\ \text { ficient dose (6 or more weeks of } 1000 \text { mg/day of chlorpromazine equivalents); demonstrated a doc- } \\ \text { umented failure to show a satisfactory clinical response to an adequate trial of clozapine (3 or more } \\ \text { months of at least } 600 \text { mg/day of oral clozapine or a plasma drug level of } 350 \text { ng/mL or higher); and had } \\ \text { persistent psychotic symptoms. }\end{array}$

$\begin{array}{ll}\text { Interventions } & \text { Combination therapy: clozapine + risperidone }(N=20) . \\ \text { 2. Monotherapy: clozapine + placebo }(N=20) .\end{array}$

Schedule: risperidone $1 \mathrm{mg}$ to $6 \mathrm{mg}$ /day, clozapine baseline doses established by treating psychiatrists and remained stable throughout the study.

- Usable data -
1. Leaving the study early.
2. Clinical response: not clinically improved.
3. Mental state: SANS, BPRS.
4. Adverse events: movement disorders.

\section{- Unable to use -}

1. Adverse events: SAS (no SDs reported, no suitable mean to impute data); white cell counts (Not reported).

\section{Notes}

\section{Risk of bias}

\begin{tabular}{lll}
\hline Bias & Authors' judgement & Support for judgement \\
\hline $\begin{array}{l}\text { Random sequence genera- } \\
\text { tion (selection bias) }\end{array}$ & Unclear risk & $\begin{array}{l}\text { Patients were randomly assigned in a 1:1 ratio to augmentation with risperi- } \\
\text { done or matching placebo. }\end{array}$ \\
\hline $\begin{array}{l}\text { Allocation concealment } \\
\text { (selection bias) }\end{array}$ & Low risk & $\begin{array}{l}\text { Each patient's study medication dose was managed by a non-blinded research } \\
\text { fellow not involved in any aspect of patient care who acted as intermediary be- } \\
\text { tween the study investigators, treating psychiatrist, and pharmacy. }\end{array}$ \\
\hline
\end{tabular}


C +risperidone 2005b (Continued)

Blinding (performance Low risk The raters, treating psychiatrist, and patient remained blinded throughout the bias and detection bias) study.

All outcomes
Incomplete outcome data Low risk All included patients completed the trial. (attrition bias)

All outcomes

Selective reporting (re- $\quad$ High risk $\quad$ Not all expected outcomes reported.
porting bias)

Other bias

High risk
This study was funded by Johnson \& Johnson Pharmaceutical Research \& Development.

\section{C +risperidone 2006}

$\begin{array}{ll}\text { Methods } & \text { Allocation: randomised. } \\ \text { Blinding: double-blind. } \\ \text { Duration: } 8 \text { weeks. } \\ \text { Setting: inpatients and outpatients. } \\ \text { Design: parallel. } \\ \text { Country: Canada, Germany, China, and the UK. }\end{array}$
Participants Diagnosis: schizophrenia or schizoaffective disorder (DSM-IV).
$\mathbf{N}=68$.
Sex: M 50, F 18.
Age: average 35 years.
History: treatment with clozapine for the indication of poor response to other antipsychotic agents; treatment for at least 12 weeks at a stable dose of $400 \mathrm{mg}$ or more per day, unless the size of the dose was limited by side effects.

Interventions

1. Combination therapy: clozapine + risperidone $(N=34)$.

2. Monotherapy: clozapine + placebo $(\mathrm{N}=34)$.

Schedule: risperidone $1 \mathrm{mg}$ to $3 \mathrm{mg}$ tablets/day, clozapine average $490 \mathrm{mg} /$ day.

- Usable data -
1. Leaving the study early.
2. Clinical response: not clinically improved, CGI-S.
3. Mental state: PANSS.
4. Adverse events: serious or requiring discontinuation, weight gain, ESRS, drowsiness, white cell counts.

\section{- Unable to use -}

1. Clinical response: $\mathrm{CGI}-\mathrm{I}$ (Not reported).

2. Adverse events: BAS (Skewed data).

\section{- Not used in review -}

1. Brown-Peterson procedure, Letter-Number Sequencing (LNS) task, fasting blood glucose, total cholesterol, low-density lipoprotein (LDL) cholesterol, high-density lipoprotein (HDL) cholesterol, and triglycerides. 
C +risperidone 2006 (Continued)

Protocol: ClinicalTrials.gov number NCT00272584.

\section{Risk of bias}

\begin{tabular}{lll}
\hline Bias & Authors' judgement & Support for judgement \\
\hline $\begin{array}{l}\text { Random sequence genera- } \\
\text { tion (selection bias) }\end{array}$ & Low risk & $\begin{array}{l}\text { Randomisation was performed according to a computer-generated schedule } \\
\text { with a permuted-block design. The fixed block size was four patients. }\end{array}$ \\
\hline $\begin{array}{l}\text { Allocation concealment } \\
\text { (selection bias) }\end{array}$ & Low risk & $\begin{array}{l}\text { The site investigators did not know the block size. The person generating the } \\
\text { randomisation schedule was not involved in determining the patients' eligibil- } \\
\text { ity, administering treatment, or determining outcome. The patients were as- } \\
\text { signed in sequence at each site. }\end{array}$
\end{tabular}

Blinding (performance Low risk

bias and detection bias)

Throughout the study, the patients, sight investigators, and raters remained

All outcomes blinded.

\begin{tabular}{lll}
\hline $\begin{array}{l}\text { Incomplete outcome data } \\
\text { (attrition bias) } \\
\text { All outcomes }\end{array}$ & Low risk & Primary analysis was performed according to the ITT principle. \\
\hline $\begin{array}{l}\text { Selective reporting (re- } \\
\text { porting bias) }\end{array}$ & High risk & Not all stated outcomes reported. \\
\hline Other bias & Unclear risk & $\begin{array}{l}\text { Supported by a grant from the Stanley Medical Research Institute. Risperidone } \\
\text { and placebo were provided by Janssen, Canada. }\end{array}$
\end{tabular}

\section{C +risperidone 2007}

$\begin{array}{ll}\text { Methods } & \text { Allocation: randomised. } \\ & \text { Blinding: double-blind. } \\ & \text { Duration: } 6 \text { weeks. } \\ \text { Setting: outpatients. } \\ \text { Design: parallel. } \\ \text { Country: USA. }\end{array}$
Participants Diagnosis: refractory schizophrenia (DSM-IV).
$\mathbf{N}=24$.
Sex: $21 \mathrm{M}, 3 \mathrm{~F}$.
Age: average 42.3 years (range $27-55$ ).
History: displayed stable, residual psychiatric symptoms; failed at least two previous trials of antipsy- chotics prior to clozapine and currently treated with clozapine monotherapy for at least 6 months, at a stable dose for at least 8 weeks and with clozapine plasma levels of at $200 \mathrm{ng} / \mathrm{mL}$, unless the clozapine dose necessary to achieve that level was not tolerated.

$\begin{array}{ll}\text { Interventions } & \text { 1. Combination therapy: clozapine + risperidone }(N=11) . \\ \text { 2. Monotherapy: clozapine + placebo }(N=13) .\end{array}$

Schedule: risperidone $4 \mathrm{mg} /$ day, clozapine $456 \mathrm{mg}$ /day average (200 mg to $700 \mathrm{mg} /$ day). Subjects received one capsule twice daily for three days, then two capsules twice daily for the rest of the study.

$\begin{array}{ll}\text { Outcomes } & \text { - Usable data - } \\ \text { 1. Leaving the study early. } \\ \text { 2. Clinical response: not clinically improved. }\end{array}$


3. Mental state: PANSS, SANS.

\section{- Unable to use -}

1. Adverse events: AIMS, SAS, BAS (Skewed data); prolactin level (no SD reported, not able to impute as no similar mean); Systematic Assessment for Treatment Emergent Events (SAFTEE; no data reported).

\section{- Not used in review -}

1. Calgary Depression Scale for Schizophrenia (CDSS).

Notes

*The active 6-week treatment period was preceded by a 2-week single-blind placebo lead-in period to eliminate potential placebo-responders.

ClinicalTrials.gov identifier NCT00289861.

\section{Risk of bias}

\begin{tabular}{lll}
\hline Bias & Authors' judgement & Support for judgement \\
\hline $\begin{array}{l}\text { Random sequence genera- } \\
\text { tion (selection bias) }\end{array}$ & Unclear risk & Participants randomised in blocks of 10. No further details. \\
\hline $\begin{array}{l}\text { Allocation concealment } \\
\text { (selection bias) }\end{array}$ & Unclear risk & No details provided. \\
\hline $\begin{array}{l}\text { Blinding (performance } \\
\text { bias and detection bias) } \\
\text { All outcomes }\end{array}$ & Low risk & $\begin{array}{l}\text { An independent research pharmacy prepared matching capsules that con- } \\
\text { tained either 1 mg risperidone or placebo. }\end{array}$ \\
\hline $\begin{array}{l}\text { Incomplete outcome data } \\
\text { (attrition bias) } \\
\text { All outcomes }\end{array}$ & High risk & $\begin{array}{l}\text { Twopatients in the placebo group and three patients in the risperidone group } \\
\text { did not finish the study. }\end{array}$ \\
\hline $\begin{array}{l}\text { Selective reporting (re- } \\
\text { porting bias) }\end{array}$ & High risk & Not all expected outcomes reported. \\
\hline \begin{tabular}{l} 
Other bias \\
\hline
\end{tabular} & Unclear risk & Trial supported by a grant from the Stanley Medical Research Institute. \\
\hline
\end{tabular}

C +sertindole 2006

$\begin{array}{ll}\text { Methods } & \text { Allocation: randomised. } \\ \text { Blinding: double-blind. } & \\ & \text { Duration: } 12 \text { weeks. } \\ \text { Setting: unclear. } \\ \text { Design: parallel. } \\ \text { Country: Denmark. } \\ \text { Dignosis: schizophrenia (ICD10, F20.0-3). } \\ \text { N }=50 . \\ \text { Sex: M 30, F 20. } \\ \text { Age: average 42 years. } \\ \text { History: clozapine treatment minimum } 6 \text { months, total PANSS > 65, no antipsychotic other than cloza- } \\ \text { pine drug } 1 \text { month prior. }\end{array}$




\section{- Usable data -}

1. Leaving the study early.

2. Service use: hospital admission.

3. Clinical response: GAF.

4. Mental state: PANSS.

5. Adverse events: serious or requiring discontinuation, death, weight gain, average weight gain, drowsiness, tremor.

\section{- Unable to use -}

1. Clinical response: $\mathrm{CGI-S} \mathrm{(reported} \mathrm{as} 0$ mean change ( $\mathrm{Cl} 0$ to 0$)$ for both treatment groups).

2. Clinical response: $\mathrm{CGI}-\mathrm{I}$ (reported as 0 mean change ( $\mathrm{Cl}-1$ to 0$)$ for both treatment groups).

3. Quality of life: World Health Organization Quality of Life Brief Questionnaire (QoL-BREF) (no overall scores reported)..

4. Adverse events: UKU side effects rating scale (no overall scores reported).

\section{- Not used in review -}

1. Cogntive tests, Drug Attitude Inventory, fasting glucose, lipids, Hb1Ac.

Notes ClinicalTrials.gov identifier NCT00345982.

\section{Risk of bias}

\begin{tabular}{lll}
\hline Bias & Authors' judgement & Support for judgement \\
\hline $\begin{array}{l}\text { Random sequence genera- } \\
\text { tion (selection bias) }\end{array}$ & Low risk & Computer-generated randomisation. \\
\hline $\begin{array}{l}\text { Allocation concealment } \\
\text { (selection bias) }\end{array}$ & Low risk & $\begin{array}{l}\text { Central allocation, "Randomization [...] was administered by the local phar- } \\
\text { macy that had no affiliation to the study". }\end{array}$ \\
\hline $\begin{array}{l}\text { Blinding (performance } \\
\text { bias and detection bias) } \\
\text { All outcomes }\end{array}$ & Low risk & $\begin{array}{l}\text { Participants, personnel and assessors were blinded, "Blinding was concealed } \\
\text { until the end of the 12-week visit, and disclosure of the blinding was first done } \\
\text { after reporting all PANSS values to the pharmacy to ensure that the prima- } \\
\text { ry outcome data could not be changed after unblinding". Blinded raters per- } \\
\text { formed all clinical research assessments. }\end{array}$
\end{tabular}

\begin{tabular}{|c|c|c|}
\hline $\begin{array}{l}\text { Incomplete outcome data } \\
\text { (attrition bias) }\end{array}$ & Low risk & $\begin{array}{l}\text { All analyses were performed as completers analyses and ITT analyses, using } \\
\text { the LOCF principle. }\end{array}$ \\
\hline
\end{tabular}

$$
\begin{aligned}
& \text { All analyses were performed as completers analyses and ITT analyses, using } \\
& \text { the LOCF principle. }
\end{aligned}
$$

All pre-specified outcomes were reported.

Selective reporting (re- Low risk $\quad$ All pre-specified outcomes were reported.
porting bias)

\begin{tabular}{|c|c|c|}
\hline Other bias & High risk & $\begin{array}{l}\text { H. Lundbeck supported the study with a study grant and study medication. } \\
\text { They had no influence on conduct of the study or preparation of the manu- } \\
\text { script. }\end{array}$ \\
\hline
\end{tabular}

\section{C +sulpiride 1997}

$\begin{array}{ll}\text { Methods } & \text { Allocation: randomised. } \\ \text { Blinding: double-blind. } \\ \text { Duration: } 10 \text { weeks. }\end{array}$


C +sulpiride 1997 (Continued)

Setting: not reported.

Design: parallel.

Country: Israel.

\begin{tabular}{ll}
\hline Participants & Diagnosis: schizophrenia (DSM-IV). \\
$\mathbf{N}=28$. \\
Sex: $\mathrm{M} 19, \mathrm{~F} 9$. \\
Age: average $~$ \\
History: failed to respond to at least three types of typical antipsychotics; partial and unsatisfactory \\
response to clozapine following at least 12 weeks of treatment in an adequate dose.
\end{tabular}

Interventions $\quad$ Combination therapy: clozapine + sulpiride $(N=16)$.

2. Monotherapy: clozapine + placebo $(\mathrm{N}=12)$.

Schedule: sulpiride was raised to $600 \mathrm{mg} /$ day by $100 \mathrm{mg} /$ day increments and thereafter remained constant for the rest of the study period. Clozapine dosage (range $400 \mathrm{mg}$ to $450 \mathrm{mg} /$ day) remained unchanged for the entire study.

- Usable data -
1. Leaving the study early.
2. Cinical response: not clinically improved.
3. Mental state: BPRS, SAPS, SANS.
4. Adverse events: serious or requiring discontinuation, change in prolactin level.

\section{- Unable to use -}

1. Adverse events: white blood cell counts (Not reported).

\section{- Not used in review -}

1. Hamilton Rating Scale for Depression (HAM-D).

\begin{tabular}{ll}
\hline Notes & The sulpiride or placebo was added for 10 consecutive weeks. All assigned participants finished the \\
study.
\end{tabular}

\section{Risk of bias}

\begin{tabular}{lll}
\hline Bias & Authors' judgement & Support for judgement \\
\hline $\begin{array}{l}\text { Random sequence genera- } \\
\text { tion (selection bias) }\end{array}$ & Low risk & $\begin{array}{l}\text { Participants were randomly allocated according to a table of random num- } \\
\text { bers. }\end{array}$ \\
\hline $\begin{array}{l}\text { Allocation concealment } \\
\text { (selection bias) }\end{array}$ & Unclear risk & No details provided. \\
\hline $\begin{array}{l}\text { Blinding (performance } \\
\text { bias and detection bias) }\end{array}$ & Low risk & $\begin{array}{l}\text { The placebo tablets were made to appear identical to the sulpiride tablets by } \\
\text { the manufacturer. }\end{array}$ \\
\hline $\begin{array}{l}\text { Incomplete outcome data } \\
\begin{array}{l}\text { (attrition bias) } \\
\text { All outcomes }\end{array}\end{array}$ & Low risk & All included patients completed the trial. \\
\hline $\begin{array}{l}\text { Selective reporting (re- } \\
\text { porting bias) }\end{array}$ & High risk & Not all expected outcomes reported. \\
\hline \begin{tabular}{l} 
Other bias \\
\hline
\end{tabular} & Unclear risk & Funding not reported. \\
\hline
\end{tabular}




\begin{tabular}{|c|c|}
\hline Methods & $\begin{array}{l}\text { Allocation: 'randomised' according to clinic admission order. } \\
\text { Blinding: not stated. } \\
\text { Duration: } 12 \text { weeks. } \\
\text { Setting: community patients. } \\
\text { Design: parallel. } \\
\text { Country: China. }\end{array}$ \\
\hline Participants & $\begin{array}{l}\text { Diagnosis: schizophrenia (CCMD-2-R). } \\
\mathbf{N}=150 . \\
\text { Sex: male and female. } \\
\text { Age: mean } 26 \text { years, SD } 7.78 \text { years. } \\
\text { History: length of illness: mean } 2.4 \text { years, SD } 1.3 \text { years.. }\end{array}$ \\
\hline Interventions & $\begin{array}{l}\text { 1. Combination therapy: sulpiride }(1390.2 \mathrm{mg} \pm 104.86 \mathrm{mg} / \text { day })+\text { clozapine }(25 \mathrm{mg} \text { to } 75 \mathrm{mg} / \text { day })(\mathrm{N} \\
=50) \text {. } \\
\text { 2. Monotherapy: clozapine }(486.17 \mathrm{mg} \pm 30.8 \mathrm{mg} / \text { day })(\mathrm{N}=50) \text {. } \\
\text { 3. Monotherapy: sulpiride }(1390.2 \mathrm{mg} \pm 104.86 \mathrm{mg} / \text { day })(\mathrm{N}=50) \text {. }\end{array}$ \\
\hline
\end{tabular}

Outcomes

\section{- Usable data -}

1. Clinical response: not clinically improved.

2. Mental state: BPRS.

3. Adverse events: white blood cell counts.

\section{- Unable to use -}

1. Adverse events: TESS (Skewed data).

\section{Notes}

\section{Risk of bias}

\begin{tabular}{|c|c|c|}
\hline Bias & Authors' judgement & Support for judgement \\
\hline $\begin{array}{l}\text { Random sequence genera- } \\
\text { tion (selection bias) }\end{array}$ & High risk & Randomised, according to clinic attendance sequence. \\
\hline $\begin{array}{l}\text { Allocation concealment } \\
\text { (selection bias) }\end{array}$ & Unclear risk & Not stated. \\
\hline $\begin{array}{l}\text { Blinding (performance } \\
\text { bias and detection bias) } \\
\text { All outcomes }\end{array}$ & Unclear risk & Not stated. \\
\hline $\begin{array}{l}\text { Incomplete outcome data } \\
\text { (attrition bias) } \\
\text { All outcomes }\end{array}$ & Low risk & No incomplete outcome data. \\
\hline $\begin{array}{l}\text { Selective reporting (re- } \\
\text { porting bias) }\end{array}$ & Low risk & All measured outcomes were reported. \\
\hline Other bias & Low risk & None obvious. \\
\hline
\end{tabular}




Allocation: randomised
Blinding: double-blind
Duration: 6 weeks.
Setting: inpatients
Design: parallel
Country: China

\begin{tabular}{ll}
\hline Participants & Diagnosis: schizophrenia (CCMD-2-R) \\
& N $=41$. \\
Age: $21-49$ years. \\
Sex: female only. \\
History: length of illness 3 months to 20 years, BPRS score greater than or equal to 38. \\
\hline Interventions \\
$\begin{array}{l}\text { 1. Combination therapy: clozapine }(350 \mathrm{mg} / \text { day }) \text {, sulpiride }(800 \mathrm{mg} / \text { day })(\mathrm{N}=20) . \\
\text { 2. Monotherapy: clozapine }(350 \mathrm{mg} / \text { day) }(\mathrm{N}=21) .\end{array}$
\end{tabular}

\begin{tabular}{ll}
\hline Outcomes & - Usable data - \\
1. Clinical response: not clinically improved. \\
2. Mental state: BPRS, SANS. \\
3. Adverse events: movement disorders, drowsiness.
\end{tabular}

\section{- Unable to use -}

1. Adverse events: TESS (Not reported).

\begin{tabular}{lll}
\hline Notes & \\
\hline Risk of bias & Authors' judgement & Support for judgement \\
\hline Bias & High risk & Randomised, according to hospital admission order. \\
\hline $\begin{array}{l}\text { Random sequence genera- } \\
\text { tion (selection bias) }\end{array}$ & Unclear risk & Not stated. \\
\hline $\begin{array}{l}\text { Allocation concealment } \\
\text { (selection bias) }\end{array}$ & Unclear risk & Double-blind. \\
\hline $\begin{array}{l}\text { Blinding (performance } \\
\text { bias and detection bias) } \\
\text { All outcomes }\end{array}$ & \\
\hline $\begin{array}{l}\text { Incomplete outcome data } \\
\begin{array}{l}\text { (attrition bias) } \\
\text { All outcomes }\end{array}\end{array}$ & Low risk & No incomplete outcome data. \\
\hline $\begin{array}{l}\text { Selective reporting (re- } \\
\text { porting bias) }\end{array}$ & High risk & TESS was measured, but not reported. \\
\hline \begin{tabular}{l} 
Other bias \\
\hline
\end{tabular} & Low risk & None obvious. \\
\hline
\end{tabular}


C +sulpiride 1999C

\begin{tabular}{|c|c|c|}
\hline Methods & \multicolumn{2}{|c|}{$\begin{array}{l}\text { Allocation: randomised. } \\
\text { Blinding: double-blind. } \\
\text { Duration: } 12 \text { weeks. } \\
\text { Setting: inpatients. } \\
\text { Design: parallel. } \\
\text { Country: China. }\end{array}$} \\
\hline Participants & \multicolumn{2}{|c|}{$\begin{array}{l}\text { Diagnosis: schizophrenia (mainly exhibiting negative symptoms). } \\
\mathbf{N}=88 \text {. } \\
\text { Sex: } M \text { and } F \text { (Not reported). } \\
\text { Age: average } \sim 35 \text { years. }\end{array}$} \\
\hline Interventions & \multicolumn{2}{|c|}{$\begin{array}{l}\text { 1. Combination therapy: clozapine } 50 \mathrm{mg} \text { to } 500 \mathrm{mg} \text { + chlorimipramine } 50 \mathrm{mg} \text { to } 150 \mathrm{mg}(\mathrm{N}=29) \text {. } \\
\text { 2. Combination therapy: clozapine }(50 \mathrm{mg} \text { to } 500 \mathrm{mg})+\text { sulpiride }(0.2 \mathrm{mg} \text { to } 1.0 \mathrm{mg})(\mathrm{N}=29) \text {. } \\
\text { 3. Monotherapy: clozapine (50mg to } 500 \mathrm{mg})(\mathrm{N}=30) \text {. } \\
\text { Schedule: daily dose. }\end{array}$} \\
\hline Outcomes & \multicolumn{2}{|c|}{$\begin{array}{l}\text { - Usable data - } \\
\text { 1. Leaving the study early. } \\
\text { 2. Clinical response: not clinically improved. } \\
\text { 3. Mental state: BPRS. } \\
\text { - Unable to use - } \\
\text { 1. Mental state: SANS (Skewed data). } \\
\text { 2. Adverse events: TESS (Skewed data). }\end{array}$} \\
\hline Notes & \multicolumn{2}{|c|}{ *Abstract in English, report in Chinese. } \\
\hline \multicolumn{3}{|l|}{ Risk of bias } \\
\hline Bias & Authors' judgement & Support for judgement \\
\hline $\begin{array}{l}\text { Random sequence genera- } \\
\text { tion (selection bias) }\end{array}$ & Unclear risk & Randomised, but randomisation method was not described. \\
\hline $\begin{array}{l}\text { Allocation concealment } \\
\text { (selection bias) }\end{array}$ & Unclear risk & No information provided. \\
\hline $\begin{array}{l}\text { Blinding (performance } \\
\text { bias and detection bias) } \\
\text { All outcomes }\end{array}$ & Unclear risk & No information provided. \\
\hline $\begin{array}{l}\text { Incomplete outcome data } \\
\text { (attrition bias) } \\
\text { All outcomes }\end{array}$ & Low risk & All included patients completed the trial. \\
\hline $\begin{array}{l}\text { Selective reporting (re- } \\
\text { porting bias) }\end{array}$ & High risk & Not all expected outcomes reported. \\
\hline Other bias & Unclear risk & Source of funding not reported. \\
\hline
\end{tabular}


C +sulpiride 2003

\begin{tabular}{|c|c|}
\hline Methods & $\begin{array}{l}\text { Allocation: randomised ('randomised' is only mentioned in abstract, not mentioned at all in th } \\
\text { text). } \\
\text { Blinding: not stated. } \\
\text { Duration: } 3 \text { months. } \\
\text { Setting: inpatients. } \\
\text { Design: parallel. } \\
\text { Country: China. }\end{array}$ \\
\hline Participants & $\begin{array}{l}\text { Diagnosis: schizophrenia (CCMD-2-R). } \\
\mathbf{N}=98 . \\
\text { Age: average age } ~ 32 \text { years. } \\
\text { Sex: male and female. } \\
\text { History: chronic treatment resistant. Length of illness: average } \sim 7 \text { years (range } 2 \text {-29 years). }\end{array}$ \\
\hline Interventions & $\begin{array}{l}\text { 1. Combination therapy: clozapine }+ \text { sulpiride; dosages are the monotherapy arms }(\mathrm{N}=30) \text {. } \\
\text { 2. Monotherapy: clozapine }(150 \mathrm{mg} \sim 300 \mathrm{mg} / \text { day })(\mathrm{N}=31) \text {. } \\
\text { 3. Monotherapy: sulpiride }(300 \mathrm{mg} \sim 600 \mathrm{mg} / \text { day })(\mathrm{N}=29) \text {. }\end{array}$ \\
\hline
\end{tabular}

\section{Outcomes}

\section{- Usable data -}

1. Clinical response: not clinically improved.

2. Mental state: BPRS, SANS.

3. Adverse events: movement disorders.

\section{- Unable to use -}

1. Mental state: SAPS (no SD, not able to impute data as no similar means).

\section{Notes}

\section{Risk of bias}

\begin{tabular}{|c|c|c|}
\hline Bias & Authors' judgement & Support for judgement \\
\hline $\begin{array}{l}\text { Random sequence genera- } \\
\text { tion (selection bias) }\end{array}$ & Unclear risk & Randomised, no further information. \\
\hline $\begin{array}{l}\text { Allocation concealment } \\
\text { (selection bias) }\end{array}$ & Unclear risk & Not stated. \\
\hline $\begin{array}{l}\text { Blinding (performance } \\
\text { bias and detection bias) } \\
\text { All outcomes }\end{array}$ & Unclear risk & Not stated. \\
\hline $\begin{array}{l}\text { Incomplete outcome data } \\
\text { (attrition bias) } \\
\text { All outcomes }\end{array}$ & Low risk & $\begin{array}{l}3 \text { cases were excluded from final analysis as they did not complete the } 3 \text { - } \\
\text { month treatment. } 4 \text { cases dropped out due to allergy to clozapine, } 1 \text { case } \\
\text { dropped out due to allergy to sulpiride. }\end{array}$ \\
\hline $\begin{array}{l}\text { Selective reporting (re- } \\
\text { porting bias) }\end{array}$ & Low risk & All measured outcomes were reported. \\
\hline Other bias & Low risk & None obvious. \\
\hline
\end{tabular}


C +sulpiride 2004

\begin{tabular}{ll}
\hline Methods & Allocation: randomised. \\
& Blinding: no. \\
& Duration: 8 weeks. \\
Setting: outpatients ("day patients"). & Design: parallel. \\
Country: Israel. \\
\hline
\end{tabular}

\begin{tabular}{ll}
\hline Participants & Diagnosis: schizophrenia (DSM-IV). \\
& N = 17. \\
& Sex: M 9, F 8. \\
& Age: average $~ 31$ years. \\
& History: chronic. Illnesses longer than 2 years duration.
\end{tabular}

Interventions 1. Combination therapy: olanzapine + sulpiride $(N=9)$.

2. Monotherapy: olanzapine $(\mathrm{N}=8)$.

Schedule: sulpiride $100 \mathrm{mg}$ to $600 \mathrm{mg} /$ day, olanzapine $20 \mathrm{mg}$ to $30 \mathrm{mg} /$ day.

\begin{tabular}{|c|c|c|}
\hline Outcomes & \multicolumn{2}{|c|}{$\begin{array}{l}\text { - Usable data - } \\
\text { 1. Leaving the study early. } \\
\text { 2. Mental state: PANSS. } \\
\text { - Unable to use - } \\
\text { 1. Adverse events: SAS, BAS (Skewed data). } \\
\text { - Not used in review - } \\
\text { 1. Hamilton Scale for Depression (HAM-D) and the Hamilton Scale for Anxiety (HAM-A). }\end{array}$} \\
\hline \multicolumn{3}{|l|}{ Notes } \\
\hline \multicolumn{3}{|l|}{ Risk of bias } \\
\hline Bias & Authors' judgement & Support for judgement \\
\hline $\begin{array}{l}\text { Random sequence genera- } \\
\text { tion (selection bias) }\end{array}$ & Unclear risk & No details provided. \\
\hline $\begin{array}{l}\text { Allocation concealment } \\
\text { (selection bias) }\end{array}$ & Unclear risk & No details provided. \\
\hline $\begin{array}{l}\text { Blinding (performance } \\
\text { bias and detection bias) } \\
\text { All outcomes }\end{array}$ & High risk & $\begin{array}{l}\text { Limitations for the study include the absence of a double-blind, placebo-con- } \\
\text { trolled study design, and non-blind rating. }\end{array}$ \\
\hline $\begin{array}{l}\text { Incomplete outcome data } \\
\text { (attrition bias) } \\
\text { All outcomes }\end{array}$ & High risk & $\begin{array}{l}\text { Only those } 16 \text { patients who completed the study were included in the final sta- } \\
\text { tistical analysis. }\end{array}$ \\
\hline $\begin{array}{l}\text { Selective reporting (re- } \\
\text { porting bias) }\end{array}$ & High risk & Not all expected outcomes reported. \\
\hline Other bias & Unclear risk & $\begin{array}{l}\text { This study was not supported by any funding. No protocol- a priori was pub- } \\
\text { lished. }\end{array}$ \\
\hline
\end{tabular}


C +sulpiride 2006

Methods
Blinding: not stated.
Duration: 8 weeks.
Setting: unclear if it's inpatients or community.
Design: parallel.
Country: China.

Diagnosis: schizophrenia (CCMD-III).
N $=64$.
Age: $16-60$ years.
Sex: male and female.
History: length of illness: $8.48 \pm 5.42$ years in combination group; $8.79 \pm 6.73$ years in clozapine group.

Interventions Combination therapy: clozapine (25 mg, twice a day) + sulpride (200-600 mg/d) $(\mathrm{N}=32)$.
2. Monotherapy: clozapine: $25 \mathrm{mg}$, twice a day ( $\mathrm{N}=32)$.

\section{Outcomes}

\section{- Usable data -}

1. Clinical response: not clinically improved.

2. Mental state: SANS.

3. Adverse events: weight gain.

\section{Notes}

\section{Risk of bias}

\begin{tabular}{|c|c|c|}
\hline Bias & Authors' judgement & Support for judgement \\
\hline $\begin{array}{l}\text { Random sequence genera- } \\
\text { tion (selection bias) }\end{array}$ & Unclear risk & Randomised, no further information. \\
\hline $\begin{array}{l}\text { Allocation concealment } \\
\text { (selection bias) }\end{array}$ & Unclear risk & Not stated. \\
\hline $\begin{array}{l}\text { Blinding (performance } \\
\text { bias and detection bias) } \\
\text { All outcomes }\end{array}$ & Unclear risk & Not stated. \\
\hline $\begin{array}{l}\text { Incomplete outcome data } \\
\text { (attrition bias) } \\
\text { All outcomes }\end{array}$ & Unclear risk & It is unclear if there are incomplete outcome data. \\
\hline $\begin{array}{l}\text { Selective reporting (re- } \\
\text { porting bias) }\end{array}$ & Low risk & All measured outcomes were reported. \\
\hline Other bias & Low risk & None obvious. \\
\hline
\end{tabular}

C +sulpiride 2013

$\begin{array}{ll}\text { Methods } & \text { Allocation: randomised. } \\ \text { Blinding: double-blind. }\end{array}$


C +sulpiride 2013 (Continued)

Duration: 6 weeks.

Setting: inpatient.

Design: parallel.

Country: Taiwan.

\begin{tabular}{ll}
\hline Participants & Diagnosis: schizophrenia (DSM IV). \\
$\mathbf{N}=96^{\star}$. & \\
Sex: $\mathrm{M} 54, \mathrm{~F} 38$. & Age: $38.6 \pm 9.0$. \\
History: Hospitalised schizophrenic patients with acute exacerbation, had a baseline Clinical Global \\
Impression-Severity of Illness Scale (CGI- S) of at least 4 . After a washout period of at least 3 days, pa- \\
tients were assigned randomly to either treatment for 6 weeks.
\end{tabular}

Interventions

1. Combination therapy: combination of low-dose amisulpride ( $400 \mathrm{mg} /$ day) plus low-dose sulpiride $(800 \mathrm{mg} /$ day). Drugs were administered orally in two divided doses $(\mathrm{N}=49)$.

2. Monotherapy: full-dose amisulpride $(800 \mathrm{mg} /$ day). Drugs were administered orally in two divided doses $(\mathrm{N}=47)$.

Outcomes

\section{- Usable data -}

1. Clinical Response: not clinically improved, CGI-S, GAF.

2. Leaving the study early.

3. Mental State: PANSS (Total, Positive, Negative).

4. Adverse events: serious event, AIMS, BAS, SAS, tremor, prolactin levels, weight gain (Binary and average kg).

5. Economic burden: drug cost.

6. Quality of life: Short Form-36 (mental component summary and physical component summary).

\section{- Unable to use -}

Adverse events: prolactin levels (Skewed data).

\section{- Not used in this review -}

1. Metabolic parameters, CDSS.

\begin{tabular}{|c|c|}
\hline \multirow{3}{*}{ Notes } & * 92 patients are included in the ITT analysis. \\
\hline & Unpublished data were provided by the investigator. \\
\hline & ClinicalTrials.gov identifier NCT01615185. \\
\hline
\end{tabular}

\section{Risk of bias}

\begin{tabular}{lll}
\hline Bias & Authors' judgement & Support for judgement \\
\hline $\begin{array}{l}\text { Random sequence genera- } \\
\text { tion (selection bias) }\end{array}$ & Low risk & $\begin{array}{l}\text { From correspondence: Randomisation was made by creating a list with soft- } \\
\text { ware. }\end{array}$ \\
\hline $\begin{array}{l}\text { Allocation concealment } \\
\text { (selection bias) }\end{array}$ & Unclear risk & No information provided. \\
\hline $\begin{array}{l}\text { Blinding (performance } \\
\text { bias and detection bias) } \\
\text { All outcomes }\end{array}$ & Unclear risk & "Double blind”. No other information provided. \\
\hline
\end{tabular}


C +sulpiride 2013 (Continued)

Incomplete outcome data High risk "Study completion rates were similar in the two groups: 31 (67.4\%) of 46 pa(attrition bias)

All outcomes

\section{tients in the antipsychotic combination group and 32 (69.6\%) of 46 patients in} the monotherapy group-"

Selective reporting (re- Low risk All pre-specified outcomes were reported. Protocol available.

porting bias)

\begin{tabular}{ll}
\hline Other bias & $\begin{array}{l}\text { Funded by the Kai-Syuan Psychiatric Hospital in 2011, the Food and Drug Ad- } \\
\text { ministration, Department of Health, Taiwan, the National Science Council, Tai- } \\
\text { wan, China Medical University Hospital, Taiwan and the Taiwan Department of } \\
\text { Health Clinical Trial and Research Center of Excellence. }\end{array}$
\end{tabular}

AIMS - Abnormal Involuntary Movement Scale.

ASEX - Arizona Sexual Experience Scale.

BACS - Brief Assessment of Cognition in Schizophrenia.

BAS - Barnes Akathisia Scale.

BMI - Body Mass Index.

BPRS - Brief Psychiatric Rating Scale.

CCMD - Chinese Classification of Mental Disorders.

CDSS - Calgary Depression Scale for Schizophrenia.

CGI-I - Clinical Global Impression - Improvement.

CGI-S - Clinical Global Impression - Severity.

$\mathrm{Cl}$ - confidence interval.

DAI - Drug Attitude Inventory.

DIEPSS - Drug-Induced Extrapyramidal Symptoms Scale.

DSM-IV - Diagnostic and Statistical Manual of Mental Disorders.

ECG - Electrocardiogram.

ECT- Electroconvulsive therapy.

ESRS - Extrapyramidal Symptom Rating Scale.

ESS - Epworth Sleepiness Scale.

FSI - Fatigue Symptom Inventory.

GAF - Global Assessment of Functioning Scale.

GEOPTE - Scale of social cognition for psychosis.

HAM-A - Hamilton Scale for Anxiety.

HAM-D - Hamilton Rating Scale for Depression.

HDL - high density lipoprotein.

HVA - plasma homovanillic acid.

IAQ - Investigator Assessment Questionnaire.

ICD - International Classification of Diseases.

IM - intramuscular.

ITT - intention-to-treat.

LOCF - last-observation-carried forward.

LDL - low-density lipoprotein.

MADRS - Montgomery-Åsberg Depression Rating Scale.

NIMH - National Institute of Mental Health.

NOSIE - Nurses Observation Scale for Inpatient Evaluation.

PANSS - Positive and Negative Syndrome Scale.

QLS - Quality of Life Scale.

QoL-BREF- Quality of Life Brief Questionnaire.

SAFTEE - Systematic Assessment for Treatment Emergent Events.

SAI -Schedule for Assessment of Insight.

SANS - Scale for the Assessment of Negative Symptoms.

SAPS - Scale for the Assessment of Positive Symptoms.

SAS - Simpson Angus Scale.

$\mathrm{SD}$ - standard deviation.

SF-36 - Short Form health survey.

SG - glucose effectiveness.

$\mathrm{SI}$ - insulin sensitivity index.

SLOF - Specific Level of Function scale. 
SMP- Slow moving proteinase a common gastric aid.

SWN - Subjective Well Being under Neuroleptics.

TESS - Treatment Emergent Symptom Scale

UKU - Udvalg for Kliniske Undersøgelser.

VLDL - very low-density lipoprotein.

WCST - Wisconsin Card Sorting Test.

YBOCS - Yale-Brown Obsessive Compulsive Scale.

Characteristics of excluded studies [ordered by study ID]

\begin{tabular}{ll}
\hline Study & Reason for exclusion \\
\hline Ahn 2002 & Allocation: randomised. \\
& Participants: adult patients with schizophrenia. \\
& Interventions: direct switching method vs start-tapering switching method. \\
\hline Alptekin 2012 & $\begin{array}{l}\text { Allocation: randomised. } \\
\text { Participants: adults with DSM-IV diagnosis of schizophrenia. } \\
\text { Interventions: slowly increasing the dose of sertindole with immediate discontinuation of the cur- } \\
\text { rent antipsychotic after randomisation vs slowly increasing the dose of sertindole with decreasing } \\
\text { the dose of the current antipsychotic. } \\
\text { Outcomes: Drop out, PANSS, EPS, metabolic parameters and Qtc prolongation. }\end{array}$ \\
\hline
\end{tabular}

Awad 2014

Allocation: randomised.

Participants: adults with clinically stable DSM-IV defined schizophrenia or schizoaffective disorder.

Interventions: lurasidone vs different doses of lurasidone.

Allocation: randomised.

Participants: people with schizophrenia.

Interventions: combination treatment clozapine plus aripiprazole vs combination treatment clozapine plus haloperidol.

Allocation: randomised.

Participants: adults with DSM-IV diagnosis of schizophrenia.

Interventions: switching to asenapine vs switching to olanzapine.

Outcomes: stability, PANSS, discontinuation, BMI and adverse events.

Allocation: randomised.

Participants: adults with schizophrenia.

Interventions: paliperidone extended-release tablets vs olanzapine.

\section{Citrome 2012}

\section{Allocation: randomised.}

Participants: outpatients with a DSM IV-TR diagnosis of schizophrenia.

Interventions: switch immediately to iloperidone or to gradually taper their prior antipsychotic dose over the first 2 weeks of iloperidone use.

Dai $2012 \quad$ Allocation: randomised.

Participants: adults with schizophrenia and hyperlipidaemia.

Interventions: antipsychotic medication and synthetical intervention vs antipsychotic medication.

Participants: schizophrenia. 


\begin{tabular}{|c|c|}
\hline Study & Reason for exclusion \\
\hline & Interventions: group therapy and risperidone vs risperidone. \\
\hline \multirow[t]{4}{*}{ DRKS00008018 } & Allocation: randomised. \\
\hline & Participants: patients with a first episode of schizophrenia according to DSM-V. \\
\hline & $\begin{array}{l}\text { Interventions: treatment with any antipsychotic drug for at least } 12 \text { months vs treatment with any } \\
\text { antipsychotic drug only for first episode of schizophrenia, tapering-off medication after remission } \\
\text { of positive symptoms. }\end{array}$ \\
\hline & Outcomes: Total grey matter volume, assessment of safety, drop out rates, PANSS, BACS, SF-36. \\
\hline
\end{tabular}

\section{Fang 2012}

Allocation: randomised.

Participants: people with schizophrenia.

Interventions: risperidone and clonazepam (a benzodiazepine, not an antipsychotic) vs haloperidol.

\section{Fleischhacker 2012}

Allocation: randomised.

Participants: subjects with schizophrenia requiring chronic treatment with an antipsychotic. Interventions: aripiprazole (400 mg/month) vs aripiprazole (50 mg/month) versus oral aripiprazole (10 $\mathrm{mg}$ to $30 \mathrm{mg} /$ day).

Outcomes: time to relapse and adverse events.

\section{Fleischhacker 2013}

Allocation: randomised.

Participants: adults with DSM-IV diagnosis of schizophrenia.

Interventions: aripiprazole once monthly IM depot vs placebo once monthly IM depot.

Outcomes: Leaving the study early, SAS, BAS.

\begin{abstract}
Goff 2008
Allocation: randomised.

Participants: people with schizophrenia.

Interventions: antipsychotic (clozapine, olanzapine or risperidone) plus add on therapy CX516 (AMPA-receptor-positive modulator, potential treatment for cognitive deficits in schizophrenia) vs antipsychotic plus placebo.
\end{abstract}

Allocation: randomised, cross-over.

Participants: people with schizophrenia.

Interventions: olanzapine plus aripiprazole vs olanzapine plus placebo.

Outcomes: PANSS, SANS, HDRS, GAF, Fatigue Scale Inventory, Quality of Life Scale, SAS, BAS, AIMS, and anthropomorphic, blood pressure and metabolic assessments.

No usable data: results not given for each phase of the trial separately.
Hwang 2015

Allocation: randomised.

Participants: diagnosis of schizophrenia or schizoaffective disorder according to the DSM IV. Interventions: fast tapering off the current medication within 1 week after initiating aripiprazole vs slow tapering off the current medication within 4 weeks after initiating aripiprazole.

\begin{tabular}{ll}
\hline JPRN-UMIN000011710 & The trial could not start and ended before patients were enrolled. \\
\hline JPRN-UMIN000012729 & $\begin{array}{l}\text { Allocation: randomised. } \\
\text { Participants: schizophrenia. } \\
\text { Interventions: blonaserin (atypical antipsychotic) vs aripiprazole. } \\
\text { Outcomes: PANSS, DAI-10, CGI. }\end{array}$ \\
\hline JPRN-UMIN000017047 & $\begin{array}{l}\text { Allocation: randomised. } \\
\text { Participants: adults with DSM-IV diagnosis of schizophrenia. } \\
\text { Interventions: blonanserin as add-on therapy vs olanzapine as add-on therapy. }\end{array}$ \\
\hline
\end{tabular}




\begin{tabular}{ll}
\hline Study & Reason for exclusion \\
\hline Kelly 2005 & Outcome: change in total score of the PANSS between baseline and endpoint (12 months). \\
\hline Participants: people with schizophrenia. \\
Interventions: divalproex extended release (DV-ER) and lithium vs DV-ER and quetiapine versus \\
DV-ER and placebo.
\end{tabular}

Allocation: not randomised.

Participants: people with schizophrenia.

Outcomes: nocturnal hypersalivation rating scale, PANSS, CGI, SAS, plasma prolactin levels.

No usable data: results not given for each phase of the trial separately.

\section{Kwon 2012}

Allocation: randomised.

Participants: adults with DSM-IV diagnosis of schizophrenia.

Interventions: sertindole vs olanzapine.

Outcomes: leaving the study early, PANSS, CGI, adverse events.

\section{Lerner 2004}

Li 2013

Allocation: not randomised.

Allocation: randomised.

Participants: adults with schizophrenia.

Interventions: liqi xingshen decoction vs risperidone.

\begin{abstract}
Lieberman 2009
Allocation: randomised.

Participants: people with schizophrenia.

Interventions: antipsychotic (quetiapine or risperidone) plus TC-5619 (partial agonist at the $\alpha 7$ subtype of the neural nicotinic acetylcholine receptors, potential treatment for cognitive dysfunction in schizophrenia) vs antipsychotic plus placebo.
\end{abstract}

Allocation: randomised.

Participants: patients aged 18-60 years DSM-IV-TR defined schizophrenia.

Interventions: cariprazine vs aripiprazole vs placebo.

Outcomes: Leaving the study early, PANSS, CGI-S.

\begin{tabular}{ll}
\hline Lundbeck 2004 & Allocation: randomised. \\
Participants: adults with DSM-IV diagnosis of schizophrenia. \\
Interventions: bifeprunox ( $30 \mathrm{mg}$ or $40 \mathrm{mg}$ once daily) vs risperidone (4 mg or $6 \mathrm{mg}$ once daily).
\end{tabular}

Lundbeck 2008

Allocation: randomised.

Participants: people with schizophrenia.

Interventions: risperidone and Lu AE58054 (selective 5-HT6 antagonist, potential treatment for cognitive deficits in schizophrenia) vs risperidone and placebo.
Allocation: randomised.

Participants: patients who presented with an acute agitation state requiring medication for rapid tranquillization.

Interventions: haloperidol and promethazine vs haloperidol and midazolam versus olanzapine vs ziprasidone. ziprasidone. 


\begin{tabular}{ll}
\hline Study & Reason for exclusion \\
\hline Intervention: paliperidone (6 mg, $9 \mathrm{mg}$, and $12 \mathrm{mg} / \mathrm{day})$ vs olanzapine (10 mg daily) vs placebo. \\
\hline Altzer 2012 & $\begin{array}{l}\text { Participants: patients who met DSM-IV criteria for schizophrenia, with a recent acute exacerba- } \\
\text { tion of psychotic symptoms. } \\
\text { Intervention: risperidone and pimavanserin (inverse agonist of the 5-HT2A receptor) vs haloperi- } \\
\text { dol and pimavanserin vs risperidone and placebo vs haloperidol and placebo. }\end{array}$ \\
\hline
\end{tabular}

\begin{tabular}{ll} 
Mi 2013 & Allocation: randomised. \\
& Participants: schizophrenia. \\
& Interventions: antipsychotic medication plus psychotherapy vs antipsychotic medication. \\
\hline Mir 2008 & Allocation: not randomised. \\
\hline Mythri 2013 & $\begin{array}{l}\text { Allocation: randomised. } \\
\text { Participants: acute agitation in patients with psychiatric disorders. }\end{array}$ \\
\hline NCT01003379 & $\begin{array}{l}\text { Allocation: randomised. } \\
\text { Participants: people with schizophrenia. } \\
\text { Interventions: antipsychotic (quetiapine or risperidone) and TC-5619 (partial agonist at the } \alpha 7 \\
\text { subtype of the neural nicotinic acetylcholine receptors, potential treatment for cognitive dysfunc- } \\
\text { tion in schizophrenia) vs antipsychotic and placebo. }\end{array}$
\end{tabular}

NCT01234779
Allocation: randomised.
Participants: people with schizophrenia.
$\begin{aligned} & \text { Intervention: bitopertin } 10 \mathrm{mg} / \text { day or } 30 \mathrm{mg} / \text { day (RO4917838 is glycine reuptake inhibitor) vs } \\ & \text { olanzapine } 15 \mathrm{mg} / \text { day vs placebo. }\end{aligned}$

NCT01939548

Participants: psychiatrically stable patients with schizophrenia who have had a suboptimal response to current treatment.

Interventions: PF-02545920 (PDE10 inhibitor) vs placebo.
NCT02477670 Participants: patients who meet DSM IV-TR diagnostic criteria for schizophrenia.
Interventions: AVP-786 (Deuterated [d6]-Dextromethorphan Hydrobromide [d6-DM]/Quinidine Sulfate, not an antipsychotic) vs placebo.

Pfizer $2009 \quad \begin{aligned} & \text { Allocation: randomised. } \\ & \text { Participants: people with schizophrenia. } \\ & \text { Interventions: antipsychotic (risperidone, olanzapine, quetiapine, ziprasidone, paliperidone, or } \\ & \text { aripiprazole) and PF-03463275 (glycine transporter } 1 \text { (GlyT1) inhibitor vs antipsychotic (risperi- } \\ & \text { done, olanzapine, quetiapine, ziprasidone, paliperidone, or aripiprazole) and placebo. }\end{aligned}$

\begin{tabular}{ll}
\hline Ruiz-Doblado 2010 & Allocation: narrative review. \\
\hline Rupnow 2005 & Allocation: randomised. \\
& $\begin{array}{l}\text { Participants: people with schizophrenia. } \\
\text { Interventions: in the 14-day monotherapy phase patients randomised to risperidone, quetiapine, } \\
\text { or placebo, followed by 28-day additive-therapy phase during which clinicians allowed to add psy- } \\
\text { chotropic medications. }\end{array}$ \\
\hline
\end{tabular}

Sacchetti $2006 \quad$ Allocation: randomised.

Participants: people with schizophrenia.. 


\begin{tabular}{|c|c|}
\hline Study & Reason for exclusion \\
\hline & Interventions: ziprasidone vs clozapine. \\
\hline Semenikhin 2013 & $\begin{array}{l}\text { Allocation: randomised } \\
\text { Participants: people with schizophrenia } \\
\text { Interventions: cortexin vs placebo. }\end{array}$ \\
\hline Sofronov 2013 & $\begin{array}{l}\text { Allocation: randomised. } \\
\text { Participants: patients with paranoid schizophrenia according to the criteria of ICD } 10 . \\
\text { Interventions: sertindole monotherapy vs paliperidone monotherapy vs fluvoxamine in combina- } \\
\text { tion with zuclopenthixole. }\end{array}$ \\
\hline
\end{tabular}

Allocation: pharmacokinetics study.
Participants: participants in this study were male and female patients between 18 and 65 years of
age, who were on a stable, oral, chronic (more than 2 months) antipsychotic medication.

Stahl 2010b

Allocation: post-hoc extension evaluating monotherapy after combination treatment.

Sukegawa 2008

Allocation: randomised.

Participants: adults with schizophrenia or related disorders.

Interventions: reduction of antipsychotics using the method of RAS vs continuation of polypharmacy.

Sukegawa 2014

Allocation: randomised.

Participants: were inpatients or outpatients diagnosed with schizophrenia according to the DSMIV-TR.

Interventions: safe correction of antipsychotic polypharmacy (SCAP) method versus the doses of antipsychotics were not changed for 3 months if clinically feasible.

UMIN000004931 Allocation: not randomised.

Wang $2013 \quad$ Allocation: randomised.

Participants: schizophrenia.

Interventions: antipsychotic medication plus acupuncture vs antipsychotic medication.

Weiden 2013

Allocation: randomised.

Participants: adults with schizophrenia or related disorders.

Interventions: gradual-switch from risperidone to iloperidone vs immediate switch to open-label iloperidone.

Outcomes: Integrated Clinical Global Impression of Change.

\begin{tabular}{ll}
\hline Wilson 1994 & Allocation: not randomised; cohort study. \\
\hline Winseck 2013 & $\begin{array}{l}\text { Allocation: randomised. } \\
\text { Participants: adults with schizophrenia or related disorders. } \\
\text { Interventions: gradual-switch from aripiprazole to iloperidone vs immediate switch to iloperi- } \\
\text { done. } \\
\text { Outcomes: Integrated Clinical Global Impression of Change. }\end{array}$ \\
\hline Wu 2002 & Allocation: not randomised. \\
\hline Wu 2015 & Allocation: randomised. \\
Participants: schizophrenia. \\
Interventions: aripiprazole combined with ziprasidone and comprehensive and systematic nurs- \\
ing vs aripiprazole combined with ziprasidone and routine nursing.
\end{tabular}




\begin{tabular}{ll}
\hline Study & Reason for exclusion \\
\hline
\end{tabular}

Allocation: randomised.

Participants: adults with DSM-IV diagnosis of schizophrenia.

Interventions: antipsychotics and wuji powder vs antipsychotics and aripiprazole.

Allocation: randomised.

Participants: people with schizophrenia.

Interventions: sertraline plus sulpiride vs risperidone.

$\begin{array}{ll}\text { Yamanouchi } 2015 & \text { Allocation: randomised. } \\ \text { Participants: adults with schizophrenia (DSM IV). } \\ \text { Interventions: safe correction of antipsychotic polypharmacy method vs stable dose of the cur- } \\ \text { rent antipsychotics. }\end{array}$

Allocation: not randomised.

Participants: adults with schizophrenia.

Interventions: shugan-jieyu capsule and aripiprazole vs aripiprazole.

Outcomes: clinical response, PANSS, adverse events.

Allocation: randomised.

Participants: adults with schizophrenia.

Interventions: venlafaxine with other antipsychotic vs antipsychotic medication.

Outcomes: SANS, BPRS, TESS.

Participants: people with schizophrenia.

Interventions: clozapine plus ziprasidone vs clozapine plus risperidone..

AIMS - Abnormal Involuntary Movement Scale

BACS - Brief Assessment of Cognition in Schizophrenia

BAS - Barnes Akathisia Scale

BMI - Body Mass Index

BPRS - Brief Psychiatric Rating Scale

CGI-S - Clinical Global Impression-Severity

DAI - Drug Attitude Inventory

DSM-IV - Diagnostic and Statistical Manual of Mental Disorders

EPS - extrapyramidal side effects

GAF - Global Assessment Scale of Functioning Scale

ICD - International Classification of Diseases

IM - intramuscular

PANSS - Positive and Negative Syndrome Scale

HDRS - Hamilton Depression Rating Scale

RAS - Reduction and Simplification

SANS - Scale for the Assessment of Negative Symptoms

SAS - Simpson Angus Scale

SF-36 - SF-form health survey

TESS - Treatment Emergent Symptom Scale 
Characteristics of studies awaiting assessment [ordered by study ID]

\section{NCT01450514}

Methods

Participants

Allocation: randomised. Blindness: double-blind.

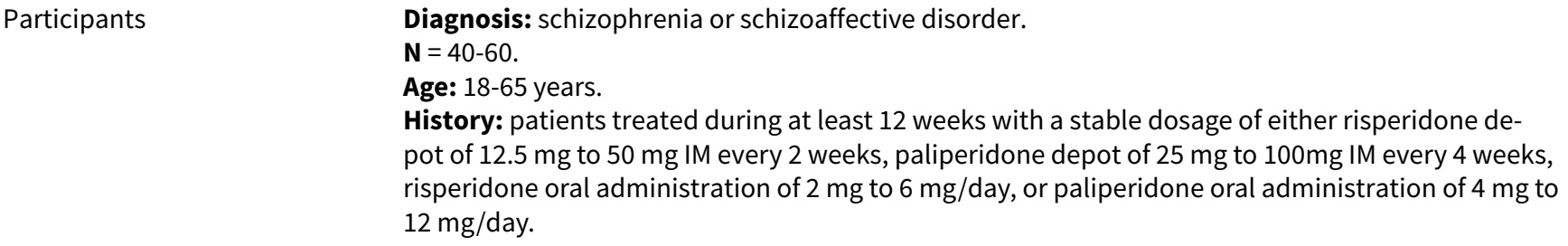

Interventions

1. Combination therapy: risperidone or paliperidone plus pipamperone ( $15 \mathrm{mg} / \mathrm{day})$.

2. Monotherapy: risperidone or paliperidone plus placebo.

\section{Outcomes}

1. Functional MRI tests.

2. Positive and Negative Syndrome Scale (PANSS) items.

3. Subjective Well-being under Neuroleptics questionnaire (SWN) score.

4. Intrinsic Motivation Inventory for Schizophrenia Research (IMI-SR) questionnaire.

5. Clinical Global Impression if Improvement (CGI-I) score.

6. Barnes Akathisia Rating Scale (BARS) total and sub-item scores.

7. Brief Assessment of Cognition Scale (BACS) score and sub-item scores.

\section{Notes}

We contacted the principal investigator by e-mail to obtain data from this study, this clinical trial was carried out but the sponsor decided not to release the data as they wanted to keep them confidential.

Sponsor: PharmaNeuroBoost N.V.

ClinicalTrials.gov identifier: NCT01450514.

\section{Xu 2006}

Methods Allocation: randomised.

Blindness: single-blind.

Participants Diagnosis: schizophrenia (CCMD-3).

$\mathbf{N}=60$.

Age: average $25 \pm 6$ years.

Sex: Female.

Interventions

1. Combination therapy: risperidone or sulpiride plus aripiprazole $5 \mathrm{mg} / \mathrm{day}$.

2. Monotherapy: risperidone or sulpiride plus placebo (vitamin C $100 \mathrm{mg} / \mathrm{day}$ ).

Outcomes Leaving the study early.
2. Mental state: BPRS.
3. Prolactin levels.
4. Adverse events.


Xu 2006 (Continued)

Notes Vitamin C might have an effect on the symptoms of schizophrenia.

Yuan 2014

\begin{tabular}{ll}
\hline Methods & $\begin{array}{l}\text { Allocation: randomised. } \\
\text { Blindness: double-blind. }\end{array}$ \\
\hline Participants & Diagnosis: first episode schizophrenia (DSM-IV). \\
\hline Interventions & Intervention: \\
1. Stage $1:$ Risperidone (3 $\mathrm{mg}$ to $6 \mathrm{mg} /$ day), aripiprazole (15 $\mathrm{mg}$ to $30 \mathrm{mg} /$ day) or olanzapine (10 $\mathrm{mg}$ \\
to $25 \mathrm{mg} /$ day). \\
$\begin{array}{l}\text { 2. Stage 2: Change the current antipsychotic to one of the other two study drugs. } \\
\text { 3. Stage 3: pharmacotherapy could be switched to any other atypical antipsychotic including long- } \\
\text { acting medications. Furthermore, a combination with other antipsychotics or a certain adjunctive } \\
\text { antidepressant was allowed for patients who still could not reach satisfactory outcome after the } \\
\text { previous } 2 \text { stages. }\end{array}$
\end{tabular}

If the treatment failed as judged by the investigators and/or the patient, the patients entered the next stage of the trial.
Outcomes
1. Clinical response: CGI-S and CGI-I and $<50 \%$ reduction on the total score of the PANSS.
2. Mental state: PANSS.
3. Adverse events: UKU side-effect scale.
4. Treatment adherence: DAI.
5. Social functions: Personal and Social Performance scale.
6. Cognitive performance.

BACS - Brief Assessment of Cognition in Schizophrenia.

BAS - Barnes Akathisia Scale.

BPRS - Brief Psychiatric Rating Scale.

CCMD - Chinese Classification of Mental Disorders.

CGI-I - Clinical Global Impression - Improvement.

CGI-S - Clinical Global Impression - Severity.

DAI - Drug Attitude Inventory.

DSM - Diagnostic and Statistical Manual of Mental Disorders.

IM - intramuscular.

IMI-SR - Intrinsic Motivation Inventory for Schizophrenia Research.

MRI - magnetic resonance imaging.

PANSS - Positive and Negative Syndrome Scale.

SWN - Subjective Well Being under Neuroleptics.

UKU - Udvalg for Kliniske Undersøgelser.

\section{Characteristics of ongoing studies [ordered by study ID]}

CTRI-02-003397

Trial name or title

Randomised, double-blind, placebo-controlled trial to evaluate the effects of adjunctive treatment with aripiprazole on body weight, metabolic parameters, clinical efficacy, and adverse events in people with psychotic disorders on treatment with Clozapine. 
CTRI-02-003397 (Continued)

Methods
Allocation: randomised.
Duration: 16 weeks.
Country: India.

\begin{tabular}{|c|c|}
\hline Participants & $\begin{array}{l}\text { Diagnosis: schizophrenia and schizoaffective (ICD-10). } \\
\text { Target sample size: } 60 . \\
\text { History: Participants on a stable dose of clozapine (100 mg to } 900 \mathrm{mg}) \text {, with residual positive or } \\
\text { negative psychotic or psychiatric symptoms with a minimum score on the Brief Psychiatric Rating } \\
\text { Scale (BPRS) > 31, experiencing troublesome weight gain, and are overweight or obese. } \\
\text { Age: } 18 \text { years or above. }\end{array}$ \\
\hline Interventions & $\begin{array}{l}\text { 1. Combination therapy: clozapine + aripiprazole ( } 10 \mathrm{mg} \text { to } 20 \mathrm{mg} / \mathrm{day}) \text {. } \\
\text { 2. Monotherapy: clozapine + placebo.. }\end{array}$ \\
\hline Outcomes & $\begin{array}{l}\text { 1. Clinical response: not clinical improved, and CGI (Efficacy index score). } \\
\text { 2. Mental state: BPRS. } \\
\text { 3. Adverse events: weight gain, SAS, sedation, hypersalivation. } \\
\text { 4. Others: BMI, fasting sugars, triglycerides, lipids from baseline, compliance with diet, compliance } \\
\text { with exercise. }\end{array}$ \\
\hline
\end{tabular}

\begin{tabular}{ll}
\hline Starting date & First enrolment on February 2013. \\
\hline Contact information & Prince Rajamanickam (princer@cmcvellore.ac.in). \\
\hline Notes & Clinical trial registry India ID: CTRI/2013/02/003397. \\
& Sponsor: Christian Medical College. \\
\hline
\end{tabular}

\section{ISRCTN68824876}

\section{Methods}

Allocation: randomised.

Blindness: double-blind.

Duration: 12 months.

\begin{tabular}{ll}
\hline Participants & Diagnosis: schizophrenia. \\
Target sample size: 230. \\
History: persistent symptom severity despite adequate \\
( $400 \mathrm{mg}$ or more of clozapine/day), total score of 80 or \\
SOFAS score of 40 or less. \\
Age: $18-65$ years. \\
\hline Interventions & 2. Combination therapy: amisulpiride + clozapine. \\
& 2. Monotherapy: placebo + clozapine.
\end{tabular}


ISRCTN68824876 (Continued)

Outcomes

1. Clinical response: not clinical improved (20\% reduction in total PANSS score).

2. Mental state: PANSS negative symptom sub-scale score, CDSS.

3. Functioning: SOFAS.

4. Service use: Service engagement scale.

5. Adverse effects: Antipsychotic side effect measures.

6. Quality of life: Euroqol EQ-5D.

7. Economic: resource use data questionnaire.

\begin{tabular}{ll}
\hline Starting date & September 2010 \\
\hline Contact information & Prof. Thomas Barnes (t.r.barnes@imperial.ac.uk) \\
& Centre for Mental Health \\
& Imperial College \\
& Charing Cross Campus \\
& St Dunstan's Road \\
& London \\
& W6 8RP \\
\hline Notes & ISRCTN trial registration ID: ISRCTN68824876 \\
& Sponsor: Imperial College London (UK) \\
\hline
\end{tabular}

Schmidt-Kraepelin 2013

Trial name or title Is an antipsychotic combination treatment of olanzapine and amisulpiride more effective than
monotherapy.

\begin{tabular}{ll}
\hline Methods & $\begin{array}{l}\text { Allocation: randomised. } \\
\text { Blindness: double-blind. }\end{array}$ \\
\hline Participants & $\begin{array}{l}\text { Diagnosis: schizophrenia or schizoaffective disorder. } \\
\text { Expected sample size: } 399 . \\
\text { Age: } 18-65 \text { years. } \\
\text { History: no details. }\end{array}$ \\
\hline Interventions & $\begin{array}{l}\text { 1. Combination therapy: olanzapine and amisulpiride. } \\
\text { 2. Monotherapy: olanzapine. } \\
\text { 3. Monotherapy: amisulpiride. }\end{array}$ \\
\hline Outcomes & $\begin{array}{l}\text { 1. Symptomatic improvement of schizophrenia in comparison to time of inclusion of patient mea- } \\
\text { sured by PANSS. }\end{array}$ \\
$\begin{array}{l}\text { 2. Serious adverse drug reactions. } \\
\text { 3. Change of clinical condition measured by CGI scale. } \\
\text { 4. Change of the subjective well-being measured by SWN scale. }\end{array}$ \\
\hline Starting date & June 2012. \\
\hline Contact information & Joachim Cordes (joachim.cordes@lvr.de). \\
Sandra Feyerabend (sandra.feyerabend@lvr.de).
\end{tabular}


Schmidt-Kraepelin 2013 (Continued)

Notes

ClinicalTrials.gov identifier: NCT01609153.

DRKS-ID: DRKS00003603.

Sponsor: Heinrich-Heine University, Duesseldor.

BPRS - Brief Psychiatric Rating Scale

BMI - Body Mass Index

CGI - Clinical Global Impression

CDSS - Calgary Depression Scale for Schizophrenia

ICD - International Classification of Diseases

PANSS - Positive and Negative Syndrome Scale

SAS - Simpson Angus Scale

SOFAS - Social and Occupational Functioning Assessment Scale

SWN - Subjective Well Being under Neuroleptics

DATA AND ANALYSES

COmparison 1. ANTIPSYCHOTIC COMBINATIONS vS ANTIPSYCHOTIC MONOTHERAPY

\begin{tabular}{|c|c|c|c|c|}
\hline Outcome or subgroup title & No. of studies & $\begin{array}{l}\text { No. of partici- } \\
\text { pants }\end{array}$ & Statistical method & Effect size \\
\hline $\begin{array}{l}1 \text { Clinical response: } 1 \text {. No clinically im- } \\
\text { portant response - not improved }\end{array}$ & 29 & 2398 & $\begin{array}{l}\text { Risk Ratio (M-H, Random, } \\
95 \% \mathrm{Cl})\end{array}$ & $0.73[0.64,0.83]$ \\
\hline 1.1 clozapine in both groups & 17 & 1127 & $\begin{array}{l}\text { Risk Ratio (M-H, Random, } \\
95 \% \mathrm{Cl} \text { ) }\end{array}$ & $0.66[0.53,0.83]$ \\
\hline 1.2 other atypical in both groups & 7 & 674 & $\begin{array}{l}\text { Risk Ratio (M-H, Random, } \\
95 \% \mathrm{Cl} \text { ) }\end{array}$ & $0.95[0.83,1.09]$ \\
\hline 1.3 typical drugs in both groups & 5 & 597 & $\begin{array}{l}\text { Risk Ratio (M-H, Random, } \\
95 \% \mathrm{Cl})\end{array}$ & $0.64[0.49,0.84]$ \\
\hline 2 Clinical response: 2 . Relapse & 3 & 512 & $\begin{array}{l}\text { Risk Ratio (M-H, Random, } \\
95 \% \mathrm{Cl})\end{array}$ & $0.63[0.31,1.29]$ \\
\hline 2.1 clozapine in both groups & 1 & 70 & $\begin{array}{l}\text { Risk Ratio (M-H, Random, } \\
95 \% \mathrm{Cl})\end{array}$ & $0.85[0.54,1.33]$ \\
\hline 2.2 typical drugs in both groups & 2 & 442 & $\begin{array}{l}\text { Risk Ratio (M-H, Random, } \\
95 \% \mathrm{Cl})\end{array}$ & $0.54[0.16,1.81]$ \\
\hline 3 Leaving the study early & 43 & 3137 & $\begin{array}{l}\text { Risk Ratio (M-H, Random, } \\
95 \% \mathrm{Cl} \text { ) }\end{array}$ & $0.90[0.76,1.07]$ \\
\hline 3.1 clozapine in both groups & 18 & 932 & $\begin{array}{l}\text { Risk Ratio (M-H, Random, } \\
95 \% \mathrm{Cl} \text { ) }\end{array}$ & $1.28[0.88,1.86]$ \\
\hline 3.2 other atypical drugs in both groups & 15 & 1247 & $\begin{array}{l}\text { Risk Ratio (M-H, Random, } \\
95 \% \mathrm{Cl} \text { ) }\end{array}$ & $1.03[0.83,1.27]$ \\
\hline 3.3 typical drugs in both groups & 6 & 628 & $\begin{array}{l}\text { Risk Ratio (M-H, Random, } \\
95 \% \mathrm{Cl})\end{array}$ & $0.69[0.43,1.12]$ \\
\hline
\end{tabular}




\begin{tabular}{|c|c|c|c|c|}
\hline Outcome or subgroup title & No. of studies & $\begin{array}{l}\text { No. of partici- } \\
\text { pants }\end{array}$ & Statistical method & Effect size \\
\hline 3.4 any antipsychotics in both groups & 4 & 330 & $\begin{array}{l}\text { Risk Ratio (M-H, Random, } \\
95 \% \mathrm{Cl} \text { ) }\end{array}$ & $0.61[0.30,1.25]$ \\
\hline 4 Service utilisation: Hospital admission & 3 & 202 & $\begin{array}{l}\text { Risk Ratio (M-H, Random, } \\
95 \% \mathrm{Cl})\end{array}$ & $0.96[0.36,2.55]$ \\
\hline 4.1 clozapine in both groups & 2 & 88 & $\begin{array}{l}\text { Risk Ratio (M-H, Random, } \\
95 \% \mathrm{Cl})\end{array}$ & $2.85[0.31,26.41]$ \\
\hline 4.2 any antipsychotics in both groups & 1 & 114 & $\begin{array}{l}\text { Risk Ratio (M-H, Random, } \\
95 \% \mathrm{Cl})\end{array}$ & $0.74[0.25,2.19]$ \\
\hline $\begin{array}{l}5 \text { Clinical response: } 3 \text {. Global state }- \text { i. av- } \\
\text { erage severity score (CGI-S scale, high = } \\
\text { bad) }\end{array}$ & 7 & 496 & $\begin{array}{l}\text { Mean Difference (IV, Ran- } \\
\text { dom, 95\% CI) }\end{array}$ & $-0.13[-0.31,0.06]$ \\
\hline 5.1 clozapine in both groups & 3 & 179 & $\begin{array}{l}\text { Mean Difference (IV, Ran- } \\
\text { dom, } 95 \% \mathrm{CI} \text { ) }\end{array}$ & $-0.04[-0.55,0.47]$ \\
\hline 5.2 other atypical drugs in both groups & 3 & 263 & $\begin{array}{l}\text { Mean Difference (IV, Ran- } \\
\text { dom, 95\% CI) }\end{array}$ & $-0.16[-0.37,0.05]$ \\
\hline 5.3 typical drugs in both groups & 1 & 54 & $\begin{array}{l}\text { Mean Difference (IV, Ran- } \\
\text { dom, } 95 \% \mathrm{CI} \text { ) }\end{array}$ & $-0.10[-0.42,0.22]$ \\
\hline $\begin{array}{l}6 \text { Clinical response: } 3 \text {. Global state - ii. } \\
\text { change in severity score (CGI-S scale, } \\
\text { high = bad) }\end{array}$ & 3 & 233 & $\begin{array}{l}\text { Mean Difference (IV, Ran- } \\
\text { dom, } 95 \% \mathrm{CI} \text { ) }\end{array}$ & $0.11[-0.09,0.32]$ \\
\hline 6.1 clozapine in both groups & 1 & 53 & $\begin{array}{l}\text { Mean Difference (IV, Ran- } \\
\text { dom, } 95 \% \mathrm{CI} \text { ) }\end{array}$ & $0.21[-0.09,0.51]$ \\
\hline 6.2 other atypical drugs in both groups & 2 & 180 & $\begin{array}{l}\text { Mean Difference (IV, Ran- } \\
\text { dom, } 95 \% \mathrm{CI} \text { ) }\end{array}$ & $0.03[-0.24,0.31]$ \\
\hline $\begin{array}{l}7 \text { Clinical response: } 4 \text {. Global state - av- } \\
\text { erage improvement score (CGI-I scale, } \\
\text { high = bad) }\end{array}$ & 4 & 336 & $\begin{array}{l}\text { Mean Difference (IV, Ran- } \\
\text { dom, } 95 \% \mathrm{CI} \text { ) }\end{array}$ & $\begin{array}{l}-0.36[-0.58 \\
-0.13]\end{array}$ \\
\hline 7.1 clozapine in both groups & 2 & 259 & $\begin{array}{l}\text { Mean Difference (IV, Ran- } \\
\text { dom, } 95 \% \mathrm{CI} \text { ) }\end{array}$ & $\begin{array}{l}-0.33[-0.56 \\
-0.10]\end{array}$ \\
\hline 7.2 other atypical drugs in both groups & 2 & 77 & $\begin{array}{l}\text { Mean Difference (IV, Ran- } \\
\text { dom, } 95 \% \mathrm{CI} \text { ) }\end{array}$ & $-0.62[-1.34,0.10]$ \\
\hline $\begin{array}{l}8 \text { Clinical response: } 5 \text {. Global state }- \text { i. av- } \\
\text { erage functioning score (GAF scale, high } \\
=\text { good) }\end{array}$ & 3 & & $\begin{array}{l}\text { Mean Difference (IV, Ran- } \\
\text { dom, } 95 \% \mathrm{CI} \text { ) }\end{array}$ & Subtotals only \\
\hline 8.1 Clozapine in both groups & 1 & 30 & $\begin{array}{l}\text { Mean Difference (IV, Ran- } \\
\text { dom, 95\% CI) }\end{array}$ & $-4.5[-8.38,-0.62]$ \\
\hline 8.2 Other atypical drugs in both groups & 2 & 77 & $\begin{array}{l}\text { Mean Difference (IV, Ran- } \\
\text { dom, } 95 \% \mathrm{CI} \text { ) }\end{array}$ & $8.73[1.56,15.90]$ \\
\hline
\end{tabular}




\begin{tabular}{|c|c|c|c|c|}
\hline Outcome or subgroup title & No. of studies & $\begin{array}{l}\text { No. of partici- } \\
\text { pants }\end{array}$ & Statistical method & Effect size \\
\hline $\begin{array}{l}9 \text { Clinical response: } 5 \text {. Global state - ii. } \\
\text { change in functioning score (GAF scale, } \\
\text { high = good) }\end{array}$ & 3 & 349 & $\begin{array}{l}\text { Mean Difference (IV, Ran- } \\
\text { dom, } 95 \% \mathrm{CI} \text { ) }\end{array}$ & $0.27[-1.42,1.97]$ \\
\hline 9.1 Clozapine in both groups & 2 & 257 & $\begin{array}{l}\text { Mean Difference (IV, Ran- } \\
\text { dom, } 95 \% \mathrm{CI} \text { ) }\end{array}$ & $0.27[-1.56,2.10]$ \\
\hline 9.2 Other atypical drugs in both groups & 1 & 92 & $\begin{array}{l}\text { Mean Difference (IV, Ran- } \\
\text { dom, } 95 \% \mathrm{CI} \text { ) }\end{array}$ & $0.30[-4.21,4.81]$ \\
\hline $\begin{array}{l}10 \text { Mental state: } 1 . \text { Overall - a.i average } \\
\text { total score (PANSS scale, high = bad) }\end{array}$ & 11 & & $\begin{array}{l}\text { Mean Difference (IV, Ran- } \\
\text { dom, } 95 \% \mathrm{CI} \text { ) }\end{array}$ & $\begin{array}{l}\text { Totals not select- } \\
\text { ed }\end{array}$ \\
\hline 10.1 Clozapine in both groups & 6 & & $\begin{array}{l}\text { Mean Difference (IV, Ran- } \\
\text { dom, } 95 \% \mathrm{CI} \text { ) }\end{array}$ & $0.0[0.0,0.0]$ \\
\hline 10.2 Other atypical drugs in both groups & 4 & & $\begin{array}{l}\text { Mean Difference (IV, Ran- } \\
\text { dom, } 95 \% \mathrm{CI} \text { ) }\end{array}$ & $0.0[0.0,0.0]$ \\
\hline 10.3 Any antipsychotic in both groups & 1 & & $\begin{array}{l}\text { Mean Difference (IV, Ran- } \\
\text { dom, } 95 \% \mathrm{Cl} \text { ) }\end{array}$ & $0.0[0.0,0.0]$ \\
\hline $\begin{array}{l}11 \text { Mental state: } 1 . \text { Overall - a.ii change in } \\
\text { total score (PANSS scale, high = bad) }\end{array}$ & 8 & 406 & $\begin{array}{l}\text { Mean Difference (IV, Ran- } \\
\text { dom, } 95 \% \mathrm{CI} \text { ) }\end{array}$ & $-1.05[-3.42,1.32]$ \\
\hline 11.1 Clozapine in both groups & 3 & 133 & $\begin{array}{l}\text { Mean Difference (IV, Ran- } \\
\text { dom, } 95 \% \mathrm{CI} \text { ) }\end{array}$ & $-0.55[-3.54,2.43]$ \\
\hline 11.2 Other atypical drugs in both groups & 5 & 273 & $\begin{array}{l}\text { Mean Difference (IV, Ran- } \\
\text { dom, } 95 \% \mathrm{CI} \text { ) }\end{array}$ & $-2.26[-6.71,2.18]$ \\
\hline $\begin{array}{l}12 \text { Mental state: } 1 . \text { Overall - b.i. average } \\
\text { total score (BPRS scale, high = bad) }\end{array}$ & 21 & 1082 & $\begin{array}{l}\text { Mean Difference (IV, Ran- } \\
\text { dom, } 95 \% \mathrm{CI} \text { ) }\end{array}$ & $\begin{array}{l}-4.15[-6.17 \\
-2.13]\end{array}$ \\
\hline 12.1 clozapine in both groups & 16 & 820 & $\begin{array}{l}\text { Mean Difference (IV, Ran- } \\
\text { dom, } 95 \% \mathrm{CI} \text { ) }\end{array}$ & $\begin{array}{l}-5.19[-7.08, \\
-3.30]\end{array}$ \\
\hline 12.2 other atypical drugs in both groups & 2 & 68 & $\begin{array}{l}\text { Mean Difference (IV, Ran- } \\
\text { dom, } 95 \% \mathrm{CI} \text { ) }\end{array}$ & $\begin{array}{l}-3.09[-13.26 \\
7.08]\end{array}$ \\
\hline 12.3 typical drugs in both groups & 3 & 194 & $\begin{array}{l}\text { Mean Difference (IV, Ran- } \\
\text { dom, } 95 \% \mathrm{CI} \text { ) }\end{array}$ & $0.06[-1.36,1.48]$ \\
\hline $\begin{array}{l}13 \text { Mental state: } 1 \text {. Overall - b.ii change } \\
\text { total score (BPRS scale, high = bad) }\end{array}$ & 1 & 100 & $\begin{array}{l}\text { Mean Difference (IV, Ran- } \\
\text { dom, } 95 \% \mathrm{CI} \text { ) }\end{array}$ & $\begin{array}{l}-2.72[-5.37 \\
-0.07]\end{array}$ \\
\hline 13.1 clozapine in both groups & 1 & 100 & $\begin{array}{l}\text { Mean Difference (IV, Ran- } \\
\text { dom, } 95 \% \mathrm{Cl} \text { ) }\end{array}$ & $\begin{array}{l}-2.72[-5.37 \\
-0.07]\end{array}$ \\
\hline $\begin{array}{l}14 \text { Mental state: } 2 \text {. Specific - a. positive } \\
\text { symptoms - no clinical improvement }\end{array}$ & 2 & & $\begin{array}{l}\text { Risk Ratio (M-H, Random, } \\
95 \% \mathrm{Cl})\end{array}$ & $\begin{array}{l}\text { Totals not select- } \\
\text { ed }\end{array}$ \\
\hline 14.1 clozapine in both groups & 2 & & $\begin{array}{l}\text { Risk Ratio (M-H, Random, } \\
95 \% \mathrm{Cl})\end{array}$ & $0.0[0.0,0.0]$ \\
\hline
\end{tabular}




\begin{tabular}{|c|c|c|c|c|}
\hline Outcome or subgroup title & No. of studies & $\begin{array}{l}\text { No. of partici- } \\
\text { pants }\end{array}$ & Statistical method & Effect size \\
\hline $\begin{array}{l}15 \text { Mental state: } 2 \text {. Specific - b. positive } \\
\text { symptoms - i. average score (PANSS } \\
\text { scale, high = bad) }\end{array}$ & 4 & 158 & $\begin{array}{l}\text { Mean Difference (IV, Ran- } \\
\text { dom, } 95 \% \mathrm{CI} \text { ) }\end{array}$ & $2.02[0.90,3.14]$ \\
\hline 15.1 clozapine in both groups & 3 & 119 & $\begin{array}{l}\text { Mean Difference (IV, Ran- } \\
\text { dom, } 95 \% \mathrm{CI} \text { ) }\end{array}$ & $2.19[0.92,3.45]$ \\
\hline 15.2 any antipsychotics in both groups & 1 & 39 & $\begin{array}{l}\text { Mean Difference (IV, Ran- } \\
\text { dom, } 95 \% \mathrm{CI} \text { ) }\end{array}$ & $1.40[-1.03,3.83]$ \\
\hline $\begin{array}{l}16 \text { Mental state: } 2 \text {. Specific - b. positive } \\
\text { symptoms - ii. change score (PANSS } \\
\text { scale, high = bad) }\end{array}$ & 9 & 891 & $\begin{array}{l}\text { Mean Difference (IV, Ran- } \\
\text { dom, } 95 \% \mathrm{CI} \text { ) }\end{array}$ & $0.01[-0.45,0.47]$ \\
\hline 16.1 clozapine in both groups & 3 & 308 & $\begin{array}{l}\text { Mean Difference (IV, Ran- } \\
\text { dom, } 95 \% \mathrm{CI} \text { ) }\end{array}$ & $-0.39[-1.08,0.29]$ \\
\hline 16.2 other atypical drugs in both groups & 6 & 583 & $\begin{array}{l}\text { Mean Difference (IV, Ran- } \\
\text { dom, } 95 \% \mathrm{CI} \text { ) }\end{array}$ & $0.33[-0.28,0.95]$ \\
\hline $\begin{array}{l}17 \text { Mental state: } 2 \text {. Specific - b. positive } \\
\text { symptoms - iii. average score (BPRS } \\
\text { scale, high = bad) }\end{array}$ & 3 & 133 & $\begin{array}{l}\text { Mean Difference (IV, Ran- } \\
\text { dom, } 95 \% \mathrm{CI} \text { ) }\end{array}$ & $-1.02[-2.42,0.38]$ \\
\hline 17.1 clozapine in both groups & 2 & 93 & $\begin{array}{l}\text { Mean Difference (IV, Ran- } \\
\text { dom, } 95 \% \mathrm{CI} \text { ) }\end{array}$ & $\begin{array}{l}-1.66[-3.32 \\
-0.00]\end{array}$ \\
\hline 17.2 other atypical drugs in both groups & 1 & 40 & $\begin{array}{l}\text { Mean Difference (IV, Ran- } \\
\text { dom, } 95 \% \mathrm{CI} \text { ) }\end{array}$ & $-0.07[-1.61,1.47]$ \\
\hline $\begin{array}{l}18 \text { Mental state: } 2 \text {. Specific - b. positive } \\
\text { symptoms - iv. change data (BPRS scale, } \\
\text { high = bad) }\end{array}$ & 1 & 17 & $\begin{array}{l}\text { Mean Difference (IV, Ran- } \\
\text { dom, } 95 \% \mathrm{CI} \text { ) }\end{array}$ & $-0.30[-1.16,0.56]$ \\
\hline 18.1 typical drugs in both groups & 1 & 17 & $\begin{array}{l}\text { Mean Difference (IV, Ran- } \\
\text { dom, } 95 \% \mathrm{CI} \text { ) }\end{array}$ & $-0.30[-1.16,0.56]$ \\
\hline $\begin{array}{l}19 \text { Mental state: } 2 \text {. Specific - b. posi- } \\
\text { tive symptoms - v. average score (SAPS } \\
\text { scale, high = bad) }\end{array}$ & 1 & 28 & $\begin{array}{l}\text { Mean Difference (IV, Ran- } \\
\text { dom, } 95 \% \mathrm{CI} \text { ) }\end{array}$ & $\begin{array}{l}-6.76[-11.91 \\
-1.61]\end{array}$ \\
\hline 19.1 other atypical drugs in both groups & 1 & 28 & $\begin{array}{l}\text { Mean Difference (IV, Ran- } \\
\text { dom, } 95 \% \mathrm{CI} \text { ) }\end{array}$ & $\begin{array}{l}-6.76[-11.91 \\
-1.61]\end{array}$ \\
\hline $\begin{array}{l}20 \text { Mental state: } 2 \text {. Specific - b. posi- } \\
\text { tive symptoms - vi. change score (SAPS } \\
\text { scale, high = bad) }\end{array}$ & 1 & 28 & $\begin{array}{l}\text { Mean Difference (IV, Ran- } \\
\text { dom, } 95 \% \mathrm{CI} \text { ) }\end{array}$ & $\begin{array}{l}-5.80[-11.33 \\
-0.27]\end{array}$ \\
\hline 20.1 clozapine in both groups & 1 & 28 & $\begin{array}{l}\text { Mean Difference (IV, Ran- } \\
\text { dom, } 95 \% \mathrm{CI} \text { ) }\end{array}$ & $\begin{array}{l}-5.80[-11.33 \\
-0.27]\end{array}$ \\
\hline $\begin{array}{l}21 \text { Mental state: } 3 \text {. Specific - a. negative } \\
\text { symptoms - no clinical improvement }\end{array}$ & 3 & & $\begin{array}{l}\text { Risk Ratio (M-H, Random, } \\
95 \% \mathrm{Cl})\end{array}$ & $\begin{array}{l}\text { Totals not select- } \\
\text { ed }\end{array}$ \\
\hline
\end{tabular}




\begin{tabular}{|c|c|c|c|c|}
\hline Outcome or subgroup title & No. of studies & $\begin{array}{l}\text { No. of partici- } \\
\text { pants }\end{array}$ & Statistical method & Effect size \\
\hline 21.1 clozapine in both groups & 2 & & $\begin{array}{l}\text { Risk Ratio (M-H, Random, } \\
95 \% \mathrm{Cl})\end{array}$ & $0.0[0.0,0.0]$ \\
\hline 21.2 typical drugs in both groups & 1 & & $\begin{array}{l}\text { Risk Ratio (M-H, Random, } \\
95 \% \mathrm{Cl})\end{array}$ & $0.0[0.0,0.0]$ \\
\hline $\begin{array}{l}22 \text { Mental state: } 3 \text {. Specific - b. nega- } \\
\text { tive symptoms - i. average score (PANSS } \\
\text { scale, high = bad) }\end{array}$ & 5 & 194 & $\begin{array}{l}\text { Mean Difference (IV, Ran- } \\
\text { dom, } 95 \% \mathrm{CI} \text { ) }\end{array}$ & $1.16[-0.49,2.80]$ \\
\hline 22.1 clozapine in both groups & 3 & 119 & $\begin{array}{l}\text { Mean Difference (IV, Ran- } \\
\text { dom, } 95 \% \mathrm{CI} \text { ) }\end{array}$ & $0.31[-1.18,1.80]$ \\
\hline 22.2 other atypical drugs in both groups & 1 & 36 & $\begin{array}{l}\text { Mean Difference (IV, Ran- } \\
\text { dom, } 95 \% \mathrm{CI} \text { ) }\end{array}$ & $1.20[-1.51,3.91]$ \\
\hline 22.3 Any antipsychotic in both groups & 1 & 39 & $\begin{array}{l}\text { Mean Difference (IV, Ran- } \\
\text { dom, } 95 \% \mathrm{CI} \text { ) }\end{array}$ & $3.30[1.60,5.00]$ \\
\hline $\begin{array}{l}23 \text { Mental state: } 3 \text {. Specific - b. nega- } \\
\text { tive symptoms - ii. change score (PANSS } \\
\text { scale, high = bad) }\end{array}$ & 9 & 891 & $\begin{array}{l}\text { Mean Difference (IV, Ran- } \\
\text { dom, } 95 \% \mathrm{CI} \text { ) }\end{array}$ & $0.02[-0.54,0.58]$ \\
\hline 23.1 clozapine in both groups & 3 & 308 & $\begin{array}{l}\text { Mean Difference (IV, Ran- } \\
\text { dom, } 95 \% \mathrm{CI} \text { ) }\end{array}$ & $0.31[-1.11,1.74]$ \\
\hline 23.2 other atypical drugs in both groups & 6 & 583 & $\begin{array}{l}\text { Mean Difference (IV, Ran- } \\
\text { dom, } 95 \% \mathrm{CI} \text { ) }\end{array}$ & $0.01[-0.69,0.72]$ \\
\hline $\begin{array}{l}24 \text { Mental state: } 3 \text {. Specific - b. nega- } \\
\text { tive symptoms - iii. average score (BPRS } \\
\text { scale, high = bad) }\end{array}$ & 2 & & $\begin{array}{l}\text { Mean Difference (IV, Ran- } \\
\text { dom, } 95 \% \mathrm{CI} \text { ) }\end{array}$ & Subtotals only \\
\hline 24.1 clozapine in both groups & 1 & 61 & $\begin{array}{l}\text { Mean Difference (IV, Ran- } \\
\text { dom, } 95 \% \mathrm{CI} \text { ) }\end{array}$ & $\begin{array}{l}-4.30[-12.25 \\
3.65]\end{array}$ \\
\hline 24.2 other atypical drugs in both groups & 1 & 40 & $\begin{array}{l}\text { Mean Difference (IV, Ran- } \\
\text { dom, } 95 \% \mathrm{CI} \text { ) }\end{array}$ & $1.90[0.69,3.11]$ \\
\hline $\begin{array}{l}25 \text { Mental state: } 3 \text {. Specific - b. nega- } \\
\text { tive symptoms - iv. change score (BPRS } \\
\text { scale, high = bad) }\end{array}$ & 1 & & $\begin{array}{l}\text { Mean Difference (IV, Ran- } \\
\text { dom, } 95 \% \mathrm{CI} \text { ) }\end{array}$ & Subtotals only \\
\hline 25.1 typical drugs in both groups & 1 & 12 & $\begin{array}{l}\text { Mean Difference (IV, Ran- } \\
\text { dom, } 95 \% \mathrm{CI} \text { ) }\end{array}$ & $0.2[-0.29,0.69]$ \\
\hline $\begin{array}{l}26 \text { Mental state: } 3 \text {. Specific - b. nega- } \\
\text { tive symptoms - v. average score (SANS } \\
\text { scale, high = bad) }\end{array}$ & 11 & & $\begin{array}{l}\text { Mean Difference (IV, Ran- } \\
\text { dom, } 95 \% \mathrm{CI} \text { ) }\end{array}$ & $\begin{array}{l}\text { Totals not select- } \\
\text { ed }\end{array}$ \\
\hline 26.1 clozapine in both groups & 9 & & $\begin{array}{l}\text { Mean Difference (IV, Ran- } \\
\text { dom, } 95 \% \mathrm{CI} \text { ) }\end{array}$ & $0.0[0.0,0.0]$ \\
\hline 26.2 other atypical drugs in both groups & 1 & & $\begin{array}{l}\text { Mean Difference (IV, Ran- } \\
\text { dom, } 95 \% \mathrm{CI} \text { ) }\end{array}$ & $0.0[0.0,0.0]$ \\
\hline
\end{tabular}




\begin{tabular}{|c|c|c|c|c|}
\hline Outcome or subgroup title & No. of studies & $\begin{array}{l}\text { No. of partici- } \\
\text { pants }\end{array}$ & Statistical method & Effect size \\
\hline 26.3 typical drugs in both groups & 1 & & $\begin{array}{l}\text { Mean Difference (IV, Ran- } \\
\text { dom, } 95 \% \mathrm{CI} \text { ) }\end{array}$ & $0.0[0.0,0.0]$ \\
\hline $\begin{array}{l}27 \text { Mental state: } 3 \text {. Specific - b. nega- } \\
\text { tive symptoms - vi. average score (SANS } \\
\text { scale, high = bad) }\end{array}$ & 1 & 28 & $\begin{array}{l}\text { Mean Difference (IV, Ran- } \\
\text { dom, } 95 \% \mathrm{Cl} \text { ) }\end{array}$ & $\begin{array}{l}-6.80[-12.65 \\
-0.95]\end{array}$ \\
\hline 27.1 clozapine in both groups & 1 & 28 & $\begin{array}{l}\text { Mean Difference (IV, Ran- } \\
\text { dom, } 95 \% \mathrm{CI} \text { ) }\end{array}$ & $\begin{array}{l}-6.80[-12.65 \\
-0.95]\end{array}$ \\
\hline $\begin{array}{l}28 \text { Mental state: } 4 \text {. Specific - aggres- } \\
\text { sion/agitation - average score (BPRS } \\
\text { scale, high = bad) }\end{array}$ & 1 & & $\begin{array}{l}\text { Mean Difference (IV, Ran- } \\
\text { dom, } 95 \% \mathrm{Cl} \text { ) }\end{array}$ & Subtotals only \\
\hline 28.1 typical drugs in both groups & 1 & 12 & $\begin{array}{l}\text { Mean Difference (IV, Ran- } \\
\text { dom, } 95 \% \mathrm{CI} \text { ) }\end{array}$ & $-1.3[-2.32,-0.28]$ \\
\hline $\begin{array}{l}29 \text { Adverse events: } 1 \text {. General - a. serious } \\
\text { event or requiring discontinuation }\end{array}$ & 30 & 2398 & $\begin{array}{l}\text { Risk Ratio (M-H, Random, } \\
95 \% \mathrm{Cl})\end{array}$ & $1.05[0.65,1.69]$ \\
\hline 29.1 clozapine in both groups & 14 & 766 & $\begin{array}{l}\text { Risk Ratio (M-H, Random, } \\
95 \% \mathrm{Cl})\end{array}$ & $1.83[0.82,4.08]$ \\
\hline 29.2 other atypical in both groups & 10 & 1016 & $\begin{array}{l}\text { Risk Ratio (M-H, Random, } \\
95 \% \mathrm{Cl})\end{array}$ & $0.65[0.37,1.14]$ \\
\hline 29.3 typical drugs in both groups & 3 & 403 & $\begin{array}{l}\text { Risk Ratio (M-H, Random, } \\
95 \% \mathrm{Cl})\end{array}$ & $0.45[0.09,2.30]$ \\
\hline 29.4 any antipsychotics in both groups & 3 & 213 & $\begin{array}{l}\text { Risk Ratio (M-H, Random, } \\
95 \% \mathrm{Cl})\end{array}$ & $2.84[0.79,10.29]$ \\
\hline $\begin{array}{l}30 \text { Adverse events: } 1 \text {. General - b. death } \\
\text { (suicide or non-suicide deaths) }\end{array}$ & 4 & 897 & $\begin{array}{l}\text { Risk Ratio (M-H, Random, } \\
95 \% \mathrm{Cl})\end{array}$ & $3.0[0.13,70.30]$ \\
\hline 30.1 clozapine in both groups & 2 & 257 & $\begin{array}{l}\text { Risk Ratio (M-H, Random, } \\
95 \% \mathrm{Cl})\end{array}$ & $3.0[0.13,70.30]$ \\
\hline 30.2 other atypical in both groups & 1 & 323 & $\begin{array}{l}\text { Risk Ratio (M-H, Random, } \\
95 \% \mathrm{Cl})\end{array}$ & $0.0[0.0,0.0]$ \\
\hline 30.3 typical drugs in both groups & 1 & 317 & $\begin{array}{l}\text { Risk Ratio (M-H, Random, } \\
95 \% \mathrm{Cl})\end{array}$ & $0.0[0.0,0.0]$ \\
\hline $\begin{array}{l}31 \text { Adverse events: } 2 \text {. Movement disor- } \\
\text { ders - a. any }\end{array}$ & 20 & 1868 & $\begin{array}{l}\text { Risk Ratio (M-H, Random, } \\
95 \% \mathrm{Cl})\end{array}$ & $1.07[0.92,1.25]$ \\
\hline 31.1 clozapine in both groups & 8 & 545 & $\begin{array}{l}\text { Risk Ratio (M-H, Random, } \\
95 \% \mathrm{Cl})\end{array}$ & $1.53[0.69,3.38]$ \\
\hline 31.2 other atypical in both groups & 9 & 846 & $\begin{array}{l}\text { Risk Ratio (M-H, Random, } \\
95 \% \mathrm{Cl})\end{array}$ & $0.97[0.80,1.18]$ \\
\hline 31.3 typical drugs in both groups & 2 & 363 & $\begin{array}{l}\text { Risk Ratio (M-H, Random, } \\
95 \% \mathrm{Cl})\end{array}$ & $1.30[0.82,2.05]$ \\
\hline
\end{tabular}




\begin{tabular}{|c|c|c|c|c|}
\hline Outcome or subgroup title & No. of studies & $\begin{array}{l}\text { No. of partici- } \\
\text { pants }\end{array}$ & Statistical method & Effect size \\
\hline 31.4 any antipsychotics in both groups & 1 & 114 & $\begin{array}{l}\text { Risk Ratio (M-H, Random, } \\
95 \% \mathrm{Cl})\end{array}$ & $0.99[0.60,1.61]$ \\
\hline $\begin{array}{l}32 \text { Adverse events: } 2 \text {. Movement disor- } \\
\text { ders - b.i. average scores (SAS, high = } \\
\text { bad) }\end{array}$ & 2 & & $\begin{array}{l}\text { Mean Difference (IV, Ran- } \\
\text { dom, } 95 \% \mathrm{CI} \text { ) }\end{array}$ & $\begin{array}{l}\text { Totals not select- } \\
\text { ed }\end{array}$ \\
\hline 32.1 clozapine in both groups & 1 & & $\begin{array}{l}\text { Mean Difference (IV, Ran- } \\
\text { dom, } 95 \% \mathrm{CI} \text { ) }\end{array}$ & $0.0[0.0,0.0]$ \\
\hline 32.2 other atypical drugs in both groups & 1 & & $\begin{array}{l}\text { Mean Difference (IV, Ran- } \\
\text { dom, } 95 \% \mathrm{Cl} \text { ) }\end{array}$ & $0.0[0.0,0.0]$ \\
\hline $\begin{array}{l}33 \text { Adverse events: } 2 \text {. Movement disor- } \\
\text { ders - b.ii. change scores (SAS, high = } \\
\text { bad) }\end{array}$ & 1 & & $\begin{array}{l}\text { Mean Difference (IV, Ran- } \\
\text { dom, 95\% CI) }\end{array}$ & $\begin{array}{l}\text { Totals not select- } \\
\text { ed }\end{array}$ \\
\hline 33.1 other atypical drugs in both groups & 1 & & $\begin{array}{l}\text { Mean Difference (IV, Ran- } \\
\text { dom, } 95 \% \mathrm{CI} \text { ) }\end{array}$ & $0.0[0.0,0.0]$ \\
\hline $\begin{array}{l}34 \text { Adverse events: } 2 \text {. Movement disor- } \\
\text { ders - b.iii. average scores (TESS, high = } \\
\text { bad) }\end{array}$ & 2 & & $\begin{array}{l}\text { Mean Difference (IV, Ran- } \\
\text { dom, } 95 \% \mathrm{CI} \text { ) }\end{array}$ & $\begin{array}{l}\text { Totals not select- } \\
\text { ed }\end{array}$ \\
\hline 34.1 clozapine in both groups & 1 & & $\begin{array}{l}\text { Mean Difference (IV, Ran- } \\
\text { dom, } 95 \% \mathrm{CI} \text { ) }\end{array}$ & $0.0[0.0,0.0]$ \\
\hline 34.2 other atypical drugs in both groups & 1 & & $\begin{array}{l}\text { Mean Difference (IV, Ran- } \\
\text { dom, } 95 \% \mathrm{CI} \text { ) }\end{array}$ & $0.0[0.0,0.0]$ \\
\hline $\begin{array}{l}35 \text { Adverse events: } 2 \text {. Movement disor- } \\
\text { ders - b.iv. average scores (DIEPSS, high } \\
=\text { bad) }\end{array}$ & 1 & 61 & $\begin{array}{l}\text { Mean Difference (IV, Ran- } \\
\text { dom, } 95 \% \mathrm{CI} \text { ) }\end{array}$ & $0.30[-0.49,1.09]$ \\
\hline 35.1 clozapine in both groups & 1 & 61 & $\begin{array}{l}\text { Mean Difference (IV, Ran- } \\
\text { dom, } 95 \% \mathrm{CI} \text { ) }\end{array}$ & $0.30[-0.49,1.09]$ \\
\hline $\begin{array}{l}36 \text { Adverse events: } 2 \text {. Movement disor- } \\
\text { ders - b.v. change scores (BAS, high = } \\
\text { bad) }\end{array}$ & 2 & 91 & $\begin{array}{l}\text { Mean Difference (IV, Ran- } \\
\text { dom, } 95 \% \mathrm{CI} \text { ) }\end{array}$ & $-0.7[-1.54,0.14]$ \\
\hline 36.1 Other atypical drugs in both groups & 2 & 91 & $\begin{array}{l}\text { Mean Difference (IV, Ran- } \\
\text { dom, } 95 \% \mathrm{CI} \text { ) }\end{array}$ & $-0.7[-1.54,0.14]$ \\
\hline $\begin{array}{l}37 \text { Adverse events: } 2 \text {. Movement disor- } \\
\text { ders - b.vi. change scores (AIMS, high = } \\
\text { bad) }\end{array}$ & 1 & 63 & $\begin{array}{l}\text { Mean Difference (IV, Ran- } \\
\text { dom, } 95 \% \mathrm{CI} \text { ) }\end{array}$ & $0.10[-0.84,1.04]$ \\
\hline 37.1 Other antipsychotic in both groups & 1 & 63 & $\begin{array}{l}\text { Mean Difference (IV, Ran- } \\
\text { dom, } 95 \% \mathrm{CI} \text { ) }\end{array}$ & $0.10[-0.84,1.04]$ \\
\hline $\begin{array}{l}38 \text { Adverse events: } 3 \text {. Endocrine - pro- } \\
\text { lactin level (high = bad) }\end{array}$ & 7 & & $\begin{array}{l}\text { Mean Difference (IV, Ran- } \\
\text { dom, } 95 \% \mathrm{Cl} \text { ) }\end{array}$ & $\begin{array}{l}\text { Totals not select- } \\
\text { ed }\end{array}$ \\
\hline
\end{tabular}




\begin{tabular}{|c|c|c|c|c|}
\hline Outcome or subgroup title & No. of studies & $\begin{array}{l}\text { No. of partici- } \\
\text { pants }\end{array}$ & Statistical method & Effect size \\
\hline 38.1 clozapine in both groups & 4 & & $\begin{array}{l}\text { Mean Difference (IV, Ran- } \\
\text { dom, } 95 \% \mathrm{CI} \text { ) }\end{array}$ & $0.0[0.0,0.0]$ \\
\hline 38.2 other atypical drugs in both groups & 1 & & $\begin{array}{l}\text { Mean Difference (IV, Ran- } \\
\text { dom, } 95 \% \mathrm{CI} \text { ) }\end{array}$ & $0.0[0.0,0.0]$ \\
\hline 38.3 typical drugs in both groups & 2 & & $\begin{array}{l}\text { Mean Difference (IV, Ran- } \\
\text { dom, } 95 \% \mathrm{CI})\end{array}$ & $0.0[0.0,0.0]$ \\
\hline $\begin{array}{l}39 \text { Adverse events: } 4 \text {. Metabolic - a. } \\
\text { weight gain (binary) }\end{array}$ & 6 & 804 & $\begin{array}{l}\text { Risk Ratio (M-H, Random, } \\
95 \% \mathrm{Cl})\end{array}$ & $1.00[0.66,1.53]$ \\
\hline 39.1 clozapine in both groups & 4 & 389 & $\begin{array}{l}\text { Risk Ratio (M-H, Random, } \\
95 \% \mathrm{Cl})\end{array}$ & $0.63[0.34,1.19]$ \\
\hline 39.2 other atypical in both groups & 2 & 415 & $\begin{array}{l}\text { Risk Ratio (M-H, Random, } \\
95 \% \mathrm{Cl})\end{array}$ & $1.33[0.89,1.99]$ \\
\hline $\begin{array}{l}40 \text { Adverse events: } 4 \text {. Metabolic - b. aver- } \\
\text { age weight gain (kg) }\end{array}$ & 9 & & $\begin{array}{l}\text { Mean Difference (IV, Ran- } \\
\text { dom, } 95 \% \mathrm{CI} \text { ) }\end{array}$ & $\begin{array}{l}\text { Totals not select- } \\
\text { ed }\end{array}$ \\
\hline 40.1 clozapine in both groups & 6 & & $\begin{array}{l}\text { Mean Difference (IV, Ran- } \\
\text { dom, } 95 \% \mathrm{CI} \text { ) }\end{array}$ & $0.0[0.0,0.0]$ \\
\hline 40.2 other atypical drugs in both groups & 3 & & $\begin{array}{l}\text { Mean Difference (IV, Ran- } \\
\text { dom, } 95 \% \mathrm{CI} \text { ) }\end{array}$ & $0.0[0.0,0.0]$ \\
\hline $\begin{array}{l}41 \text { Adverse events: } 5 \text {. Blood - a. de- } \\
\text { creased white cell counts (binary) }\end{array}$ & 2 & 315 & $\begin{array}{l}\text { Risk Ratio (M-H, Random, } \\
95 \% \mathrm{Cl})\end{array}$ & $0.18[0.04,0.82]$ \\
\hline 41.1 clozapine in both groups & 2 & 315 & $\begin{array}{l}\text { Risk Ratio (M-H, Random, } \\
95 \% \mathrm{Cl})\end{array}$ & $0.18[0.04,0.82]$ \\
\hline $\begin{array}{l}42 \text { Adverse events: } 5 \text {. Blood - b. average } \\
\text { white cell counts }\left(10^{-3} / \mathrm{mm}^{3}\right)\end{array}$ & 1 & & $\begin{array}{l}\text { Mean Difference (IV, Ran- } \\
\text { dom, } 95 \% \mathrm{CI} \text { ) }\end{array}$ & Subtotals only \\
\hline 42.1 clozapine in both groups & 1 & 61 & $\begin{array}{l}\text { Mean Difference (IV, Ran- } \\
\text { dom, } 95 \% \mathrm{CI} \text { ) }\end{array}$ & $0.66[-0.20,1.52]$ \\
\hline $\begin{array}{l}43 \text { Adverse events: } 6 \text {. Central nervous } \\
\text { system (CNS) - a. drowsiness }\end{array}$ & 12 & & $\begin{array}{l}\text { Risk Ratio (M-H, Random, } \\
95 \% \mathrm{Cl})\end{array}$ & $\begin{array}{l}\text { Totals not select- } \\
\text { ed }\end{array}$ \\
\hline 43.1 clozapine in both groups & 6 & & $\begin{array}{l}\text { Risk Ratio (M-H, Random, } \\
95 \% \mathrm{Cl})\end{array}$ & $0.0[0.0,0.0]$ \\
\hline 43.2 other atypical in both groups & 3 & & $\begin{array}{l}\text { Risk Ratio (M-H, Random, } \\
95 \% \mathrm{Cl})\end{array}$ & $0.0[0.0,0.0]$ \\
\hline 43.3 typical drugs in both groups & 3 & & $\begin{array}{l}\text { Risk Ratio (M-H, Random, } \\
95 \% \mathrm{Cl})\end{array}$ & $0.0[0.0,0.0]$ \\
\hline $\begin{array}{l}44 \text { Adverse events: } 6 \text {. Central nervous } \\
\text { system (CNS) - b. tremor }\end{array}$ & 4 & 222 & $\begin{array}{l}\text { Risk Ratio (M-H, Random, } \\
95 \% \mathrm{Cl})\end{array}$ & $0.87[0.47,1.62]$ \\
\hline
\end{tabular}




\begin{tabular}{|c|c|c|c|c|}
\hline Outcome or subgroup title & No. of studies & $\begin{array}{l}\text { No. of partici- } \\
\text { pants }\end{array}$ & Statistical method & Effect size \\
\hline 44.1 clozapine in both groups & 3 & 130 & $\begin{array}{l}\text { Risk Ratio (M-H, Random, } \\
95 \% \mathrm{Cl} \text { ) }\end{array}$ & $0.61[0.12,3.13]$ \\
\hline 44.2 Other atypical in both groups & 1 & 92 & $\begin{array}{l}\text { Risk Ratio (M-H, Random, } \\
95 \% \mathrm{Cl})\end{array}$ & $0.92[0.47,1.80]$ \\
\hline $\begin{array}{l}45 \text { Quality of life: 1a. Average score (QLS } \\
\text { high=good) }\end{array}$ & 1 & 30 & $\begin{array}{l}\text { Mean Difference (IV, Ran- } \\
\text { dom, } 95 \% \mathrm{CI} \text { ) }\end{array}$ & $0.80[-5.44,7.04]$ \\
\hline 45.1 clozapine in both groups & 1 & 30 & $\begin{array}{l}\text { Mean Difference (IV, Ran- } \\
\text { dom, } 95 \% \mathrm{CI} \text { ) }\end{array}$ & $0.80[-5.44,7.04]$ \\
\hline $\begin{array}{l}46 \text { Quality of life: } 1 \text { b. Average score } \\
\text { (SWN, high=good) }\end{array}$ & 2 & 248 & $\begin{array}{l}\text { Mean Difference (IV, Ran- } \\
\text { dom, } 95 \% \mathrm{CI} \text { ) }\end{array}$ & $2.05[-1.08,5.18]$ \\
\hline 46.1 clozapine in both groups & 2 & 248 & $\begin{array}{l}\text { Mean Difference (IV, Ran- } \\
\text { dom, } 95 \% \mathrm{CI} \text { ) }\end{array}$ & $2.05[-1.08,5.18]$ \\
\hline $\begin{array}{l}47 \text { Quality of Life: } 1 \text { c. Average score - } \\
\text { Mental component summary (SF-36, } \\
\text { high = good) }\end{array}$ & 1 & 60 & $\begin{array}{l}\text { Mean Difference (IV, Ran- } \\
\text { dom, } 95 \% \mathrm{CI} \text { ) }\end{array}$ & $0.60[-4.28,5.48]$ \\
\hline 47.1 Other atypical in both groups & 1 & 60 & $\begin{array}{l}\text { Mean Difference (IV, Ran- } \\
\text { dom, } 95 \% \mathrm{CI} \text { ) }\end{array}$ & $0.60[-4.28,5.48]$ \\
\hline $\begin{array}{l}48 \text { Quality of Life: } 1 \mathrm{~d} \text {. Average score - } \\
\text { Physical component summary (SF-36, } \\
\text { high = good) }\end{array}$ & 1 & 60 & $\begin{array}{l}\text { Mean Difference (IV, Ran- } \\
\text { dom, } 95 \% \mathrm{CI} \text { ) }\end{array}$ & $-1.70[-4.71,1.31]$ \\
\hline 48.1 Other atypical in both groups & 1 & 60 & $\begin{array}{l}\text { Mean Difference (IV, Ran- } \\
\text { dom, } 95 \% \mathrm{CI} \text { ) }\end{array}$ & $-1.70[-4.71,1.31]$ \\
\hline $\begin{array}{l}49 \text { SUBGROUP ANALYSIS Clinical Re- } \\
\text { sponse: Not clinically improved - Pa- } \\
\text { tients enrolled in the studies }\end{array}$ & 18 & 1244 & $\begin{array}{l}\text { Risk Ratio (M-H, Random, } \\
95 \% \mathrm{Cl})\end{array}$ & $0.81[0.70,0.93]$ \\
\hline 49.1 Chronic & 14 & 987 & $\begin{array}{l}\text { Risk Ratio (M-H, Random, } \\
95 \% \mathrm{Cl})\end{array}$ & $0.77[0.63,0.93]$ \\
\hline 49.2 Acute & 4 & 257 & $\begin{array}{l}\text { Risk Ratio (M-H, Random, } \\
95 \% \mathrm{Cl})\end{array}$ & $0.87[0.73,1.05]$ \\
\hline $\begin{array}{l}50 \text { SUBGROUP ANALYSIS Clinical Re- } \\
\text { sponse: Not clinically improved - Treat- } \\
\text { ment duration }\end{array}$ & 28 & 2344 & $\begin{array}{l}\text { Risk Ratio (M-H, Random, } \\
95 \% \mathrm{Cl})\end{array}$ & $0.73[0.64,0.83]$ \\
\hline $50.1 \leq 12$ weeks & 21 & 1604 & $\begin{array}{l}\text { Risk Ratio (M-H, Random, } \\
95 \% \mathrm{Cl})\end{array}$ & $0.77[0.66,0.89]$ \\
\hline $50.2>12$ weeks & 7 & 740 & $\begin{array}{l}\text { Risk Ratio (M-H, Random, } \\
95 \% \mathrm{Cl})\end{array}$ & $0.64[0.51,0.80]$ \\
\hline $\begin{array}{l}51 \text { SUBGROUP ANALYSIS Clinical Re- } \\
\text { sponse: Not clinically improved - Use of } \\
\text { clozapine in both groups }\end{array}$ & 28 & 2344 & $\begin{array}{l}\text { Risk Ratio (M-H, Random, } \\
95 \% \mathrm{Cl})\end{array}$ & $0.73[0.64,0.83]$ \\
\hline
\end{tabular}




\begin{tabular}{|c|c|c|c|c|}
\hline Outcome or subgroup title & No. of studies & $\begin{array}{l}\text { No. of partici- } \\
\text { pants }\end{array}$ & Statistical method & Effect size \\
\hline 51.1 clozapine & 17 & 1127 & $\begin{array}{l}\text { Risk Ratio (M-H, Random, } \\
95 \% \mathrm{Cl} \text { ) }\end{array}$ & $0.66[0.53,0.83]$ \\
\hline 51.2 other antipsychotic & 11 & 1217 & $\begin{array}{l}\text { Risk Ratio (M-H, Random, } \\
95 \% \mathrm{Cl} \text { ) }\end{array}$ & $0.78[0.69,0.89]$ \\
\hline $\begin{array}{l}52 \text { SUBGROUP ANALYSIS Clinical Re- } \\
\text { sponse: Not clinically improved - Drug } \\
\text { added to clozapine }\end{array}$ & 17 & 1127 & $\begin{array}{l}\text { Risk Ratio (M-H, Random, } \\
95 \% \mathrm{Cl})\end{array}$ & $0.66[0.53,0.83]$ \\
\hline 52.1 Sulpirirde & 8 & 486 & $\begin{array}{l}\text { Risk Ratio (M-H, Random, } \\
95 \% \mathrm{Cl} \text { ) }\end{array}$ & $0.54[0.43,0.68]$ \\
\hline 52.2 Risperidone & 7 & 507 & $\begin{array}{l}\text { Risk Ratio (M-H, Random, } \\
95 \% \mathrm{Cl} \text { ) }\end{array}$ & $0.89[0.68,1.15]$ \\
\hline 52.3 Pipotazine & 2 & 134 & $\begin{array}{l}\text { Risk Ratio (M-H, Random, } \\
95 \% \mathrm{Cl} \text { ) }\end{array}$ & $0.50[0.34,0.74]$ \\
\hline $\begin{array}{l}53 \text { SUBGROUP ANALYSIS Leaving the } \\
\text { study early - Patients enrolled in the } \\
\text { studies }\end{array}$ & 28 & 2208 & $\begin{array}{l}\text { Risk Ratio (M-H, Random, } \\
95 \% \mathrm{Cl})\end{array}$ & $0.92[0.74,1.16]$ \\
\hline 53.1 Chronic & 25 & 2075 & $\begin{array}{l}\text { Risk Ratio (M-H, Random, } \\
95 \% \mathrm{Cl} \text { ) }\end{array}$ & $0.87[0.69,1.10]$ \\
\hline 53.2 Acute & 3 & 133 & $\begin{array}{l}\text { Risk Ratio (M-H, Random, } \\
95 \% \mathrm{Cl})\end{array}$ & $1.15[0.53,2.51]$ \\
\hline $\begin{array}{l}54 \text { SUBGROUP ANALYSIS Leaving the } \\
\text { study early - Treatment duration }\end{array}$ & 32 & 2454 & $\begin{array}{l}\text { Risk Ratio (M-H, Random, } \\
95 \% \mathrm{Cl} \text { ) }\end{array}$ & $0.96[0.77,1.20]$ \\
\hline $54.1 \leq 12$ weeks & 23 & 1426 & $\begin{array}{l}\text { Risk Ratio (M-H, Random, } \\
95 \% \mathrm{Cl} \text { ) }\end{array}$ & $1.06[0.78,1.45]$ \\
\hline $54.2>12$ weeks & 9 & 1028 & $\begin{array}{l}\text { Risk Ratio (M-H, Random, } \\
95 \% \mathrm{Cl})\end{array}$ & $0.94[0.66,1.35]$ \\
\hline $\begin{array}{l}55 \text { SUBGROUP ANALYSIS Leaving the } \\
\text { study early - Use of clozapine in both } \\
\text { groups }\end{array}$ & 32 & 2424 & $\begin{array}{l}\text { Risk Ratio (M-H, Random, } \\
95 \% \mathrm{Cl})\end{array}$ & $0.96[0.77,1.20]$ \\
\hline 55.1 Clozapine & 16 & 878 & $\begin{array}{l}\text { Risk Ratio (M-H, Random, } \\
95 \% \mathrm{Cl} \text { ) }\end{array}$ & $1.25[0.85,1.86]$ \\
\hline 55.2 Other antipsychotics & 16 & 1546 & $\begin{array}{l}\text { Risk Ratio (M-H, Random, } \\
95 \% \mathrm{Cl})\end{array}$ & $0.89[0.66,1.20]$ \\
\hline $\begin{array}{l}56 \text { SUBGROUP ANALYSIS Leaving the } \\
\text { study early - Drug added to clozapine }\end{array}$ & 15 & 850 & $\begin{array}{l}\text { Risk Ratio (M-H, Random, } \\
95 \% \mathrm{Cl} \text { ) }\end{array}$ & $1.22[0.82,1.82]$ \\
\hline 56.1 Risperidone & 6 & 297 & $\begin{array}{l}\text { Risk Ratio (M-H, Random, } \\
95 \% \mathrm{Cl})\end{array}$ & $1.32[0.66,2.67]$ \\
\hline
\end{tabular}




\begin{tabular}{|c|c|c|c|c|}
\hline Outcome or subgroup title & No. of studies & $\begin{array}{l}\text { No. of partici- } \\
\text { pants }\end{array}$ & Statistical method & Effect size \\
\hline 56.2 Amisulpiride & 1 & 16 & $\begin{array}{l}\text { Risk Ratio (M-H, Random, } \\
95 \% \mathrm{Cl} \text { ) }\end{array}$ & $0.46[0.06,3.57]$ \\
\hline 56.3 Aripiprazole & 4 & 347 & $\begin{array}{l}\text { Risk Ratio (M-H, Random, } \\
95 \% \mathrm{Cl} \text { ) }\end{array}$ & $1.41[0.78,2.56]$ \\
\hline 56.4 Pimozide & 1 & 53 & $\begin{array}{l}\text { Risk Ratio (M-H, Random, } \\
95 \% \mathrm{Cl})\end{array}$ & $0.67[0.18,2.53]$ \\
\hline 56.5 Sertindole & 1 & 50 & $\begin{array}{l}\text { Risk Ratio (M-H, Random, } \\
95 \% \mathrm{Cl} \text { ) }\end{array}$ & $1.25[0.38,4.12]$ \\
\hline 56.6 Sulpiride & 2 & 87 & $\begin{array}{l}\text { Risk Ratio (M-H, Random, } \\
95 \% \mathrm{Cl})\end{array}$ & $0.0[0.0,0.0]$ \\
\hline $\begin{array}{l}57 \text { SENSITIVITY ANALYSIS Clinical Re- } \\
\text { sponse: Not clinically improved - Ran- } \\
\text { domisation }\end{array}$ & 28 & 2344 & $\begin{array}{l}\text { Risk Ratio (M-H, Random, } \\
95 \% \mathrm{Cl})\end{array}$ & $0.73[0.64,0.83]$ \\
\hline 57.1 Low / unclear risk of bias & 25 & 2140 & $\begin{array}{l}\text { Risk Ratio (M-H, Random, } \\
95 \% \mathrm{Cl})\end{array}$ & $0.75[0.66,0.85]$ \\
\hline 57.2 High & 3 & 204 & $\begin{array}{l}\text { Risk Ratio (M-H, Random, } \\
95 \% \mathrm{Cl})\end{array}$ & $0.44[0.27,0.71]$ \\
\hline $\begin{array}{l}58 \text { SENSITIVITY ANALYSIS Clinical Re- } \\
\text { sponse: Not clinically improved - Double } \\
\text { blind }\end{array}$ & 28 & 2344 & $\begin{array}{l}\text { Risk Ratio (M-H, Random, } \\
95 \% \mathrm{Cl})\end{array}$ & $0.73[0.64,0.83]$ \\
\hline 58.1 Low / unclear & 25 & 2197 & $\begin{array}{l}\text { Risk Ratio (M-H, Random, } \\
95 \% \mathrm{Cl} \text { ) }\end{array}$ & $0.71[0.62,0.82]$ \\
\hline $58.2 \mathrm{High}$ & 3 & 147 & $\begin{array}{l}\text { Risk Ratio (M-H, Random, } \\
95 \% \mathrm{Cl} \text { ) }\end{array}$ & $0.80[0.64,1.01]$ \\
\hline $\begin{array}{l}59 \text { SENSITIVITY ANALYSIS Clinical re- } \\
\text { sponse: } 1 \text {. No clinically important re- } \\
\text { sponse - not improved - Fixed effect }\end{array}$ & 29 & 2398 & $\begin{array}{l}\text { Risk Ratio (M-H, Fixed, 95\% } \\
\mathrm{Cl})\end{array}$ & $0.74[0.68,0.81]$ \\
\hline 59.1 clozapine in both groups & 17 & 1127 & $\begin{array}{l}\text { Risk Ratio (M-H, Fixed, 95\% } \\
\mathrm{Cl} \text { ) }\end{array}$ & $0.64[0.56,0.74]$ \\
\hline 59.2 other atypical in both groups & 7 & 674 & $\begin{array}{l}\text { Risk Ratio (M-H, Fixed, 95\% } \\
\mathrm{Cl})\end{array}$ & $0.96[0.83,1.11]$ \\
\hline 59.3 typical drugs in both groups & 5 & 597 & $\begin{array}{l}\text { Risk Ratio (M-H, Fixed, 95\% } \\
\mathrm{Cl} \text { ) }\end{array}$ & $0.69[0.59,0.81]$ \\
\hline $\begin{array}{l}60 \text { SENSITIVITY ANALYSIS Leaving the } \\
\text { study early - Randomisation }\end{array}$ & 30 & 2326 & $\begin{array}{l}\text { Risk Ratio (M-H, Random, } \\
95 \% \mathrm{Cl} \text { ) }\end{array}$ & $0.94[0.74,1.18]$ \\
\hline 60.1 Low / unclear risk of bias & 29 & 2298 & $\begin{array}{l}\text { Risk Ratio (M-H, Random, } \\
95 \% \mathrm{Cl} \text { ) }\end{array}$ & $0.94[0.74,1.18]$ \\
\hline
\end{tabular}




\begin{tabular}{|c|c|c|c|c|}
\hline Outcome or subgroup title & No. of studies & $\begin{array}{l}\text { No. of partici- } \\
\text { pants }\end{array}$ & Statistical method & Effect size \\
\hline 60.2 High risk & 1 & 28 & $\begin{array}{l}\text { Risk Ratio (M-H, Random, } \\
95 \% \mathrm{Cl} \text { ) }\end{array}$ & $0.0[0.0,0.0]$ \\
\hline $\begin{array}{l}61 \text { SENSITIVITY ANALYSIS Leaving the } \\
\text { study early - Double blind }\end{array}$ & 30 & 2326 & $\begin{array}{l}\text { Risk Ratio (M-H, Random, } \\
95 \% \mathrm{Cl})\end{array}$ & $0.94[0.74,1.18]$ \\
\hline 61.1 Low / unclear risk & 27 & 2156 & $\begin{array}{l}\text { Risk Ratio (M-H, Random, } \\
95 \% \mathrm{Cl})\end{array}$ & $0.94[0.74,1.19]$ \\
\hline 61.2 High risk of bias & 3 & 170 & $\begin{array}{l}\text { Risk Ratio (M-H, Random, } \\
95 \% \mathrm{Cl})\end{array}$ & $0.96[0.33,2.82]$ \\
\hline $\begin{array}{l}62 \text { SENSITIVITY ANALYSIS Leving the } \\
\text { study early - Fixed effect }\end{array}$ & 43 & 3137 & $\begin{array}{l}\text { Risk Ratio (M-H, Fixed, 95\% } \\
\mathrm{Cl})\end{array}$ & $0.90[0.78,1.04]$ \\
\hline 62.1 clozapine in both groups & 18 & 932 & $\begin{array}{l}\text { Risk Ratio (M-H, Fixed, 95\% } \\
\mathrm{Cl})\end{array}$ & $1.33[0.92,1.92]$ \\
\hline 62.2 other atypical drugs in both groups & 15 & 1247 & $\begin{array}{l}\text { Risk Ratio (M-H, Fixed, 95\% } \\
\mathrm{Cl})\end{array}$ & $1.01[0.82,1.25]$ \\
\hline 62.3 typical drugs in both groups & 6 & 628 & $\begin{array}{l}\text { Risk Ratio (M-H, Fixed, 95\% } \\
\text { Cl) }\end{array}$ & $0.70[0.53,0.92]$ \\
\hline 62.4 any antipsychotics in both groups & 4 & 330 & $\begin{array}{l}\text { Risk Ratio (M-H, Fixed, 95\% } \\
\mathrm{Cl} \text { ) }\end{array}$ & $0.57[0.39,0.84]$ \\
\hline
\end{tabular}

\section{Analysis 1.1. Comparison 1 ANTIPSYCHOTIC COMBINATIONS vs ANTIPSYCHOTIC MONOTHERAPY, Outcome 1 Clinical response: 1 . No clinically important response - not improved.}

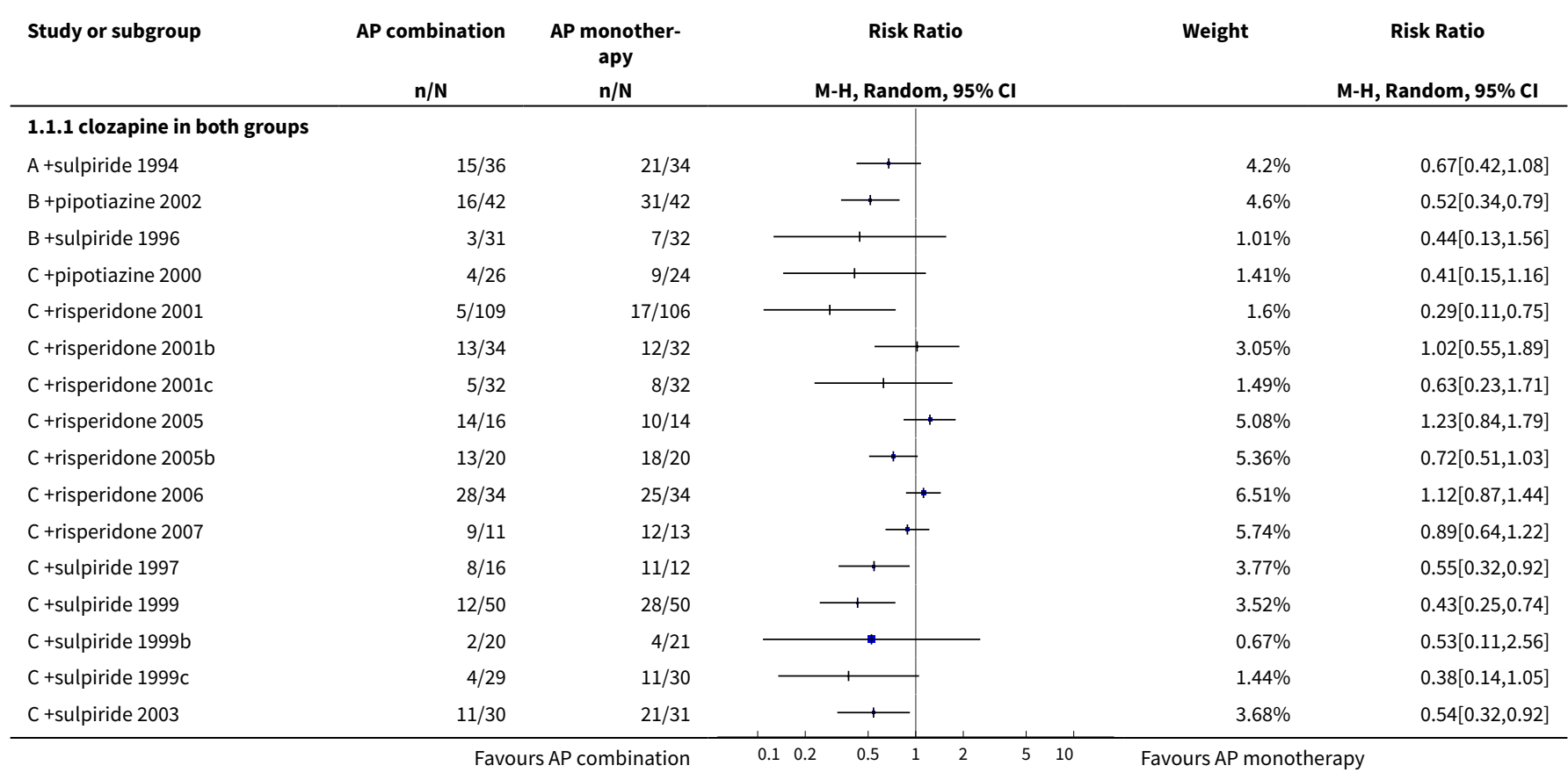




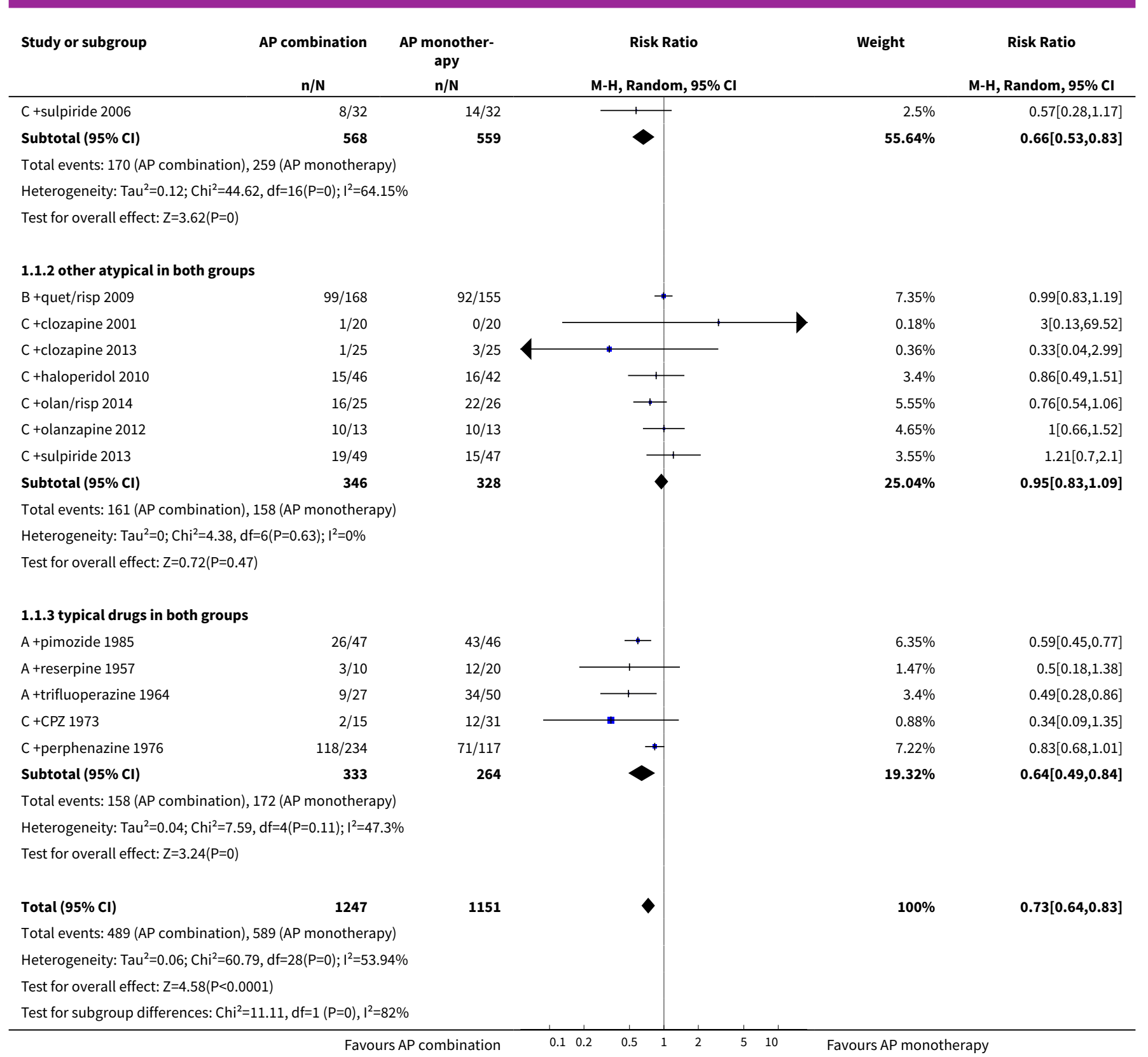

Analysis 1.2. Comparison 1 ANTIPSYCHOTIC COMBINATIONS vs ANTIPSYCHOTIC MONOTHERAPY, Outcome 2 Clinical response: 2. Relapse.

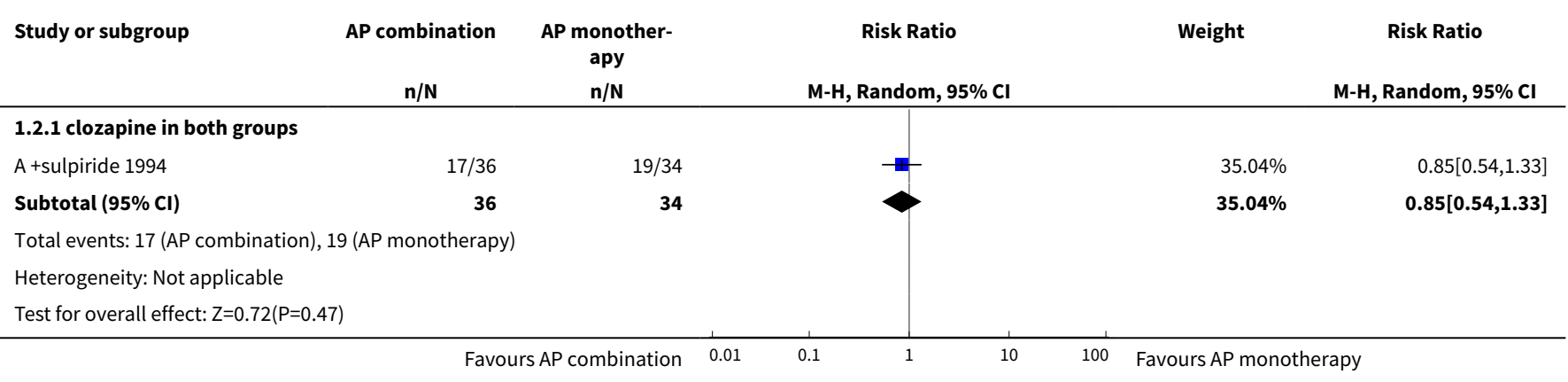




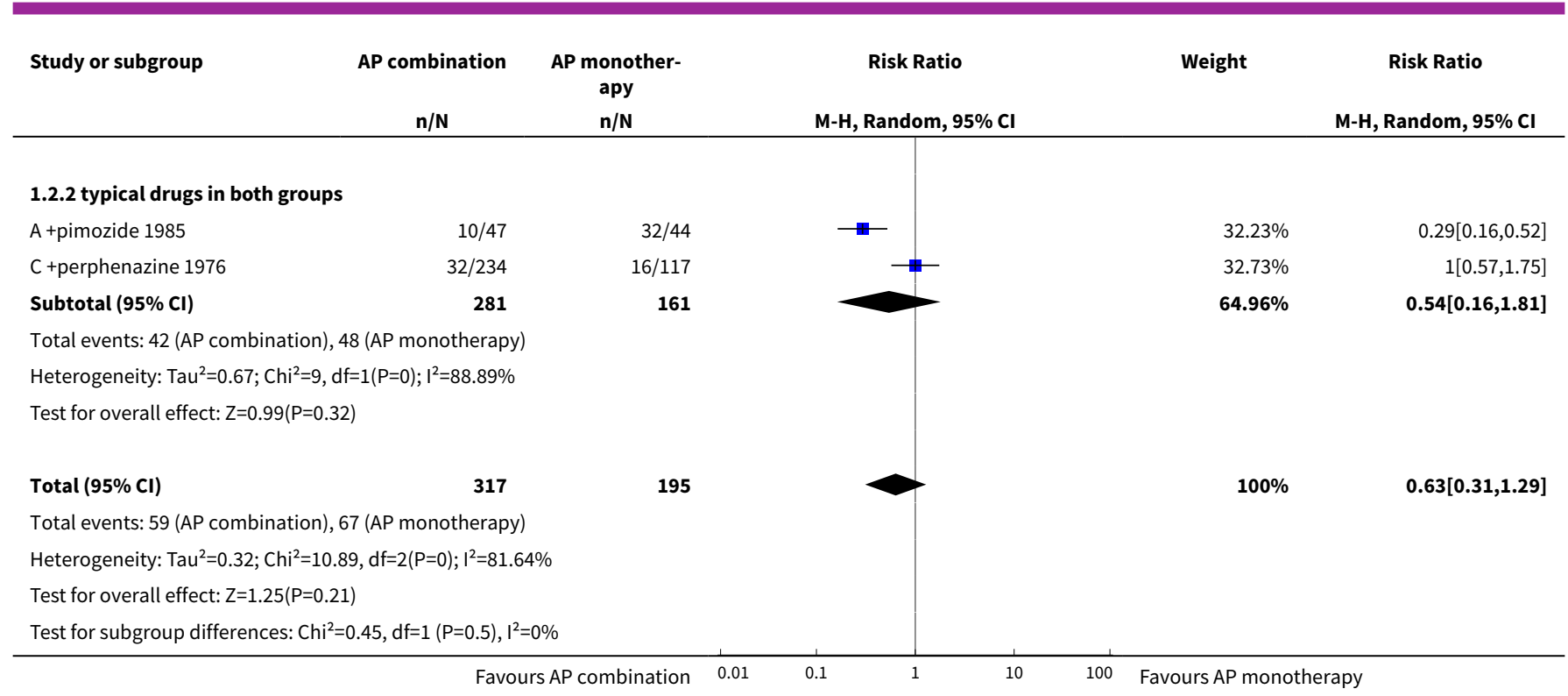

Analysis 1.3. Comparison 1 ANTIPSYCHOTIC COMBINATIONS vs ANTIPSYCHOTIC MONOTHERAPY, Outcome 3 Leaving the study early.

\begin{tabular}{|c|c|c|c|c|c|}
\hline \multirow[t]{2}{*}{ Study or subgroup } & \multirow{2}{*}{$\begin{array}{l}\text { AP combination } \\
n=\mathbf{N}\end{array}$} & \multirow{2}{*}{$\begin{array}{l}\text { AP monother- } \\
\text { apy } \\
n / N \\
\end{array}$} & Risk Ratio & \multirow[t]{2}{*}{ Weight } & \multirow{2}{*}{$\begin{array}{c}\text { Risk Ratio } \\
\text { M-H, Random, } 95 \% \mathrm{CI}\end{array}$} \\
\hline & & & M-H, Random, 95\% Cl & & \\
\hline \multicolumn{6}{|c|}{ 1.3.1 clozapine in both groups } \\
\hline B +aripiprazole 2008 & $11 / 108$ & $6 / 99$ & + & $2.91 \%$ & $1.68[0.65,4.37]$ \\
\hline B +aripiprazole 2011 & $6 / 20$ & $3 / 20$ & + & $1.83 \%$ & $2[0.58,6.91]$ \\
\hline B +risperidone 2010 & $8 / 33$ & $8 / 36$ & + & $3.5 \%$ & $1.09[0.46,2.57]$ \\
\hline C + amisulpride 2008 & $2 / 13$ & $1 / 3$ & +- & $0.71 \%$ & $0.46[0.06,3.57]$ \\
\hline C +aripiprazole 2008 & $3 / 30$ & $3 / 32$ & 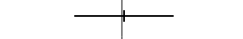 & $1.25 \%$ & $1.07[0.23,4.88]$ \\
\hline C +aripiprazole 2013 & $4 / 20$ & $4 / 18$ & - & $1.85 \%$ & $0.9[0.26,3.08]$ \\
\hline C +olanzapine $2012 b$ & $1 / 7$ & $0 / 7$ & — & $0.33 \%$ & $3[0.14,63.15]$ \\
\hline C + pimozide 2011 & $3 / 25$ & $5 / 28$ & 1 & $1.62 \%$ & $0.67[0.18,2.53]$ \\
\hline C + pimozide 2013 & $2 / 14$ & $0 / 14$ & & $0.35 \%$ & $5[0.26,95.61]$ \\
\hline C +risperidone 2005 & $1 / 16$ & $0 / 14$ & 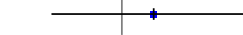 & $0.31 \%$ & $2.65[0.12,60.21]$ \\
\hline C +risperidone $2005 b$ & $0 / 20$ & $0 / 20$ & & & Not estimable \\
\hline C +risperidone 2006 & $2 / 34$ & $1 / 34$ & $\rightarrow$ & $0.54 \%$ & $2[0.19,21.03]$ \\
\hline C +risperidone 2007 & $3 / 11$ & $2 / 13$ & 1 & $1.14 \%$ & $1.77[0.36,8.77]$ \\
\hline C +sertindole 2006 & $5 / 25$ & $4 / 25$ & 1 & $1.97 \%$ & $1.25[0.38,4.12]$ \\
\hline C +sulpiride 1997 & $0 / 16$ & $0 / 12$ & & & Not estimable \\
\hline C +sulpiride 1999 c & $0 / 29$ & $0 / 30$ & & & Not estimable \\
\hline Subtotal $(95 \% \mathrm{CI})$ & 473 & 459 & t & $19.84 \%$ & $1.28[0.88,1.86]$ \\
\hline \multicolumn{6}{|c|}{ Total events: 55 (AP combination), 40 (AP monotherapy) } \\
\hline \multicolumn{6}{|c|}{ Heterogeneity: $\mathrm{Tau}^{2}=0 ; \mathrm{Chi}^{2}=4.84, \mathrm{df}=13(\mathrm{P}=0.98) ; \mathrm{I}^{2}=0 \%$} \\
\hline \multicolumn{6}{|c|}{ Test for overall effect: $Z=1.28(P=0.2)$} \\
\hline \multicolumn{6}{|c|}{ 1.3.2 other atypical drugs in both groups } \\
\hline C +arip/pali 2014 & F & tion $\quad 0.001$ & 0.1 & $1000 \quad$ Favo & \\
\hline
\end{tabular}




\begin{tabular}{|c|c|c|c|c|c|}
\hline \multirow[t]{2}{*}{ Study or subgroup } & \multirow{2}{*}{$\begin{array}{c}\text { AP combination } \\
n / N\end{array}$} & \multirow{2}{*}{$\begin{array}{l}\text { AP monother- } \\
\text { apy } \\
n / N \\
\end{array}$} & \multirow{2}{*}{$\begin{array}{c}\text { Risk Ratio } \\
\text { M-H, Random, } 95 \% \mathrm{CI}\end{array}$} & \multirow[t]{2}{*}{ Weight } & \multirow{2}{*}{$\begin{array}{c}\text { Risk Ratio } \\
\text { M-H, Random, } 95 \% \mathrm{CI}\end{array}$} \\
\hline & & & & & \\
\hline C +aripiprazole 2014 & $4 / 59$ & $3 / 57$ & 1 & $1.36 \%$ & $1.29[0.3,5.5]$ \\
\hline C +aripiprazole 2015 & $7 / 89$ & $4 / 30$ & 1 & $2.08 \%$ & $0.59[0.19,1.88]$ \\
\hline C +aripiprazole 2015b & $0 / 15$ & $2 / 15$ & - & $0.35 \%$ & $0.2[0.01,3.85]$ \\
\hline C +aripiprazole 2015 c & $2 / 56$ & $4 / 57$ & \begin{tabular}{l|l}
1 & \\
1
\end{tabular} & $1.06 \%$ & $0.51[0.1,2.67]$ \\
\hline C +aripiprazole 2016 & $5 / 30$ & $7 / 30$ & + & $2.56 \%$ & $0.71[0.25,2]$ \\
\hline C+clozapine 2001 & $0 / 20$ & $0 / 20$ & & & Not estimable \\
\hline C +clozapine 2013 & $0 / 25$ & $0 / 25$ & & & Not estimable \\
\hline C +fluphen dec 2009 & $0 / 14$ & $0 / 14$ & & & Not estimable \\
\hline C +haloperidol 2010 & $13 / 46$ & $10 / 42$ & + & $4.78 \%$ & $1.19[0.58,2.42]$ \\
\hline C +olan/risp 2014 & $13 / 25$ & $14 / 26$ & - & $7.56 \%$ & $0.97[0.58,1.62]$ \\
\hline C +olanzapine 2012 & $8 / 13$ & $5 / 13$ & + & $3.86 \%$ & $1.6[0.71,3.6]$ \\
\hline C +sulpiride 2004 & $1 / 9$ & $0 / 8$ & $\rightarrow$ & $0.32 \%$ & $2.7[0.13,58.24]$ \\
\hline C +sulpiride 2013 & $18 / 49$ & $15 / 47$ & 4 & $6.87 \%$ & $1.15[0.66,2.01]$ \\
\hline Subtotal $(95 \% \mathrm{CI})$ & 678 & 569 & 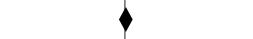 & $43.47 \%$ & $1.03[0.83,1.27]$ \\
\hline \multicolumn{6}{|c|}{ Total events: 124 (AP combination), 112 (AP monotherapy) } \\
\hline \multicolumn{6}{|c|}{ Heterogeneity: $\mathrm{Tau}^{2}=0 ; \mathrm{Chi}^{2}=5.26, \mathrm{df}=10(\mathrm{P}=0.87) ; \mathrm{I}^{2}=0 \%$} \\
\hline \multicolumn{6}{|c|}{ Test for overall effect: $Z=0.26(P=0.79)$} \\
\hline \multicolumn{6}{|c|}{ 1.3.3 typical drugs in both groups } \\
\hline A +pimozide 1985 & $27 / 47$ & $42 / 46$ & + & $14.93 \%$ & $0.63[0.48,0.82]$ \\
\hline A +reserpine 1957 & $0 / 10$ & $2 / 22$ & & $0.35 \%$ & $0.42[0.02,7.99]$ \\
\hline A +trifluoperazine 1964 & $0 / 27$ & $0 / 50$ & & & Not estimable \\
\hline C +aripiprazole 2007b & $4 / 28$ & $0 / 28$ & & $0.36 \%$ & $9[0.51,159.7]$ \\
\hline C +levomepromazine 2004 & $0 / 9$ & $4 / 10$ & - & $0.39 \%$ & $0.12[0.01,2]$ \\
\hline C +perphenazine 1976 & $21 / 234$ & $13 / 117$ & $\rightarrow$ & $5.41 \%$ & $0.81[0.42,1.56]$ \\
\hline Subtotal $(95 \% \mathrm{Cl})$ & 355 & 273 & & $21.44 \%$ & $0.69[0.43,1.12]$ \\
\hline \multicolumn{6}{|c|}{ Total events: 52 (AP combination), 61 (AP monotherapy) } \\
\hline \multicolumn{6}{|c|}{ Heterogeneity: $\mathrm{Tau}^{2}=0.08 ; \mathrm{Chi}^{2}=5.48, \mathrm{df}=4(\mathrm{P}=0.24) ; \mathrm{I}^{2}=27.04 \%$} \\
\hline \multicolumn{6}{|c|}{ Test for overall effect: $Z=1.5(P=0.13)$} \\
\hline \multicolumn{6}{|c|}{ 1.3.4 any antipsychotics in both groups } \\
\hline A +any antipsychotic 2011 & $8 / 62$ & $18 / 65$ & 1 & $4.32 \%$ & $0.47[0.22,0.99]$ \\
\hline A +any antipsychotic 2012 & $11 / 30$ & $7 / 30$ & + & $3.93 \%$ & $1.57[0.71,3.5]$ \\
\hline A +any antipsychotic 2015 & $11 / 52$ & $27 / 52$ & $\rightarrow-$ & $6.38 \%$ & $0.41[0.23,0.73]$ \\
\hline B + any antipsychotic 2013 & $1 / 19$ & $3 / 20$ & $\rightarrow-1-$ & $0.63 \%$ & $0.35[0.04,3.09]$ \\
\hline Subtotal $(95 \% \mathrm{Cl})$ & 163 & 167 & & $15.26 \%$ & $0.61[0.3,1.25]$ \\
\hline \multicolumn{6}{|c|}{ Total events: 31 (AP combination), 55 (AP monotherapy) } \\
\hline \multicolumn{6}{|c|}{ Heterogeneity: $\operatorname{Tau}^{2}=0.3 ; \mathrm{Chi}^{2}=7.87, \mathrm{df}=3(\mathrm{P}=0.05) ; \mathrm{I}^{2}=61.9 \%$} \\
\hline \multicolumn{6}{|c|}{ Test for overall effect: $Z=1.35(P=0.18)$} \\
\hline Total $(95 \% \mathrm{Cl})$ & 1669 & 1468 & $\uparrow$ & $100 \%$ & $0.9[0.76,1.07]$ \\
\hline \multicolumn{6}{|c|}{ Total events: 262 (AP combination), 268 (AP monotherapy) } \\
\hline \multicolumn{6}{|c|}{ Heterogeneity: $\operatorname{Tau}^{2}=0.04 ; \mathrm{Chi}^{2}=39.4, \mathrm{df}=33(\mathrm{P}=0.21) ; \mathrm{I}^{2}=16.25 \%$} \\
\hline \multicolumn{6}{|c|}{ Test for overall effect: $Z=1.16(P=0.24)$} \\
\hline Test for subgroup difference & 5.79, $d f=1(P=0.12)$, & $48.21 \%$ & & & \\
\hline
\end{tabular}


Analysis 1.4. Comparison 1 ANTIPSYCHOTIC COMBINATIONS vs ANTIPSYCHOTIC MONOTHERAPY, Outcome 4 Service utilisation: Hospital admission.

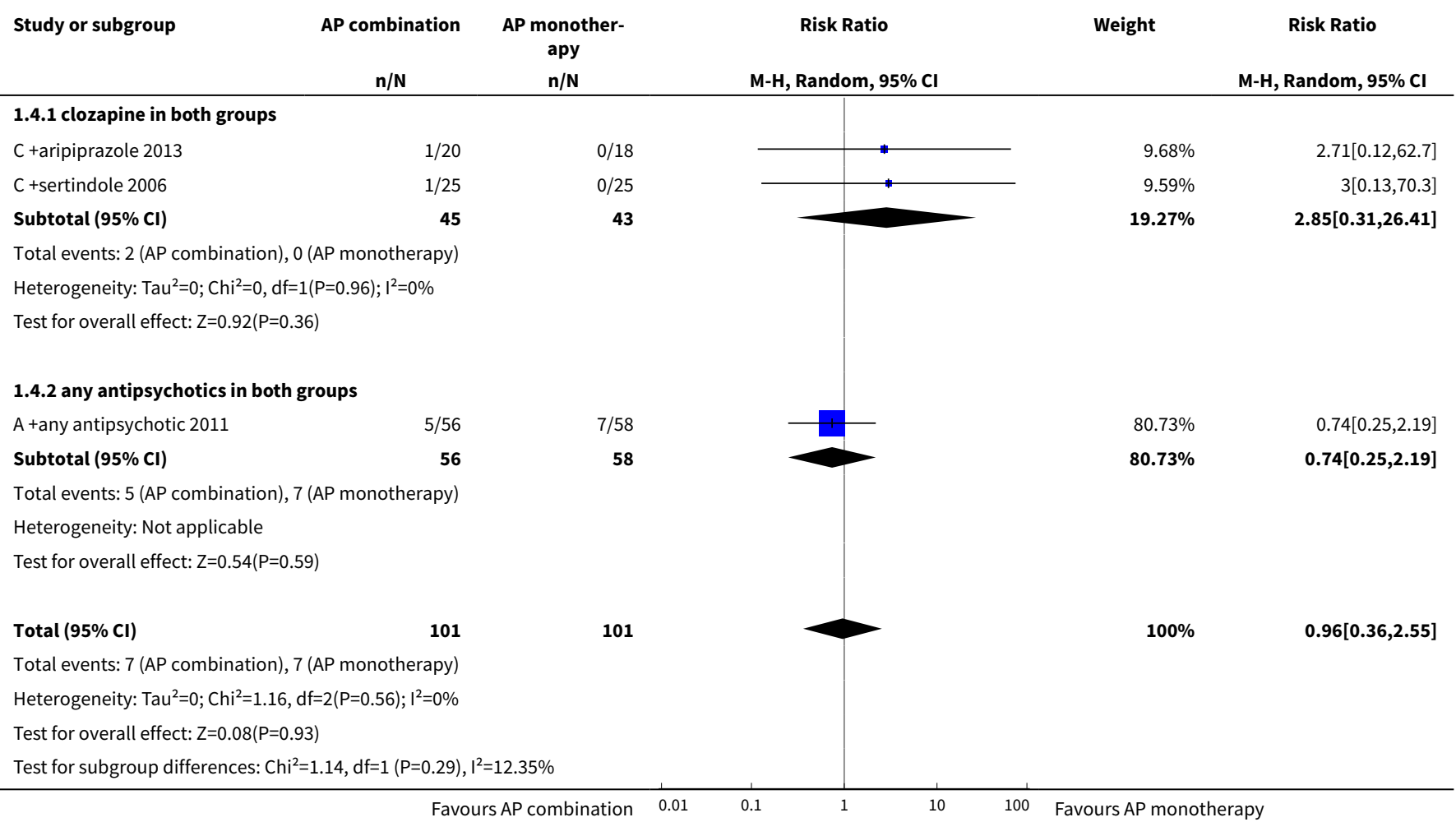

\section{Analysis 1.5. Comparison 1 ANTIPSYCHOTIC COMBINATIONS vs ANTIPSYCHOTIC MONOTHERAPY, Outcome 5 Clinical response: 3 . Global state $-i$. average severity score (CGI-S scale, high $=$ bad).}

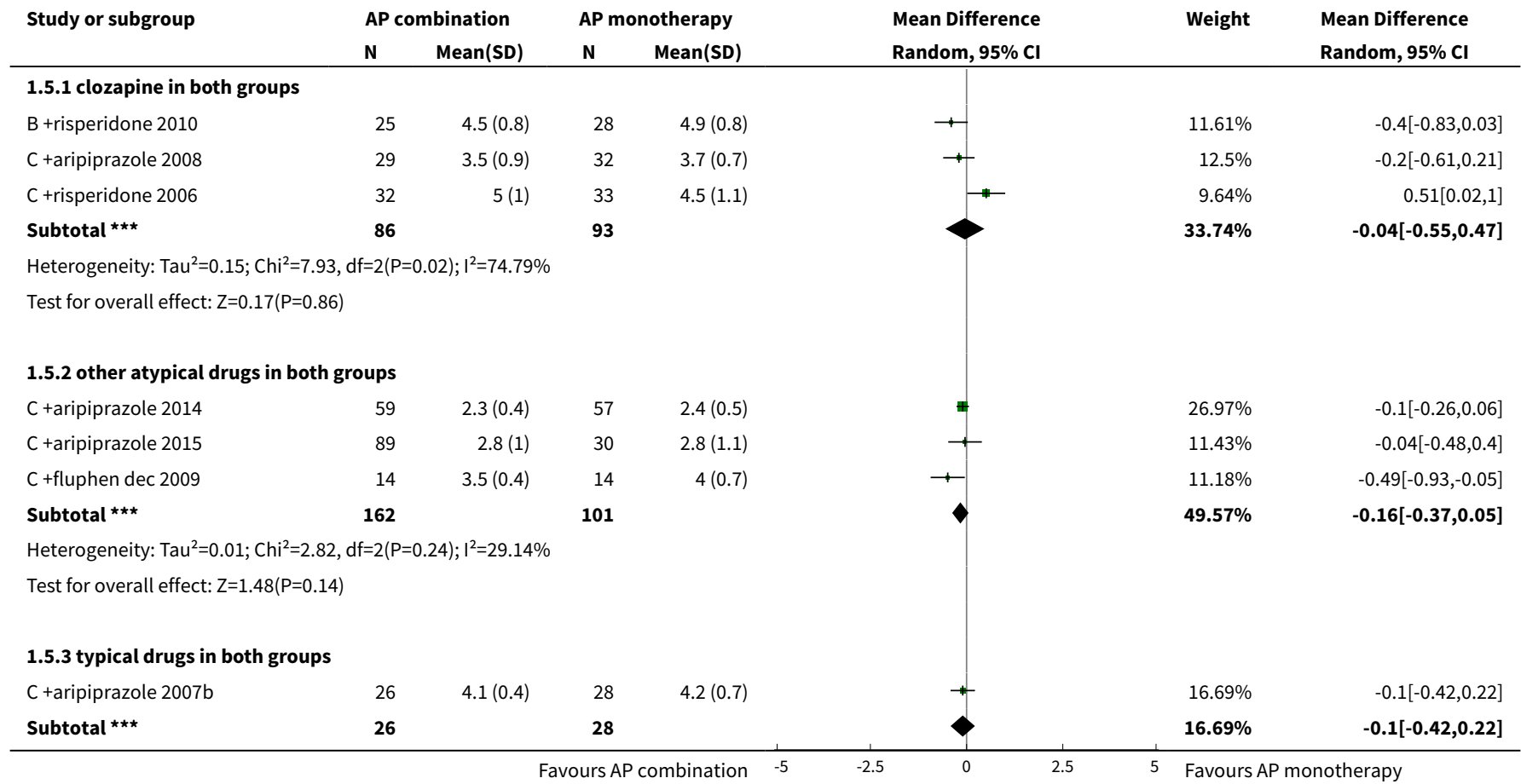




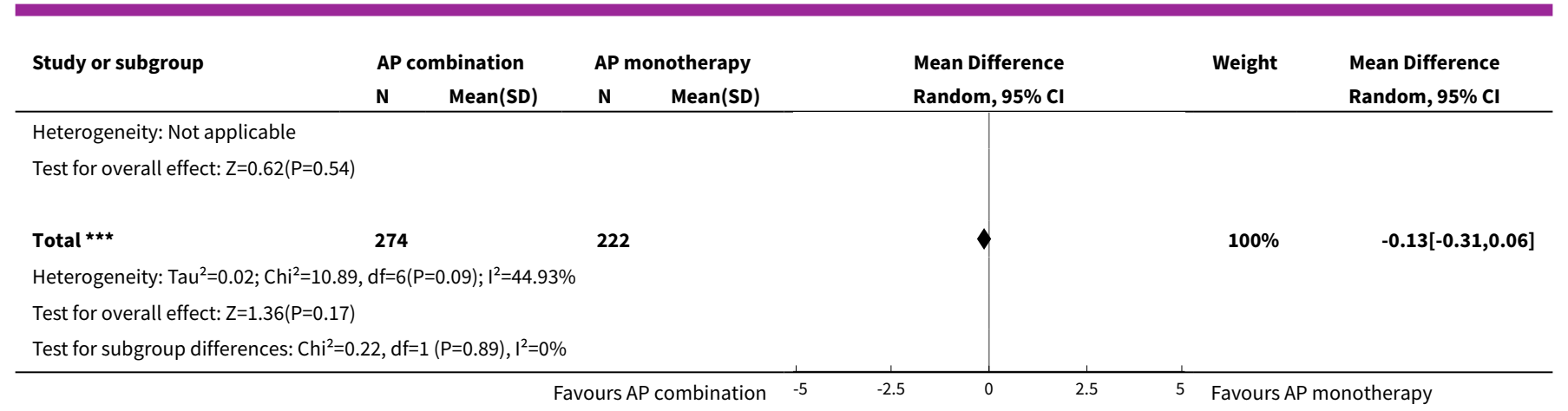

Analysis 1.6. Comparison 1 ANTIPSYCHOTIC COMBINATIONS vS ANTIPSYCHOTIC MONOTHERAPY, Outcome 6 Clinical response: 3 . Global state - ii. change in severity score (CGI-S scale, high = bad).

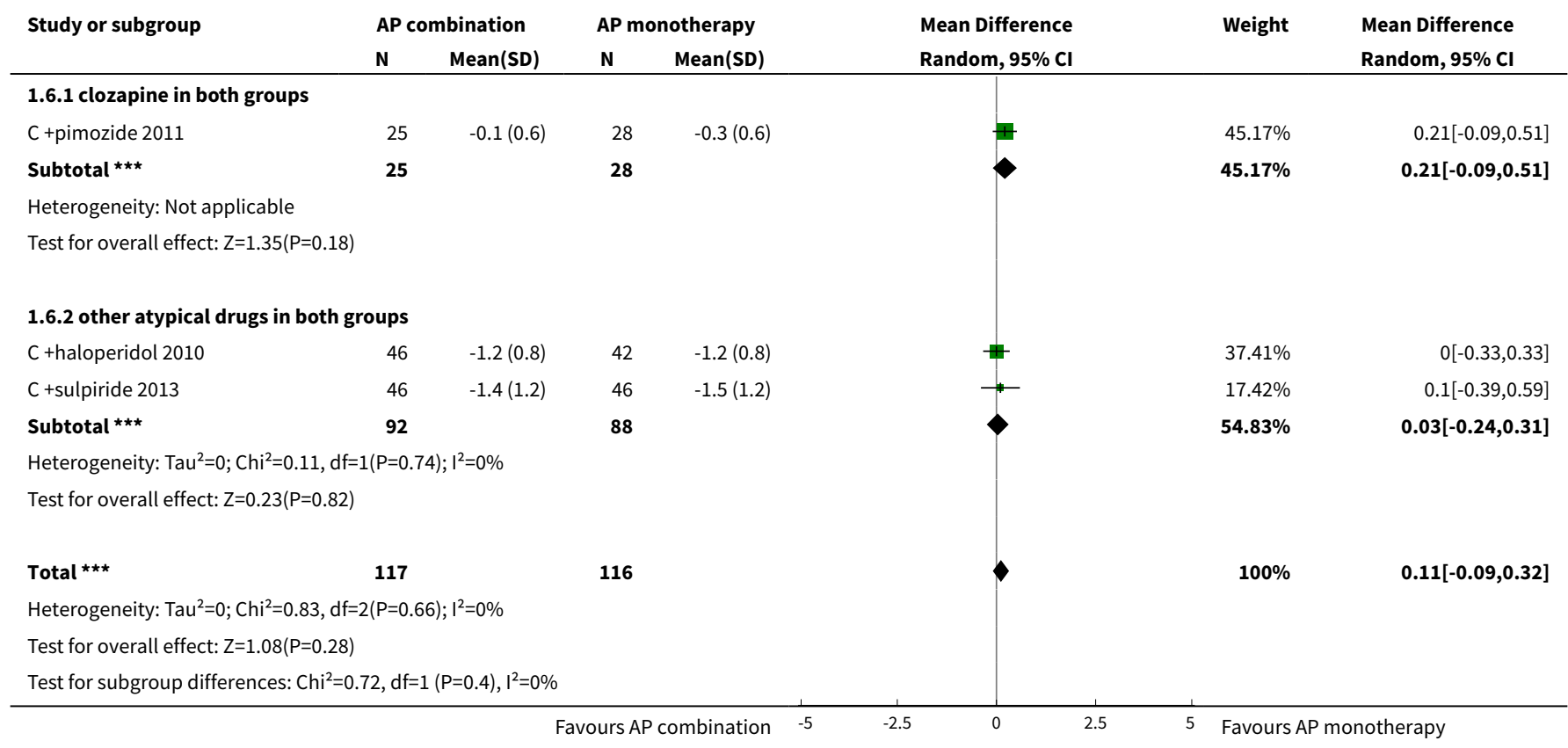

Analysis 1.7. Comparison 1 ANTIPSYCHOTIC COMBINATIONS vs ANTIPSYCHOTIC MONOTHERAPY, Outcome 7 Clinical response: 4. Global state - average improvement score (CGI-I scale, high = bad).

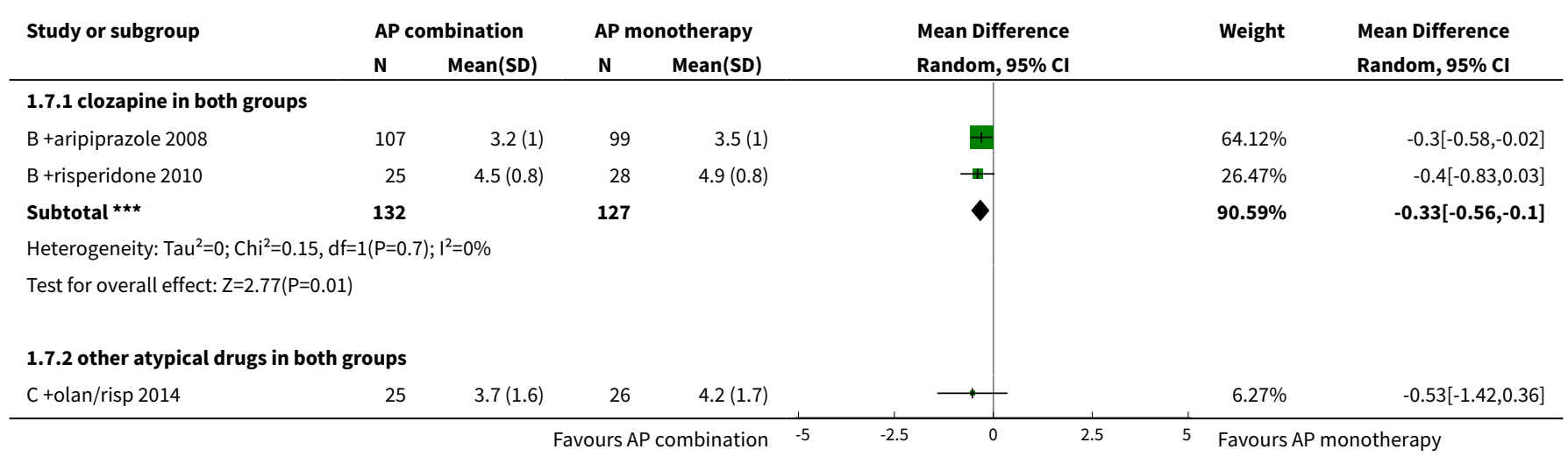




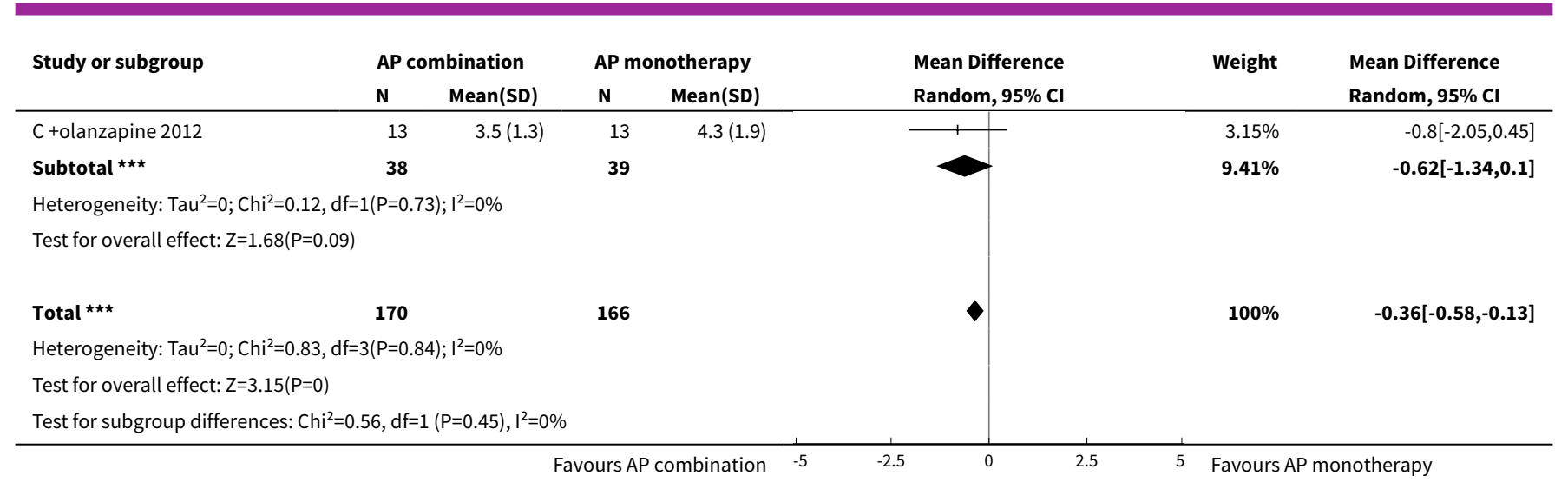

Analysis 1.8. Comparison 1 ANTIPSYCHOTIC COMBINATIONS vs ANTIPSYCHOTIC MONOTHERAPY,
Outcome 8 Clinical response: 5 . Global state - i. average functioning score (GAF scale, high = good).

\begin{tabular}{|c|c|c|c|c|c|c|c|}
\hline \multirow[t]{2}{*}{ Study or subgroup } & \multicolumn{2}{|c|}{ AP combination } & \multicolumn{2}{|c|}{ AP monotherapy } & \multirow{2}{*}{$\begin{array}{l}\text { Mean Difference } \\
\text { Random, } 95 \% \mathrm{Cl}\end{array}$} & \multirow[t]{2}{*}{ Weight } & \multirow{2}{*}{$\begin{array}{l}\text { Mean Difference } \\
\text { Random, } 95 \% \mathrm{CI}\end{array}$} \\
\hline & $\mathbf{N}$ & Mean(SD) & $\mathbf{N}$ & Mean(SD) & & & \\
\hline \multicolumn{5}{|c|}{ 1.8.1 Clozapine in both groups } & & & \\
\hline C + risperidone 2005 & 16 & $50.3(5.6)$ & 14 & $54.8(5.2)$ & & $100 \%$ & $-4.5[-8.38,-0.62]$ \\
\hline Subtotal *** & 16 & & 14 & & & $100 \%$ & $-4.5[-8.38,-0.62]$ \\
\hline \multicolumn{8}{|c|}{ Heterogeneity: Not applicable } \\
\hline \multicolumn{8}{|c|}{ Test for overall effect: $\mathrm{Z}=2.27(\mathrm{P}=0.02)$} \\
\hline \multicolumn{8}{|c|}{ 1.8.2 Other atypical drugs in both groups } \\
\hline C +olan/risp 2014 & 25 & $45.3(17.1)$ & 26 & $35.6(14.5)$ & & $67.51 \%$ & $9.71[0.99,18.43]$ \\
\hline C +olanzapine 2012 & 13 & $42.8(19.4)$ & 13 & $36.1(12.6)$ & T & $32.49 \%$ & $6.7[-5.87,19.27]$ \\
\hline Subtotal $* \star \star$ & 38 & & 39 & & & $100 \%$ & $8.73[1.56,15.9]$ \\
\hline \multicolumn{8}{|c|}{ Heterogeneity: $\mathrm{Tau}^{2}=0 ; \mathrm{Chi}^{2}=0.15, \mathrm{df}=1(\mathrm{P}=0.7) ; \mathrm{I}^{2}=0 \%$} \\
\hline \multicolumn{8}{|c|}{ Test for overall effect: $Z=2.39(P=0.02)$} \\
\hline
\end{tabular}

Analysis 1.9. Comparison 1 ANTIPSYCHOTIC COMBINATIONS vS ANTIPSYCHOTIC MONOTHERAPY, Outcome 9 Clinical response: 5 . Global state - ii. change in functioning score (GAF scale, high = good).

\begin{tabular}{|c|c|c|c|c|c|c|c|}
\hline \multirow[t]{2}{*}{ Study or subgroup } & \multicolumn{2}{|c|}{ AP combination } & \multicolumn{2}{|c|}{ AP monotherapy } & \multirow{2}{*}{$\begin{array}{l}\text { Mean Difference } \\
\text { Random, } 95 \% \mathrm{Cl}\end{array}$} & \multirow[t]{2}{*}{ Weight } & \multirow{2}{*}{$\begin{array}{l}\text { Mean Difference } \\
\text { Random, } 95 \% \mathrm{Cl}\end{array}$} \\
\hline & $\mathbf{N}$ & $\operatorname{Mean}(S D)$ & $\mathbf{N}$ & Mean(SD) & & & \\
\hline \multicolumn{8}{|c|}{ 1.9.1 Clozapine in both groups } \\
\hline B +aripiprazole 2008 & 108 & $6(9.4)$ & 99 & $5.5(9)$ & $\#$ & $46.11 \%$ & $0.5[-1.99,2.99]$ \\
\hline$C+$ sertindole 2006 & 25 & $2(4.8)$ & 25 & $2(4.8)$ & \# & $39.77 \%$ & $0[-2.69,2.69]$ \\
\hline Subtotal ${ }^{\star \star \star}$ & 133 & & 124 & & 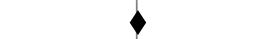 & $85.88 \%$ & $0.27[-1.56,2.1]$ \\
\hline \multicolumn{8}{|c|}{ Heterogeneity: $\operatorname{Tau}^{2}=0 ; \mathrm{Chi}^{2}=0.07, \mathrm{df}=1(\mathrm{P}=0.79) ; \mathrm{I}^{2}=0 \%$} \\
\hline \multicolumn{8}{|c|}{ 1.9.2 Other atypical drugs in both groups } \\
\hline C +sulpiride 2013 & 46 & $15.7(10.2)$ & 46 & $15.4(11.8)$ & & $14.12 \%$ & $0.3[-4.21,4.81]$ \\
\hline Subtotal ${ }^{\star \star \star}$ & 46 & & 46 & & & $14.12 \%$ & $0.3[-4.21,4.81]$ \\
\hline \multicolumn{8}{|c|}{ Heterogeneity: Not applicable } \\
\hline & & & Irs $\mathrm{AP}$ & notherapy $\quad-50$ & -25 & 50 Favours & mbination \\
\hline
\end{tabular}




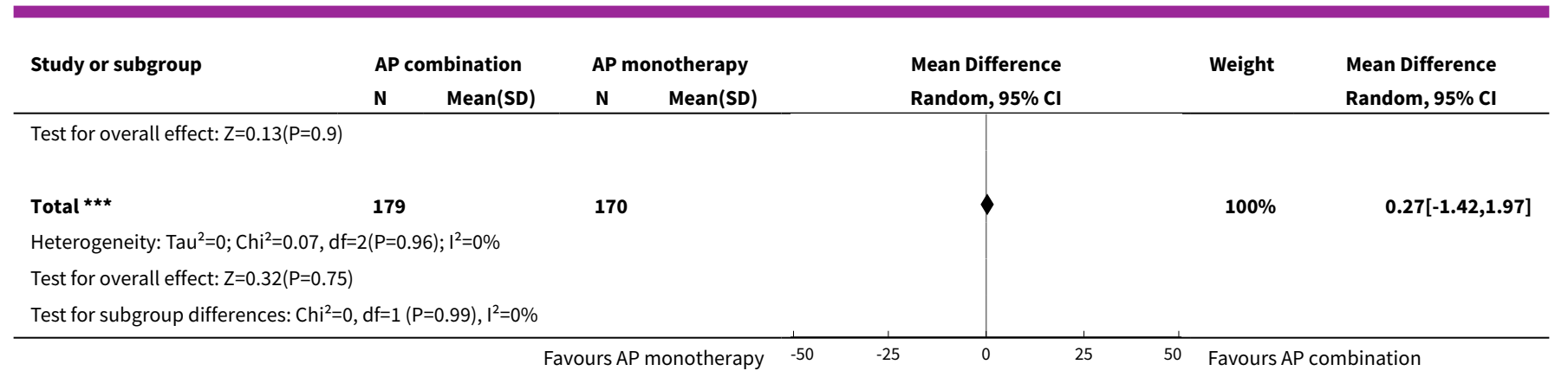

Analysis 1.10. Comparison 1 ANTIPSYCHOTIC COMBINATIONS vs ANTIPSYCHOTIC MONOTHERAPY, Outcome 10 Mental state: 1. Overall - a.i average total score (PANSS scale, high = bad).

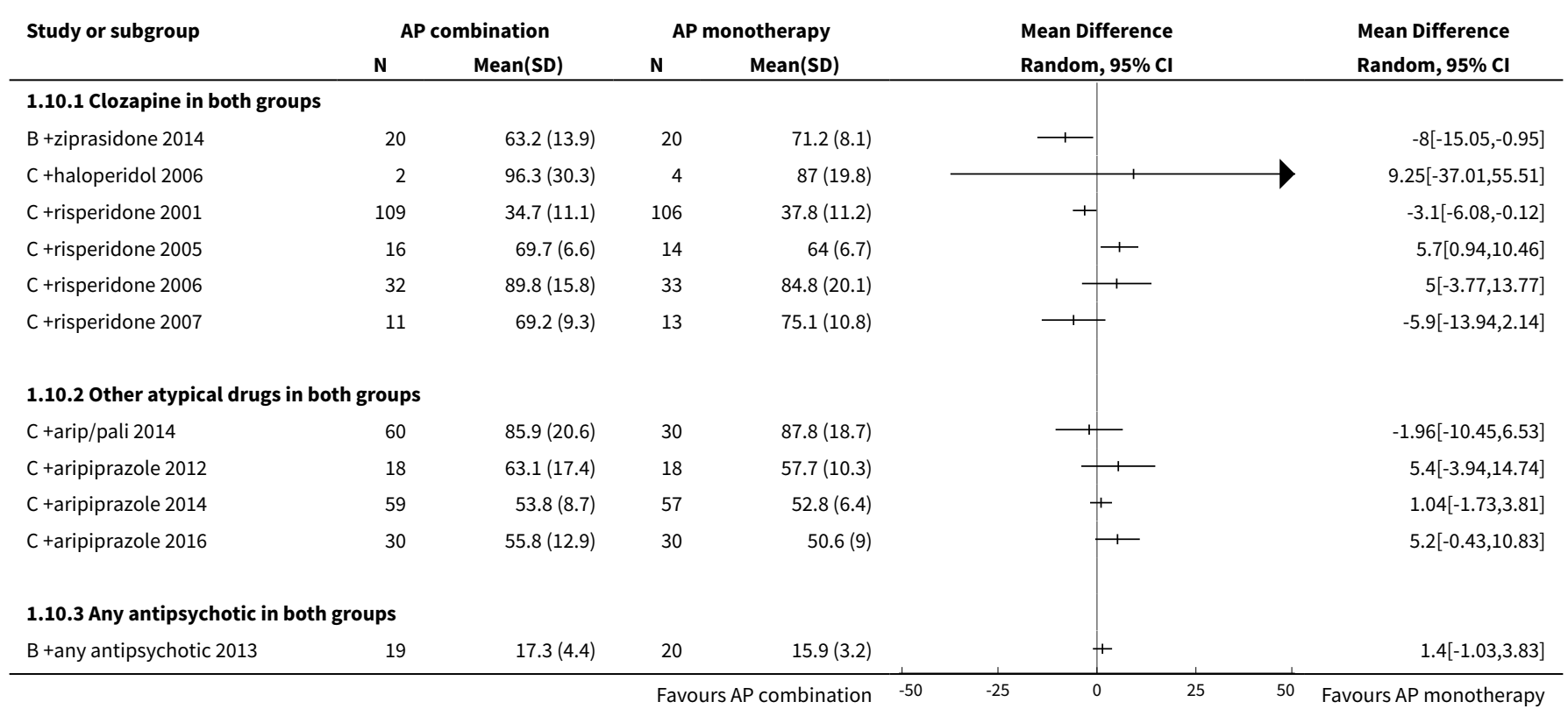

\section{Analysis 1.11. Comparison 1 ANTIPSYCHOTIC COMBINATIONS vs ANTIPSYCHOTIC MONOTHERAPY, Outcome 11 Mental state: 1. Overall - a.ii change in total score (PANSS scale, high = bad).}

\begin{tabular}{|c|c|c|c|c|c|c|c|}
\hline \multirow[t]{2}{*}{ Study or subgroup } & \multicolumn{2}{|c|}{ AP combination } & \multicolumn{2}{|c|}{ AP monotherapy } & \multirow{2}{*}{$\begin{array}{l}\text { Mean Difference } \\
\text { Random, } 95 \% \mathrm{Cl}\end{array}$} & \multirow{2}{*}{ Weight } & \multirow{2}{*}{$\begin{array}{l}\text { Mean Difference } \\
\text { Random, } 95 \% \mathrm{Cl}\end{array}$} \\
\hline & $\mathbf{N}$ & Mean(SD) & $\mathbf{N}$ & Mean(SD) & & & \\
\hline \multicolumn{8}{|c|}{ 1.11.1 Clozapine in both groups } \\
\hline C +aripiprazole 2013 & 16 & $-5.6(8.8)$ & 14 & $-2.6(6.3)$ & $*$ & $19.06 \%$ & $-3[-8.43,2.43]$ \\
\hline C+pimozide 2011 & 25 & $-0.7(10.6)$ & 28 & $-3.6(10.2)$ & + & $17.9 \%$ & $2.84[-2.76,8.44]$ \\
\hline C + sertindole 2006 & 25 & $-6(7.3)$ & 25 & $-5(7.3)$ & $\#$ & $34.62 \%$ & $-1[-5.03,3.03]$ \\
\hline Subtotal $\star \star \star$ & 66 & & 67 & & $>$ & $71.58 \%$ & $-0.55[-3.54,2.43]$ \\
\hline \multicolumn{8}{|c|}{ Test for overall effect: $Z=0.36(P=0.72)$} \\
\hline \multicolumn{8}{|c|}{ 1.11.2 Other atypical drugs in both groups } \\
\hline C +haloperidol 2010 & 46 & $-20.1(20.4)$ & 42 & $-22.2(19.8)$ & 廿- & $7.96 \%$ & $2.1[-6.3,10.5]$ \\
\hline C +olan/risp 2014 & 25 & $-28.7(25)$ & 26 & $-17.6(19.1)$ & 1 & $3.76 \%$ & $-11.06[-23.29,1.17]$ \\
\hline
\end{tabular}




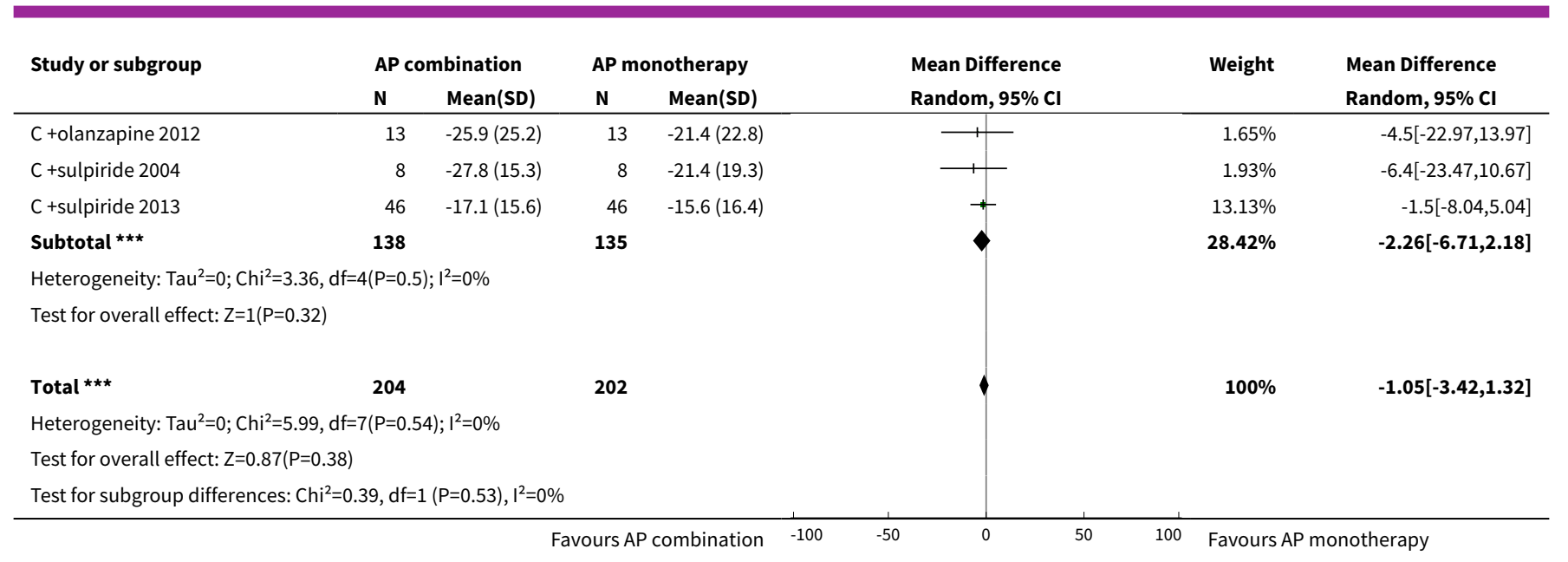

\section{Analysis 1.12. Comparison 1 ANTIPSYCHOTIC COMBINATIONS vS ANTIPSYCHOTIC MONOTHERAPY, Outcome 12 Mental state: 1. Overall - b.i. average total score (BPRS scale, high = bad).}

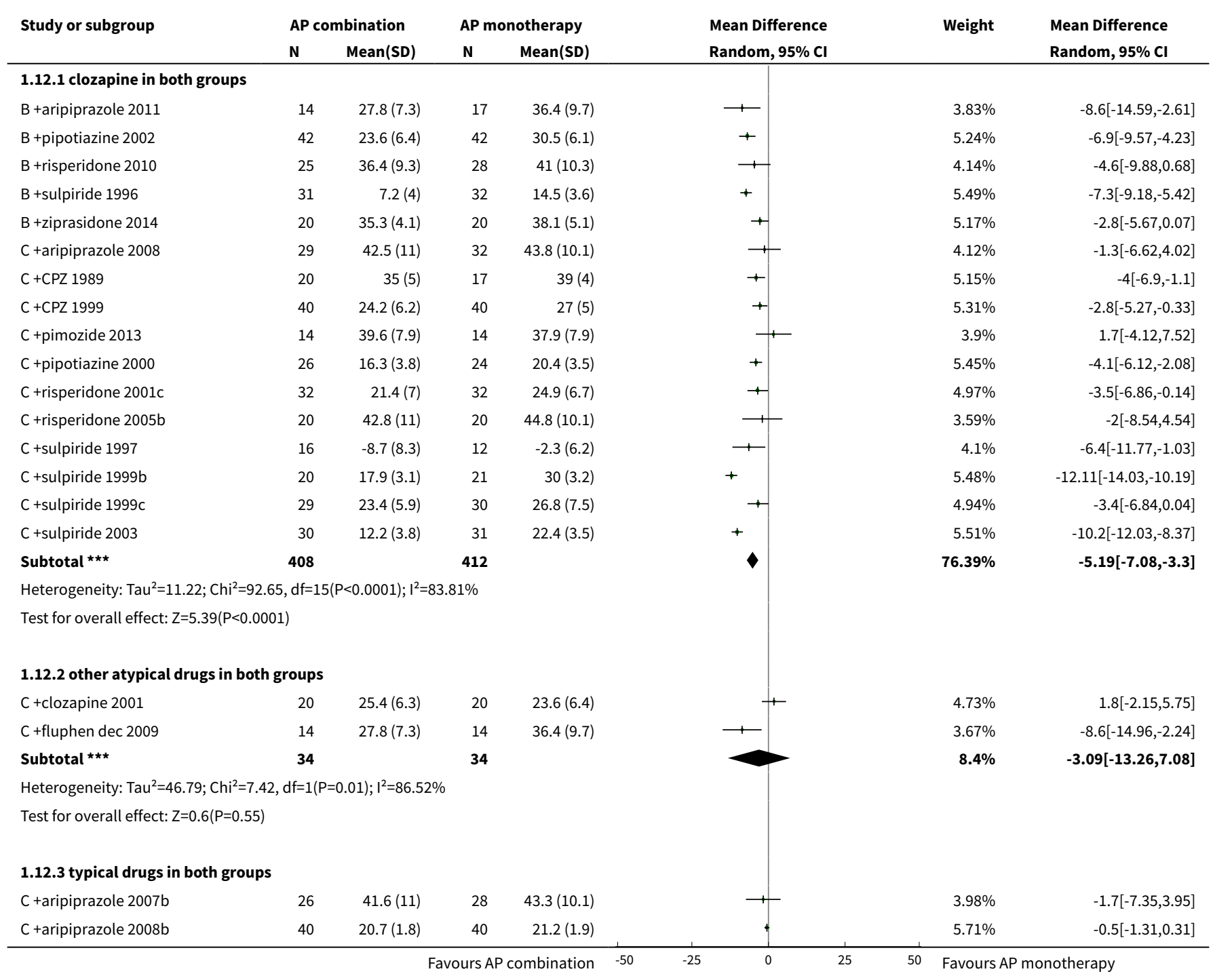




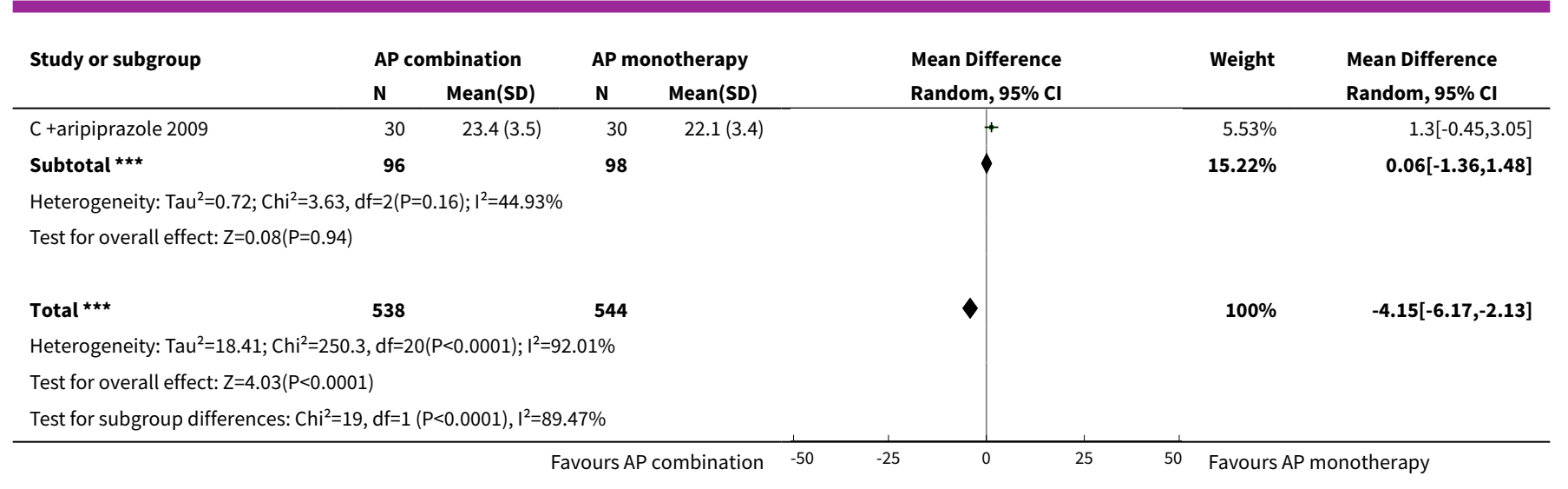

\section{Analysis 1.13. Comparison 1 ANTIPSYCHOTIC COMBINATIONS vs ANTIPSYCHOTIC MONOTHERAPY, Outcome 13 Mental state: 1. Overall - b.ii change total score (BPRS scale, high = bad).}

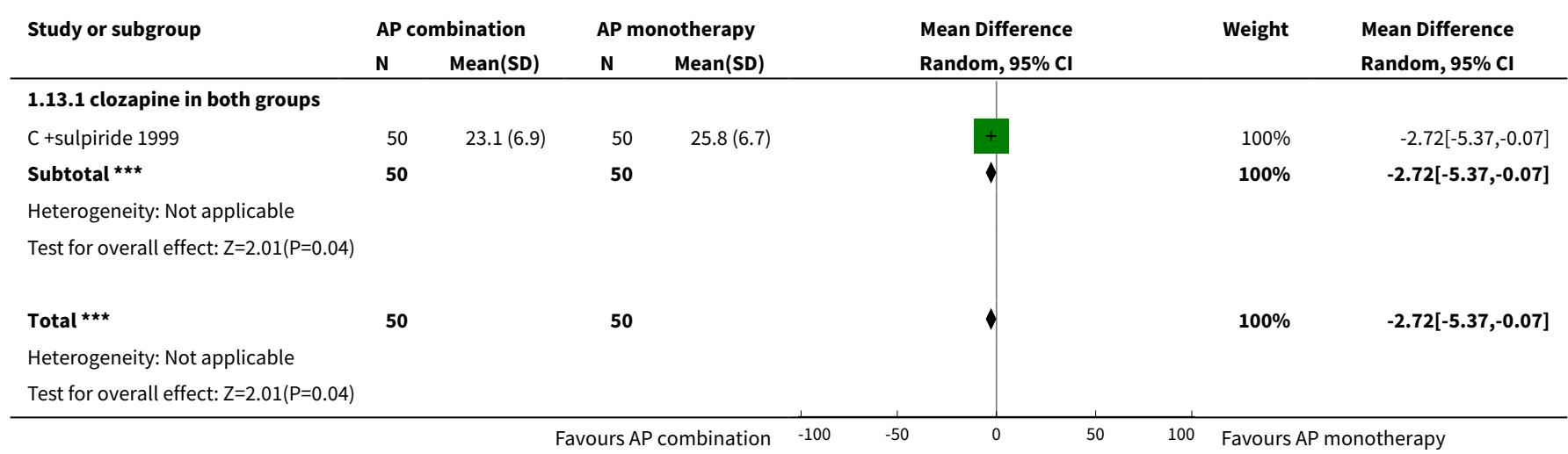

Analysis 1.14. COmparison 1 ANTIPSYCHOTIC COMBINATIONS vs ANTIPSYCHOTIC MONOTHERAPY, Outcome 14 Mental state: 2 . Specific - a. positive symptoms - no clinical improvement.

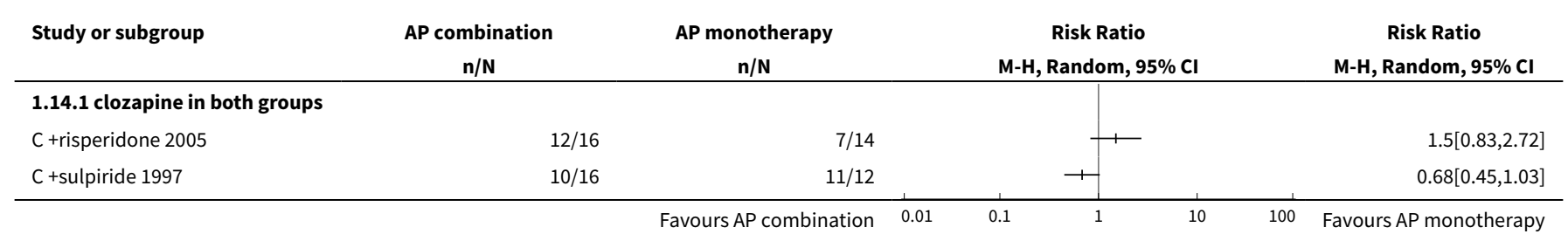

Analysis 1.15. Comparison 1 ANTIPSYCHOTIC COMBINATIONS vs ANTIPSYCHOTIC MONOTHERAPY, Outcome 15 Mental state: 2 . Specific - b. positive symptoms - $i$. average score (PANSS scale, high = bad).

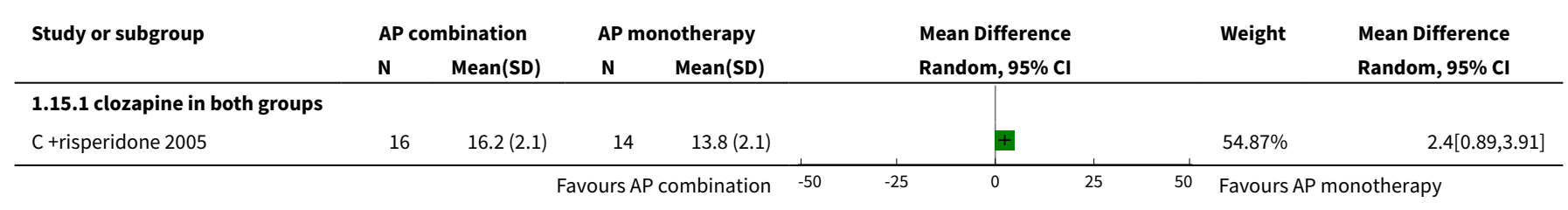




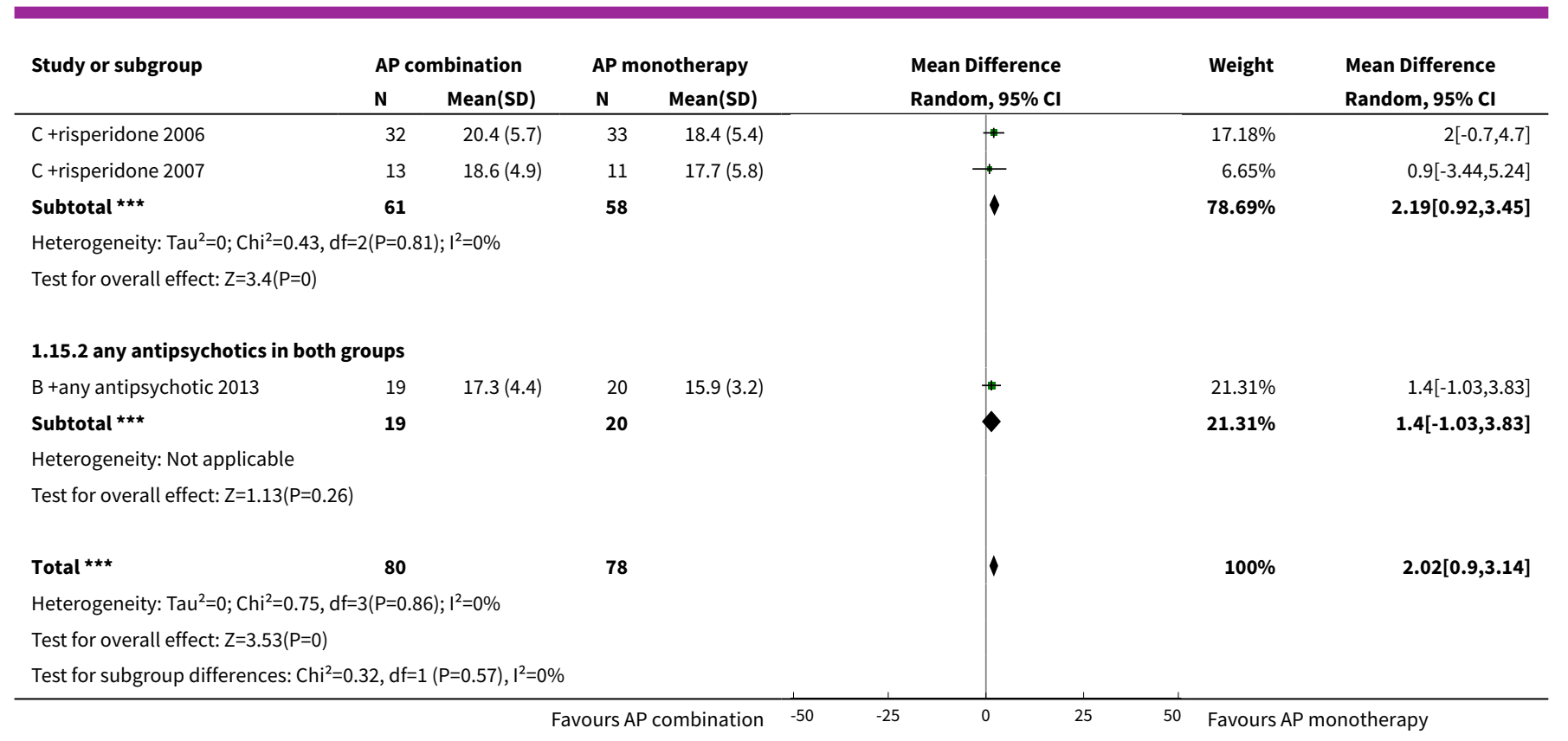

Analysis 1.16. Comparison 1 ANTIPSYCHOTIC COMBINATIONS vS ANTIPSYCHOTIC MONOTHERAPY, Outcome 16 Mental state: 2 . Specific - b. positive symptoms - ii. change score (PANSS scale, high = bad).

\begin{tabular}{|c|c|c|c|c|c|c|c|}
\hline \multirow[t]{2}{*}{ Study or subgroup } & \multicolumn{2}{|c|}{ AP combination } & \multicolumn{2}{|c|}{ AP monotherapy } & \multirow{2}{*}{$\begin{array}{l}\text { Mean Difference } \\
\text { Random, } 95 \% \mathrm{Cl}\end{array}$} & \multirow[t]{2}{*}{ Weight } & \multirow{2}{*}{$\begin{array}{l}\text { Mean Difference } \\
\text { Random, } 95 \% \mathrm{Cl}\end{array}$} \\
\hline & $\mathbf{N}$ & Mean(SD) & $\mathbf{N}$ & Mean(SD) & & & \\
\hline \multicolumn{8}{|c|}{ 1.16.1 clozapine in both groups } \\
\hline B +aripiprazole 2008 & 106 & $-2.2(3.1)$ & 99 & $-1.7(3)$ & 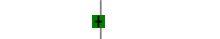 & $30.39 \%$ & $-0.5[-1.33,0.33]$ \\
\hline C+pimozide 2011 & 25 & $-1.3(2.6)$ & 28 & $-1(3)$ & * & $9.44 \%$ & $-0.25[-1.74,1.24]$ \\
\hline C + sertindole 2006 & 25 & $-2(4.8)$ & 25 & $-2(2.4)$ & + & $4.66 \%$ & $0[-2.12,2.12]$ \\
\hline Subtotal $* \star \star$ & 156 & & 152 & & 1 & $44.49 \%$ & $-0.39[-1.08,0.29]$ \\
\hline \multicolumn{8}{|c|}{ Test for overall effect: $Z=1.13(P=0.26)$} \\
\hline \multicolumn{8}{|c|}{ 1.16.2 other atypical drugs in both groups } \\
\hline B +quet/risp 2009 & 160 & $-2.6(3.1)$ & 150 & $-3.1(3)$ & $\Phi$ & $45.96 \%$ & $0.5[-0.18,1.18]$ \\
\hline C +haloperidol 2010 & 46 & $-6.3(6.5)$ & 42 & $-7.2(6)$ & 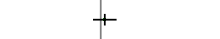 & $3.08 \%$ & $0.9[-1.71,3.51]$ \\
\hline C +olan/risp 2014 & 25 & $-9.6(8.1)$ & 26 & $-6.5(6.3)$ & + & $1.31 \%$ & $-3.07[-7.08,0.94]$ \\
\hline C +sulpiride 2004 & 8 & $-8.4(6)$ & 8 & $-6.6(7.9)$ & $\longrightarrow$ & $0.44 \%$ & $-1.8[-8.67,5.07]$ \\
\hline C +sulpiride 2013 & 46 & $-6.4(5.8)$ & 46 & $-5.8(5)$ & + & $4.29 \%$ & $-0.6[-2.81,1.61]$ \\
\hline 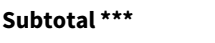 & 298 & & 285 & & 1 & $55.51 \%$ & $0.33[-0.28,0.95]$ \\
\hline \multicolumn{8}{|c|}{ Heterogeneity: $\operatorname{Tau}^{2}=0 ; \mathrm{Chi}^{2}=4.25, \mathrm{df}=5(\mathrm{P}=0.51) ; \mathrm{I}^{2}=0 \%$} \\
\hline \multicolumn{8}{|c|}{ Test for overall effect: $Z=1.05(P=0.29)$} \\
\hline 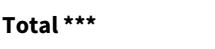 & 454 & & 437 & & 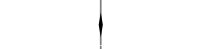 & $100 \%$ & $0.01[-0.45,0.47]$ \\
\hline \multicolumn{8}{|c|}{ Heterogeneity: $\mathrm{Tau}^{2}=0 ; \mathrm{Chi}^{2}=6.85, \mathrm{df}=8(\mathrm{P}=0.55) ; \mathrm{I}^{2}=0 \%$} \\
\hline \multicolumn{8}{|c|}{ Test for overall effect: $Z=0.03(P=0.97)$} \\
\hline Test for subgroup diff & $8, \mathrm{df}=$ & $P=0.12), I^{2}=5$ & & & & & \\
\hline
\end{tabular}


Analysis 1.17. Comparison 1 ANTIPSYCHOTIC COMBINATIONS vs ANTIPSYCHOTIC MONOTHERAPY, Outcome 17 Mental state: 2 . Specific - b. positive symptoms - iii. average score (BPRS scale, high = bad).

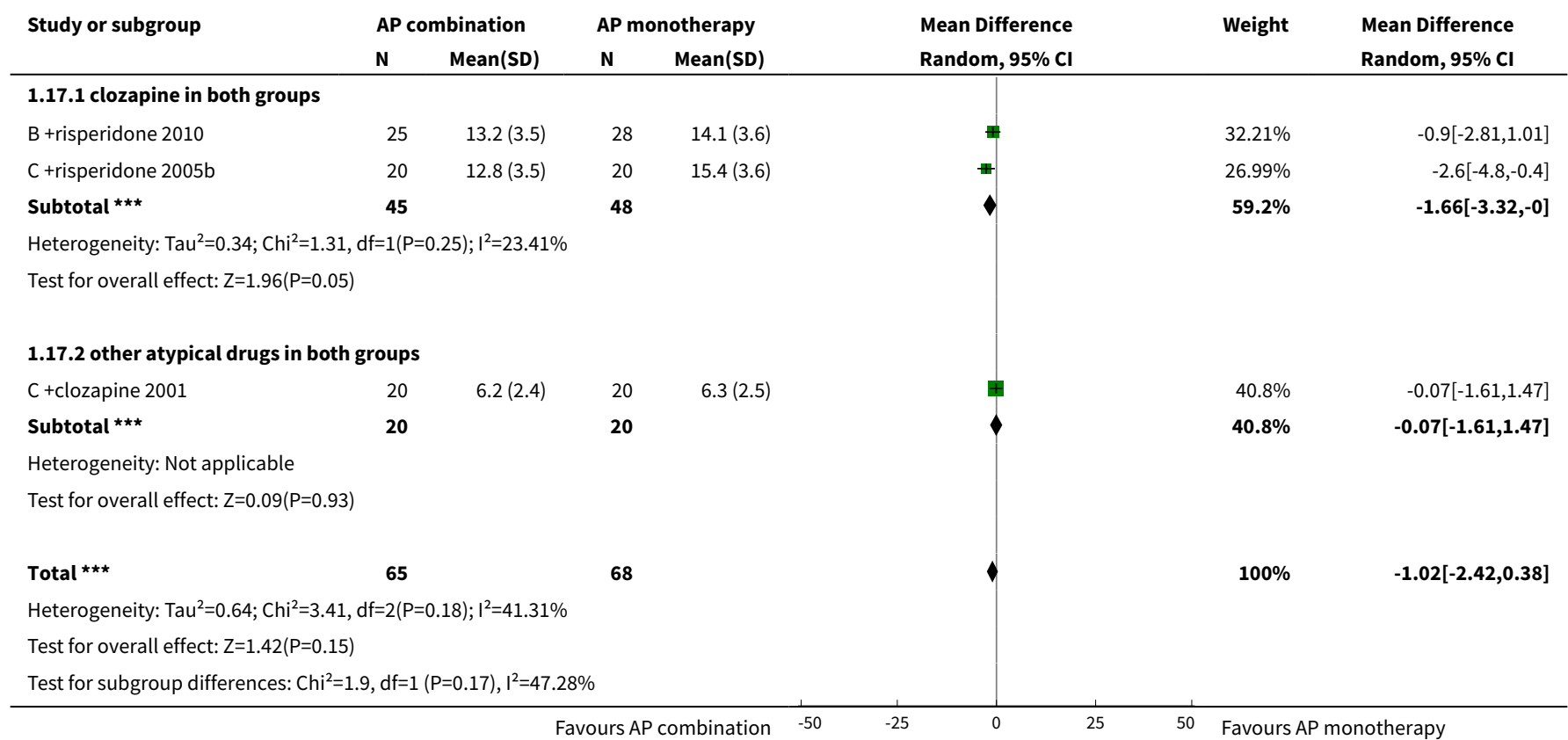

Analysis 1.18. Comparison 1 ANTIPSYCHOTIC COMBINATIONS vS ANTIPSYCHOTIC MONOTHERAPY, Outcome 18 Mental state: 2 . Specific - b. positive symptoms - iv. change data (BPRS scale, high = bad).

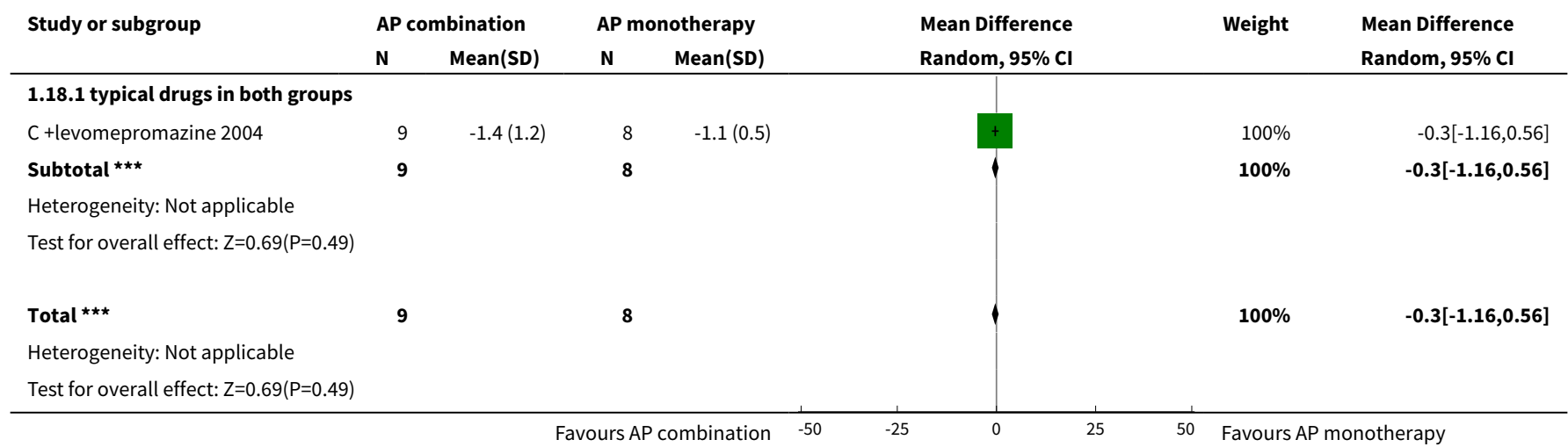

Analysis 1.19. Comparison 1 ANTIPSYCHOTIC COMBINATIONS vs ANTIPSYCHOTIC MONOTHERAPY, Outcome 19 Mental state: 2 . Specific - b. positive symptoms - v. average score (SAPS scale, high = bad).

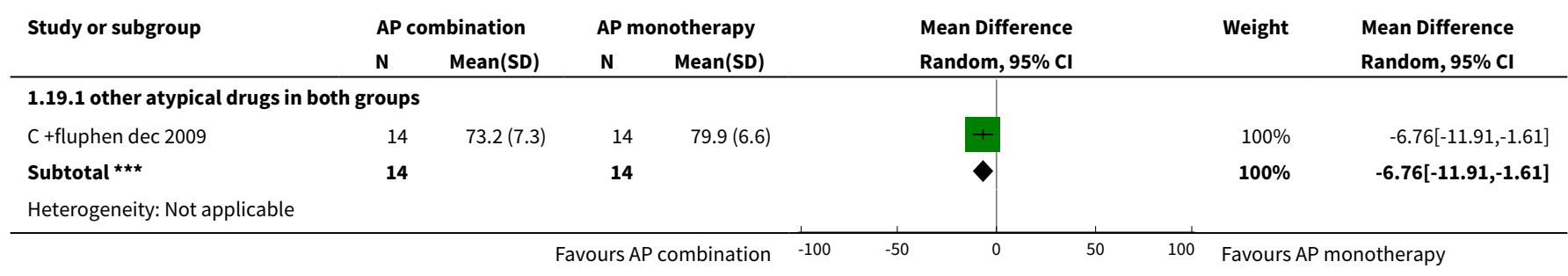




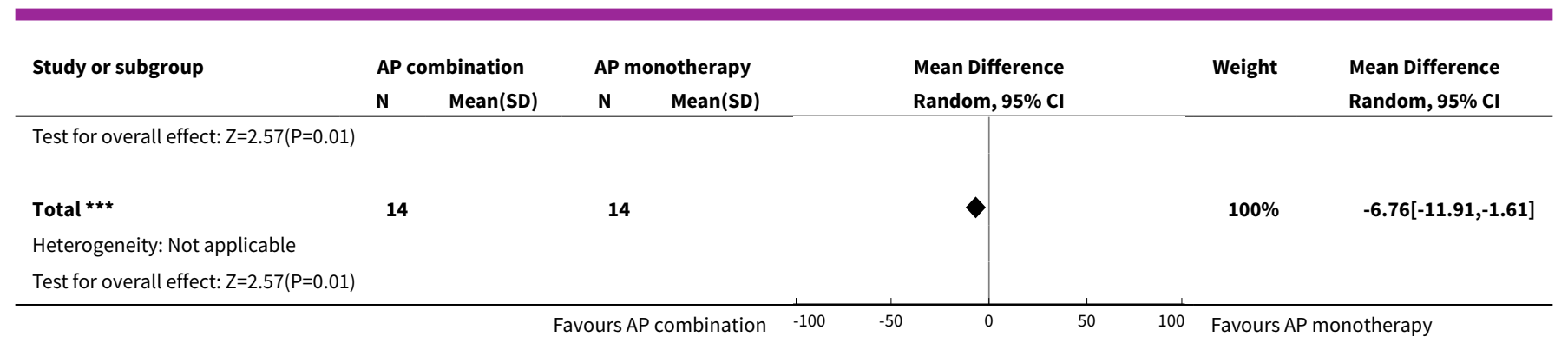

Analysis 1.20. Comparison 1 ANTIPSYCHOTIC COMBINATIONS vs ANTIPSYCHOTIC MONOTHERAPY, Outcome 20 Mental state: 2 . Specific - b. positive symptoms - vi. change score (SAPS scale, high = bad).

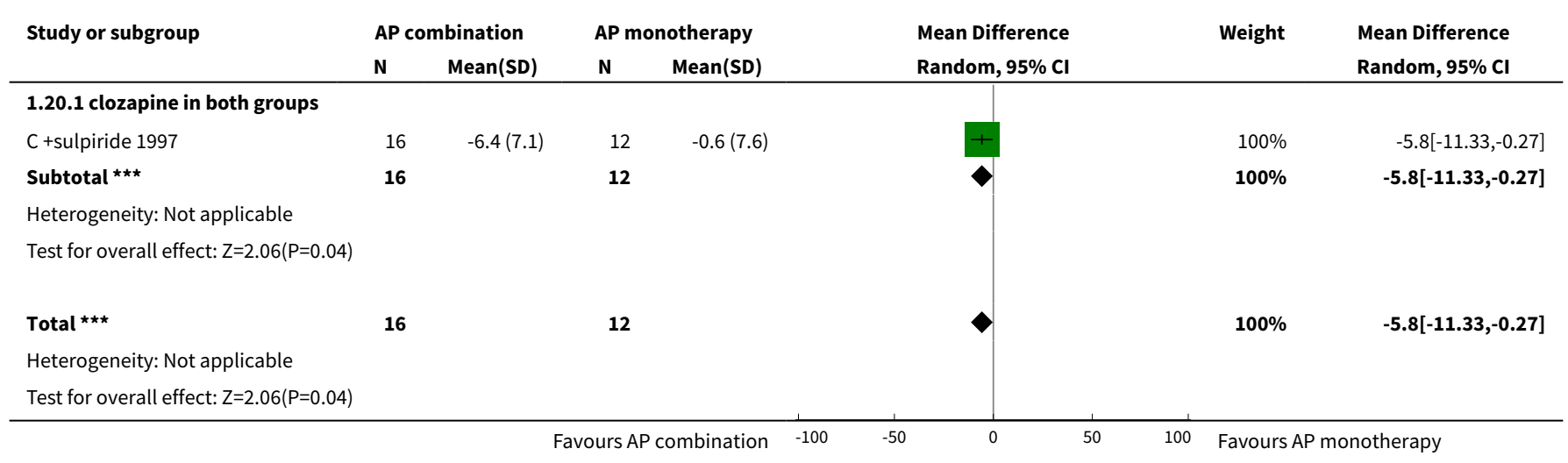

Analysis 1.21. Comparison 1 ANTIPSYCHOTIC COMBINATIONS vS ANTIPSYCHOTIC MONOTHERAPY, Outcome 21 Mental state: 3 . Specific - a. negative symptoms - no clinical improvement.

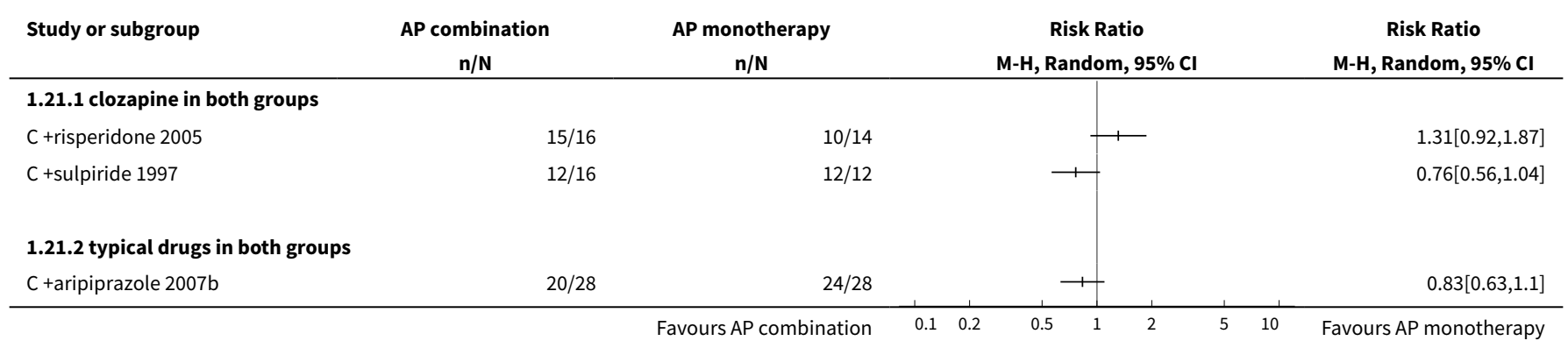

Analysis 1.22. Comparison 1 ANTIPSYCHOTIC COMBINATIONS vS ANTIPSYCHOTIC MONOTHERAPY, Outcome 22 Mental state: 3 . Specific - b. negative symptoms - $i$. average score (PANSS scale, high = bad).

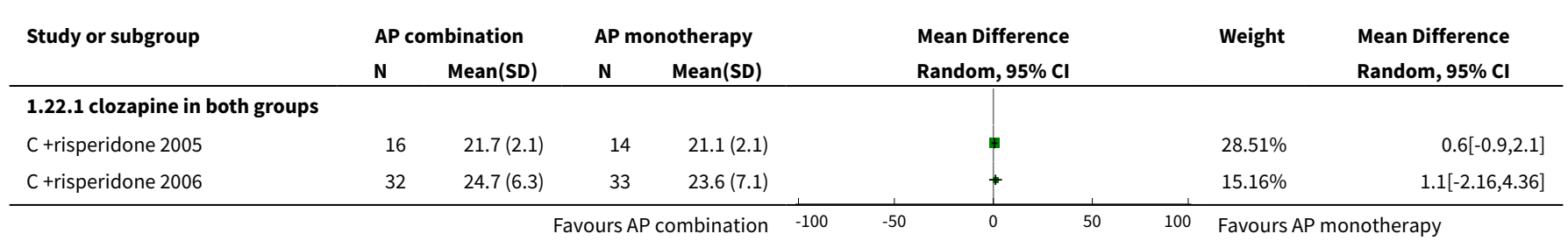




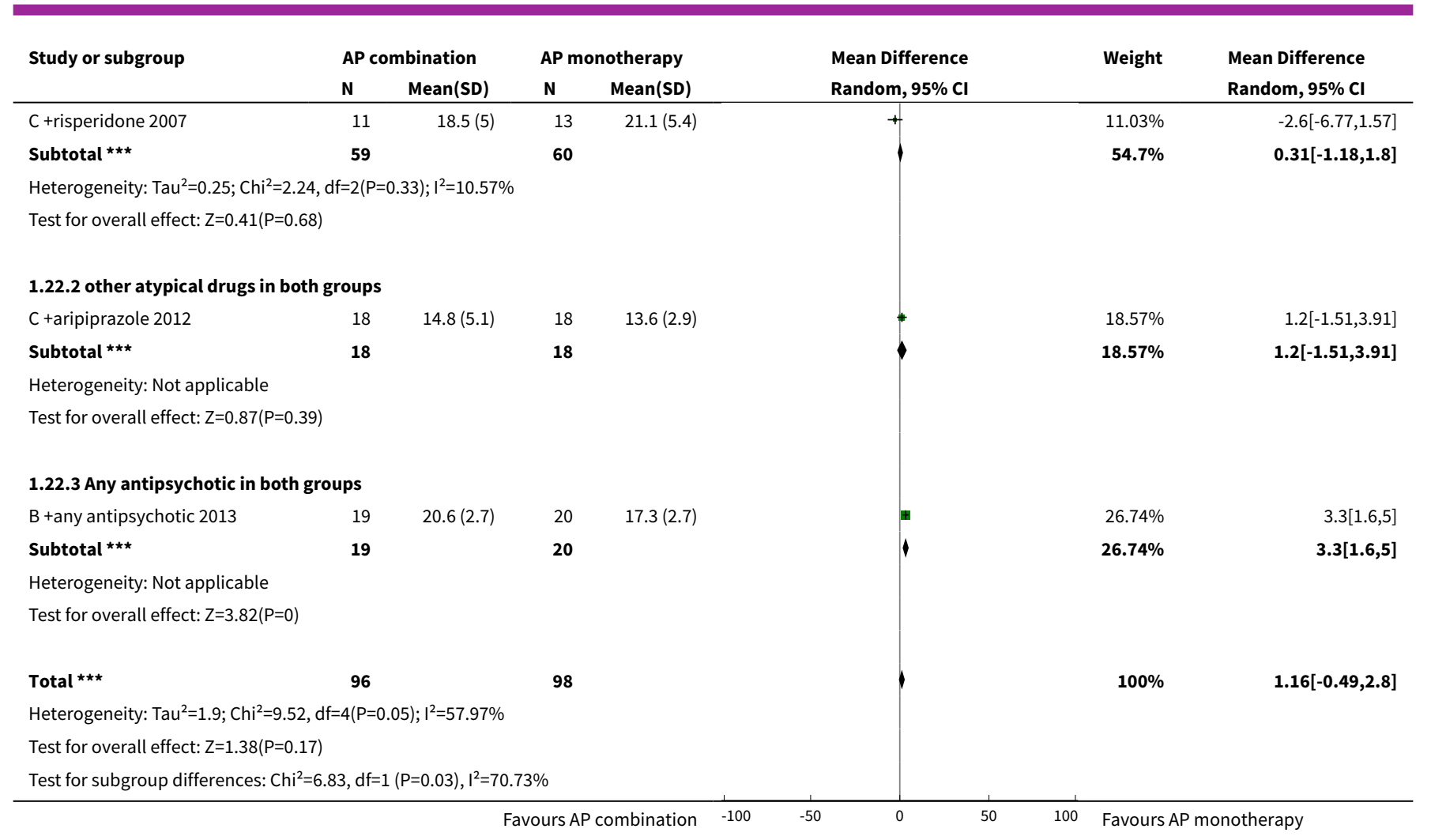

Analysis 1.23. Comparison 1 ANTIPSYCHOTIC COMBINATIONS vS ANTIPSYCHOTIC MONOTHERAPY, Outcome 23 Mental state: 3. Specific - b. negative symptoms - ii. change score (PANSS scale, high = bad).

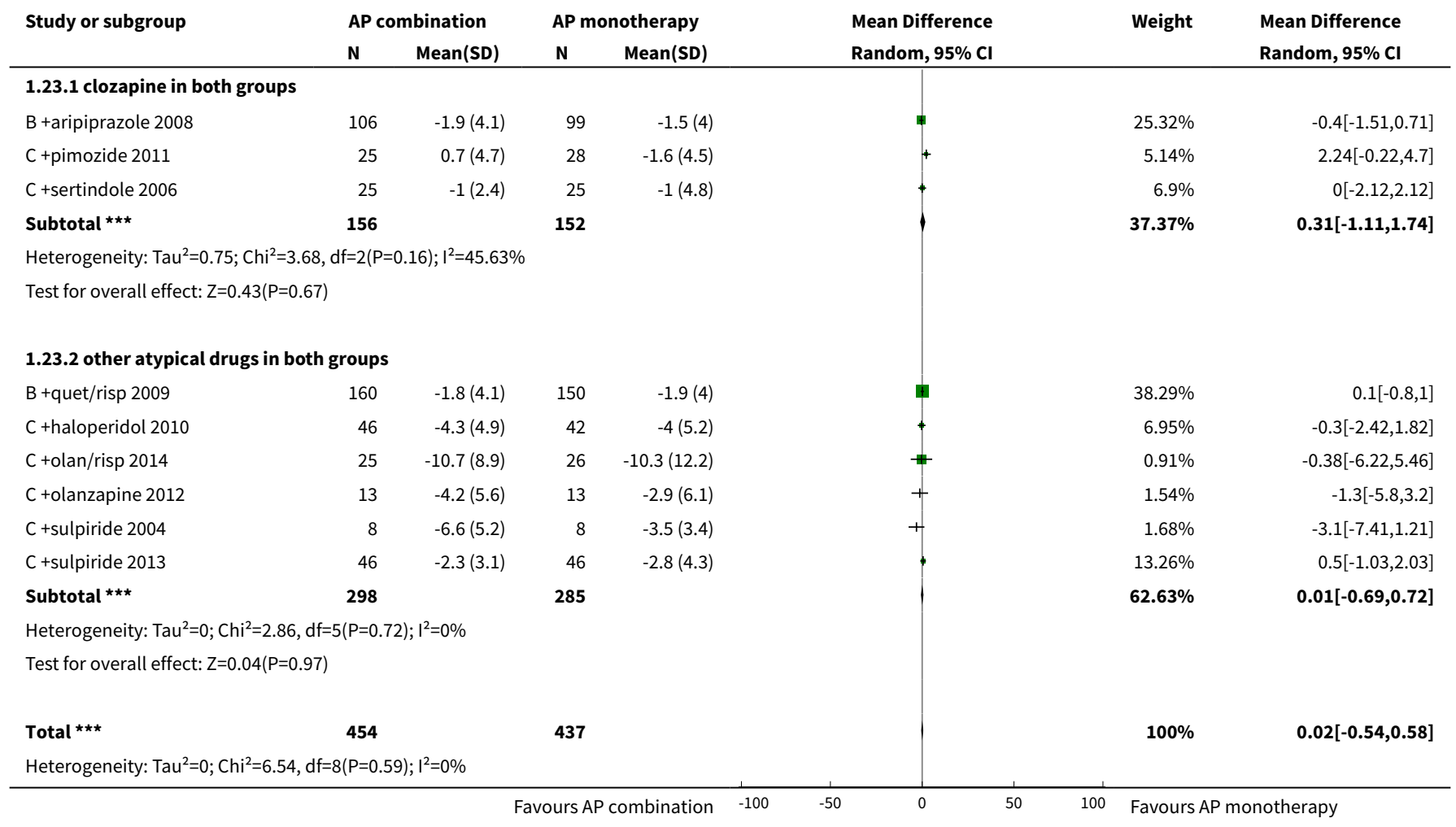




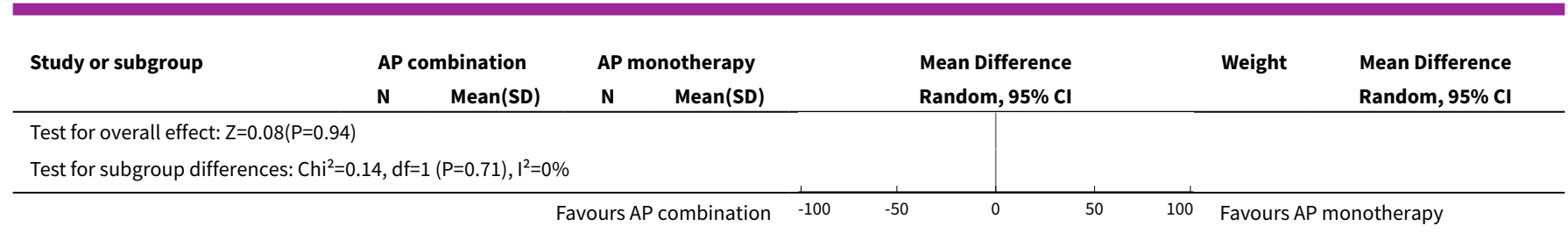

Analysis 1.24. Comparison 1 ANTIPSYCHOTIC COMBINATIONS vs ANTIPSYCHOTIC MONOTHERAPY, Outcome 24 Mental state: 3. Specific - b. negative symptoms - iii. average score (BPRS scale, high = bad).

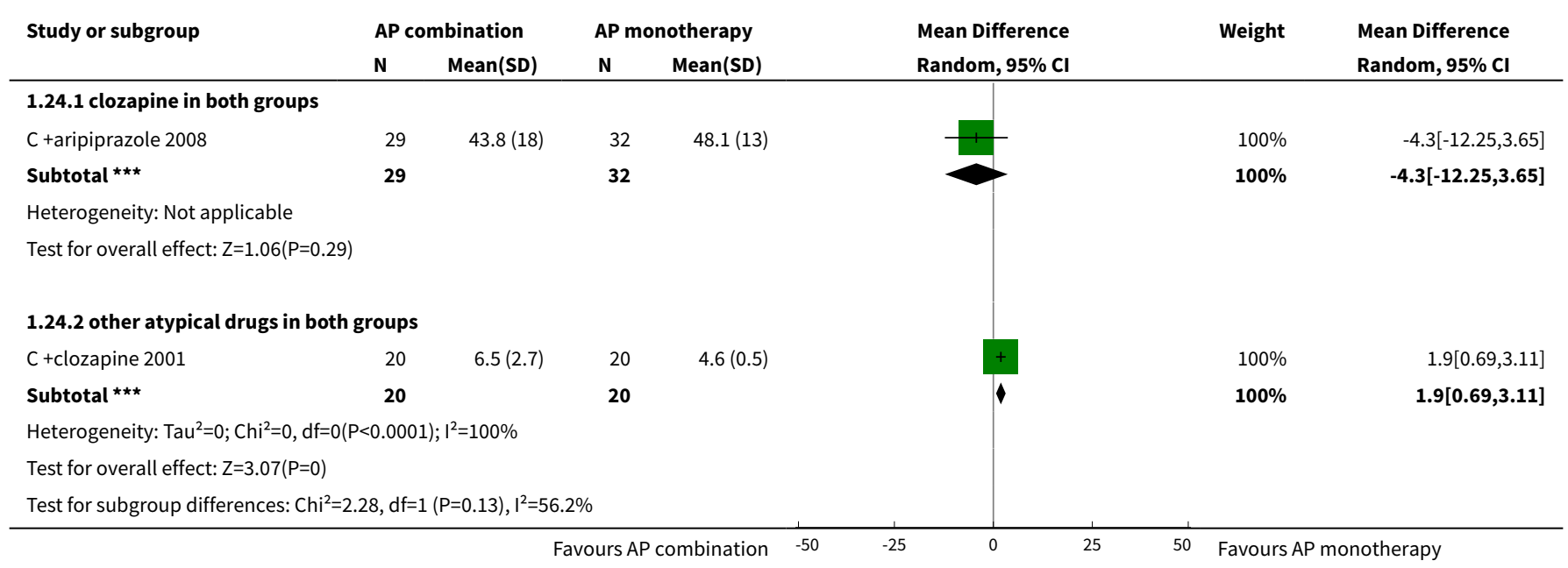

Analysis 1.25. Comparison 1 ANTIPSYCHOTIC COMBINATIONS vs ANTIPSYCHOTIC MONOTHERAPY, Outcome 25 Mental state: 3. Specific - b. negative symptoms - iv. change score (BPRS scale, high = bad).

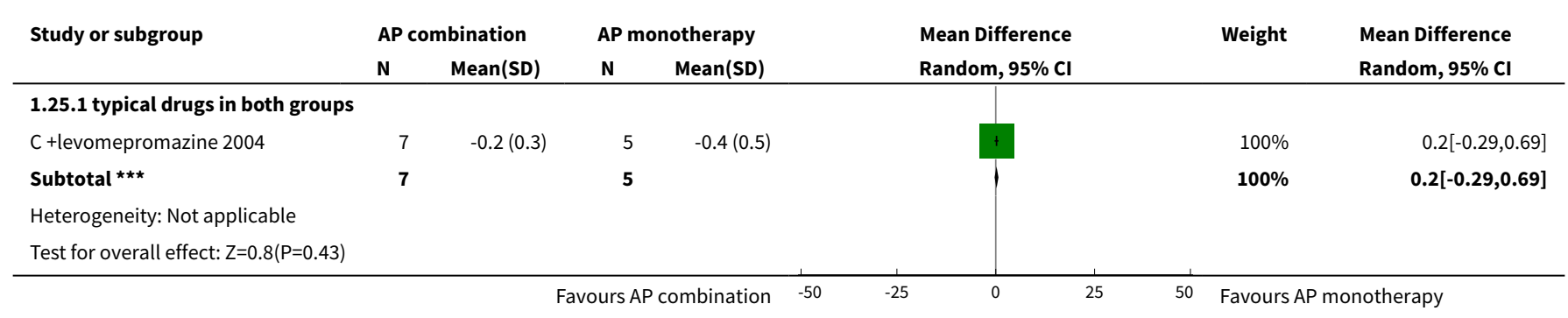

Analysis 1.26. Comparison 1 ANTIPSYCHOTIC COMBINATIONS vS ANTIPSYCHOTIC MONOTHERAPY, Outcome 26 Mental state: 3. Specific - b. negative symptoms - v. average score (SANS scale, high = bad).

\begin{tabular}{|c|c|c|c|c|c|c|}
\hline \multirow[t]{2}{*}{ Study or subgroup } & \multicolumn{2}{|c|}{ AP combination } & \multicolumn{2}{|c|}{ AP monotherapy } & \multirow{2}{*}{$\begin{array}{l}\text { Mean Difference } \\
\text { Random, } 95 \% \mathrm{Cl}\end{array}$} & \multirow{2}{*}{$\begin{array}{l}\text { Mean Difference } \\
\text { Random, 95\% Cl }\end{array}$} \\
\hline & $\mathbf{N}$ & Mean(SD) & $\mathbf{N}$ & Mean(SD) & & \\
\hline \multicolumn{7}{|c|}{ 1.26.1 clozapine in both groups } \\
\hline B +risperidone 2010 & 25 & $31.3(11.9)$ & 28 & $34.4(14.8)$ & + & $-3.1[-10.3,4.1]$ \\
\hline B +sulpiride 1996 & 31 & $17.8(3)$ & 32 & $31(3.8)$ & + & $-13.2[-14.89,-11.51]$ \\
\hline C +aripiprazole 2008 & 29 & $43.8(18)$ & 32 & $48.1(13)$ & + & $-4.3[-12.25,3.65]$ \\
\hline
\end{tabular}




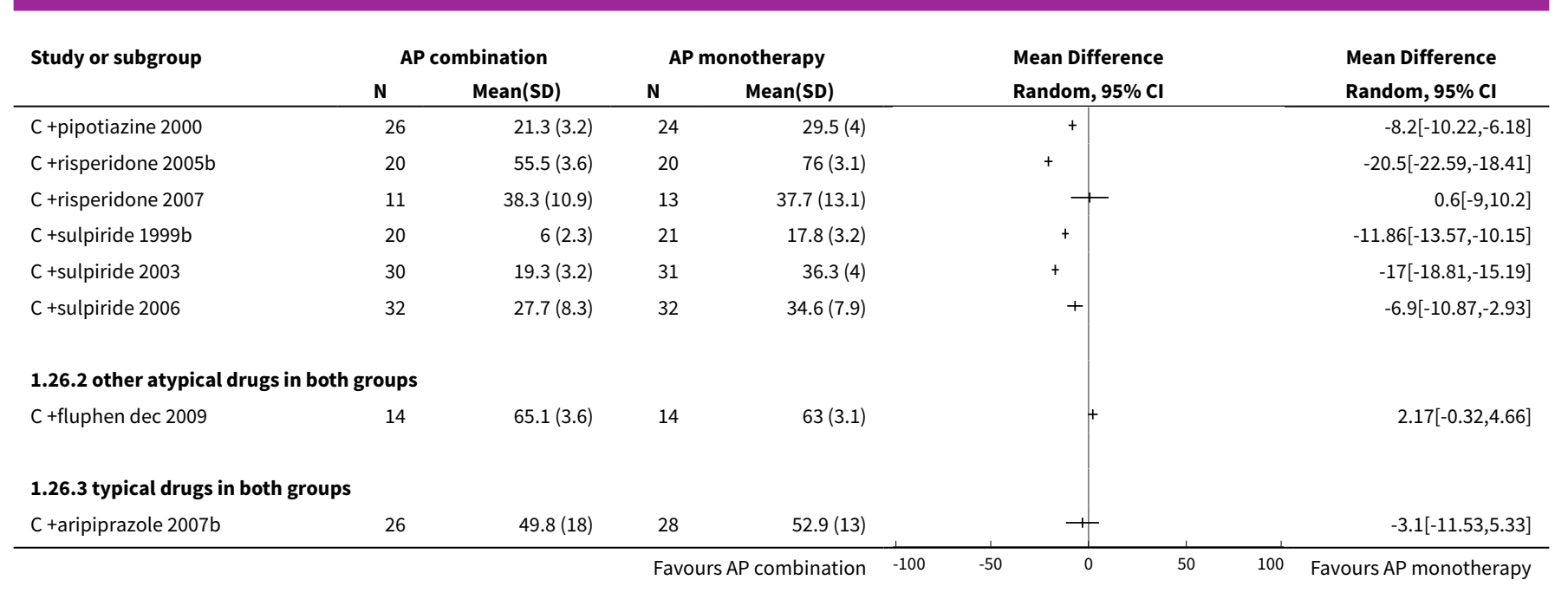

Analysis 1.27. Comparison 1 ANTIPSYCHOTIC COMBINATIONS vS ANTIPSYCHOTIC MONOTHERAPY, Outcome 27 Mental state: 3. Specific - b. negative symptoms - vi. average score (SANS scale, high = bad).

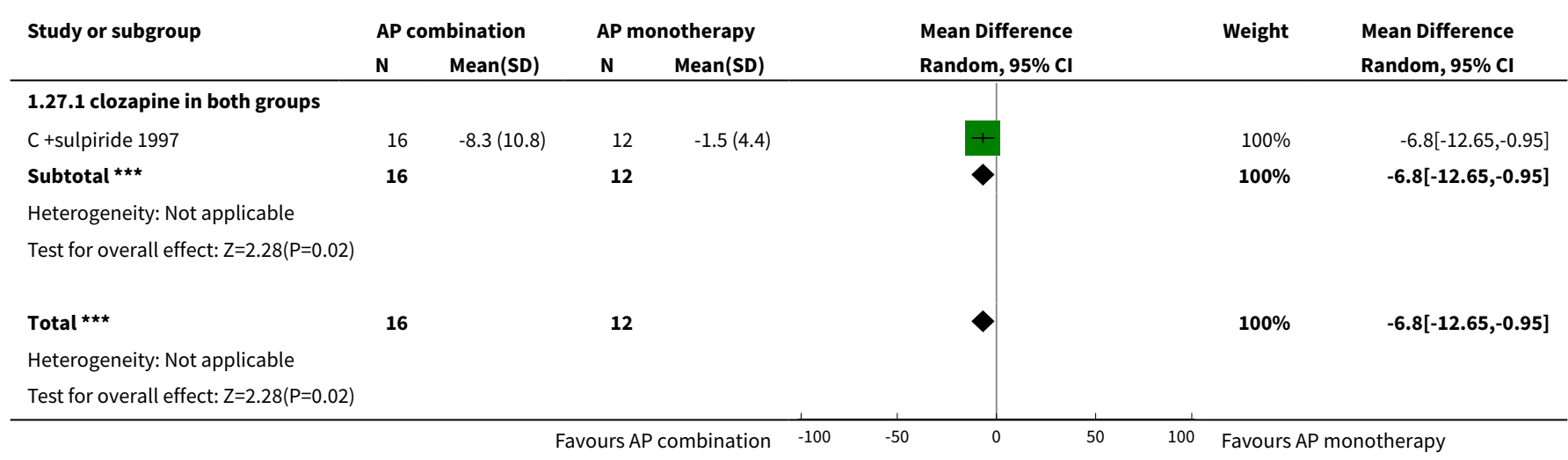

Analysis 1.28. Comparison 1 ANTIPSYCHOTIC COMBINATIONS vs ANTIPSYCHOTIC MONOTHERAPY, Outcome 28 Mental state: 4. Specific - aggression/agitation - average score (BPRS scale, high = bad).

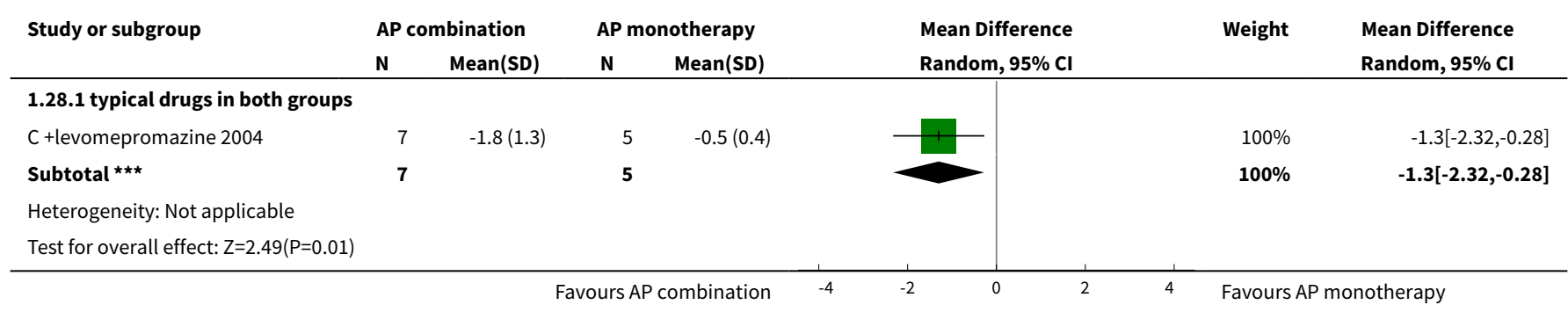


Analysis 1.29. Comparison 1 ANTIPSYCHOTIC COMBINATIONS vS ANTIPSYCHOTIC MONOTHERAPY, Outcome 29 Adverse events: 1. General - a. serious event or requiring discontinuation.

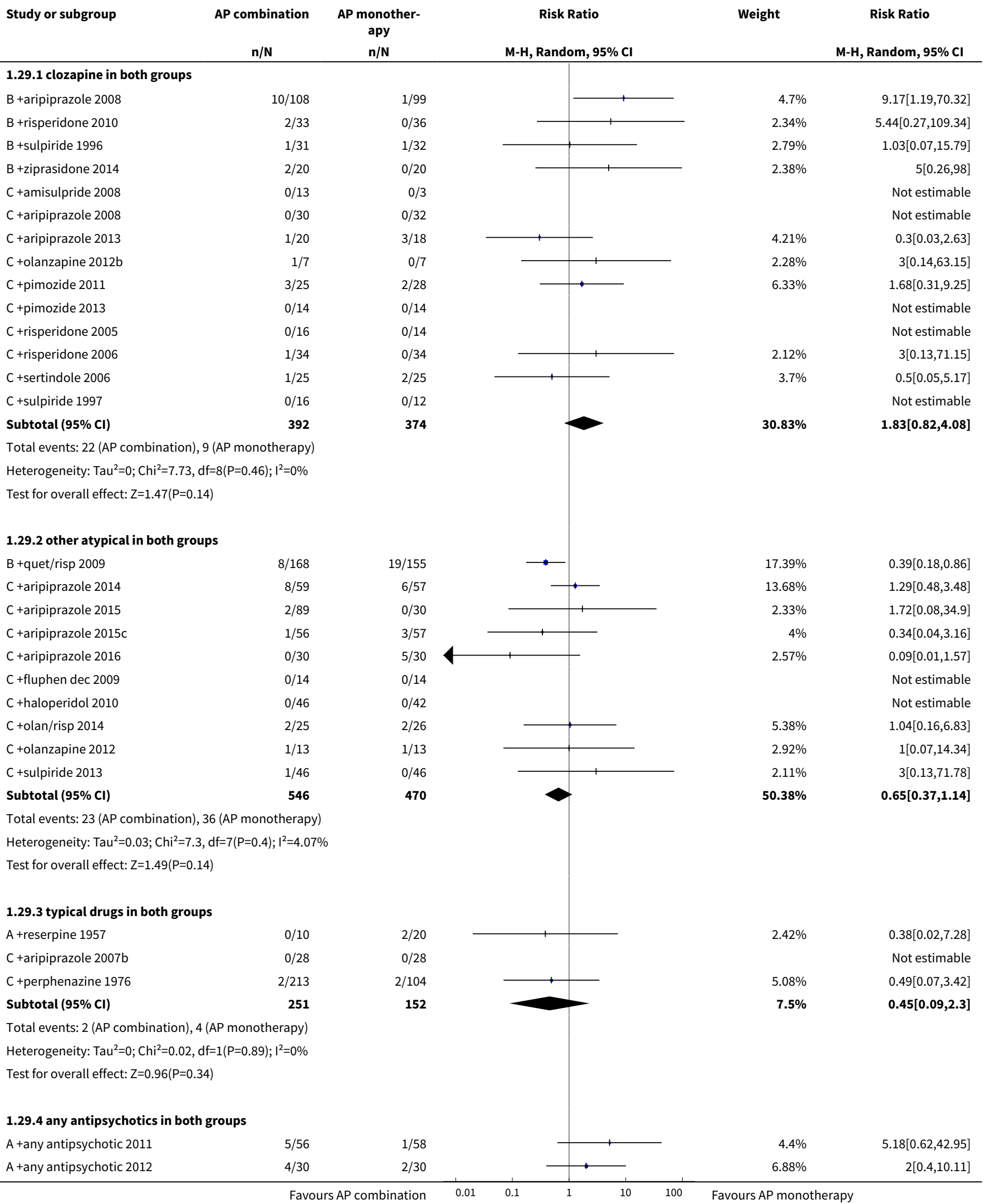




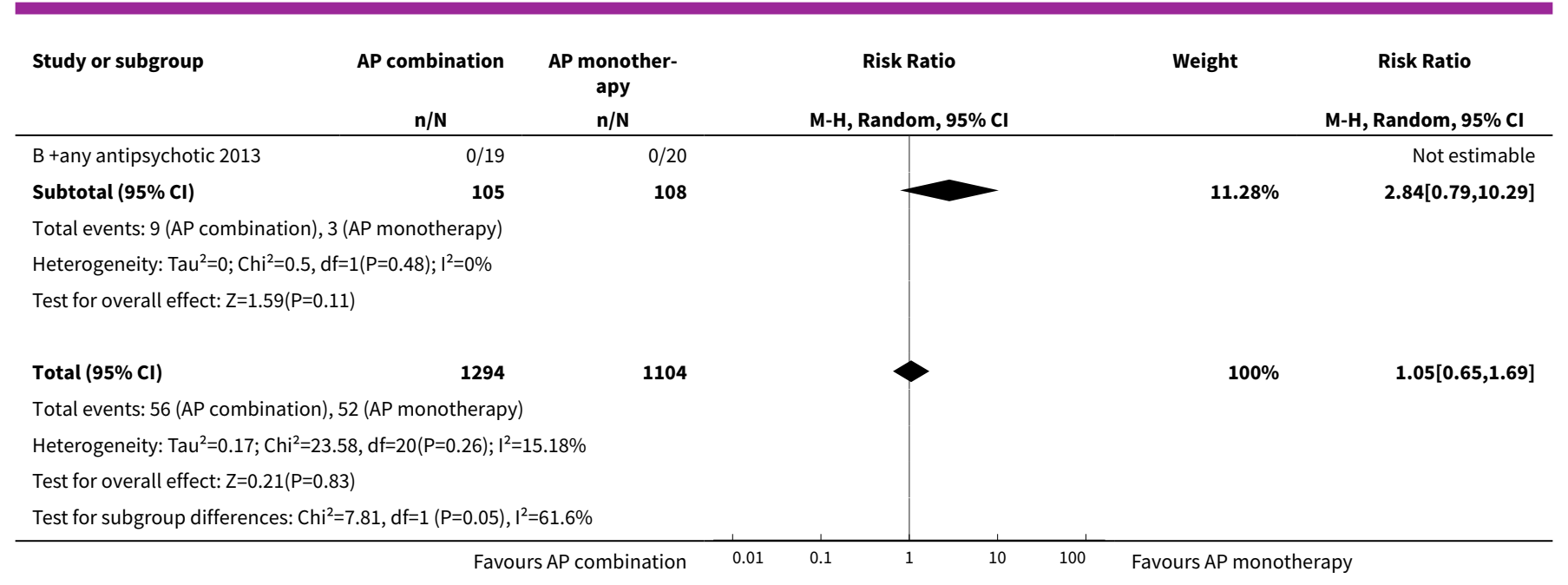

\section{Analysis 1.30. Comparison 1 ANTIPSYCHOTIC COMBINATIONS vs ANTIPSYCHOTIC MONOTHERAPY, Outcome 30 Adverse events: 1 . General - b. death (suicide or non-suicide deaths).}

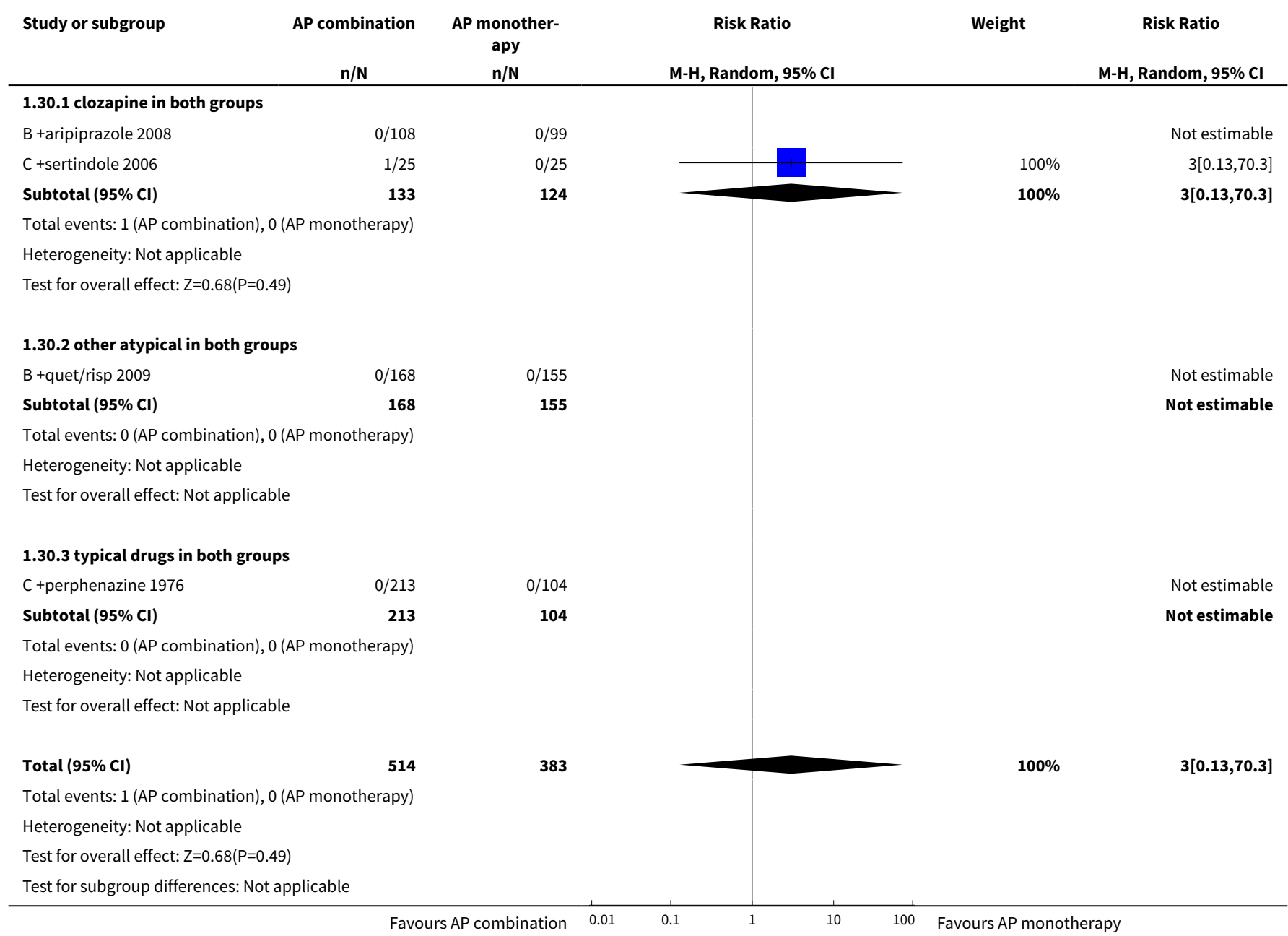


Analysis 1.31. Comparison 1 ANTIPSYCHOTIC COMBINATIONS vs ANTIPSYCHOTIC MONOTHERAPY, Outcome 31 Adverse events: 2. Movement disorders - a. any.

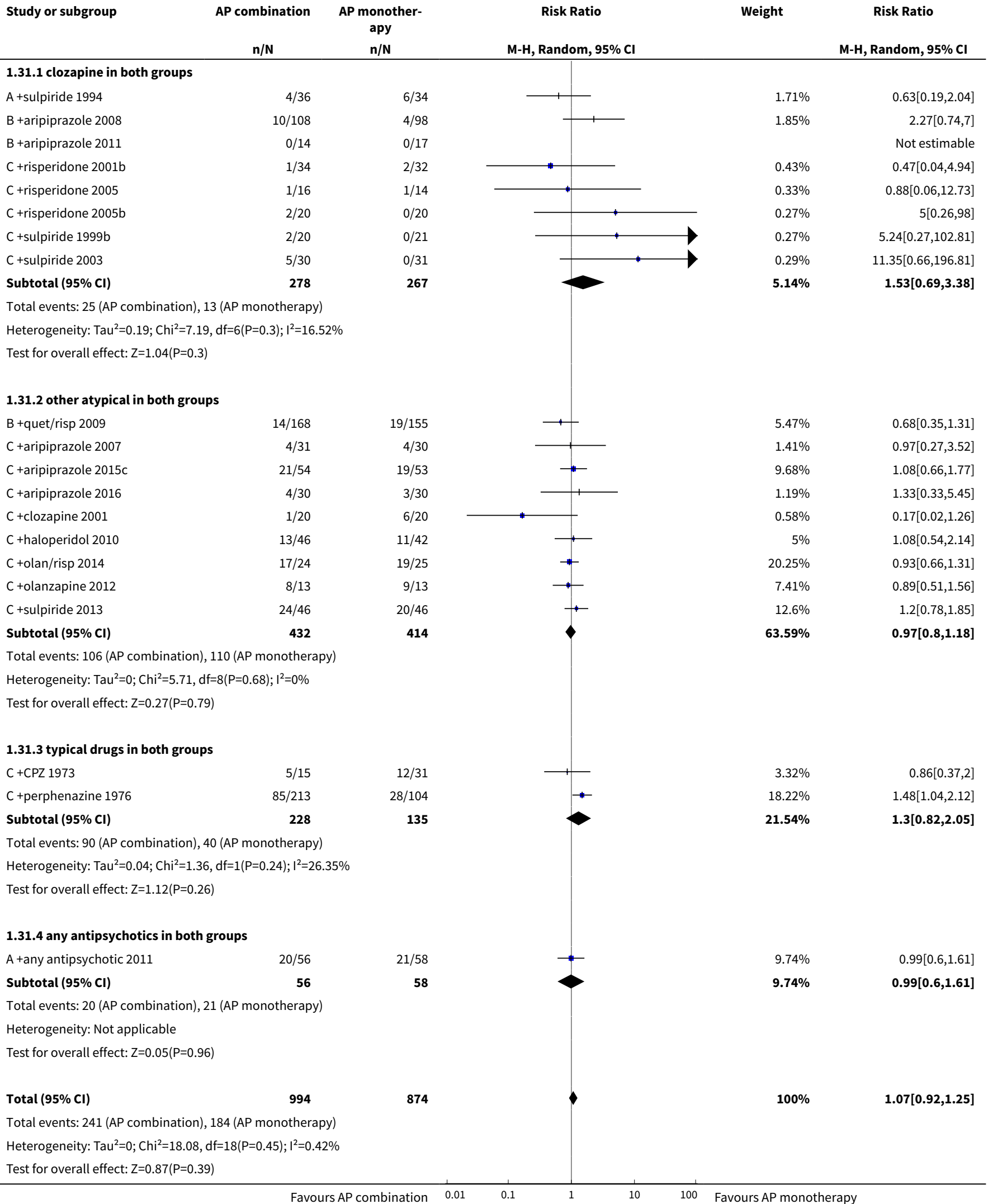




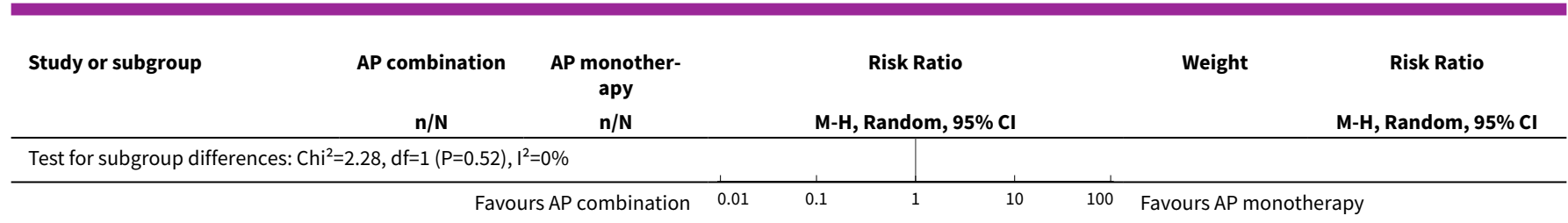

Analysis 1.32. Comparison 1 ANTIPSYCHOTIC COMBINATIONS vs ANTIPSYCHOTIC MONOTHERAPY, Outcome 32 Adverse events: 2. Movement disorders - b.i. average scores (SAS, high = bad).

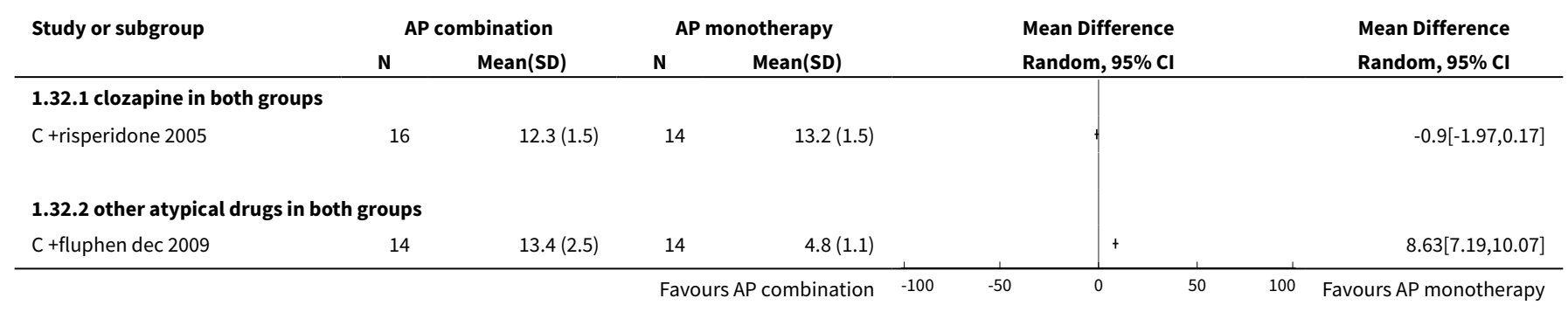

Analysis 1.33. Comparison 1 ANTIPSYCHOTIC COMBINATIONS VS ANTIPSYCHOTIC MONOTHERAPY, Outcome 33 Adverse events: 2. Movement disorders - b.ii. change scores (SAS, high = bad).

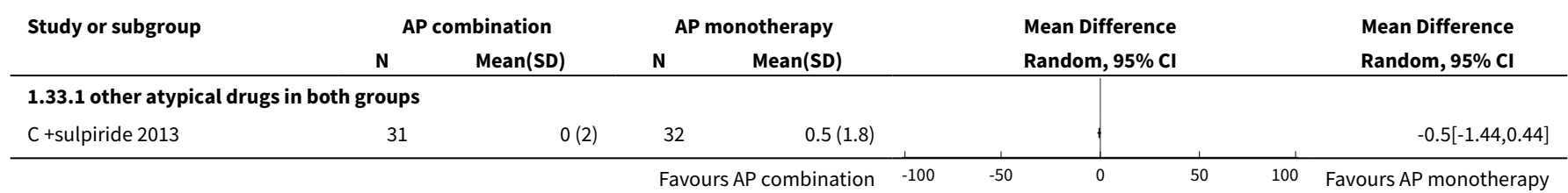

Analysis 1.34. Comparison 1 ANTIPSYCHOTIC COMBINATIONS vs ANTIPSYCHOTIC MONOTHERAPY, Outcome 34 Adverse events: 2 . Movement disorders - b.iii. average scores (TESS, high = bad).

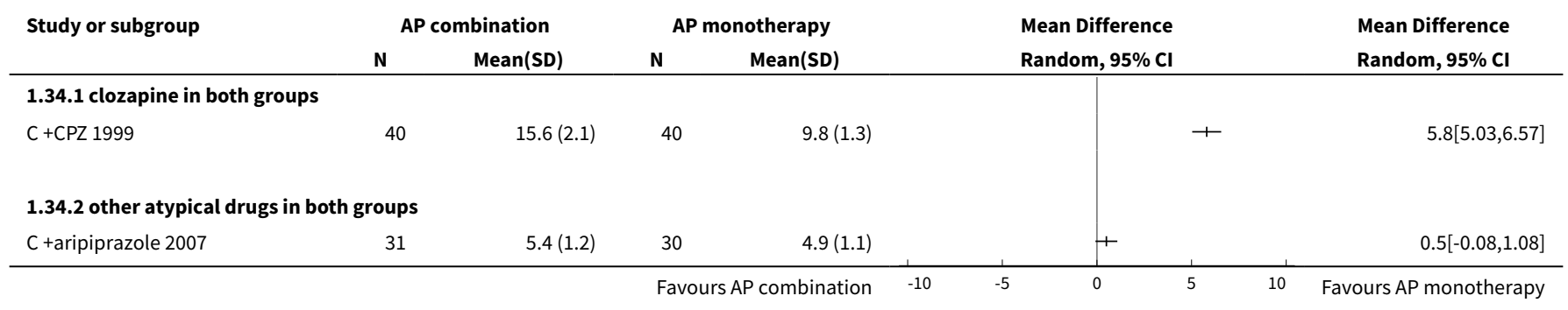


Analysis 1.35. Comparison 1 ANTIPSYCHOTIC COMBINATIONS vs ANTIPSYCHOTIC MONOTHERAPY, Outcome 35 Adverse events: 2. Movement disorders - b.iv. average scores (DIEPSS, high = bad).

\begin{tabular}{|c|c|c|c|c|c|c|c|}
\hline \multirow[t]{2}{*}{ Study or subgroup } & \multicolumn{2}{|c|}{ AP combination } & \multicolumn{2}{|c|}{ AP monotherapy } & \multirow{2}{*}{$\begin{array}{l}\text { Mean Difference } \\
\text { Random, } 95 \% \mathrm{Cl}\end{array}$} & \multirow[t]{2}{*}{ Weight } & \multirow{2}{*}{$\begin{array}{l}\text { Mean Difference } \\
\text { Random, } 95 \% \mathrm{Cl}\end{array}$} \\
\hline & $\mathbf{N}$ & $\operatorname{Mean}(S D)$ & $\mathbf{N}$ & $\operatorname{Mean}(S D)$ & & & \\
\hline \multicolumn{8}{|l|}{ 1.35.1 clozapine in both groups } \\
\hline C +aripiprazole 2008 & 29 & $3.6(1.9)$ & 32 & $3.3(1.1)$ & & $100 \%$ & $0.3[-0.49,1.09]$ \\
\hline Subtotal $* \star \star$ & 29 & & 32 & & & $100 \%$ & $0.3[-0.49,1.09]$ \\
\hline \multicolumn{8}{|l|}{ Heterogeneity: Not applicable } \\
\hline \multicolumn{8}{|l|}{ Test for overall effect: $Z=0.74(P=0.46)$} \\
\hline Total $\star \star \star ~$ & 29 & & 32 & & & $100 \%$ & $0.3[-0.49,1.09]$ \\
\hline \multicolumn{8}{|l|}{ Heterogeneity: Not applicable } \\
\hline \multicolumn{8}{|l|}{ Test for overall effect: $Z=0.74(P=0.46)$} \\
\hline & & & urs $A$ & mbination & -2 & Favours & notherapy \\
\hline
\end{tabular}

\section{Analysis 1.36. Comparison 1 ANTIPSYCHOTIC COMBINATIONS vs ANTIPSYCHOTIC MONOTHERAPY,} Outcome 36 Adverse events: 2 . Movement disorders - b.v. change scores (BAS, high = bad).

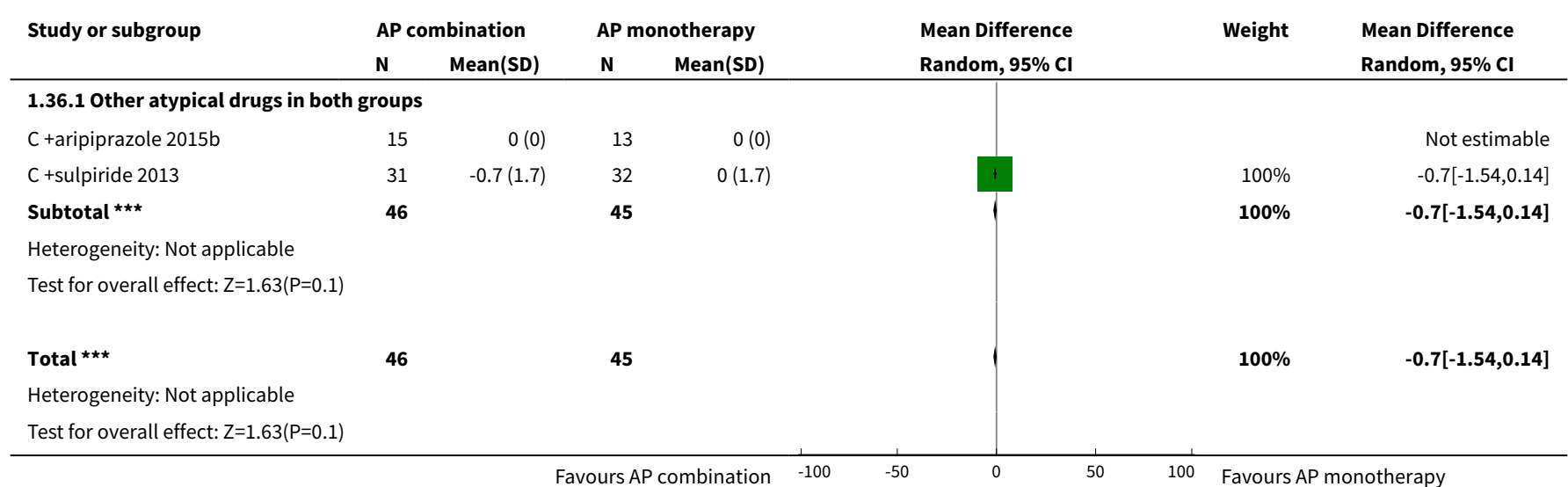

\section{Analysis 1.37. Comparison 1 ANTIPSYCHOTIC COMBINATIONS vs ANTIPSYCHOTIC MONOTHERAPY,} Outcome 37 Adverse events: 2. Movement disorders - b.vi. change scores (AIMS, high = bad).

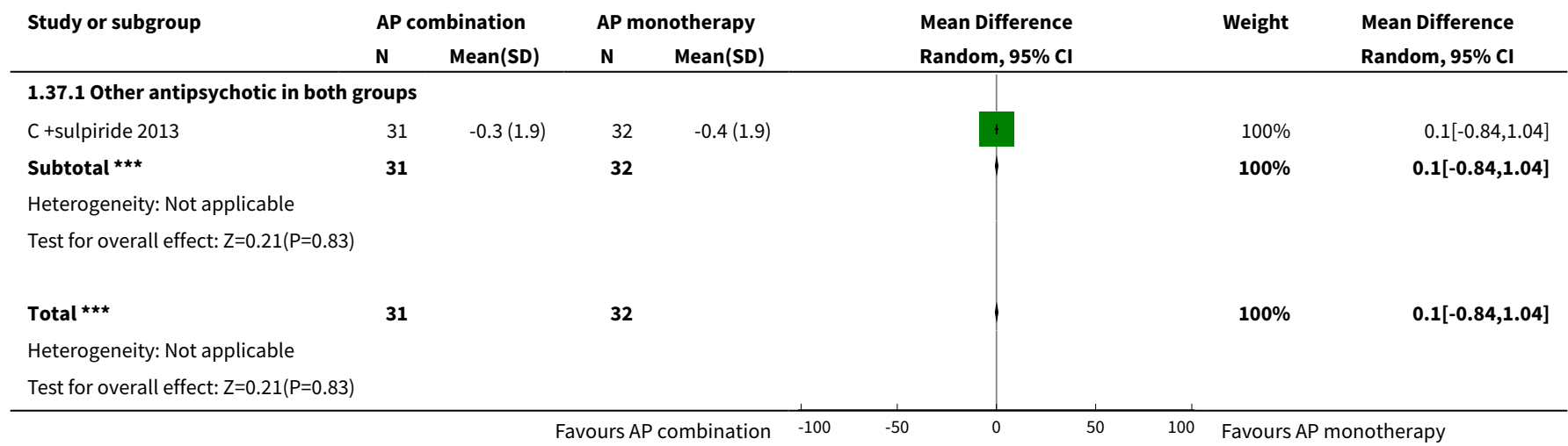


Analysis 1.38. Comparison 1 ANTIPSYCHOTIC COMBINATIONS vs ANTIPSYCHOTIC MONOTHERAPY, Outcome 38 Adverse events: 3 . Endocrine - prolactin level (high = bad).

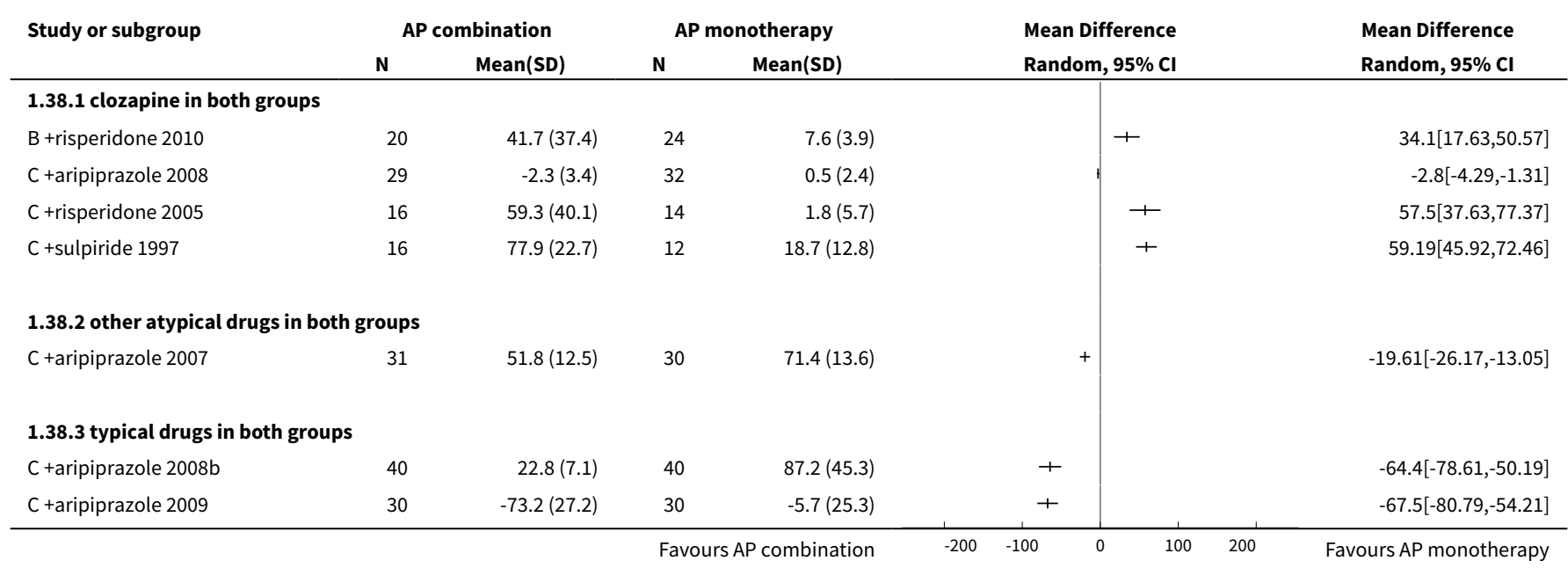

Analysis 1.39. Comparison 1 ANTIPSYCHOTIC COMBINATIONS vS ANTIPSYCHOTIC MONOTHERAPY, Outcome 39 Adverse events: 4. Metabolic - a. weight gain (binary).

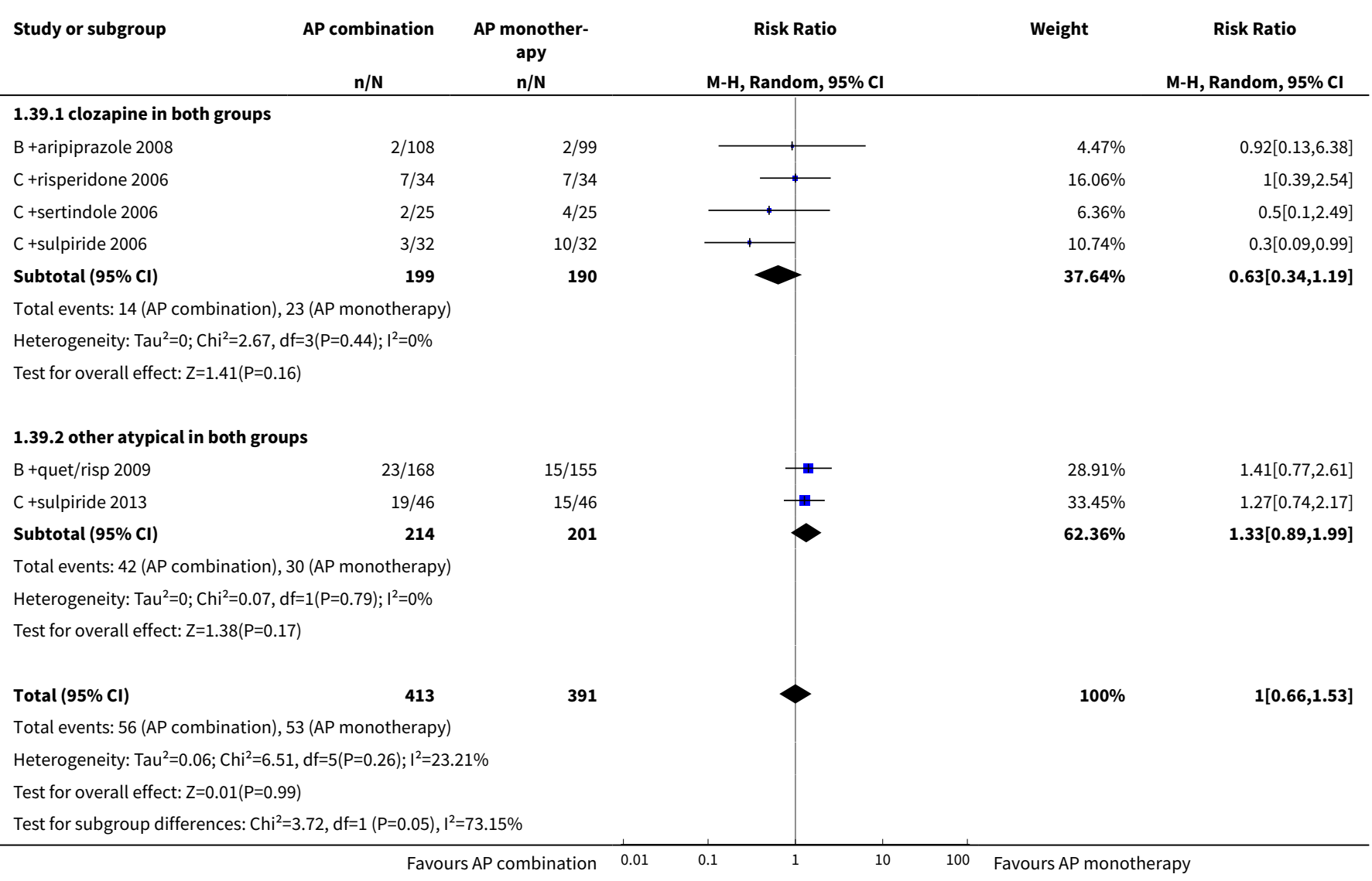


Analysis 1.40. Comparison 1 ANTIPSYCHOTIC COMBINATIONS vs ANTIPSYCHOTIC MONOTHERAPY, Outcome 40 Adverse events: 4. Metabolic - b. average weight gain (kg).

\begin{tabular}{|c|c|c|c|c|c|c|}
\hline \multirow[t]{2}{*}{ Study or subgroup } & \multicolumn{2}{|c|}{ AP combination } & \multicolumn{2}{|c|}{ AP monotherapy } & \multirow{2}{*}{$\begin{array}{l}\text { Mean Difference } \\
\text { Random, 95\% Cl }\end{array}$} & \multirow{2}{*}{$\begin{array}{l}\text { Mean Difference } \\
\text { Random, } 95 \% \mathrm{Cl}\end{array}$} \\
\hline & $\mathbf{N}$ & $\operatorname{Mean}(S D)$ & $\mathbf{N}$ & Mean(SD) & & \\
\hline \multicolumn{7}{|c|}{ 1.40.1 clozapine in both groups } \\
\hline B +aripiprazole 2008 & 108 & $-2.5(3.7)$ & 99 & $-0.4(3.7)$ & - & $-2.15[-3.17,-1.13]$ \\
\hline B +risperidone 2010 & 24 & $0.1(2.9)$ & 26 & $1.1(4.4)$ & & $-1[-3.05,1.05]$ \\
\hline C +aripiprazole 2008 & 29 & $-1.2(2.3)$ & 32 & $-0.6(1.7)$ & + & $-0.6[-1.62,0.42]$ \\
\hline C +aripiprazole 2013 & 16 & $-1.5(2.3)$ & 14 & $0.3(2.3)$ & & $-1.8[-3.45,-0.15]$ \\
\hline C +sertindole 2006 & 25 & $0.1(2.7)$ & 25 & $0.1(2.9)$ & & $0[-1.55,1.55]$ \\
\hline \multicolumn{7}{|c|}{ 1.40.2 other atypical drugs in both groups } \\
\hline C +olan/risp 2014 & 25 & $-0.3(3.6)$ & 26 & $0.4(3.5)$ & + & $-0.75[-2.7,1.2]$ \\
\hline C +olanzapine 2012 & 13 & $2(3.2)$ & 13 & $1(2.8)$ & & $1[-1.31,3.31]$ \\
\hline C +sulpiride 2013 & 31 & $-0.8(3)$ & 32 & $-1.3(2.6)$ & & $0.5[-0.89,1.89]$ \\
\hline
\end{tabular}

Analysis 1.41. Comparison 1 ANTIPSYCHOTIC COMBINATIONS vS ANTIPSYCHOTIC MONOTHERAPY, Outcome 41 Adverse events: 5. Blood - a. decreased white cell counts (binary).

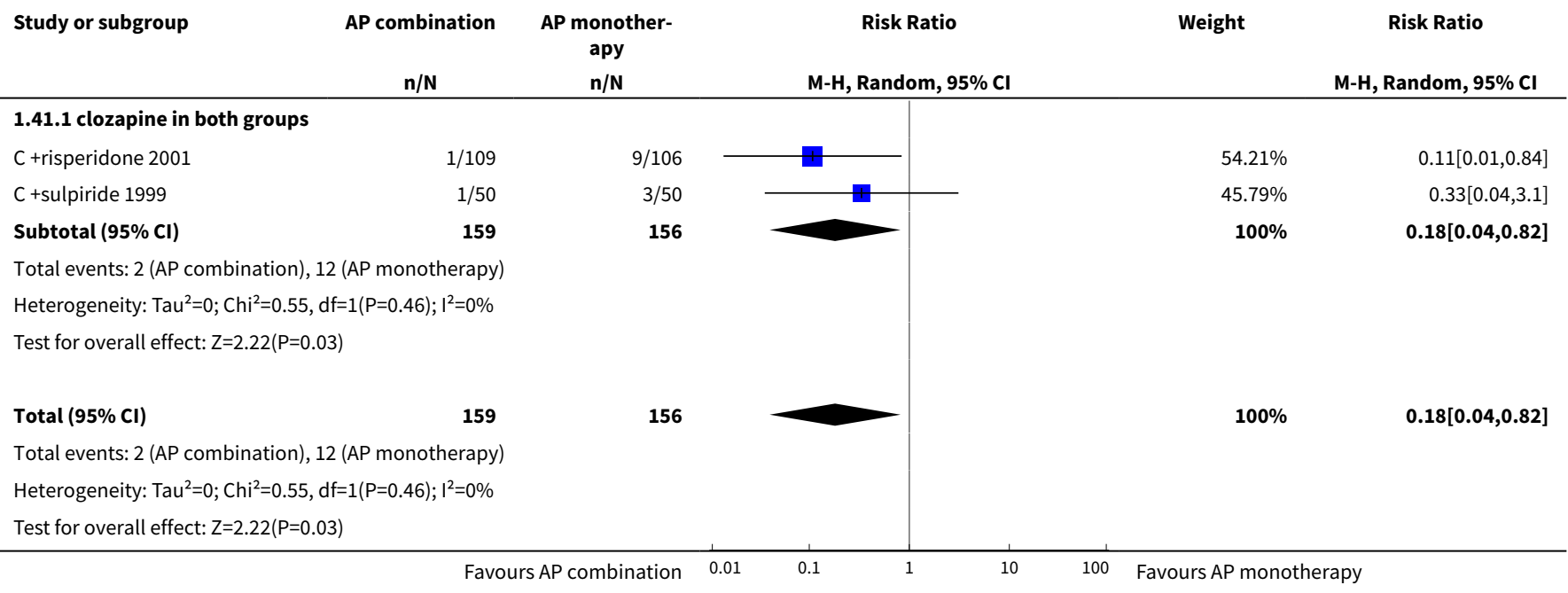

Analysis 1.42. Comparison 1 ANTIPSYCHOTIC COMBINATIONS vS ANTIPSYCHOTIC MONOTHERAPY, Outcome 42 Adverse events: 5 . Blood - b. average white cell counts $\left(10^{-3} / \mathrm{mm}^{3}\right)$.

\begin{tabular}{|c|c|c|c|c|c|c|c|}
\hline \multirow[t]{2}{*}{ Study or subgroup } & \multicolumn{2}{|c|}{ AP combination } & \multicolumn{2}{|c|}{ AP monotherapy } & \multirow{2}{*}{$\begin{array}{l}\text { Mean Difference } \\
\text { Random, 95\% Cl }\end{array}$} & \multirow[t]{2}{*}{ Weight } & \multirow{2}{*}{$\begin{array}{l}\text { Mean Difference } \\
\text { Random, 95\% CI }\end{array}$} \\
\hline & $\mathbf{N}$ & $\operatorname{Mean}(S D)$ & $\mathbf{N}$ & $\operatorname{Mean}(S D)$ & & & \\
\hline \multicolumn{8}{|c|}{ 1.42.1 clozapine in both groups } \\
\hline C +risperidone 2006 & 30 & $7.5(1.7)$ & 31 & $6.9(1.7)$ & & $100 \%$ & $0.66[-0.2,1.52]$ \\
\hline Subtotal $\star \star \star$ & 30 & & 31 & & & $100 \%$ & $0.66[-0.2,1.52]$ \\
\hline \multicolumn{8}{|c|}{ Heterogeneity: Not applicable } \\
\hline \multicolumn{8}{|c|}{ Test for overall effect: $Z=1.5(P=0.13)$} \\
\hline
\end{tabular}


Analysis 1.43. Comparison 1 ANTIPSYCHOTIC COMBINATIONS vs ANTIPSYCHOTIC MONOTHERAPY, Outcome 43 Adverse events: 6. Central nervous system (CNS) - a. drowsiness.

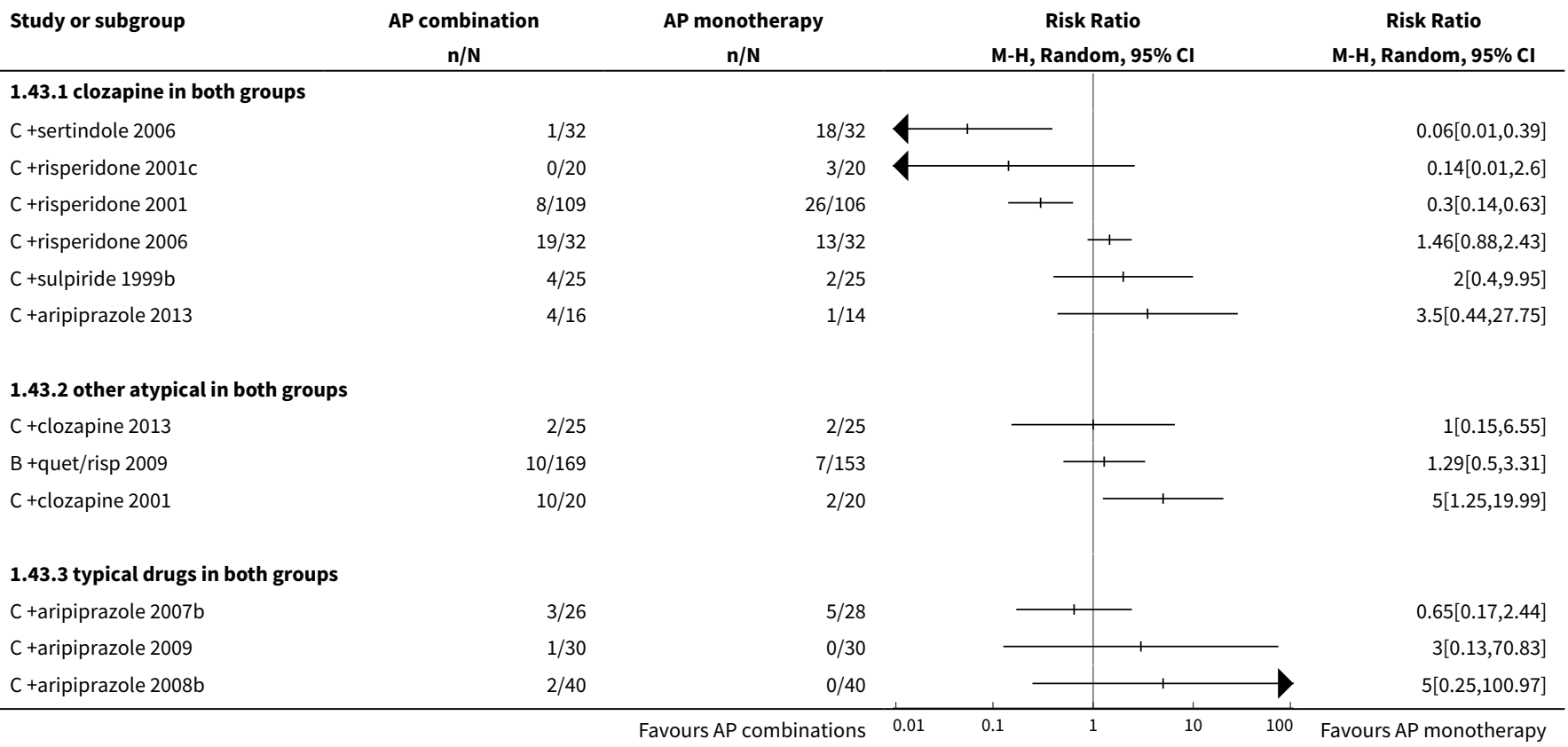

Analysis 1.44. Comparison 1 ANTIPSYCHOTIC COMBINATIONS vs ANTIPSYCHOTIC MONOTHERAPY, Outcome 44 Adverse events: 6. Central nervous system (CNS) - b. tremor.

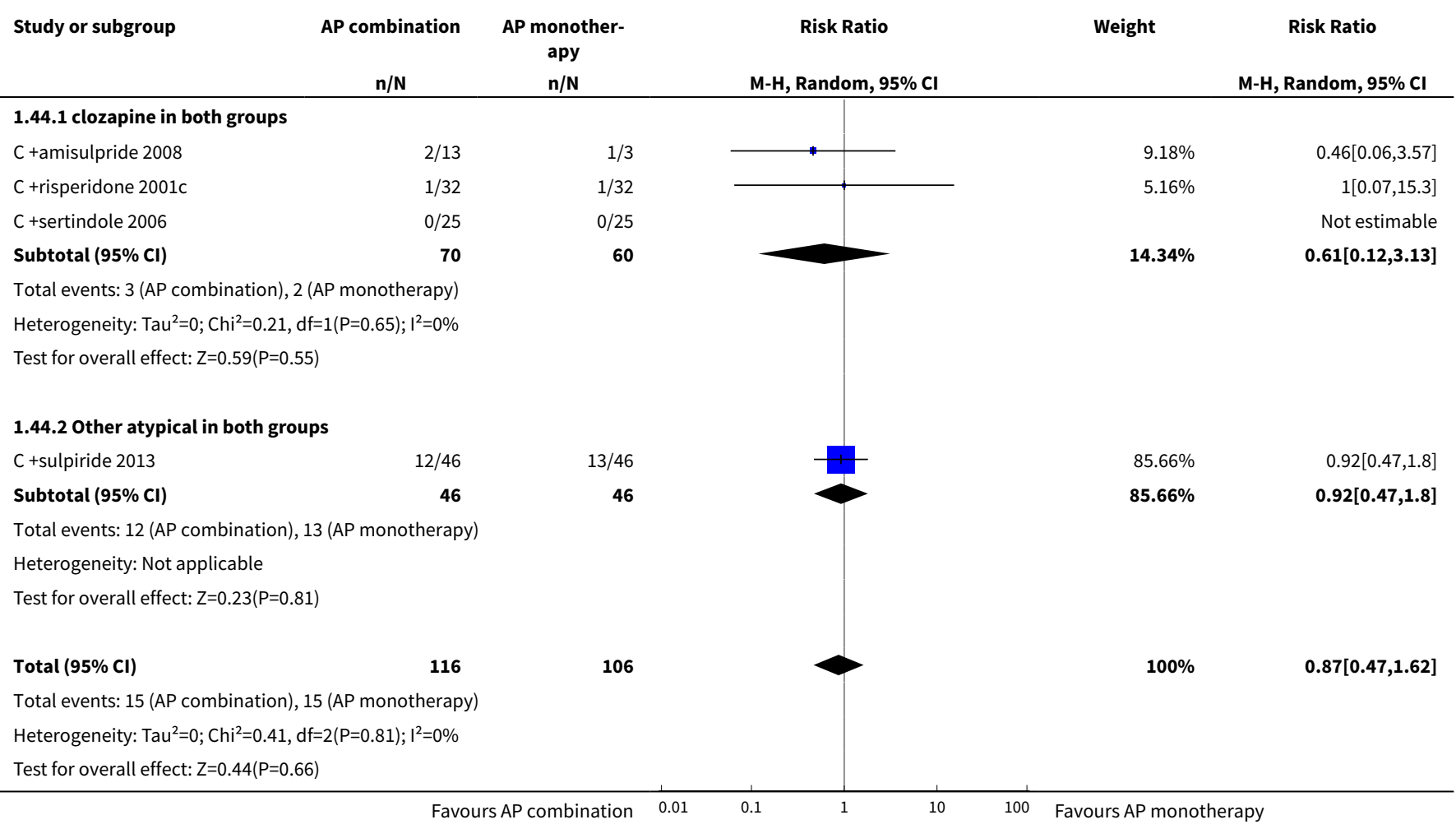




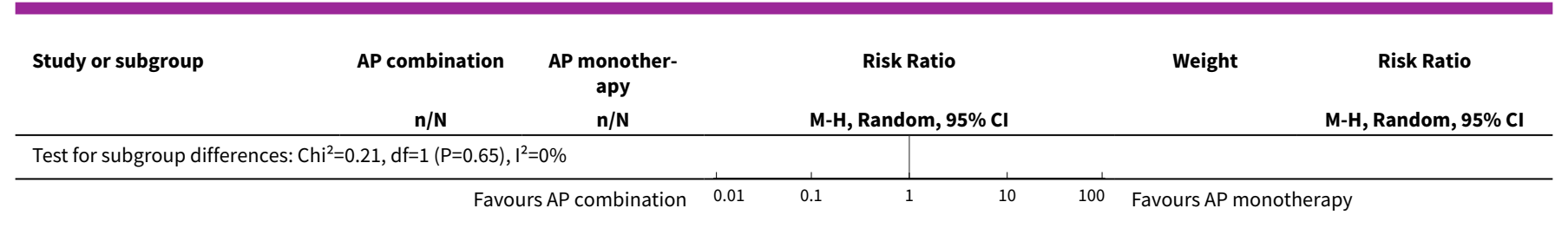

\section{Analysis 1.45. Comparison 1 ANTIPSYCHOTIC COMBINATIONS vs ANTIPSYCHOTIC} MONOTHERAPY, Outcome 45 Quality of life: 1a. Average score (QLS high=good).

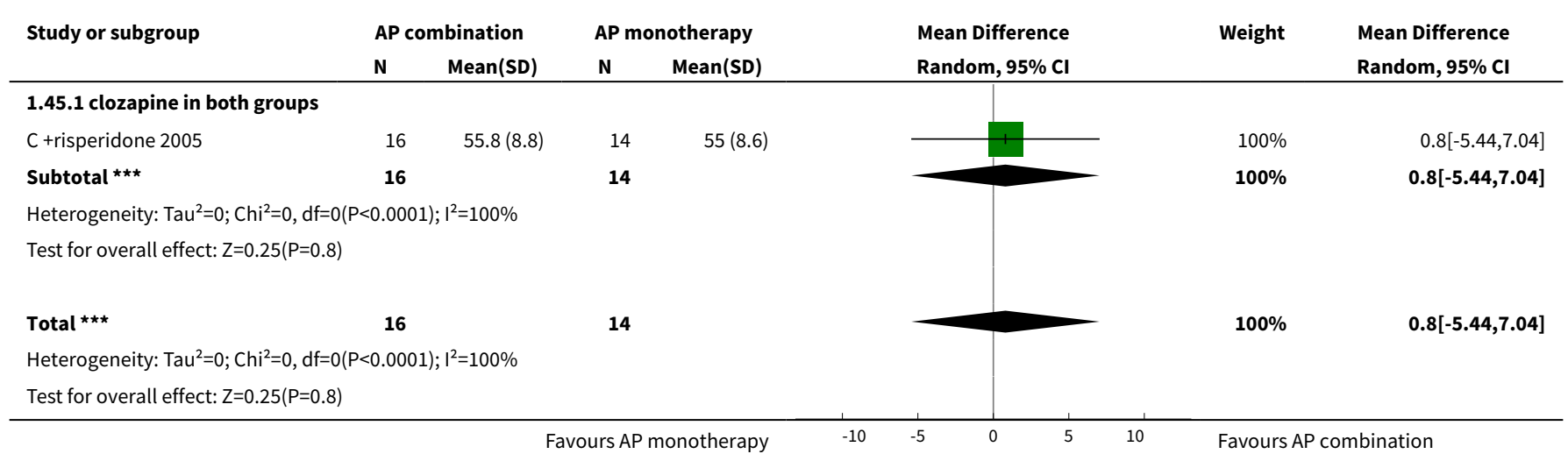

Analysis 1.46. Comparison 1 ANTIPSYCHOTIC COMBINATIONS vS ANTIPSYCHOTIC MONOTHERAPY, Outcome 46 Quality of life: 1b. Average score (SWN, high=good).

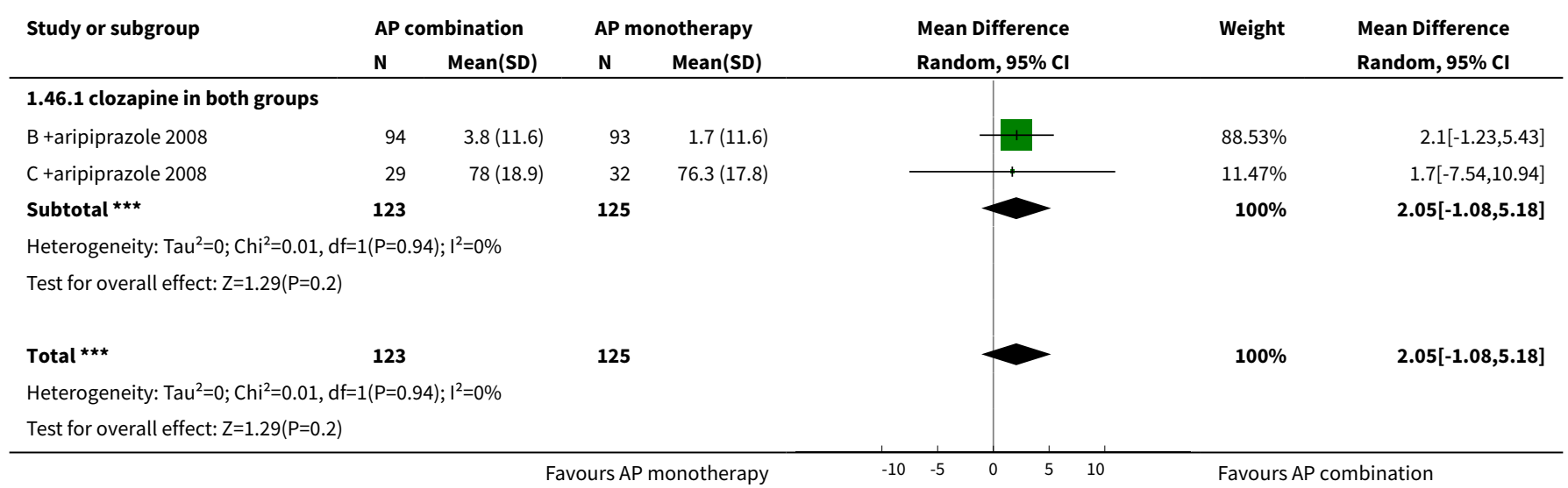

Analysis 1.47. Comparison 1 ANTIPSYCHOTIC COMBINATIONS vS ANTIPSYCHOTIC MONOTHERAPY, Outcome 47 Quality of Life: 1c. Average score - Mental component summary (SF-36, high = good).

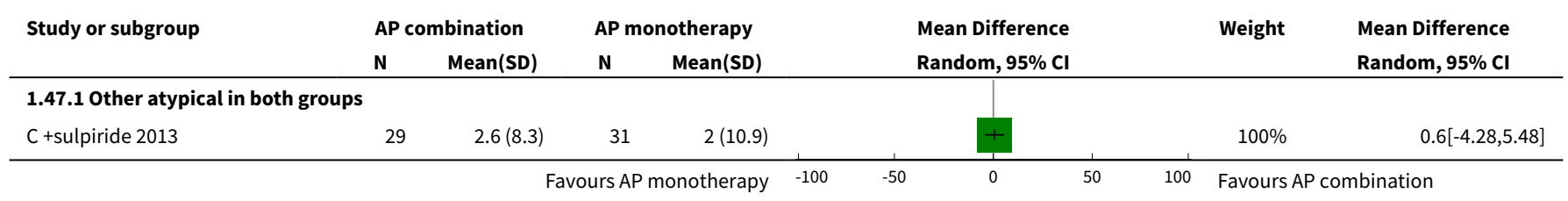




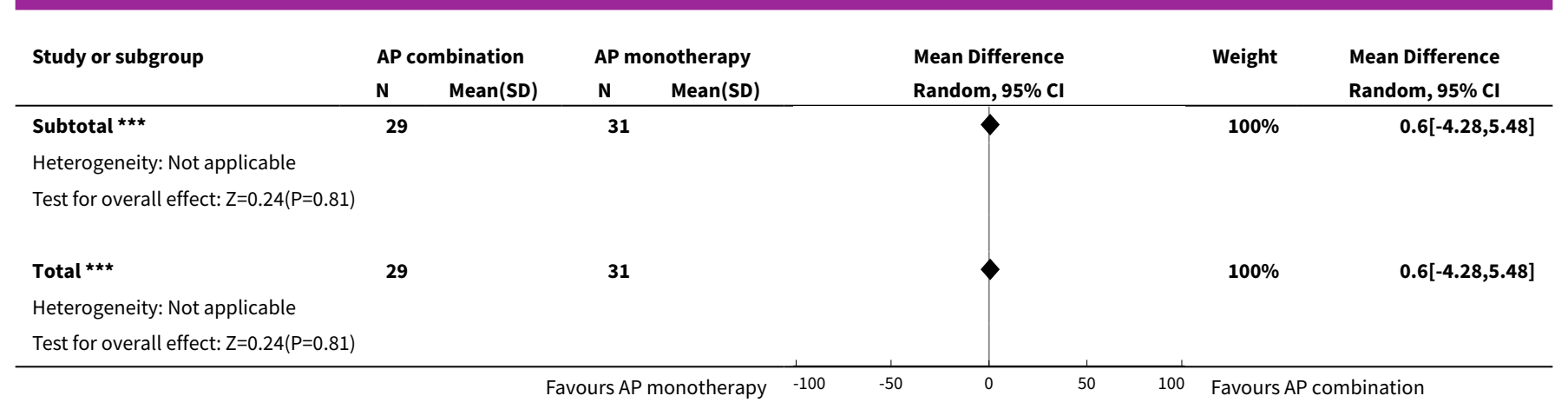

Analysis 1.48. Comparison 1 ANTIPSYCHOTIC COMBINATIONS vS ANTIPSYCHOTIC MONOTHERAPY, Outcome 48 Quality of Life: 1d. Average score - Physical component summary (SF-36, high = good).

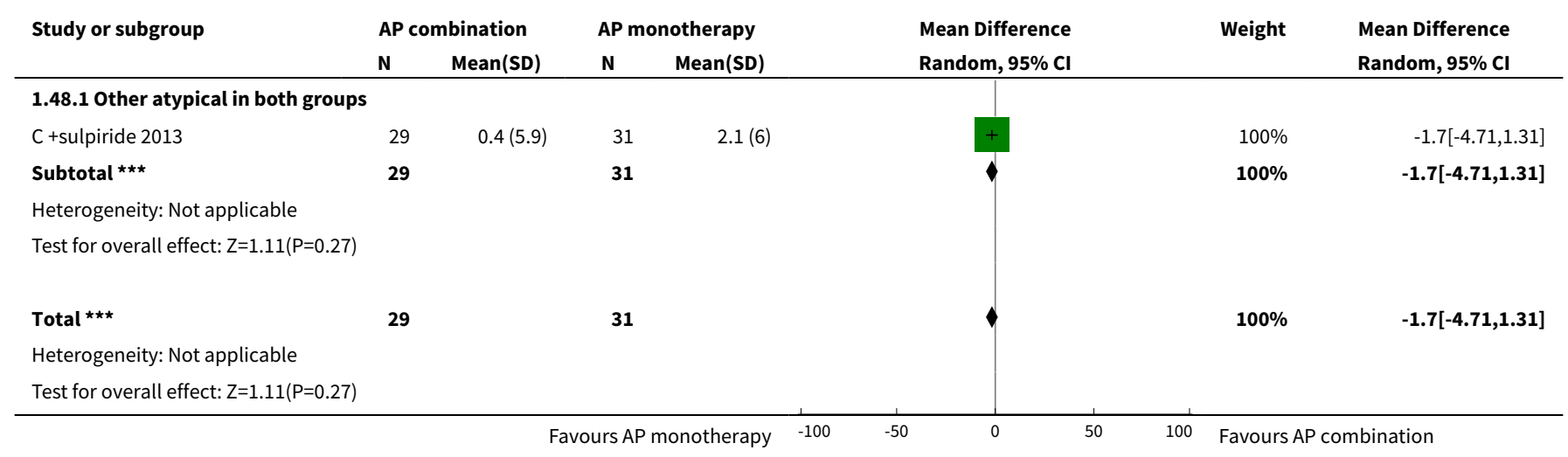

\section{Analysis 1.49. Comparison 1 ANTIPSYCHOTIC COMBINATIONS vs ANTIPSYCHOTIC MONOTHERAPY, Outcome 49 SUBGROUP ANALYSIS Clinical Response: Not clinically improved - Patients enrolled in the studies.}

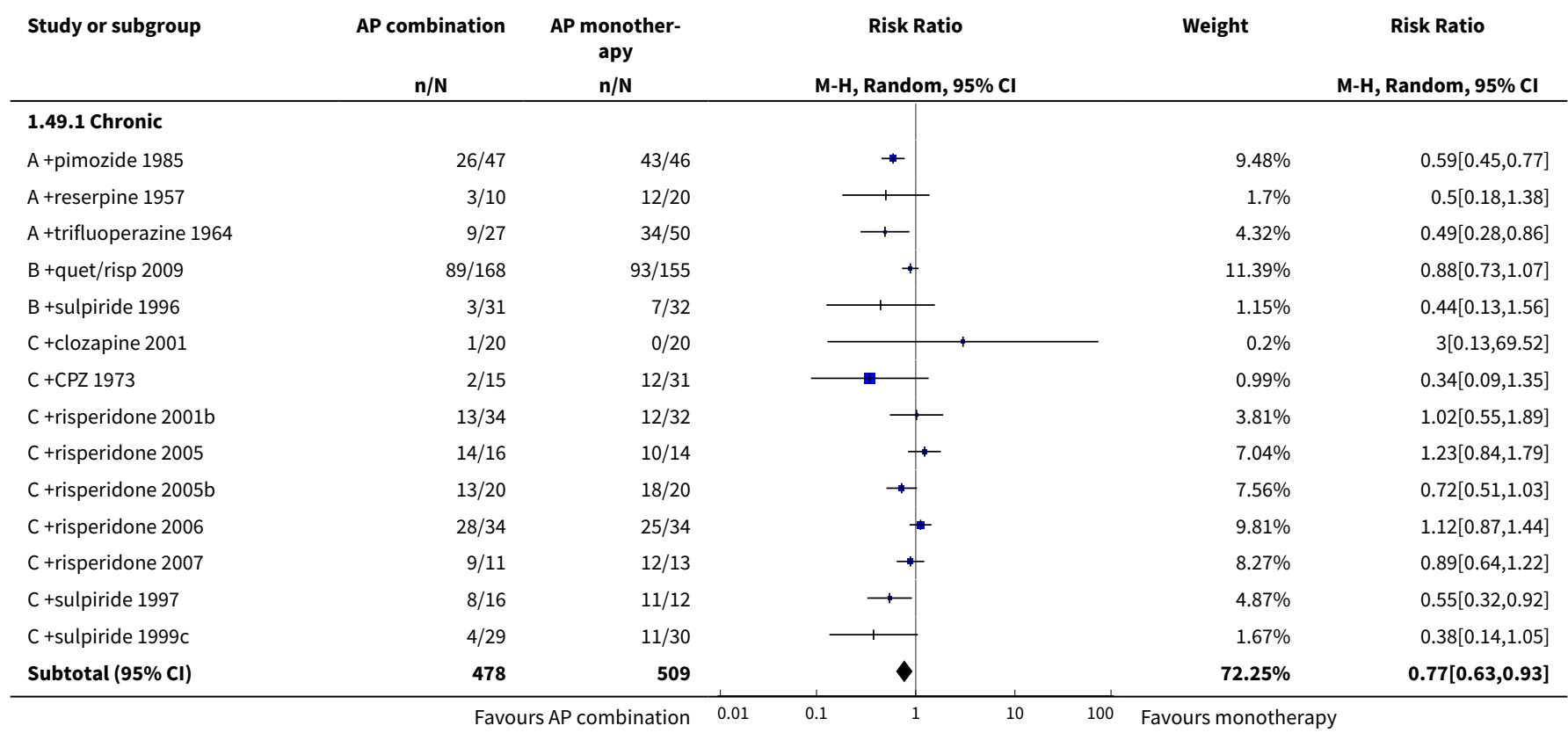




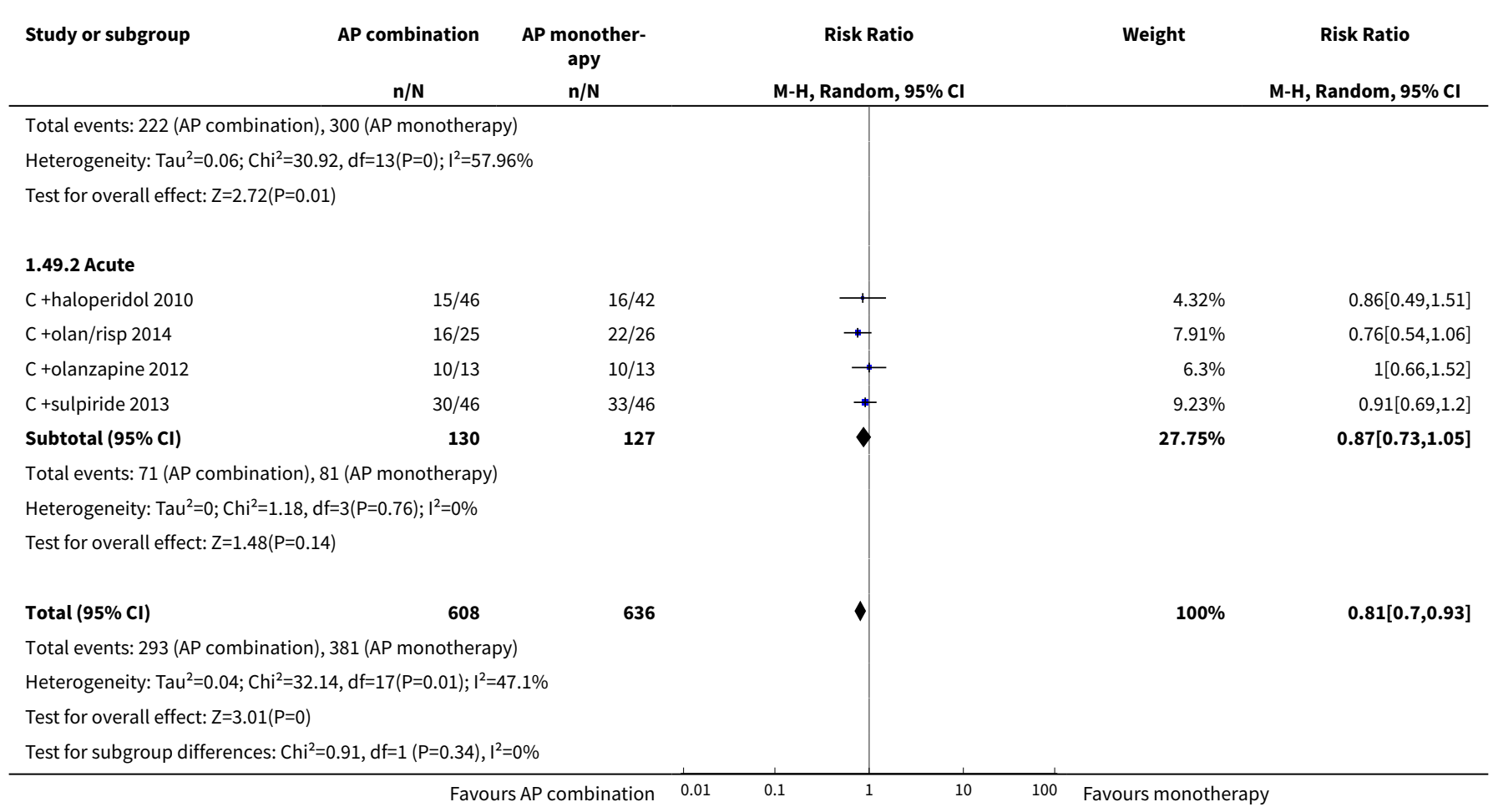
Analysis 1.50. Comparison 1 ANTIPSYCHOTIC COMBINATIONS vs ANTIPSYCHOTIC MONOTHERAPY,
Outcome 50 SUBGROUP ANALYSIS Clinical Response: Not clinically improved - Treatment duration.

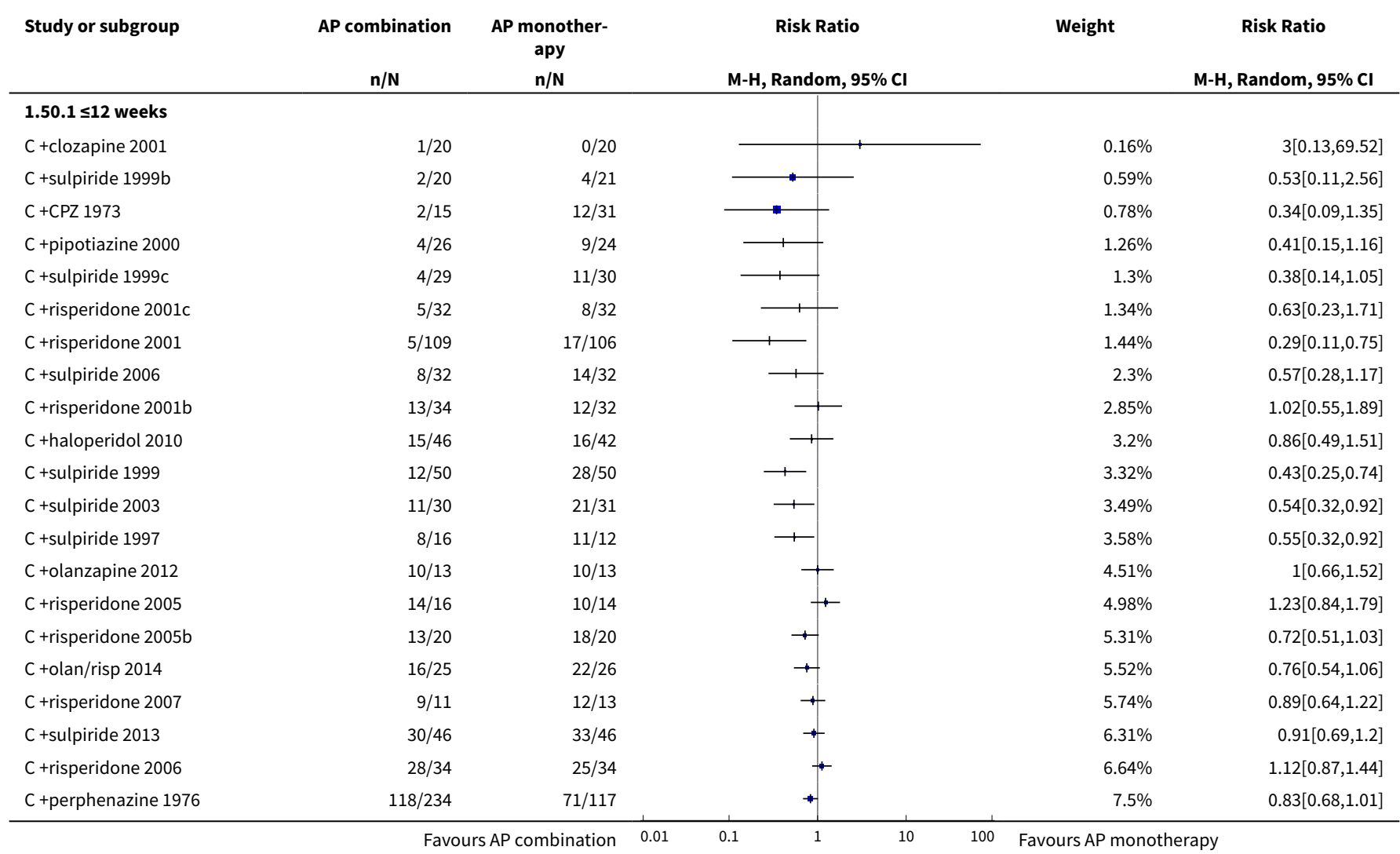




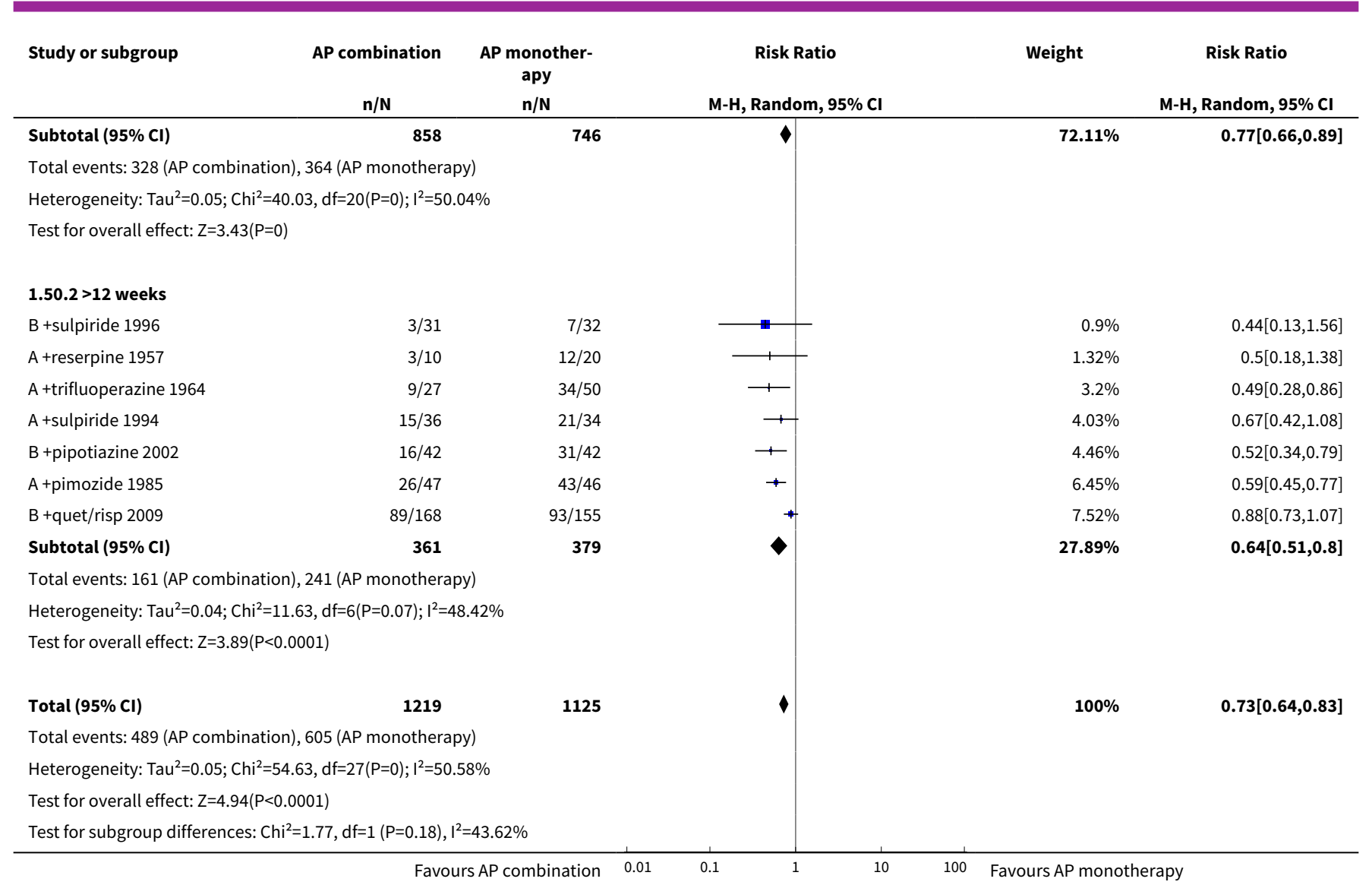

\section{Analysis 1.51. Comparison 1 ANTIPSYCHOTIC COMBINATIONS vs ANTIPSYCHOTIC MONOTHERAPY, Outcome 51 SUBGROUP ANALYSIS Clinical Response: Not clinically improved - Use of clozapine in both groups.}

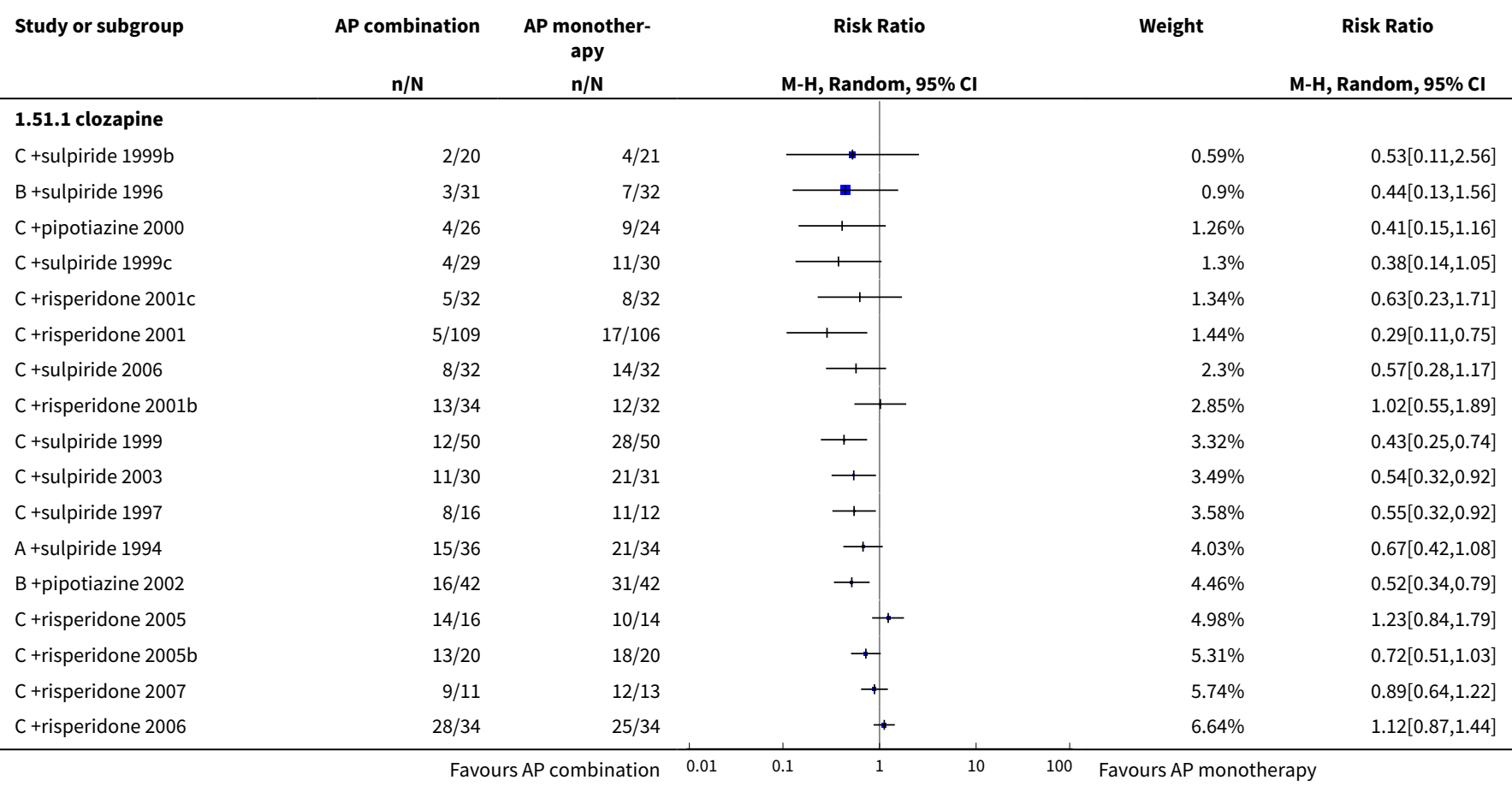




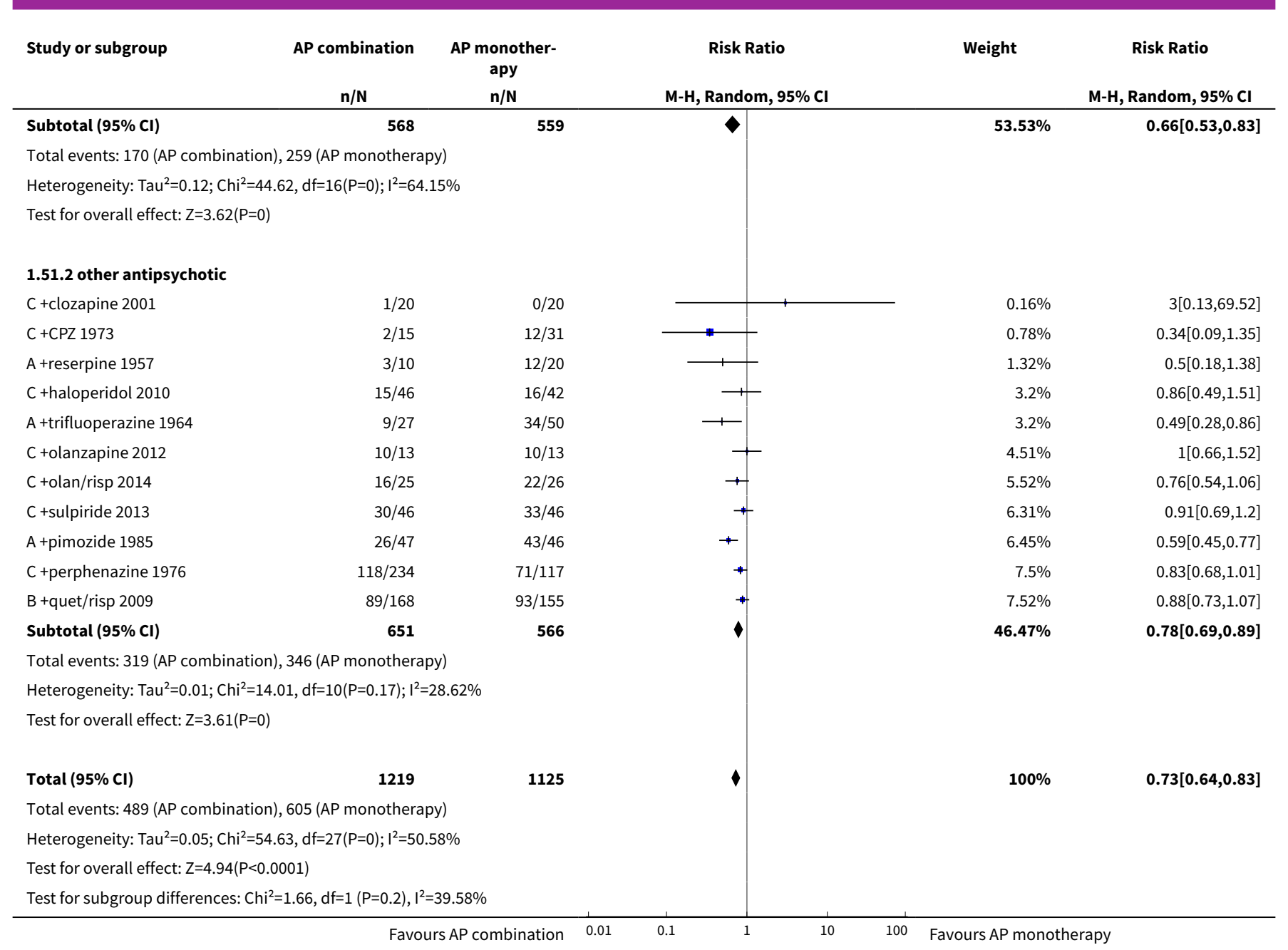

\section{Analysis 1.52. Comparison 1 ANTIPSYCHOTIC COMBINATIONS vS ANTIPSYCHOTIC MONOTHERAPY, Outcome 52 SUBGROUP ANALYSIS Clinical Response: Not clinically improved - Drug added to clozapine.}

\begin{tabular}{|c|c|c|c|c|c|}
\hline Study or subgroup & $\begin{array}{c}\text { AP combination } \\
n / N\end{array}$ & $\begin{array}{l}\text { AP monother- } \\
\text { apy } \\
n / N\end{array}$ & $\begin{array}{c}\text { Risk Ratio } \\
\text { M-H, Random, } 95 \% \mathrm{CI}\end{array}$ & Weight & $\begin{array}{c}\text { Risk Ratio } \\
\text { M-H, Random, } 95 \% \mathrm{CI}\end{array}$ \\
\hline \multicolumn{6}{|l|}{ 1.52.1 Sulpirirde } \\
\hline C +sulpiride 1999b & $2 / 20$ & $4 / 21$ & & $1.69 \%$ & $0.53[0.11,2.56]$ \\
\hline B +sulpiride 1996 & $3 / 31$ & $7 / 32$ & 1 & $2.46 \%$ & $0.44[0.13,1.56]$ \\
\hline C +sulpiride 1999 c & $4 / 29$ & $11 / 30$ & (1 & $3.33 \%$ & $0.38[0.14,1.05]$ \\
\hline C +sulpiride 1999 & $12 / 50$ & $28 / 50$ & $\longrightarrow$ & $6.59 \%$ & $0.43[0.25,0.74]$ \\
\hline C +sulpiride 2003 & $11 / 30$ & $21 / 31$ & $\rightarrow$ & $6.8 \%$ & $0.54[0.32,0.92]$ \\
\hline C +sulpiride 1997 & $8 / 16$ & $11 / 12$ & $\rightarrow$ & $6.9 \%$ & $0.55[0.32,0.92]$ \\
\hline A +sulpiride 1994 & $15 / 36$ & $21 / 34$ & $\rightarrow$ & $7.41 \%$ & $0.67[0.42,1.08]$ \\
\hline Subtotal (95\% CI) & 244 & 242 & $\diamond$ & $40.34 \%$ & $0.54[0.43,0.68]$ \\
\hline
\end{tabular}

Total events: 63 (AP combination), 117 (AP monotherapy)

Heterogeneity: $\mathrm{Tau}^{2}=0 ; \mathrm{Chi}^{2}=2.21, \mathrm{df}=7(\mathrm{P}=0.95) ; \mathrm{I}^{2}=0 \%$

Test for overall effect: $Z=5.31(P<0.0001)$ 


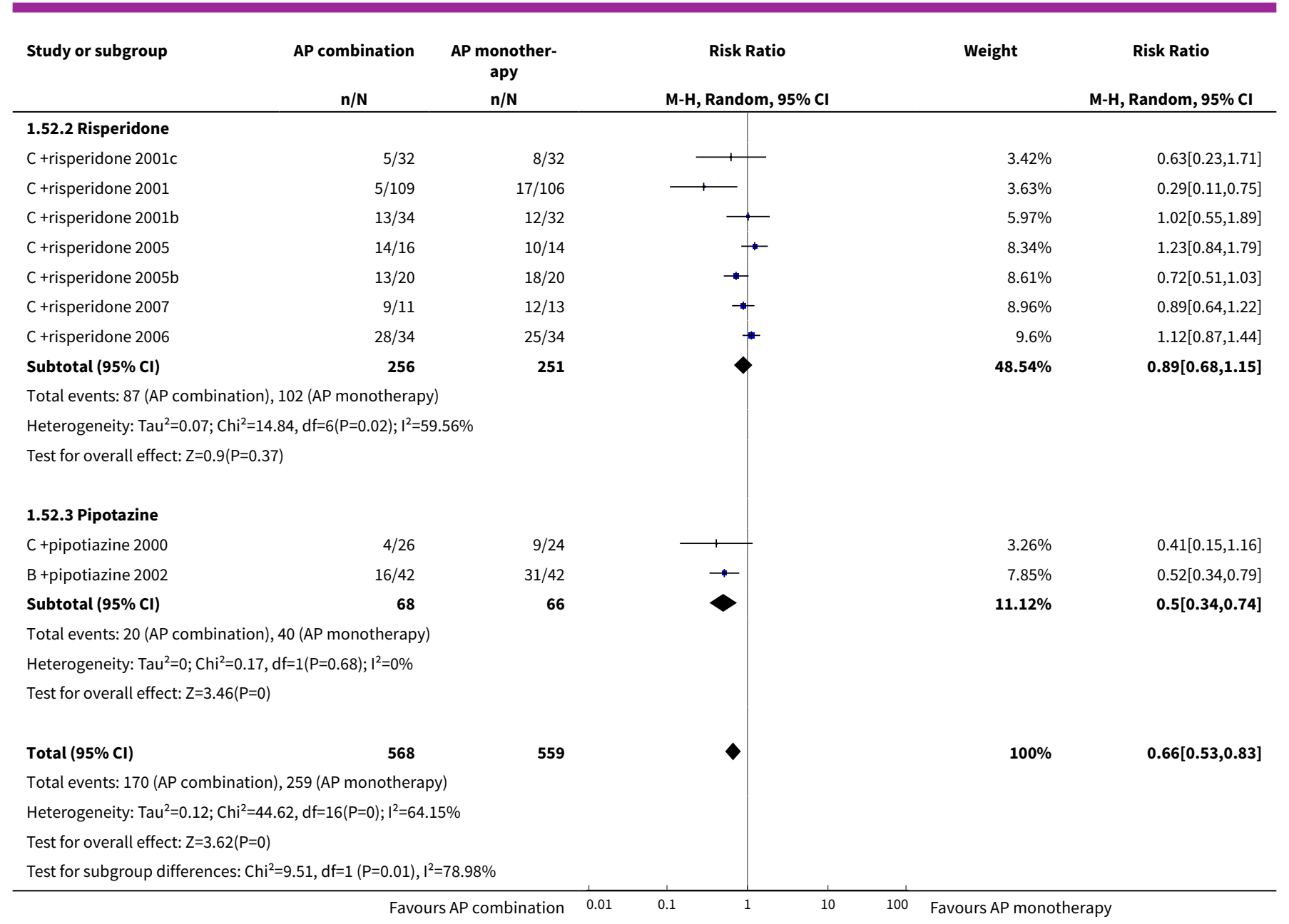

\section{Analysis 1.53. Comparison 1 ANTIPSYCHOTIC COMBINATIONS VS ANTIPSYCHOTIC MONOTHERAPY, Outcome 53 SUBGROUP ANALYSIS Leaving the study early - Patients enrolled in the studies.}

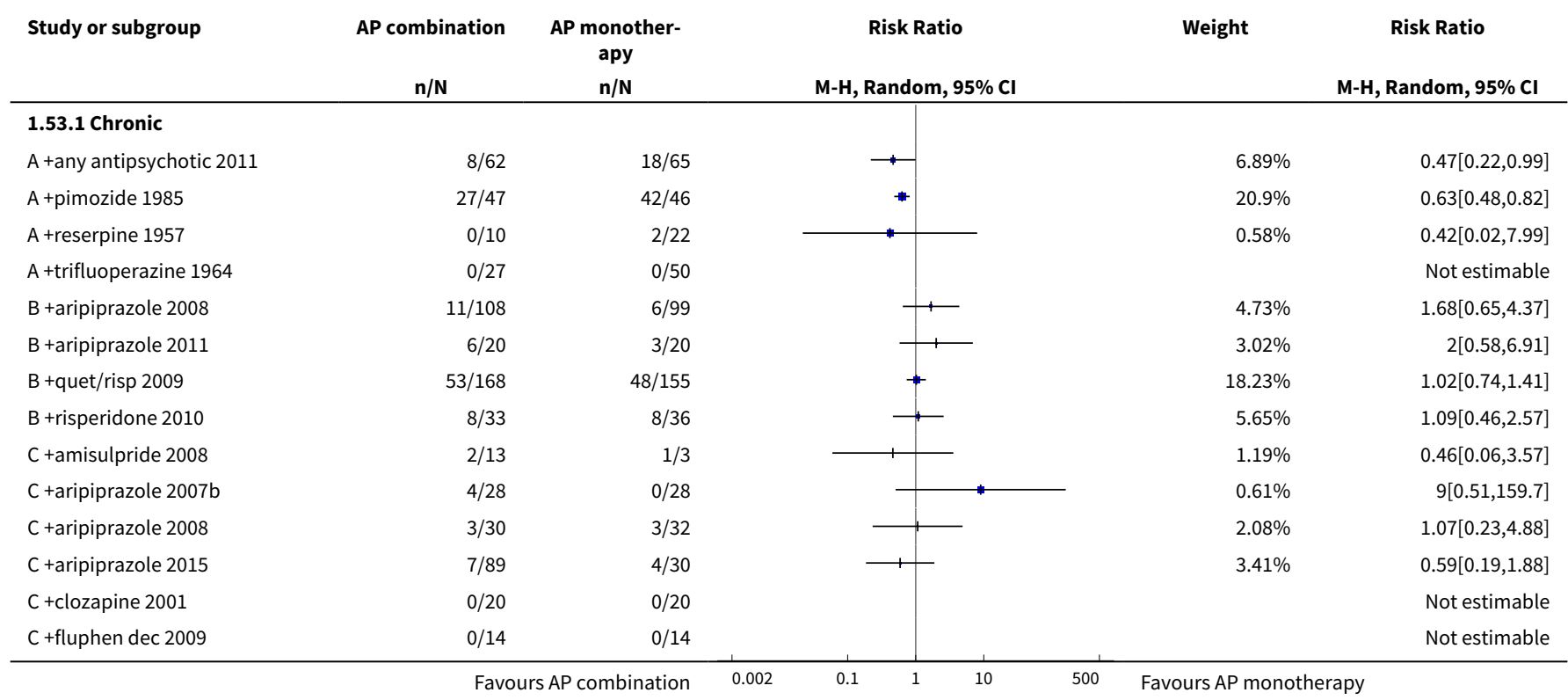




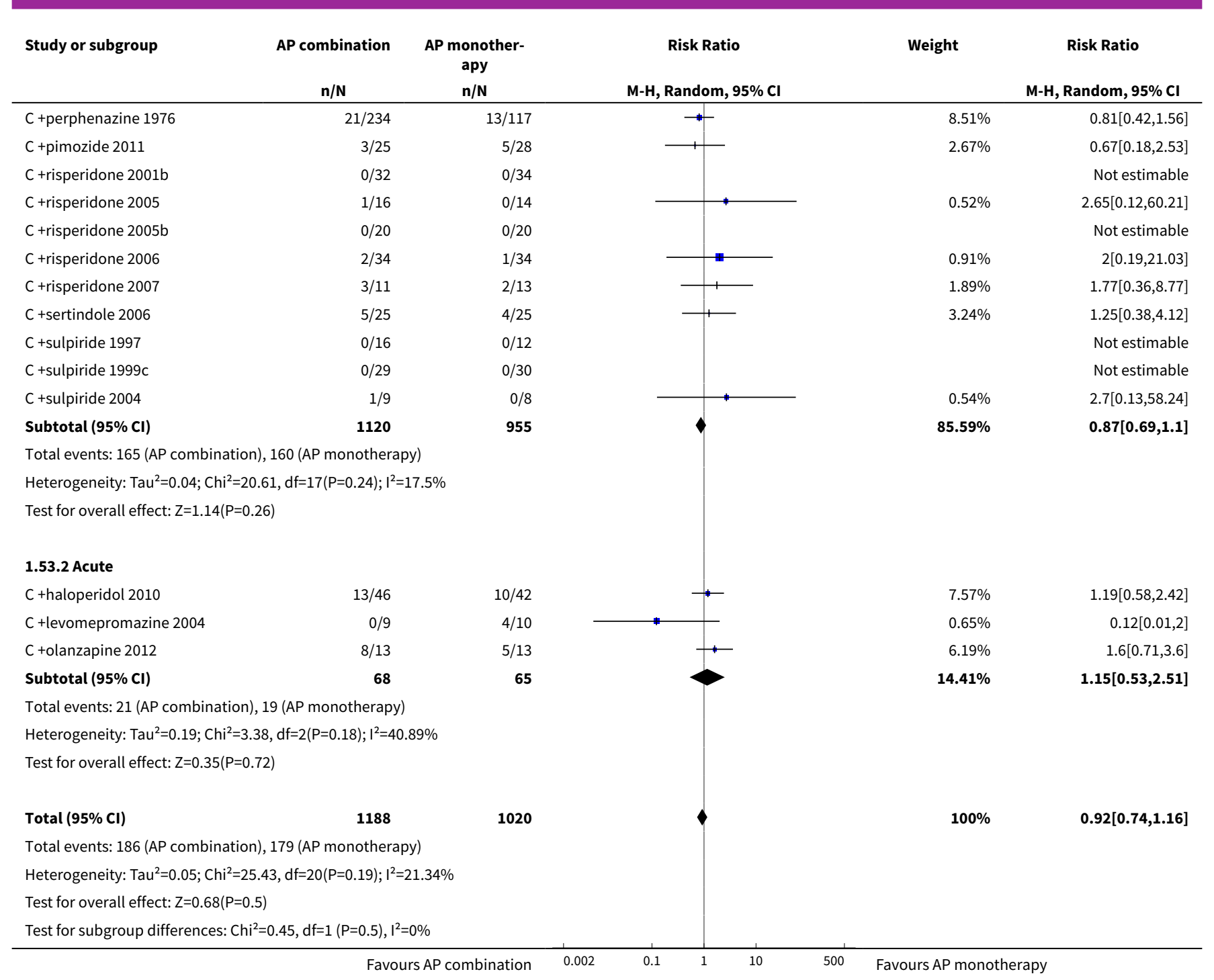

Analysis 1.54. Comparison 1 ANTIPSYCHOTIC COMBINATIONS vs ANTIPSYCHOTIC MONOTHERAPY, Outcome 54 SUBGROUP ANALYSIS Leaving the study early - Treatment duration.

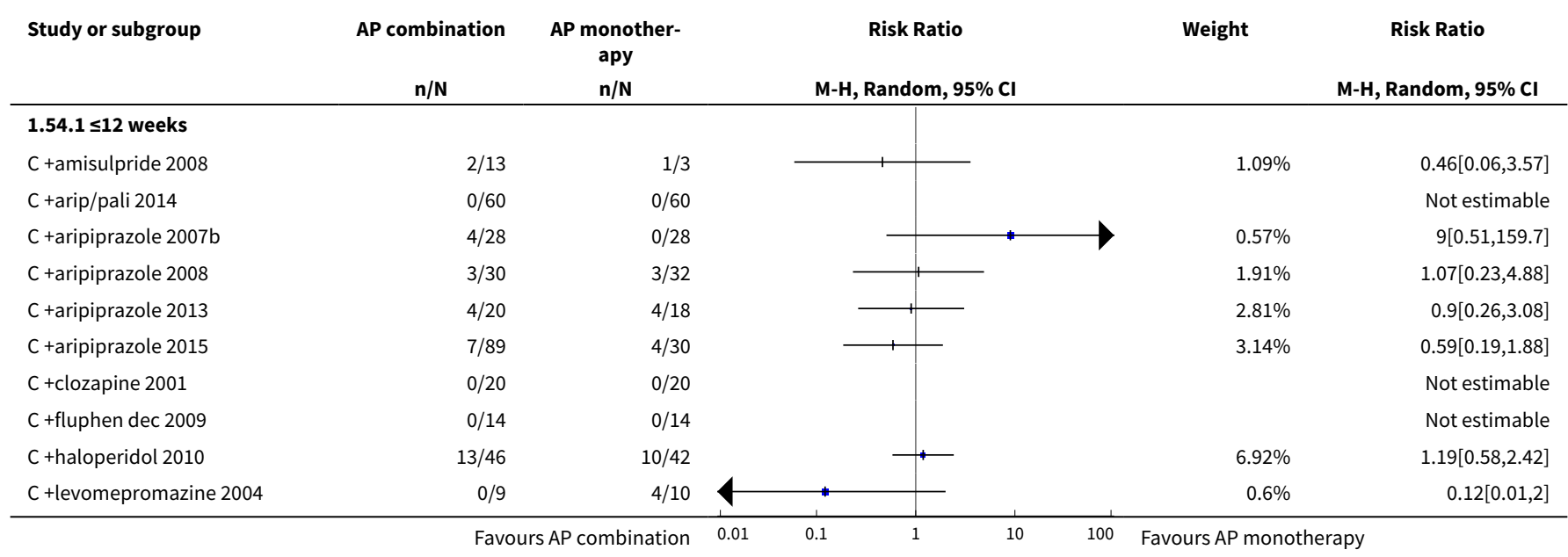




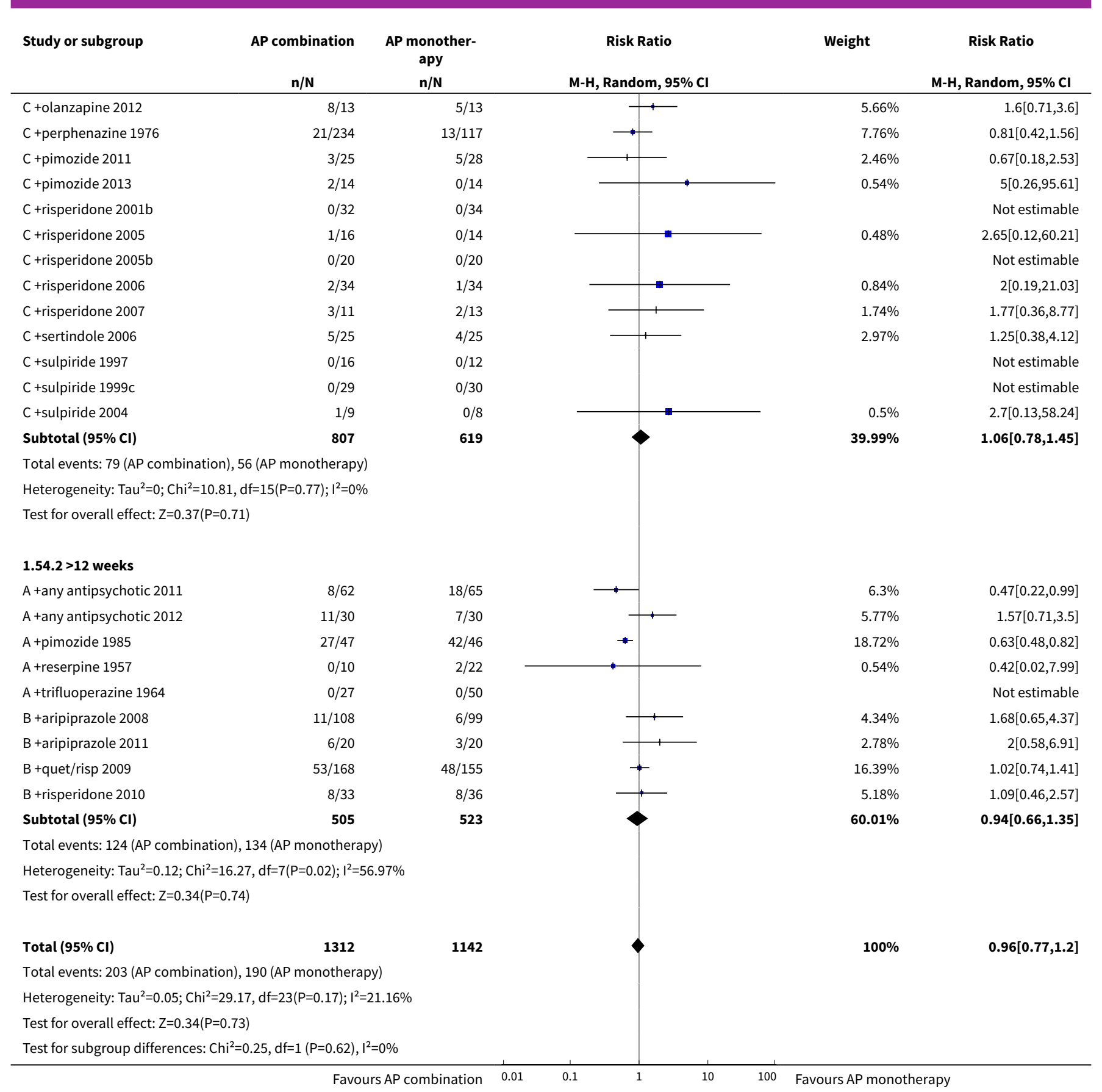

\section{Analysis 1.55. Comparison 1 ANTIPSYCHOTIC COMBINATIONS VS ANTIPSYCHOTIC MONOTHERAPY, Outcome 55 SUBGROUP ANALYSIS Leaving the study early - Use of clozapine in both groups.}

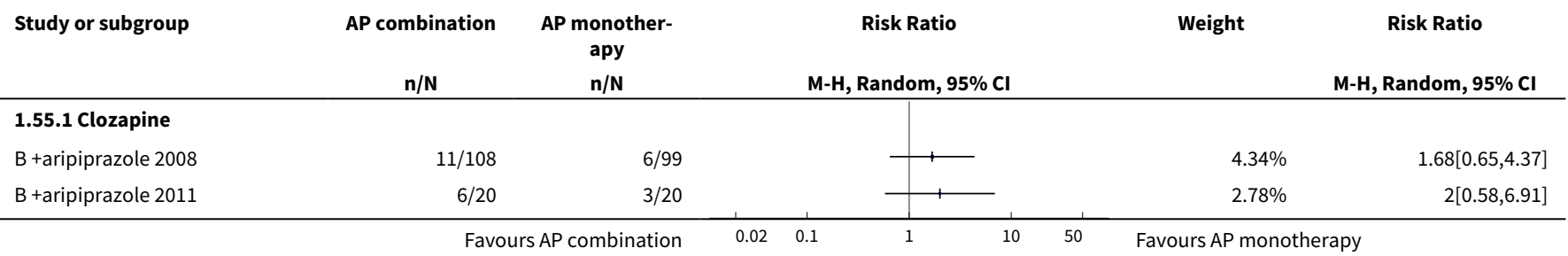




\begin{tabular}{|c|c|c|c|c|c|}
\hline \multirow[t]{2}{*}{ Study or subgroup } & AP combination & $\begin{array}{c}\text { AP monother- } \\
\text { apy }\end{array}$ & Risk Ratio & \multirow[t]{2}{*}{ Weight } & \multirow[t]{2}{*}{$\begin{array}{c}\text { Risk Ratio } \\
\text { M-H, Random, } 95 \% \mathrm{Cl}\end{array}$} \\
\hline & $n / N$ & $n / N$ & M-H, Random, 95\% Cl & & \\
\hline B + risperidone 2010 & $8 / 33$ & $8 / 36$ & 1 & $5.18 \%$ & $1.09[0.46,2.57]$ \\
\hline C +amisulpride 2008 & $2 / 13$ & $1 / 3$ & & $1.09 \%$ & $0.46[0.06,3.57]$ \\
\hline C +aripiprazole 2008 & $3 / 30$ & $3 / 32$ & & $1.91 \%$ & $1.07[0.23,4.88]$ \\
\hline C +aripiprazole 2013 & $4 / 20$ & $4 / 18$ & 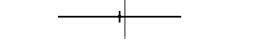 & $2.81 \%$ & $0.9[0.26,3.08]$ \\
\hline C +pimozide 2011 & $3 / 25$ & $5 / 28$ & & $2.46 \%$ & $0.67[0.18,2.53]$ \\
\hline C +pimozide 2013 & $2 / 14$ & $0 / 14$ & & $0.54 \%$ & $5[0.26,95.61]$ \\
\hline$C$ +risperidone $2001 \mathrm{~b}$ & $0 / 32$ & $0 / 34$ & & & Not estimable \\
\hline C +risperidone 2005 & $1 / 16$ & $0 / 14$ & & $0.48 \%$ & $2.65[0.12,60.21]$ \\
\hline$C$ +risperidone $2005 b$ & $0 / 20$ & $0 / 20$ & & & Not estimable \\
\hline C +risperidone 2006 & $2 / 34$ & $1 / 34$ & - & $0.84 \%$ & $2[0.19,21.03]$ \\
\hline C +risperidone 2007 & $3 / 11$ & $2 / 13$ & - & $1.74 \%$ & $1.77[0.36,8.77]$ \\
\hline C +sertindole 2006 & $5 / 25$ & $4 / 25$ & 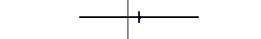 & $2.97 \%$ & $1.25[0.38,4.12]$ \\
\hline C +sulpiride 1997 & $0 / 16$ & $0 / 12$ & & & Not estimable \\
\hline C +sulpiride 1999 C & $0 / 29$ & $0 / 30$ & & & Not estimable \\
\hline Subtotal $(95 \% \mathrm{CI})$ & 446 & 432 & & $27.14 \%$ & $1.25[0.85,1.86]$ \\
\hline \multicolumn{6}{|c|}{ Total events: 50 (AP combination), 37 (AP monotherapy) } \\
\hline \multicolumn{6}{|c|}{ Heterogeneity: $\mathrm{Tau}^{2}=0 ; \mathrm{Chi}^{2}=4.53, \mathrm{df}=11(\mathrm{P}=0.95) ; \mathrm{I}^{2}=0 \%$} \\
\hline \multicolumn{6}{|c|}{ Test for overall effect: $Z=1.13(P=0.26)$} \\
\hline \multicolumn{6}{|l|}{ 1.55.2 Other antipsychotics } \\
\hline A +any antipsychotic 2011 & $8 / 62$ & $18 / 65$ & & $6.3 \%$ & $0.47[0.22,0.99]$ \\
\hline A +any antipsychotic 2012 & $11 / 30$ & $7 / 30$ & $\rightarrow$ & $5.77 \%$ & $1.57[0.71,3.5]$ \\
\hline A +pimozide 1985 & $27 / 47$ & $42 / 46$ & $\rightarrow$ & $18.72 \%$ & $0.63[0.48,0.82]$ \\
\hline A +reserpine 1957 & $0 / 10$ & $2 / 22$ & & $0.54 \%$ & $0.42[0.02,7.99]$ \\
\hline A +trifluoperazine 1964 & $0 / 27$ & $0 / 50$ & & & Not estimable \\
\hline B +quet/risp 2009 & $53 / 168$ & $48 / 155$ & - & $16.39 \%$ & $1.02[0.74,1.41]$ \\
\hline C +arip/pali 2014 & $0 / 30$ & $0 / 60$ & & & Not estimable \\
\hline C +aripiprazole 2007b & $4 / 28$ & $0 / 28$ & & $0.57 \%$ & $9[0.51,159.7]$ \\
\hline C +aripiprazole 2015 & $7 / 89$ & $4 / 30$ & . & $3.14 \%$ & $0.59[0.19,1.88]$ \\
\hline C+clozapine 2001 & $0 / 20$ & $0 / 20$ & & & Not estimable \\
\hline C +fluphen dec 2009 & $0 / 14$ & $0 / 14$ & & & Not estimable \\
\hline C +haloperidol 2010 & $13 / 46$ & $10 / 42$ & - & $6.92 \%$ & $1.19[0.58,2.42]$ \\
\hline C +levomepromazine 2004 & $0 / 9$ & $4 / 10$ & & $0.6 \%$ & $0.12[0.01,2]$ \\
\hline C +olanzapine 2012 & $8 / 13$ & $5 / 13$ & $\rightarrow$ & $5.66 \%$ & $1.6[0.71,3.6]$ \\
\hline C +perphenazine 1976 & $21 / 234$ & $13 / 117$ & $\longrightarrow$ & $7.76 \%$ & $0.81[0.42,1.56]$ \\
\hline C +sulpiride 2004 & $1 / 9$ & $0 / 8$ & & $0.5 \%$ & $2.7[0.13,58.24]$ \\
\hline Subtotal $(95 \% \mathrm{Cl})$ & 836 & 710 & & $72.86 \%$ & $0.89[0.66,1.2]$ \\
\hline \multicolumn{6}{|c|}{ Total events: 153 (AP combination), 153 (AP monotherapy) } \\
\hline \multicolumn{6}{|c|}{ Heterogeneity: $\mathrm{Tau}^{2}=0.09 ; \mathrm{Chi}^{2}=20.13, \mathrm{df}=11(\mathrm{P}=0.04) ; \mathrm{I}^{2}=45.35 \%$} \\
\hline \multicolumn{6}{|c|}{ Test for overall effect: $Z=0.78(P=0.43)$} \\
\hline Total $(95 \% \mathrm{Cl})$ & 1282 & 1142 & & $100 \%$ & $0.96[0.77,1.2]$ \\
\hline \multicolumn{6}{|c|}{ Total events: 203 (AP combination), 190 (AP monotherapy) } \\
\hline \multicolumn{6}{|c|}{ Heterogeneity: $\mathrm{Tau}^{2}=0.05 ; \mathrm{Chi}^{2}=29.17, \mathrm{df}=23(\mathrm{P}=0.17) ; \mathrm{I}^{2}=21.16 \%$} \\
\hline \multicolumn{6}{|c|}{ Test for overall effect: $Z=0.34(P=0.73)$} \\
\hline Test for subgroup difference & 1.89, $d f=1(P=0.17)$, & $7.18 \%$ & & & \\
\hline
\end{tabular}


Analysis 1.56. COmparison 1 ANTIPSYCHOTIC COMBINATIONS VS ANTIPSYCHOTIC MONOTHERAPY, Outcome 56 SUBGROUP ANALYSIS Leaving the study early - Drug added to clozapine.

\section{Study or subgroup}

\section{AP combination}

AP monotherapy

$\mathrm{n} / \mathrm{N}$

$n / N$

Risk Ratio

M-H, Random, 95\% Cl

\subsubsection{Risperidone}

B +risperidone 2010

C +risperidone $2001 \mathrm{~b}$

C +risperidone 2005

$\mathrm{C}+$ risperidone $2005 \mathrm{~b}$

C +risperidone 2006

C +risperidone 2007

Subtotal $(95 \% \mathrm{Cl})$

Total events: 14 (AP combination), 11 (AP monotherapy) Heterogeneity: $\mathrm{Tau}^{2}=0 ; \mathrm{Chi}^{2}=0.64, \mathrm{df}=3(\mathrm{P}=0.89) ; \mathrm{I}^{2}=0 \%$

Test for overall effect: $Z=0.78(P=0.44)$

\subsubsection{Amisulpiride}

C +amisulpride 2008

Subtotal $(95 \% \mathrm{CI})$

Total events: 2 (AP combination), 1 (AP monotherapy)

Heterogeneity: Not applicable

Test for overall effect: $\mathrm{Z}=0.74(\mathrm{P}=0.46)$

$\begin{array}{ll}8 / 33 & 8 / 36 \\ 0 / 32 & 0 / 34 \\ 1 / 16 & 0 / 14 \\ 0 / 20 & 0 / 20 \\ 2 / 34 & 1 / 34 \\ 3 / 11 & 2 / 13 \\ 146 & 151\end{array}$

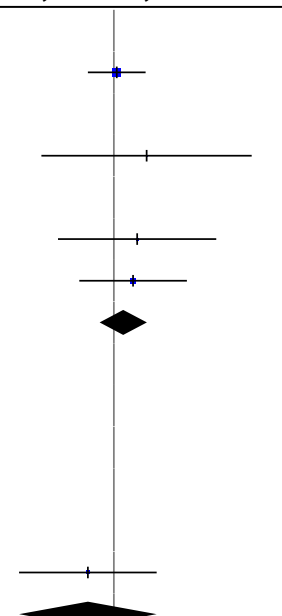

Weight

Risk Ratio

M-H, Random, 95\% Cl

$21.27 \%$

$1.09[0.46,2.57]$

Not estimable

$1.61 \%$

$2.65[0.12,60.21]$

Not estimable

$2.83 \%$

$2[0.19,21.03]$

$6.13 \%$

$31.84 \%$

$1.77[0.36,8.77]$

$1.32[0.66,2.67]$

\subsubsection{Aripiprazole}

B +aripiprazole 2008

B +aripiprazole 2011

C +aripiprazole 2008

C +aripiprazole 2013

Subtotal $(95 \% \mathrm{CI})$

Total events: 24 (AP combination), 16 (AP monotherapy) Heterogeneity: $\mathrm{Tau}^{2}=0 ; \mathrm{Chi}^{2}=1.08, \mathrm{df}=3(\mathrm{P}=0.78) ; \mathrm{I}^{2}=0 \%$

Test for overall effect: $Z=1.14(P=0.26)$

\subsubsection{Pimozide}

C +pimozide 2011 Subtotal $(95 \% \mathrm{CI})$

Total events: 3 (AP combination), 5 (AP monotherapy)

Heterogeneity: Not applicable

Test for overall effect: $Z=0.59(P=0.56)$

\subsubsection{Sertindole}

C +sertindole 2006

Subtotal $(95 \% \mathrm{CI})$

Total events: 5 (AP combination), 4 (AP monotherapy) Heterogeneity: Not applicable

Test for overall effect: $\mathrm{Z}=0.37(\mathrm{P}=0.71)$

\subsubsection{Sulpiride}

C +sulpiride 1997

C +sulpiride 1999 C

Subtotal $(95 \% \mathrm{CI})$

Total events: 0 (AP combination), 0 (AP monotherapy) 


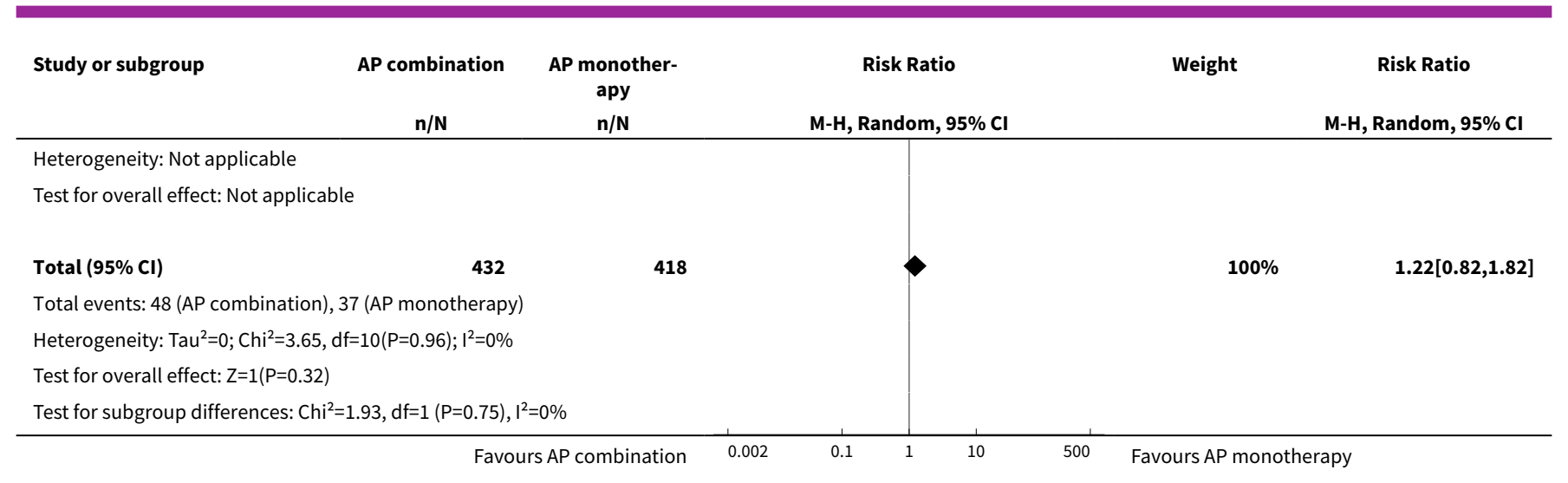

\section{Analysis 1.57. Comparison 1 ANTIPSYCHOTIC COMBINATIONS VS ANTIPSYCHOTIC MONOTHERAPY, Outcome 57 SENSITIVITY ANALYSIS Clinical Response: Not clinically improved - Randomisation.}

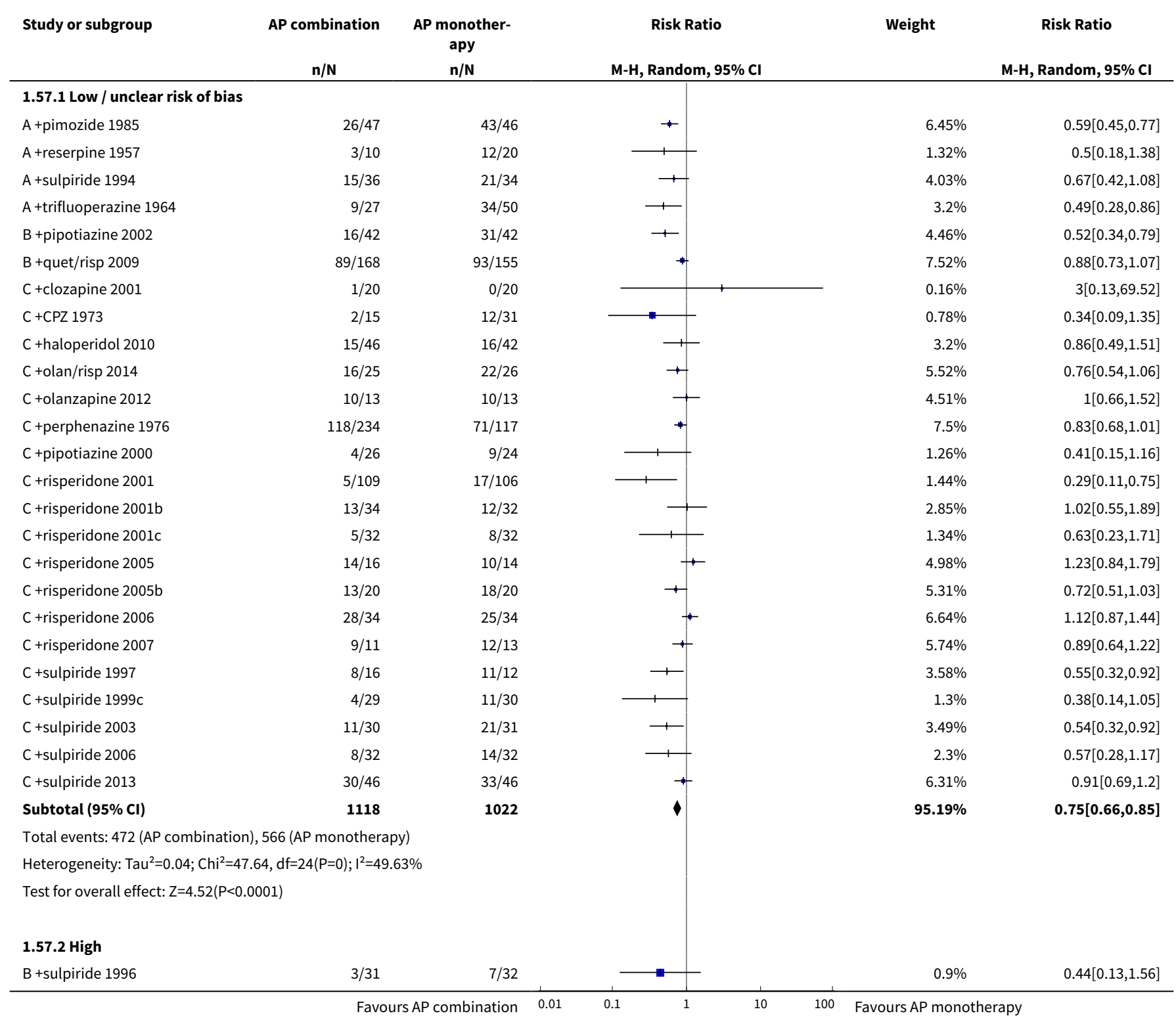




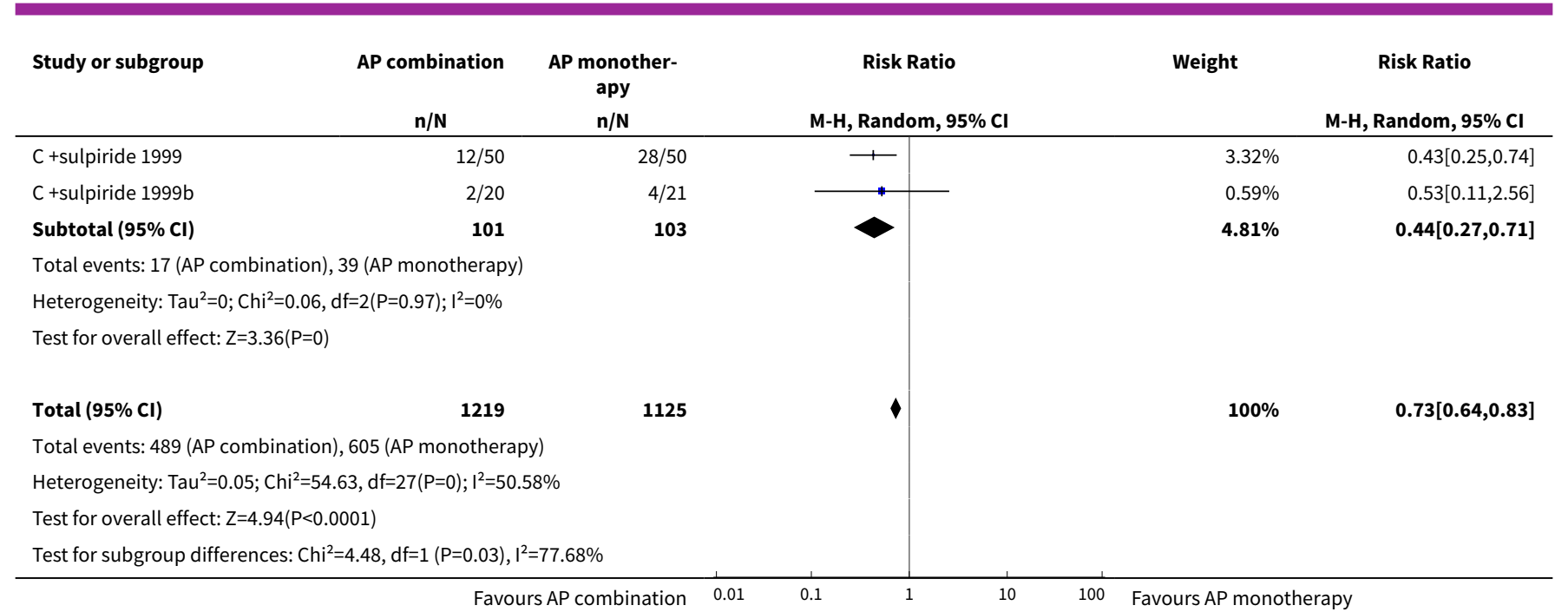

\section{Analysis 1.58. Comparison 1 ANTIPSYCHOTIC COMBINATIONS vS ANTIPSYCHOTIC MONOTHERAPY, Outcome 58 SENSITIVITY ANALYSIS Clinical Response: Not clinically improved - Double blind.}

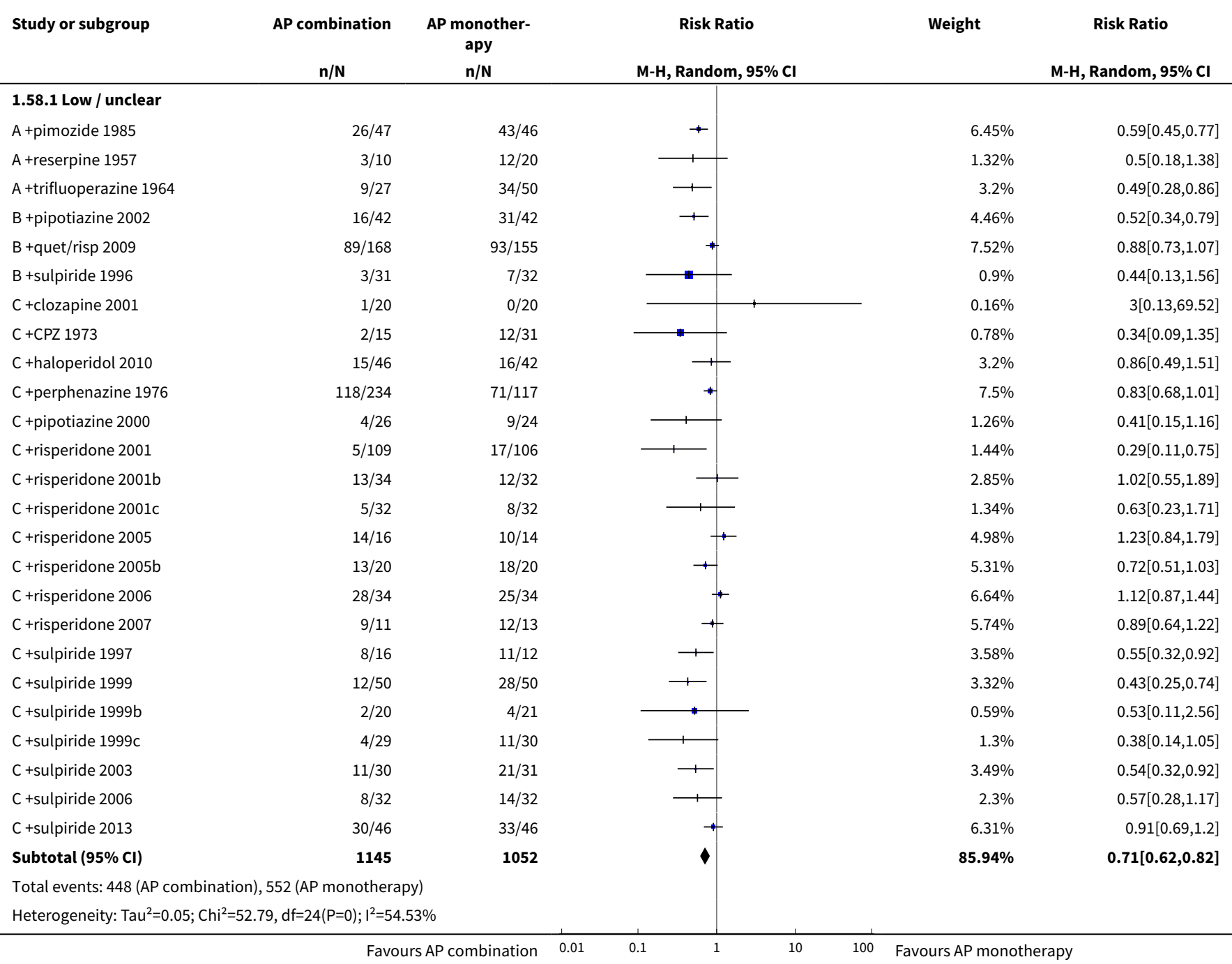




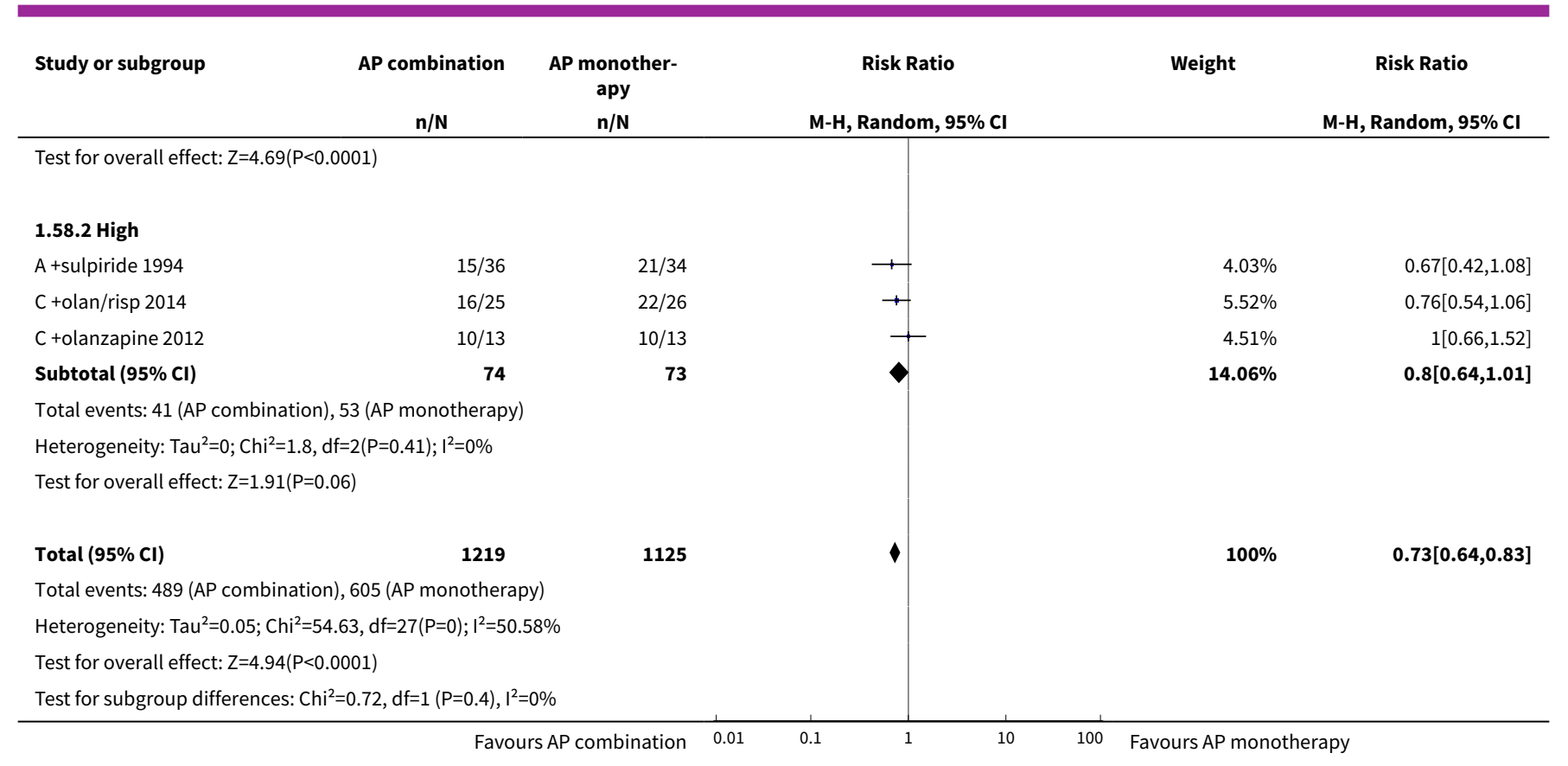

\section{Analysis 1.59. Comparison 1 ANTIPSYCHOTIC COMBINATIONS vs ANTIPSYCHOTIC MONOTHERAPY, Outcome 59 SENSITIVITY ANALYSIS Clinical response: 1 . No clinically important response - not improved - Fixed effect.}

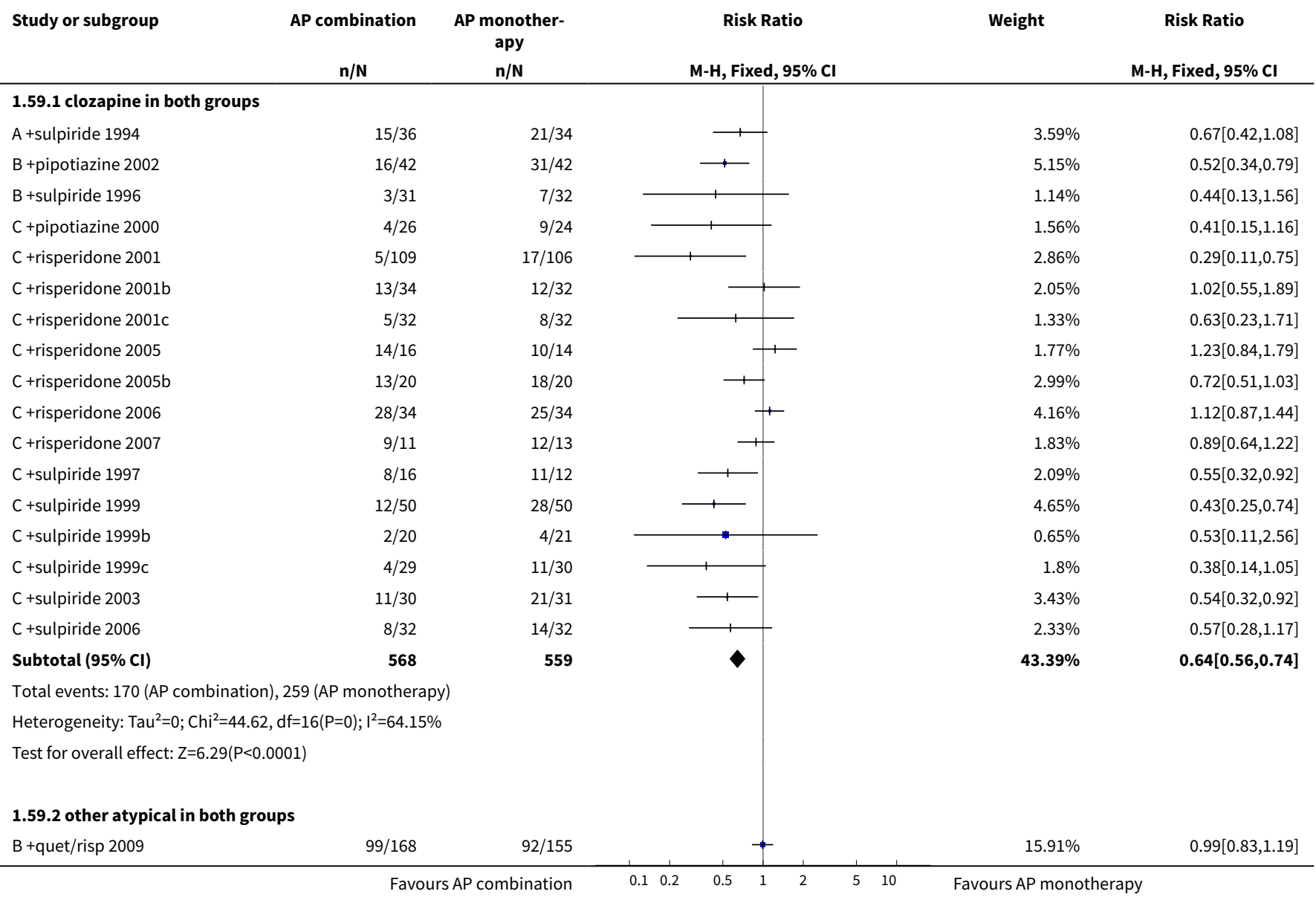




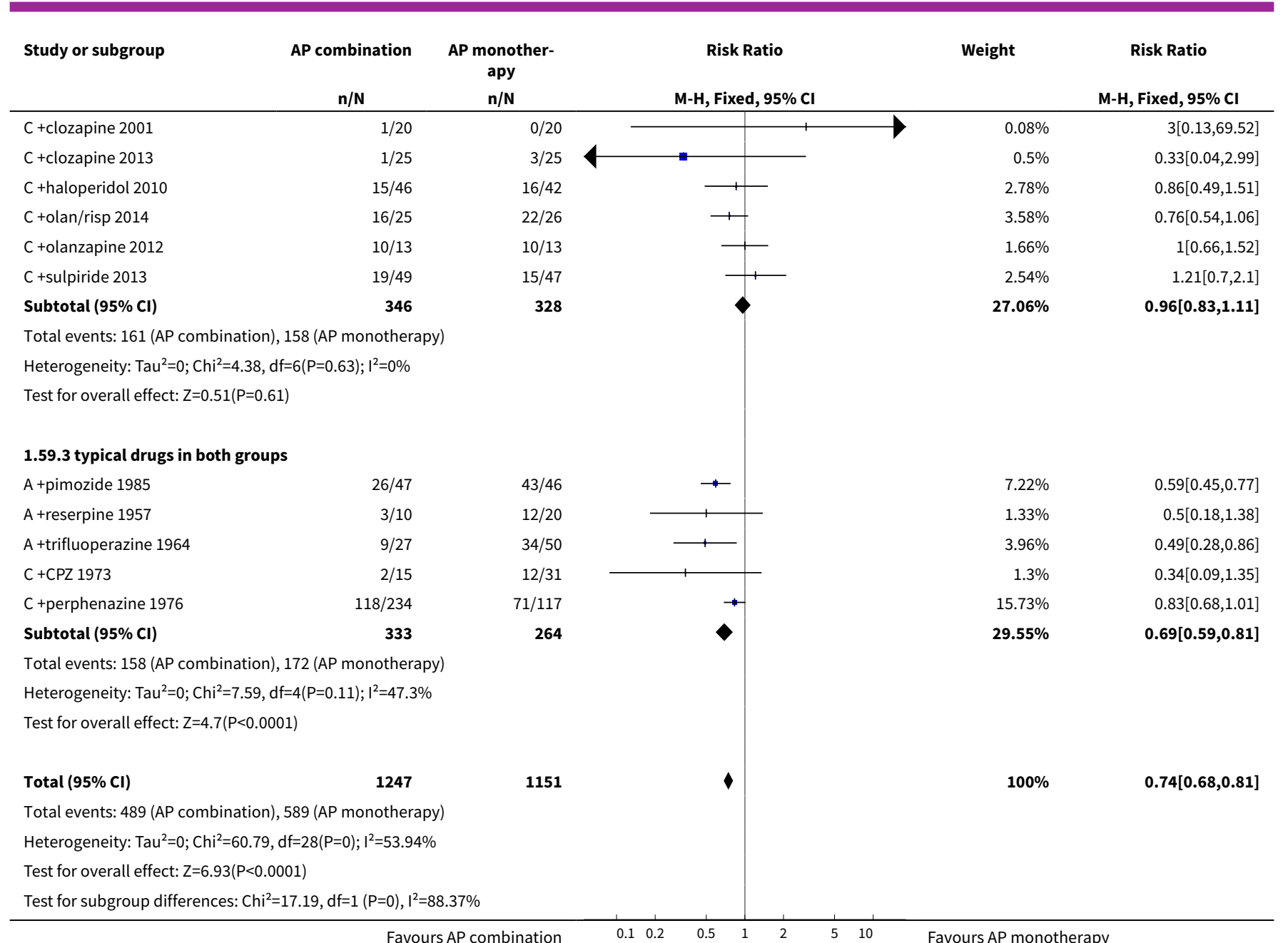

Analysis 1.60. Comparison 1 ANTIPSYCHOTIC COMBINATIONS vS ANTIPSYCHOTIC MONOTHERAPY, Outcome 60 SENSITIVITY ANALYSIS Leaving the study early - Randomisation.

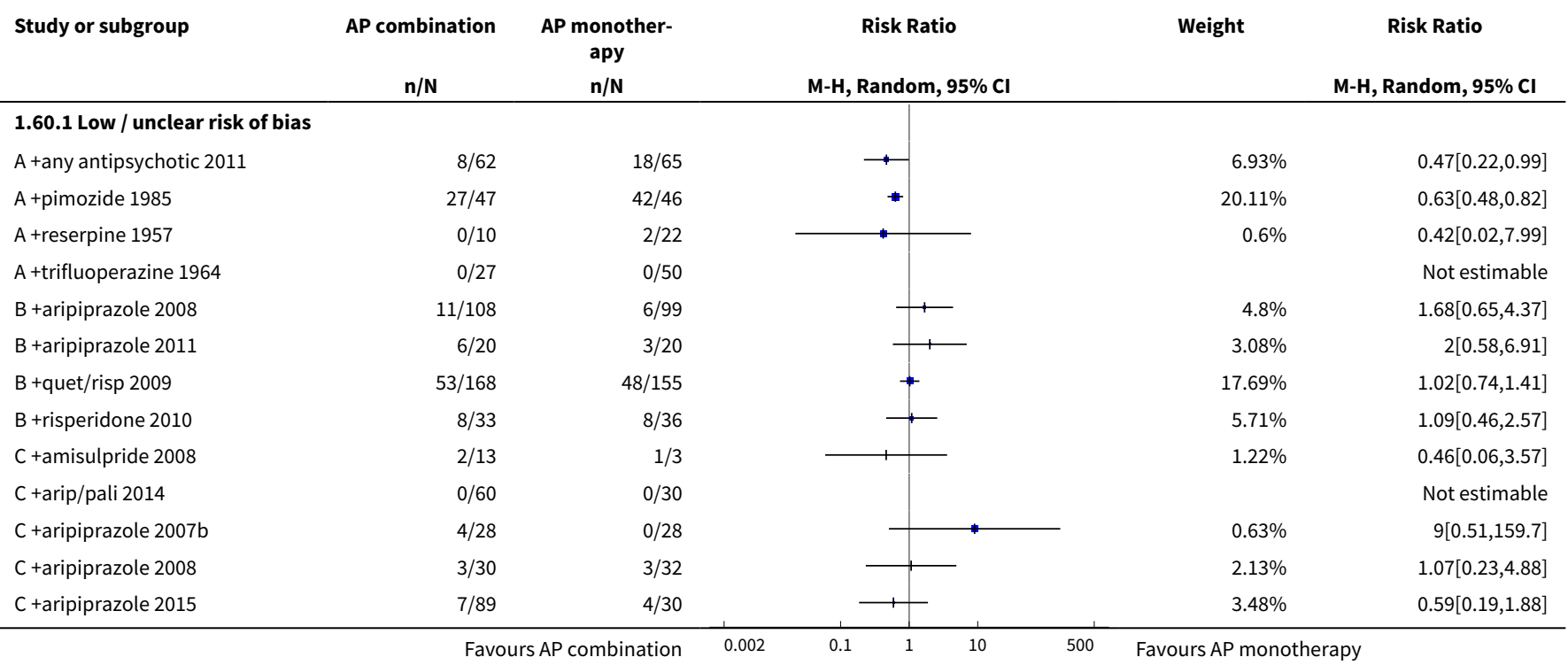




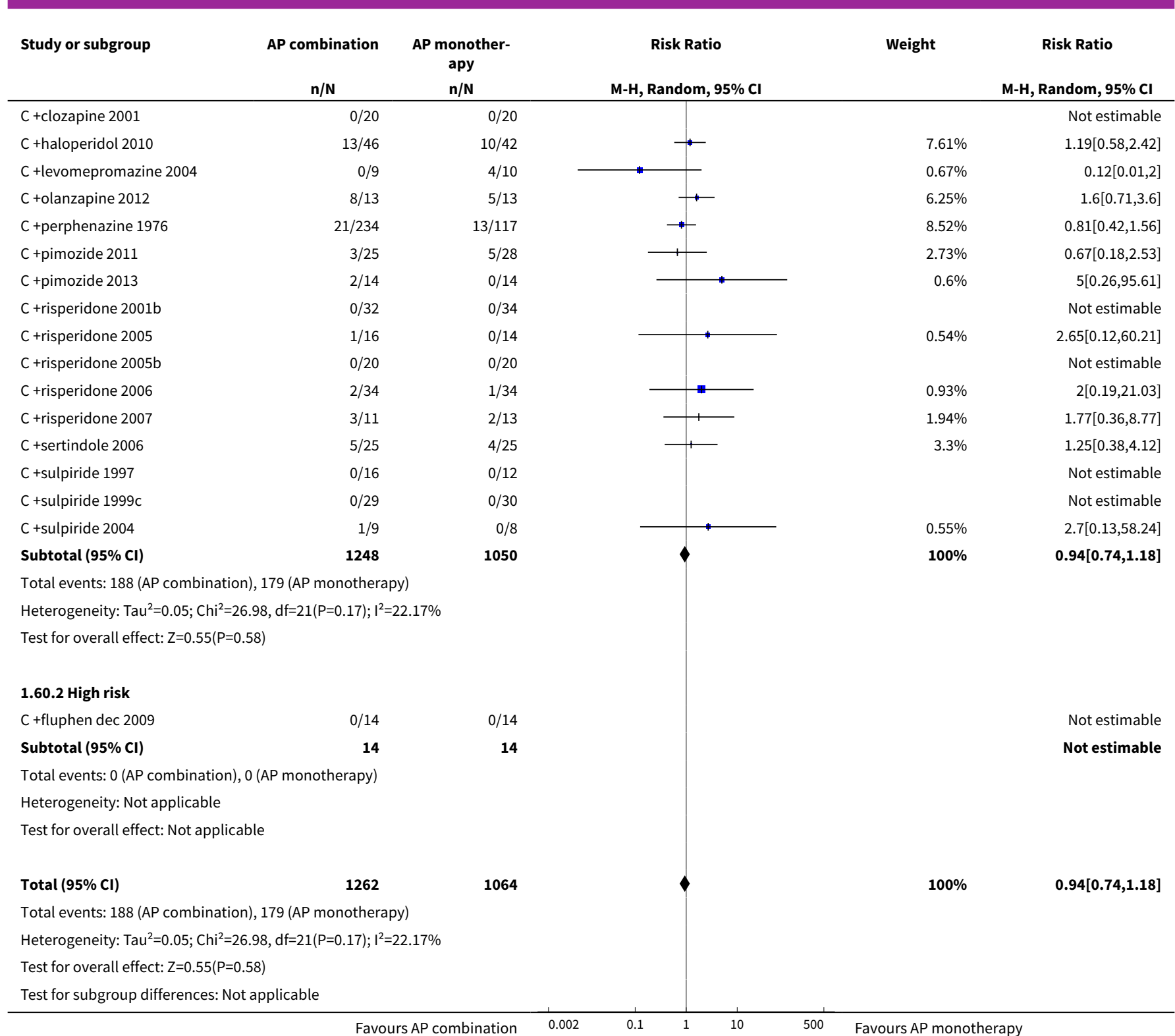

Analysis 1.61. Comparison 1 ANTIPSYCHOTIC COMBINATIONS vs ANTIPSYCHOTIC MONOTHERAPY, Outcome 61 SENSITIVITY ANALYSIS Leaving the study early - Double blind.

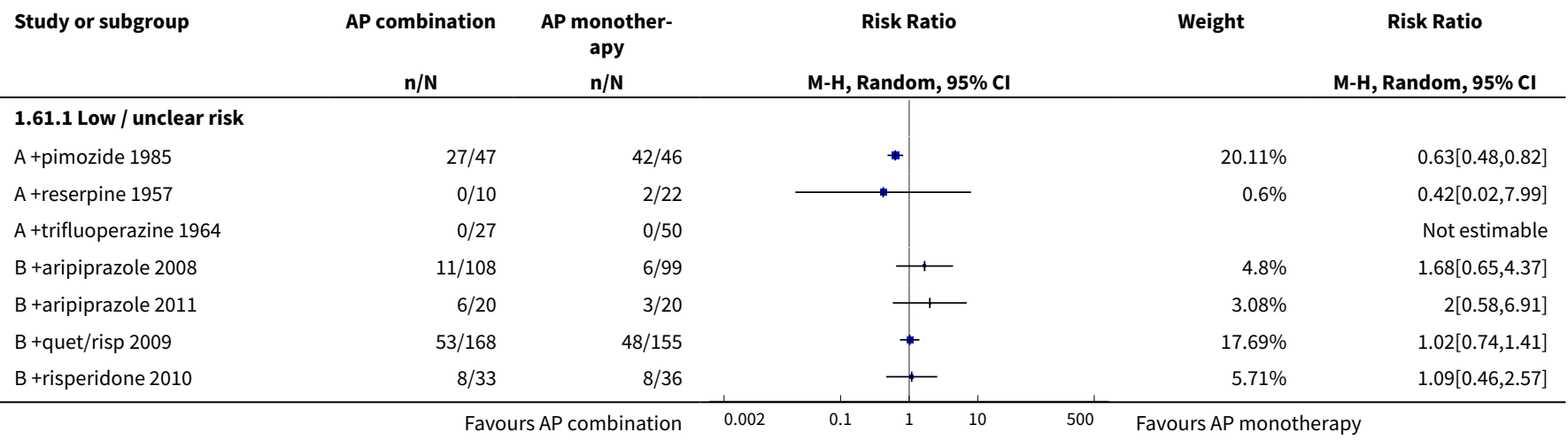




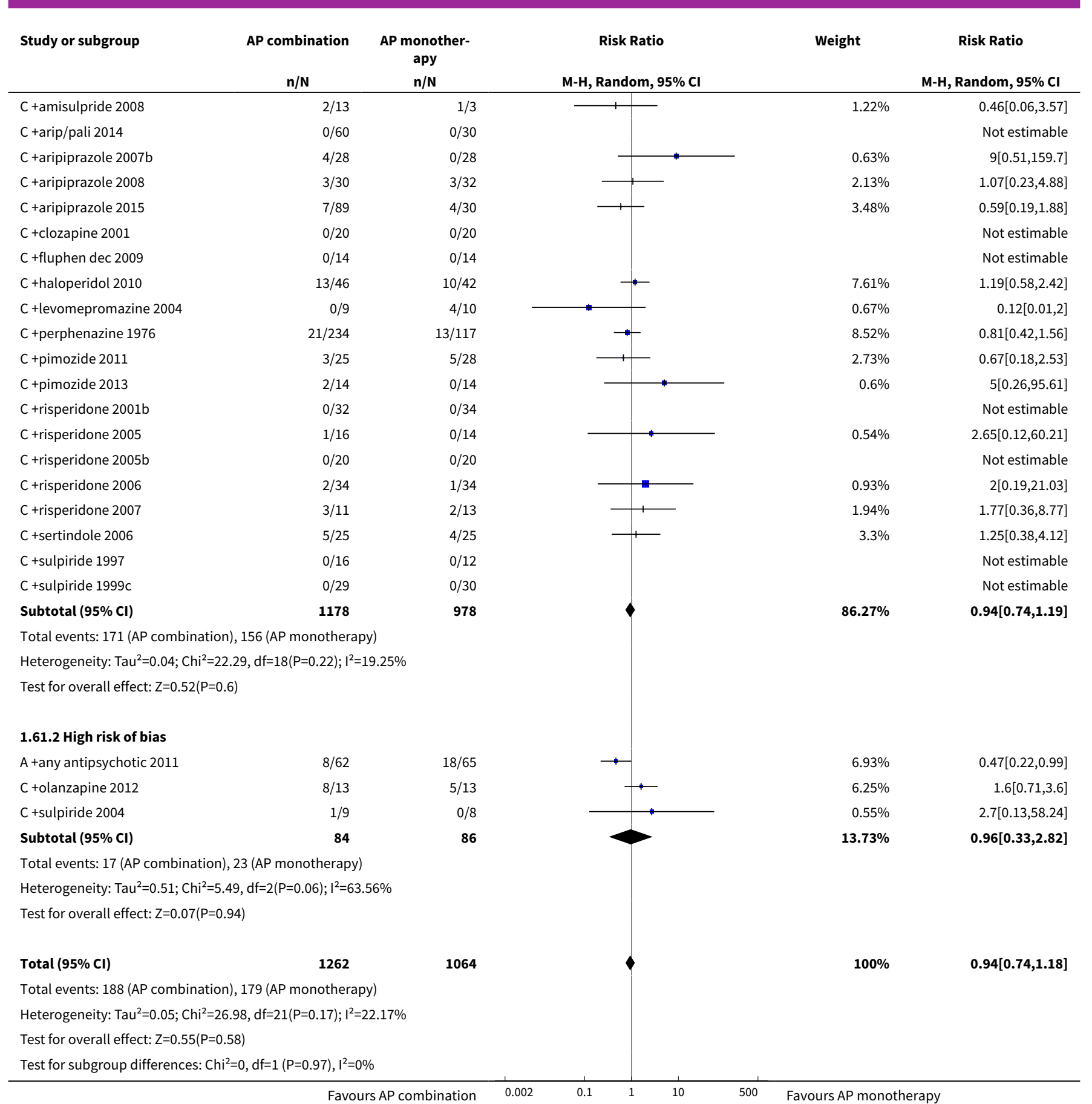

Analysis 1.62. Comparison 1 ANTIPSYCHOTIC COMBINATIONS vs ANTIPSYCHOTIC MONOTHERAPY, Outcome 62 SENSITIVITY ANALYSIS Leving the study early - Fixed effect.

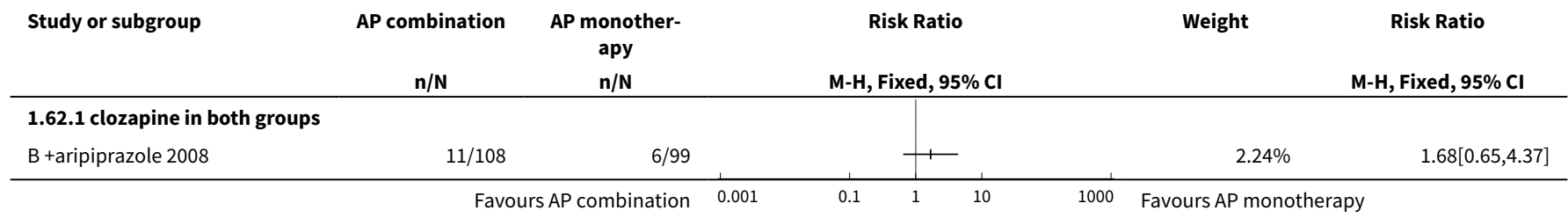




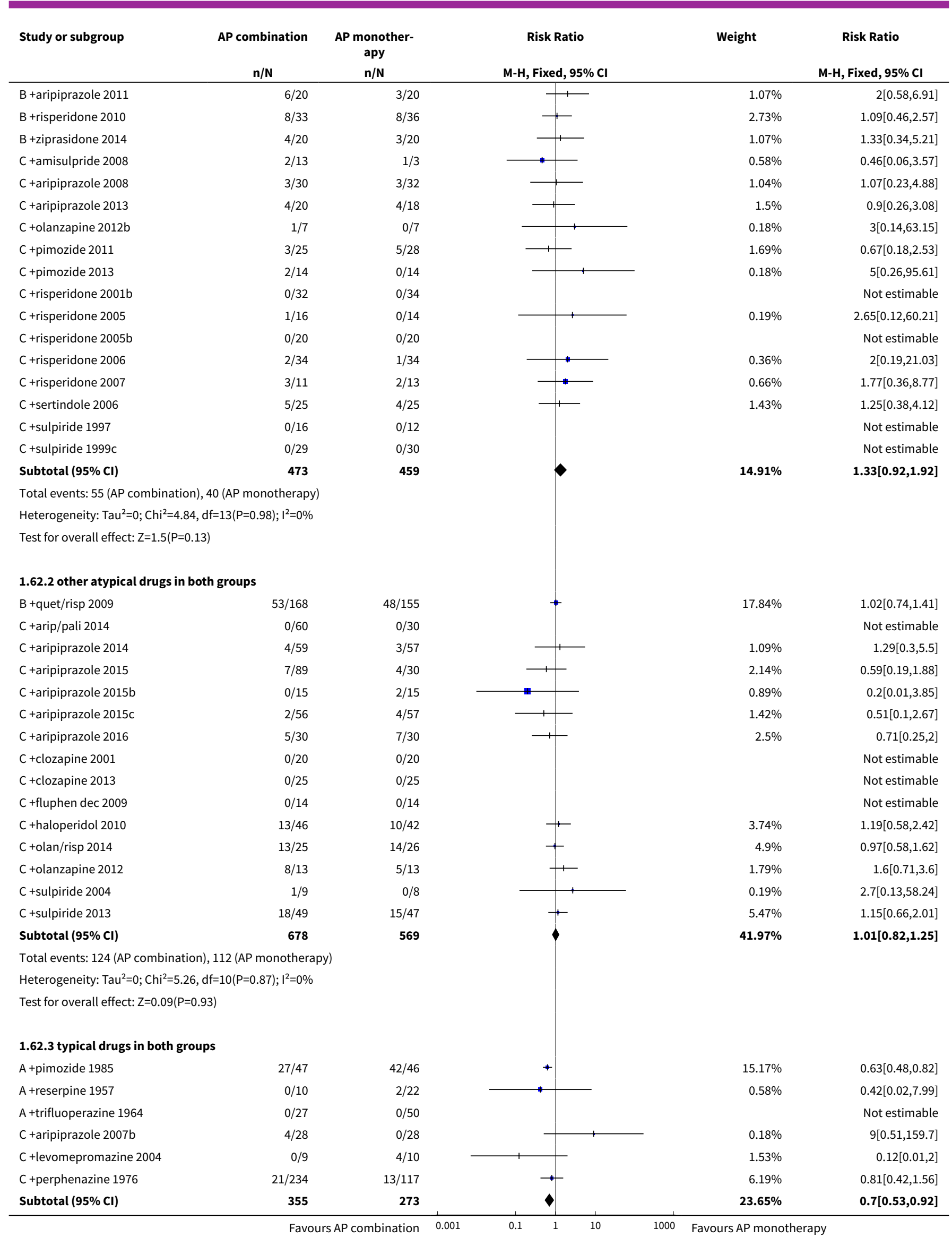




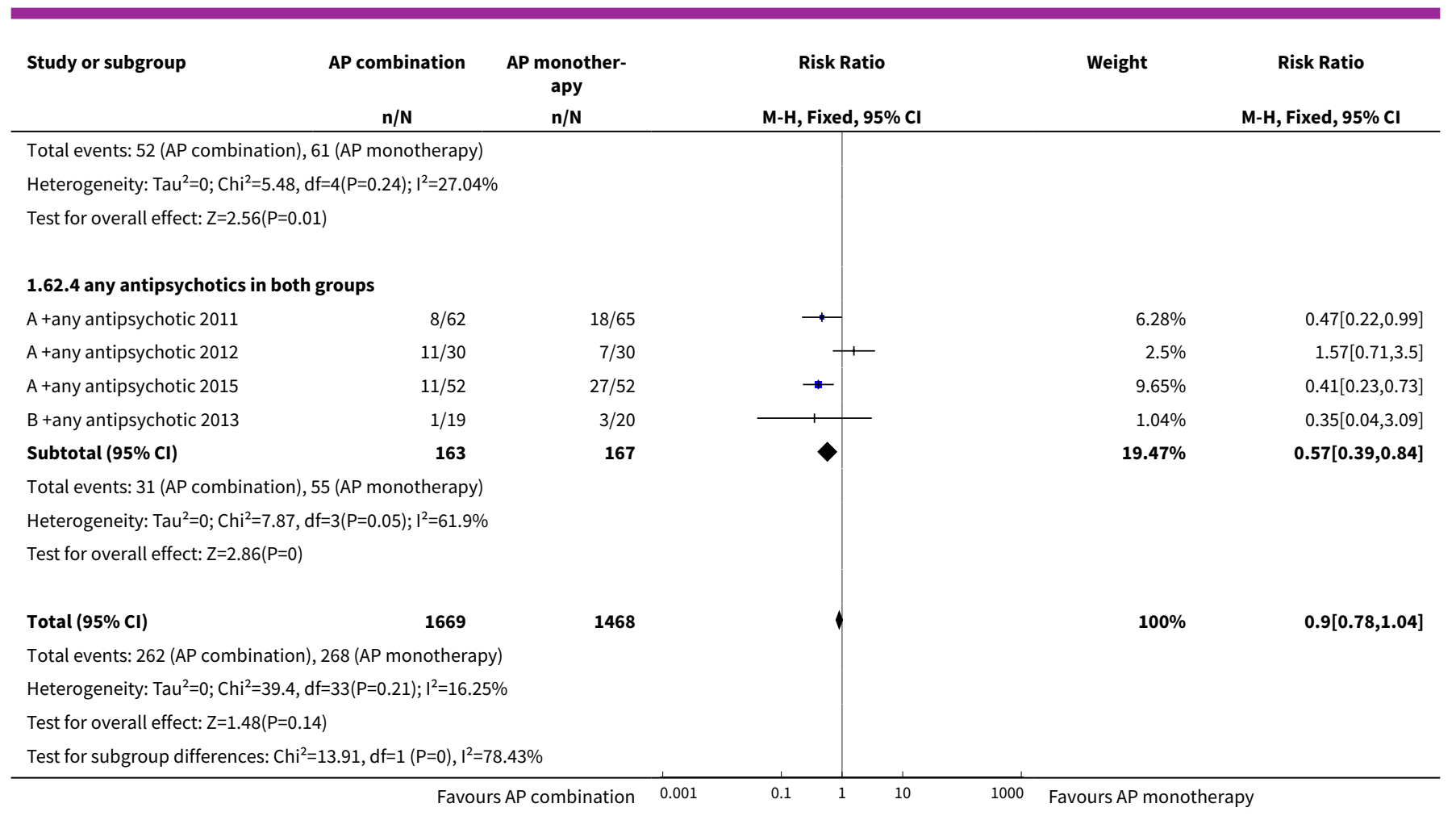

\section{ADDITIONAL TABLES}

Table 1. Meta-regression

\begin{tabular}{llllll} 
& Estimate & SE & \multicolumn{2}{l}{$95 \%$ Cl } & P value \\
\cline { 3 - 5 } & & & Lower & Upper \\
\hline Intercept & -29.5401 & 26.5608 & -81.5983 & 22.5181 & 0.2661 \\
\hline Year & 0.0147 & 0.0133 & -0.0114 & 0.0408 & 0.2706 \\
\hline Chinese & 64.7592 & 120.7041 & -171.8166 & 301.3349 & 0.5916 \\
\hline $\begin{array}{l}\text { Year }{ }^{*} \text { Chinese (interac- } \\
\text { tion) }\end{array}$ & -0.0326 & 0.0603 & -0.1508 & 0.0857 & 0.5895 \\
\hline
\end{tabular}

$\operatorname{tau}^{2}$ (estimated amount of residual heterogeneity): 0.8884 (SE = 0.4106)

tau (square root of estimated tau ${ }^{2}$ value): 0.9426

$I^{2}$ (residual heterogeneity / unaccounted variability): $97.91 \%$

$\mathrm{H}^{2}$ (unaccounted variability / sampling variability): $47.80 \%$

$\mathrm{R}^{2}$ (amount of heterogeneity accounted for): $0.00 \%$

\section{Table 2. Suggested design of future study}

$\begin{array}{ll}\text { Methods } & \text { Allocation: randomised - clearly described generation of sequence and concealment of allocation. } \\ \text { Blindness: double - described and tested. } \\ \text { Duration: } 12 \text { months minimum. }\end{array}$


Table 2. Suggested design of future study (Continued)

\begin{tabular}{ll} 
Participants & $\begin{array}{l}\text { Diagnosis: schizophrenia (operational criteria). } \\
\mathbf{N}=600 .^{*} \\
\text { Age: any. } \\
\text { Gender: both. } \\
\text { History: any. }\end{array}$ \\
\hline Interventions & 1. Antipsychotic combination. $\mathrm{N}=300$. \\
& 2. Antipsychotic of choice. $\mathrm{N}=300$. \\
\hline Outcomes & - Global impression: $\mathrm{CGI}{ }^{\star \star}$, relapse, clinical improvement. \\
& - Leaving study early (any reason, adverse events, inefficacy). \\
- Adverse events: major and minor problems as perceived by participant and clinician. & Employment, family satisfaction, patient satisfaction. \\
- Quality of life: simple binary rating.
\end{tabular}

* power calculation suggested 300/group would allow good chance of showing a 10\% difference between groups for primary outcome. ** Primary outcome.

CGI - Clinical Global Impression.

\section{APPEN DICES}

Appendix 1. Checklist to aid consistency and reproducibility of GRADE assessments

\begin{tabular}{|c|c|c|c|c|c|}
\hline $\begin{array}{l}\text { Trial limita- } \\
\text { tions }\end{array}$ & & $\begin{array}{l}\text { SoF outcome } \\
1 \text { (Clinical re- } \\
\text { sponse: No } \\
\text { clinical im- } \\
\text { provement) }\end{array}$ & $\begin{array}{l}\text { SoF outcome } \\
3 \text { (Leaving the } \\
\text { study early) }\end{array}$ & $\begin{array}{l}\text { SoF outcome } \\
4 \text { (Service uti- } \\
\text { lization: Hos- } \\
\text { pital admis- } \\
\text { sion) }\end{array}$ & $\begin{array}{l}\text { SoF outcome } \\
6 \text { (Adverse } \\
\text { events: Seri- } \\
\text { ous event or } \\
\text { requiring dis- } \\
\text { continuation) }\end{array}$ \\
\hline \multirow[t]{6}{*}{ Risk of biasa } & $\begin{array}{l}\text { Was random sequence generation used (i.e. } \\
\text { no potential for selection bias)? }\end{array}$ & Unclear & Yes & Unclear & Unclear \\
\hline & $\begin{array}{l}\text { Was allocation concealment used (i.e. no } \\
\text { potential for selection bias)? }\end{array}$ & Unclear & Unclear & Yes & Yes \\
\hline & $\begin{array}{l}\text { Was there blinding of participants and per- } \\
\text { sonnel (i.e. no potential for performance } \\
\text { bias) or outcome not likely to be influenced } \\
\text { by lack of blinding? }\end{array}$ & Unclear & Unclear & Unclear & Unclear \\
\hline & $\begin{array}{l}\text { Was there blinding of outcome assessment } \\
\text { (i.e. no potential for detection bias) or was } \\
\text { outcome measurement not likely to be influ- } \\
\text { enced by lack of blinding? }\end{array}$ & Unclear & Unclear & Unclear & No $(*)$ \\
\hline & Was an objective outcome used? & No $\left({ }^{\star}\right)$ & Yes & Yes & Yes \\
\hline & $\begin{array}{l}\text { Were more than } 80 \% \text { of participants en- } \\
\text { rolled in trials included in the analysis (i.e. } \\
\text { no potential reporting bias)? }\end{array}$ & Unclear & Yes & Unclear & Unclear \\
\hline
\end{tabular}


(Continued)

Were data reported consistently for the out- Unclear $\quad$ Yes Unclear $\quad$ Yes come of interest (i.e. no potential selective reporting)?

\begin{tabular}{|c|c|c|c|}
\hline $\begin{array}{l}\text { No other biases reported (i.e. no potential of } \\
\text { other bias)? }\end{array}$ & Yes & Yes & Yes \\
\hline
\end{tabular}

\begin{tabular}{lllll}
\hline $\begin{array}{l}\text { Did the trials end up as scheduled (i.e. not } \\
\text { stopped early)? }\end{array}$ & Yes & Yes & Yes & Yes
\end{tabular}

\begin{tabular}{|c|c|c|c|c|c|}
\hline \multirow{7}{*}{$\begin{array}{l}\text { Inconsisten- } \\
\text { cyb }\end{array}$} & Point estimates did not vary widely? & Yes & No( $\left.{ }^{*}\right)$ & Yes & No(*) \\
\hline & $\begin{array}{l}\text { To what extent did confidence intervals } \\
\text { overlap (substantial: all confidence intervals } \\
\text { overlap at least one of the included studies } \\
\text { point estimate; }\end{array}$ & Some & Some & Some & Some \\
\hline & $\begin{array}{l}\text { some: confidence intervals overlap but not } \\
\text { all overlap at least one point estimate; no: at } \\
\text { least one outlier: where the confidence in- } \\
\text { terval of some }\end{array}$ & & & & \\
\hline & $\begin{array}{l}\text { of the studies do not overlap with those of } \\
\text { most included studies)? }\end{array}$ & & & & \\
\hline & Was the direction of effect consistent? & Yes & No $\left(^{\star}\right)$ & No $\left(^{*}\right)$ & No $\left(^{*}\right)$ \\
\hline & $\begin{array}{l}\text { What was the magnitude of statistical het- } \\
\left.\text { erogeneity (as measured by } I^{2}\right) \text { - low }\left(I^{2}<\right. \\
40 \%) \text {, moderate }\left(I^{2} 40 \%-60 \%\right) \text {, high } I^{2}> \\
60 \%) ?\end{array}$ & High $\left(^{\star}\right)$ & Low & Low & Low \\
\hline & $\begin{array}{l}\text { Was the test for heterogeneity statistically } \\
\text { significant }(P<0.1) \text { ? }\end{array}$ & $\begin{array}{l}\text { Statistically } \\
\text { significant }\left({ }^{\star}\right)\end{array}$ & $\begin{array}{l}\text { Not statisti- } \\
\text { cally signifi- } \\
\text { cant }\end{array}$ & $\begin{array}{l}\text { Not statisti- } \\
\text { cally signifi- } \\
\text { cant }\end{array}$ & $\begin{array}{l}\text { Not statisti- } \\
\text { cally signifi- } \\
\text { cant }\end{array}$ \\
\hline \multirow[t]{5}{*}{ Indirectness } & $\begin{array}{l}\text { Were the populations in included studies ap- } \\
\text { plicable to the decision context? }\end{array}$ & Applicable & Applicable & Applicable & Applicable \\
\hline & $\begin{array}{l}\text { Were the interventions in the included stud- } \\
\text { ies applicable to the decision context? }\end{array}$ & $\begin{array}{l}\text { Highly applic- } \\
\text { able }\end{array}$ & Applicable & Applicable & Applicable \\
\hline & $\begin{array}{l}\text { Was the included outcome not a surrogate } \\
\text { outcome? }\end{array}$ & Yes & Yes & Yes & Yes \\
\hline & Was the outcome timeframe sufficient? & $\begin{array}{l}\text { Insufficient } \\
\left({ }^{\star}\right)\end{array}$ & Sufficient & $\begin{array}{l}\text { Insufficient } \\
\left({ }^{\star}\right)\end{array}$ & $\begin{array}{l}\text { Insufficient } \\
\left({ }^{\star}\right)\end{array}$ \\
\hline & $\begin{array}{l}\text { Were the conclusions based on direct com- } \\
\text { parisons? }\end{array}$ & Yes & Yes & Yes & Yes \\
\hline \multirow[t]{2}{*}{ Imprecisionc } & $\begin{array}{l}\text { Was the confidence interval for the pooled } \\
\text { estimate not consistent with benefit and } \\
\text { harm? }\end{array}$ & Yes & No $\left(^{*}\right)$ & No $\left(^{*}\right)$ & No $\left(^{*}\right)$ \\
\hline & $\begin{array}{l}\text { What is the magnitude of the median sam- } \\
\text { ple size (high: } 300 \text { participants, intermedi- }\end{array}$ & High & High & Intermediate & High \\
\hline
\end{tabular}


(Continued)

ate: $100-300$ participants, low: $<100$ partici-

pants)?e

\begin{tabular}{|c|c|c|c|c|}
\hline $\begin{array}{l}\text { What was the magnitude of the number of } \\
\text { included studies (large: }>10 \text { studies, moder- } \\
\text { ate: } 5-10 \text { studies, small: }<5 \text { studies)?e }\end{array}$ & Large & Large & Small $\left(^{\star}\right)$ & Large \\
\hline $\begin{array}{l}\text { Was the outcome a common event (e.g. oc- } \\
\text { curs more than } 1 / 100) \text { ? }\end{array}$ & Yes & Yes & Yes & Yes \\
\hline Was a comprehensive search conducted? & Yes & Yes & Yes & Yes \\
\hline Was grey literature searched? & Yes & Yes & Yes & Yes \\
\hline $\begin{array}{l}\text { Were no restrictions applied to study selec- } \\
\text { tion on the basis of language? }\end{array}$ & Yes & Yes & Yes & Yes \\
\hline $\begin{array}{l}\text { There was no industry influence on studies } \\
\text { included in the review? }\end{array}$ & Yes & Yes & Yes & No $\left(^{*}\right)$ \\
\hline $\begin{array}{l}\text { There was no evidence of funnel plot asym- } \\
\text { metry? }\end{array}$ & No $\left(^{*}\right)$ & Yes & N/A & Yes \\
\hline $\begin{array}{l}\text { There was no discrepancy in findings be- } \\
\text { tween published and unpublished trials? }\end{array}$ & Unclear & Unclear & Unclear & Unclear \\
\hline
\end{tabular}

\section{Footnotes}

aQuestions on risk of bias are answered in relation to the majority of the aggregated evidence in the meta-analysis rather than to individual trials

${ }^{b}$ Questions on inconsistency are primarily based on visual assessment of forest plots and the statistical quantification of heterogeneity based on $\mathrm{I}^{2}$ statistic

cWhen judging the width of the confidence interval it is recommended to use a clinical decision threshold to assess whether the imprecision is clinically meaningful

dQuestions address comprehensiveness of the search strategy, industry influence, funnel plot asymmetry and discrepancies between published and unpublished trials

eDepends on the context of the systematic review area

$\left({ }^{*}\right)$ : key item for potential downgrading the quality of the evidence (GRADE) as shown in the footnotes of the 'Summary of findings' table; GRADE: Grading of Recommendations Assessment, Development and Evaluation; N/A: not applicable

\section{Appendix 2. Initial trial selection and data extraction}

Two review authors (NM, KSW) inspected all abstracts of studies identified as above and identified potentially relevant reports. JX screened the Chinese language studies. Where disagreement occurred this was resolved by discussion, or where there was still doubt, the full article was acquired for further inspection. We acquired the full articles of relevant reports for reassessment and carefully inspected for a final decision on inclusion (see Criteria for considering studies for this review). NM and KSW were not blinded to the names of the authors, institutions or journal of publication. Where difficulties or disputes arose, we asked author CEA for help and where it was impossible to decide or if adequate information was not available to make a decision, we added these studies to those awaiting assessment and the authors of the papers contacted for clarification.

\section{Extraction}

Review authors NM and KSW extracted data from all included studies. In addition, JX extracted data for all Chinese studies and one study in Japanese was inspected by IO. To ensure reliability, CEA independently extracted data from a random sample of these studies, comprising $30 \%$ of the total. Again, any disagreement was discussed, decisions documented and, if necessary, authors of studies contacted for 
clarification. With remaining problems CEA helped clarify issues and those final decisions were documented. We extracted data presented only in graphs and figures whenever possible, but only included if two review authors independently had the same result. Where possible, we extracted data relevant to each component centre of multi-centre studies separately.

\section{Management}

\subsection{Forms}

A form for data collection was created in Microsoft InfoPath 2007, piloted in three trials independently by two authors, and revised after author discussion. We extracted data onto these forms.

\section{Assessment of risk of bias in included studies}

KSW and NM independently assessed the risk of bias of each trial published in English and JX assessed trials published in Chinese using Cochrane's 'Risk of bias' tool (Higgins 2011). This set of criteria is based on evidence of associations between overestimate of effect and high risk of bias of the article such as sequence generation, allocation concealment, blinding, incomplete outcome data and selective reporting. If the raters disagreed, the final rating was made by consensus, with the involvement of another member of the review group. Where inadequate details of randomisation and other characteristics of trials were provided, authors of the studies were contacted in order to obtain further information. Non-concurrence in quality assessment was reported, but if disputes arise as to which category a trial is to be allocated, again, resolution was made by discussion with CEA. The level of risk of bias was noted in both the text of the review and in the 'Summary of findings' table 1.

\section{Data synthesis}

We understand that there is no closed argument for preference for use of fixed-effect or random-effects models. The random-effects method incorporates an assumption that the different studies are estimating different, yet related, intervention effects. This often seems to be true to us and the random-effects model takes into account differences between studies even if there is no statistically significant heterogeneity. There is, however, a disadvantage to the random-effects model. It puts added weight onto small studies which often are the most biased ones. Depending on the direction of effect these studies can either inflate or deflate the effect size. A fixed-effect model was used, unless we demonstrated statistically significant heterogeneity $(P<0.10)$ for a specific outcome, in which case the random-effects models was preferred.

\section{Subgroup analysis and investigation of heterogeneity}

\section{Subgroup analyses - only primary outcomes}

For subgroup and sensitivity analyses the random-effects model was used for all analyses for which an 12 was above $50 \%$. Subgroup and sensitivity analyses were undertaken only where there were at least 10 trials. In addition, trials with zero events were not accounted for in the subgroup analyses.

\subsection{Leaving the study early}

We undertook four subgroup analyses using data from the primary outcome 'leaving the study early':

- enrolment of acutely exacerbated or chronically ill patients;

- treatment duration < 12 weeks versus $\geq 12$ weeks;

- clozapine versus non-clozapine combinations; and

- drug added to co-treatment of the same antipsychotics in combination or monotherapy groups.

\section{Appendix 3. Participant's country of origin}

\begin{tabular}{lll}
\hline Country of origin & Number of trials & Number of participants \\
\hline China & 24 & 2222 \\
\hline Europe & 6 & 367 \\
\hline Iran & 1 & 28 \\
\hline Israel & 2 & 45 \\
\hline Japan & 7 & 594 \\
\hline
\end{tabular}


(Continued)

\begin{tabular}{lcc} 
Korea & 2 & 96 \\
\hline Multinational & 1 & 68 \\
\hline Taiwan & 2 & 184 \\
\hline Turkey & 1 & 30 \\
\hline USA & 12 & 975 \\
\hline Unclear & 2 & 70
\end{tabular}

\section{Appendix 4. Operational criteria}

\begin{tabular}{lll}
\hline Operational criteria & & Number of trials \\
\hline Diagnostic and Statistical Manual of Mental Disorders & DSM-IV & 31 \\
\cline { 2 - 3 } & DSM-III & 1 \\
\cline { 2 - 3 } & DSM-II & 2 \\
\hline International Classification of Diseases & ICD-10 & 5 \\
\hline Chinese Classification of Mental Disorders & CCMD-III & 8 \\
\cline { 2 - 3 } & CCMD-2R & 13 \\
\hline Unclear & & 2 \\
\hline
\end{tabular}

\section{Appendix 5. Antipsychotic doses}




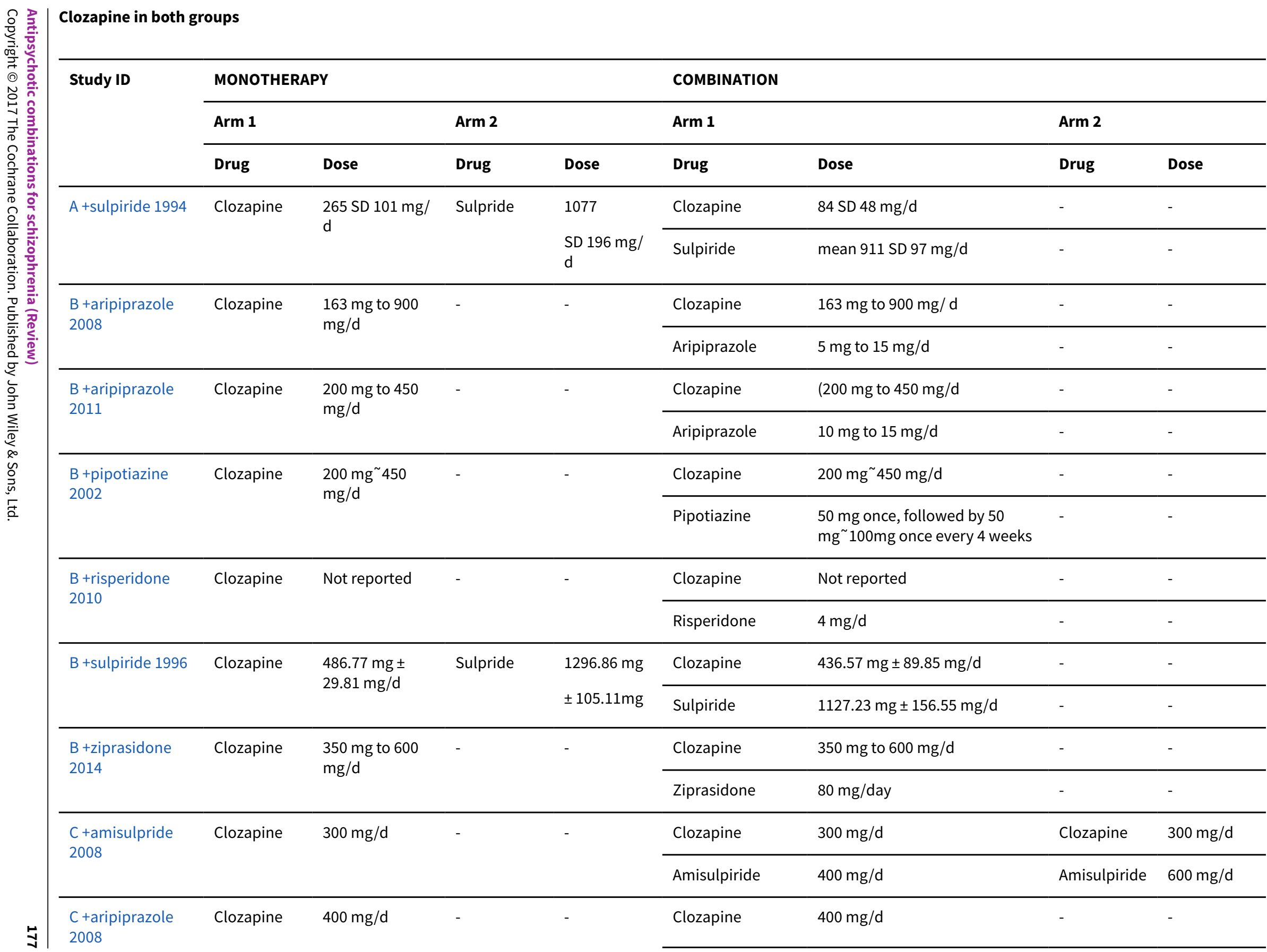




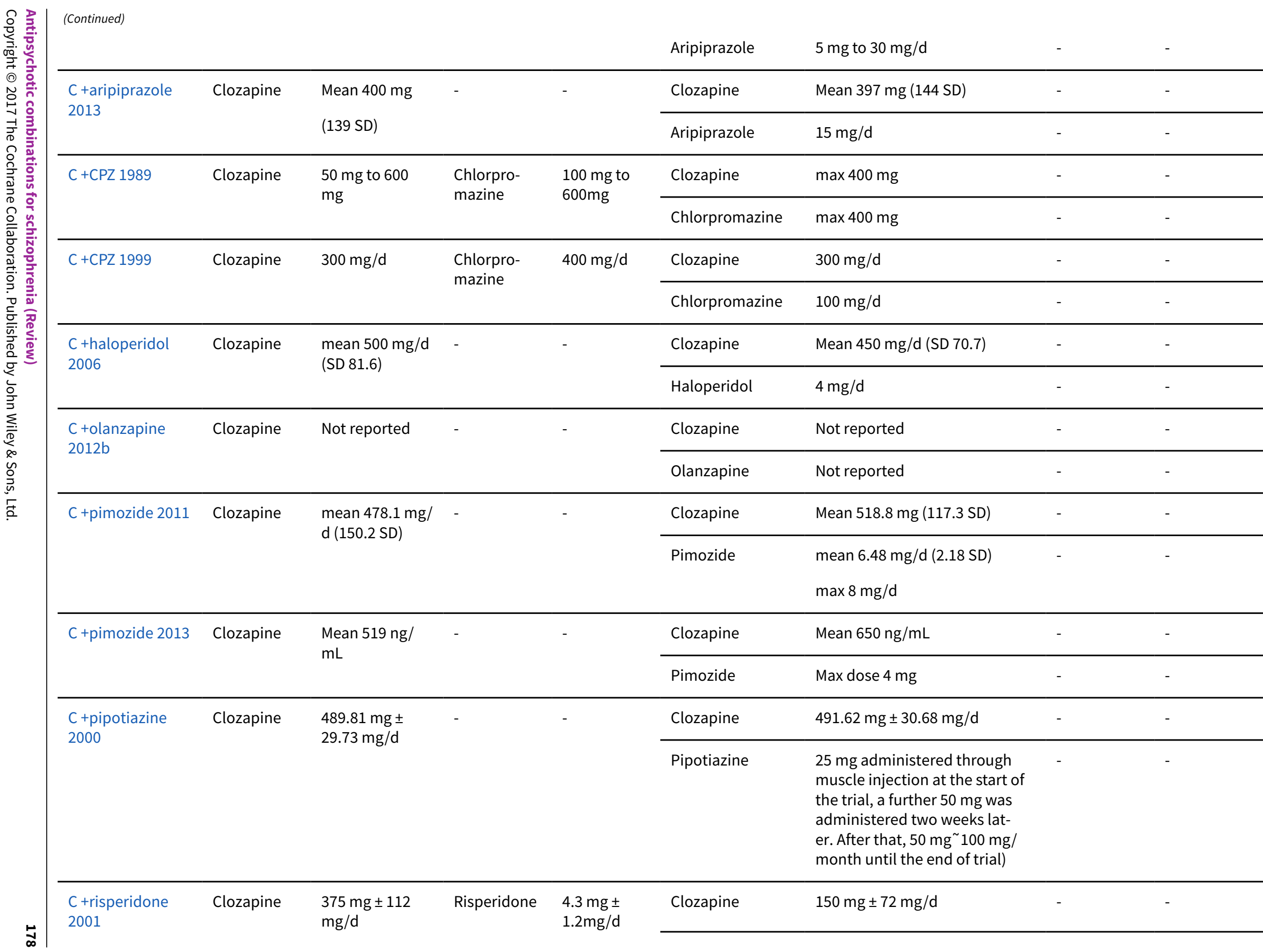




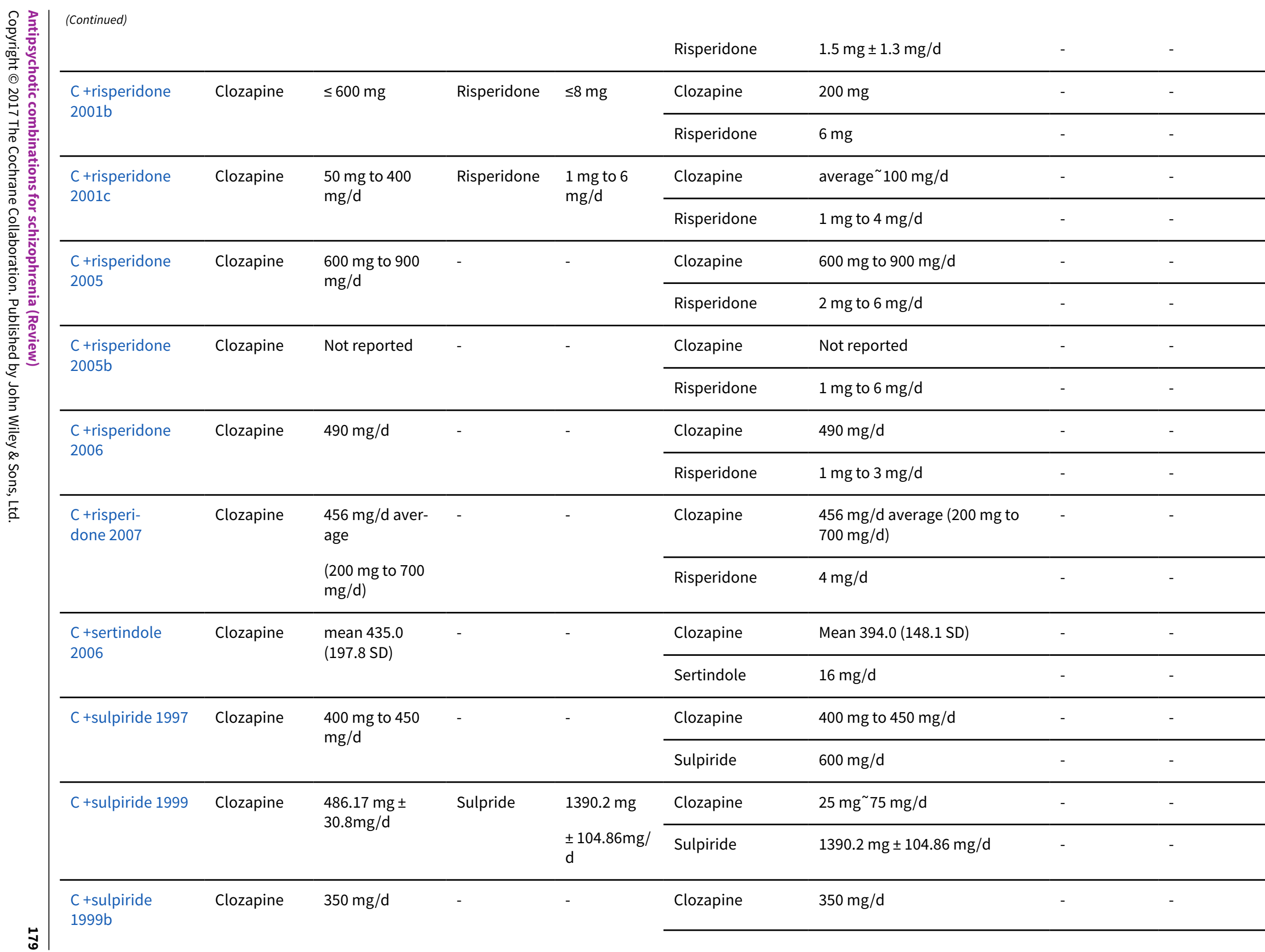




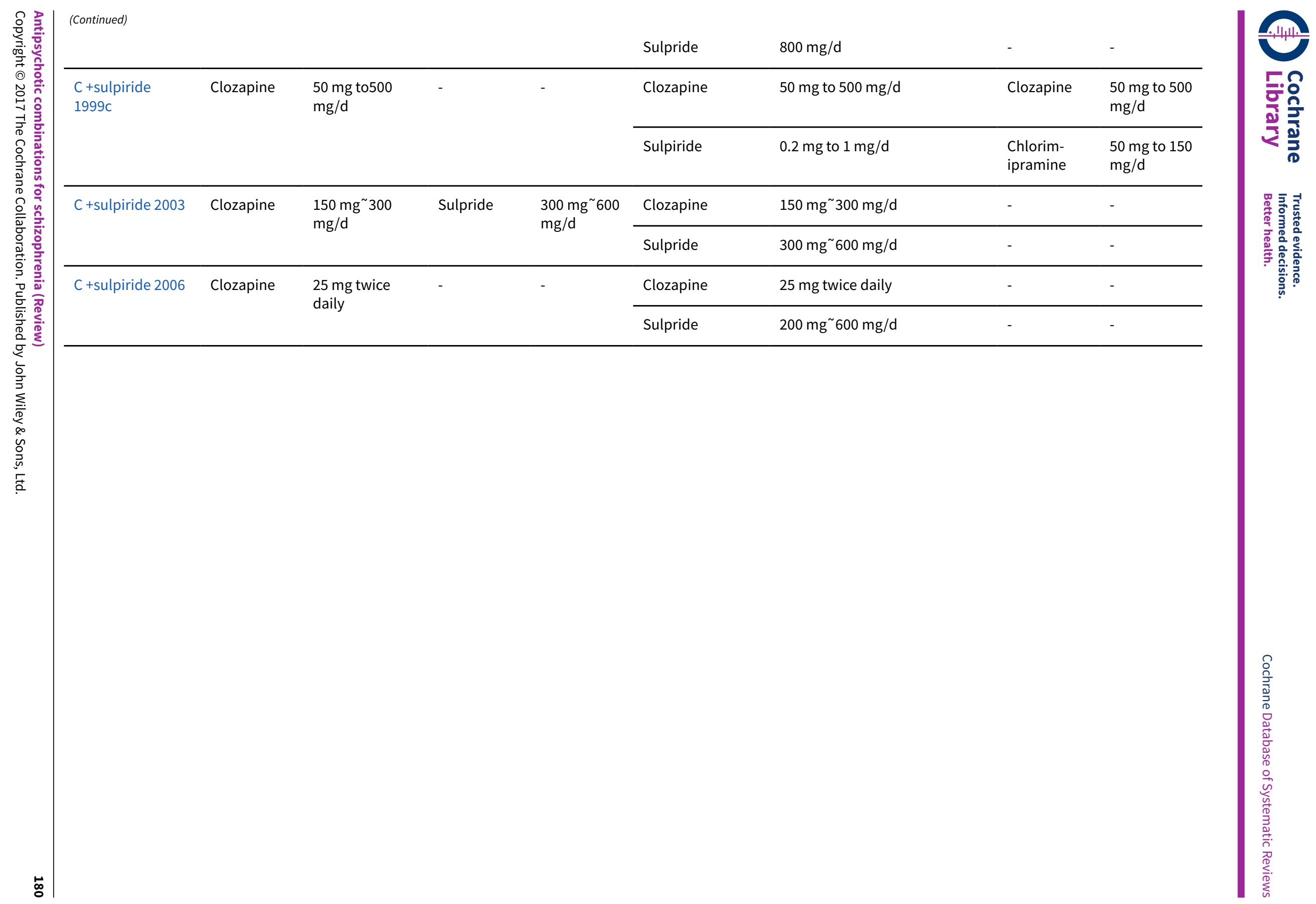




\begin{tabular}{|c|c|c|c|c|c|c|c|c|c|c|}
\hline \multirow[t]{3}{*}{ Study ID } & \multicolumn{4}{|c|}{ MONOTHERAPY } & \multicolumn{6}{|c|}{ COMBINATION } \\
\hline & \multicolumn{2}{|l|}{ Arm 1} & \multicolumn{2}{|l|}{ Arm 2} & \multicolumn{2}{|l|}{ Arm 1} & \multicolumn{2}{|l|}{ Arm 2} & \multicolumn{2}{|l|}{ Arm 3} \\
\hline & Drug & Dose & Drug & Dose & Drug & Dose & Drug & Dose & Drug & Dose \\
\hline \multirow[t]{2}{*}{$\begin{array}{l}\text { B +quet/risp } \\
2009\end{array}$} & \multirow[t]{2}{*}{$\begin{array}{l}\text { Quetiap- } \\
\text { ine }\end{array}$} & \multirow[t]{2}{*}{$\begin{array}{l}400 \mathrm{mg} \text { to } \\
800 \mathrm{mg} / \mathrm{d}\end{array}$} & \multirow[t]{2}{*}{$\begin{array}{l}\text { Risperi- } \\
\text { done }\end{array}$} & \multirow[t]{2}{*}{$\begin{array}{l}4 \mathrm{mg} \text { to } 8 \\
\mathrm{mg} / \mathrm{d}\end{array}$} & Quetiapine & $400 \mathrm{mg}$ to $800 \mathrm{mg} / \mathrm{d}$ & $\begin{array}{l}\text { Risperi- } \\
\text { done }\end{array}$ & $\begin{array}{l}4 \mathrm{mg} \text { to } 8 \\
\mathrm{mg} / \mathrm{d}\end{array}$ & - & - \\
\hline & & & & & Aripiprazole & $2 \mathrm{mg}$ to $15 \mathrm{mg} / \mathrm{d}$ & $\begin{array}{l}\text { Aripipra- } \\
\text { zole }\end{array}$ & $\begin{array}{l}2 \mathrm{mg} \text { to } 15 \\
\mathrm{mg} / \mathrm{d}\end{array}$ & - & - \\
\hline \multirow{2}{*}{$\begin{array}{l}\text { C +aripipra- } \\
\text { zole } 2007\end{array}$} & \multirow[t]{2}{*}{ Sulpiride } & \multirow{2}{*}{$\begin{array}{l}500 \mathrm{mg} \text { to } \\
900 \mathrm{mg}\end{array}$} & \multirow[t]{2}{*}{-} & \multirow[t]{2}{*}{-} & Sulpiride & $600 \mathrm{mg}$ to $900 \mathrm{mg}$ & - & - & - & - \\
\hline & & & & & Aripiprazole & $10 \mathrm{mg}$ & - & - & - & - \\
\hline \multirow[t]{2}{*}{$\begin{array}{l}\text { C +aripipra- } \\
\text { zole } 2012\end{array}$} & \multirow[t]{2}{*}{$\begin{array}{l}\text { Risperi- } \\
\text { done }\end{array}$} & \multirow[t]{2}{*}{$\begin{array}{l}2 \mathrm{mg} \text { to } 12 \\
\mathrm{mg} / \mathrm{d}\end{array}$} & \multirow[t]{2}{*}{$\begin{array}{l}\text { Olanzap- } \\
\text { ine }\end{array}$} & \multirow[t]{2}{*}{$\begin{array}{l}2.5 \mathrm{mg} \text { to } \\
20 \mathrm{mg} / \mathrm{d}\end{array}$} & Risperidone & $2 \mathrm{mg}$ to $12 \mathrm{mg} / \mathrm{d}$ & $\begin{array}{l}\text { Olanzap- } \\
\text { ine }\end{array}$ & $\begin{array}{l}2.5 \mathrm{mg} \text { to } \\
20 \mathrm{mg} / \mathrm{d}\end{array}$ & - & - \\
\hline & & & & & Aripiprazole & $6 \mathrm{mg}$ to $30 \mathrm{mg} / \mathrm{d}$ & $\begin{array}{l}\text { Aripipra- } \\
\text { zole }\end{array}$ & $\begin{array}{l}6 \mathrm{mg} \text { to } 30 \\
\mathrm{mg} / \mathrm{d}\end{array}$ & - & - \\
\hline \multirow[t]{2}{*}{$\begin{array}{l}\text { C +aripipra- } \\
\text { zole 2013b }\end{array}$} & \multirow[t]{2}{*}{$\begin{array}{l}\text { Risperi- } \\
\text { done }\end{array}$} & \multirow[t]{2}{*}{$\begin{array}{l}3 \mathrm{mg} \text { to } 6 \\
\mathrm{mg}\end{array}$} & \multirow[t]{2}{*}{-} & \multirow[t]{2}{*}{-} & Risperidone & $3 \mathrm{mg}$ to $6 \mathrm{mg}$ & - & - & - & - \\
\hline & & & & & Aripiprazole & $10 \mathrm{mg} /$ day & - & - & - & - \\
\hline \multirow[t]{2}{*}{$\begin{array}{l}\text { C +aripipra- } \\
\text { zole } 2014\end{array}$} & \multirow[t]{2}{*}{$\begin{array}{l}\text { Risperi- } \\
\text { done }\end{array}$} & \multirow[t]{2}{*}{$\begin{array}{l}3 \mathrm{mg} \text { to } 8 \\
\mathrm{mg} / \text { day }\end{array}$} & \multirow[t]{2}{*}{-} & \multirow[t]{2}{*}{-} & Risperidone & $3 \mathrm{mg}$ to $8 \mathrm{mg} /$ day & - & - & - & - \\
\hline & & & & & Aripiprazole & $10 \mathrm{mg}$ to $20 \mathrm{mg} /$ day & - & - & - & - \\
\hline \multirow[t]{2}{*}{$\begin{array}{l}\text { C +aripipra- } \\
\text { zole } 2015\end{array}$} & \multirow[t]{2}{*}{$\begin{array}{l}\text { Risperi- } \\
\text { done }\end{array}$} & $\begin{array}{l}\text { mean }=4.93 \\
\mathrm{mg} / \text { day SD } \\
1.05\end{array}$ & - & - & Risperidone & $\begin{array}{l}\text { mean }=4.63 \mathrm{mg} / \text { day SD } \\
1.10\end{array}$ & $\begin{array}{l}\text { Risperi- } \\
\text { done }\end{array}$ & $\begin{array}{l}\text { mean }= \\
4.79 \mathrm{mg} / \\
\text { day SD } \\
1.01\end{array}$ & $\begin{array}{l}\text { Risperi- } \\
\text { done }\end{array}$ & $\begin{array}{l}\text { mean }= \\
5.07 \mathrm{mg} / \\
\text { day SD } \\
1.12\end{array}$ \\
\hline & & & & & Aripiprazole & $5 \mathrm{mg} / \mathrm{d}$ & $\begin{array}{l}\text { Aripipra- } \\
\text { zole }\end{array}$ & $10 \mathrm{mg} / \mathrm{d}$ & $\begin{array}{l}\text { Aripipra- } \\
\text { zole }\end{array}$ & $20 \mathrm{mg} / \mathrm{d}$ \\
\hline $\begin{array}{l}\text { C +aripipra- } \\
\text { zole } 2015 b\end{array}$ & $\begin{array}{l}\text { Risperi- } \\
\text { done }\end{array}$ & $\begin{array}{l}\text { mean } 6 \mathrm{mg} / \\
\text { day }\end{array}$ & - & - & Risperidone & mean $6 \mathrm{mg} /$ day & - & - & - & - \\
\hline & & & & & Aripiprazole & $10 \mathrm{mg} /$ day & - & - & - & - \\
\hline
\end{tabular}




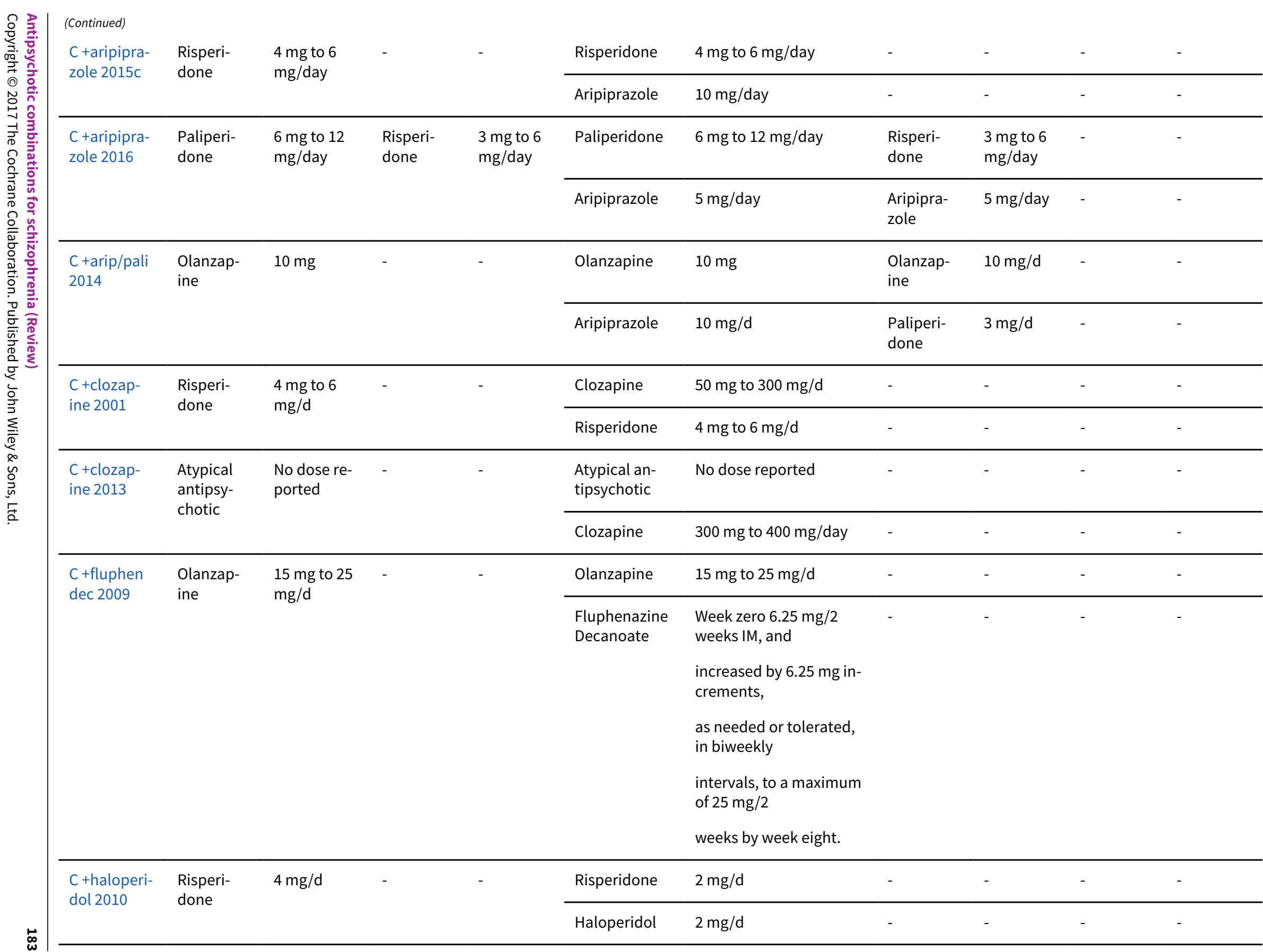




\begin{tabular}{|c|c|c|c|c|c|c|c|c|c|c|}
\hline \multirow{3}{*}{$\begin{array}{l}\text { C +olanzap- } \\
\text { ine } 2012\end{array}$} & \multirow{3}{*}{$\begin{array}{l}\text { Risperi- } \\
\text { done }\end{array}$} & \multirow{3}{*}{$\begin{array}{l}\text { Starting at } 3 \\
\mathrm{mg} / \mathrm{d} \text {, } \\
\text { at } 2 \text { weeks < } \\
6 \mathrm{mg} / \mathrm{d} \\
\text { was allowed } \\
\text { and at } 8 \\
\text { weeks }<12 \\
\mathrm{mg} / \mathrm{d}\end{array}$} & \multirow[t]{3}{*}{-} & \multirow[t]{3}{*}{-} & Risperidone & $<6 \mathrm{mg} / \mathrm{d}$ & - & - & - & - \\
\hline & & & & & Olanzapine & $<20 \mathrm{mg} / \mathrm{d}$ & - & - & - & - \\
\hline & & & & & & & & & & \\
\hline \multirow[t]{2}{*}{$\begin{array}{l}\text { C tolan/risp } \\
2014\end{array}$} & \multirow[t]{2}{*}{$\begin{array}{l}\text { Olanzap- } \\
\text { ine }\end{array}$} & \multirow[t]{2}{*}{$\begin{array}{l}\text { Max } 18.8 \\
\mathrm{mg} / \text { day }\end{array}$} & \multirow[t]{2}{*}{$\begin{array}{l}\text { Risperi- } \\
\text { done }\end{array}$} & \multirow[t]{2}{*}{$\begin{array}{l}\text { Max } 8.2 \\
\mathrm{mg} / \text { day }\end{array}$} & Olanzapine & Mean 19.0 mg/day & $\begin{array}{l}\text { Risperi- } \\
\text { done }\end{array}$ & $\begin{array}{l}\text { Mean } 8.7 \\
\text { mg/day }\end{array}$ & - & - \\
\hline & & & & & Risperidone & Max $8.1 \mathrm{mg} /$ day & $\begin{array}{l}\text { Olanzap- } \\
\text { ine }\end{array}$ & $\begin{array}{l}\text { Max. } 16.1 \\
\mathrm{mg} / \text { day }\end{array}$ & - & - \\
\hline \multirow{2}{*}{$\begin{array}{l}\text { C+sulpiride } \\
2004\end{array}$} & \multirow{2}{*}{$\begin{array}{l}\text { Olanzap- } \\
\text { ine }\end{array}$} & \multirow{2}{*}{$\begin{array}{l}20 \mathrm{mg} \text { to } 30 \\
\mathrm{mg} / \mathrm{d}\end{array}$} & \multirow[t]{2}{*}{-} & \multirow[t]{2}{*}{-} & Olanzapine & $20 \mathrm{mg}$ to $30 \mathrm{mg} / \mathrm{d}$ & - & - & - & - \\
\hline & & & & & Sulpiride & $100 \mathrm{mg}$ to $600 \mathrm{mg} / \mathrm{d}$ & - & - & - & - \\
\hline \multirow{2}{*}{$\begin{array}{l}\text { C+sulpiride } \\
2013\end{array}$} & \multirow{2}{*}{$\begin{array}{l}\text { Amisul- } \\
\text { pride }\end{array}$} & \multirow[t]{2}{*}{$800 \mathrm{mg} /$ day } & \multirow[t]{2}{*}{-} & \multirow[t]{2}{*}{-} & Amisulpride & $400 \mathrm{mg} /$ day & - & - & - & - \\
\hline & & & & & Sulpiride & 800 mg/day & - & - & - & - \\
\hline
\end{tabular}




\section{Footnotes}

$d$ - day 


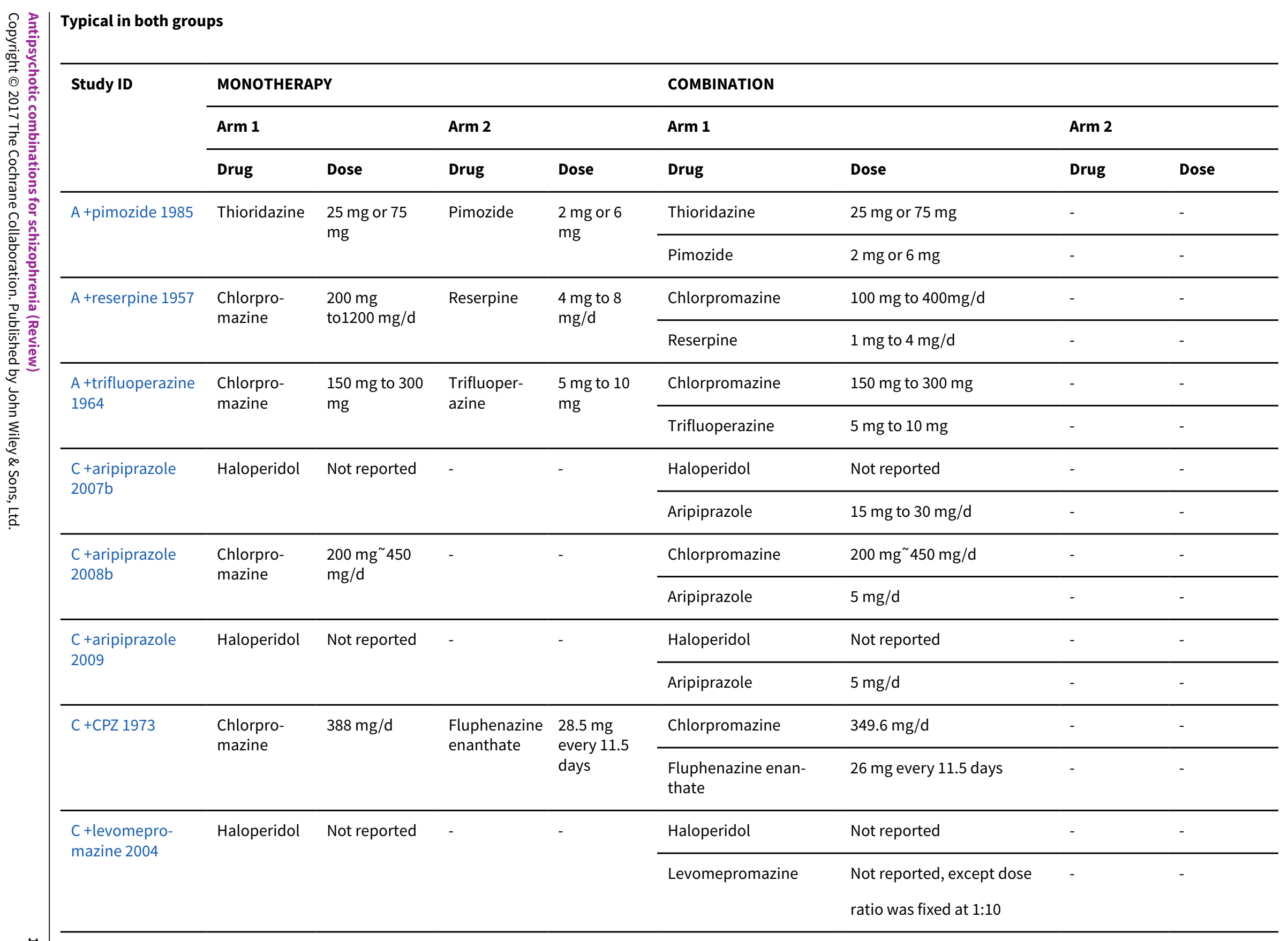




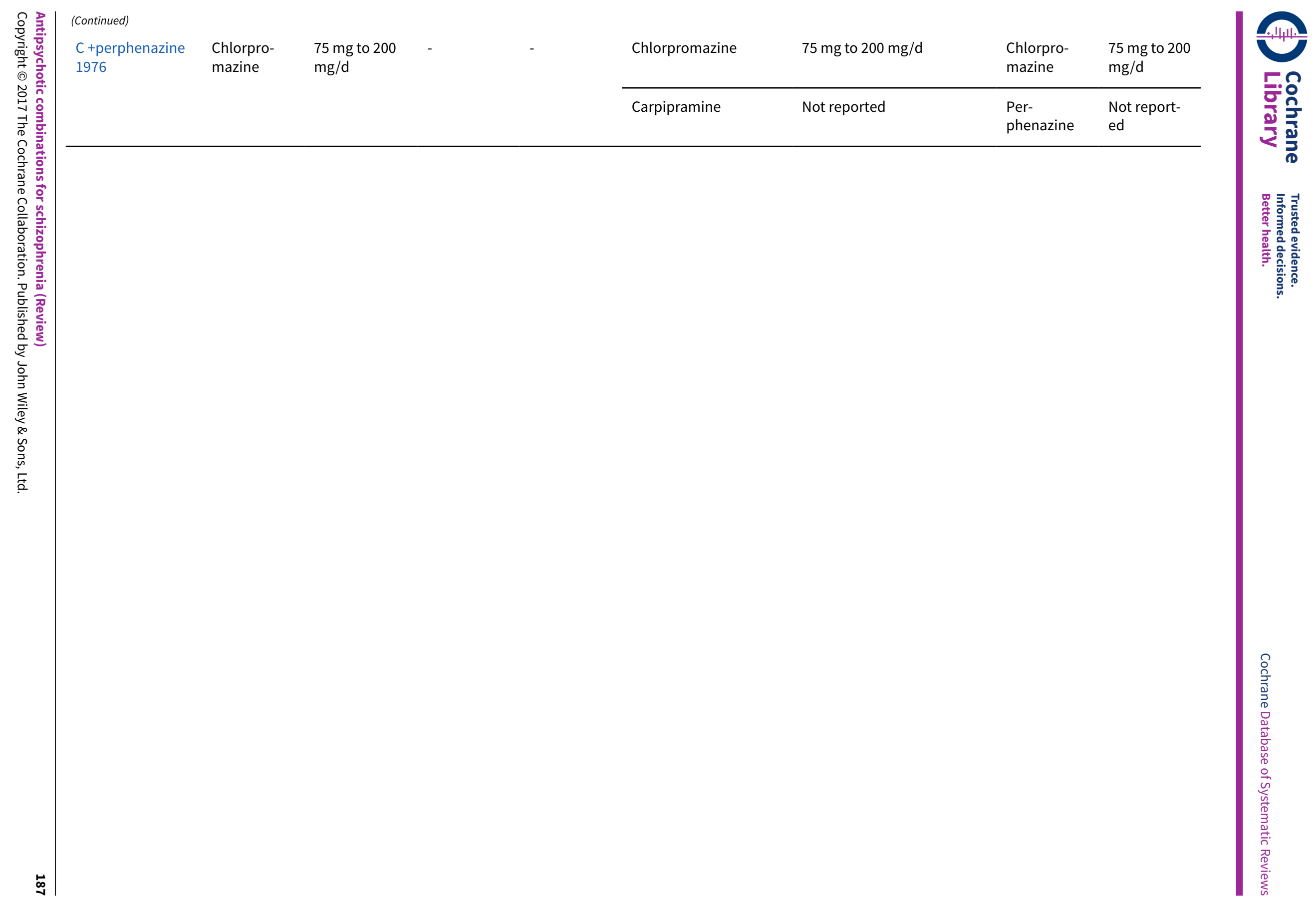




\section{Footnotes}

d - day

Any antipsychotic in both groups

\begin{tabular}{|c|c|c|c|c|}
\hline \multirow[t]{3}{*}{ Study ID } & \multicolumn{2}{|l|}{ MONOTHERAPY } & \multicolumn{2}{|l|}{ COMBINATION } \\
\hline & \multicolumn{2}{|l|}{ Arm 1} & \multicolumn{2}{|l|}{ Arm 1} \\
\hline & Drug & Dose & Drug & Dose \\
\hline \multirow[t]{2}{*}{$\begin{array}{l}\text { A +any antipsy- } \\
\text { chotic } 2011\end{array}$} & \multirow[t]{2}{*}{$\begin{array}{l}\text { Switch to an- } \\
\text { tipsychotic } \\
\text { monotherapy }\end{array}$} & $\begin{array}{l}\text { Mean haloperi- } \\
\text { dol equivalent }= \\
7.2 \mathrm{mg} / \text { day }\end{array}$ & \multirow{2}{*}{$\begin{array}{l}\text { Most common antipsychotic combinations were } \\
\text { quetiapine and risperidone, quetiapine and a first- } \\
\text { generation antipsychotic, risperidone and a first- } \\
\text { generation antipsychotic, olanzapine and a first- } \\
\text { generation antipsychotic, ziprasidone and a first- } \\
\text { generation antipsychotic, aripiprazole and queti- } \\
\text { apine and olanzapine and risperidone. }\end{array}$} & $\begin{array}{l}\text { Mean haloperi- } \\
\text { dol equivalent } \\
6.1 \mathrm{mg} / \text { day }\end{array}$ \\
\hline & & $\begin{array}{l}\text { Mean chlorpro- } \\
\text { mazine equiva- } \\
\text { lent }=387.8 \mathrm{mg} / \\
\mathrm{d}\end{array}$ & & $\begin{array}{l}\text { Mean chlorpro- } \\
\text { mazine equiva- } \\
\text { lent } 325.8 \mathrm{mg} / \mathrm{d}\end{array}$ \\
\hline \multirow{4}{*}{$\begin{array}{l}\text { A +any antipsy- } \\
\text { chotic } 2012\end{array}$} & \multirow{2}{*}{$\begin{array}{l}\text { Switch to dual } \\
\text { antipsychotics } \\
\text { by adding up } \\
\text { another medica- } \\
\text { tion, choice of } \\
\text { medication to }\end{array}$} & \multirow{4}{*}{$\begin{array}{l}\text { No dose report- } \\
\text { ed }\end{array}$} & Previous monotherapy & \multirow{4}{*}{$\begin{array}{l}\text { Dose of all drugs } \\
\text { could be raised } \\
\text { or } \\
\text { lowered at the } \\
\text { discretion of the } \\
\text { prescriber }\end{array}$} \\
\hline & & & \multirow[t]{3}{*}{$\begin{array}{l}\text { Choice of medication to add was left to prescriber } \\
\text { and patient }\end{array}$} & \\
\hline & $\begin{array}{l}\text { add was left to } \\
\text { prescriber and } \\
\text { patient a }\end{array}$ & & & \\
\hline & $\begin{array}{l}\text { the dose of all } \\
\text { drug could be } \\
\text { raised or lowered }\end{array}$ & & & \\
\hline $\begin{array}{l}\text { B +any antipsy- } \\
\text { chotic } 2013\end{array}$ & $\begin{array}{l}\text { Switch to an- } \\
\text { tipsychotic } \\
\text { monotherapy }\end{array}$ & $\begin{array}{l}\text { Mean chlorpro- } \\
\text { mazine equiva- } \\
\text { lent }=552.9 \mathrm{mg} / \\
\mathrm{d}\end{array}$ & $\begin{array}{l}\text { Most common baseline polypharmacy combina- } \\
\text { tion were: } \\
\text { risperidone and a first-generation antipsychotic, } \\
\text { olanzapine and a first generation antipsychotic } \\
\text { olanzapine and risperidone, risperidone and que- } \\
\text { tiapine olanzapine and aripiprazole, aripiprazole } \\
\text { and a first generation antipsychotic, quetiapine } \\
\text { and aripiprazole, blonanserin and a first generation } \\
\text { antipsychotic, blonanserin and olanzapine, blo- } \\
\text { nanserin and quetiapine. }\end{array}$ & $\begin{array}{l}\text { Mean chlorpro- } \\
\text { mazine equiva- } \\
\text { lent }=635.0 \mathrm{mg} / \\
\text { day }\end{array}$ \\
\hline \multirow[t]{2}{*}{$\begin{array}{l}\text { A +any antipsy- } \\
\text { chotic } 2015\end{array}$} & \multirow[t]{2}{*}{$\begin{array}{l}\text { Switch to an- } \\
\text { tipsychotic } \\
\text { monotherapy }\end{array}$} & \multirow[t]{2}{*}{$\begin{array}{l}\text { Mean olanzapine } \\
\text { equivalent }=32.9 \\
\text { mg/day }\end{array}$} & $\begin{array}{l}\text { Half the patients were receiving either clozapine } \\
\text { or a long-acting injectable antipsychotic as one of } \\
\text { their } 2 \text { antipsychotics at baseline. }\end{array}$ & \multirow[t]{2}{*}{$\begin{array}{l}\text { Mean olanzapine } \\
\text { equivalent }=41.2 \\
\mathrm{mg} / \text { day }\end{array}$} \\
\hline & & & $\begin{array}{l}\text { No other information regarding which combina- } \\
\text { tions were used. }\end{array}$ & \\
\hline
\end{tabular}

Footnotes

d-day 


\section{Appendix 6. Missing data}

\begin{tabular}{|c|c|c|c|c|}
\hline Study ID & Subgroup & Outcome & Data missing & \\
\hline \multirow[t]{8}{*}{$\begin{array}{l}\text { A +any antipsy- } \\
\text { chotic } 2015\end{array}$} & \multirow{8}{*}{$\begin{array}{l}\text { Any antipsy- } \\
\text { chotics in both } \\
\text { groups }\end{array}$} & $\begin{array}{l}\text { Clinical response: Global state - severity (CGI-S } \\
\text { scale, high = bad) }\end{array}$ & \multirow[t]{8}{*}{$\begin{array}{l}\text { No means and } \\
\text { SDs reported. }\end{array}$} & \multirow[t]{8}{*}{$\begin{array}{l}\text { Not added, no da- } \\
\text { ta }\end{array}$} \\
\hline & & $\begin{array}{l}\text { Clinical response: } 4 \text {. Global state - average im- } \\
\text { provement score (CGI-I scale, high = bad) }\end{array}$ & & \\
\hline & & Mental state: Total score (PANSS scale, high = bad) & & \\
\hline & & $\begin{array}{l}\text { Mental state: Positive symptoms (PANSS scale, } \\
\text { high = bad) }\end{array}$ & & \\
\hline & & $\begin{array}{l}\text { Mental state: Negative symptoms (PANSS scale, } \\
\text { high = bad) }\end{array}$ & & \\
\hline & & $\begin{array}{l}\text { Adverse events: Movement disorders (AIMS, high } \\
=\text { bad) }\end{array}$ & & \\
\hline & & $\begin{array}{l}\text { Adverse events: Movement disorders (SAS, high } \\
=\text { bad) }\end{array}$ & & \\
\hline & & $\begin{array}{l}\text { Adverse events: Movement disorders (BAS, high= } \\
\text { bad) }\end{array}$ & & \\
\hline \multirow[t]{8}{*}{$\begin{array}{l}\text { B +aripiprazole } \\
2008\end{array}$} & \multirow[t]{8}{*}{$\begin{array}{l}\text { Clozapine in } \\
\text { both groups }\end{array}$} & Adverse events: Average weight gain (kg) & $\begin{array}{l}\text { No SD, mean dif- } \\
\text { ference and Cls } \\
\text { reported }\end{array}$ & $\begin{array}{l}\text { Added using } \\
\text { Revman calculator }\end{array}$ \\
\hline & & $\begin{array}{l}\text { Clinical response: Global state - severity (CGI-I } \\
\text { scale, high = bad) }\end{array}$ & $\begin{array}{l}\text { SE reported not } \\
\text { SD }\end{array}$ & $\begin{array}{l}\text { Added using } \\
\text { Revman calculator }\end{array}$ \\
\hline & & $\begin{array}{l}\text { Clinical response: Global state - severity (CGI-S } \\
\text { scale, high = bad) }\end{array}$ & SE reported as 0 & $\begin{array}{l}\text { Not added, not } \\
\text { able to impute da- } \\
\text { ta }\end{array}$ \\
\hline & & $\begin{array}{l}\text { Clinical response: } 5 \text {. Global state - average func- } \\
\text { tioning score (GAF scale, high = good }\end{array}$ & $\begin{array}{l}\text { SE reported not } \\
\text { SD }\end{array}$ & $\begin{array}{l}\text { Added using } \\
\text { Revman calculator }\end{array}$ \\
\hline & & Mental state: Total score (PANSS scale, high = bad) & SE reported as 0 & $\begin{array}{l}\text { Not added, not } \\
\text { able to impute da- } \\
\text { ta }\end{array}$ \\
\hline & & $\begin{array}{l}\text { Mental state: Positive symptoms (PANSS scale, } \\
\text { high = bad) }\end{array}$ & $\begin{array}{l}\text { SE reported not } \\
\text { SD }\end{array}$ & $\begin{array}{l}\text { Added using } \\
\text { Revman calculator }\end{array}$ \\
\hline & & $\begin{array}{l}\text { Mental state: Negative symptoms (PANSS scale, } \\
\text { high = bad) }\end{array}$ & & \\
\hline & & $\begin{array}{l}\text { Quality of life: 1b. Average score (SWN, high = } \\
\text { good) }\end{array}$ & & \\
\hline $\begin{array}{l}\text { B +quet/risp } \\
2009\end{array}$ & $\begin{array}{l}\text { Other atypical } \\
\text { drugs in both } \\
\text { groups }\end{array}$ & Adverse events: Average weight gain (kg) & $\begin{array}{l}\text { No SD, } \mathrm{P} \text { values } \\
\text { reported }\end{array}$ & $\begin{array}{l}\text { Not added, no } \\
\text { similar mean, not }\end{array}$ \\
\hline
\end{tabular}


able to impute data

$\begin{aligned} & \text { Mental state: Positive symptoms (PANSS scale, } \\ & \text { high = bad) }\end{aligned}$
$\begin{aligned} & \text { Mental state: Negative symptoms (PANSS scale, } \\ & \text { high = bad) }\end{aligned}$
$\begin{aligned} & \text { Adverse events: Movement disorders (AIMS, high } \\ & =\text { bad) }\end{aligned}$

Adverse events: Movement disorders (BAS, high = bad)

Adverse events: Movement disorders (SAS, high = bad)
No SD, mean re- Not added - mean ported in a graph in Figure 2, no similar mean to impute
Data imputed from $B$ +aripiprazole 2008

\begin{tabular}{lll}
\hline Adverse events: Prolactin level (high = bad) & $\begin{array}{l}\text { No SD, P values } \\
\text { reported }\end{array}$ & $\begin{array}{l}\text { Not added, no } \\
\text { similar mean, not } \\
\text { able to impute da- } \\
\text { ta }\end{array}$ \\
$\begin{array}{l}\text { Clinical response: Global state - severity (CGI-S } \\
\text { scale, high = bad) }\end{array}$ &
\end{tabular}

Mental state: Total score (PANSS scale, high = bad)

Quality of life (SWN, high = good)

\begin{tabular}{|c|c|c|c|c|}
\hline \multirow{5}{*}{$\begin{array}{l}\text { C +amisulpride } \\
2008\end{array}$} & \multirow{5}{*}{$\begin{array}{l}\text { Clozapine in } \\
\text { both groups }\end{array}$} & Adverse events: Prolactin level (high = bad) & \multirow{5}{*}{$\begin{array}{l}\text { No SD, no other } \\
\text { data }\end{array}$} & \multirow{5}{*}{$\begin{array}{l}\text { Not added - } 2 \\
\text { combination } \\
\text { groups with dif- } \\
\text { ferent doses, no } \\
\text { similar means, not } \\
\text { able to impute da- } \\
\text { ta }\end{array}$} \\
\hline & & $\begin{array}{l}\text { Clinical response: Global state - severity (CGI-S } \\
\text { scale, high = bad) }\end{array}$ & & \\
\hline & & $\begin{array}{l}\text { Clinical response: Global state (GAF scale, high = } \\
\text { good) }\end{array}$ & & \\
\hline & & $\begin{array}{l}\text { Mental state: Depressive symptoms (MADRS scale, } \\
\text { high = bad) }\end{array}$ & & \\
\hline & & Mental state: Total score (BPRS scale, high = bad) & & \\
\hline \multirow[t]{3}{*}{$\begin{array}{l}\text { C +aripiprazole } \\
2007 b\end{array}$} & \multirow[t]{3}{*}{$\begin{array}{l}\text { Typicals in both } \\
\text { groups }\end{array}$} & $\begin{array}{l}\text { Clinical response: Global state - severity (CGI-S } \\
\text { scale, high = bad) }\end{array}$ & \multirow[t]{3}{*}{$\begin{array}{l}\text { No SD, no other } \\
\text { data }\end{array}$} & $\begin{array}{l}\text { Data imputed } \\
\text { from C +fluphen } \\
\text { dec } 2009\end{array}$ \\
\hline & & Mental state: Total score (BPRS scale, high = bad) & & \multirow{2}{*}{$\begin{array}{l}\text { Data imputed } \\
\text { from C +aripipra- } \\
\text { zole } 2008\end{array}$} \\
\hline & & $\begin{array}{l}\text { Mental state: Negative symptoms (SANS scale, } \\
\text { high = bad) }\end{array}$ & & \\
\hline $\begin{array}{l}\text { C +aripiprazole } \\
2008\end{array}$ & $\begin{array}{l}\text { Clozapine in } \\
\text { both groups }\end{array}$ & $\begin{array}{l}\text { Adverse events: Movement disorders (UKU, high = } \\
\text { bad) }\end{array}$ & $\begin{array}{l}\text { No means and } \\
\text { SDs reported }\end{array}$ & $\begin{array}{l}\text { Not added, no da- } \\
\text { ta }\end{array}$ \\
\hline \multirow{2}{*}{$\begin{array}{l}\text { C +aripiprazole } \\
\text { 2013b }\end{array}$} & \multirow{2}{*}{$\begin{array}{l}\text { Other atypical } \\
\text { drugs in both } \\
\text { groups }\end{array}$} & Mental state: Total score (PANSS scale, high = bad) & \multirow{2}{*}{$\begin{array}{l}\text { SE reported not } \\
\text { SD }\end{array}$} & \multirow{2}{*}{$\begin{array}{l}\text { Added using } \\
\text { Revman calculator }\end{array}$} \\
\hline & & $\begin{array}{l}\text { Mental state: positive symptoms (PANSS scale, } \\
\text { high = bad) }\end{array}$ & & \\
\hline
\end{tabular}




\begin{tabular}{|c|c|c|c|c|}
\hline & & $\begin{array}{l}\text { Mental state: Negative symptoms (PANSS scale, } \\
\text { high = bad) }\end{array}$ & & \\
\hline & & $\begin{array}{l}\text { Adverse events: Movement disorders (ESRS, high } \\
=\text { bad) }\end{array}$ & & \\
\hline \multirow{4}{*}{$\begin{array}{l}\text { C +aripiprazole } \\
2015 b\end{array}$} & \multirow{4}{*}{$\begin{array}{l}\text { Other atypical } \\
\text { drugs in both } \\
\text { groups }\end{array}$} & Mental state: Total score (BPRS scale, high = bad) & \multirow{4}{*}{$\begin{array}{l}\text { No means and } \\
\text { SDs reported. }\end{array}$} & \multirow{4}{*}{$\begin{array}{l}\text { Not added, no da- } \\
\text { ta }\end{array}$} \\
\hline & & $\begin{array}{l}\text { Adverse events: Movement disorders (SAS, high = } \\
\text { bad) }\end{array}$ & & \\
\hline & & $\begin{array}{l}\text { Adverse events: Movement disorders (BAS, high = } \\
\text { bad) }\end{array}$ & & \\
\hline & & Adverse events: Prolactin level (high = bad) & & \\
\hline \multirow[t]{4}{*}{$\begin{array}{l}\text { C +aripiprazole } \\
2015 \mathrm{c}\end{array}$} & \multirow{4}{*}{$\begin{array}{l}\text { Other atypical } \\
\text { drugs in both } \\
\text { groups }\end{array}$} & Mental state: Total score (PANSS scale, high=bad) & \multirow[t]{4}{*}{$\begin{array}{l}\text { Reported in a } \\
\text { graph }\end{array}$} & \multirow[t]{3}{*}{$\begin{array}{l}\text { Measured from } \\
\text { figure } 4\end{array}$} \\
\hline & & $\begin{array}{l}\text { Mental state: Positive symptoms (PANSS scale, } \\
\text { high = bad) }\end{array}$ & & \\
\hline & & $\begin{array}{l}\text { Mental state: Negative symptoms (PANSS scale, } \\
\text { high = bad) }\end{array}$ & & \\
\hline & & Adverse events: Prolactin level (high = bad) & & $\begin{array}{l}\text { Measured from } \\
\text { figure } 2\end{array}$ \\
\hline \multirow[t]{2}{*}{$\begin{array}{l}\text { C +aripiprazole } \\
2016\end{array}$} & \multirow[t]{2}{*}{$\begin{array}{l}\text { Other atypical } \\
\text { drugs in both } \\
\text { groups }\end{array}$} & Adverse event: Movement disorders - Any & $\begin{array}{l}\text { Reported as per- } \\
\text { centage not as } \\
\text { absolute value }\end{array}$ & $\begin{array}{l}\text { Added using excel } \\
2011\end{array}$ \\
\hline & & Adverse events: Prolactin level (high = bad) & $\begin{array}{l}\text { Median and } \\
\text { quartile reported } \\
\text { not median and } \\
\text { SD. }\end{array}$ & Not able to impute \\
\hline$C+C P Z 1989$ & $\begin{array}{l}\text { Clozapine in } \\
\text { both groups }\end{array}$ & Mental state: Total score (BPRS scale, high = bad) & $\begin{array}{l}\text { SE reported not } \\
\text { SD }\end{array}$ & $\begin{array}{l}\text { Added using } \\
\text { Revman calculator }\end{array}$ \\
\hline \multirow[t]{3}{*}{$\begin{array}{l}\text { C +levomepro- } \\
\text { mazine } 2004\end{array}$} & \multirow[t]{3}{*}{$\begin{array}{l}\text { Typicals in both } \\
\text { groups }\end{array}$} & $\begin{array}{l}\text { Mental state: Avarage score (BPRS scale, high = } \\
\text { bad) }\end{array}$ & \multirow[t]{3}{*}{$\begin{array}{l}\text { Reported in a } \\
\text { graph }\end{array}$} & \multirow[t]{3}{*}{$\begin{array}{l}\text { Measured from } \\
\text { Figure } 1\end{array}$} \\
\hline & & $\begin{array}{l}\text { Mental state: Positive symptoms (BPRS scale, high } \\
=\text { bad) }\end{array}$ & & \\
\hline & & $\begin{array}{l}\text { Mental state: Negative symptoms (BPRS scale, } \\
\text { high = bad) }\end{array}$ & & \\
\hline \multirow[t]{3}{*}{$\begin{array}{l}\text { C + pimozide } \\
2013\end{array}$} & \multirow[t]{3}{*}{$\begin{array}{l}\text { Clozapine in } \\
\text { both groups }\end{array}$} & Mental state: Total score (BPRS scale, high=bad) & \multirow[t]{3}{*}{$\begin{array}{l}\text { SE reported not } \\
\text { SD }\end{array}$} & \multirow[t]{3}{*}{$\begin{array}{l}\text { Added using } \\
\text { Revman calculator }\end{array}$} \\
\hline & & $\begin{array}{l}\text { Clinical response: Global state - severity (CGI-S } \\
\text { scale, high=bad) }\end{array}$ & & \\
\hline & & $\begin{array}{l}\text { Clinical response: Global state - severity (CGI-I } \\
\text { scale, low = bad) }\end{array}$ & & \\
\hline
\end{tabular}


(Continued)

Mental state: Negative symptoms (SANS scale,

high = bad)

\begin{tabular}{|c|c|c|c|c|}
\hline \multirow[t]{10}{*}{$\begin{array}{l}\text { C +risperidone } \\
2005\end{array}$} & \multirow[t]{10}{*}{$\begin{array}{l}\text { Clozapine in } \\
\text { both groups }\end{array}$} & Mental state: Total score (PANSS scale, high = bad) & \multirow[t]{10}{*}{$\begin{array}{l}\text { SE reported not } \\
\text { SD }\end{array}$} & \multirow[t]{10}{*}{$\begin{array}{l}\text { Added using } \\
\text { Revman calculator }\end{array}$} \\
\hline & & $\begin{array}{l}\text { Mental state: Positive symptoms (PANSS scale, } \\
\text { high = bad) }\end{array}$ & & \\
\hline & & $\begin{array}{l}\text { Mental state: Negative symptoms (PANSS scale, } \\
\text { high = bad) }\end{array}$ & & \\
\hline & & $\begin{array}{l}\text { Clinical response: Global state - severity (CGI-S } \\
\text { scale, high = bad) }\end{array}$ & & \\
\hline & & $\begin{array}{l}\text { Clinical response: Global state (GAF scale, high = } \\
\text { good) }\end{array}$ & & \\
\hline & & Quality of life (QLS high = good) & & \\
\hline & & $\begin{array}{l}\text { Adverse events: Movement disorders (AIMS, high } \\
=\text { bad) }\end{array}$ & & \\
\hline & & $\begin{array}{l}\text { Adverse events: Movement disorders (SAS, high = } \\
\text { bad) }\end{array}$ & & \\
\hline & & $\begin{array}{l}\text { Adverse events: Movement disorders (BAS, high = } \\
\text { bad) }\end{array}$ & & \\
\hline & & $\begin{array}{l}\text { Adverse events: Movement disorders (UKU, high = } \\
\text { bad) }\end{array}$ & & \\
\hline \multirow[t]{4}{*}{$\begin{array}{l}\text { C +risperidone } \\
2005 b\end{array}$} & \multirow[t]{4}{*}{$\begin{array}{l}\text { Clozapine in } \\
\text { both groups }\end{array}$} & Mental state: Total score (BPRS scale, high = bad) & \multirow[t]{3}{*}{$\begin{array}{l}\text { No SD, no other } \\
\text { data }\end{array}$} & $\begin{array}{l}\text { Data imputed } \\
\text { from C +aripipra- } \\
\text { zole } 2008\end{array}$ \\
\hline & & $\begin{array}{l}\text { Mental state: Positive symptoms (BPRS scale, high } \\
=\text { bad) }\end{array}$ & & $\begin{array}{l}\text { Data imputed } \\
\text { from B +risperi- } \\
\text { done } 2010\end{array}$ \\
\hline & & $\begin{array}{l}\text { Mental state: Negative symptoms (SANS scale, } \\
\text { high = bad) }\end{array}$ & & $\begin{array}{l}\text { Data imputed } \\
\text { from C +fluphen } \\
\text { dec } 2009\end{array}$ \\
\hline & & $\begin{array}{l}\text { Adverse events: Movement disorders (SAS, high = } \\
\text { bad) }\end{array}$ & $\begin{array}{l}\text { No SD, mean in a } \\
\text { graph }\end{array}$ & $\begin{array}{l}\text { Not added, mean } \\
\text { in Figure } 4 \text {, no } \\
\text { similar mean to } \\
\text { impute }\end{array}$ \\
\hline $\begin{array}{l}\text { C +risperi- } \\
\text { done } 2007\end{array}$ & $\begin{array}{l}\text { Clozapine in } \\
\text { both groups }\end{array}$ & Adverse events: Prolactin level (high $=$ bad) & $\begin{array}{l}\text { No SD, no other } \\
\text { data }\end{array}$ & $\begin{array}{l}\text { Not added, no } \\
\text { similar mean, not } \\
\text { able to impute da- } \\
\text { ta }\end{array}$ \\
\hline \multirow[t]{2}{*}{$\begin{array}{l}\text { C + sertindole } \\
2006\end{array}$} & \multirow[t]{2}{*}{$\begin{array}{l}\text { Clozapine in } \\
\text { both groups }\end{array}$} & Mental state: Total score (PANSS scale, high = bad) & \multirow[t]{2}{*}{$\begin{array}{l}\text { No SD, Cl's re- } \\
\text { ported }\end{array}$} & \multirow[t]{2}{*}{$\begin{array}{l}\text { Added using } \\
\text { Revman calculator }\end{array}$} \\
\hline & & $\begin{array}{l}\text { Mental state: Positive symptoms (PANSS scale, } \\
\text { high = bad) }\end{array}$ & & \\
\hline
\end{tabular}


(Continued)

Mental state: Negative symptoms (PANSS scale, high = bad)

Clinical response: Global state (GAF scale, high = good)

Clinical response: Global state - improvement

$($ CGI-I scale, high = bad)

Not added

means reported as

0 and no SD, $\mathrm{Cl}$ in-

tervals -1 to 0

Clinical response: Global state - severity (CGI-S

scale, high = bad)

Not added -

means reported as

0 and no $\mathrm{SD}, \mathrm{Cl}$ in-

tervals 0 to 0

Adverse events: Average weight gain (kg)

Added using

Revman calculator

C +sulpiride 2003 Clozapine in both groups
Mental state: Total score (BPRS scale, high = bad)

Mental state: Negative symptoms (SANS scale, high $=$ bad)

Mental state: Negative symptoms (SAPS scale, high = bad)

\begin{tabular}{ll}
\begin{tabular}{l} 
No SD, no other $\begin{array}{l}\text { data } \\
\text { data imputed } \\
\text { from C + pipoti- } \\
\text { azine 2000 }\end{array}$ \\
\cline { 2 - 2 }
\end{tabular} & $\begin{array}{l}\text { Data imputed } \\
\text { from C +pipoti- } \\
\text { azine 2000 }\end{array}$ \\
\cline { 2 - 2 } & $\begin{array}{l}\text { Not added, no } \\
\text { similar mean, not } \\
\text { able to impute da- } \\
\text { ta }\end{array}$ \\
\hline $\begin{array}{l}\text { Reported as per- } \\
\text { centage not as } \\
\text { absolute value }\end{array}$ & $\begin{array}{l}\text { Added using excel } \\
\text { 2011 }\end{array}$ \\
\hline
\end{tabular}

centage not as

solute value
C +sulpiride 2013 Other atypical Clinical Response: Not clinically improved drugs in both groups

\section{Footnotes}

AIMS - Abnormal Involuntary Movement Scale.

BAS - Barnes Akathisia Scale.

BPRS - Brief Psychiatric Rating Scale.

CGI-I - Clinical Global Impression - Improvement.

CGI-S - Clinical Global Impression - Severity

$\mathrm{Cl}$ - confidence interval.

ESRS - Extrapyramidal Symptom Rating Scale.

GAF - Global Assessment of Functioning Scale.

MADRS - Montgomery-Åsberg Depression Rating Scale.

PANSS - Positive and Negative Syndrome Scale.

QLS - Quality of Life Scale.

SANS - Scale for the Assessment of Negative Symptoms.

SAPS - Scale for the Assessment of Positive Symptoms.

SAS - Simpson Angus Scale.

SD - standard deviation.

SE- standard error.

SWN - Subjective Well Being under Neuroleptics.

UKU - Udvalg for Kliniske Undersøgelser. 


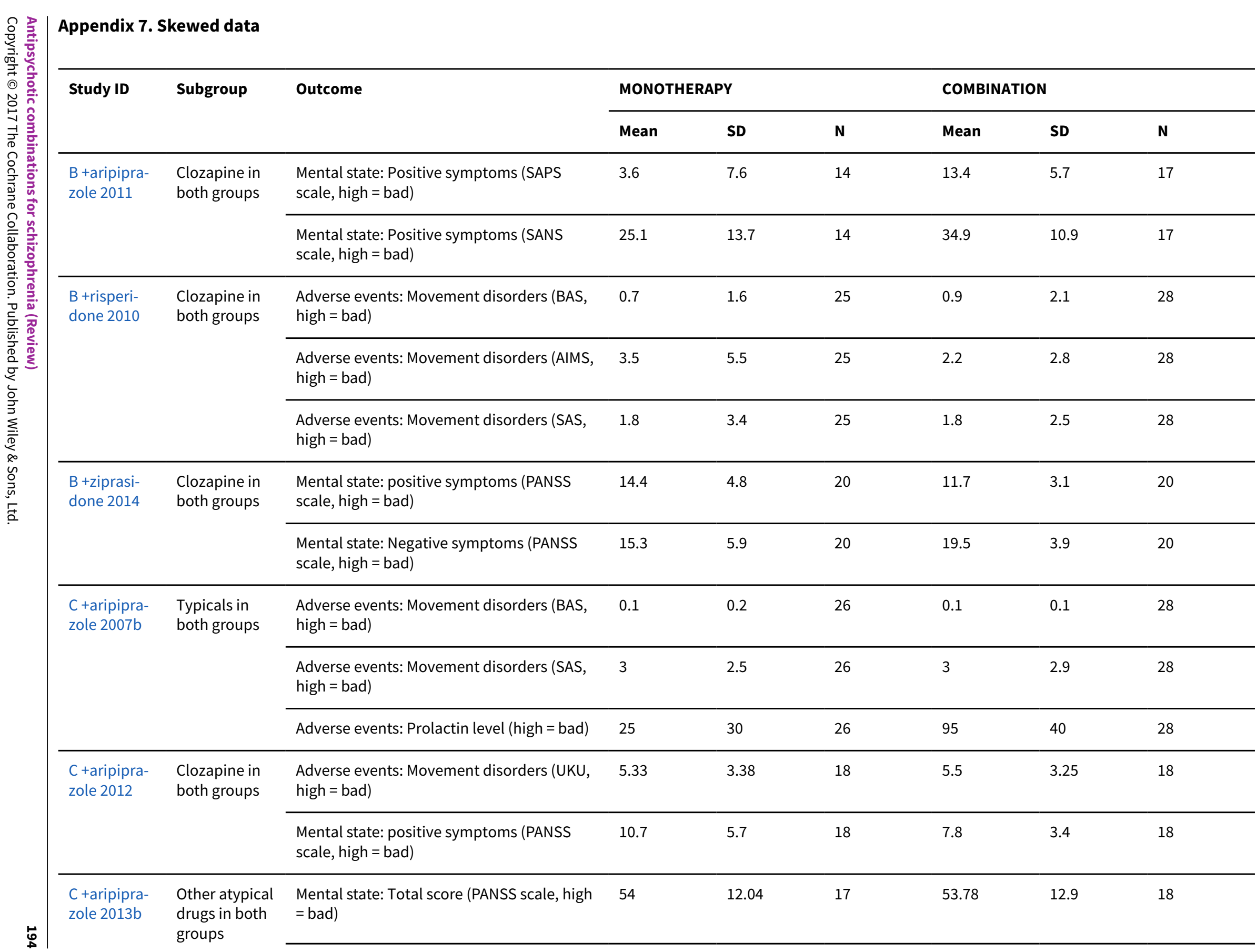




\begin{tabular}{|c|c|c|c|c|c|c|c|c|}
\hline \multirow[t]{3}{*}{ 官蛋 } & & $\begin{array}{l}\text { Mental state: positive symptoms (PANSS } \\
\text { scale, high = bad) }\end{array}$ & 12.47 & 3.63 & 17 & 12.33 & 2.97 & 18 \\
\hline & & $\begin{array}{l}\text { Mental state: Negative symptoms (PANSS } \\
\text { scale, high = bad) }\end{array}$ & 14.71 & 4 & 17 & 15.17 & 4.58 & 18 \\
\hline & & $\begin{array}{l}\text { Adverse events: Movement disorders (ESRS, } \\
\text { high = bad) }\end{array}$ & 8.53 & 9.73 & 17 & 8.67 & 9.72 & 18 \\
\hline $\begin{array}{l}\text { C +aripipra- } \\
\text { zole } 2014\end{array}$ & $\begin{array}{l}\text { Other atypical } \\
\text { drugs in both } \\
\text { groups }\end{array}$ & Adverse events: Prolactin level (high = bad) & 20.98 & 16.34 & 59 & 94.1 & 64.84 & 57 \\
\hline $\begin{array}{l}\text { C +aripipra- } \\
\text { zole } 2015\end{array}$ & $\begin{array}{l}\text { Other atypical } \\
\text { drugs in both }\end{array}$ & $\begin{array}{l}\text { Mental state: Total score (PANSS scale, high } \\
=\text { bad) }\end{array}$ & 49.28 & 11.49 & 89 & 51.23 & 11.91 & 30 \\
\hline ¿ & & $\begin{array}{l}\text { Adverse events: Movement disorders (SAS, } \\
\text { high = bad) }\end{array}$ & 1.01 & 1.38 & 89 & 1.26 & 1.48 & 30 \\
\hline$\underset{\infty}{\infty}$ & & $\begin{array}{l}\text { Adverse events: Movement disorders (BAS, } \\
\text { high = bad) }\end{array}$ & 0.26 & 0.58 & 89 & 0.23 & 0.5 & 30 \\
\hline & & Adverse events: Prolactin level (high = bad) & 32.91 & 28.93 & 89 & 87.72 & 57.24 & 30 \\
\hline $\begin{array}{l}\text { C +aripipra- } \\
\text { zole } 2015 b\end{array}$ & $\begin{array}{l}\text { Other atypical } \\
\text { drugs in both }\end{array}$ & $\begin{array}{l}\text { Mental state: Positive symptoms (BPRS } \\
\text { scale, high = bad) }\end{array}$ & 9.53 & 5.317 & 15 & 7.15 & 2.544 & 13 \\
\hline & & $\begin{array}{l}\text { Adverse events: Movement disorders (SAS, } \\
\text { high = bad) }\end{array}$ & 3 & 1.732 & 15 & 3.62 & 1.387 & 13 \\
\hline & & Adverse events: Prolactin level (high = bad) & 55.427 & 558.938 & 15 & 2170.46 & 1405.217 & 13 \\
\hline $\begin{array}{l}\text { C+aripipra- } \\
\text { zole } 2015 c\end{array}$ & $\begin{array}{l}\text { Other atypical } \\
\text { drugs in both }\end{array}$ & $\begin{array}{l}\text { Mental state: Total score (PANSS scale, high } \\
=\text { bad) }\end{array}$ & 44 & 11 & 54 & 49 & 8 & 53 \\
\hline & & $\begin{array}{l}\text { Mental state: Positive symptoms (PANSS } \\
\text { scale, high = bad) }\end{array}$ & 10 & 3 & 54 & 11 & 4 & 53 \\
\hline & & $\begin{array}{l}\text { Mental state: Negative symptoms (PANSS } \\
\text { scale, high = bad) }\end{array}$ & 11 & 4 & 54 & 15 & 4 & 53 \\
\hline & & Adverse events: Prolactin level (high = bad) & 500 & 430 & 54 & 2000 & 1430 & 53 \\
\hline
\end{tabular}




\begin{tabular}{|c|c|c|c|c|c|c|c|c|}
\hline $\begin{array}{l}\text { (Continued) } \\
\text { C +aripipra- } \\
\text { zole } 2016\end{array}$ & $\begin{array}{l}\text { Other atypical } \\
\text { drugs in both } \\
\text { groups }\end{array}$ & $\begin{array}{l}\text { Mental state: Negative symptoms (PANSS } \\
\text { scale, high = bad) }\end{array}$ & 16.2 & 5.3 & 30 & 14.4 & 3.8 & 30 \\
\hline \multirow[t]{2}{*}{$\begin{array}{l}\text { C+clozap- } \\
\text { ine } 2013\end{array}$} & \multirow{2}{*}{$\begin{array}{l}\text { Other atypical } \\
\text { drugs in both } \\
\text { groups }\end{array}$} & $\begin{array}{l}\text { Mental state: Total score (PANSS scale, high } \\
=\text { bad) }\end{array}$ & 51 & 16 & 25 & 52.96 & 14.39 & 25 \\
\hline & & $\begin{array}{l}\text { Mental state: Negative symptoms (PANSS } \\
\text { scale, high = bad) }\end{array}$ & 10.84 & 4.24 & 25 & 13.56 & 5.08 & 25 \\
\hline \multirow[t]{5}{*}{$\begin{array}{l}\text { C+haloperi- } \\
\text { dol } 2010\end{array}$} & \multirow{5}{*}{$\begin{array}{l}\text { Other atypical } \\
\text { drugs in both } \\
\text { groups }\end{array}$} & $\begin{array}{l}\text { Adverse events: Movement disorders (BAS, } \\
\text { high = bad) }\end{array}$ & 0.87 & 1.61 & 46 & 0.9 & 1.43 & 42 \\
\hline & & $\begin{array}{l}\text { Adverse events: Movement disorders (AIMS, } \\
\text { high = bad) }\end{array}$ & 0.33 & 0.67 & 46 & 0.19 & 0.77 & 42 \\
\hline & & $\begin{array}{l}\text { Adverse events: Movement disorders (SAS, } \\
\text { high = bad) }\end{array}$ & 1.09 & 1.74 & 46 & 2.31 & 2.94 & 42 \\
\hline & & Adverse events: Prolactin level (high = bad) & 55.47 & 64.91 & 28 & 83.88 & 52.65 & 27 \\
\hline & & $\begin{array}{l}\text { Clinical response: Global state - severity } \\
\text { (GAF scale, high = good) }\end{array}$ & 21 & 12.9 & 46 & 20.1 & 13.6 & 42 \\
\hline $\begin{array}{l}\text { C +olan/risp } \\
2014\end{array}$ & $\begin{array}{l}\text { Other atypical } \\
\text { drugs in both } \\
\text { groups }\end{array}$ & Adverse events: Prolactin level (high = bad) & 119.68 & 68.04 & 25 & 98.3 & 88.25 & 25 \\
\hline $\begin{array}{l}\text { C+pipoti- } \\
\text { azine } 2000\end{array}$ & $\begin{array}{l}\text { Clozapine in } \\
\text { both groups }\end{array}$ & $\begin{array}{l}\text { Adverse events: Movement disorders (TESS, } \\
\text { high = bad) }\end{array}$ & 4.9 & 5 & 26 & 5 & 4.9 & 24 \\
\hline \multirow[t]{3}{*}{$\begin{array}{l}\text { C+pimozide } \\
2013\end{array}$} & \multirow[t]{3}{*}{$\begin{array}{l}\text { Clozapine in } \\
\text { both groups }\end{array}$} & $\begin{array}{l}\text { Clinical response: Global state - severity } \\
\text { (CGI-S scale, high = bad) }\end{array}$ & 3.9 & 1.87 & 14 & 3.9 & 2.99 & 14 \\
\hline & & $\begin{array}{l}\text { Clinical response: Global state - severity } \\
\text { (CGI-I scale, low = bad) }\end{array}$ & 3.4 & 1.87 & 14 & 2.8 & 3.74 & 14 \\
\hline & & $\begin{array}{l}\text { Mental state: Negative symptoms (SANS } \\
\text { scale, high = bad) }\end{array}$ & 37.3 & 22.08 & 14 & 36.3 & 22.8 & 14 \\
\hline $\begin{array}{l}\text { C+risperi- } \\
\text { done } 2005\end{array}$ & $\begin{array}{l}\text { Clozapine in } \\
\text { both groups }\end{array}$ & $\begin{array}{l}\text { Clinical response: Global state - severity } \\
\text { (CGI-S scale, high = bad) }\end{array}$ & 4.3 & 4.48 & 16 & 4 & 0.4864 & 14 \\
\hline
\end{tabular}




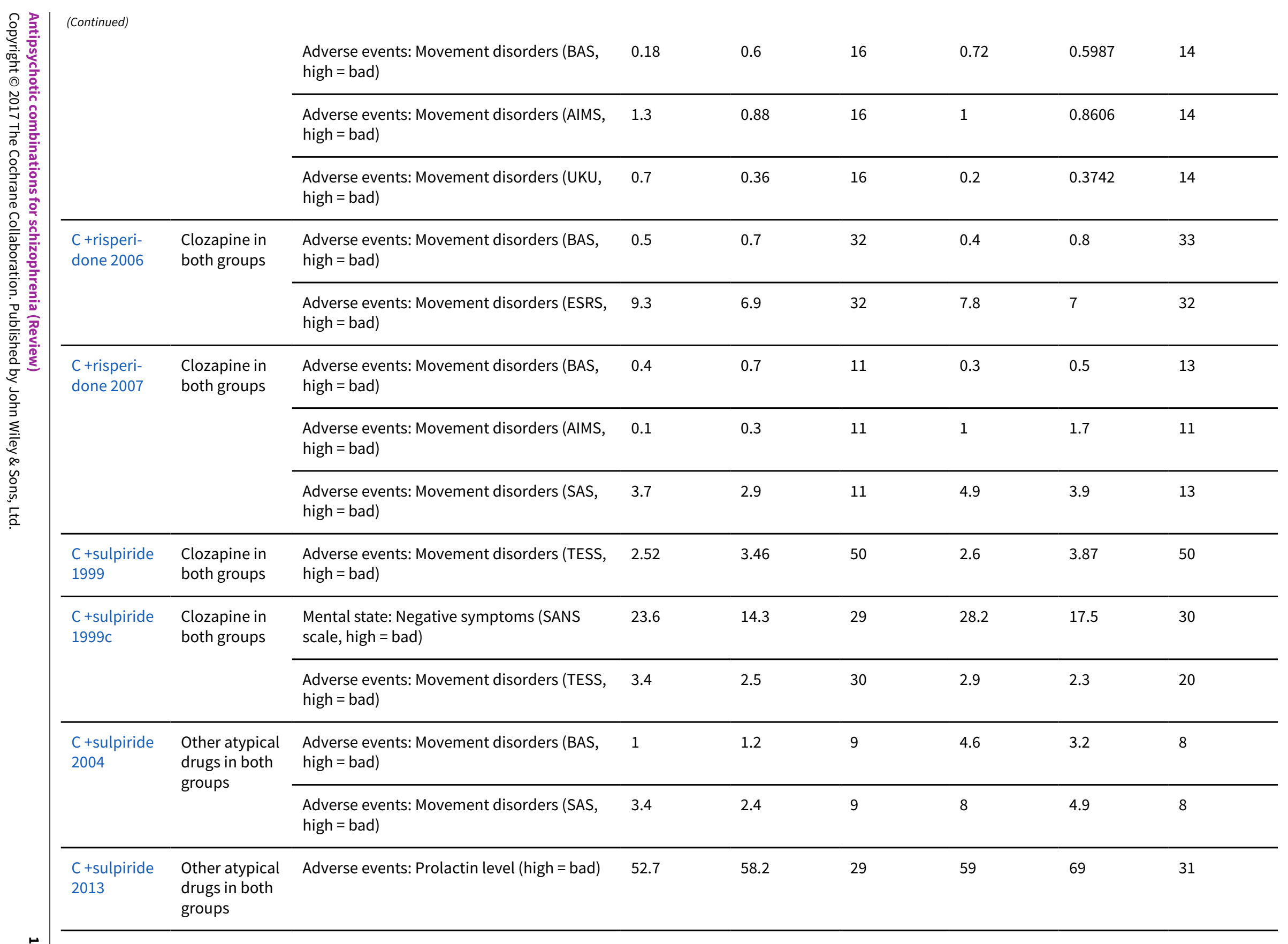



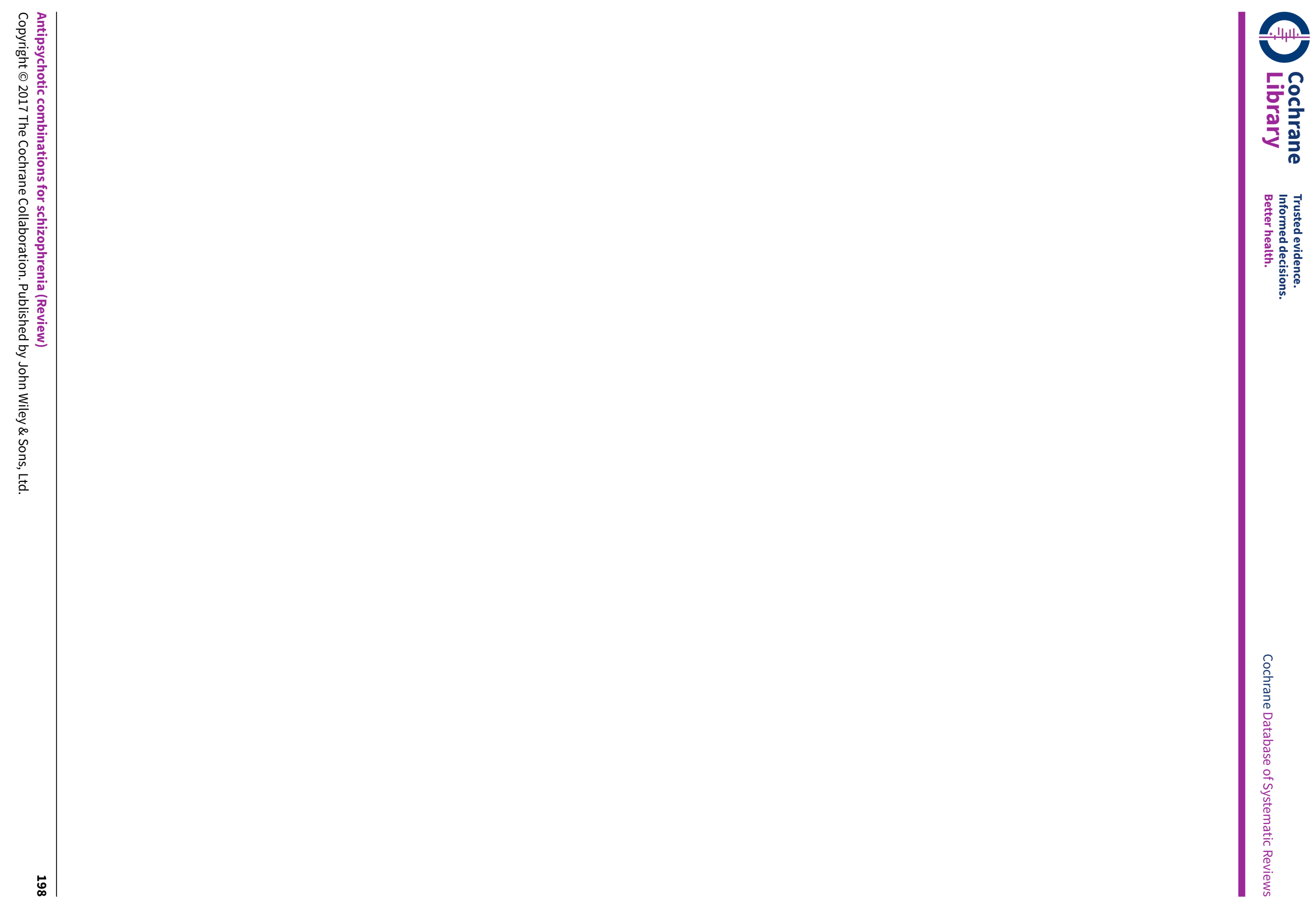
Footnotes

AIMS - Abnormal Involuntary Movement Scale.

BAS - Barnes Akathisia Scale.

BPRS - Brief Psychiatric Rating Scale.

CGI-I - Clinical Global Impression - Improvement.

CGI-S - Clinical Global Impression - Severity.

ESRS - Extrapyramidal Symptom Rating Scale.

GAF - Global Assessment of Functioning Scale.

PANSS - Positive and Negative Syndrome Scale.

SANS - Scale for the Assessment of Negative Symptoms.

SAPS - Scale for the Assessment of Positive Symptoms.

SAS - Simpson Angus Scale.

SD - Standard deviation.

TESS - Treatment Emergent Symptom Scale.

UKU - Udvalg for Kliniske Undersøgelser.

\section{WHAT'S NEW}

\begin{tabular}{|c|c|c|}
\hline Date & Event & Description \\
\hline 18 September 2017 & Amended & $\begin{array}{l}\text { We have made several amendments to the data and text of the } \\
\text { review since publication. These changes have not substantively } \\
\text { altered the overall conclusions of this review } \\
\text { - Updated and corrected data extraction from C +perphenazine } \\
\text { 1976, a Japanese trial. This changed the results for the out- } \\
\text { come 'clinical response: not clinically improved' for the sub- } \\
\text { group 'typical antipsychotics in both groups'. } \\
\text { - Decided to not pool the data of the outcome 'relapse' due to } \\
\text { high heterogeneity. } \\
\text { - Amended Summary of findings for the main comparison } \\
\text { - Separated endpoint data from change data. (see Differences } \\
\text { between protocol and review). } \\
\text { - Minor changes to PRISMA, conclusions and discussion sections. } \\
\text { - Completed meta-regression for the primary outcome to inves- } \\
\text { tigate heterogeneity (see Differences between protocol and re- } \\
\text { view). }\end{array}$ \\
\hline
\end{tabular}

\section{H I S T O R Y}

Protocol first published: Issue 2, 2011

Review first published: Issue 6, 2017

\begin{tabular}{lll}
\hline Date & Event & Description \\
\hline 8 May 2017 & $\begin{array}{l}\text { New citation required but conclusions } \\
\text { have not changed }\end{array}$ & $\begin{array}{l}\text { Review fully updated with new data from search update. New re- } \\
\text { sults did not alter the overall conclusions of the review. }\end{array}$ \\
\hline 29 June 2016 & New search has been performed & $\begin{array}{l}\text { Results of update search added to review, minor changes to dis- } \\
\text { cussion and conclusions. }\end{array}$ \\
\hline 25 January 2016 & Amended & $\begin{array}{l}\text { Search updated and 1344 references were sent to the review au- } \\
\text { thors for screening. }\end{array}$ \\
\hline
\end{tabular}




\section{CONTRIBUTIONS OF AUTHORS}

Javier Ortiz-Orendain - selection of studies, data extraction, 'Summary of findings' table, completion of report (2016).

Santiago Castiello - selection of studies, data extraction, 'Summary of findings' table, completion of report (2016).

Luis Enrique Colunga - referee for disagreement, 'Summary of findings' table, completion of report (2016).

Yue $\mathrm{Hu}$ - translation and data extraction of the Chinese papers.

Nicola Mayaan - protocol writing, selection of studies, data extraction, 'Summary of findings' table, completion of report (2012).

Clive Adams - protocol writing, completion of reports $(2012,2016)$.

\section{DECLARATIONSOF INTEREST}

Javier Ortiz-Orendain - participated in clinical trials sponsored by drug companies but received no payment for his contribution.

Santiago Castiello-de Obeso - none known.

Luis Enrique Colunga-Lozano - none known.

Yue Hu - works for Systematic Review Solutions Ltd, a company that carries out systematic reviews.

Nicola Mayaan - worked for Enhance Reviews Ltd during the preparation of this review, a company that carried out systematic reviews mostly for the public sector. Now works for Cochrane Respose.

Clive Adams - none known.

\section{SOURCES OFSUPPORT}

\section{Internal sources}

- University of Nottingham, UK.

\section{External sources}

- NIHR Programme Grant, UK.

NIHR Programme Grant, 2010 (10/401/15)

\section{DIFFERENCES BETWEEN PROTOCOL ANDREVIEW}

The background was updated with more up-to-date references since the protocol publication in 2011.

We had planned to exclude studies that were quasi-randomised. However, of 62 trials analysed, only 18 reported an adequate generation of allocation sequence. Certainly, in two studies (B +sulpiride 1996; C +fluphen dec 2009) sequence generation was a quasi-randomised method (which we rated as having a high risk of bias), and three studies (C +CPZ 1999; C +sulpiride 1999; C +sulpiride 1999b) also posed a high risk of bias as they randomised according to hospital admission order or time. In all remaining studies, however, the method of assignment was unclear and we could not be sure these too had not used some form of quasi-random method. After debate, we decided not to specifically exclude the five studies named above as this could penalise authors for clarity and unfairly emphasise the findings of trials where methods of allocation were not well described. We do not think we were biased by the knowledge of results, and in no case did inclusion of the five trials materially alter the findings.

The order of the primary outcomes was changed because we consider 'Clinical Response' more relevant for patients (consumers), the general public, administrators and policy makers than 'Leaving the study early.' We also changed wording from clinically significant to clinically important, and clarified the 'Summary of findings' outcomes 'adverse effects' and 'quality of life' need to be clinically important data - as now specified as necessary by Cochrane Schizophrenia for all 'summary of findings' outcomes.

We planned to report data on subgroups of people in the same clinical state, stage, and with similar problems. However, most studies included participants with chronic schizophrenia, and we decided instead to present data in the analyses grouped by the type of antipsychotic used: trials with clozapine in both the monotherapy arm and in the combination arm of the trial, trials with other atypical drugs in both the monotherapy and combination arms, trials with typical antipsychotic drugs in both arms, or any antipsychotics in both groups, in order to facilitate subgroup analyses. 
Due to a high heterogeneity in the outcome 'No clinically important response', we made a meta-regression with the random effect described by DerSimonian and Laird (DerSimonian 1986) to assess two potential effect modifiers: year of publication and Chinese studies. A metaregression is similar to a simple regression but it consider different weights of studies depending on their inverse variance of each (Higgins 2011). We used the function 'rma.uni()' in the package 'metafor', for The R Project for Statistical Computing program.

Originally we had decided to use change data and baseline data in the same analysis, but after debate, we decided to present endpoint data and change data separately because we considered it problematic and full of assumptions that we were not comfortably taking.

One of the included trials (C +aripiprazole 2015b), was found by methods not reported in the protocol; this trial was found in an Internet search while working on one of the included studies (C +aripiprazole 2015).

We decided to establish an appendix 'Checklist to aid consistency and reproducibility of GRADE assessments' (Meader 2014) in order to aid with the standardisation of the 'Summary of findings' tables (Appendix 1).

\section{N DEX TERMS}

\section{Medical Subject Headings (MeSH)}

Antipsychotic Agents [*therapeutic use]; Clozapine [therapeutic use]; Drug Therapy, Combination; Patient Dropouts [statistics \& numerical data]; Quality of Life; Randomized Controlled Trials as Topic; Recurrence; Schizophrenia [ ${ }^{\star}$ drug therapy]; Treatment Outcome

\section{MeSH check words}

Humans 